SLAC-R-951

June 2010

Papers from U.S. Department of Energy

Science Undergraduate Laboratory Internship Program (SULI 2009)

SLAC National Accelerator Laboratory, Stanford University, Menlo Park, CA 94025

Work supported by Department of Energy contract DE-AC02-76SF00515. 


\title{
Optimization of the LCLS Single Pulse Shutter
}

\author{
Solomon Adera \\ Office of Science, Science Undergraduate Laboratory Internship (SULI) Program \\ Georgia Institute of Technology, Atlanta \\ Stanford Linear Accelerator Center (SLAC) \\ Menlo Park, California
}

14 August, 2009

Prepared in partial fulfillment of the requirements of the Office of Science, U.S. Department of Energy's SULI under the direction of Dr. David Fritz at the Linac Coherent Light Source (LCLS) project.

Participant:

\section{Signature}

Research Advisor:

Signature 


\section{Table of Contents}

$\begin{array}{ll}\text { Abstract } & 2\end{array}$

Introduction $\quad 3$

$\begin{array}{lc}\text { Methods and Results } & 6\end{array}$

Discussion and Conclusions $\quad 9$

$\begin{array}{ll}\text { Acknowledgement } & 10\end{array}$

$\begin{array}{ll}\text { Reference } & 11\end{array}$

Tables and Figures 12 


\begin{abstract}
A mechanical shutter which operates on demand is used to isolate a single pulse from a $120 \mathrm{~Hz}$ X-ray source. This is accomplished with a mechanical shutter which is triggered on demand with frequencies ranging from 0 to $10 \mathrm{~Hz}$. The single pulse shutter is an iron blade that oscillates on a pivot in response to a force generated by a pair of pulsed electromagnets (current driven teeter-totter). To isolate an individual pulse from the X-ray beam, the motion of the mechanical shutter should be synchronized in such a way that it allows a single pulse to pass through the aperture and blocks the other incoming pulses. Two consecutive pulses are only $\sim 8 \mathrm{~ms}$ apart and the shutter is required to complete one full cycle such that no two pulses pass through the opening. Also the opening of the shutter blade needs to be at least $4 \mathrm{~mm}$ so that a $1 \mathrm{~mm}$ diameter rms Gaussian beam can pass through without modulation. However, the $4 \mathrm{~mm}$ opening is difficult to obtain due to blade rebound and oscillation of the blade after colliding with the electromagnet. The purpose of this project is to minimize and/or totally eliminate the rebound of the shutter blade in pursuit of maximizing the aperture while keeping the open window interval $<\sim 12 \mathrm{~ms}$.
\end{abstract}




\section{INTRODUCTION}

The current single pulse shutter design is an iron blade (ferromagnetic material) onto which a shaft with end bearings is force fitted. The flat blade rocks about the shaft in response to the periodically exerted force by a pair of pulsed electromagnets. Depending on the amount of current flowing through the electromagnets, they magnetize and demagnetize alternately which induces an oscillatory motion of the shutter blade (see Fig. $1)$.

One face of the blade is exposed to the X-ray beam. This face of the blade is coated with a material which not only absorbs the irradiation coming from the X-ray source but also resists the high peak power from the X-ray beam. To attain this effect the blade is bonded with silicon nitride $\mathrm{Si}_{3} \mathrm{~N}_{4}$ strip.

\section{Definition of terms:}

Exposure time $\mathrm{t}_{0}$ : is the time the shutter blade stays open for the FWHM (Full Width at Half Maximum) X-ray beam to pass through. It depends on beam size, beam position, and repetition rate. For this experiment, $\mathrm{t}_{0}$ is defined at the position where the motion of the blade is steeper (see Fig. 2).

Opening delay $\mathrm{t}_{\mathrm{d}}$ : is the time elapsed before the shutter blade responds to an input pulse. It depends on the responsiveness of the shutter blade in addition to the device used to generate the pulse (see Fig. 2).

$\underline{\text { Static aperture sa: }}$ is the difference between maximum and minimum vertical positions of the shutter blade when the pulse picker is not operating (see Fig. 3). It can easily be calculated from the displacement vs. time plot of the shutter by locating the maximum and minimum points on the graph. It can also be measured by taking readings at the 
respective positions using a laser detector. The laser detector emits a beam (see Fig. 3) and displays the distance between the laser source and the obstacle (in this case the shutter blade). The readings are taken when the shutter blade is fully closed and when it's fully open from the display. The difference between these two readings gives the static aperture sa,

$$
s a=Z_{\max , s}-z_{\min , s},
$$

where $\mathrm{z}_{\max , \mathrm{s}}$ and $\mathrm{z}_{\min , \mathrm{s}}$ are the readings when the shutter is fully closed and fully open respectively while the shutter is not operating (see Fig. 4).

Even if the shutter blade is fully open, the laser beam detects an obstacle because the laser source is positioned vertically above it as can be seen from Fig. 3. The laser source is set up to measure the vertical position of the blade. However, in the actual set up when the pulse picker starts operating in the LCLS, in which case the laser beam is positioned horizontally, the X-ray beam will pass through without any obstacle when the shutter blade is fully open.

Dynamic aperture da: is defined in the same way as static aperture except that the pulse picker is operating when the maximum $\left(\mathrm{z}_{\max , \mathrm{d}}\right)$ and minimum $\left(\mathrm{z}_{\min , \mathrm{d}}\right)$ points are located. It's given by the formula,

$$
d a=Z_{\text {max }, d}-Z_{\min , d},
$$

where $\mathrm{z}_{\max , \mathrm{d}}$ and $\mathrm{z}_{\text {min,d }}$ are located on the displacement vs. time plot by excluding the oscillation and rebound of the shutter blade as shown in Fig. $4\left(\mathrm{z}_{\text {max,d }}\right.$ is the trough of the upper oscillation and $\mathrm{z}_{\min , \mathrm{d}}$ is located at the position where exposure time is defined). It's less than the static aperture because of the oscillation and rebound. 
Gaussian beam: is a beam whose intensity distribution can be described by a Gaussian function (see Fig. 5).

When fully open, the shutter blade is required to have a $4 \mathrm{~mm}$ dynamic aperture to effectively transmit the beam without modulation of the wave front. The rms Gaussian beam size $\sigma(\mathrm{rms})$ that can be transmitted without significant modulation is given by the formula,

$$
\sigma(r m s)=\frac{d a}{4}
$$

Where da is the dynamic aperture. For a $4 \mathrm{~mm}$ dynamic aperture, $\sigma(\mathrm{rms})=1 \mathrm{~mm}$.

FWHM (Full Width at Half Maximum): also called Full Duration at Half Maximum is the width of the pulse for which the intensity of the beam is not less than half the maximum value (see Fig. 5). Mathematically, it's given by the formula,

$$
F W H M=2.36 * \sigma(r m s)
$$

For $1 \mathrm{~mm}$ rms Gaussian beam, FWHM $=2.36 \mathrm{~mm}$.

N.B. This calculation shows that a $4 \mathrm{~mm}$ dynamic aperture is required to obtain a $1 \mathrm{~mm}$ rms Gaussian beam or a $2.36 \mathrm{~mm}$ FWHM beam to pass through. However, in actuality the beam won't be as thick as $1 \mathrm{~mm}$. For a $50 \mu \mathrm{m}$ diameter X-ray beam, only $4 * .05=0.2$ $\mathrm{mm}$ aperture is required to avoid significant distortion.

Period $\tau$ : is the time between two consecutive pulses (see Fig. 6),

$$
\tau=\frac{1}{f},
$$

where $\tau$ is the period and $f$ is the frequency. The frequency of the source of the X-ray beam is $120 \mathrm{~Hz}$. The period is, therefore; 


$$
\tau=\frac{1}{120}=8.33^{*} 10^{-3} \mathrm{~s}=8.33 \mathrm{~ms}
$$

Two consecutive pulses are, therefore, $8.33 \mathrm{~ms}$ apart.

Note that not more than one pulse will pass through the aperture as long as the exposure time of the shutter blade is a little less than twice the period, that is $t_{0}<2 * 8.33$ $=16.66 \mathrm{~ms}$. Theoretically, the exposure time is in the order of $f \mathrm{~s}$, but to accommodate the uncertainties in timing, it's required to be around $\sim 12 \mathrm{~ms}$.

Inclination angle $\theta$ : is the angle the shutter blade makes with the horizontal (see Fig. 9).

$$
\theta=\tan ^{-1}\left(\frac{s a}{L-l}\right)
$$

where $\mathrm{L}$ is half of the length of the shutter blade and 1 is the distance of the beam from the tip of the shutter blade.

\section{METHODS AND RESULTS}

Although not as effective as anticipated, previously two approaches were employed to address the rebound problem of the shutter blade. The first approach was to damp the vibration created due to the collision between shutter blade and the electromagnet by using a torsion spring. This method resulted in a bigger aperture, but it introduced a new problem: delayed response and longer exposure time. The other problem encountered in implementing this method was the unavailability of enough room underneath the shutter blade to accommodate a torsion spring.

The other approach tried so far is to manufacture the blade from a material that does not rebound as much. Rebounding is not a material property and therefore, such solutions are not easy to come by as experiments should be done to see the response. This approach is still in progress and no result is found up until this report is written. 
This research project addresses the bouncing back problem from a different perspective: adjusting the electromagnet in such a way that the blade does not oscillate after colliding with the electromagnet. One of the electromagnets is fixed and so its position can not be altered. However, the other electromagnet can move vertically up and down in addition to tilting sideways from the vertical.

Tilting the electromagnet (bigger inclination angle) increases the aperture (see Fig 7). However, it decreases the exposure time significantly (see Fig 8). This is because, when the inclination angle increases, the shutter blade accelerates more and gains kinetic energy before colliding with the electromagnet. As a result it rebounds with high velocity as soon as it hits the electromagnet (see Fig. 21).This problem can be addressed by slowing down the return motion using a stronger electromagnet or a damper. A stronger electromagnet can exert enough force to slow down the rebound and a damper can absorb the impact energy and reduces the energy available for rebound.

During collision, full contact between the shutter blade and the electromagnet is required to guarantee maximum energy absorption. Full contact is achieved by tilting the electromagnet while moving it up and down vertically. Therefore, all experiments executed in this research are done in such a way that the shutter blade touches the electromagnet fully. Another reason to demand full contact between the two parts is to avoid damage which otherwise will result from single point contact.

\section{Experiments 1 through 6}

In pursuit of meeting the requirement of the LCLS ( $\mathrm{da} \sim 4 \mathrm{~mm}$ and $\mathrm{t}_{0} \sim 12 \mathrm{~ms}$ ) six experiments are executed at different positions of the electromagnet without changing the frequency $(f=1 \mathrm{~Hz})$ and the results are summarized in Table 1 . The data in Table 1 is 
plotted to show how the aperture (both static and dynamic) and response time depend on inclination angle (see Fig. 7 and 8). Static aperture increases with inclination angle. So does dynamic aperture.

In experiment $1, \mathrm{z}_{\max , \mathrm{s}}$ and $\mathrm{z}_{\mathrm{min}, \mathrm{s}}$ are respectively 1.76 and $-2.16 \mathrm{~mm}$ before setting the shutter blade in motion. Therefore,

$$
s a=Z_{\text {max }, s}-Z_{\text {min }, s}=[1.76-(-2.16)] *\left(\frac{25.05}{21.75}\right)=4.6 \mathrm{~mm} .
$$

The overall shutter blade is $50.1 \mathrm{~mm}$ long $(\mathrm{L}=25.05 \mathrm{~mm})$ and the laser beam is positioned $3.3 \mathrm{~mm}$ from the tip of the blade $(1=3.3 \mathrm{~mm}$ ). The inclination angle $\theta$ (see Fig. 9) is, therefore;

$$
\theta=\tan ^{-1}\left(\frac{3.92}{25.05-3.3}\right)=10.2^{\circ}
$$

The plot for the above set up is shown in Fig. 10 with the screen capture from the oscilloscope shown in Fig. 11. Data points are taken at $50 \mu$ interval. Reading points from the plot (see Fig. 10);

$$
\text { Dynamic aperture: } \mathrm{da}=[1.3-(-1.5)] * 25.05 / 21.75=3.3 \mathrm{~mm}
$$

Exposure time: $\mathrm{t}_{0}=755.6-749.2=6.4 \mathrm{~ms}$

The experimental results obtained from the other experiments (experiments 2 through 6) and similar calculations of da and $t_{0}$ are shown in the Tables and Figures along with the corresponding plot and screen capture.

\section{Experiments 7 through 11}

Without changing the set up, experiment 6 is repeated for different frequencies of the shutter blade. The data obtained are summarized in Table 2 and the plots are shown in Fig. 12 and 13. As can be seen from the plots, frequency has an insignificant or no 
effect on both the exposure time and dynamic aperture. The individual plots are very similar to the plot obtained from experiment 6.

\section{DISCUSSION AND CONCLUSIONS}

The maximum inclination for which the shutter blade can oscillate is $12.7^{\circ}$. When the inclination exceeds $12.7^{\circ}$, the electromagnet does not exert the enough force to pull the shutter blade back and as a result the shutter blade sticks at one position. A $3.0 \mathrm{~mm}$ dynamic aperture is obtained for this set up. This dynamic aperture can be increased significantly (to about $5.0 \mathrm{~mm}$ ) if the motion of the blade from point ' $b$ ' to point ' $c$ ' (see Fig. 21) takes longer time. Therefore, this research project boils down to ways of increasing this exposure time without sacrificing the dynamic aperture.

As can be seen from Fig. 25, the shutter starts to open after the input voltage has decreased to 12 from $150 \mathrm{~V}$. This is because of the short life (about $4 \mathrm{~ms}$ ) of the input voltage. If the duration of this input voltage is longer (about $6 \mathrm{~ms}$ ) and the $12 \mathrm{~V}$ lasts shorter (about $2 \mathrm{~ms}$ ), the blade moves faster in response to the magnetic force of attraction. This will minimize the time delay $\mathrm{t}_{\mathrm{d}}$ and increases the exposure time $\mathrm{t}_{0}$ meeting the LCLS's requirement of sharper response (see ideal response in Fig. 16). However, the duration of the input volt can not be adjusted manually, and therefore the vendor should be contacted about modifying the product to LCLS's need.

Another way of increasing the exposure time is by decreasing the delay time which requires the use of a stronger electromagnet. When the force exerted by the electromagnet is bigger, the displacement vs. time plot looks like Fig. 14 (a). For smaller force of attraction, the response looks like Fig 14 (b). Therefore, stronger electromagnet guarantee that the motion (referring to Fig. 14) (i) from point ' $a$ ' to point ' $b$ ' is faster and 
steeper, (ii) from point ' $b$ ' to point ' $c$ ' takes longer time because of a combined effect of reduced delay time and a bigger force which can resist immediate rebound, and (c) from point ' $\mathrm{c}$ ' to point ' $\mathrm{d}$ ' is faster and steeper. Moreover, employing stronger electromagnets reduce the oscillation. This method of solving the problem requires replacing the electromagnet by a bigger one in addition to adding a spacer/washer between the top cover of the pulse picker and the housing to support the bigger aperture. Design modification may not be required if the physical size of the new electromagnet matches with the existing one.

The oscillation of the blade can also be eliminated by employing a damping element (compression spring or a hydraulic damper) between the plate on which the electromagnet rests and the bottom housing of the pulse picker. This method requires design modifications to make a room to accommodate the damping element.

Both exposure time and dynamic aperture remain almost constant when the frequency is varied from 0 to $10 \mathrm{~Hz}$. Therefore, frequency has none or minimal effect on the two parameters $\mathrm{t}_{0}$ and da.

\section{ACKNOWLEDGEMENT}

First and foremost I would like to thank God for he made this possible. This work was supported by the Department of Energy at the Stanford Linear Accelerator Center. I would like to thank the DoE, Office of Science and SLAC for giving me the opportunity to partake in the SULI program. My special thanks go to my mentor David Fritz for his time and mentorship. I would also like to thank Eliazar Ortiz and Marc Campell for their help, and Stephen Rock for his follow up and concern. 


\section{REFERENCE}

[1] Guenther, Robert. Modern Optics. Canada. New Jersey: John Wiley \& Sons, Inc., 1990. pp. $569-590$.

[2] Kuo, and Farid Golnaraghi. Automatic Control Systems. New Jersey: John Wiley \& Sons, Inc., 2003.

[3] Nise, Norman. Control Systems Engineering. New York: John Wiley \& Sons, Inc., 2000. 


\section{TABLES AND FIGURES}

Table 1: Effect of Inclination

\begin{tabular}{|c|c|c|c|}
\hline \multirow{2}{*}{$\begin{array}{l}\text { Inclination } \\
\text { (deg) }\end{array}$} & \multicolumn{2}{|c|}{ Aperture (mm) } & \multirow{2}{*}{$\begin{array}{l}\text { Exposure } \\
\text { Time (ms) }\end{array}$} \\
\hline & Static & Dynamic & \\
\hline 6.7 & 3.1 & 1.5 & 7.3 \\
\hline 8.4 & 3.8 & 2.4 & 6.7 \\
\hline 10.2 & 4.6 & 3.3 & 6.4 \\
\hline 11.3 & 5.1 & 2.4 & 5.8 \\
\hline 11.9 & 5.5 & 3.0 & 3.8 \\
\hline 12.7 & 5.8 & 3.0 & 3.8 \\
\hline
\end{tabular}

Table 2: Effect of Frequency

\begin{tabular}{|c|c|c|}
\hline $\begin{array}{c}\text { Shutter Frequency } \\
(\mathrm{Hz})\end{array}$ & $\begin{array}{c}\text { Exposure Time } \\
(\mathrm{ms})\end{array}$ & $\begin{array}{c}\text { Dynamic Aperture } \\
(\mathrm{mm})\end{array}$ \\
\hline 1 & 3.8 & 3.0 \\
\hline 3 & 4.0 & 2.7 \\
\hline 5 & 4.0 & 2.7 \\
\hline 7 & 4.0 & 2.9 \\
\hline 9 & 4.0 & 2.9 \\
\hline 10 & 4.0 & 2.6 \\
\hline
\end{tabular}
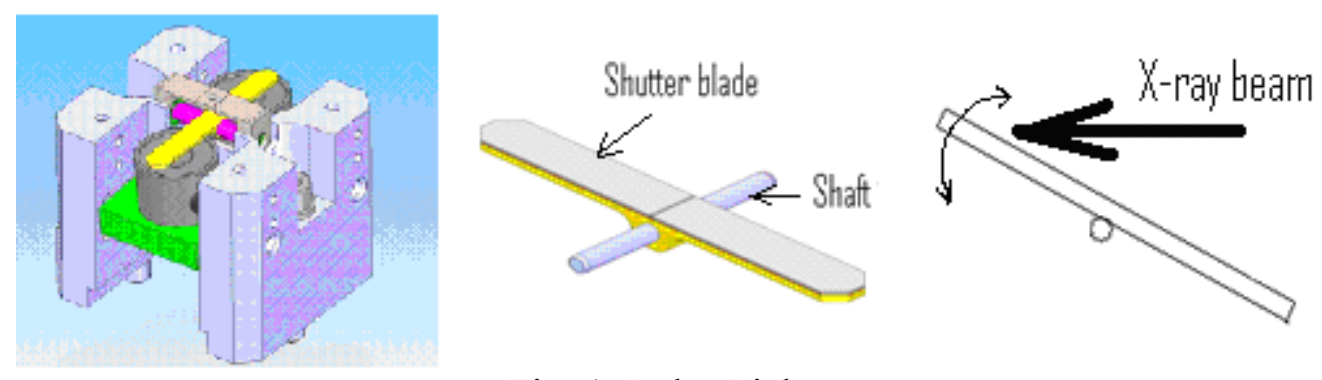

Fig. 1: Pulse Picker

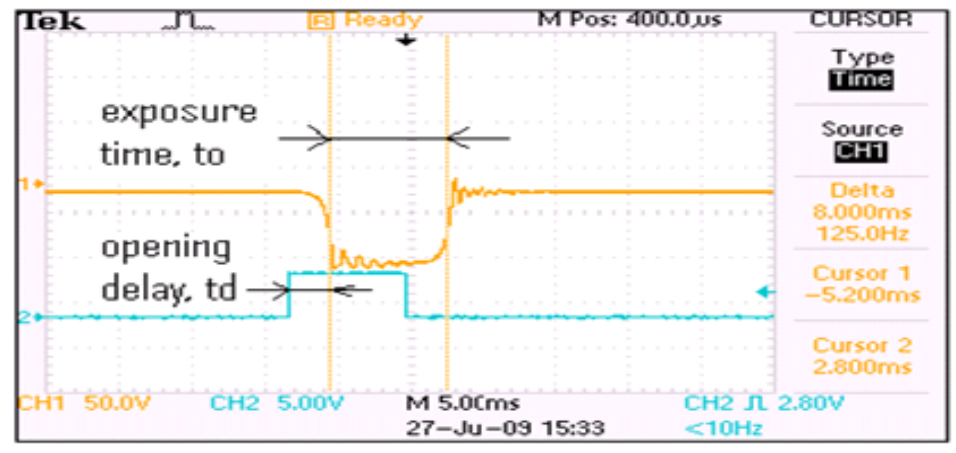

Fig. 2: Response to Input Pulse 


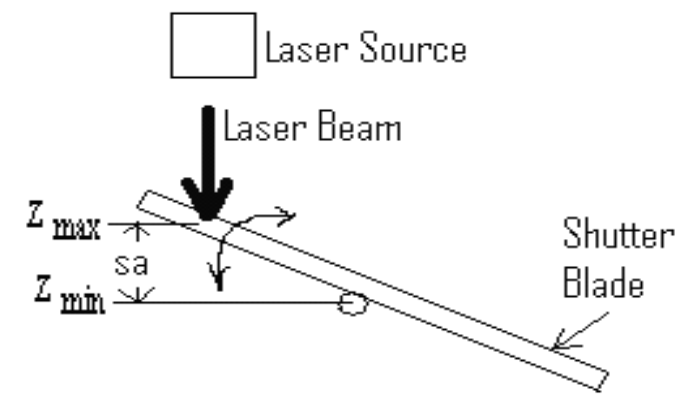

Fig. 3: Vertical Laser Beam

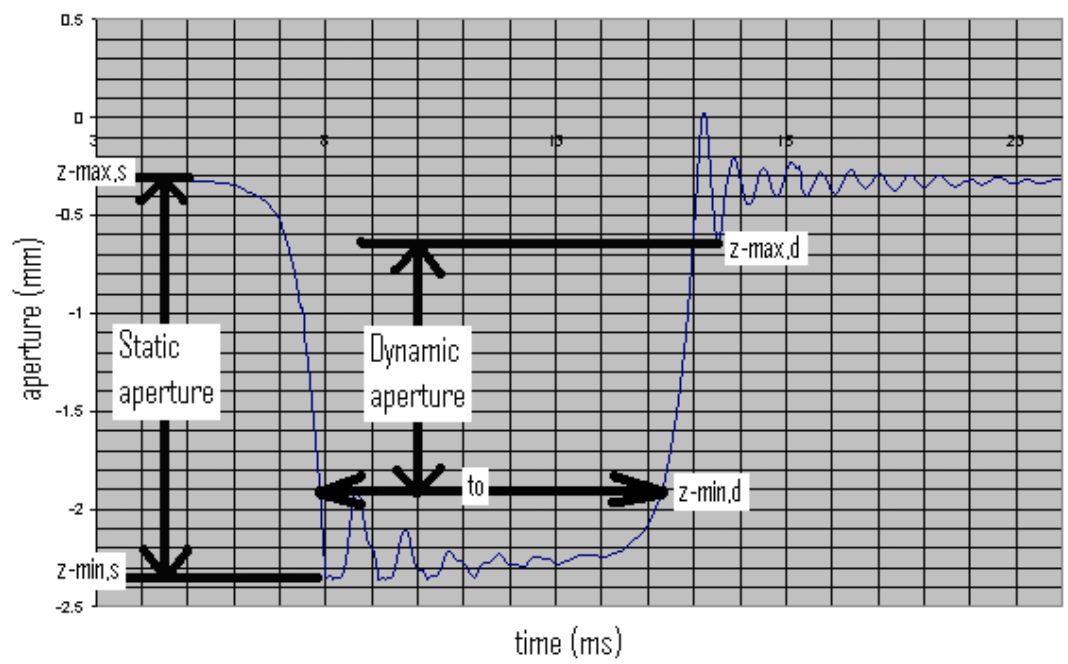

Fig. 4: Static and Dynamic Aperture

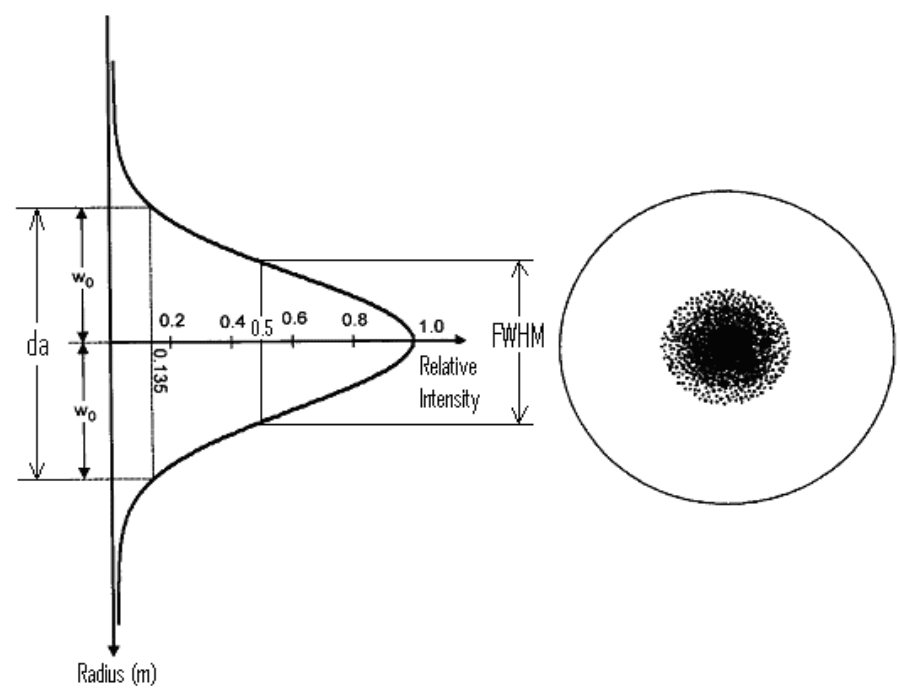

Fig. 5: Gaussian Beam 


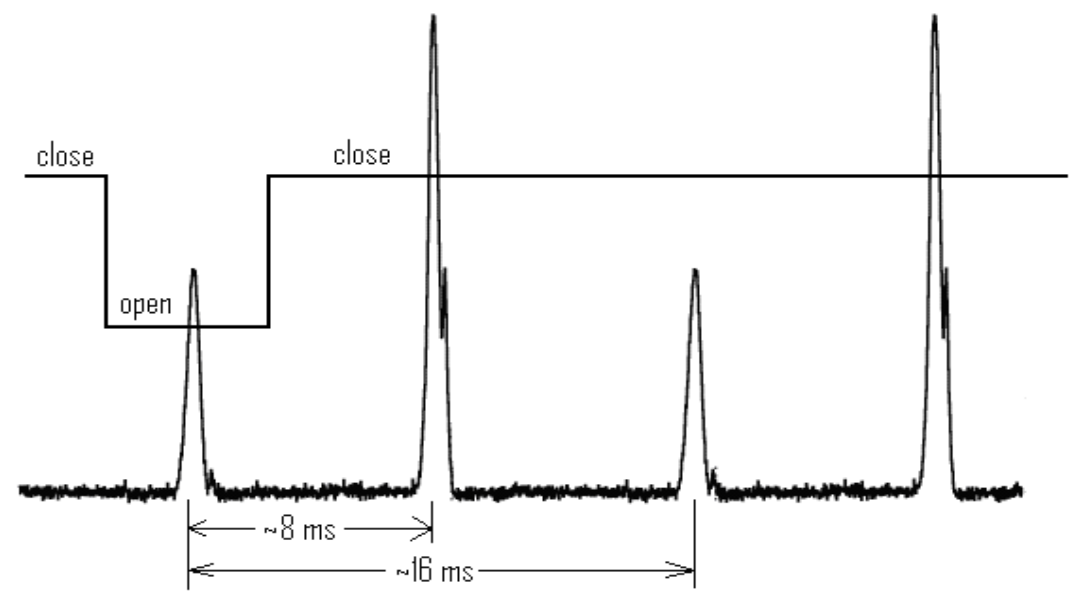

Fig. 6: X-ray Beam Profile

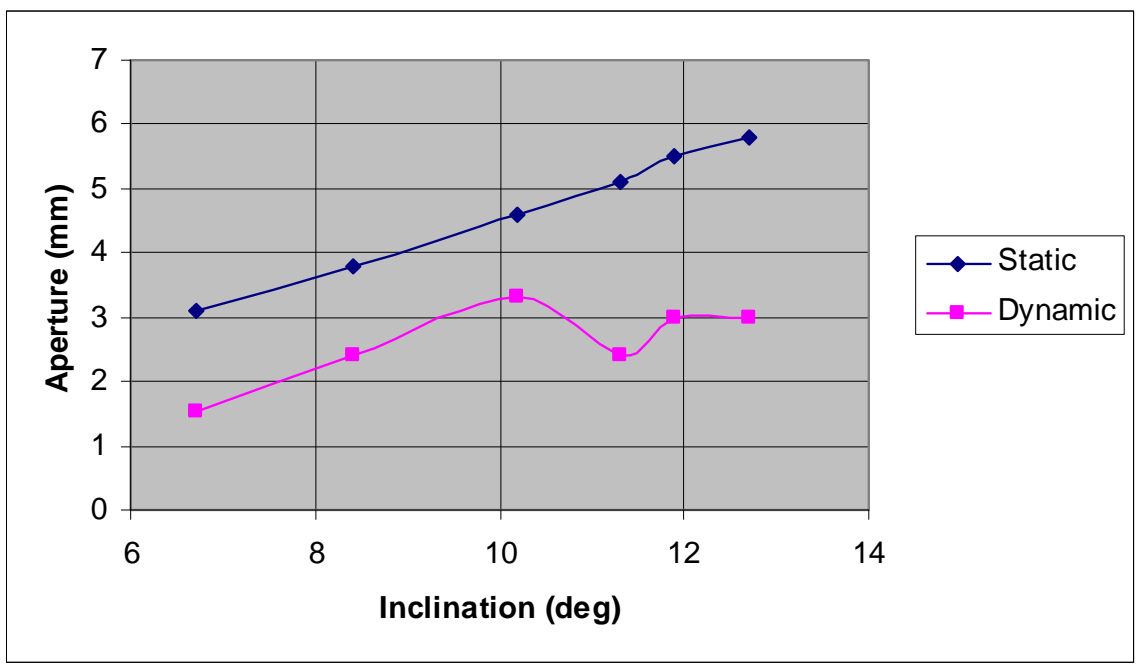

Fig. 7: Aperture vs. Inclination

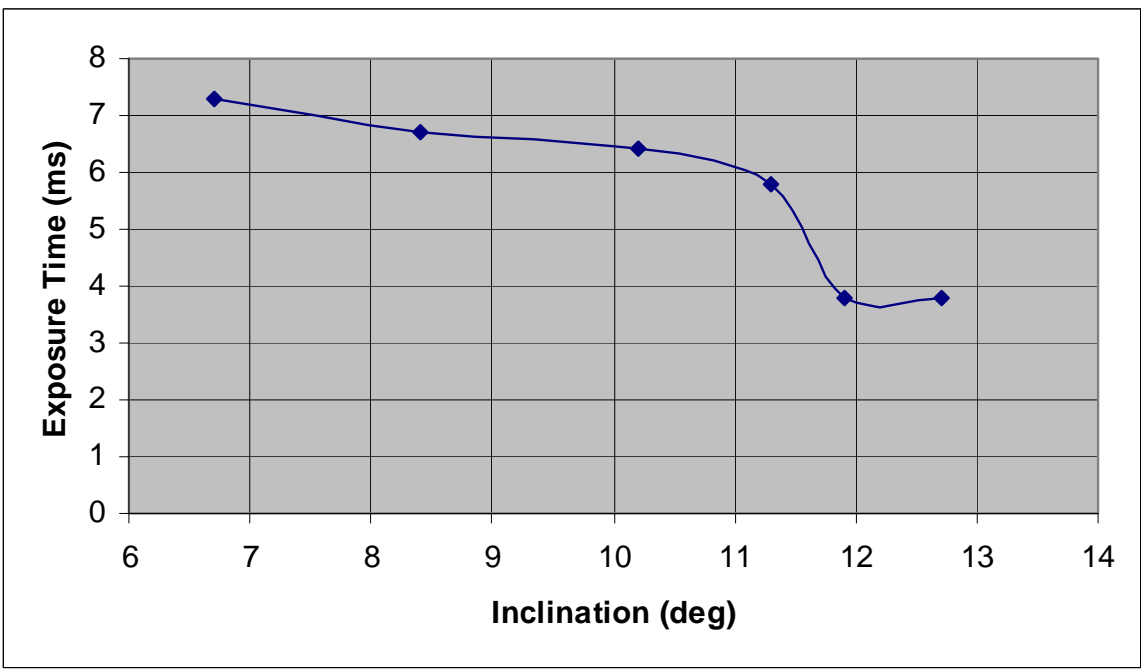

Fig. 8: Exposure Time vs. Inclination 


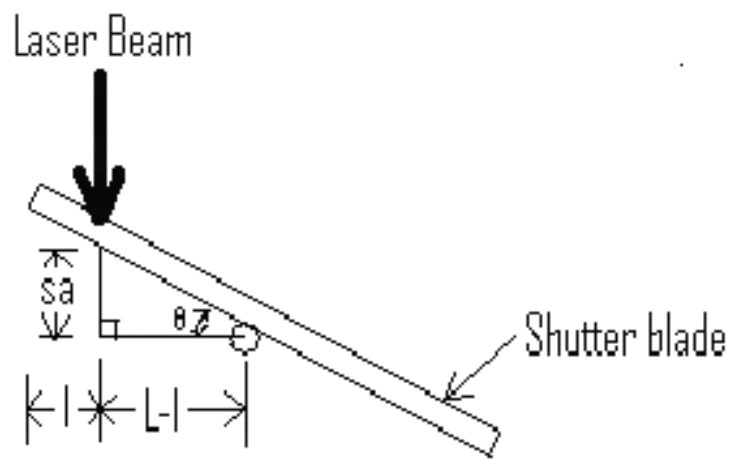

Fig. 9: Quantifying Inclination

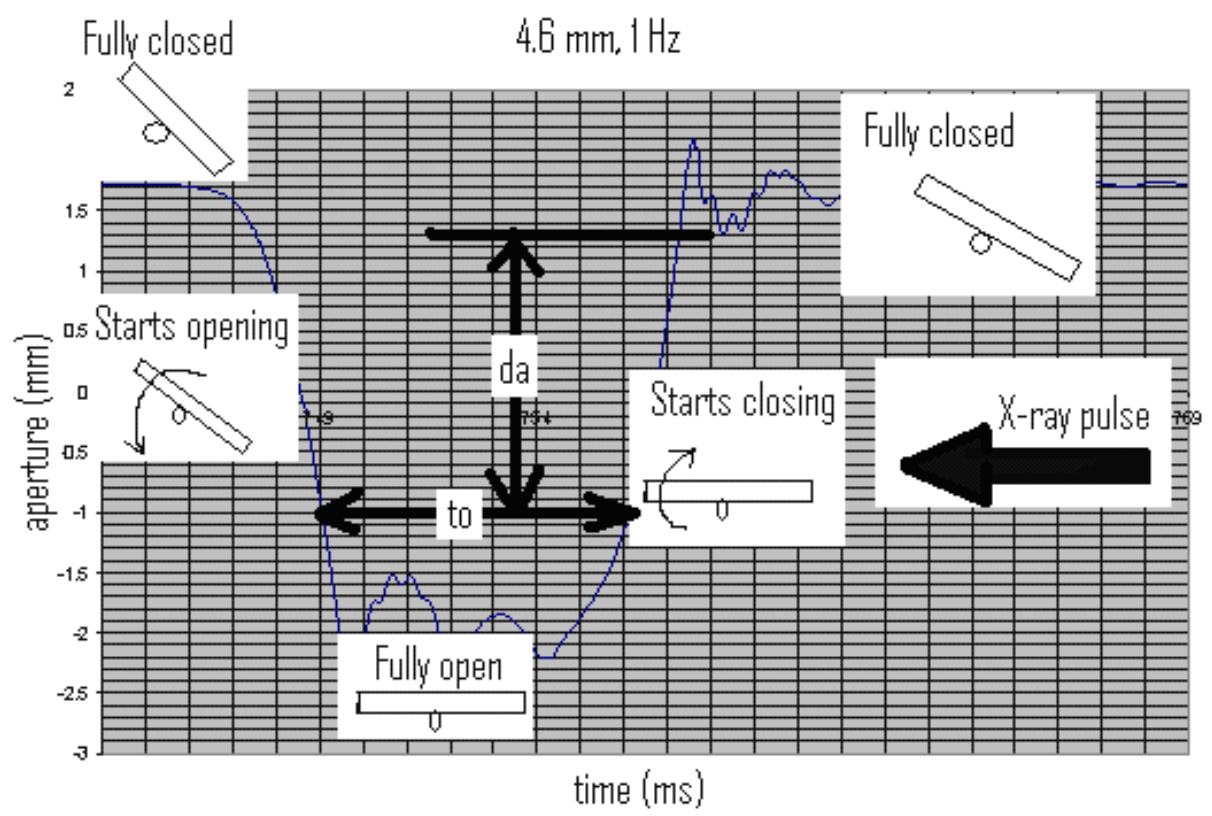

Fig. 10: Aperture vs. Time (experiment 1, $50 \mu \mathrm{m} / \mathrm{s}$ )

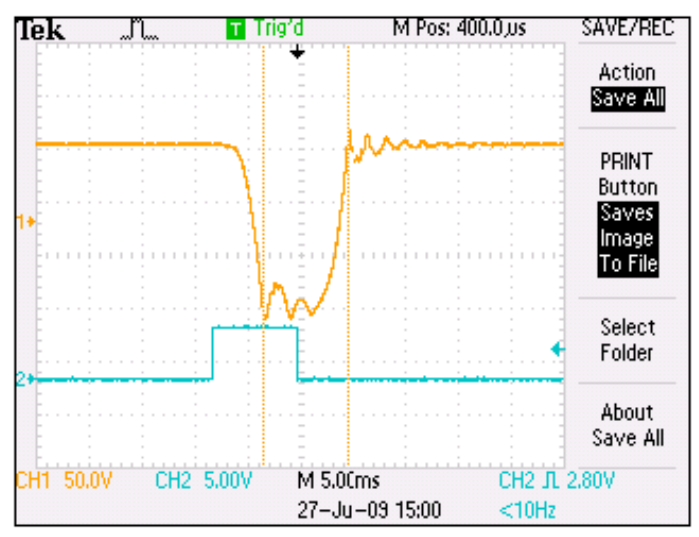

Fig. 11: Oscilloscope Screen Capture (experiment 1) 


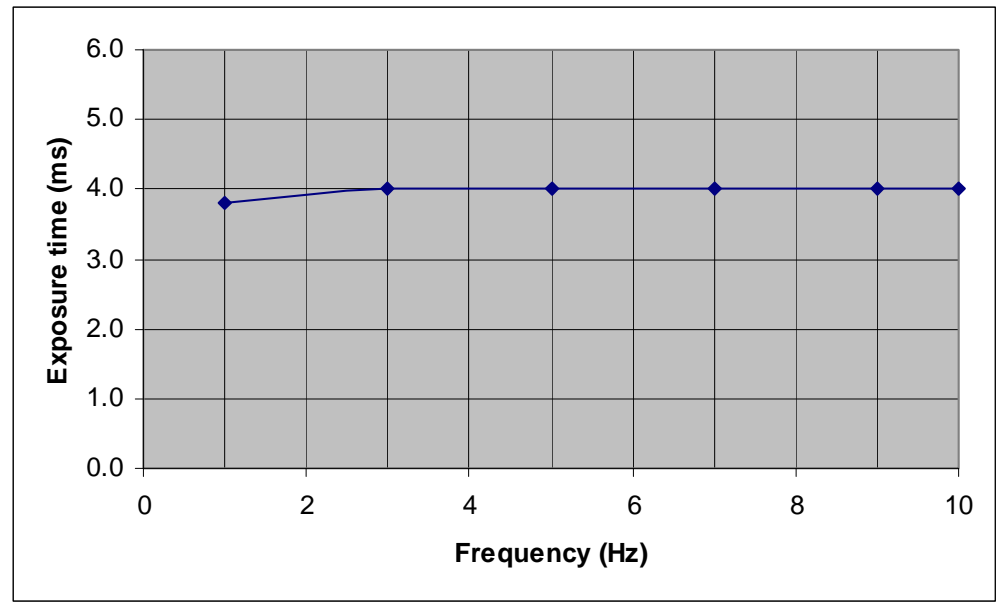

Fig 12: Exposure Time vs. Frequency

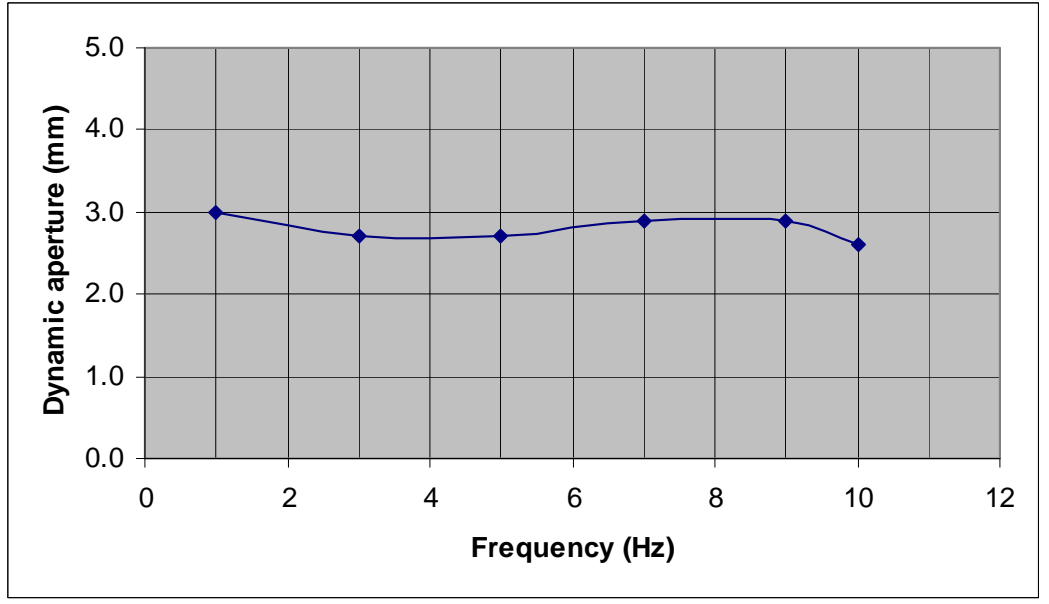

Fig 13: Dynamic Aperture vs. Frequency

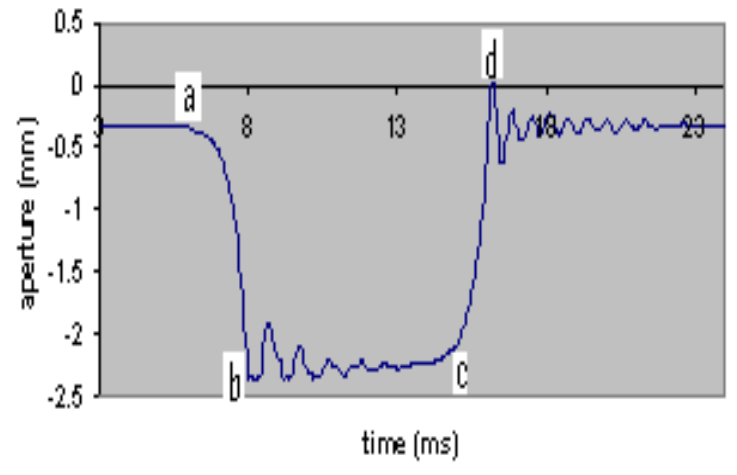

(a)

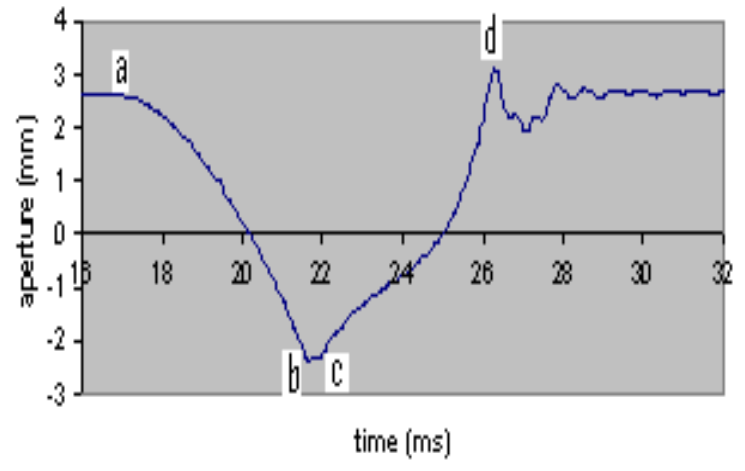

(b)

Fig 14: (a) Experiment 2 (2.57 mm, $1 \mathrm{~Hz})$ (b) Experiment $5(4.90 \mathrm{~mm}, 1 \mathrm{~Hz})$ 


\section{Experiment 2:}

$$
\mathrm{sa}=[-0.33-(-2.24)] * 25.05 / 21.75=3.1 \mathrm{~mm}
$$

$$
\theta=\tan ^{-1}\left(\frac{2.57}{25.05-3.3}\right)=6.7^{\circ}
$$

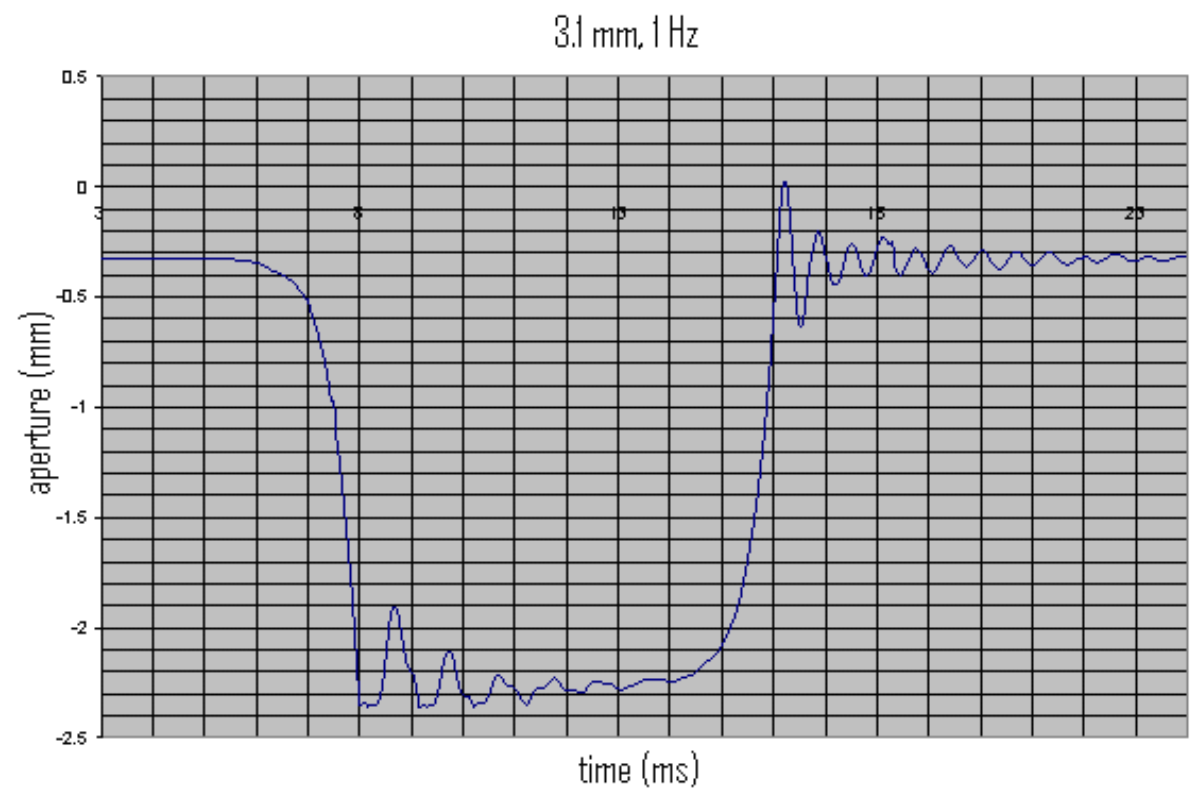

Fig. 15: Aperture vs. Time

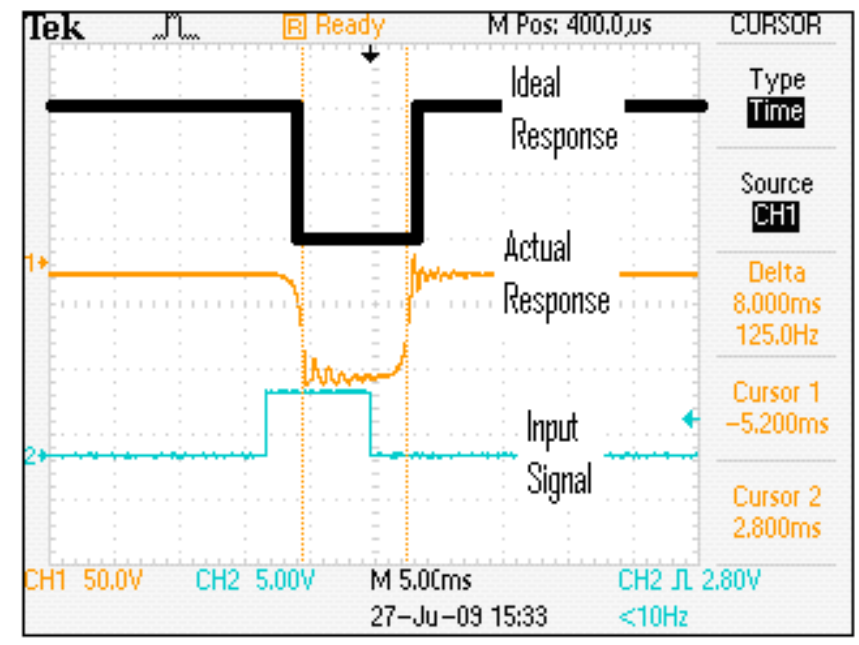

Fig. 16: Oscilloscope Screen Capture and Ideal Response

$$
\begin{aligned}
& \mathrm{da}=[-0.65-(-1.9)] * 25.05 / 21.75=1.5 \mathrm{~mm} \\
& \mathrm{t}_{0}=15.2-7.9=7.3 \mathrm{~ms}
\end{aligned}
$$




\section{Experiment 3:}

$$
\mathrm{sa}=[0.98-(-2.24)] * 25.05 / 21.75=3.8 \mathrm{~mm}
$$

$$
\theta=\tan ^{-1}\left(\frac{3.22}{25.05-3.3}\right)=8.4^{\circ}
$$

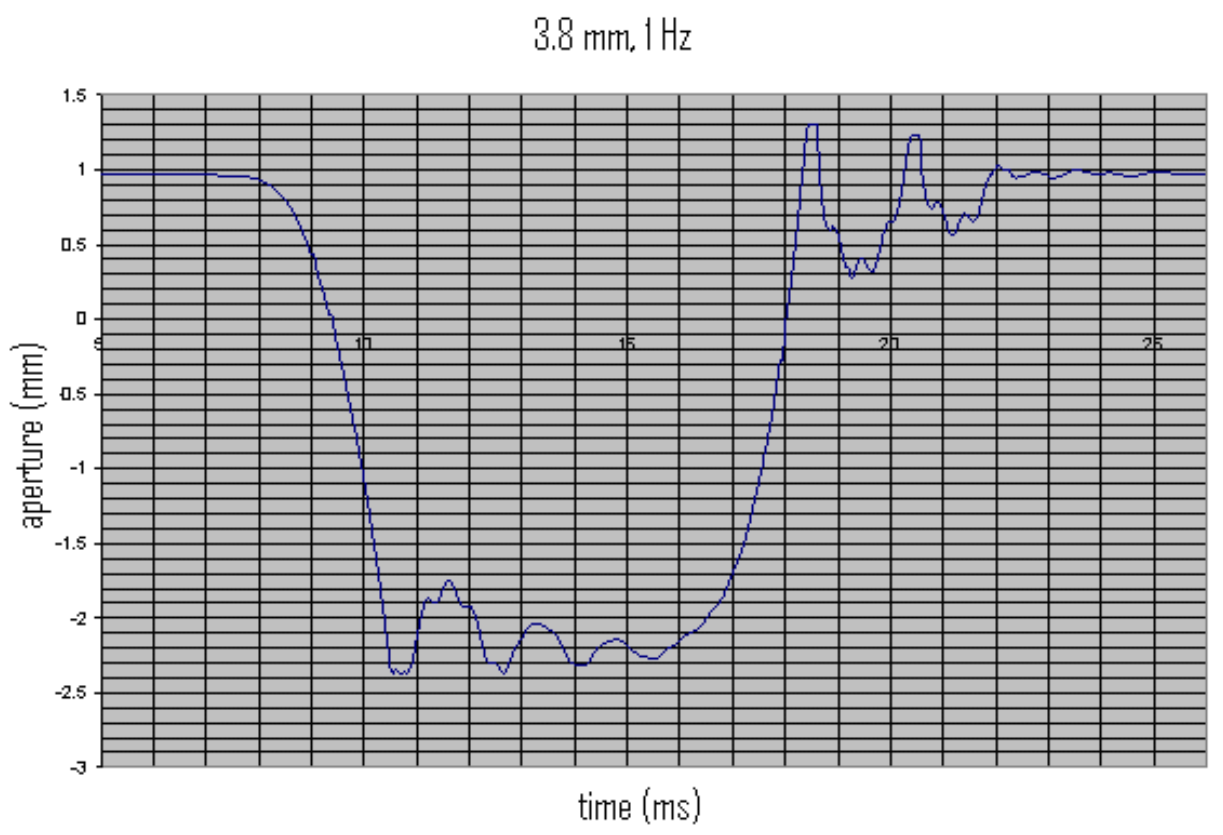

Fig. 17: Aperture vs. Time

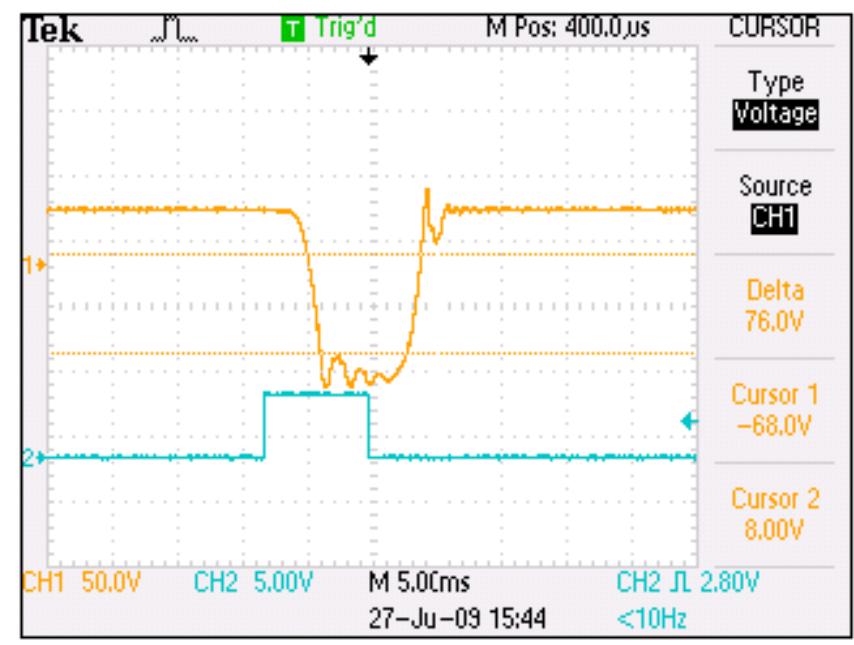

Fig. 18: Oscilloscope Screen Capture

$\mathrm{da}=[0.25-(-1.75)] * 25.05 / 21.75=2.4 \mathrm{~mm}$

$\mathrm{t}_{0}=16.9-10.2=6.7 \mathrm{~ms}$ 


\section{Experiment 4:}

$$
\begin{gathered}
\mathrm{sa}=[2.09-(-2.24)] * 25.05 / 21.75=5.1 \mathrm{~mm} \\
\theta=\tan ^{-1}\left(\frac{4.33}{25.05-3.3}\right)=11.3^{\circ} \\
5.1 \mathrm{~mm}, 1 \mathrm{~Hz}
\end{gathered}
$$

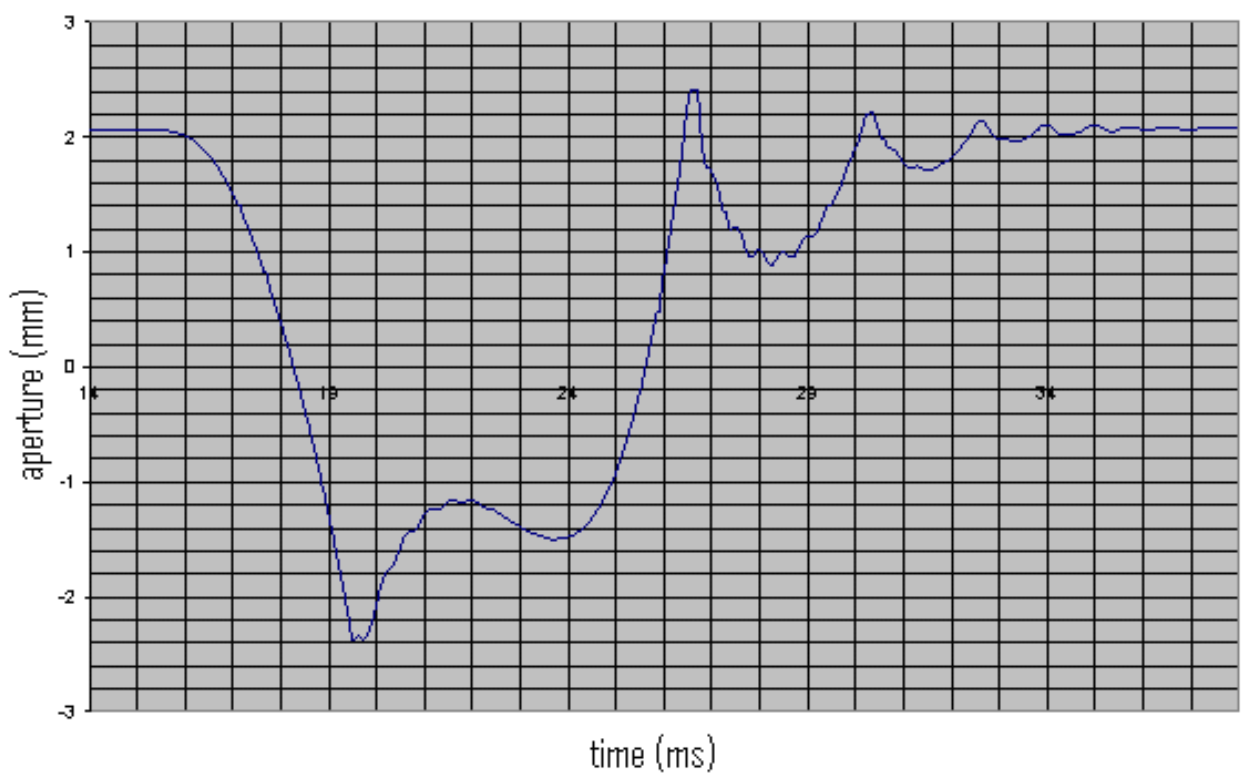

Fig. 19: Aperture vs. Time

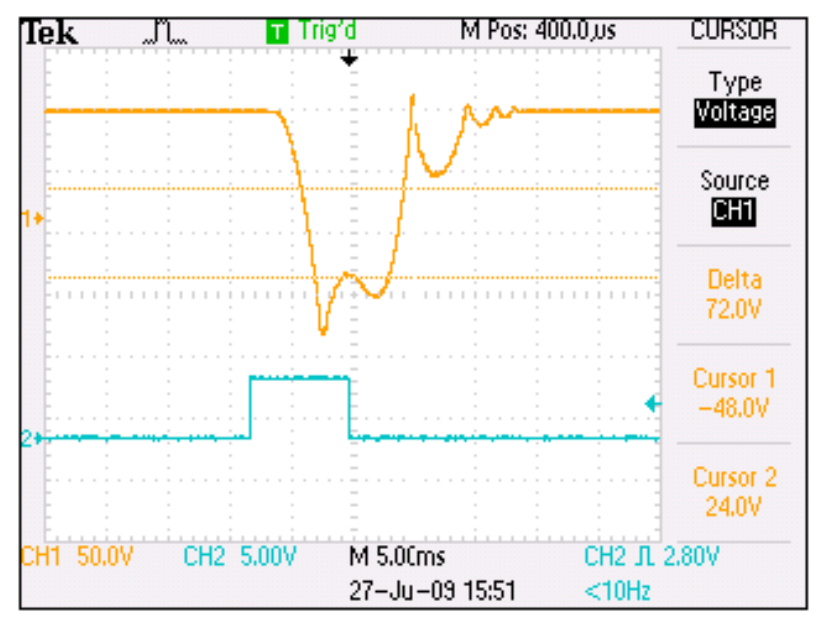

Fig. 20: Oscilloscope Screen Capture

$$
\begin{aligned}
\mathrm{da} & =[0.90-(-1.10)] * 25.05 / 21.75=2.4 \mathrm{~mm} \\
\mathrm{t}_{0} & =24.7-18.9=5.8 \mathrm{~ms}
\end{aligned}
$$




\section{Experiment 5:}

$$
\mathrm{sa}=[2.65-(-2.25)] * 25.05 / 21.75=5.8 \mathrm{~mm}
$$

$$
\theta=\tan ^{-1}\left(\frac{4.9}{25.05-3.3}\right)=12.7^{\circ}
$$

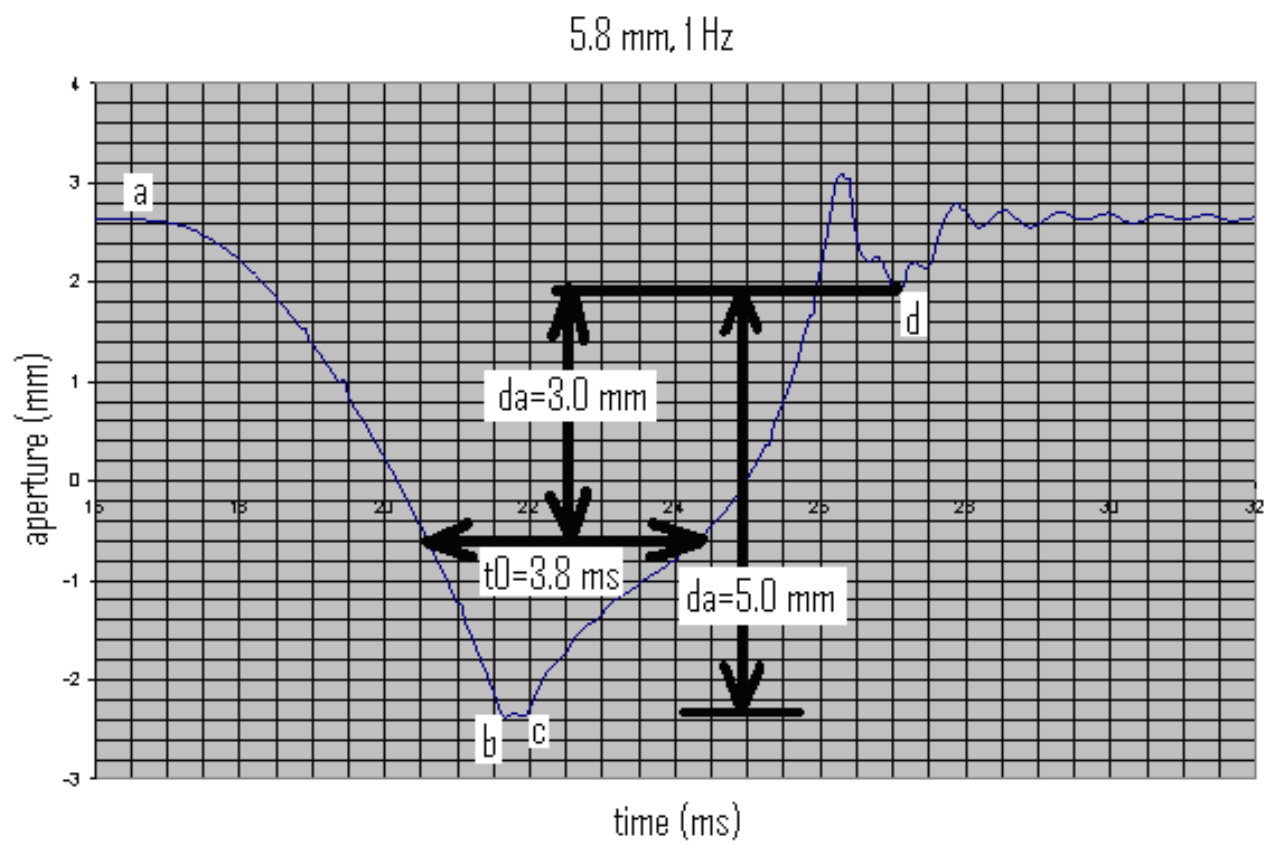

Fig. 21: Aperture vs. Time

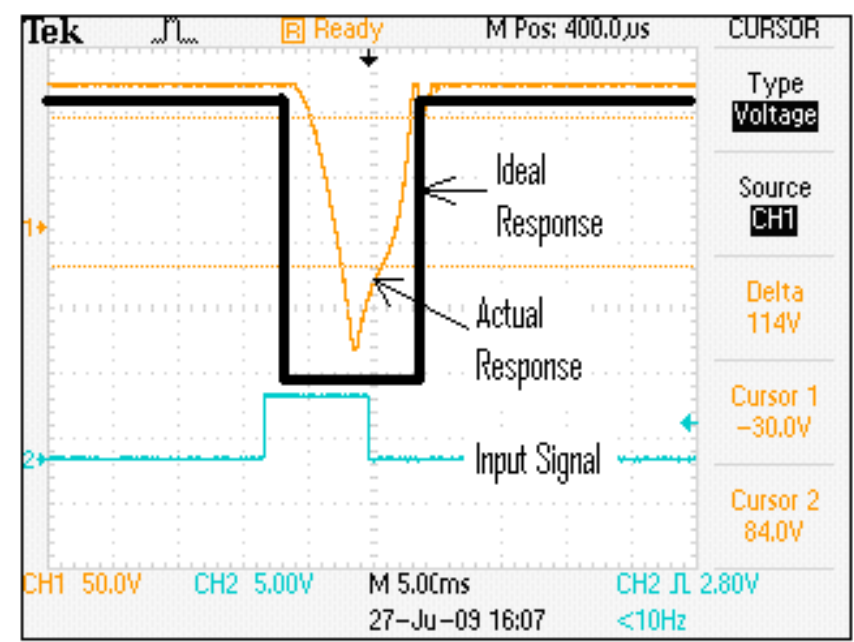

Fig. 22: Oscilloscope Screen Capture and Ideal Response

$\mathrm{da}=[1.90-(-0.6)] * 25.05 / 21.75=3.0 \mathrm{~mm}$

$\mathrm{t}_{0}=24.4-20.6=3.8 \mathrm{~ms}$ 


\section{Experiment 6:}

$$
\mathrm{sa}=[2.34-(-2.26)] * 25.05 / 21.75=5.5 \mathrm{~mm}
$$

$$
\theta=\tan ^{-1}\left(\frac{4.6}{25.05-3.3}\right)=11.9^{\circ}
$$

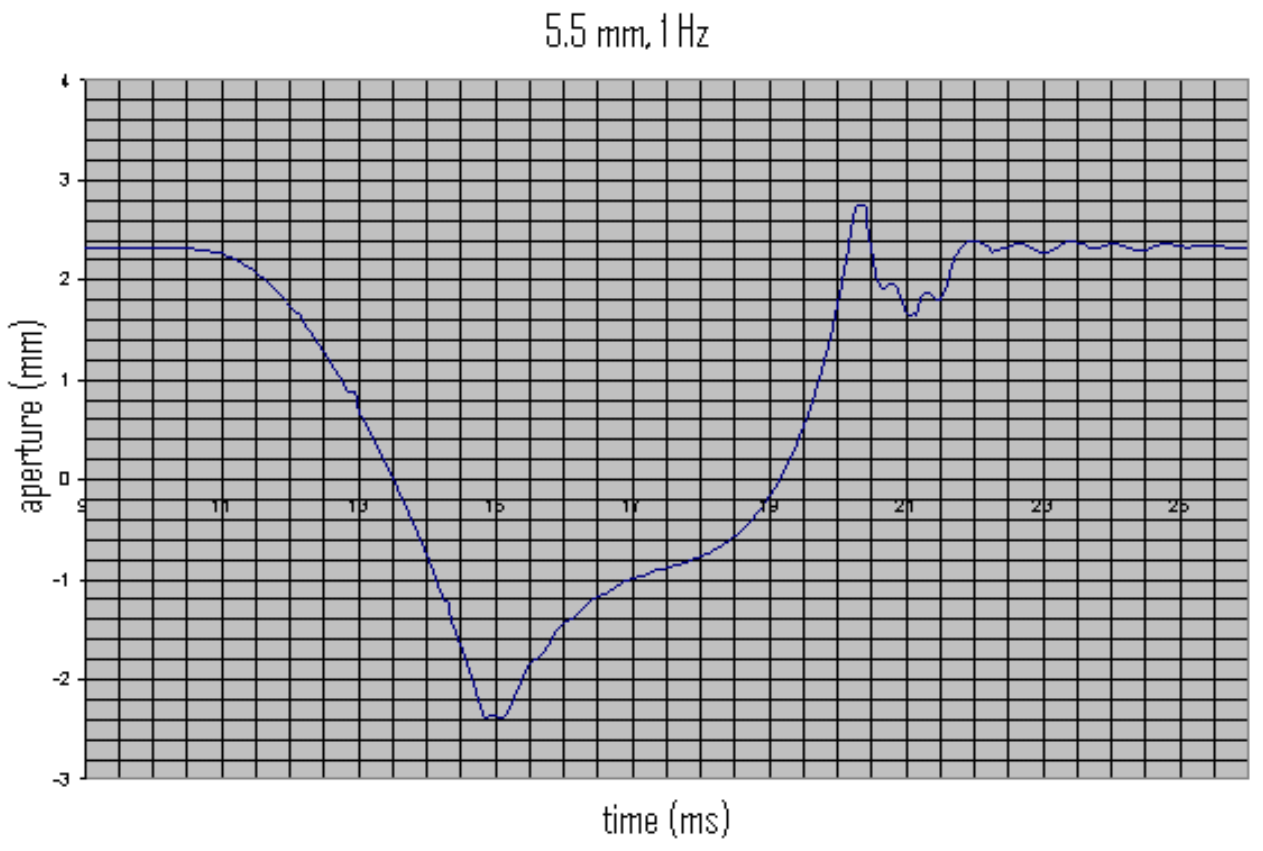

Fig. 23: Aperture vs. Time

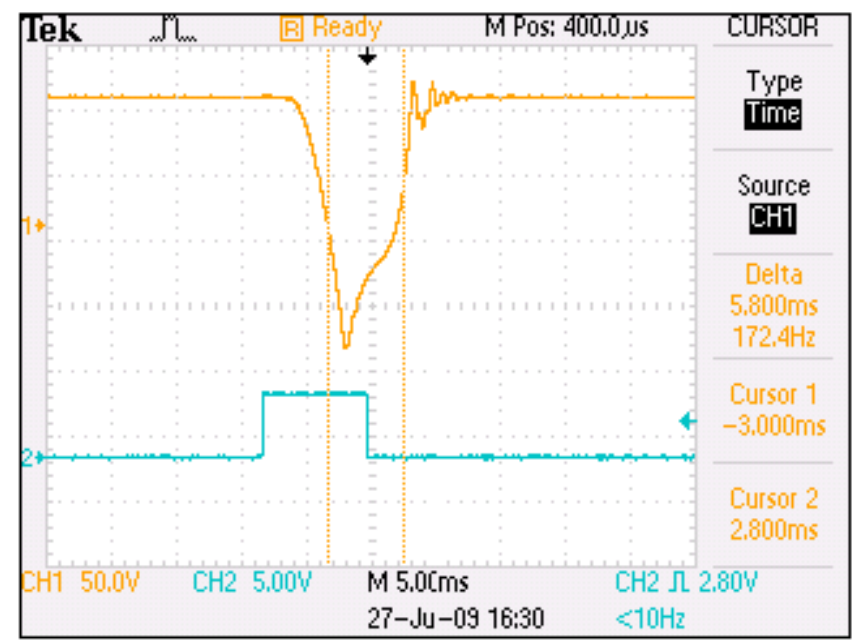

Fig. 24: Oscilloscope Screen Capture

$\mathrm{da}=[1.65-(-0.8)] * 25.05 / 21.75=3.0 \mathrm{~mm}$

$\mathrm{t}_{0}=18.75-13.75=3.8 \mathrm{~ms}$ 


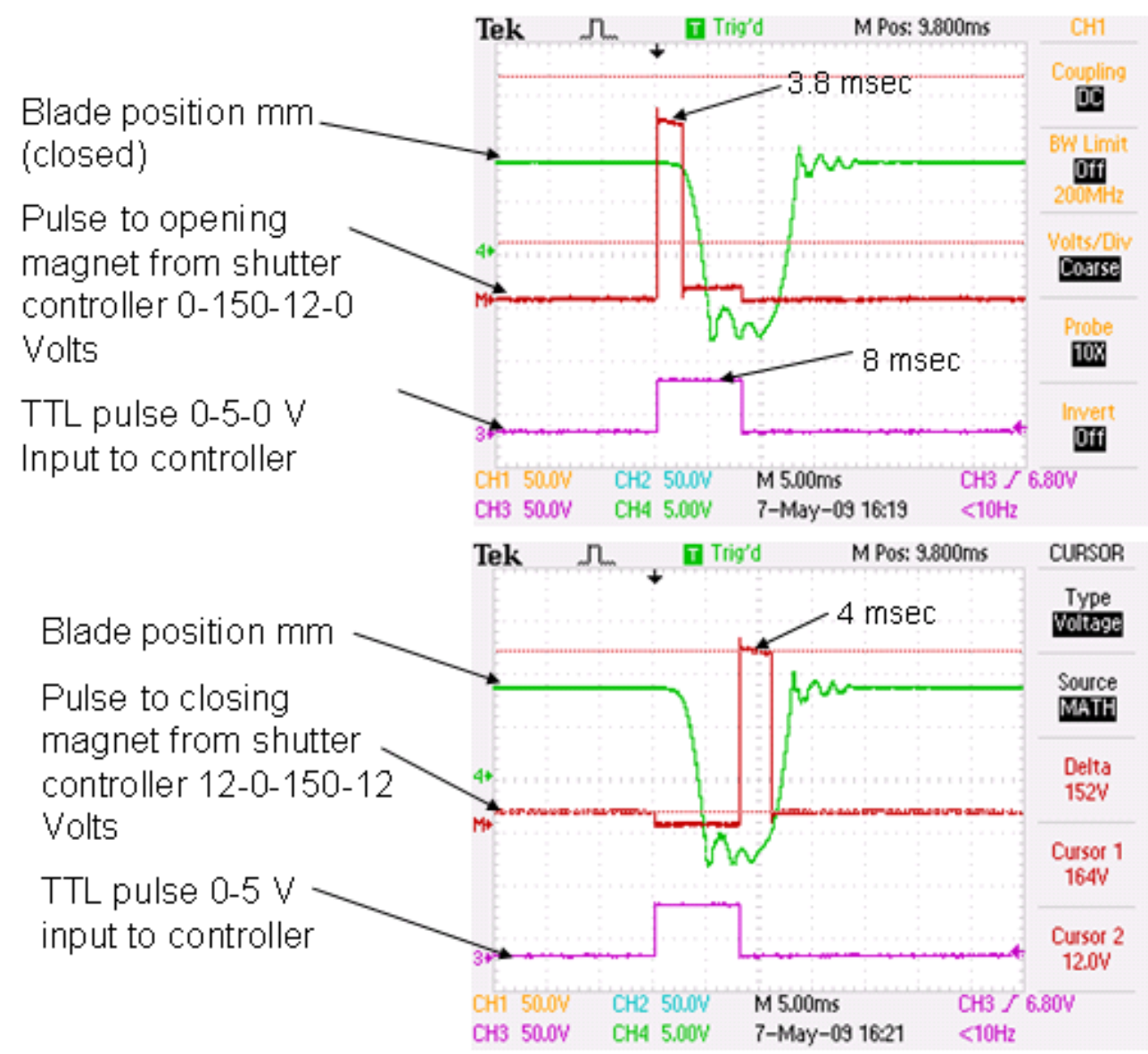

Fig. 25: Input Signal and Response Comparison 


\title{
Thermometric- and Acoustic-Based Beam Power Monitor for Ultra-Bright X-Rays
}

\author{
Gregory Bentsen \\ Office of Science, Science Undergraduate Laboratory Internship (SULI) \\ University of Rochester \\ SLAC National Accelerator Laboratory \\ Stanford, CA
}

August 14, 2009

Prepared in partial fulfillment of the requirements of the Office of Science, Department of Energy's Science Undergraduate Laboratory Internship under the direction of Joseph Frisch at the LCLS Diagnostics Group, SLAC National Accelerator Laboratory.

Participant:

Signature

Research Advisor:

Signature 


\section{TABLE OF CONTENTS}

$\begin{array}{ll}\text { Abstract } & \text { ii }\end{array}$

Introduction $\quad$ iii

Theory iv

Methods

Results

Discussion $\quad$ xiii

Conclusions $\quad$ XV

$\begin{array}{ll}\text { Acknowledgements } & \text { xvi }\end{array}$

References $\quad$ xix 


\begin{abstract}
Thermometric- and Acoustic-Based Beam Power Monitor for Ultra-Bright X-Rays. GREGORY BENTSEN (University of Rochester, Rochester, NY 14627)

JOSEPH FRISCH (LCLS Diagnostics Group, SLAC National Accelerator Laboratory, Stanford, CA 94025)

A design for an average beam power monitor for ultra-bright X-ray sources is proposed that makes simultaneous use of calorimetry and radiation acoustics. Radiation incident on a solid target will induce heating and ultrasonic vibrations, both of which may be measured to give a fairly precise value of the beam power. The monitor is intended for measuring ultrabright Free-Electron Laser (FEL) X-ray beams, for which traditional monitoring technologies such as photo-diodes or scintillators are unsuitable. The monitor consists of a Boron Carbide $\left(\mathrm{B}_{4} \mathrm{C}\right)$ target designed to absorb most of the incident beam's energy. Resistance temperature detectors (RTD) and piezoelectric actuators are mounted on the outward faces of the target to measure the temperature changes and ultrasonic vibrations induced by the incident beam. The design was tested using an optical pulsed beam (780 nm, 120 and $360 \mathrm{~Hz}$ ) from a Ti:sapphire oscillator at several energies between 0.8 and $2.6 \mathrm{~mJ}$. The RTDs measured an increase in temperature of about $10^{\circ} \mathrm{K}$ over a period of several minutes. The piezoelectric sensors recorded ringing acoustic oscillations at $580 \pm 40 \mathrm{kHz}$. Most importantly, the amplitude of the acoustic signals was observed to scale linearly with beam power up to $2 \mathrm{~mJ}$ of pulse energy. Above this pulse energy, the vibrational signals became nonlinear. Several causes for this nonlinearity are discussed, including amplifier saturation and piezoelectric saturation. Despite this nonlinearity, these measurements demonstrate the feasibility of such a beam power measurement device. The advantage of two distinct measurements (acoustic and thermometric) provides a useful method of calibration that is unavailable to current LCLS diagnostics tools.
\end{abstract}




\section{INTRODUCTION}

The Linac Coherent Light Source (LCLS) at the SLAC National Accelerator Laboratory is a new source of X-rays intended for probing molecular and atomic physics at very small length and time scales. Electrons accelerated and bunched by the existing linear accelerator are injected into undulators, which induce oscillations in the electron beam. The oscillating bunches radiate at a wavelength equal to the separation between bunches. The final X-rays have energies between $800 \mathrm{eV}$ and $9 \mathrm{keV}$. The pulse length of the beam is less than $10 \mathrm{fs}$, and is repeated at a rate of $10 \mathrm{~Hz}$. The beam diameter is about $100 \mu \mathrm{m}$. The energy per pulse is between 1 and $3 \mathrm{~mJ}$, resulting in a peak power of over $100 \mathrm{GW}$ and a peak brightness of over $10^{32}$ photons / $\mathrm{s} \mathrm{mm}^{2} \mathrm{mr}^{2}[10]$.

The exceptionally short pulse length and low emittance make the LCLS beam nearly ten billion times brighter than existing sources. As such, traditional devices and methods for measuring beam energy are not available for use in LCLS diagnostics. Photodiode circuits would, at best, be saturated by the high intensity of X-rays; at worst, they would be permanently damaged. Yttrium aluminum garnet (YAG) screens, which fluoresce when struck by incident X-ray or electron radiation, provide a possible alternative and are currently the primary diagnostic in use at LCLS [9] [14] [10]. Although intended to measure the beam's spatial profile and alignment, digital imaging processing may be applied to fluorescence images to obtain rough power measurements. Problems arise, however, when the beam energy increases above the screen's saturation point. In addition, calibration is tricky due to dependancies on the cameras used to capture the fluorescence, as well as degradation of the screen over time. Some studies have also shown inherent "blurriness" in YAG screen images that tends to enlarge the observed beam size, further reducing the viability of this technique as a precision beam power diagnostic [17].

The simplest alternative to these methods is calorimetry, a standard technique used in 
many detector applications. This technique is, in fact, already being used as one of the primary diagnostics tools for LCLS [8]. Calorimeters, however, suffer from complications of heat exchange and thermal noise, processes which are often difficult to either precisely quantify or eliminate. The proposed device aims to supplement the existing calorimetric technique with a less noisy alternative: radiation acoustics. Certain solids, when irradiated by a pulsed X-ray beam, will 'ring' ultrasonically, as if struck by a hammer [15]. These ultrasonic vibrations are relatively clean, have a narrow bandwidth $(Q>15)$, and have a ringing amplitude that varies linearly with beam energy [23]. This linear correlation may be used to determine beam energy based on a given measurement of ringing amplitude.

In order to use these phenomena in a measurement of beam energy, theoretical models were constructed. These were used both to provide proof of the device's feasibility as well as to give rough estimates of its responsiveness. Mechanical design and material choices were carried out based on the predictions of these models [16]. Design and testing of electronics were carried out alongside the mechanical design. A schematic drawing of the planned device

appears in Figure 1. A prototype device was machined and assembled with the intention of eventually installing a version in the LCLS beamline. The prototype was tested using an pulsed optical beam but was not installed.

\section{THEORY}

In order to compute a value for the beam power, theoretical models were constructed to characterize the system's thermal and acoustic properties. These models may then be used to solve for the beam energy given measurements of the thermal and acoustic excitation of the system. These models assume a $1 \mathrm{~cm}^{3}$ target composed of Boron Carbide. 


\section{Thermal Theory}

Validity of Lumped-system Model The following heat exchange model was constructed assuming a lumped system; this assumption posits that temperature gradients within objects may be ignored due to the small size and large thermal conductivity of the objects. This assumption is justified by the value of the Biot Number, $B i$, a dimensionless number that quantifies the relative rates of heating within and without an object:

$$
B i=\frac{h L}{k}
$$

where $h$ is heat transfer coefficient describing the rate of heating at a boundary, $L$ is the characteristic length of the object $(L \approx V o l . / A r e a)$, and $k$ is the thermal conductivity of the object. If $B i$ is much less than 1, the object internally equilibrates very quickly and temperature gradients may be ignored. The value of $B i$ for the proposed device was calculated to be 0.026 , indicating that temperature gradients within the $\mathrm{B}_{4} \mathrm{C}$ target are negligible.

Dynamic Heat Equation Simple heat exchange models were used to characterize the thermal behavior of the target after irradiation. To keep the models simple, the total heat flow to and from the $\mathrm{B}_{4} \mathrm{C}$ target was assumed to be approximately described by Newton's law of cooling. That is, the temperature of the target was assumed to be described by the

differential equation given by Equation (2), where solutions to the equation are given by Equation (3).

$$
\begin{gathered}
\frac{d T}{d t}=h\left(T-T_{a}\right)+k \\
T(t)=m e^{a t}+b
\end{gathered}
$$


$T$ is the temperature of the target and $h, k, m, a$, and $b$ are arbitrary coefficients. The ambient temperature, $T_{a}$, was assumed constant.

Five heat exchange processes were accounted for in the model: (a) heat flow through electrical wiring via the sensors; (b) convection currents in the ambient vacuum; (c) heat flow through the piezoelectric actuators; (d) exchange of thermal radiation between the target and the cylindrical beam line; and (e) exchange of thermal radiation between the target and tungsten collimator (labeled in Figure 1).

For processes (a) through (c), differential energy loss is governed by the same general equation as in Equation (2):

$$
\frac{d Q}{d t}_{T_{1} \rightarrow T_{2}}=h\left(T_{1}-T_{2}\right)
$$

where $\frac{d Q}{d t} T_{1} \rightarrow T_{2}$ is the thermal energy transferred between object 1 and $2, T_{1}$ and $T_{2}$ are the temperatures of the two objects, and $h$ is a constant governing the rate of heat exchange, determined by the dimensions and thermal properties of the system. These processes are thermally grounded to the ambient temperature $T_{a}$, so the variable $T_{2}$ may be replaced by the constant $T_{a}$ in Equation (4).

The radiative processes, (d) and (e), are governed by a different general equation:

$$
\frac{d Q}{d t}_{T_{1} \rightarrow T_{2}}=A_{1} \sigma\left(T_{1}^{4}-T_{2}^{4}\right) /\left[\frac{1}{\epsilon_{1}}+\frac{\left(1-\epsilon_{2}\right) A_{1}}{\epsilon_{2} A_{2}}\right]
$$

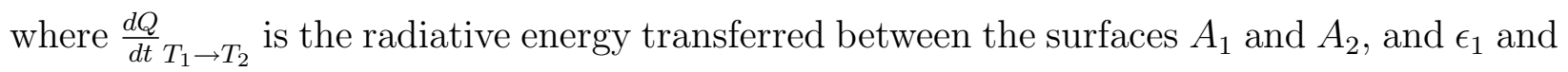
$\epsilon_{2}$ are the emissivities of the two surfaces, respectively. This equation may be approximated (via binomial expansion) by:

$$
\begin{gathered}
\frac{d Q}{d t}{ }_{T_{1} \rightarrow T_{2}, \text { approx. }}=h_{\text {rad. }}\left(T_{1}-T_{2}\right) \\
\text { where } \quad h_{\text {rad. }}=4 T_{2}^{3} A_{1} \sigma /\left[\frac{1}{\epsilon_{1}}+\frac{\left(1-\epsilon_{2}\right) A_{1}}{\epsilon_{2} A_{2}}\right] .
\end{gathered}
$$


In this way, one may quantify radiation exchange (i.e. processes (d) and (e)) in the simple form assumed in Equation (2).

Relative Significance of Heat Exchange Processes The relative significance of the processes (a) through (e) may be evaluated by comparing each process' "heat transfer coefficient," $h$. The larger this coefficient, the more heat energy is lost per unit time through a particular process. A plot of this comparison is shown in Figure 2. It is evident from this comparison that only heat loss through the piezoelectric actuators and radiation exchange with the beam line container are significant enough to warrant further consideration.

Total Heat Flow The total differential temperature change in the model is given by

$$
\frac{d T}{d t}_{\text {target }}=\frac{1}{m c_{v}}\left[\sum_{i=1}^{5} \frac{d Q_{i}}{d t}(T)+P_{\gamma}\right]
$$

where $\frac{d Q_{i}}{d t}(T)$ are the differential heat energies lost by each of the five processes, $P_{\gamma}$ is the power in the X-ray beam, and $m c_{v}$ is the target's specific heat. Solving for the temperature function $T(t)$, a limiting temperature of $301.2{ }^{\circ} \mathrm{K}$ and a time constant of $300 \mathrm{~s}$ were found (assuming a $10 \mathrm{~mW}$ average beam power). The theoretical temperature curve calculated with this model is shown in Figure 3.

Solving for Beam Power The heat losses $\frac{d Q_{i}}{d t}(T)$ are constant regardless of whether the beam is on or off. Therefore, these losses may be quantified when the beam is off and then filtered out when the beam is turned back on. The beam power is determined when these known heat losses have been corrected for. That is, given a temperature curve (like the one shown in Figure 3), one may solve for the characteristic time constant of the system using 
two points $\left\langle T_{1}, t_{1}\right\rangle$ and $\left\langle T_{2}, t_{2}\right\rangle$ on the "beam off" curve:

$$
\tau=\frac{t_{1}-t_{2}}{\log \left(T_{1}-T_{a}\right)-\log \left(T_{2}-T_{a}\right)}
$$

where $T_{a}$ is the ambient temperature (assumed to be $300^{\circ} \mathrm{K}$ in Figure 3 ). This time constant characterizes the heat losses in the system. Then the power of the beam may be solved for using this time constant and two points $\left\langle T_{3}, t_{3}\right\rangle$ and $\left\langle T_{4}, t_{4}\right\rangle$ on the "beam on" curve:

$$
P_{\gamma}=\frac{m c_{v}}{\tau}\left[T_{a}-\frac{T_{3}-T_{4} e^{\left(t_{3}-t_{4}\right) / \tau}}{1-e^{\left(t_{3}-t_{4}\right) / \tau}}\right]
$$

Complications Due to Target Clamp In the original heat exchange model, the large aluminum base supporting the target and collimators (see bottom of Figure 1) was assumed to remain at a fixed temperature, $T_{a}$, due to its large thermal mass. This assumption is not true, however, of the clamp (circled in red in Figure 1) that holds the target down on top of the aluminum mount. As the target's temperature increases, heat energy flows to the clamp, causing its temperature to rise as well. The dynamical flow of heat energy is impacted by this temperature change.

A more accurate model must consist of two coupled differential equations. This coupled system may be described by a system of matrices, analogous to the single-variable temperature equation given by Equation (2),

$$
\overrightarrow{\mathbf{T}^{\prime}}=[\mathbf{M}] \overrightarrow{\mathbf{T}}+\overrightarrow{\mathbf{b}}
$$

where $\overrightarrow{\mathbf{T}}$ is the temperature vector containing the two temperature values $T_{\text {target }}$ and $T_{\text {clamp }}$, $\overrightarrow{\mathbf{T}}^{\prime}$ is the differential temperature vector, $[\mathbf{M}]$ is the coupling matrix, and $\overrightarrow{\mathbf{b}}$ is the constant 
term vector. Solutions to this differential matrix equation are of the form:

$$
\begin{gathered}
\overrightarrow{\mathbf{T}}(t)=\overrightarrow{\mathbf{v}}[\mathbf{B}]\left[\begin{array}{cc}
e^{\lambda_{1} t} & 0 \\
0 & e^{\lambda_{2} t}
\end{array}\right][\mathbf{B}]^{-1}-[\mathbf{M}]^{-1} \overrightarrow{\mathbf{b}} \\
\text { where } \quad \overrightarrow{\mathbf{v}}=\overrightarrow{\mathbf{T}}_{\mathbf{i}}+[\mathbf{M}]^{-1} \overrightarrow{\mathbf{b}},
\end{gathered}
$$

$\overrightarrow{\mathbf{T}}_{\mathbf{i}}$ is the initial temperature vector, the columns of $[\mathbf{B}]$ are the eigenvectors of $[\mathbf{M}]$, and $\lambda_{1}$ and $\lambda_{2}$ are the eigenvalues of $[\mathbf{M}]$.

The results of this 2-variable model are shown in Figure 4. The "actual" temperature curve (i.e. temperature is affected by the clamp) is shown in blue, whereas the "nominal" curve (i.e. assuming the clamp has infinite thermal mass) is shown in brown. The clamp acts to retain heat in the system, resulting in higher limiting temperatures. Fortunately, since the second-order coupling effects are small, this variation may be compensated for by a simple multiplication factor. That is, the actual temperature curve is approximately proportional to the nominal temperature curve:

$$
T_{\text {act. }}(t)=\frac{1}{\kappa} T_{\text {nom. }}(t)
$$

where $\kappa=0.6622$ for all beam energies.

\section{Acoustic Theory}

Known theory may also be used to model acoustic phenomena. The generation of sound waves by X-ray radiation is considered in detail by Kalinichenko, et. al. [15]. For a photon beam, sound generation in a target is dominated by thermoelastic expansion due to lump energy deposition, as opposed to dynamic stresses from individual photons. In the range of X-ray energies produced by LCLS $(800 \mathrm{eV}-9 \mathrm{keV})$, energy is transferred to the target 
primarily via the photoelectric effect (as well as a smaller contributions from Rayleigh and Compton scattering). These effects generate secondary electrons, which continue to transfer energy to the target via ionization of target atoms. These mechanisms primarily increase the thermal energy of the target, causing thermal expansion that is responsible for wave generation.

For the vibrational measurement to have any significance, the fraction of beam energy absorbed by the target must be known. The most energetic X-rays in the FEL beam have energies less than $9 \mathrm{keV}$, giving a maximum attenuation length in $\mathrm{B}_{4} \mathrm{C}$ of about $2 \mathrm{~mm}$ [11]. Thus, roughly $95 \%$ of the incident photons have been absorbed in a target length of $6 \mathrm{~mm}$ (95\% length $\approx 3 \times$ atten. length). As a rough estimate, the attenuation length of the secondary electrons in the solid target may be given by the Bethe-Bloch formula,

$$
\left\langle\frac{d E_{e}}{d x}\right\rangle=-2 \pi\left(\frac{e^{2}}{4 \pi \epsilon_{0}}\right)^{2} \frac{z n}{E_{e}} \ln \left(\frac{\delta E_{e}}{I}\right)
$$

where $e$ is the electron charge, $z$ is the atomic number of the target material, $n$ is the atomic density, $I$ is the mean atomic potential, and $E_{e}$ is the initial electron energy [3]. Using this formula, the maximum stopping length for the most energetic electrons (i.e. $9 \mathrm{keV}$ ) may be calculated:

$$
d x=d E_{e} /\left[2 \pi\left(\frac{e^{2}}{4 \pi \epsilon_{0}}\right)^{2} \frac{z n}{E_{e}} \ln \left(\frac{\delta E_{e}}{I}\right)\right] \approx 6.6 \mu \mathrm{m} .
$$

Since the length of the target along the beam path is $10 \mathrm{~mm}$, it may be assumed that nearly all of the incident X-rays and secondary electrons are absorbed thermally by the target. That is,

$$
d E_{\text {target }} \approx E_{\text {pulse }} .
$$

Assuming that the target heats uniformly (see calculation of Biot number above), the amplitude of the thermoelastic wave may be approximately described as uniform expansion 
of the target in all dimensions due to heating. Using known values of specific heat $c_{v}$ and coefficient of thermal expansion $\alpha$ for Boron Carbide, the expected vibrational amplitude is:

$$
u=\frac{1}{2} \alpha L_{0} \frac{d E}{m c_{v}}
$$

where $u$ is the amplitude of vibration, $L_{0}$ is the initial length of the target, and $m c_{v}$ is the specific heat of the target. Given the assumption stated in Equation (14), the vibrational amplitude is then proportional to the pulse energy:

$$
\begin{gathered}
u=\zeta E_{\text {pulse }} \\
\text { where } \quad \zeta=\frac{\alpha L_{0}}{2 m c_{v}} .
\end{gathered}
$$

For the Boron Carbide target, $\zeta=11 \mathrm{pm} / \mathrm{mJ}$.

In addition, the resonant frequency of the target may also be calculated from the physical characteristics of the target:

$$
f=\frac{s}{2 L_{0}} \approx 545 \mathrm{kHz}
$$

where $s$ is the speed of sound in Boron Carbide and $L_{0}$ is the initial length of the target.

\section{METHODS}

Several proof-of-concept tests were carried out on a test target to determine the feasibility of the device and to verify theoretical calculations. A $0.8 \times 1 \times 1.25 \mathrm{~cm}^{3}$ Boron Carbide block was placed in the path of a pulsed $780 \mathrm{~nm}$ Q-switched Ti:sapphire beam. The target was oriented with the $1.25 \mathrm{~cm}$ face normal to the beam. The pulse length of the beam was roughly $10 \mathrm{~ns}$ at repetition rates of 120 and $360 \mathrm{~Hz}$. The bandwidth was $30 \mathrm{~nm}$, and the spatial profile was roughly Gaussian. Two monolithic ceramic piezoelectric actuators were 
glued to the target using Torr-Seal epoxy and clamped in place with an aluminum bar. Two 100 Ohm platinum RTDs were also mounted on the sides of the target.

The beam was attenuated and shaped by a wave plate and polarizer, and the spot size was controlled by a lens with a $0.5 \mathrm{~m}$ focus length. Before placing the target in the beam path, a power meter was used to measure the beam's average power. Two methods were used to vary the beam power: modification of the laser cavity length and attenuation via optical filters.

The signal from each piezoelectric actuator was fed to a 1:4 transformer, a $1.9 \mathrm{MHz}$ lowpass filter, a BJT preamplifier, and an oscilloscope. The oscilloscope was triggered on the beam pulse signal. Waveforms of the vibration signal were saved to disk for later analysis. The resistance (i.e. temperature) of the RTD was measured by a RTD temperature monitor using a 4-wire ratiometric measurement.

Several of the beam's parameters were varied over successive trials. The spot size was varied from 0.5 to $1 \mathrm{~cm} \varnothing$. The position of the beam on the target was varied from the bottom of the target to the top (roughly $4 \mathrm{~mm}$ off center in each case). The beam power was varied from $360 \mathrm{~mW}$ to $920 \mathrm{~mW}$ at $360 \mathrm{~Hz}$ repetition, and from $100 \mathrm{~mW}$ to $320 \mathrm{~mW}$ at $120 \mathrm{~Hz}$ repetition (these powers correspond to a range of roughly 0.8 to $2.6 \mathrm{~mJ}$ of pulse energy).

\section{RESULTS}

Plots of typical vibration signals from the irradiated block are shown in Figure 5. For low pulse energies $(\leq 2 \mathrm{~mJ})$, the amplitude of vibration was observed to vary linearly with beam power. This linearity disappears for pulse energies above $2 \mathrm{~mJ}$, where the vibrational

amplitude falls below the linear trend exhibited by the system at lower energies. A plot of vibration voltage versus beam power that exhibits this roll-off behavior is shown in Figure 
6. As seen in the figure, the magnitude of roll-off is dependent on the level of amplification, indicating that amplifier saturation may be to blame to for the observed nonlinearity. In an attempt to eliminate this effect, the amplifiers were removed from the system for three trials. This data is shown in Figure 7. Signal roll-off is still observed in these trials, indicating that factors other than amplifier saturation must contribute to nonlinearity in the vibration signal.

The resonant frequency of the block was observed to be $580 \pm 40 \mathrm{kHz}$, in good agreement with theory. When the diameter of the spot was halved, the vibrational amplitude was observed to increase by about 25\%. A detectable phase shift (on the order of $0.5 \mu \mathrm{s}$ ) was observed for trials in which the beam was aligned off-center. The RTDs detected a significant increase in temperature during each trial on the order of $10{ }^{\circ} \mathrm{C}$ after several minutes. This disagreed significantly with theory, which predicts a temperature rise of $43{ }^{\circ} \mathrm{C}$ over the same time period.

\section{DISCUSSION}

That the amplitude of vibration in fact varied linearly with beam power demonstrates the device's fundamental viability as a beam power monitor. It also places the device in

excellent agreement with similar experiments carried out by Kalinechenko and White [21] [23]. Unfortunately, the observed nonlinearity at high pulse energies is a significant problem. This effect could be due to several factors. The first could be a nonlinearity inherent in the piezoelectric actuators. The actuators may saturate above some maximum compression, resulting in reduced voltage signals for large compressions. Another, and more likely, source of disagreement stems from the properties of the beam used to test the device. While an X-ray beam penetrates through a good deal of the target material, inducing heating over a large depth, an optical laser is largely absorbed within a few micrometers, inducing large 
temperature gradients at the boundary of the target. The sound waves generated from this type of heating may differ significantly from those generated by the X-ray beam; this difference may be the cause of the unexpected nonlinearity.

In addition, the data disagrees with theory by a factor of 3 or more. A comparison of experimental data and theoretical predictions is shown in Figure 8. This disagreement could be due to several factors. The most sensible explanation is that the theoretical calculations do not account for resonances in the block. After irradiation, pressure waves must be expected to bounce around inside the target, constructively interfering to create compressions that are larger than those calculated from Equation (15). Another possible explanation considers again the differences between the FEL beam and the optical test beam. While theoretical calculations were carried out assuming a coherent, narrow $(\sim 100 \mu \mathrm{m})$ beam comprised of hard X-rays, the beam used to test the device was an optical beam with a much wider spot $(\sim 1 \mathrm{~cm})$. Since the details of pressure wave generation depend on the energy density and wavelength of incident radiation, these differing beam characteristics could have led to the creation of pressure waves with different amplitudes. Another suspected cause is a resonance in the cables that acted to amplify the piezoelectric signals they carried. These hypothesis must be investigated further in order to determine the true sources of discrepancy.

The dependence of vibration amplitude on spot size is also a significant impediment to the device's prospects as a power monitor. Ideally, the device should be able to measure average power regardless of the size of the spot, without any additional calibration. The literature, however, suggests that this effect is inherent to the phenomenon of wave generation by radiation beams. Calculations show that the peak vibrational amplitude depends critically on the beam radius as $u \propto R^{-3 / 2}$, where $u$ is the vibration amplitude and $R$ is the beam radius. Future work should focus on understanding and compensating for this effect.

The resonant frequency remained constant over all trials, in support of the simple wave propagation calculation shown in Equation (17). The stability of the resonant frequency 
is fortunate because it allows easy processing of the vibrational signal via either analog or digital filtering.

The phase shift in the vibration signal due to changes in beam position agrees with expectations based on the propagation of sound waves in Boron Carbide. The magnitude of phase difference, moreover, was large enough to justify using the device to make precision measurements of beam position in addition to its power measurement capabilities.

Although the behavior of the RTDs during irradiation qualitatively matched expectations, the amount of heating measured disagreed with expectations by nearly a factor of 5 . This difference may be explained by the dominant effect of convection currents in open air that acted to cool the device. In vacuum, such convection currents contribute to a negligible amount of cooling, resulting in the device reaching higher limiting temperatures. The device, however, must be tested in vacuum for the thermal technique to be truly verified.

\section{CONCLUSIONS}

As FEL X-ray sources reach higher brightness and peak power capabilities, new measurement techniques must be developed to withstand such high-power beams. The proposed device combines traditional calorimetry with the phenomenon of radiation acoustics in order to make a self-calibrated measurement of beam power. Such a technique may easily be adapted for use in future FELs with high beam brightness as well as in other pulsed sources such as hadron or electron beams.

Similar acoustic techniques may also be used to make precision measurements of other beam characteristics. These measurements include beam spot size, beam alignment, energy spectrum, spatial profile, and pulse duration. In addition, acoustic techniques may be used to study material properties such as mechanisms of radiation energy transfer, speed of sound, vibrational modes, and thermodynamic properties. Other applications include 
detection of solitary high-energy particles and modification of material properties during irradiation/acoustic wave generation [21].

Future studies must be carried out in order for the device to be considered ready for application. Theoretical studies should include understanding the discrepancies involved in the correlation between beam power and vibrational amplitude (especially with respect to the discussed nonlinearity) and more detailed models of wave generation and propagation in solids under irradiation. In addition, the application of radiation acoustics to beam position measurements must be quantified and studied further in order to use this technique with confidence.

\section{ACKNOWLEDGEMENTS}

This research was carried out at the SLAC National Accelerator Laboratory between May 18 and August 14, 2009 under the direction of Joseph Frisch. The author would like to thank the United States Department of Energy, the SLAC National Accelerator Laboratory, and the directors and administrators of the SULI internship program for making this research possible. He would also like to thank his mentors Joe Frisch and Tonee Smith as well as his partner Jennifer Loos. Thanks also go to Bill White, Steve Edstrom, and Ryan Coffee for their expertise and use of the NEH laser, Mark Petree and Phil Cutino for aid in mechanical design, Alan Fisher for guidance in electronics design, and Lesley Wolf for her indispensable help in acquiring research materials. 


\section{REFERENCES}

[1] A.F Adadurov and V.T Lazurik. Calculation of the yield of fast secondary electrons by the action of x radiation. Atomnaya Energiya, 50(3):211-212, 1981.

[2] R Akre, D Dowell, P Emma, J Frisch, S Gilevich, G Hays, Ph Hering, R Iverson, C Limborg-Deprey, H Loos, A Miahnahri, J Schmerge, J Turner, J Welch, W White, and J Wu. Commissioning the linac coherent light source injector. Phys. Rev. ST Accel. Beams, 11:30703, Mar 2008.

[3] H. Bethe. Zur theorie des durchgangs schneller korpuskularstrahlen durch materie. Annalen der Physik, 397(3):325-400, 1930.

[4] I.A Borshkovskii, V.D Volovik, I.A Grishaev, G.P Dubovik, I.I Zalyubovskii, and V.V Petrenko. Excitation of ultrasonic waves by passage of fast electrons through a metal. Zhurnal Eksperimentalnoi i Teoreticheskoi Fiziki, 13(10):546-549, Jul 1971.

[5] H.S. Carslaw and J.C. Jaeger. Conduction of Heat in Solids. Oxford University Press, 2nd edition, 1959.

[6] Alexander Wu Chao and Maury Tigner. Handbook of Accelerator Physics and Engineering. World Scientific Publishing Co., 1998.

[7] L. B da Silva, B. J MacGowan, S Mrowka, J. A Koch, R. A London, D. L Matthews, and J. H Underwood. Power measurements of a saturated yttrium x-ray laser. Optics Letters (ISSN 0146-9592), 18:1174, Jul 1993.

[8] S Friedrich, L Li, L. L Ott, Rajeswari M Kolgani, G. J Yong, Z. A Ali, O. B Drury, E Ables, and R. M Bionta. Design of a bolometer for total-energy measurement of the linear coherent light source pulsed x-ray laser. Nuclear Instruments and Methods in Physics Research Section A, 559:772, Apr 2006. 
[9] W. S Graves, E. D Johnson, and S Ulc. A high resolution electron beam profile monitor and its applications. The eighth beam instrumentation workshop. AIP Conference Proceedings, 451:206, Dec 1998.

[10] The LCLS Design Study Group. Linac coherent light source (lcls) design study report. SLAC Internal Report, 1998.

[11] B.L. Henke, E.M. Gullikson, and J.C. Davis. X-ray interactions: Photoabsorption, scattering, transmission, and reflection at $\mathrm{e}=50-30,000 \mathrm{ev}, \mathrm{z}=1-92$. Atomic Data and Nuclear Data Tables, 54(2):181-342, July 1993.

[12] Paul Horowitz and Winfield Hill. The Art of Electronics. Cambridge University Press, 2nd edition, 1989.

[13] Zhirong Huang and Kwang-Je Kim. Review of x-ray free-electron laser theory. Physical Review Special Topics - Accelerators and Beams, 10:34801, Mar 2007.

[14] E. D Johnson, W. S Graves, and K. E Robinson. Periscope pop-in beam monitor. The eighth beam instrumentation workshop. AIP Conference Proceedings, 451:479, Dec 1998.

[15] A.I. Kalinichenko, V.T. Lazurik, and I.I. Zalyubovsky. Introduction to Radiation Acoustics, volume 9 of The Physics and Technology of Particle and Photon Beams. Harwood Academic Publishers, 2001.

[16] Jennifer Loos. Design and implementation of a thermal and acoustic x-ray detector to measure the lcls beam energy. SLAC Internal Report, Aug 2009.

[17] A. H Lumpkin, B. X Yang, W. J Berg, M White, J. W Lewellen, and S. V Milton. Optical techniques for electron-beam characterizations on the aps sase fel project. Nuclear Instruments and Methods in Physics Research Section A, 429:336, Jun 1999. 
[18] John F. O'Hanlon. A User's Guide to Vacuum Technology. John Wiley Sons, Inc., 3rd edition, 2003.

[19] James Rosenzweig and Luca Serafini, editors. The Physics of High Brightness Beams. Proceedings of the 2nd ICFA Advanced Accelerator Workshop. World Scientific Publishing Co., 2000.

[20] Yoshihiko Shoji. Measurement of the time structure of a coherent synchrotron radiation burst in newsubaru. Infrared Physics \& Technology, 51:367, May 2008.

[21] V.D Volovik, A.I Kalinichenko, V.I Kobizskoi, and V.T Lazurik-El'tsufin. Thermoelastic effect of a fast particle in a solid. Zhurnal Eksperimentalnoi i Teoreticheskoi Fiziki, 19(2):135-138, 1974.

[22] X.J Wang, I Ben-Zvi, R Malone, and V Yakimenko. High-resolution beam profile monitor r\&d at the bnl atf. Brookhaven Internal Report, Sep 2000.

[23] R. M White. Elastic wave generation by electron bombardment or electromagnetic wave absorption. Journal of Applied Physics, 34:2123, Jul 1963. 


\section{FIGURES}

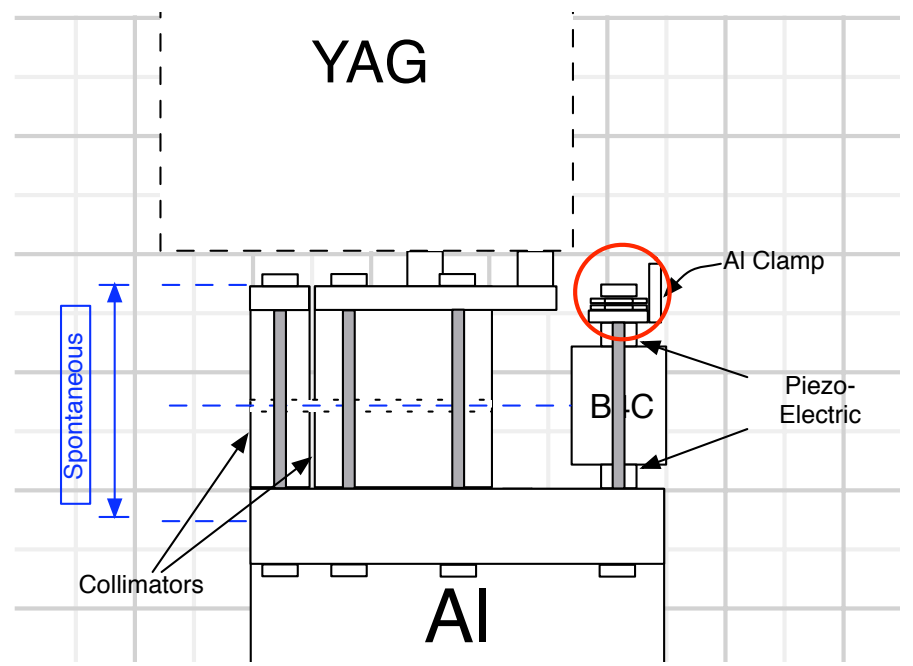

(a) View from the side of the device. The coherent spot passes through a slit in the Boron Carbide and Tungsten collimators to hit the $\mathrm{B}_{4} \mathrm{C}$ target.

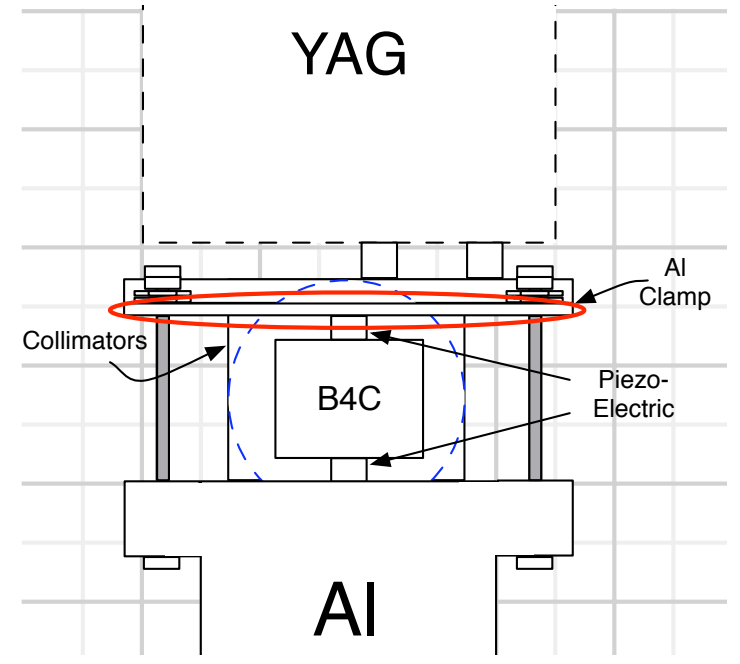

(b) View from the back of the device. The spontaneous radiation may be seen as the dotted blue line filling the front collimator.

Figure 1: (Color) Schematic diagrams of the proposed LCLS beam power monitor. Red circles mark the target clamp that gives rise to second-order thermal behavior in the system. 


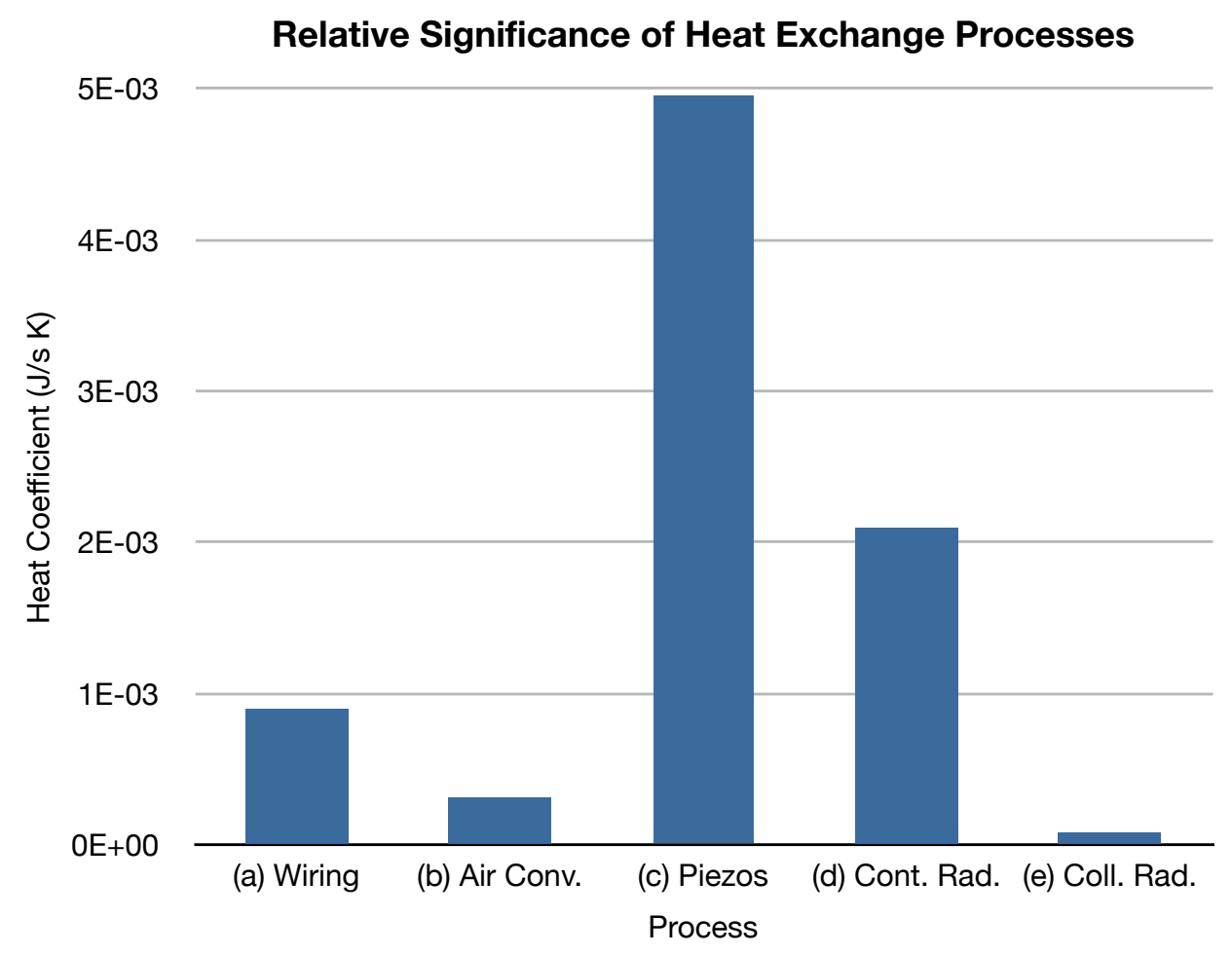

Figure 2: Relative significance of heat exchange processes in the power monitor system. Only the heat loss through the piezoelectric actuators and ambient radiation are significant enough to be regarded in future calculations. 
$\mathrm{T}(\mathrm{K})$

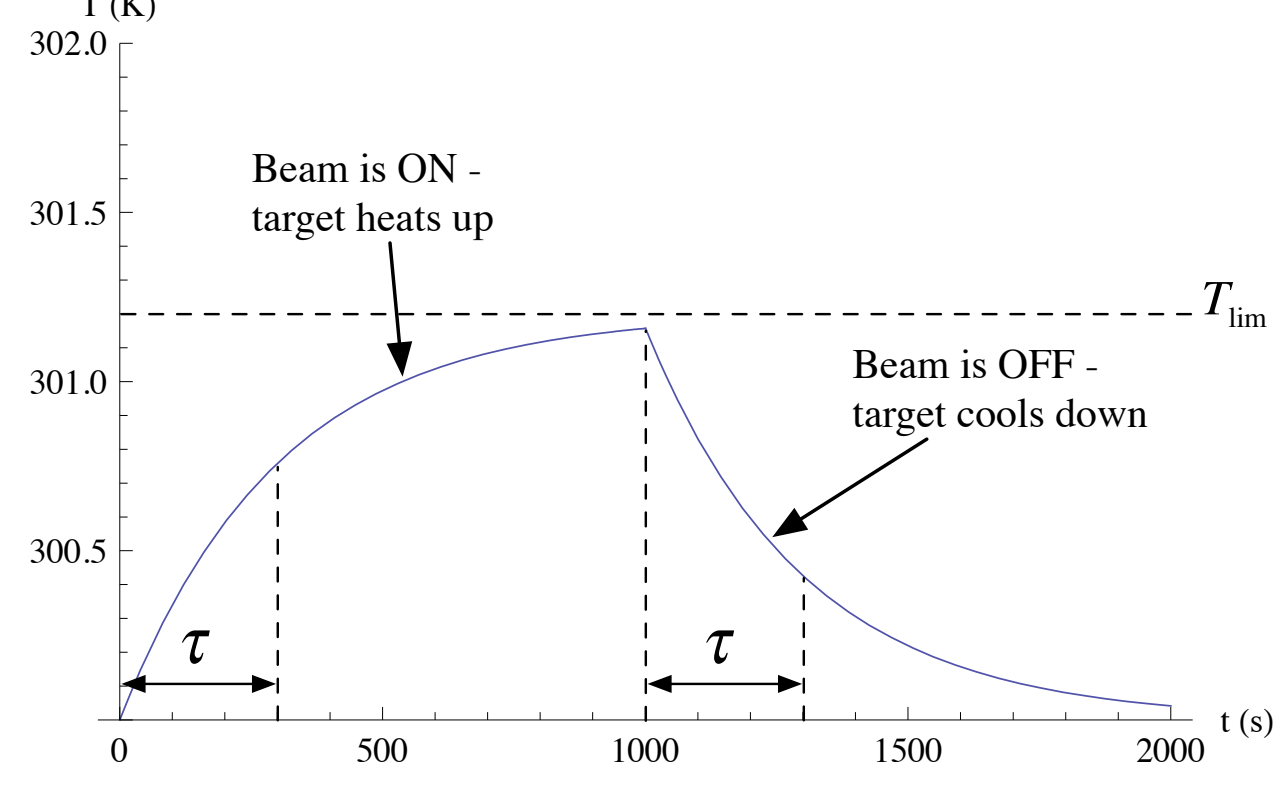

Figure 3: Theoretical temperature curve for a Boron Carbide target irradiated by a pulsed $\mathrm{X}$-ray beam. The beam is turned on at time $\mathrm{t}=0 \mathrm{~s}$, and is turned off at time $\mathrm{t}=1000 \mathrm{~s}$. The target is assumed to reside in an ambient temperature of $300{ }^{\circ} \mathrm{K}$. 


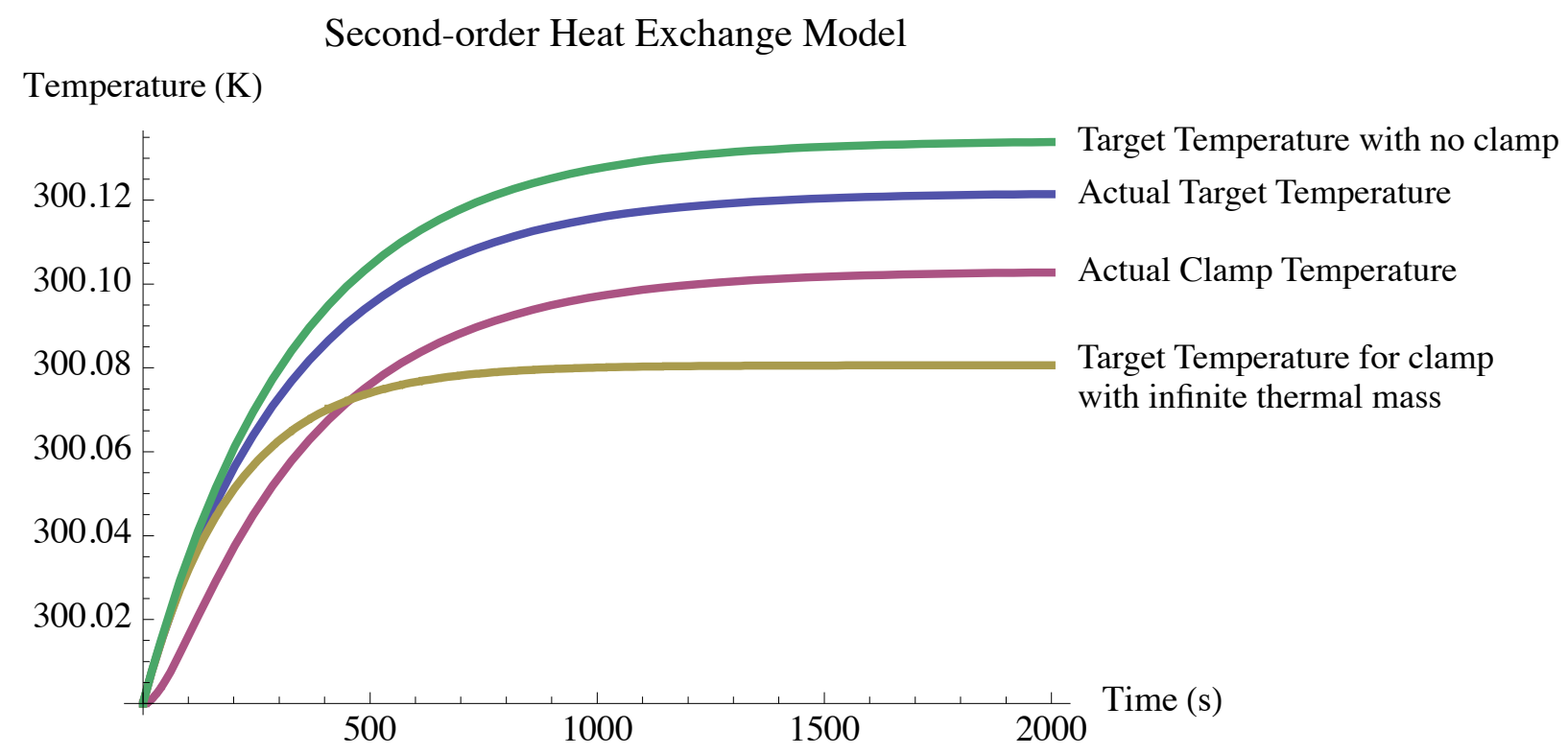

Figure 4: (Color) Temperature curves for the target and its associated clamp. The Blue and Red lines are the temperatures of the target and clamp, respectively. (Note the second-order behavior of the clamp between $t=0 \mathrm{~s}$ and $\mathrm{t}=100 \mathrm{~s}$ due to coupling effects). The Green line is the target's temperature curve if the target clamp were removed, and the Brown line is the target's temperature curve assuming a clamp with infinite thermal mass (i.e. the clamp remains at constant temperature regardless of the target's temperature). 


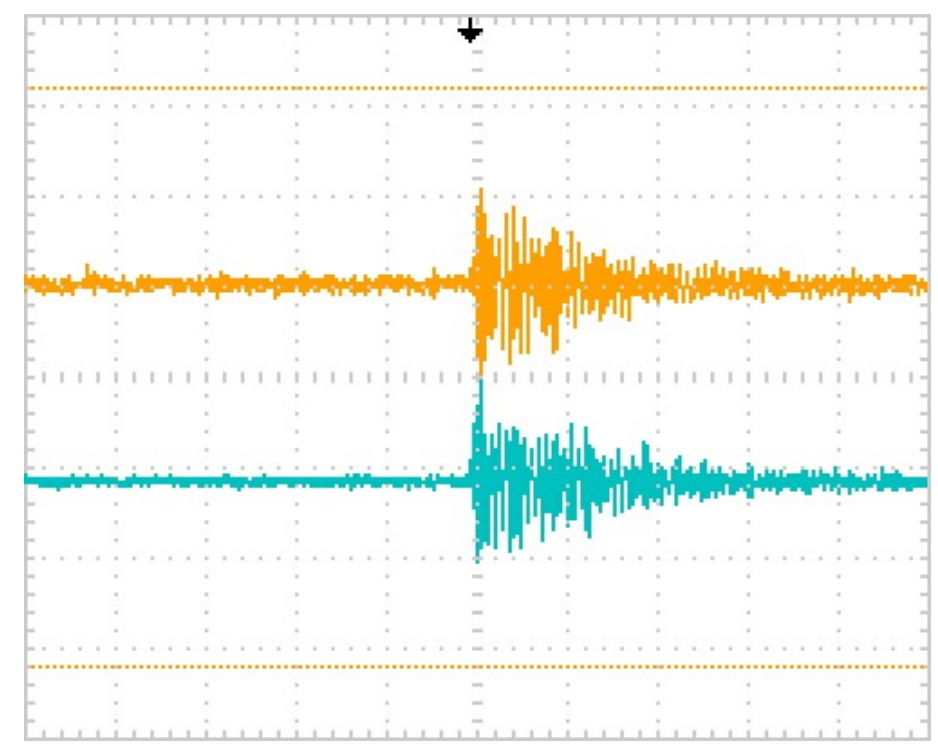

(a) Vibrational signals in irradiated test target. Units: 25 $\mu$ s/div. $\times 200 \mathrm{mV} /$ div.

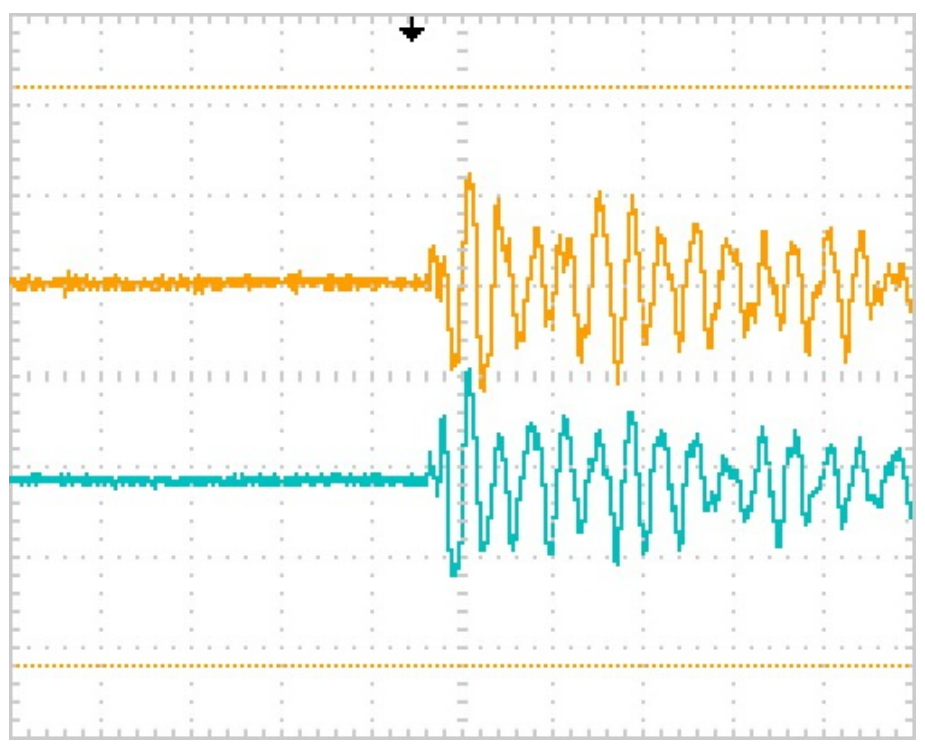

(b) Time detail of Figure (a). Units: $5 \mu \mathrm{s} /$ div. $\times 200 \mathrm{mV} /$ div.

Figure 5: (Color) Typical signals produced by radiation acoustic waves in the test target. Orange and Blue waveforms were generated by the two piezoelectric actuators mounted on either side of the target. Figure (b) shows time detail of Figure (a). Waveforms are triggered on the beam pulse. Note the exponential decay of vibration in Figure (a). 


\section{Vibrational Amplitude vs. Beam Power at various Amplifications}

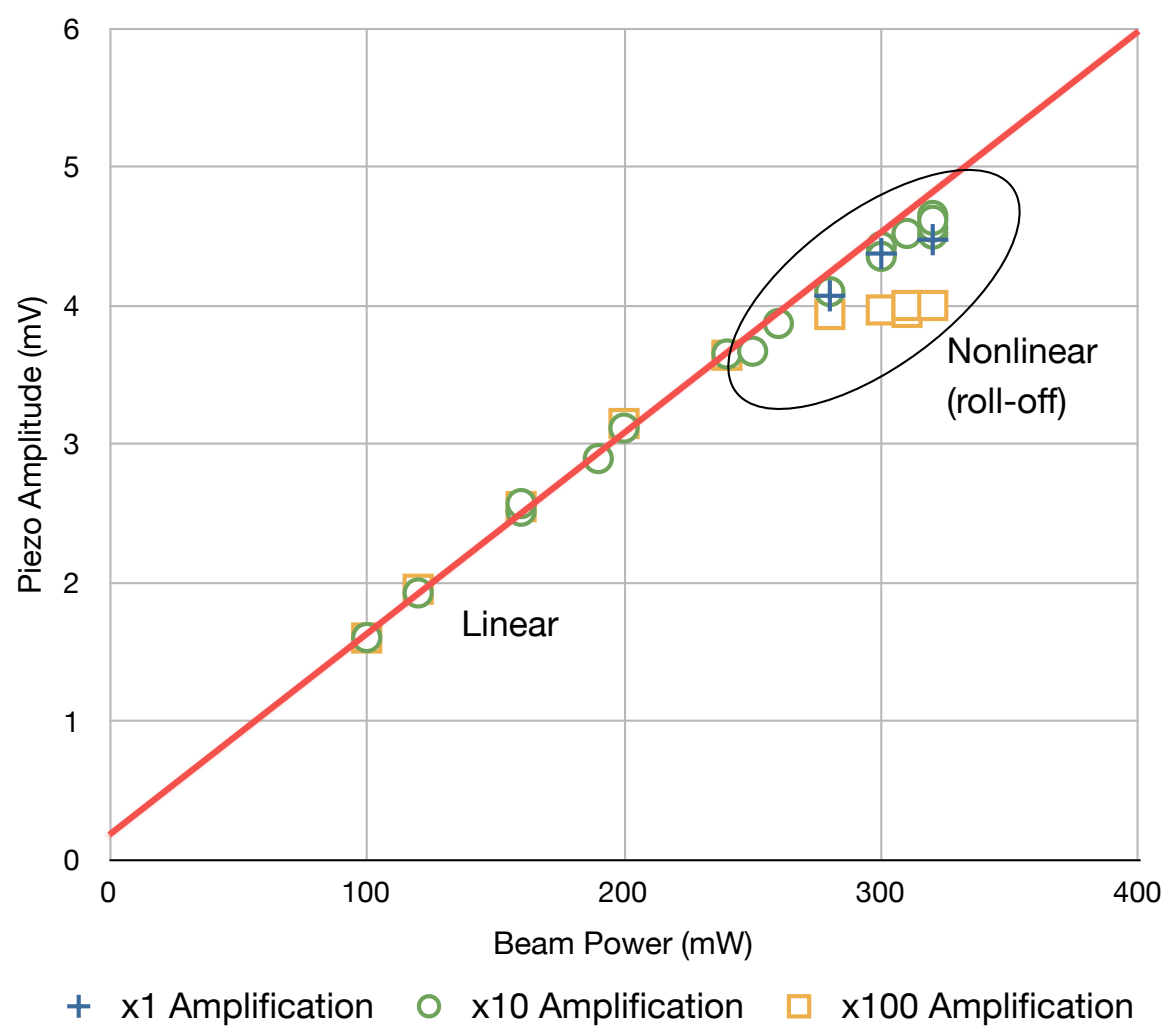

Figure 6: (Color) Plot of vibrational signal amplitude versus beam power for the proof-ofconcept test of the power monitor. The data shows linearity at low beam power (shown by the red trendline), but exhibits nonlinear behavior for pulse energies larger than $2 \mathrm{~mJ}$ (i.e. $240 \mathrm{~mW}$ ). Roll-off is more pronounced for larger amplifications, indicating that amplifier saturation may be a partial cause of nonlinearity. 


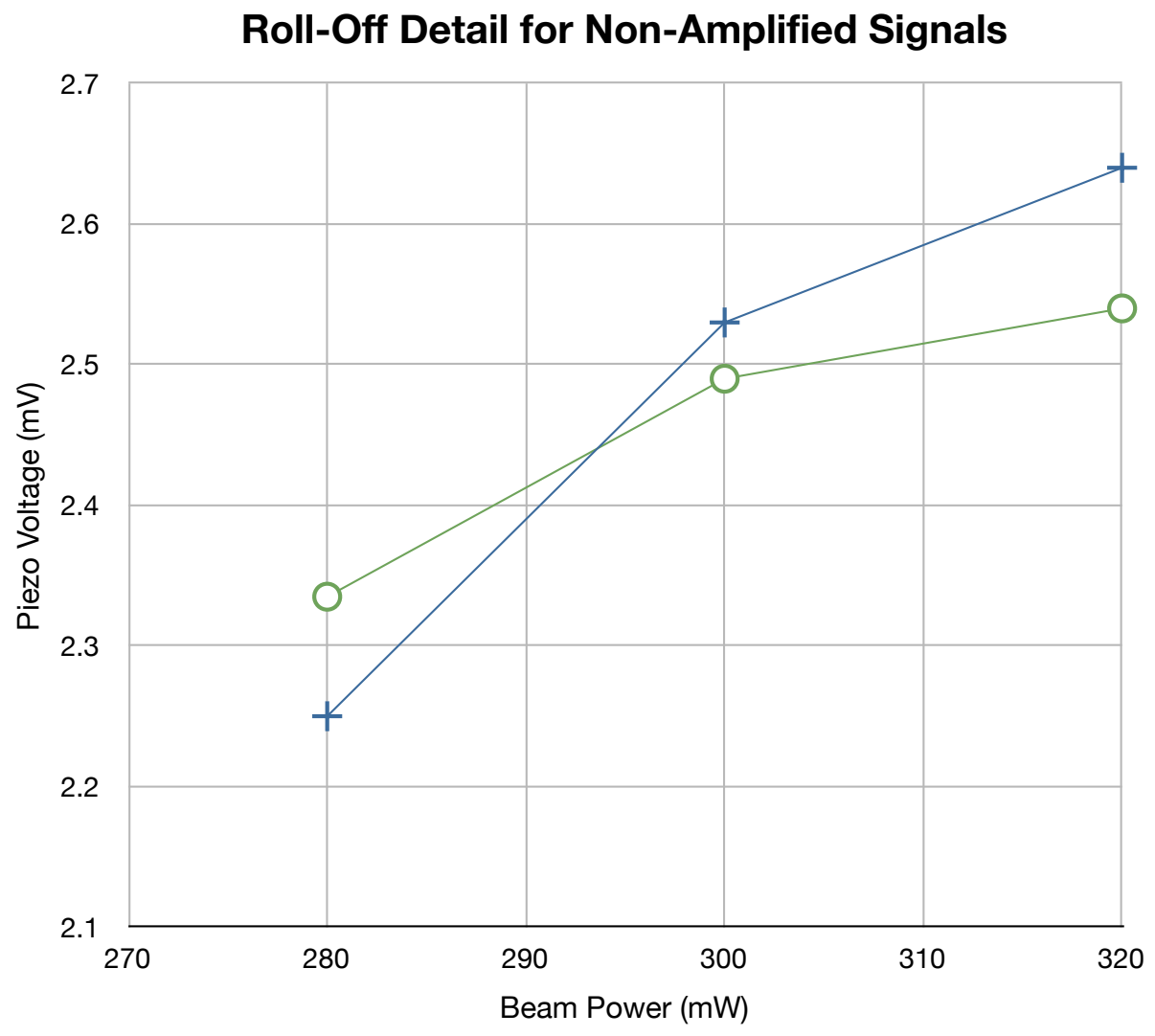

+ Ch. 1 o- Ch. 2

Figure 7: (Color) Plot of vibrational signal amplitude versus beam power for trials in which the amplifier was removed from the signal line. Signal roll-off is still observed, indicating that the phenomena is not due singularly to amplifier saturation. Other effects, still to be studied, must be responsible for the observed roll-off. 


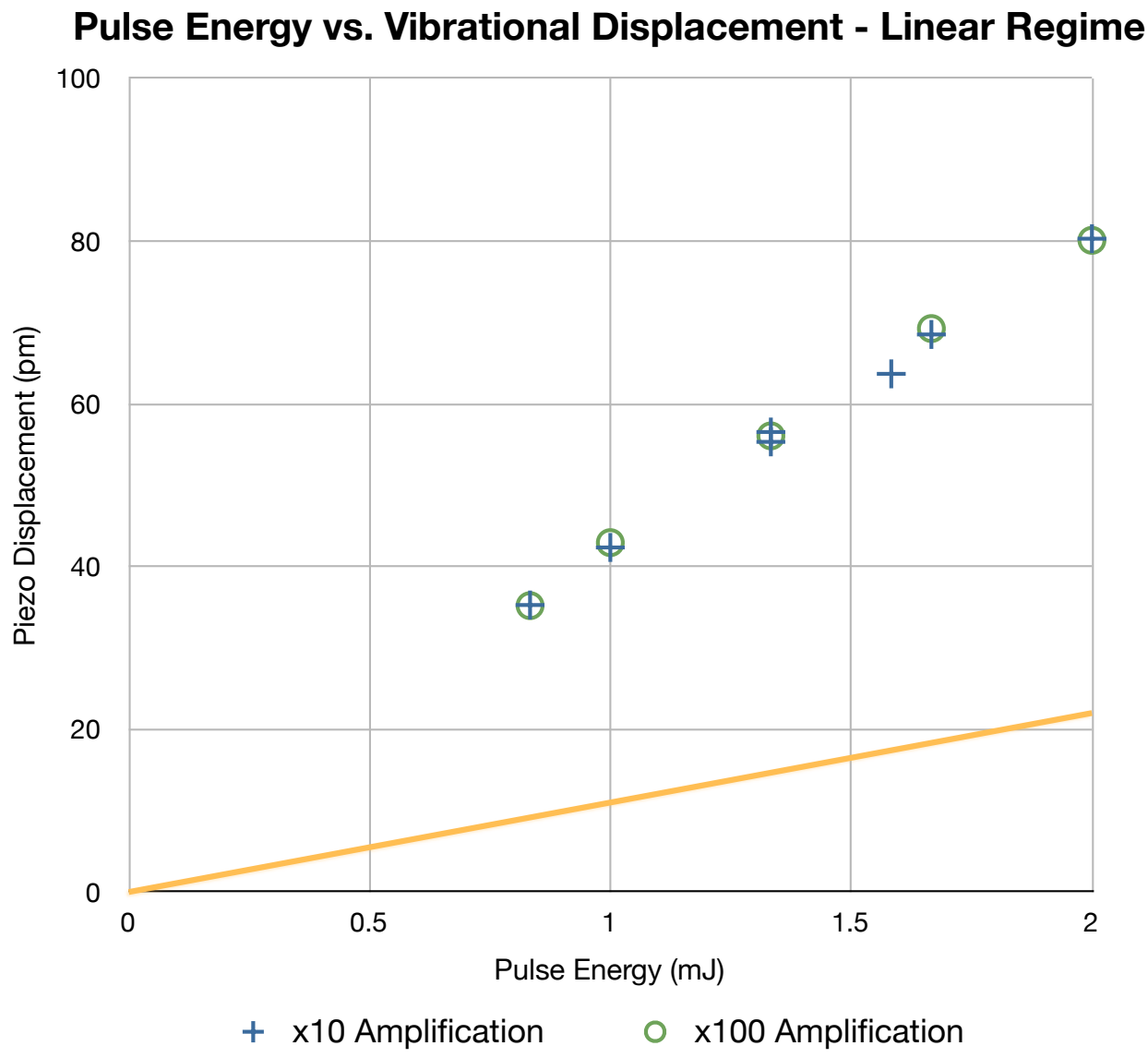

Figure 8: (Color) Plot of vibrational amplitude in the target versus beam pulse energy for the proof-of-concept test. Only data below $2 \mathrm{~mJ}$ are included (i.e. in which linearity is conserved). Theoretical predictions are shown by the yellow line. The slopes of the theoretical and experimental curves differ by a factor of 3 or more, indicating severe errors in the theoretical model. 


\title{
Fitting Out-of-Focus Star Images to Measure the Focus and Alignment of the Dark Energy Camera
}

\author{
Gary A. Binder \\ Office of Science, Science Undergraduate Laboratory Internship (SULI) Program \\ California Institute of Technology \\ SLAC National Laboratory \\ Menlo Park, California
}

August 13, 2009

Prepared in partial fulfillment of the requirements of the Office of Science, Department of Energy's Science

Undergraduate Laboratory Internship under the direction of Aaron Roodman at the Kavli Institute for Particle Astrophysics and Cosmology, SLAC National Laboratory

Participant:

Research Adviser: 


\section{Contents}

- Abstract $\quad 3$

- Introduction $\quad 4$

- Methods $\quad 4$

- Results $\quad 7$

- Conclusions $\quad 8$

- Acknowledgements $\quad 9$

$\begin{array}{lr}\text { - Literature Cited } & 9\end{array}$

$\begin{array}{ll}\text { - Figures and Tables } & 10\end{array}$ 


\section{Abstract}

Fitting Out-of-Focus Star Images to Measure the Focus and Alignment of the Dark Energy Camera. GARY A. BINDER (California Institute of Technology, Pasadena, CA 91125) AARON ROODMAN (SLAC National Laboratory, Menlo Park, CA 94204)

In order to make accurate measurements of dark energy, a system is needed to monitor the focus and alignment of the Dark Energy Camera (DECam) to be located on the Blanco 4m Telescope for the upcoming Dark Energy Survey. One new approach under development is to fit out-of-focus star images to a point spread function from which information about the focus and tilt of the camera can be obtained. As a first test of a new algorithm using this idea, simulated star images produced from a model of DECam in the optics software Zemax were fitted. Then, real images from the Mosaic II imager currently installed on the Blanco telescope were used to investigate the algorithm's capabilities. A number of problems with the algorithm were found, and more work is needed to understand its limitations and improve its capabilities so it can reliably predict camera alignment and focus. 


\section{Introduction}

The Dark Energy Survey represents an international effort to investigate the nature of the mysterious accelerated expansion of the universe, commonly attributed to a dark energy that permeates all space. The main goal of the survey is to measure the parameter $w$ appearing in the equation of state for dark energy. To accomplish this goal, the survey will use the 4 meter Victor M. Blanco Telescope at the Cerro Tololo Inter-American Observatory in Chile, and a camera specifically designed for this purpose, the Dark Energy Camera (DECam). Approximately 300 million galaxies over 5 years will be imaged across 5000 square degrees of the southern sky. The astronomical methods used to measure dark energy include galaxy cluster counts, baryon oscillations, supernovae, and weak gravitational lensing. Critical to the accuracy of these measurements is a sound understanding of the physical limitations of the telescope and camera optics due to atmospheric distortion, camera alignment and focus, and imperfections in the CCDs (charged-coupled devices) used to capture images, among other things. This is particularly important for weak gravitational lensing measurements; understanding how images are affected by the above effects is essential to get accurate results.[1] A variety of systems are in place to understand and counteract optical systematic effects.

One particular system involves the focus and alignment sensors located on the periphery of the focal plane of DECam. These are $82 \mathrm{k} \times 2 \mathrm{k}$ CCDs located alternately above and below the optimal focal plane of DECam by 1.5 millimeters as shown in Figure 1.[2] The CCDs will image stars and provide information on the positioning of the camera with respect to the primary mirror axis of the telescope. Because of fluctuations in the mechanical structure supporting the camera, small displacements in camera position are expected along the 6 degrees of freedom of the camera (3 in rotation and 3 in position). A hexapod system can maneuver the camera to correct for these fluctuations, using information provided by the focus and alignment sensors on the focal plane as well as from other monitoring systems. A fitting algorithm is under development that will take star images from the focus and alignment CCDs and provide information on the tilt of the camera, its position along the axis of the telescope i.e. how well in focus it is, and other relevant parameters to be discussed later. The primary purpose of the project was to test this new algorithm, first with artificial images made with the aid of the optics simulation software Zemax, and secondly with real images from the Mosaic II imager currently installed on the telescope. The results of the fits were compared against expected values, improvements were implemented, and problems noted.

\section{Methods}

The primary tools of investigation were all computer-based. Perhaps the most valuable tools for analysis of the reliability of the algorithm were the models of the Blanco Telescope, Dark Energy Camera, and the 
Mosaic II imager in Zemax. A diagram taken from Zemax illustrates the model of DECam and the Blanco Telescope in Figure 2. With these models, a procedure was devised to create artificial images to be used in the first test. Zemax performs ray tracing on the model the user creates to determine many useful quantities and functions describing the optical system. Among these are the point spread function (PSF), wavefront map, and Zernike coefficients. The PSF represents the image of a hypothetically infinitely distant point source at a chosen plane in the optical system. Since stars are so distant, the PSF effectively represents the image of a star at the chosen location in the camera, so it is the desired object to simulate star images. The wavefront represents the surface of equal phase for light waves passing through the exit pupil of the camera. The Zernike coefficients come from an orthonormal polynomial expansion of the wavefront on the unit disk in terms of the normalized radial coordinate $\rho$ and the azimuthal coordinate $\theta$. Zemax outputs the first 37 Zernike coefficients of the wavefront by default. In order to create artificial images of stars, the PSF must be obtained at the location of the focus and alignment CCDs in the model. Zemax can output the PSF and wavefront directly into a discrete array, however the Zernike coefficients were used instead. They provide a concise approximation of the wavefront with only 37 numbers, which can then be converted to the PSF via a Fourier transform.

A macro was created in the ZPL language used by Zemax to automate the process of extracting Zernike coefficients to text files. Python code already developed by Aaron Roodman was then used to convert the Zernike coefficients to realistic images. The code binned the wavefront into an array and applied a discrete Fourier transform from the SciPy library to convert to a PSF. To finally convert to a realistic image, three more effects needed to be added, atmospheric seeing, pixelation, and noise. Atmospheric seeing can be added by convoluting the pure PSF with the inverse transform of the modular transfer function, $e^{-3.44\left(\frac{r}{r_{0}}\right)^{5 / 3}}$, describing the Kolmogorov model of atmospheric turbulence.[3] $r_{0}$, called the Fried parameter, expresses the degree of atmospheric seeing. Similarly, pixelation can be incorporated by convoluting the PSF with the box function, defined to be 1 inside a square the same size of a pixel, and 0 elsewhere.[4] Noise can be included by adding two normally distributed random variables; the first is photostatistics noise with a Poisson distribution, and second is read noise of order 10 photons. Finally, using the PyFITS module, a standard astronomical FITS (Flexible Image Transport System) format image can be created. A typical image is shown Figure 3. Note that the hole in the center is due to the obscuration of the camera inside the telescope.

The primary tool and object of study is, of course, the code used to fit images of stars. The code was written in Python and previously written by Aaron Roodman. The code makes use of a number Python libraries located on the KIPAC software directory including NumPy, SciPy, ROOT, PyFITS, and FFTW. The routine was run on a typical Linux desktop at KIPAC. The code was split into two parts. The first 
FitFunc, takes an input FITS image, defines constants used in the fit, and provides the machinery used to calculate the parameters to be fitted and the value from the fit. The FFTW (Fastest Fourier Transform in the West) package is used to increase the speed of fitting. The second part, called focusfit, uses the TMinuit package from CERN's ROOT library to perform the minimization for the fit and returns statistical data about the fit, including parameters and errors, a correlation matrix, and the value. The parameters returned by the fit are listed in Table 1. nPhotons represents the total flux of the star received in units of photons (or rather electrons, since that is what is actually counted in a CCD). $x O f f s e t$ and $y O f f s e t$ describe the coordinates of the center of the star in units of pixels. $A_{d}, A_{t_{x}}$, and $A_{t_{y}}$ are Seidel coefficients of aberration for defocus, and $x$ and $y$ tilt respectively. In addition, the capability to fit for a background and $r_{0}$ were added later to accomodate the real images from Mosaic II. The fit works much in the same way that artificial images are created. Using a starting set of Zernike coefficients and the initial parameters given to the fit, a default PSF is created using the same procedure described to create artificial images. Then, as the fit moves through parameter space, the PSF is recalculated as neccessary, and the difference between the real and calculated image is used to calculate a $\chi^{2}$ value. This fit is unique in that photostatistics Poisson errors plus read noise are used as the errors in the $\chi^{2}$ formula. The fit iterates this calculation until a minimum $\chi^{2}$ value is found.

$A_{d}$ is the coefficient in front of the term proportional to $\rho^{2}$ in a series expansion of the wavefront. It is particularly useful since it is related to the shift in position of the camera along the longitudinal axis of the telescope via the formula,

$$
A_{d}=\frac{n}{8 F^{2} \lambda} \Delta z
$$

where $\Delta z$ is the shift in camera position along the longitudinal $z$ axis $^{1}, \lambda$ is the wavelength of light, $n$ is the index of refraction (which can be taken to be effectively 1), and $F$ is the f-number, the ratio of the focal length (the distance from the exit pupil to the focal point) to the diameter of the exit pupil.[5] Note that this formula is approximate and only holds for small $\Delta z . A_{t_{x}}$ and $A_{t_{y}}$ are the coefficients of the terms proportional $\rho \cos \theta$ to $\rho \sin \theta$ respectively. They are useful because they can be related directly to the angle of tilt about the $y$ and $x$ axes respectively, namely they are directly proportional to the angle of tilt by the factor $n a / \lambda$ where $a$ is the radius of the exit pupil of the camera.[6]

For the next test of the fit, images were provided by David Burke from the Mosaic II imager currently installed on the Blanco telescope. Images were taken in a variety of telescope orientations to see if the fit could detect any mechanical fluctuations. For a given telescope orientation, sets of star images were taken using the entire detector plane of Mosaic with a special procedure to gauge the ability to detect shift in the

\footnotetext{
${ }^{1}$ A note on signs: Here the convention will be used that "plus" means a camera shift toward the primary mirror and "minus" means away from it.
} 
$z$ direction. The camera was placed either plus or minus 1200 microns out of focus. After the first image was taken in such a configuration, the camera was shifted by 100 microns, and the charge on the CCDs was shifted by 200 pixels, maintaing the previous image. Then another image was taken, and the charge was shifted 100 pixels. The same was done 3 more times, so there were 5 images of the same set of stars lined up on the same CCD, spaced by increments of 200-100-100-100 pixels. A typical set of 5 images of the same star is shown in Figure 4. The first 200 pixel shift is done so there is a way of determining which of the images of the same star was taken first. Additionally, in-focus images were taken to test the abilities to detect variations in tilt. To handle the real images, some changes needed to be made to the fitting code. All images have a background level, so the capability to fit for a constant background was added. In addition, the $r_{0}$ fluctuates considerably over time, so it is beneficial to fit for it as well. After flat-fielding the images and cutting out individual star images into square "postage stamps", they are ready to fit.

\section{Results}

To quantify the reliability of the algorithm to detect defocus, i.e. shifts in $z$ position along the longitudinal axis of the telescope, plots of $A_{d}$ vs. $\Delta z$ were made and the slope of a linear fit compared to the theoretical value $\frac{n}{8 F^{2} \lambda}$ predicted by (1). The $F$ number used in this formula was obtained from Zemax and has a value of 2.990 for DECam and 2.908 for Mosaic II. For artificial images, an average wavelength of $\lambda=756 \mathrm{~nm}$ was used to create the image. For images from Mosaic II, the filter used has a central value of $\lambda=644 \mathrm{~nm}$.[7] Issues regarding the use of these numbers will be discussed later.

Using the Zemax model of DECam and the Blanco Telescope, images were created in the vicinity of the focus and alignment sensors at \pm 1.5 millimeters out of focus with 20 micron increments in $\Delta z$. The images were fit, and the values of $A_{d}$ returned by the fit were plotted against the shifts in $z$ used in the Zemax model. The plots are shown in Figures 5 and 6 along with statistical data. Similarly, the same procedure was done for sets of 5 star images taken from Mosaic II. An example of fitting $A_{d}$ vs. $\Delta z$ for a set of star images at $+1.2 \mathrm{~mm}$ out of focus and moving out of focus by steps of 100 microns is shown in Figure 7. (This is the same set of stars as shown in Figure 4.) An example of the data returned by the fit of a single image is shown in Table 1, in this case the first star image in Figure 4. Figure 8 shows the calculated best-fit image, a difference image between the calculated and real images, and the real image of a single star, the same as was fitted from Table 1 . Table 2 shows the value of the slope of $A_{d}$ vs. $\Delta z$ for fits of star image sets at both plus or minus 1200 microns. The value of $r_{0}$ returned by the fit of the first star image and the measured value of $r_{0}$ at the time the image was taken are shown in Table 3 for both sets plus and minus 1200 microns out of focus. 


\section{Conclusions}

The $A_{d}$ vs. $\Delta z$ fits of artificial images in Figures 5 and 6 show an excellent linear fit, but the slope is strangely high for both cases. However, the slopes produced by both are consistent. The $A_{d}$ vs. $\Delta z$ plot in Figure 7 from the Mosaic image does show a linear relationship, however, the high $\chi^{2}$ value indicates there is a systematic effect that is not being taken account. This is universal of all star sets from Mosaic so far fitted. Table 2 shows that the slope of $A_{d}$ vs. $\Delta z$ is being consistently being overestimated regardless of location. The excessive slope therefore seems to be a plague of all fits. Either the value of $\frac{n}{8 F^{2} \lambda}$ being used as comparison is wrong or there is a disease on the algorithm that must be found. The simple formula from (1) can be ambiguous. An 8 wavelength set from $700 \mathrm{~nm}$ to $825 \mathrm{~nm}$ in Zemax was used to create artificial images, and the average wavelength was used in (1). This may be a false assumption. Similarly, the red filter used on Mosaic to take images extends over a relatively large range of wavelengths of FWHM $151 \mathrm{~nm}$ (a transmission diagram illustrates this in Figure 9).[8] The central wavelength was used in (1), however this may also be false. Also problematic may be the f-number. Zemax returns the effective f-number of the entire optical system; however, it is the f-number of the light cone converging on the focal point to which (1) refers.

Furthermore, there is a clear pattern to the difference plot shown in Figure 8. This pattern is persistent for each image in a set of 5 , but it is unique to each set of images. What systematic effect causes these difference pattern needs to be investigated. This will lead to an improvement of fit quality for all sets of images, which all seem to share similarly poor $\chi^{2}$ values so far. Finally, Table 3 shows that the fit consistently does not estimate $r_{0}$ to within the error bounds returned, which may be related to unmodeled systematic effects discussed before or it may be that the fit is not sensitive to fitting for $r_{0}$.

To summarize the problems and future issues:

1. Why are the fits of real images so poor? What effect is not being modeled by the fit?

2. In fits from real images, what causes the large deviations from the theoretical straight line relation for $A_{d}$ vs. $\Delta z$ as well as deviations from the measured values of $r_{0}$ ? Why is the slope of $A_{d}$ vs. $\Delta z$ consistently larger than expected?

3. Investigations of the reliability of the tilt coefficients $A_{t_{x}}$ and $A_{t_{y}}$ have yet to be done.

4. Heretofore not mentioned, timing is a critical issue as focus and alignment data will be used after every other image, which will be read out in approximately 17 seconds and have exposure times of $\approx 100$ 
seconds[9]. Currently, fits of real images take 3 to 6 minutes to complete. Optimization strategies will have to be investigated.

Each of these problems will require further work; however, the method itself is very promising. The development of the algorithm is only in its beginning stages, and much more work needs to be done to realize its full potential.

\section{Acknowledgments}

This work was conducted at SLAC National Laboratory and supported by the United States Department of Energy, Office of Science and SLAC. Thanks go to my mentor Aaron Roodman for his generous guidance, patience, and support, David Burke for collecting the data from Mosaic II and providing support in analyzing that data, Reyna Garcia for preparing images for fitting, and the SULI staff who work to make the program run smoothly.

\section{Literature Cited}

[1] "The Dark Energy Survey Science Program," [Online]. Available: http://web.hep.uiuc.edu/home/jjt/DES_docs/DES_Science_Program.pdf. [Accessed: Aug. 12, 2009].

[2] "Instrument," Dark Energy Survey Data Managment, [Online]. Available:

http://cosmology.uiuc.edu/DES/desproject/instrument.html. [Accessed: Aug. 12, 2009].

[3] D. J. Schroeder, Astronomical Optics, 2nd ed. San Diego: Academic Press, 2000, pp. 412.

[4] D. J. Schroeder, Astronomical Optics, 2nd ed. San Diego: Academic Press, 2000, pp. 427.

[5] V. N. Mahajan, Aberration Theory Made Simple, New York: SPIE-International Society for Optical Engineers, pp. 9.

[6] V. N. Mahajan, Aberration Theory Made Simple, New York: SPIE-International Society for Optical Engineers, pp. 10.

[7] "CTIO Mosaic II Imager User Manual," Cerro Tololo Inter-American Observatory, [Online]. Available: http://www.ctio.noao.edu/mosaic/manual/index.html. [Accessed: Aug. 12, 2009].

[8] "CTIO Mosaic II Imager User Manual," Cerro Tololo Inter-American Observatory, [Online]. Available: http://www.ctio.noao.edu/mosaic/manual/index.html. [Accessed: Aug. 12, 2009].

[9] "Instrument," Dark Energy Survey Data Managment, [Online]. Available:

http://cosmology.uiuc.edu/DES/desproject/instrument.html. [Accessed: Aug. 12, 2009]. 


\section{Figures and Tables}

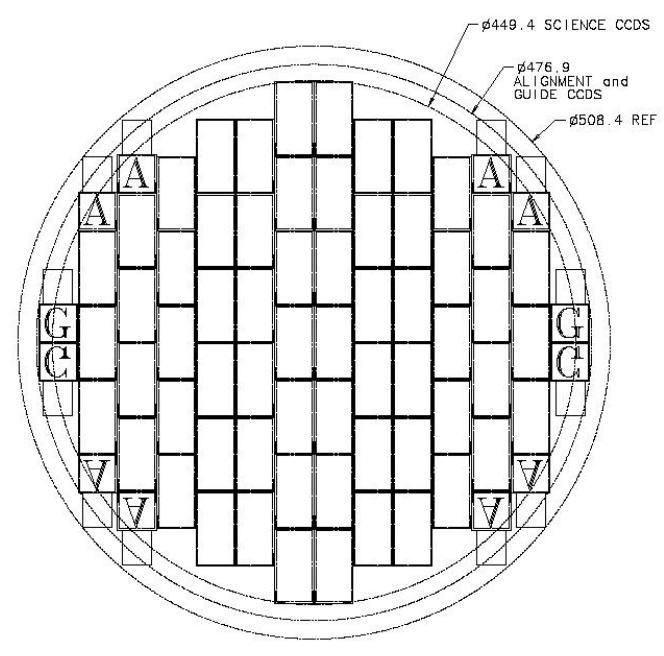

Figure 1: The focal plane of DECam; the focus and alignment sensors are indicated by the letter A.

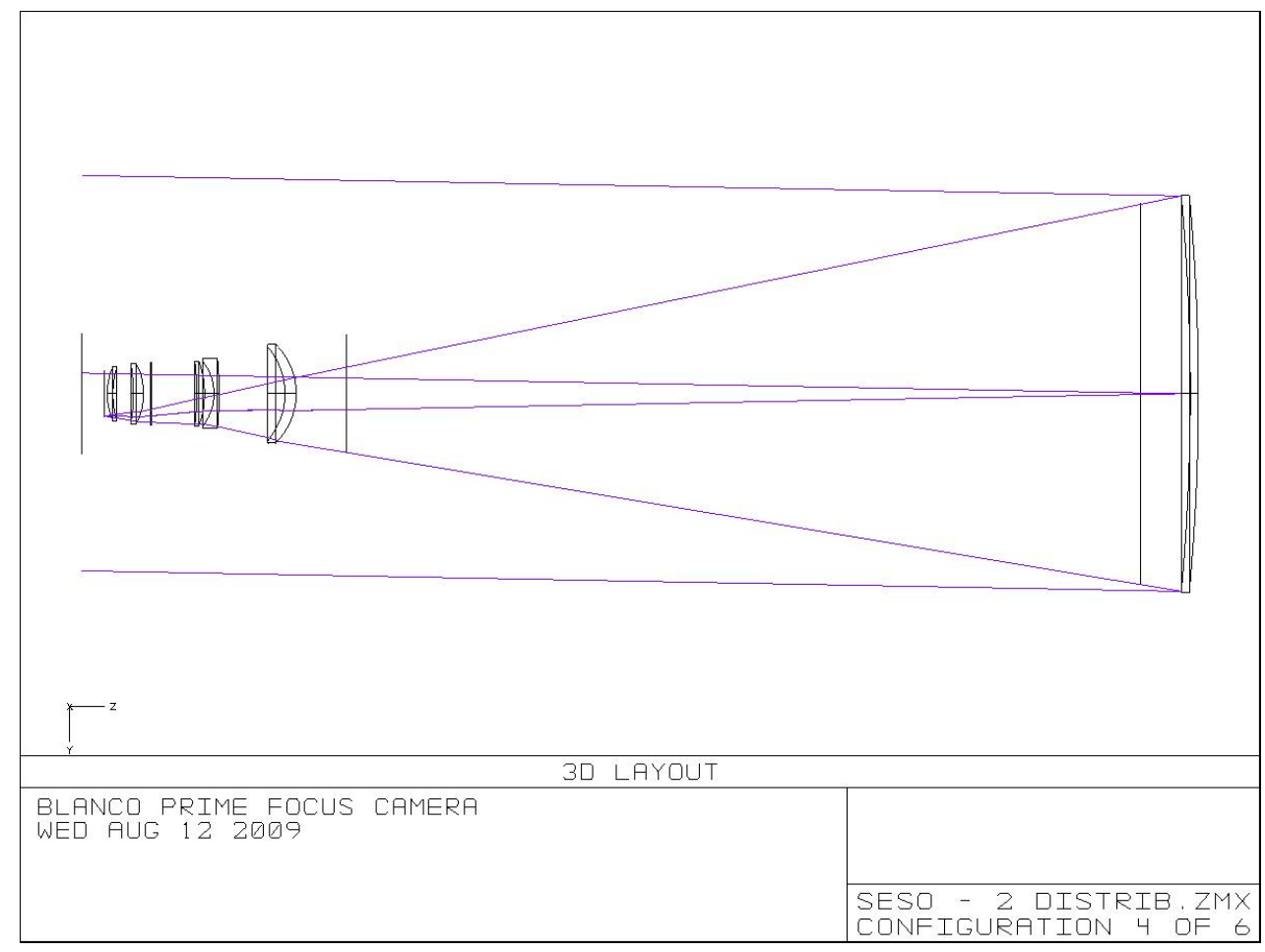

Figure 2: A diagram of DECam and Blanco in Zemax also depicting rays converging on a focus and alignment sensor. 


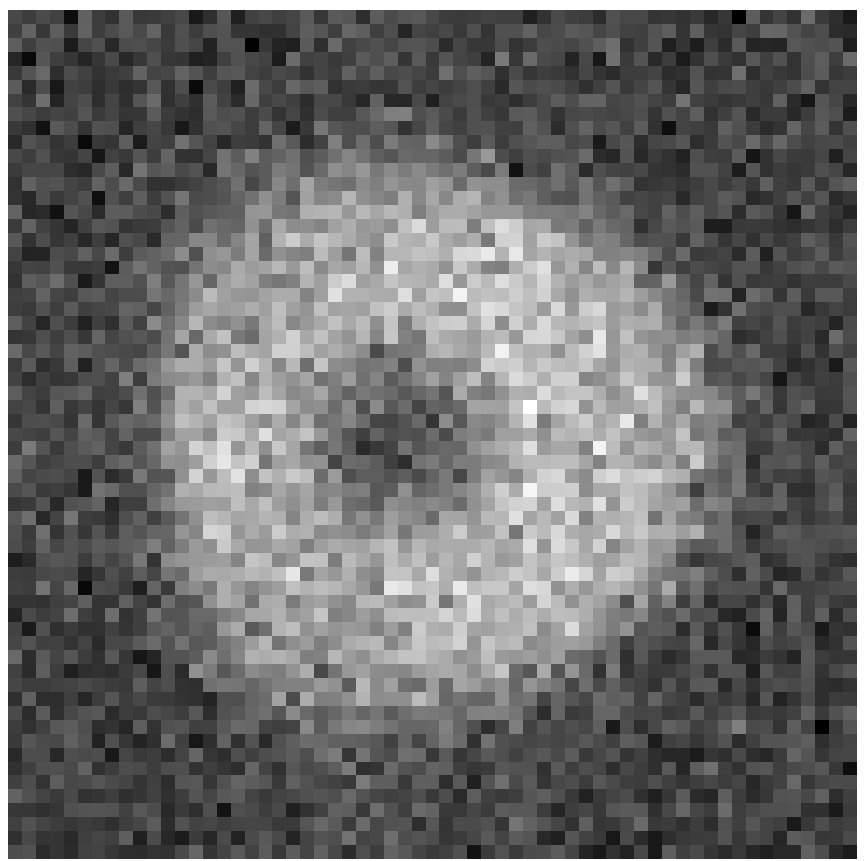

Figure 3: An artificial star image created at a sensor $1.5 \mathrm{~mm}$ below the focal plane, while the camera was in-focus.

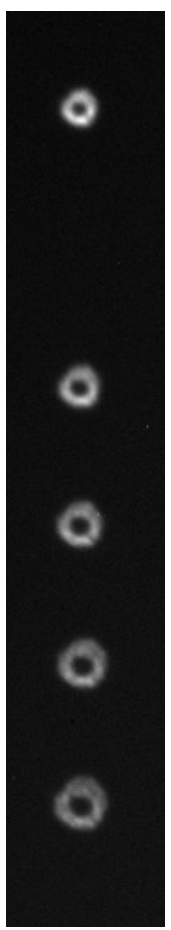

Figure 4: A cutout of a set of 5 star images from Mosaic II at $+1.2 \mathrm{~mm}$ out of focus and moving further out of focus by increments of 100 microns. Many sets of 5 star images are found on every CCD. 


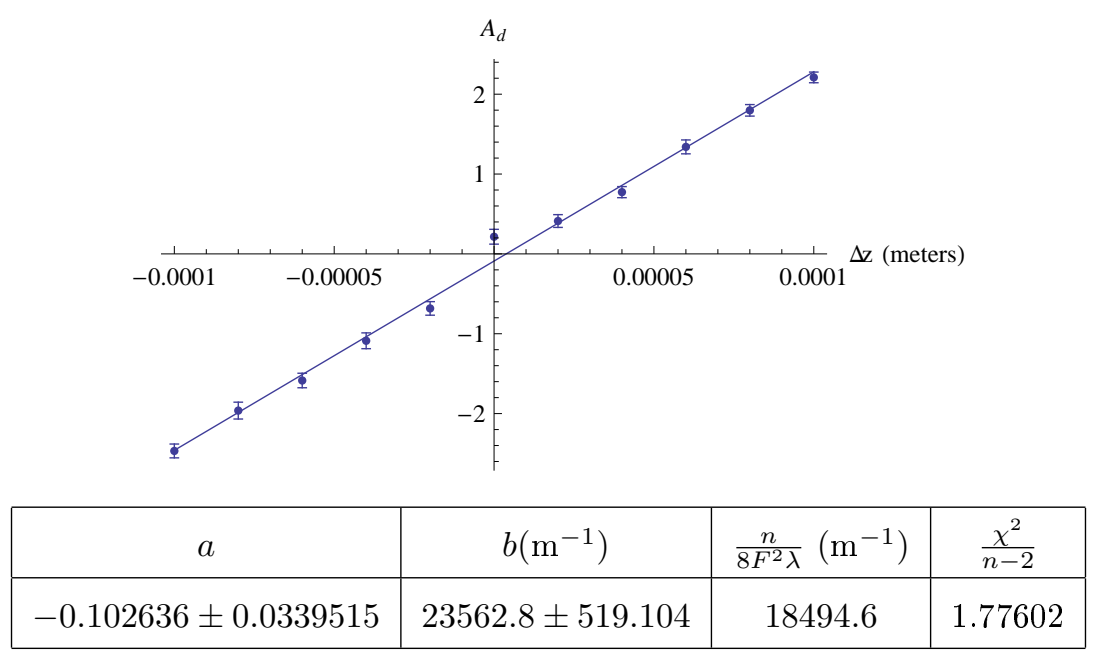

Figure 5: A linear fit of $A_{d}$ vs. $\Delta z$ to $a+b x$ with images from one of the focus and alignment sensors +1.5 $\mathrm{mm}$ above the focal plane.

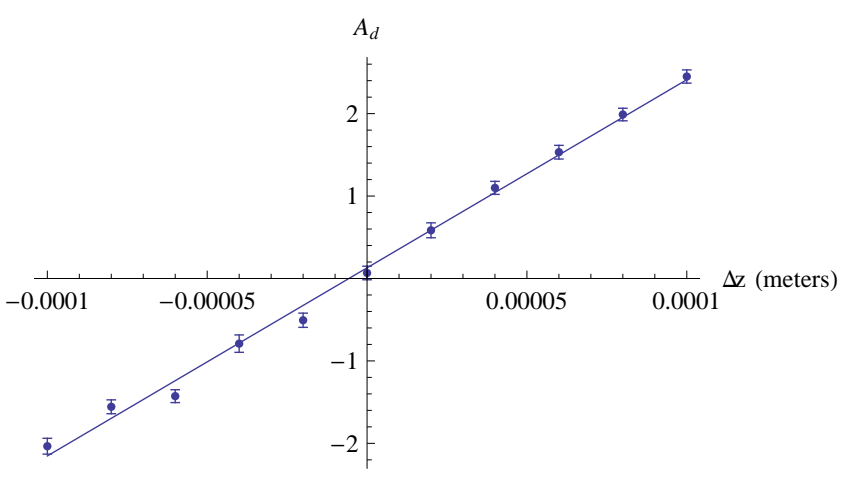

\begin{tabular}{|c|c|c|c|}
\hline$a$ & $b\left(\mathrm{~m}^{-1}\right)$ & $\frac{n}{8 F^{2} \lambda}\left(\mathrm{m}^{-1}\right)$ & $\frac{\chi^{2}}{n-2}$ \\
\hline $0.124069 \pm 0.0338838$ & $23022.7 \pm 531.66$ & 18494.6 & 1.75718 \\
\hline
\end{tabular}

Figure 6: The same plot as in Figure 5 only with images taken from a sensor $1.5 \mathrm{~mm}$ below the focal plane.

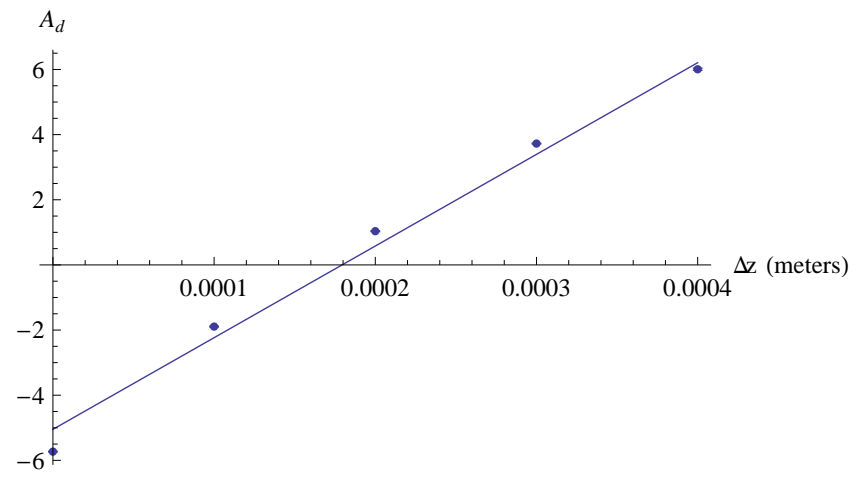

\begin{tabular}{|c|c|c|c|}
\hline$a$ & $b\left(\mathrm{~m}^{-1}\right)$ & $\frac{n}{8 F^{2} \lambda}\left(\mathrm{m}^{-1}\right)$ & $\frac{\chi^{2}}{n-2}$ \\
\hline$-5.45017 \pm 0.303405$ & $31082.0 \pm 1587.32$ & 22952.8 & 1.75718 \\
\hline
\end{tabular}

Figure 7: A fit of $A_{d}$ vs. $\Delta z$ from Mosaic II with the camera starting from $+1.2 \mathrm{~mm}$ out of focus. 


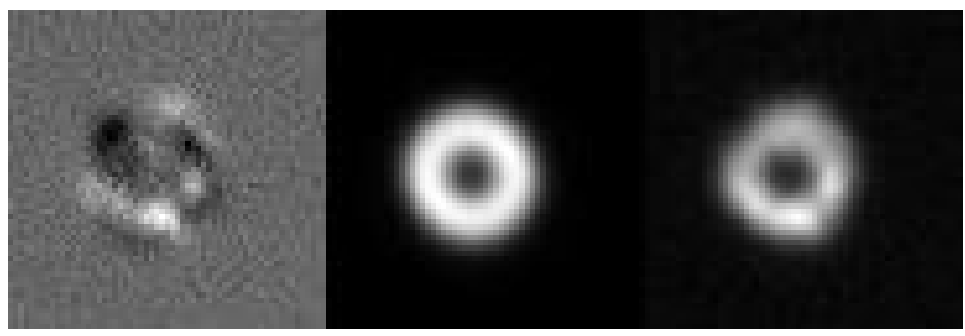

Figure 8: The difference image, the best-fit calculated image, and the actual image for the first image of the star set shown in Figure 3.

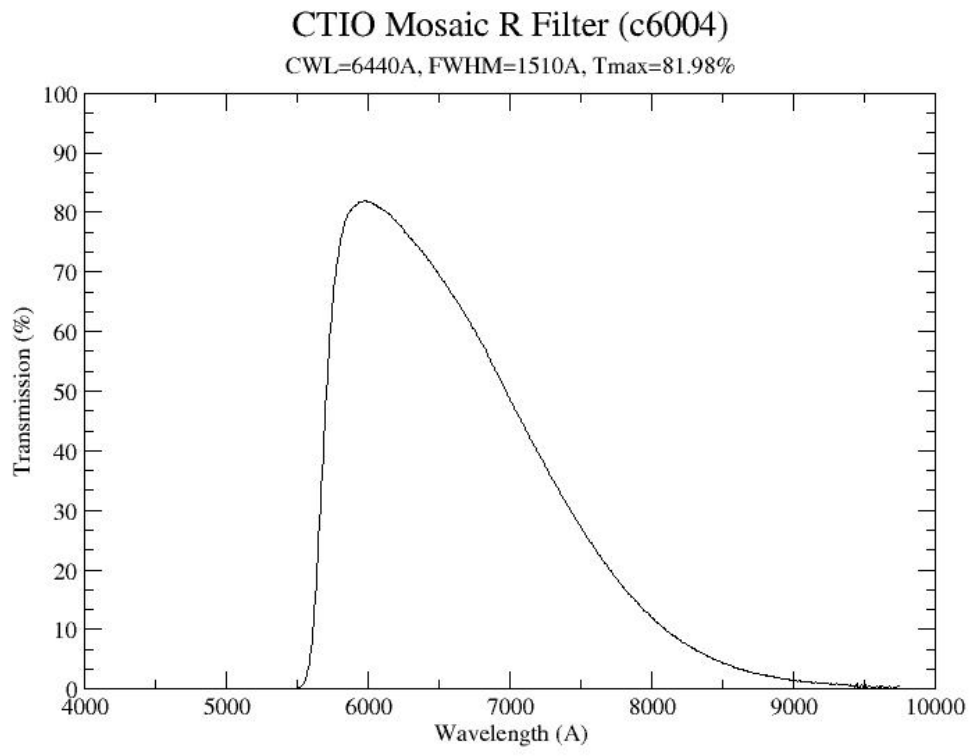

Figure 9: The transmission diagram for the red filter used on Mosaic II.

\begin{tabular}{|c|c|c|c|c|c|c|c|c|}
\hline Correlation Matrix & nPhotons & $x$ Offset & yOffset & $A_{d}$ & $A_{t_{x}}$ & $A_{t_{y}}$ & $r_{0}$ & bkgd \\
\hline nPhotons & 1 & -0.013 & -0.013 & 0.160 & 0.015 & 0.022 & -0.535 & -0.492 \\
\hline$x$ Off set & -0.013 & 1 & -0.026 & -0.094 & 0.002 & -0.030 & -0.011 & 0.008 \\
\hline$y$ Off set & -0.013 & -0.026 & 1 & -0.004 & -0.001 & -0.003 & 0.023 & 0.008 \\
\hline$A_{d}$ & 0.160 & -0.094 & -0.004 & 1 & -0.178 & 0.216 & 0.129 & -0.099 \\
\hline$A_{t_{x}}$ & 0.015 & 0.002 & -0.001 & -0.178 & 1 & 0.760 & -0.144 & -0.010 \\
\hline$A_{t_{y}}$ & 0.022 & -0.030 & -0.003 & 0.216 & 0.760 & 1 & -0.055 & -0.014 \\
\hline$r_{0}$ & -0.535 & -0.011 & 0.023 & 0.129 & -0.144 & -0.055 & 1 & 0.333 \\
\hline$b k g d$ & -0.492 & 0.008 & 0.008 & -0.099 & -0.010 & -0.014 & 0.333 & 1 \\
\hline
\end{tabular}




\begin{tabular}{|c|c|c|}
\hline Parameter & Value & Error \\
\hline nPhotons & $2.34314 \times 10^{6}$ & $3.33661 \times 10^{3}$ \\
\hline$x$ Offset & 1.77510 & $1.42374 \times 10^{-2}$ \\
\hline$y$ Offset & 5.48489 & $1.38277 \times 10^{-2}$ \\
\hline$A_{d}$ & -5.73230 & $1.00898 \times 10^{-2}$ \\
\hline$A_{t_{x}}$ & $-5.47409 \times 10^{-1}$ & $2.92589 \times 10^{-2}$ \\
\hline$A_{t_{y}}$ & $6.22443 \times 10^{-1}$ & $2.80884 \times 10^{-2}$ \\
\hline$r_{0}$ & $0.974637 \times 10^{-2}$ & $2.96489 \times 10^{-4}$ \\
\hline$b k g d$ & $5.28672 \times 10^{3}$ & 1.43845 \\
\hline
\end{tabular}

Table 1: The data returned by a typical fit, in this case that of the first image of Figure 3 . The $\chi^{2}$ value of this fit was 25396.7.

\begin{tabular}{|c|c|}
\hline Slope of $A_{d}$ vs. $\Delta z\left(\mathrm{~m}^{-1}\right)$ & Location \\
\hline $31082.0 \pm 1587.33$ & $+1.2 \mathrm{~mm}$ \\
\hline $25962.3 \pm 1874.5$ & $+1.2 \mathrm{~mm}$ \\
\hline $27663.0 \pm 1264.1$ & $+1.2 \mathrm{~mm}$ \\
\hline $32062.4 \pm 1264$ & $-1.2 \mathrm{~mm}$ \\
\hline $29736.0 \pm 1189.2$ & $-1.2 \mathrm{~mm}$ \\
\hline
\end{tabular}

Table 2: Slopes from $A_{d}$ vs. $\Delta z$ fits at a variety of locations. The expected value is.

\begin{tabular}{|c|c|}
\hline Starting $r_{0}(\mathrm{~m})$ & Measured $r_{0}(\mathrm{~m})$ \\
\hline $0.09746 \pm 0.00030$ & 0.1244 \\
\hline $0.1137 \pm 0.0048$ & 0.1301 \\
\hline $0.1223 \pm 0.0050$ & 0.1301 \\
\hline $0.12163 \pm 0.00034$ & 0.1211 \\
\hline $0.09811 \pm 0.00065$ & 0.1384 \\
\hline
\end{tabular}

Table 3: Values of $r_{0}$ from the fit of the first star image at several locations and measured $r_{0}$ values from the time of observation. 


\title{
Towards an Understanding of the Role of Aragonite in the Mechanical Properties of Nacre
}

\author{
Marc S. Davidson \\ Office of Science, Science Undergraduate Laboratory Internship (SULI) \\ Harvey Mudd College \\ Stanford Linear Accelerator Center \\ Stanford, CA
}

August 14, 2009

Prepared in partial fulfillment of the requirements of the Office of Science, Department of Energy's Science Undergraduate Laboratory Internship under the direction of Apurva Mehta at the Stanford Synchrotron Radiation Lightsource, Stanford Linear Accelerator Center.

Participant:

Signature

Research Advisor:

Signature 


\section{TABLE OF CONTENTS}

$\begin{array}{ll}\text { Abstract } & \text { ii }\end{array}$

$\begin{array}{ll}\text { Introduction } & 1\end{array}$

Materials and Methods 2

$\begin{array}{lc}\text { Results } & 5\end{array}$

$\begin{array}{ll}\text { Discussion and Conclusions } & 7\end{array}$

$\begin{array}{lr}\text { Acknowledgments } & 9\end{array}$

$\begin{array}{lr}\text { References } & 9\end{array}$ 


\begin{abstract}
Towards an Understanding of the Role of Aragonite in the Mechanical Properties of Nacre. MARC S. DAVIDSON (Harvey Mudd College, Claremont, CA, 91711) APURVA MEHTA (Stanford Synchrotron Radiation Lightsource, Stanford Linear Accelerator Center, Stanford, CA 94025)
\end{abstract}

Nacre, also known as mother-of-pearl, is a biocomposite material that exhibits higher strength and fracture toughness than its component materials. It derives its strength from the brick-and-mortar layering of aragonite $\left(\mathrm{CaCO}_{3}\right)$ platelets and organic binder. It is believed that the protein binder helps redistribute the stress throughout all tablets for optimal mechanical performance. In this study, we attempt to measure the mechanical properties of aragonite within nacre and compare them to bulk aragonite and bulk nacre and understand the redistribution of stresses. Here we show that x-ray diffraction techniques are useful for isolating and measuring strain of crystallites within a composite material. Our results show that the apparent stiffness of aragonite varies with crystallographic directions and is higher than the stiffness of bulk nacre in all cases, meaning that aragonite tablets are exposed to less than the average bulk stress. The average force applied to the bulk sample is partitioned between the aragonite and the binder, so that the protein layer bears as much as $27.2 \%$ of the total applied force. Different crystallographic directions exhibit behaviors different than bulk aragonite or bulk nacre. These are related to texture of aragonite platelets (i.e. preferred orientations within nacre). By examining nacre, we can obtain a better understanding of the mechanical relationship between the ceramic and polymer in composite materials. We expect that x-ray diffraction will become the standard method for probing the mechanical properties of composite materials. 


\section{INTRODUCTION}

Nacre, commonly known as mother-of-pearl, is a biocomposite material found lining the shells of many mollusks. It has a non-isotropic microstructure composed of aragonite $\left(\mathrm{CaCO}_{3}\right)$ platelets bound together by organic polymers. Depending on the species, these platelets can be arranged in columns or in a staggered formation. The platelets are polygonal with diameter $10 \mu \mathrm{m}$ and thickness of $0.3 \mu \mathrm{m}$ [1]. Aragonite, which constitutes approximately $95 \%$ of nacre by volume [2], has an elastic modulus between 80 and $205 \mathrm{GPa}$, depending on the crystallographic direction $[3,4,5,6]$.

Bulk aragonite has a strength of $30 \mathrm{MPa}$. Nacre, however, has a strength of $170 \mathrm{MPa}$ or more and exhibits brittle fracture upon failure $[1,2,7]$. Nacre has an extremely high interlaminar fracture toughness, believed to be created by the protein matrix [8, 9]. The elastic modulus has been measured to be $72.6 \mathrm{GPa}[10]$. Currey showed that nacre is extremely good at stopping cracks caused by external sources. The work of fracture has been measured to be $1650 \mathrm{~J} / \mathrm{m}^{2}$ - much higher than aragonite itself [1]. Fleischli et al. demonstrated that nacre has a fracture toughness of $2.9 \mathrm{MPa} \mathrm{m}^{1 / 2}$ [10], which is 3000 times higher than that of aragonite. The mechanical properties of nacre are much more desirable than those of aragonite alone, due to its composite structure.

To understand the emergent behavior in this composite material, the interactions between the individual components must be understood. To do so requires a characterization technique that allows the isolation of the properties of one material within the composite. X-ray diffraction is such a technique and therefore ideal for investigating the origin of nacre's emergent mechanical properties.

The aragonite platelets (each a single crystal) diffract an incident x-ray beam. The organic material is not crystalline in nature and makes only $5 \%$ by volume of nacre; therefore its diffraction pattern is very weak and not easy to separate from the diffraction pattern for 
aragonite. The diffraction pattern, collected with a large area detector, can be analyzed with reciprocal space techniques to determine the strain present in the aragonite tablets [11].

In this work, we are investigating why nacre has superior properties when it is composed of weak materials. To do this, we will characterize the partitioning of forces between aragonite and organic polymer in nacre. We will restrict this study to uniaxial load as it presents the simplest loading geometry. As uniaxial loads are applied, the apparent elastic modulus for aragonite in nacre can be determined and can be used to characterize force partitioning. Elastic modulus can therefore easily be extracted without making many assumptions. This will establish methods for examining crystalline components of composite materials while explaining the relationship between the structure and strength of nacre.

\section{MATERIALS AND METHODS}

\section{Epoxy Selection}

Previous studies in this lab to examine properties of nacre under tension were limited by the method to attach samples to the test rig. Samples tested during July, 2008 used A+B Epoxy Paste, experienced failure at the epoxy-sample boundary. As a result, the tensile region of the stress-strain curve could not be determined. In order to collect tensile data, a stronger epoxy was required.

Epoxies to test were selected based on shear strength published in technical data sheets. 3M 1838 B/A Green epoxy has a reported shear strength of 3000 psi [12] and Armstrong A-661 [13] has a shear strength of 3300 psi. These two epoxies were selected for testing along with epoxies used previously in this lab. Of these epoxies, A+B Fast Epoxy Paste and Loctite QuickSet 5 Minute Epoxy did not provide technical data sheets.

Epoxies were tested using aluminum holders designed to simulate the conditions present in tensile testing of nacre. The holders have slots with width and depth of $1.9 \mathrm{~mm}$ and a 
length of $5.7 \mathrm{~mm}$. This creates an aspect ratio for the slot of 3.00 , which is close to the that of the nacre grips which have an aspect ratio of 2.85. Steel wires with diameter $1.4 \mathrm{~mm}$ were prepared by sanding the surface to be bonded in order to roughen it. This surface was coated in epoxy and placed in the holders to cure overnight. A tensile load was applied to the samples with a MTS test rig with a $1 \mathrm{kN}$ load cell at Stanford University. Samples were elongated at $10 \mu \mathrm{m} \mathrm{s}^{-1}$ until failure occurred. The load at failure was compared to evaluate the relative strengths of these epoxies.

\section{Internal Standard Selection}

A powder x-ray diffraction standard was desired for calibration of x-ray diffraction images. A wrongly calibrated image can show strain in the diffraction rings that is not present in the sample. The calibration standard would be a powder applied directly to the sample. Then each image can be calibrated to the standard powder rings before strain analysis is performed. The ideal standard would have well-defined diffraction rings that don't interfere with any of the diffraction rings of nacre. Such a standard could not be found - every standard we examined overlapped with some of the nacre diffraction rings.

Diffraction data was calibrated instead with external $\mathrm{LaB}_{6}$ powder placed next to the sample. $\mathrm{LaB}_{6}$ is widely used as a diffraction standard. Silver powder applied to one edge of the gage section was selected as a secondary internal standard despite overlap with nacre rings.

\section{Sample Preparation}

Rectangular prismatic samples of red abalone were prepared by Karen Magid and coworkers in Zurich in a materials science laboratory. Sample cross-sections ranged from 88,900 $\mathrm{m}^{2}$ to $185,760 \mu \mathrm{m}^{2}$. The corresponding maximum stresses for these samples ranges from 237 to $495 \mathrm{MPa}$ at a force of $44.5 \mathrm{~N}$ (the upper limit of the load cell). Red Abalone was selected 
for its widespread use in scientific research.

\section{Experimental Setup}

The tension/compression test rig is comprised of the load cell, grips, and a strain gage. The load cell, shown in Figure 1, has an upper limit of 44.5 N. Epoxy was applied to samples and test grips and allowed to cure overnight at room temperature. Silver paint was applied as a secondary calibration standard to one edge of the gage section. Black spray paint was applied for use with a Digital Image Correlation (DIC) camera. This camera acts as an optical strain gage to find bulk strain of nacre, while diffraction techniques find microscopic strain of aragonite only. Figure 2 shows the entire experimental setup including the position of the DIC camera, large-area detector, sample, and x-ray beam.

\section{Data Analysis}

Diffraction data was analyzed using Area Diffraction Machine (ADM) [14] and Matlab. ADM was used to calibrate the data, convert circular diffraction patterns (Figure 3) to rectilinear

coordinates (Figure 4), and export peak lists showing the position of the diffraction rings. These rings were then analyzed with Matlab scripts to extract 3-dimensional strain for each load point, diffraction ring, and position along the length of the sample. Diffraction patterns were recalibrated using $\mathrm{LaB}_{6}$ standard at each load point to account for possible beam and sample movement. Stress-strain curves and elastic moduli were extracted from this data. Gaps in the diffraction rings represent a preferred orientation of the tablets. This direction preferences is called texture and is seen in many biological materials. Due to limitations on time, texture was not included in this analysis. 


\section{RESULTS}

The results from the epoxy experiment are detailed in Table 1. The epoxy adhered to two components with different adhesion areas and the tested strength was a combination of tensile and shear strength. For this reason, the data was left in units of force instead of stress. But under identical conditions, 3M and Armstrong epoxies performed significantly better than previously used epoxies.

Figure 5 shows the fracture surface of an epoxy sample. The epoxy separated from the steel sample along half of the curved surface. The flat sections along the bottom of the fracture surface show where the epoxy separated from the aluminum holder. Between those two regions is a jagged edge where the epoxy separated from itself. Steel-epoxy, epoxy-epoxy, and aluminum-epoxy failures were exhibited in this fracture surface. This fracture surface indicates that the three different bonds between materials have roughly the same strength in this failure mode.

In uniaxial loading, red abalone exhibited strain in x-ray diffraction rings as well as DIC. A sample diffraction pattern is shown in Figure 3. The best rings for tension and compression were selected based on the intensity, lack of near overlap with adjacent rings, and completeness of the ring (many rings were incomplete due to texture). Compression and tension data were collected on unloading, as recommended by ASTM standards. Unloading compression and tension data were analyzed separately, then combined to form one full data set encompassing both. Figure 4 shows diffraction rings after being "unwrapped" by ADM. Strain is seen as wiggles in the unwrapped peak lines.

Table 2 shows the apparent elastic modulus values for different $h k l$ crystallographic directions for tension and compression. Units are in GPa. Lattice parameters are as follows: $a=4.96 \AA, b=7.97 \AA$, and $c=5.74 \AA$. Values range from $79.3 \mathrm{GPa}([232]$ in tension $)$ to 119.3 GPa ([200] in compression). The value of elastic modulus as measured by DIC was 
72.4 GPa, lower than any value of aragonite elastic modulus. This result is preliminary further DIC data analysis will be published by Magid et al. in the near future.

Figure 6 shows stress-strain plots for 3 normal directions and 3 shear directions. The applied displacement is in the $y y$-direction. The x-ray beam is in the $x x$-direction where there are the fewest data points, so error on $x x$-strain is much larger and data is often skewed by small variations in the x-ray beam. The $z z$ - and $y y$-data is unaffected by small variations and the error is much smaller.

By comparing the elastic modulus in all three normal strain directions, we can determine Poisson's ratio for aragonite in nacre. This value was measured to be 0.33 . There was no correlation between measured strain and shear strain in any diffraction ring.

The force felt by each platelet can be estimated by examining the elongation in each crystallographic direction. By comparing the apparent elastic modulus measured by $\mathrm{x}-$ ray diffraction to the theoretical modulus based on the compliance tensor, we can find the stress intensity factor. This factor describes the force felt by tablets aligned in a particular direction.

$$
E_{\text {meas }} \frac{S I_{A}}{1-x}=E_{\text {theo }}
$$

where $E_{\text {meas }}$ and $E_{\text {theo }}$ are the measured and theoretical elastic moduli, $S I_{A}$ is the stress intensity factor for the protein, and $x$ is the volume fraction of protein (taken to be $5 \%$ ). This model is derived from a force balance and assumes that forces are distributed between aragonite platelets and the protein.

The stress intensity factor is used to compare the force felt by each individual component to the average applied force. A stress intensity factor less than 1 indicates that a component is exposed to less than its volume fraction of force. Data from Table 3 shows stress intensity values for aragonite. The stress intensity factor for aragonite is exactly the same as the ratio 
of the theoretical modulus of aragonite to the apparent (measured) modulus.

\section{DISCUSSION AND CONCLUSIONS}

The epoxy selected for use on the final samples was 3M 1838. The tested strength was $3 \%$ lower than the Armstrong epoxy. However, the 3M epoxy was much easier to measure, mix, and apply than the Armstrong epoxy. 3M 1838 was selected for use with the nacre samples due to its greatly increased strength over previously used epoxies.

X-ray diffraction is a valid technique for measuring strain in composite materials. Microstrain information, unavailable from conventional methods to measure strain, is easily found by examining the diffraction pattern of aragonite in nacre. This technique can be applied to a wide range of composite materials of natural and synthetic origin.

The measured value for Poisson's ratio of 0.33 fits perfectly with that of many other materials (a general rule of thumb is Poisson's ratio $\approx 0.3$ ). This falls within the range for aragonite measured by Zhang et al. [15]. More data is required to measure the Poisson's ratio in different crystallographic directions. Poisson's ratio can be observed in the left plots of Figure 6 by comparing the slopes (elastic moduli) in the $x x^{-}, y y^{-}$, and $z z$-directions. The three shear plots in the right plots of Figure 6 show that the applied force is uniaxial, as expected, and there is no relationship between stress and shear strain.

For all $h k l$ directions, the value for combined tension/compression data was closer to the tension-alone data. The data suggests that compression is consistently stiffer than tension, but the difference between the two is comparable to the error. Tension and compression data were grouped together to form one data set for further analysis. The different crystallographic directions exhibit elastic moduli anisotropically (see Table 2). This is the first indication that aragonite in nacre has an altered mechanical response.

Data tabulated in Table 3 shows that different crystallographic directions bear different 
amounts of total applied force. The first column is the calculated value for stress intensity factor of aragonite based on Equation 1. The second column is the ratio of theoretical to apparent elastic moduli. This should tell us the actual force experienced by each crystallographic direction. A value higher than 1 indicates that this direction experiences more stress than expected. The third column gives the estimated percentage of the load borne by protein based on the stress intensity factor for aragonite.

It appears that the estimated stress intensity factor is significantly different along certain crystallographic directions. For example, the [031] direction is 0.76 whereas the [202] direction is 1.29. Measurement of the stress intensity factor for aragonite in different crystallographic directions helps to better understand this effect. Future studies on the role of texture in nacre would elucidate the relationship between crystallographic direction and force partitioning. A detailed texture analysis could be used to find the crystallographic direction-averaged strain. Nacre samples taken in different directions could also be used to determine the role of texture in the mechanical properties of nacre.

Nature harnesses the anisotropic behavior of aragonite to create nacre, which exhibits anisotropic properties distinct from aragonite's. This additional anisotropic behavior is created by biological processes to create a superior composite material. From measured strains, we can deduce that the polymer layer bears different amounts of the applied force in different directions. The protein layer alone is not the sole source of nacre's strength and toughness — texture plays a crucial role as well.

Protein constitutes only $5 \%$ of nacre by volume, but it can absorb up to $27.2 \%$ of the applied force (seen in the [031] direction). The protein layer works to shield weak properties, such as strength of aragonite, from excessive force. The texture present in nacre helps ensure that the strongest crystallographic directions of aragonite absorb the most stress. Arrangement of aragonite platelets and interactions with the biopolymer helps partition forces among strong and weak directions ultimately resulting in superior material properties. 
However, to fully quantify this effect, texture studies are required.

\section{ACKNOWLEDGMENTS}

I would like to thank the Department of Energy, SLAC National Accelerator Laboratory, and the SULI program for funding this research. Special thanks go to my mentor, Apurva Mehta, for guidance and information about x-ray diffraction. I would like to thank Matt Bibee and Karen Magid for their time and assistance with running experiments and data analysis. I would like to thank Professor Reinhold Dauskardt and Ruiliang Jia for permitting the use of a tensile test rig. Finally, thanks to Alyssa Roessler for her preliminary work in $\mathrm{x}$-ray diffraction of nacre in this laboratory.

\section{REFERENCES}

[1] J. D. Currey, "Mechanical properties of mother of pearl in tension," Proc. R. Soc. Lond. B., pp. 443-463, 1977.

[2] F. Barthelat, H. Tang, P. D. Zavattieri, C.-M. Li, and H. D. Espinosa, "On the mechanics of mother-of-pearl: A key feature in the material hierarchical structure," J. Mech. Phys. Solids, pp. 306-337, 2007.

[3] J. D. Bass, Elasticity of Minerals, Glasses, and Melts. American Geophysical Union, 1995, pp. 45-63.

[4] D. R. Katti and K. S. Katti, "Three dimentional [sic] finite element modeling of microstructural development of nacre in seashells and implication of mineralization of $\mathrm{CaCO}_{3}, "$ Mat. Res. Soc. Symp. Proc., pp. 231-236, 2000.

[5] B. Ji and H. Gao, "Mechanical properties of nanostructured biological materials," J. Mech. Phys. Solids, pp. 1963-1990, 2004. 
[6] K. S. Katti, D. R. Katti, S. M. Pradhan, and A. Bhosle, "Platelet interlocks are the key to toughness and strength in nacre," J. Mater. Res., pp. 1097-1100, 2005.

[7] A. P. Jackson, J. F. V. .Vincent, and R. M. Turner, "The mechanical design of nacre," Proc. R. Soc. Lond. B, pp. 415-440, 1988.

[8] P. Calvert, "Biomimetic ceramics and hard composites," Biomimetics, Design and Processing Materials, Sarikaya and Askay, ed., pp. 145-161, 1995.

[9] M. Rubner, "Synthetic sea shell," Nature, pp. 925-926, 2003.

[10] F. D. Fleischli, M. Dietiker, C. Borgia, and R. Spolenak, "The inuence of internal length scales on mechanical properties in natural nanocomposites: A comparative study on inner layers of seashells," Acta Biomaterialia, pp. 1694-1706, 2008.

[11] D. Bronfenbrenner, Ph.D. dissertation, UC Berkeley, Berkeley, CA, 2008.

[12] (2002) 3M Scotch-Weld Epoxy Adhesive 1838. [Online]. Available: http://www. ellsworth.com/display/productdetail.html?productid=1131

[13] (2005) Armstrong A-661 Epoxy Resin Adhesive. [Online]. Available: http: //www.ellsworth.com/display/productdetail.html?productid=22

[14] J. Lande, D. Bronfenbrenner, A. Mehta, and S. Webb. (2008, Aug.) Area Diffraction Machine. Software. [Online]. Available: http://code.google.com/p/ areadiffractionmachine/

[15] J. M. Zhang, Y. Zhang, K. W. Xu, and V. Ji, "Young's modulus surface and poisson's ratio curve for orthorhombic crystals," J. Chem. Crystallogr., pp. 733-741, 2008. 


\section{FIGURES}

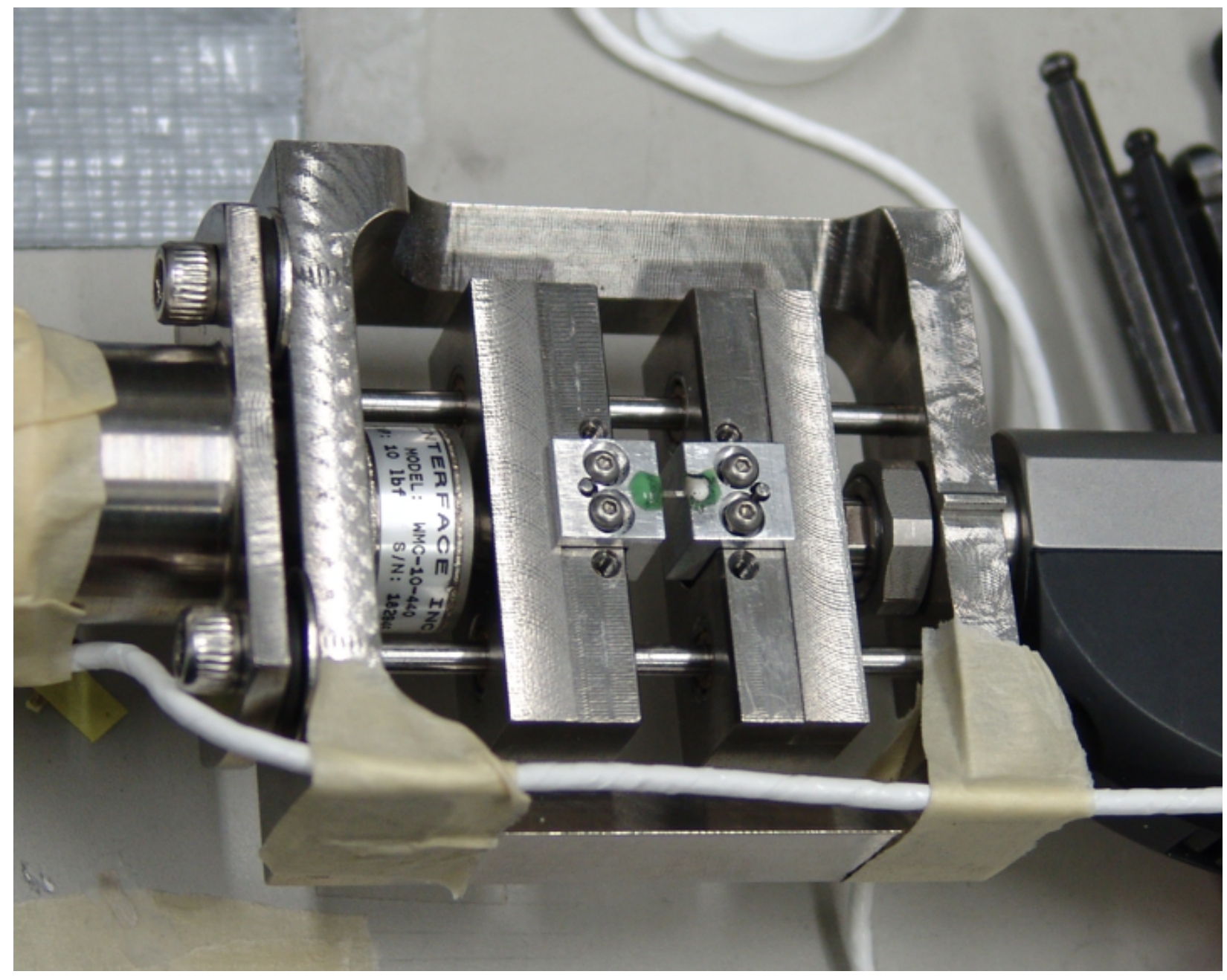

Figure 1: The test rig. The load cell is located on the left. The green epoxy and silver calibration paint are visible on the sample. 


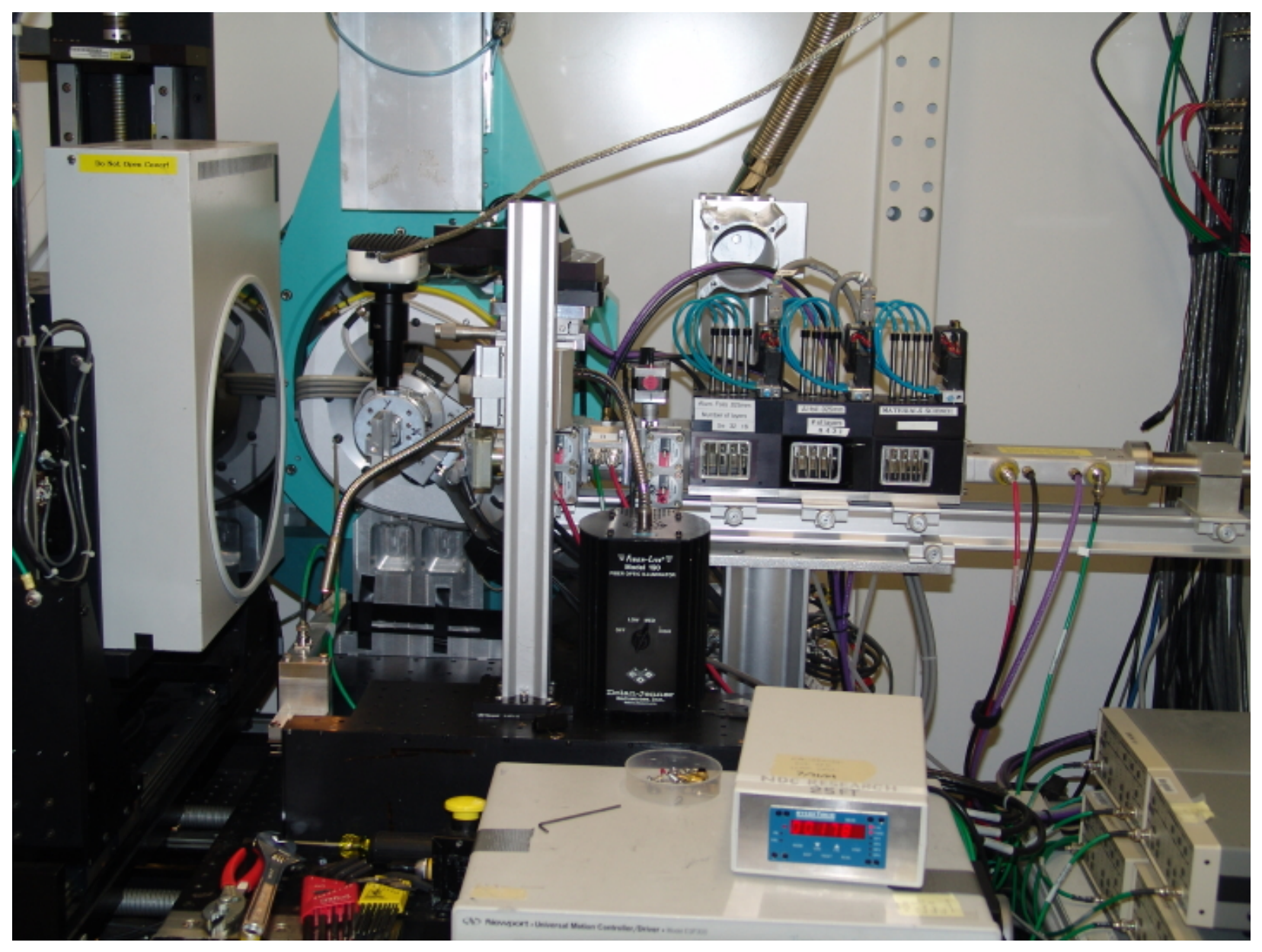

Figure 2: The x-ray diffraction setup. The x-ray beam enters from the right and diffracts off the sample. Diffraction patterns were collected using a large area detector (white box with black circle on left). The DIC camera is located above the sample. 


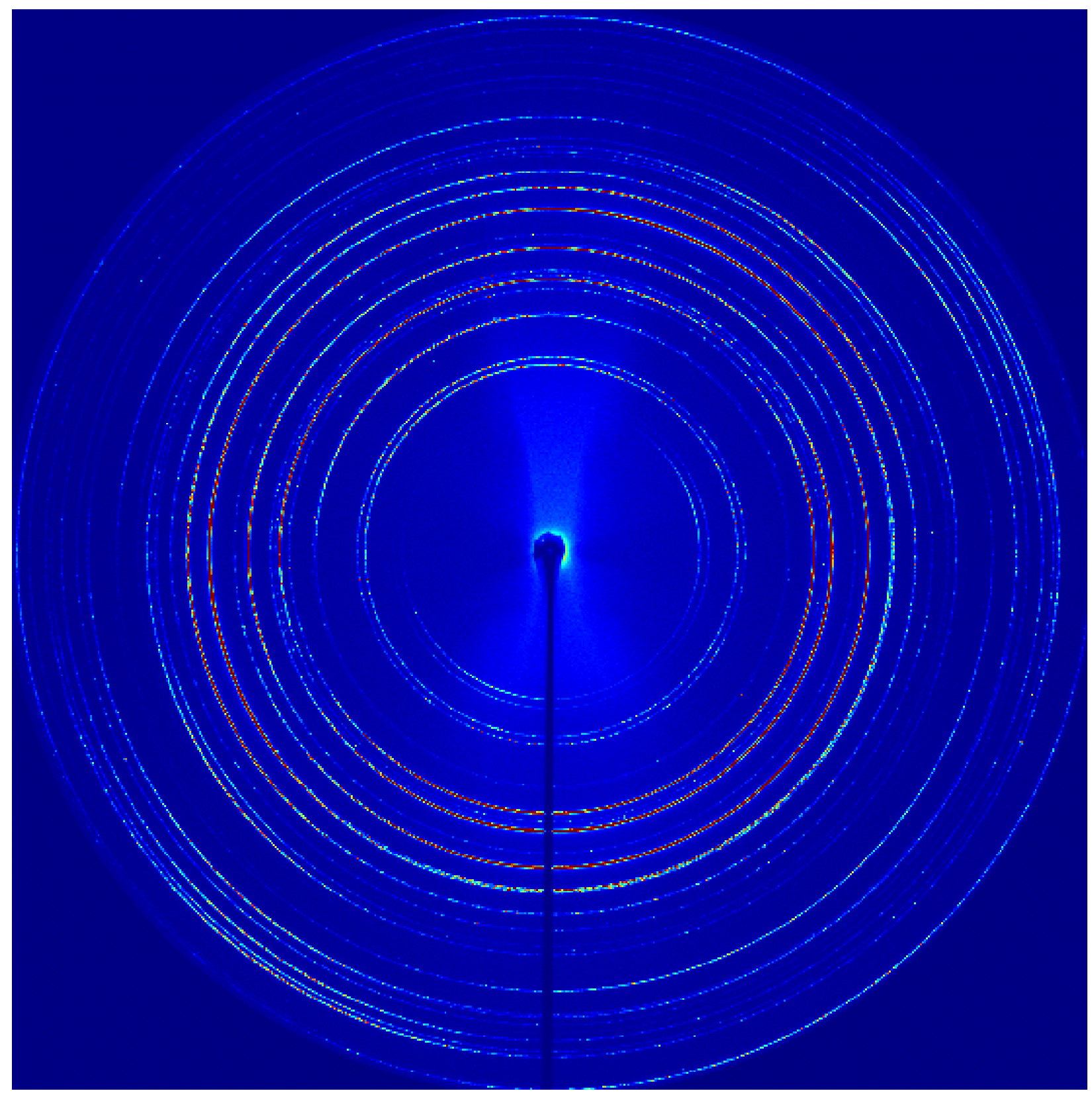

Figure 3: A circular diffraction pattern collected with a large area detector. 

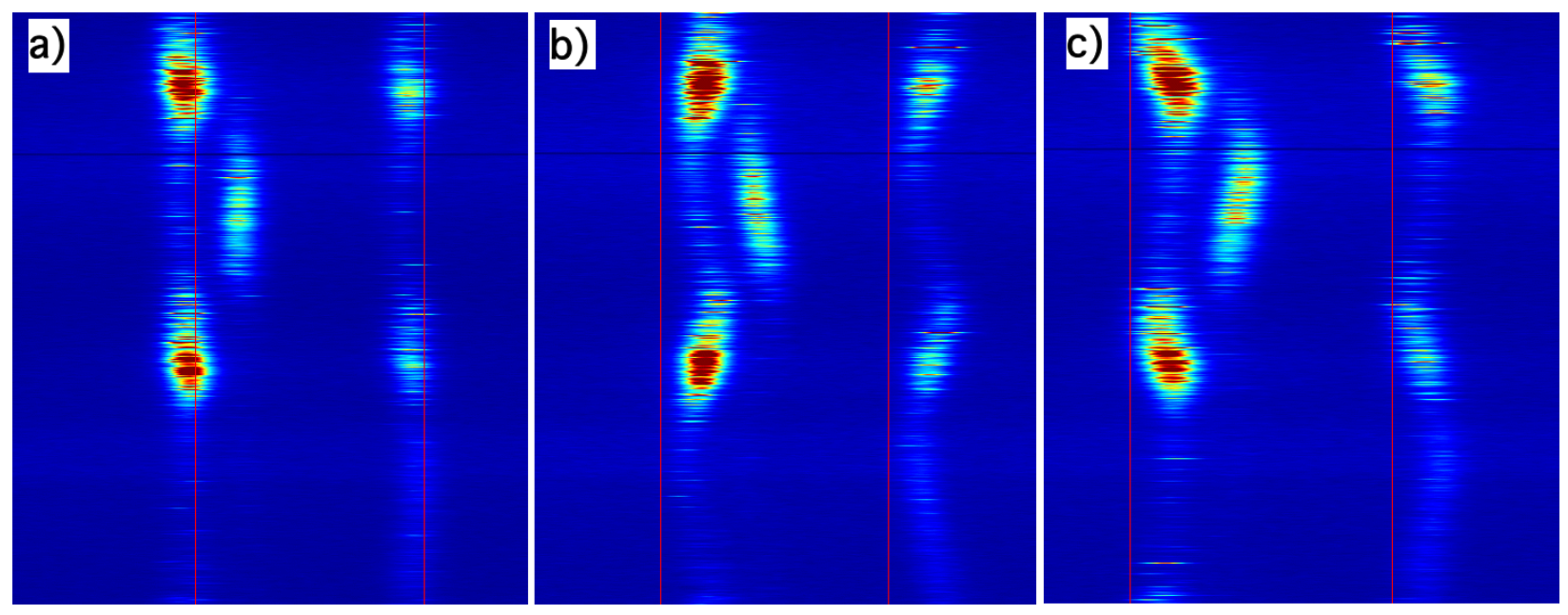

Figure 4: Diffraction rings after being "unwrapped". (a) Rings exhibiting no strain. Peaks form a straight line. (b) Rings exhibiting compression. (c) Rings exhibiting tension.

Figure 5: The fracture surface of a tensile epoxy sample. 

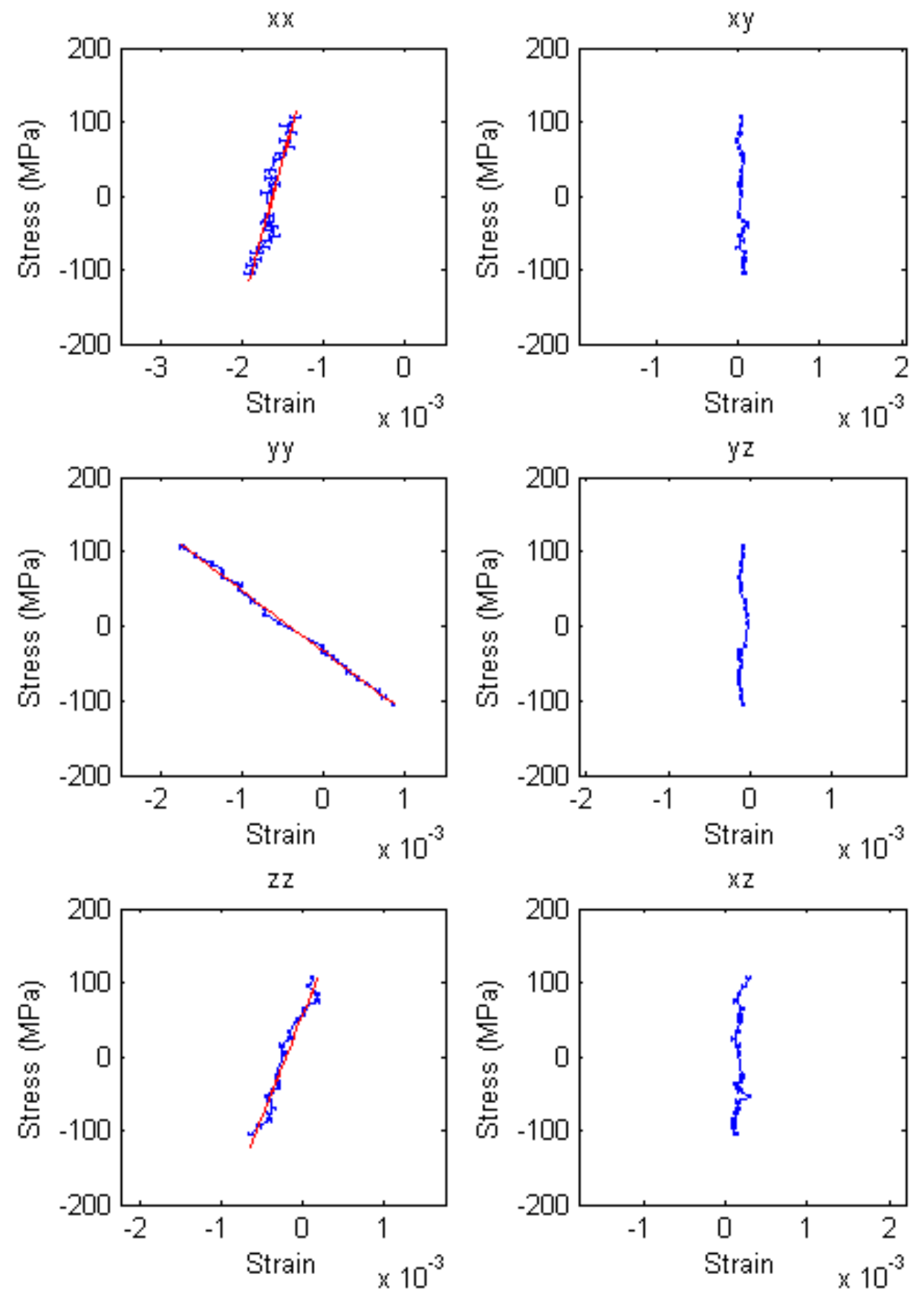

Figure 6: Stress-strain curves for 3 normal directions (left column) and 3 shear directions (right column). Scale is the same on all plots. The applied displacement is in the $y y$-direction. 


\section{TABLES}

\begin{tabular}{|c|c|}
\hline Epoxy & Yield strength \\
\hline Armstrong A-661 & $394.5 \pm 24.5$ \\
\hline 3M 1838 & $380.5 \pm 30.5$ \\
\hline A+B Epoxy Paste & $134.0 \pm 50.5$ \\
\hline Loctite & $127.7 \pm 69.8$ \\
\hline
\end{tabular}

Table 1: Yield strength for an epoxy bond between aluminum and steel. Units are Newtons.

\begin{tabular}{|c|c|c|c|c|}
\hline Crystallographic & \multicolumn{3}{|c|}{ Measured Elastic Modulus } & Theoretical \\
Direction $h k l$ & Compression & Tension & Combined & Modulus \\
\hline $200^{*}$ & $119.3 \pm 7.7$ & $100.5 \pm 4.1$ & $105.6 \pm 1.9$ & 142.9 \\
\hline 232 & $84.4 \pm 5.1$ & $79.3 \pm 3.7$ & $79.6 \pm 1.3$ & 86.7 \\
\hline 321 & $96.1 \pm 7.4$ & $95.7 \pm 6.3$ & $97.6 \pm 2.0$ & 106.2 \\
\hline 231 & $93.9 \pm 4.8$ & $89.7 \pm 3.0$ & $90.1 \pm 1.2$ & 91.3 \\
\hline 141 & $85.7 \pm 4.1$ & $85.2 \pm 2.1$ & $83.9 \pm 1.0$ & 80.5 \\
\hline 202 & $89.0 \pm 4.3$ & $84.0 \pm 2.0$ & $81.9 \pm 1.1$ & 67.7 \\
\hline 031 & $108.7 \pm 6.2$ & $97.2 \pm 3.4$ & $98.7 \pm 1.5$ & 79.6 \\
\hline
\end{tabular}

Table 2: Elastic modulus for different crystallographic directions in tension and compression, arranged in order of decreasing stress intensity (see Table 3). Units are GPa. *Near overlap with [102].

\begin{tabular}{|c|c|c|}
\hline Crystallographic Direction $h k l$ & $\mathrm{SI}_{\mathrm{A}}$ & \% load borne by protein \\
\hline $200^{*}$ & 1.29 & $-22.1 \%$ \\
\hline 232 & 1.03 & $1.7 \%$ \\
\hline 321 & 1.03 & $1.8 \%$ \\
\hline 231 & 0.96 & $8.5 \%$ \\
\hline 141 & 0.91 & $13.4 \%$ \\
\hline 202 & 0.79 & $25.4 \%$ \\
\hline 031 & 0.76 & $27.2 \%$ \\
\hline
\end{tabular}

Table 3: Values for stress intensity factor and percent load borne by protein. The stress intensity factor is the same as the ratio of theoretical elastic modulus to apparent (measured) elastic modulus of aragonite. ${ }^{*}$ Near overlap with [102]. 


\section{Proposal for a new B-Target Room Tunnel Layout}

Reza Esfandiari

Office of Science, Science Undergraduate Laboratory Internship Program

San José State University, San José

SLAC National Accelerator Laboratory

Menlo Park, California

August 14, 2009

Prepared in partial fulfillment of the requirement of the Office of Science, Department of Energy's Science Undergraduate Laboratory Internship under the direction of Carsten Hast in the Accelerator Research Division's Test Facilities Department at SLAC.

Participant:

Signature

Research Advisor:

Signature 
Table of Contents

2 Introduction

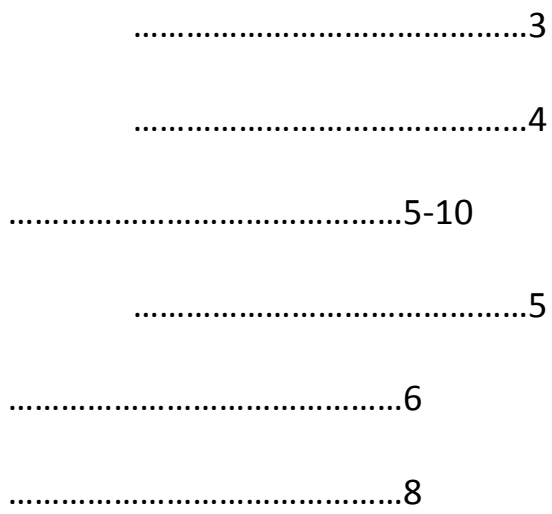

Abstract

$4 \quad$ Results

I. Results of the Calculations

11

II. The Stair

III. Matching the Results

.12

5 Conclusion

13

6 Acknowledgements

$7 \quad$ References

.14

$8 \quad$ Tables and Figures 


\section{$1 \quad$ Abstract}

Proposal for a new B-Target Room Tunnel Layout. REZA ESFANDIARI (San José State University, San José, CA 95192) CARSTEN HAST (SLAC National Accelerator Laboratory, Menlo Park, CA 94025)

Several groups at SLAC National Accelerator Laboratory are currently working on a RF Modulator prototype for a future linear collider known as the International Linear Collider (ILC). The ILC runs using about a 1000 Klystrons which create high power carrier waves for the particle acceleration. Klystrons receive their electrical input power from modulators. In order to move beyond the prototype phase, the laboratory might expand its ground base further down a tunnel located at the End Station B (ESB) in order to house four new Klystron Modulator Test Stations. This area is known as the B-Target Room Tunnel, and the task was to redesign the tunnel layout for the upcoming changes. The project first began by collecting substantial amount of information about the prototyped project, the tunnel and the researchers' feedback of what they would like to see in the upcoming design. Subsequent to numerous planning and presentations, one particular design was. Calculations for this design were then performed for the most complex aspects of the project. Based on the results of the calculations, specific sample beams, welds, bolts and materials were chosen for the possible future construction.

\section{Introduction}

The ILC is the next big thing in particle physics now that the Large Hadron Collider(LHC) has been built and is becoming operational. ILC will consists of two linear accelerators, one that has electrons and 
another that has positrons, which they collide the electrons and positrons at the speed of light [1]. Two machines, labeled as the Klystron and the Marx Modulator, play the lead role in making the particle happen. The Marx Modulator acts as the power supplier for the Klystron, Klystrons are linear beam vacuum tubes that produce high power carrier waves (RF waves) for particle accelerators [2], refer to Figure 1 for picture of the Klystron and Marx Modulator. A prototype Marx Modulator and a commercially available Klystron are currently placed and operating at the End Station B at SLAC National Accelerator Laboratory. However, in order to move beyond the current limitations, and in the hopes of pushing reliability and operational availability beyond the current values, the laboratory is in need of an expansion. At the present time, there is only one test stand that consists of the Klystron and the Marx Modulator, and the plan is to expand to four, maybe five, stations in the future. With this increase of equipments, however, comes the need for a new operating space. End Station B has a tunnel at its end that has been used for storage of depleted accelerator parts. My task was to redesign the layout of this tunnel so it could house the next generation of experiments. My work first began by putting together a proper blueprint of the place. Then, I talked to researchers, management and investors to understand not only the details about their operations, but also what they wanted in the tunnel in order to come up with an essential design. Lastly, I was to come up with the calculations needed for the construction and match those calculations with appropriate tools and materials to use.

\section{$3 \quad$ Method \& Procedure}

\section{Data Collection}

The Stanford Linear Accelerator Center (SLAC) no renamed to SLAC National Accelerator Laboratory was built in 1966 with designs and developments beginning in the 1950s. The End Station B was amongst the structures that were first built in SLAC in the late 1960s. Since at those times computer assisted drafting programs (CADs) were not yet developed, all SLAC blueprints were hand drawn by 
architects. The labeling and numbering system of the blueprints back then were not as efficient as today, and they also were not digitally stored. Within the last decade, all available blueprints were scanned and stored onto SLAC's website. As a result, the first stage was to put together a blueprint of the B-Target Room Tunnel that was as complete as possible since many pages of the blueprints were not available. Moreover, in some cases, certain measurements needed to be re-measured. One of those instances was the slope of the tunnel's floor, which according to the blueprints, had a slope of $1 \%$. With the use of Plane Surveying method and equipments, such as the theodolite, the ground slope level was measured for conformation. Additionally, certain measurements that were not provided by blueprints needed to be manually measured. For example, the cranes which were hung on the ceiling were not properly labeled and dimensioned in the blueprints, thus were in need of a measurement along with their position relative to the walls and floor. Once the blueprint packet was put together and all the blanks were filled in, the proper measurements were then used for the upcoming designs.

\section{Design Proposal \& Codes}

The second task was to come up with a design sketch that implemented the machine stations and met certain criteria specified by the researchers at the ESB along with the Departmental Manager. I spent a great deal of time talking with these people in order to gain an overall knowledge of their operations along with their visions of what they like to see in the upcoming design. Many workers addressed that it was rather difficult for them to perform maintenance on the upper portion of the Klystron since it goes as high up as eight feet. The Departmental Manager preferred a design which would place the Klystrons on a higher elevation with respect to the Marx Modulators, and part of the overall criteria was to ensure there is space available for future storage.

The next step was to continuously come up with different designs and present them to the 
departmental manager until he was satisfied with a few propositions. The chosen designs were then compiled into a PowerPoint presentation and presented to the lead managements of the ESB operation. In the final design, it was agreed to place $12^{\prime}$ long beams on the high ledge of the tunnel $\left(11^{\prime}-4^{\prime \prime}\right.$ high from the floor) placed perpendicularly to the walls. These beams were placed at $6^{\prime}$ intervals along the $108^{\prime}$ long tunnel to serve two functions; one function was to act as the base for the floor placed $11^{\prime}-4^{\prime \prime}$ high up, and second function was to act in supporting the Klystrons. At four evenly spaced intervals, beam-to-beam frames were attached to the underneath of the beams essentially creating cages which would act as the housing for the Klystrons. This housing would also partly sit on the lower ledges of the wall (7'-8" high from the floor) for extra support. The purpose of this design was not only for storage, but also to allow people to get on the floor and perform maintenance on top portions of Klystrons which now extend out about $5^{\prime}$ above the placed floor. The Marx Modulators were placed directly beneath the Klystron on the ground. Additional small details were applied such as placing a tunnel wide pipe on the top left portion of the tunnel, and also placing a stair for each end of the floor; refer to Figure 2 for the final design.

In addition, in the process of coming up with these designs, two major Codes had to be referenced and used as guidelines. These sources included the California Building Codes and the Occupational Safety and Health Administration (OSHA). The California Building Codes called a 4' clearance from the top of the machines to the ceiling, and placing the Klystron any higher than the proposed design would violate the clearance requirement. The codes require the placed floor to be split into $6^{\prime}$ segments and individually placed in the $108^{\prime}$ long tunnel. Furthermore, it is required that the placed floor would not touch the walls, and a half inch space needs to be left between the floor edges and the wall and then to be anchored in [3]. In addition to the building codes, the OSHA requirements had to be met. Any structures built for the public use, where people are expected to be going on and off the structure, will need to comply by the OSHA standards. In our case, the stairs we placed had to meet 
the OSHA standard to ensure the safety of the public. With the OSHA standards, stairs could only have an incline between $40^{\circ}-60^{\circ}$, and the stairs must have the minimum width of $22^{\prime \prime}$. In addition, any structure which requires a stair access also needs a secondary stair passage for emergencies. The emergency stair, however, could be a spiral stair whereas the main stair cannot be. As a result, 15' of space were kept clear at each end of the tunnel on the design to guarantee enough space for the stairs.

\section{Calculations}

Once the main structures of the design was finalized and set, many calculations were needed to be computed for all aspect of the design. To begin with, simple calculations were done to calculate the center of gravity (eq.1), and moment of inertia(eq.2),

$$
\begin{gathered}
\frac{\sum m_{i} r_{i}}{\sum m_{i}} \\
\int r^{2} d m
\end{gathered}
$$

and a general estimation of the Klystron weight along with the live and dead loads that will be placed into the structure. All calculations were done twice, once under static load which took into account only the live and dead loads, and a second time with the assumption of an earthquake load. It is important to note that the weight of the materials used for the structures were neglected due to the large safety margins that were provided in the calculations. SLAC National Accelerator Laboratory has its own formula for computation of earthquake loads for experimental equipment which is not covered by the building code (eq. 3),

$$
(1.5) \times \text { DeadLoad }+E
$$

where $E$ is the horizontal seismic force. All earthquake calculations were under the assumption of an 
earthquake acceleration force of $0.7 \mathrm{~g}$, a category $\mathrm{D}$ earthquake resistant measure [4].

Under these two loads assumption, the calculations were started by calculating the reactions of the beam placed on the high ledge using eq. 4,

$$
\frac{W b}{l}, \frac{W a}{l}
$$

where $W$ is the weight of the load, $l$ is the length of the beam and $a / b$ are the distances from the load to opposite ends of the beam. The bending moment (eq. 5) and Shear (eq. 6) were then calculated at the center of the beam,

$$
\begin{aligned}
\sum M & =F d \\
\tau & =\frac{F}{A}
\end{aligned}
$$

where $F$ is the force acting on the load, $d$ is the perpendicular distance of the load to the point of the moment and $A$ is the cross-section area of the applied load. The maximum bending moment and the maximum shear were also calculated for the beam and their diagrams were generated on the computer (Figures 4-7). Using Newton's second law (eq. 7), the load on each frame connected to the main beam were distributed amongst the four axial points,

$$
\sum F=m a
$$

where $m$ is the mass and a is the acceleration. Stress at loads were then calculated using eq. 8 ,

$$
\frac{W a b}{Z l}
$$

Upon completion of the calculations mentioned above, more specific earthquake resistance calculations were performed. First the Base Shear (V) was calculated (eq. 9),

$$
\frac{W S a}{g_{c}}
$$

where $W$ is the weight affected by the earthquake, Sa is the earthquake acceleration and $g$ is the gravity. 
Then, the Horizontal Seismic Force $\left(E_{h}\right)$ and the Lateral Seismic Force $\left(E_{l}\right)$ were taken into consideration, using eq. 10 and 11.

$$
\begin{gathered}
E_{h}=\rho Q_{E} \\
E_{l}=\frac{m a}{g_{c}}
\end{gathered}
$$

where $Q_{E}$ is the effect of horizontal seismic force form base shear, and $\rho$ is the redundancy factor, which is 1.3 in our case based from Minimum Design Loads for Buildings and Other Structures [5]. After understanding the Horizontal Seismic Force, one can then apply it to find whether or not the Klystron would overturn during an earthquake, and if so, with what force (eq. 12),

$$
\text { OverTurning }=\sum M_{\text {overturning }}-\sum M_{\text {resisting }}
$$

where the initial resisting moment is often the weight, and the overturning force is the earthquake. Once these main calculations were done, few additional minor calculations were completed using eq. 13-15 to determine the Torsion Moment, Building Period and Stiffness of the beam under an earthquake for future reference,

$$
\begin{gathered}
M_{\text {torsional }}(F i)=V e, \\
T=2 \Pi \sqrt{\frac{m}{g_{c}}}, \\
K=\frac{48 E I}{L^{3}} .
\end{gathered}
$$




\section{$4 \quad$ Results}

\section{Results of the Calculations}

All following results are based on earthquake load, for static load refer to Table 1.

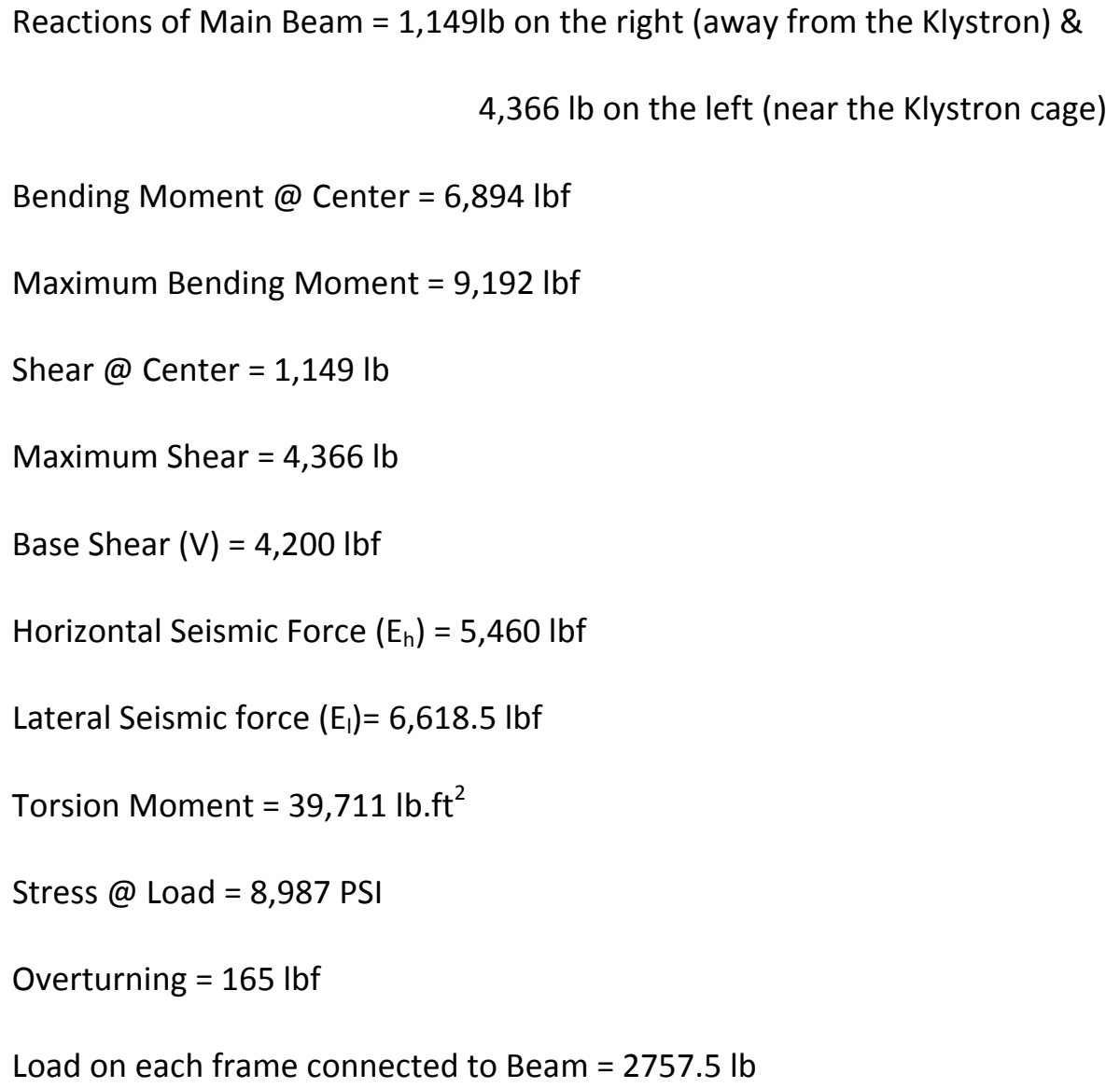

\section{The Stairs}

To meet the OSHA Standard, the stair was designed at an incline of $45^{\circ}$. Each step is $1^{\prime}$ high, $1^{\prime}$ long and the width is $25^{\prime \prime}$. The stair has the height of $11^{\prime}$ foot and $11^{\prime}$ long along the tunnel. 
Subsequent to finishing all the computations, the results are matched with the proper materials. The task at hand first started by deciding what beam to choose that could carry the heavy load of the Klystron stations during earthquakes, and could face acceptable deflections under the total load. Since the beam would go under a maximum bending moment of 9,192 lbf, a W6 20 (Fy= 36ksi) beam was chosen, refer to Table 2 for full detail on the $W 6 \times 20$ beam. These beams have an allowable bending moment of $27,000 \mathrm{lbf}$, which provides a great margin of safety. Following this decision, it had to be decided how to connect the beam-to-beam frames onto the main beam to create the cage for the Klystrons to sit in. Since each point of the beam-to-beam frame connection needed to hold $2757.5 \mathrm{lbs}$, a Weld B Capacity of Size $=1 / 4$ " was chosen which holds $14,000 \mathrm{lbs}$, leaving a large margin of safety. Due to the $5,460 \mathrm{lbf}$ Horizontal Seismic Force, the floor must be anchored into the concrete walls. Each beam set that holds a Klystron needs to be anchored at each end with a carbon steel HDI $3 / 4 " \times 4$ " anchor to resist the horizontal forces. Lastly, since the Klystron would, it will also be attached to the Beam at the point where it is at the same height as the beam. Since this force is only $165 \mathrm{lbf}$, close to negligible, no details were worked out in the proposed layout, refer to Figure 8 for the final specification of the Klystron structure.

\section{$5 \quad$ Conclusion \& Future Work}

In conclusion, under the direction of Carsten Hast, I have gained a lot of valuable experience as 
to how I can take what I have learned in school and apply it to a real life project. I have learned how to tackle a challenge which I had no idea how to solve, and what sources I should use when I hit a dead end on those challenges. And more importantly, it was the role of networking and communication which made this project a worthwhile experience. Never before had I realize the immense challenge of communication between scientists and engineers, and its importance to work towards a common goal. Looking towards the future, while this design idea is now very much developed, it is far from being built. The design and the calculations need to be checked through a licensed Structural Engineer, along with approval of the fire marshall, before the construction can begin. It is my hope that one day this design will be built primarily with the framework that I have constructed and I look forward to seeing it built someday.

\section{$6 \quad$ Acknowledgement}

This effort would not have been possible without funding from the Department of Energy and the SULI program at the SLAC National Accelerator Laboratory. I would like to thank Steve Rock, the SULI Program Director, who made it all possible, and Carsten Hast, my mentor, whose passionate support and guidance made the completion of this project feasible. Special thanks to Keith Jobe for tolerating me, along with the rest of the incredible staff working at the ESB and the Next Linear Collider Test Accelerator.

\section{$7 \quad$ References}

[1] ILC. (2002). International Linear Collider. [Online]. Available: http://www.linearcollider.org/cms/?pid=1000000

[2] E.L. Ginzton. (2009). Invention of the Klystron. VARIAN INC., CA. [Online]. Available: http://www.varianinc.com/cgi-bin/nav?corp/history/klystron\&cid=KJHOIQMJFI 
[3] International Code Council. (2007). California Building Code. ICC, United States. [Online].

Available:http://ecodes.citation.com/cgiexe/cpage.dll?pg=x\&rp=/nonindx/ST/ca/st/b200v07/index.htm \&sid=2009080509553824946\&aph=0\&cid=stan+++++++++++++++++\&ref=/nonindx/ST/ca/index.htm\&uid $=i \csc 0501 \& \operatorname{cl} A=005596 \& c|r V=005596 \& c| r X=005596$

[4] Michael R. Lindeburg. (1996). Seismic Design of building Structures. ( $7^{\text {th }}$ ed.) [Book].

[5] American Society of Civil Engineers. (2006). Minimum Design Loads for Buildings and Other Structures. ( $3^{\text {rd }}$ ed.) [Book].

[6] American Institute of Steel Construction. (1991, January). Manual of Steel Construction: Allowable Stress Design. (9 ${ }^{\text {th }}$ ed.) [Book]. 

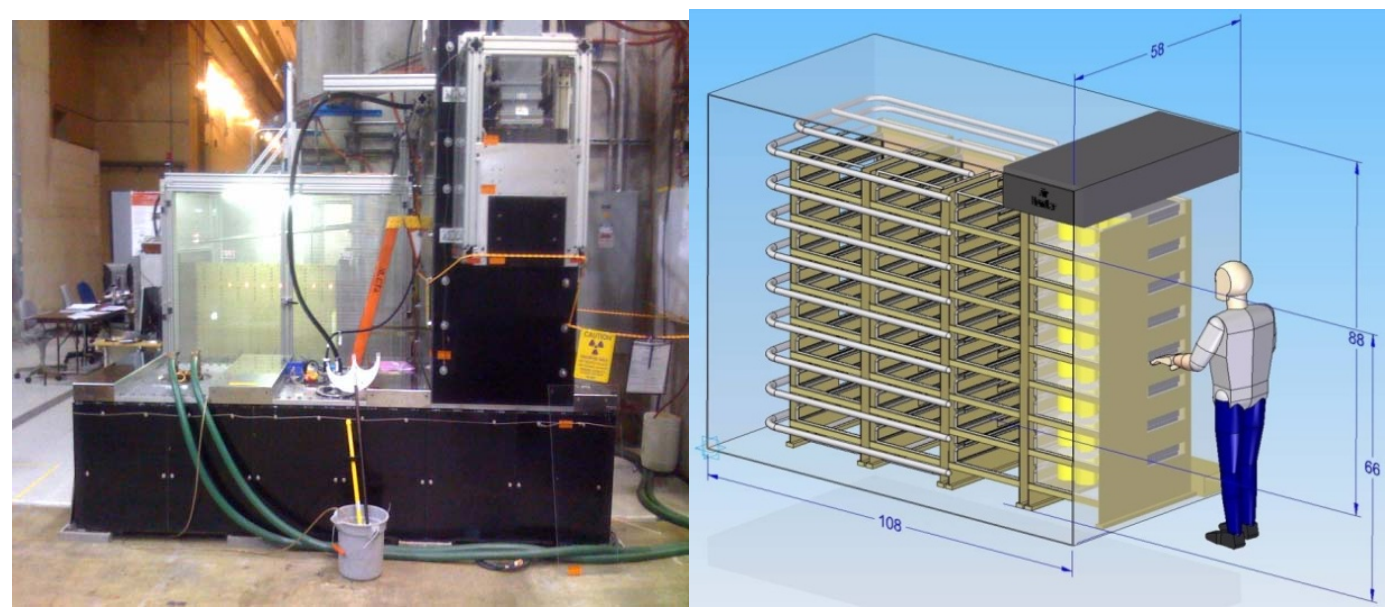

Figure 1: To the left: A Klystron with its tank that shields the radiation. To the right: Marx Modulator which powers the Klystron.
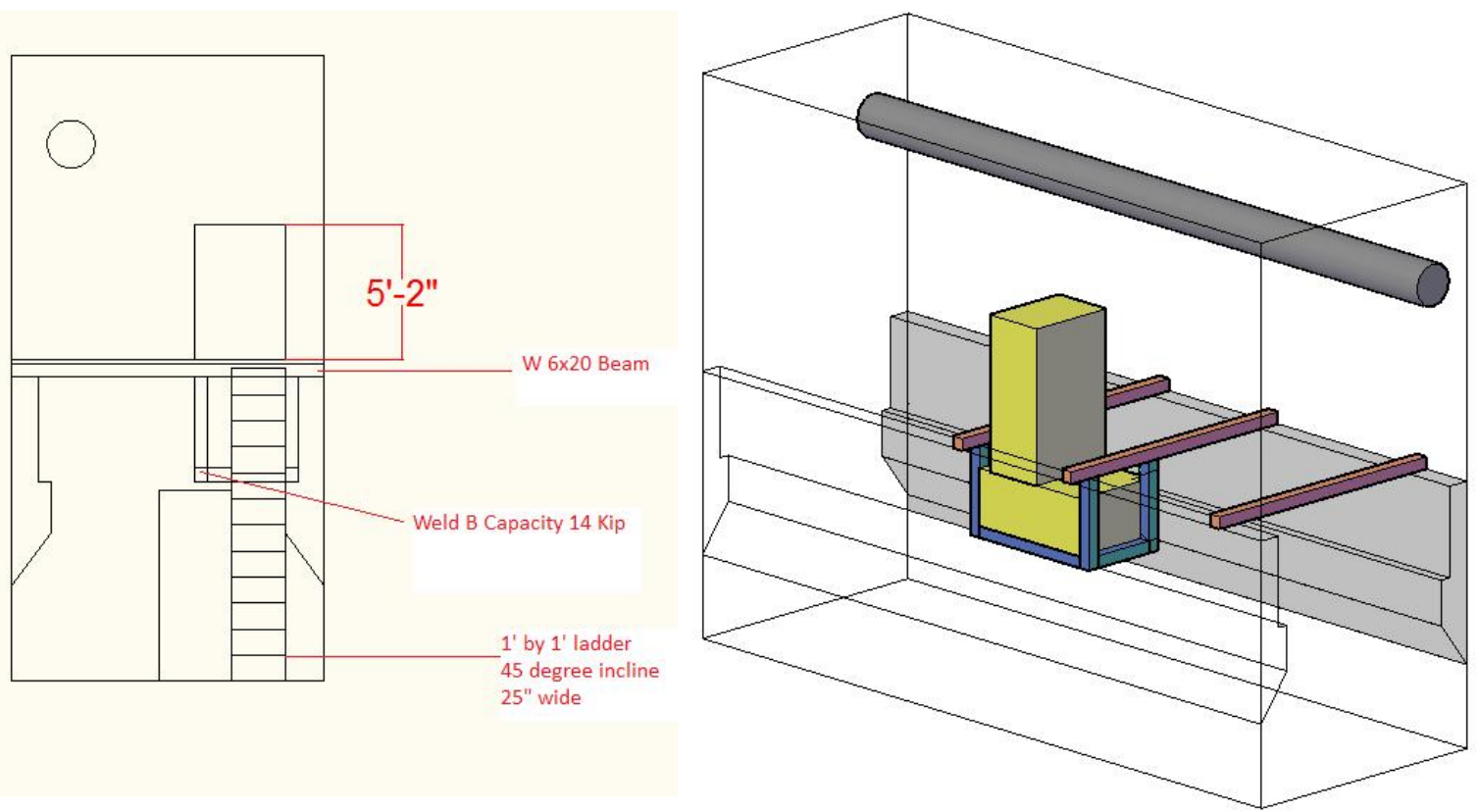

Figure 2: To the right, is the final design of one station of a Klystron in the tunnel. To the left, is the cross-sectional view of the final design looking into the tunnel. 

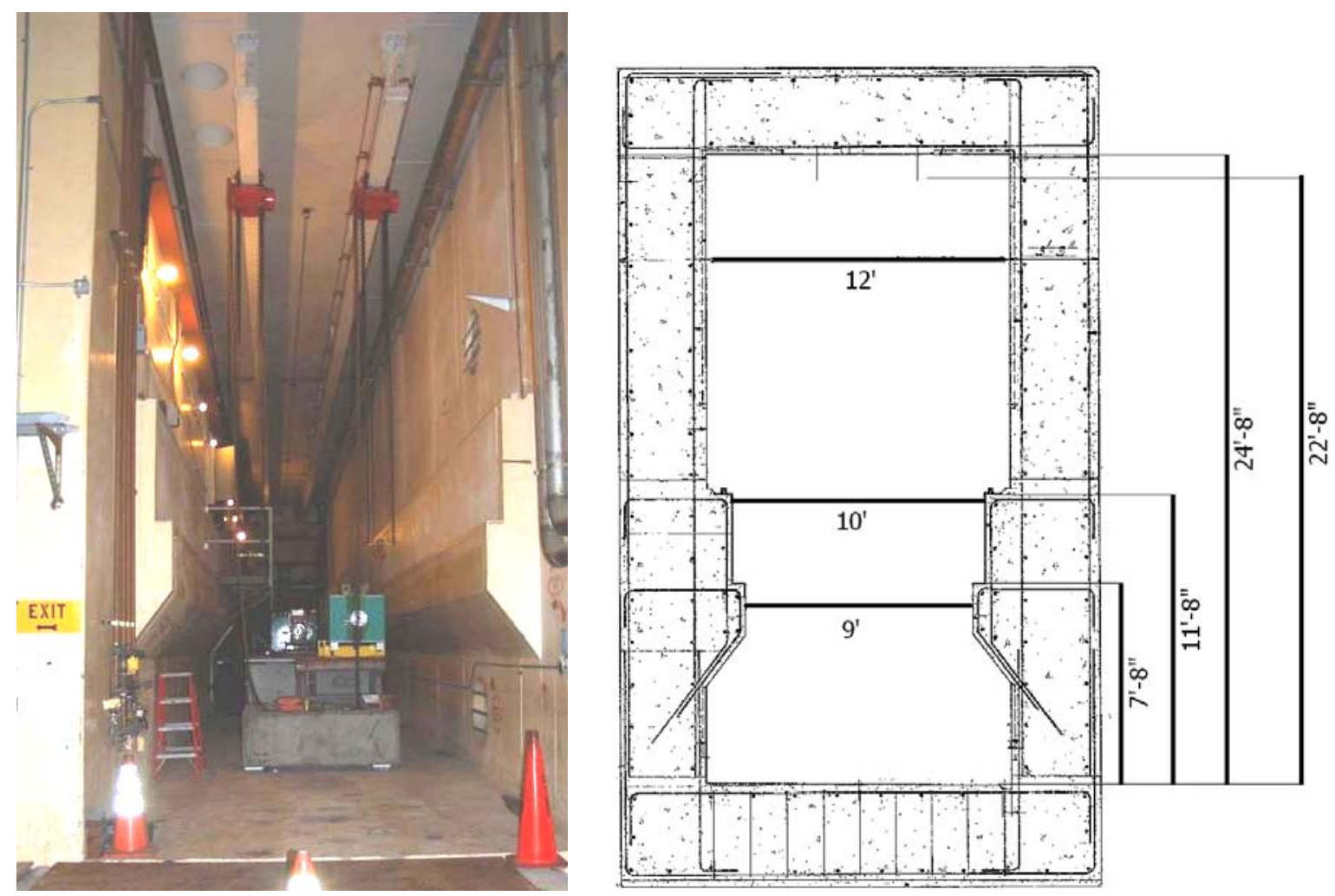

Figure 3: To the left, picture of the tunnel. To the right, the important dimensions of the tunnel. As can been seen, the cranes have the height of two feet. It was very important in the design to line up the crane with the center of gravity of the Klystron.

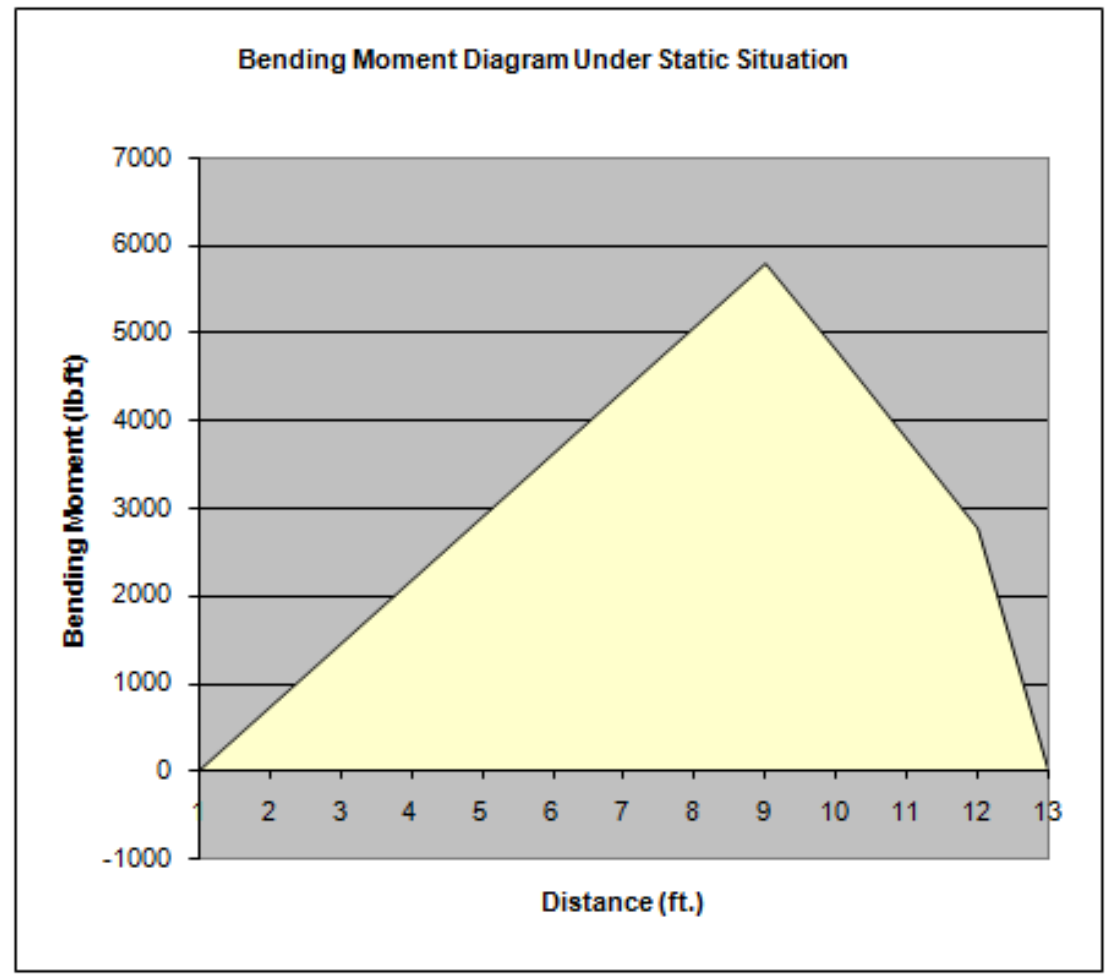

Figure 4: Bending Moment of the Beam under Static Load 


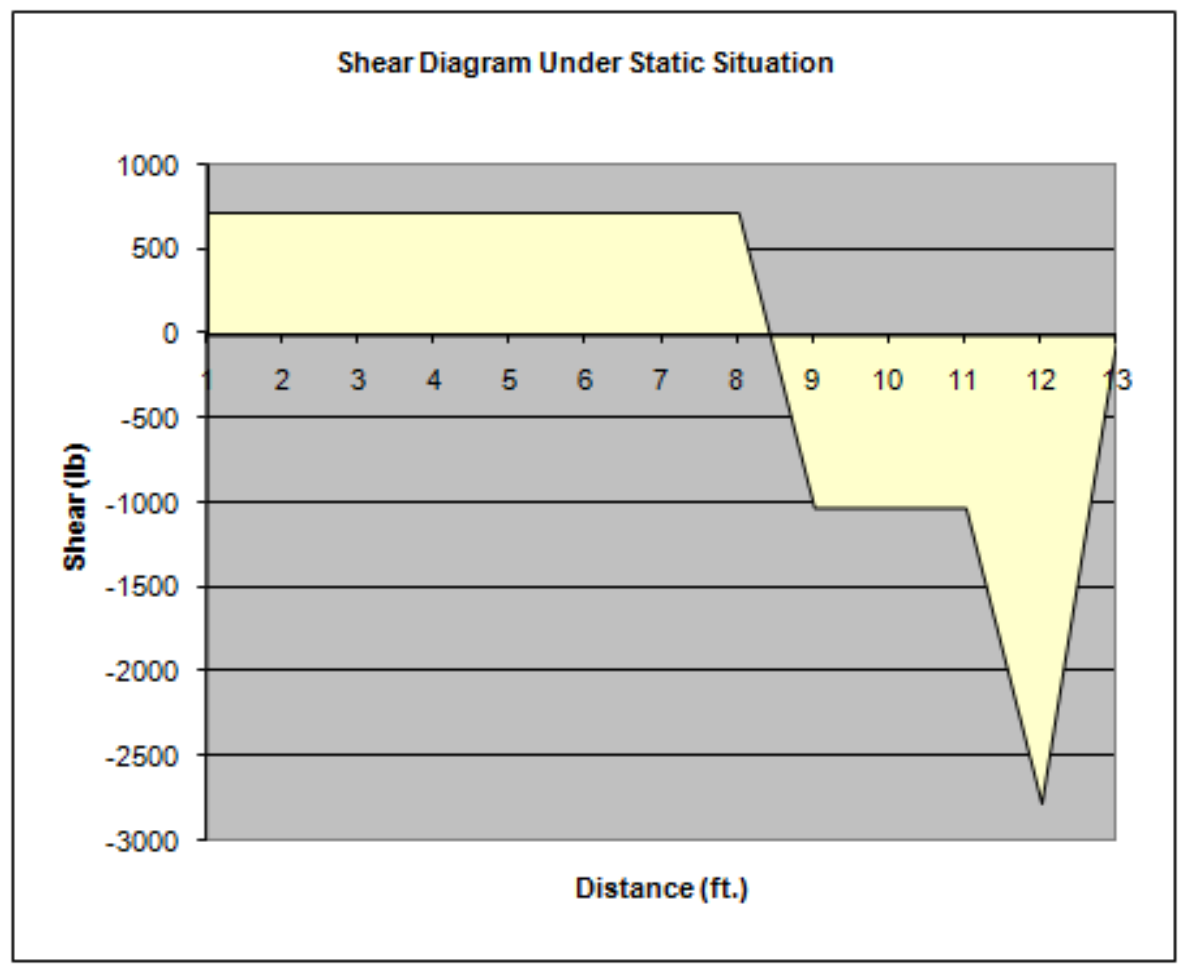

Figure 5: Applied Shear on the Beam under Static Load

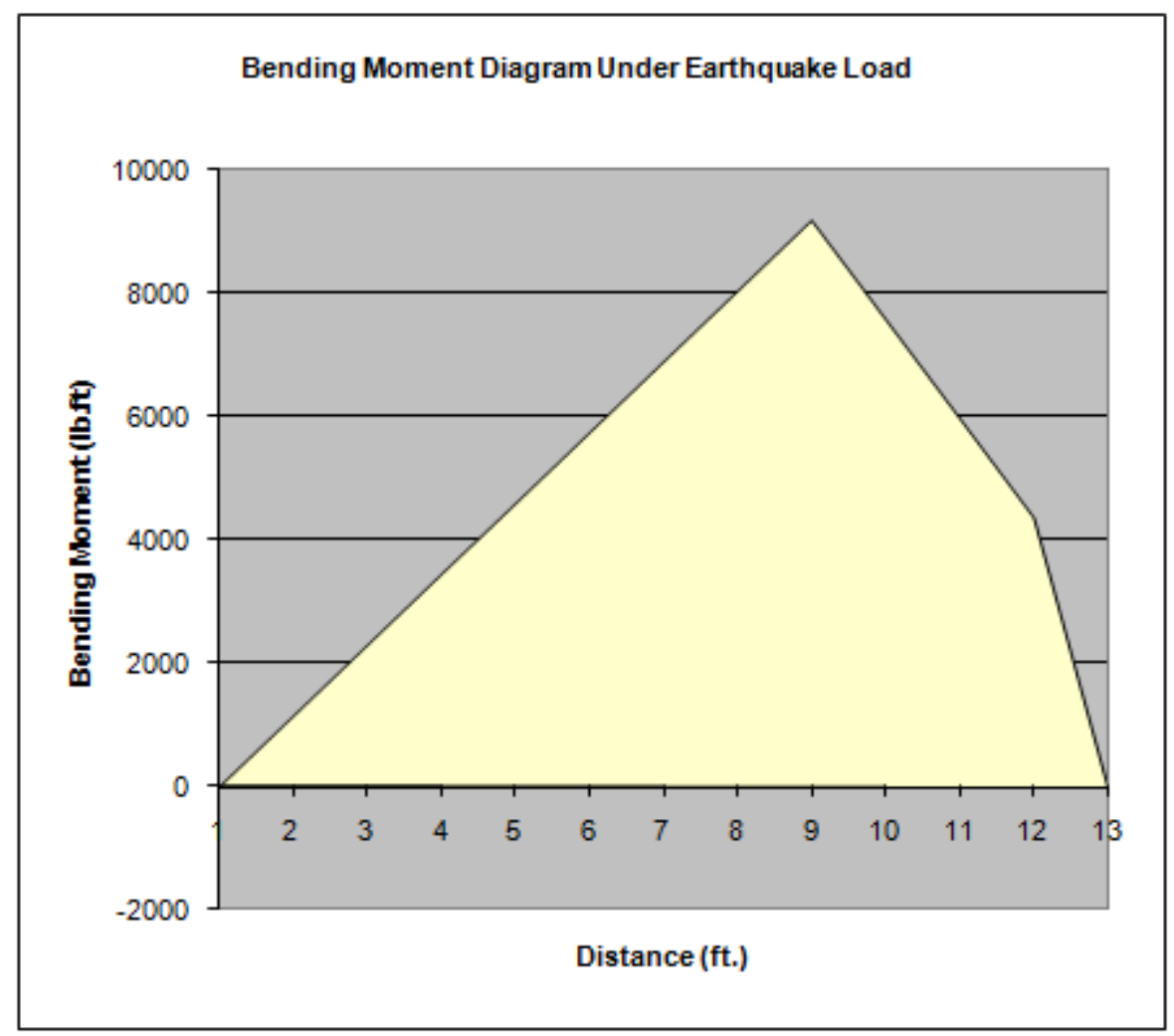

Figure 6: Bending Moment of the Beam under Earthquake Load 


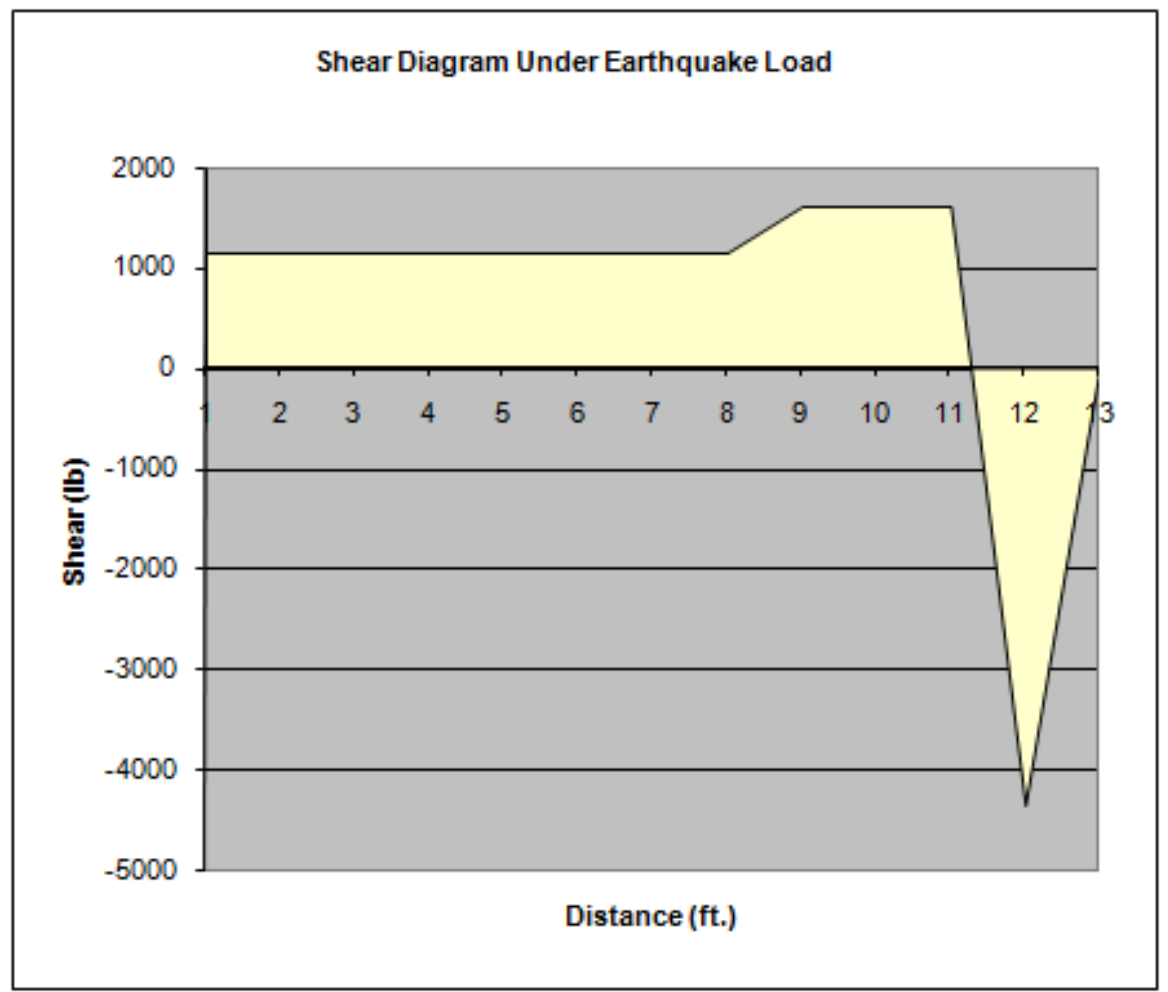

Figure 7: Applied Shear on the Beam under Earthquake Load

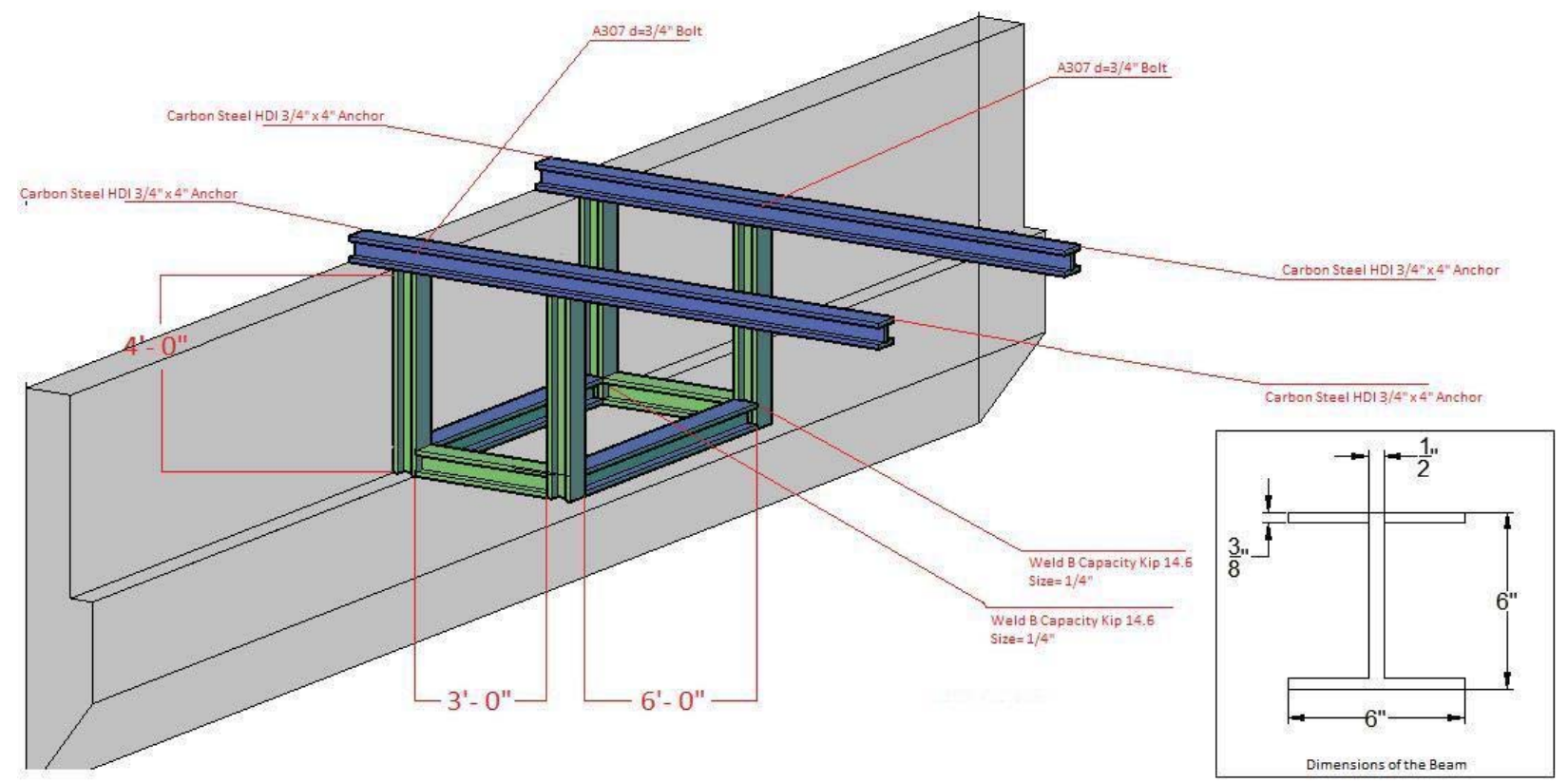

Figure 8: The final specification and dimensions for the Klystron cage hanging from the beam. 
Calculation Chart for the Tunnel B Design

\begin{tabular}{|c|c|c|}
\hline & Static & Earthquake \\
\hline Total Load & $6,000 \mathrm{lb}$ & $9,455 \mathrm{lb}$ \\
\hline $\begin{array}{l}\text { Reactions of Main Beam } \\
\text { (vert.) }\end{array}$ & $729 \mathrm{lb} \& 2771 \mathrm{lb}$ & $1,149 \mathrm{lb} \& 4,366 \mathrm{lb}$ \\
\hline Maximum Bending Moment & $5800 \mathrm{lbf}$ & $9192 \mathrm{lbf}$ \\
\hline Maximum Shear & $2,771 \mathrm{lb}$ & $4,366 \mathrm{lb}$ \\
\hline Bending Moment @ Center & $4,374 \mathrm{lbf}$ & $6,894 \mathrm{lbf}$ \\
\hline Shear@ Center & $729 \mathrm{lb}$ & $1,149 \mathrm{lb}$ \\
\hline Base Shear (V) & $4,200 \mathrm{lbf}$ & $4,200 \mathrm{lbf}$ \\
\hline $\begin{array}{l}\text { Horizontal Seismic Force } \\
\left(E_{h}\right)\end{array}$ & -------------- & $5,460 \mathrm{lbf}$ \\
\hline Lateral Seismic Force & ------------ & $6,618.5 \mathrm{lbf}$ \\
\hline Torsion Moment & 25,200 in-lbf & 39,711 in-lbf \\
\hline Stress@ Load & 5703 PSI & 8,987 PSI \\
\hline $\begin{array}{l}\text { Load on Each Frame } \\
\text { Connected to the Heavy } \\
\text { Main Beam }\end{array}$ & $1750 \mathrm{lb}$ & $2757.5 \mathrm{lb}$ \\
\hline $\begin{array}{l}\text { Overall Load on a Frame } \\
\text { Connected to the Heavy } \\
\text { Beam }\end{array}$ & $3500 \mathrm{lb}$ & $5515 \mathrm{lb}$ \\
\hline $\begin{array}{l}\text { Seismic Loading on } \\
\text { Elements of Structures }\end{array}$ & ------------- & $10,125 \mathrm{lbf}$ \\
\hline Building Period & ------------ & $.398 \mathrm{~s}$ \\
\hline $\begin{array}{l}\text { *ignore this option* } \\
\text { Bolt to hold Beam-Beam } \\
\text { Connections }\end{array}$ & $\begin{array}{l}\text { A307 } d=3 / 4^{\prime \prime} \\
\text { Angle Thickness } t=1 / 4^{\prime \prime} \\
\text { Resist } 17.7 \text { kip }\end{array}$ & $\begin{array}{l}\text { A307 } d=3 / 4^{\prime \prime} \\
\text { Angle Thickness } t=1 / 4^{\prime \prime} \\
\text { Resist } 17.7 \text { kip }\end{array}$ \\
\hline
\end{tabular}




\begin{tabular}{|l|l|l|}
\hline $\begin{array}{l}\text { Weld to hold Beam-Beam } \\
\text { Connections }\end{array}$ & $\begin{array}{l}\text { Weld B Capacity kip 14.6 } \\
\text { Size= 1/4" }\end{array}$ & $\begin{array}{l}\text { Weld B Capacity kip 14.6 } \\
\text { Size= 1/4" }\end{array}$ \\
\hline Beam Resisting Base Shear & -------------- & $\begin{array}{l}\text { 4 anchors (one at each end of the 2 } \\
\text { main beams holding the machine) } \\
\text { 4x(Carbon Steel HDI 3/4") }\end{array}$ \\
\hline Overturning & ------------- & Need to resist $165 \mathrm{lb} . \mathrm{ft}$ \\
\hline Stiffness (K) & $\frac{48 E I}{L^{3}}$ & Same for both \\
\hline Natural Period Vibration (T) & $\mathrm{E}=$ & $=2 \pi \sqrt{\frac{m}{g_{c} \times k}}=2 \pi$ \\
\hline
\end{tabular}

Table 1: Tunnel Calculations based on Static and Earthquake Loads.

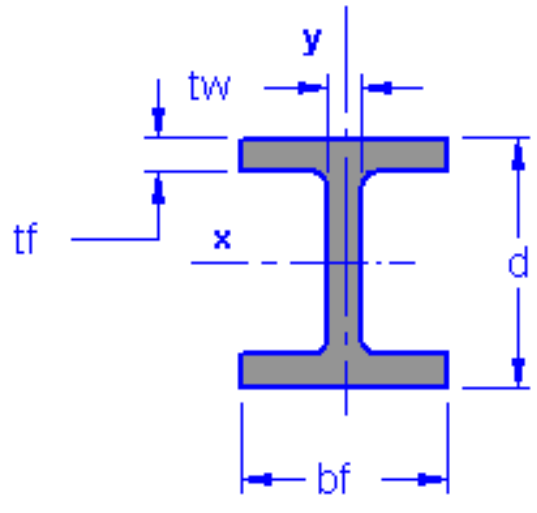

\begin{tabular}{|l|l|}
\hline iBeam & Description \\
\hline Name & $\begin{array}{l}\text { W6x20 } \\
\text { iBeam }\end{array}$ \\
\hline Dimensions & $6^{\prime \prime}$ by 6" \\
\hline X-Area (in $\left.{ }^{2}\right)$ & 5.87 \\
\hline$d$ (in) & 6.2 \\
\hline bf (in) & 6.02 \\
\hline tf (in) & 0.365 \\
\hline tw (in) & 0.26 \\
\hline $\mathrm{I}_{\mathrm{xx}}\left(\mathrm{in}^{4}\right)$ & 41.4 \\
\hline $\mathrm{I}_{\mathrm{yy}}\left(\mathrm{in}^{4}\right)$ & 13.3 \\
\hline
\end{tabular}

Table 2: Specification of the W6x20 iBeam 
Field Programmable Gate Array (FPGA) Based Trigger System for the Klystron Department

$$
\text { Darius Gray }
$$

Office of Science, Science Undergraduate Laboratory Internship Program

\author{
Texas A\&M University, College Station
}

SLAC National Accelerator Laboratory

Menlo Park, California

August 14, 2009

Prepared in partial fulfillment of the requirement of the Office of Science, Department of Energy's Science Undergraduate Laboratory Internship under the direction of Michael G. Apte in the Environmental Energy and Technology division at Lawrence Berkeley National Laboratory.

Participant:

Signature

Research Advisor:

\title{
Signature
}




\title{
Table of Contents
}

\begin{abstract}
_2
Introduction/Problem Description 3
\end{abstract}

Materials and Methods__.3

Literature Cited__. 10

Acknowledgements__11

Figures and Tables_[D 


\begin{abstract}
Field Programmable Gate Array (FPGA) Based Trigger System for the Klystron Department. Darius Gray, Alternative Paper, Science Undergraduate Laboratory Internship, SLAC National Accelerator Laboratory, Summer 2009.
\end{abstract}

The Klystron Department is in need of a new trigger system to update the laboratory capabilities. The objective of the research is to develop the trigger system using Field Programmable Gate Array (FPGA) technology with a user interface that will allow one to communicate with the FPGA via a Universal Serial Bus (USB). This trigger system will be used for the testing of klystrons. The key materials used consists of the Xilinx Integrated Software Environment (ISE) Foundation, a Programmable Read Only Memory (Prom) XCF04S, a Xilinx Spartan 3E 35S500E FPGA, Xilinx Platform Cable USB II, a Printed Circuit Board (PCB), a 100 MHz oscillator, and an oscilloscope. Key considerations include eight triggers, two of which have variable phase shifting capabilities. Once the project was completed the output signals were able to be manipulated via a Graphical User Interface by varying the delay and width of the signal. This was as planned; however, the ability to vary the phase was not completed. Future work could consist of being able to vary the phase. This project will give the operators in the Klystron Department more flexibility to run various tests. 


\section{Introduction/Problem Description}

The Klystron Department is responsible for testing klystrons, building klystrons, and for research and development of klystrons. The department has eighteen test stations that can run klystrons (see Figure 1). These test stations are equipped with various equipment to measure voltage, current, and other aspects of the klystrons. However, much of the technology used in the Klystron Department is out dated, about 60s and 70s technology. Upgrades can be implemented to increase the capabilities of the test station. My portion of the project is to program the Field Programmable Gate Array (see Figure 2) for the trigger system to test klystrons at 18 test stands.

\section{Materials and Methods}

The materials used consists of the Xilinx Integrated Software Environment (ISE) Foundation, a Programmable Read Only Memory (Prom) XCF04S, a Xilinx Spartan 3E 35S500E FPGA (Field Programmable Gate Array), Xilinx Platform Cable USB II, a regular USB cable, a Printed Circuit Board (PCB), a $100 \mathrm{MHz}$ oscillator, and an oscilloscope. The Xilinx ISE Foundation was used to write computer code in a language called Very High Speed Integrated circuits Hardware Description Language (VHDL) to perform the logic operations 
necessary. The prom was programmed with the information from the computer code and loads the FPGA when power is turned on. The Xilinx Spartan 3E 35S500E FPGA receives the code written in the Xilinx ISE Foundation and performs the logical operations necessary. It, also, takes in data from a USB and an oscillator on the PCB and sends out data to the output ports on the printed circuit board. A Xilinx Platform Cable USB II was used to send data from the Xilinx software development platform to the Prom XCF04S. When the project was completed this was replaced with a USB cable that connected to the USB port on the PCB. A PCB contains the circuit elements used including the input ports, output ports, and the oscillator. The $100 \mathrm{MHz}$ oscillator was used as the clock to make sure that the logical operations were done in synchronization. It was, also, used to generate the signals for the triggers. The oscilloscope was used to display the output signals. The goal of the project is to generate eight trigger outputs with variable delays and pulse widths by using an oscillator, or clock, input and a trigger input.

My mentor, Ron Akre, designed a test stand trigger system that would meet the needs of the test lab. A major need of the test lab, in order to meet LCLS requirements, is 50 femtoseconds of root mean squared jitter (rms) on the output of a 5045 klystron. Jitter is the deviation, in time, of a signal from a desired location. This design consisted of two parts: 1) the central timing system and 2) the FPGA based trigger generator. The central timing system has a $119 \mathrm{MHz}$ ultra low noise RF oscillator that distributes its signal with superimposed fiducials to each of the 18 test stands, which is used to create triggers. A fiducial marks a starting point in time. The operator sets the value for the delay, width, rate, and, if applicable, the phase shift value of the trigger in a user interface, developed by Kelton Stefan. The data is transported to the FPGA via a Universal Serial Bus (USB) port and the FPGA recognizes the data and sets the final attributes of the trigger. The FPGA based trigger generator will take in a signal and generate 
eight independent triggers. In order to do this, the FPGA must be programmed using digital logic, VHDL, and the Xilinx ISE Foundation. The first method used to program the chip was a schematic editor; however, a switch was made to using VHDL due to its greater flexibility. The signals that come into the FPGA include the $119 \mathrm{MHz}$ signal, a $360 \mathrm{~Hz}$ signal, and a $1 \mathrm{~Hz}$ signal. The operator indicates what the delay and width of the trigger is and, also, what the rate is. Inside the FPGA, the $119 \mathrm{MHz}$ signal is put in sync with the $360 \mathrm{~Hz}$ signal. This means that the signal that results has the same pulse width as the $119 \mathrm{MHz}$ signal, which is $8.4 \mathrm{~ns}$, but is pulsing at the frequency of the $360 \mathrm{~Hz}$ signal. That signal is used, by dividing it down, to create different rep rates of $180 \mathrm{~Hz}, 120 \mathrm{~Hz}, 60 \mathrm{~Hz}, 30 \mathrm{~Hz}, 15 \mathrm{~Hz}, 10 \mathrm{~Hz}, 5 \mathrm{~Hz}, 2 \mathrm{~Hz}$, and $1 \mathrm{~Hz}$, which are specified by the operator. The rep rates are signals that have the same pulse width of the $119 \mathrm{MHz}$ signal, $8.4 \mathrm{~ns}$, but come at the operator specified frequency. One of the desires of the test lab is to be able to run and test multiple klystrons at the same time. In order to do this, they must be in phase. This is where the $1 \mathrm{~Hz}$ signal, or $1 \mathrm{~Hz}$ fiducial, plays an important role. The $1 \mathrm{~Hz}$ fiducial is synchronized to the $60 \mathrm{~Hz}$ power line that feeds the test lab. This ensures that as long as the 360 $\mathrm{Hz}$ signal and the rep rates derived from it are in synchronization with the $1 \mathrm{~Hz}$ fiducial, then they are in synchronization with the power line too. The $1 \mathrm{~Hz}$ signal plays a similar role as a bar line that indicates when a measure starts and stops in a piece of music played by a symphony orchestra. Depending on the time signature, there are a certain amount of beats per measure and a specific note gets the beat. The first beat after a bar line is labled 1 , the second 2 , the third 3 , and so on until the number of beats fulfills the limit of the time signature. The termination of the count is indicated by a bar line. The first beat after that bar line is the beginning of a new count. The $1 \mathrm{~Hz}$ fiducial marks the starting point in time for the other signals and for the klystrons, which is synonymous to a bar line. The number of beats per measure is synonymous to the rep 
rate of the pulse. Whatever the rep rate is, there are that many pulses per $1 \mathrm{~Hz}$ pulse. For example, if the rep rate is $30 \mathrm{~Hz}$, there are 30 pulses in one second. Therefore, there are 30 pulses per $1 \mathrm{~Hz}$ fiducial. The $1 \mathrm{~Hz}$ fiducial makes sure that the triggers and the klystrons pulse at the same point in time as the bar lines in a piece of music can make sure that the musicians are at the same point in time. Having the signals pulse width the same as one cycle of the $119 \mathrm{MHz}$ signal is important. This is true because some of the digital logic elements used run on a signal that is $8.4 \mathrm{~ns}$ in width. If any other inputs to these logic elements have less or more than an 8.4 ns pulse width, the elements may work undesirably. If the other signals to the elements are less than 8.4 ns, the logic elements may ignore it. On the contrary, if it is greater than the $8.4 \mathrm{~ns}$, the logic elements may consider it more times than desired. The final signal is a signal that the operator has the freedom to vary the delay and width of as well as the rate at which it comes (see Figure 3). This is true for six of the eight triggers. The other two triggers have a unique quality to them. One of the desires of the lab is to have two triggers where the phase of the signal can be varied on the picosecond level. In order to implement this capability, the Phase Shifter and the Delay Locked Loop (DLL) functions that are a part of the Digital Clock Manager (DCM) on the Spartan 3E FPGA are used. The Phase Shifter takes in a Phase Shift Clock (PSCLK) signal, a Phase Shift Enable (PSEN) signal, and a Phase Shift Increment Decrement (PSINCDEC) signal and outputs a Phase Shift Done (PSDONE) signal, a LOCKED signal, and the desired phase shifted signal CLKO. The LOCKED output is a part of the Status Logic part of the DCM and the CLK0 output is a part of the DLL. The DLL receives its own input clock signal called CLKIN. When the PSEN is logic 0, the PSCLK is disabled and nothing happens. In order for the phase to be shifted, PSEN must be logic 1, the PSCLK must be on the rising edge, and the PSINCDEC must be logic 1 or 0 . In the later condition, a PSINCDEC value of logic 1 means to increment the 
output signal and a PSINCDEC value of logic 0 means to decrement the output signal (see Table 1 for a truth table and Table 2 for information on DCM variable phase shifting capabilities) [1]. The output signal of the DLL, which is the CLK0 signal, goes into similar logic as the six triggers without phase shift capabilities. As the output signal of the DLL gets phase shifted with respect to its input signal, the final output signal, after it goes through all of the logic, is phase shifted with respect to its zero degrees phase shift position by the same amount as the CLK0 signal. As a result, the operator is able to set the delay, width, and rate of the final output signal as well as shift its phase. This DCM functionality is utilized for two of the eight triggers. There are eight channels that take the trigger signals to various locations to run tests on the klystrons. Four of these channels include the klystron modulator, the low level RF, the scope, and the computer digitizer. The operator is able to run various tests using these triggers generated by the FPGA.

Various challenges were faced while doing the project. Among these challenges, was switching from the schematic editor in the Xilinx ISE Foundation to using a computer language called VHDL. Significant work had already been done on the project when an outside source advised the use of VHDL due to its ability to be used in other software besides the Xilinx ISE Foundation. This resulted in the need to learn VHDL and convert everything that had been done thus far from the schematic editor to VHDL. This process emphasized the importance of the skill to be able to find needed resources and to be able to learn quickly in the research field. A primary source of information was the Internet and Jeff Olsen of the controls department at the SLAC National Accelerator Laboratory. The switch turned out to be for the better as the flexibility of using VHDL instead of the schematic editor was realized quickly. Other challenges that occurred include resolving many error messages given by the compiler. This process took 
anywhere from a few seconds to over a day with help from other people to resolve. This process emphasized the importance of using good programming techniques to avoiding getting error messages from the compiler and that in the research field, things do not always go the way one might think they should the first time. Therefore, developing various skills and techniques related to the particular field is of upmost importance. One technique among many that is necessary is the constant testing of the project many times during the process of working on the project. This allows one to see undesirable things in the project as it progresses and helps in the process of isolating problems. This could include testing individual sections at a time. If one does everything at one time it might be hard to find where problems have occurred.

\section{Results}

After the programming of the FPGA had reached an ending point, the VHDL code for it was merged together with the code for the graphical user interface and the USB port. Once this process was complete it was tested to see if it worked the way it was supposed to, which it did. The next step was to perform phase jitter measurements. One of the channels that did not use the DCM was tested. This channel had 80 ps of jitter peak to peak, which is acceptable (see Figure 4). After that, one of the channels that used the DCM in the FPGA was tested for jitter using a $180 \mathrm{~Hz}$ rep rate. This channel showed 172 ps of jitter peak to peak. Furthermore, the graph on the oscilloscope had two different states each with approximately 80 ps of jitter (see Figure 5). Having two states means that instead of having one line as the signal rises to its peak amplitude, 
the signal would bounce back and forth in time between two lines. The reason for this outcome is not known. This outcome is against what was originally desired; however, whether this is acceptable or not depends on what test the operators are running.

\section{Discussion and Conclusion}

There is still work that can be done in the future. Originally, the goal was to be able to have different rep rates on different channels at the same time, but ended up only being able to have one rep rate on each channel at the same time. In the future, the code can be modified to accommodate such capabilities. Also, even though the variable phase shifter function of the DCM was programmed to be able to vary the phase of the triggers, the code was not written to be able to use it in conjunction with the user interface. Future work can be done on this to get it running.

I really enjoyed the internship and the project. I believe that much of what I learned I will be using in the future. Working on this project has further encouraged me to pursue higher levels of education. 


\section{Literature Cited}

[1] XILINX, Spartan-3 Generation FPGA User Guide- Extended Spartan-3A, Spartan-3E, and Spartan-3 FPGA Families, [UG331 (v1.5)]. January 21, 2009. 


\section{Acknowledgements}

This project was done at the SLAC National Accelerator Laboratory. My acknowledgements go out to my mentor Ron Akre for all of his assistance, Jeff Olsen, who helped me with VHDL and the Xilinx software, my partner Kelton Stefan, and Gregorio Dalit, who helped solder parts onto my PCB board. I would, also, like to thank the U.S. Department of Energy, the Office of Science, and the SLAC National Accelerator Laboratory for creating, organizing, and funding the program. Further Acknowledgements go out to Steve Rock, SueVon Gee, Vivian Lee, and Elizabeth Smith, who ran the program, for all that they have done. 
Figures and Tables

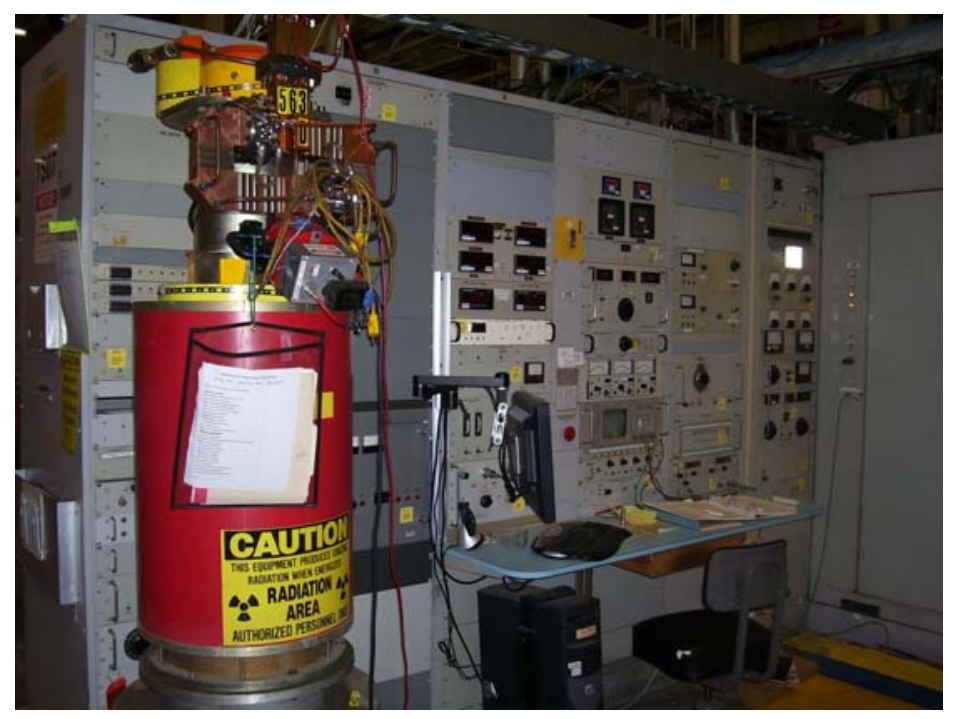

Figure 1: A test station with a klystron.

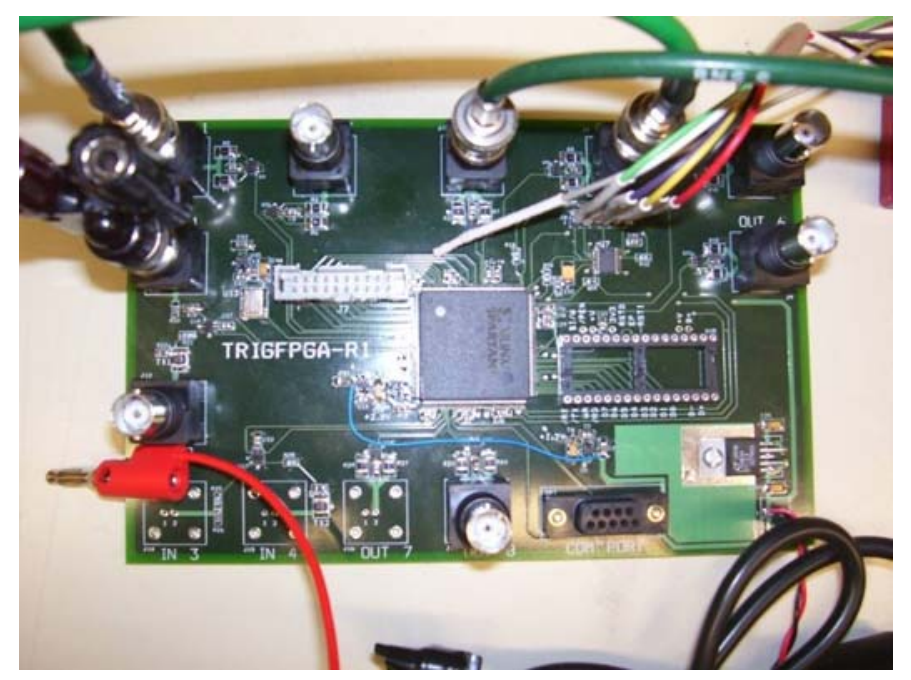

Figure 2: $P C B$ with FPGA in the center. 


\begin{tabular}{|c|c|c|c|}
\hline PSEN & PSCLK & PSINCDEC & Output \\
\hline 0 & $\mathrm{X}$ & $\mathrm{X}$ & $\begin{array}{c}\text { Nothing } \\
\text { happens }\end{array}$ \\
\hline 1 & 0 & 0 & $\begin{array}{c}\text { Nothing } \\
\text { happens }\end{array}$ \\
\hline 1 & 0 & 1 & $\begin{array}{c}\text { Nothing } \\
\text { happens }\end{array}$ \\
\hline 1 & $\begin{array}{c}\text { Rising } \\
\text { edge }\end{array}$ & 0 & $\begin{array}{c}\text { Decrement } \\
\text { phase one } \\
\text { step }\end{array}$ \\
\hline & edge & 1 & $\begin{array}{c}\text { Increment } \\
\text { phase one } \\
\text { step }\end{array}$ \\
\hline
\end{tabular}

Table 1: Truth table for the Variable phase shifter.

\begin{tabular}{|c|c|c|c|c|}
\hline $\begin{array}{c}\text { CLKIN } \\
\text { Frequency }\end{array}$ & $\begin{array}{c}\text { CLKIN } \\
\text { Period }\end{array}$ & $\begin{array}{c}\text { Maximum \# } \\
\text { of DCM } \\
\text { Delay Steps }\end{array}$ & $\begin{array}{c}\text { Typical Step } \\
\text { Size }\end{array}$ & $\begin{array}{c}\text { Maximum } \\
\text { Variable } \\
\text { Phase Shift }\end{array}$ \\
\hline $119 \mathrm{MHz}$ & $8.403 \mathrm{~ns}$ & $+/-81$ steps & $25 \mathrm{ps}$ & $+/-2.025 \mathrm{~ns}$ \\
\hline
\end{tabular}

Table 2: Capabilities of the Variable Phase Shifter at $119 \mathrm{MHz}$. 


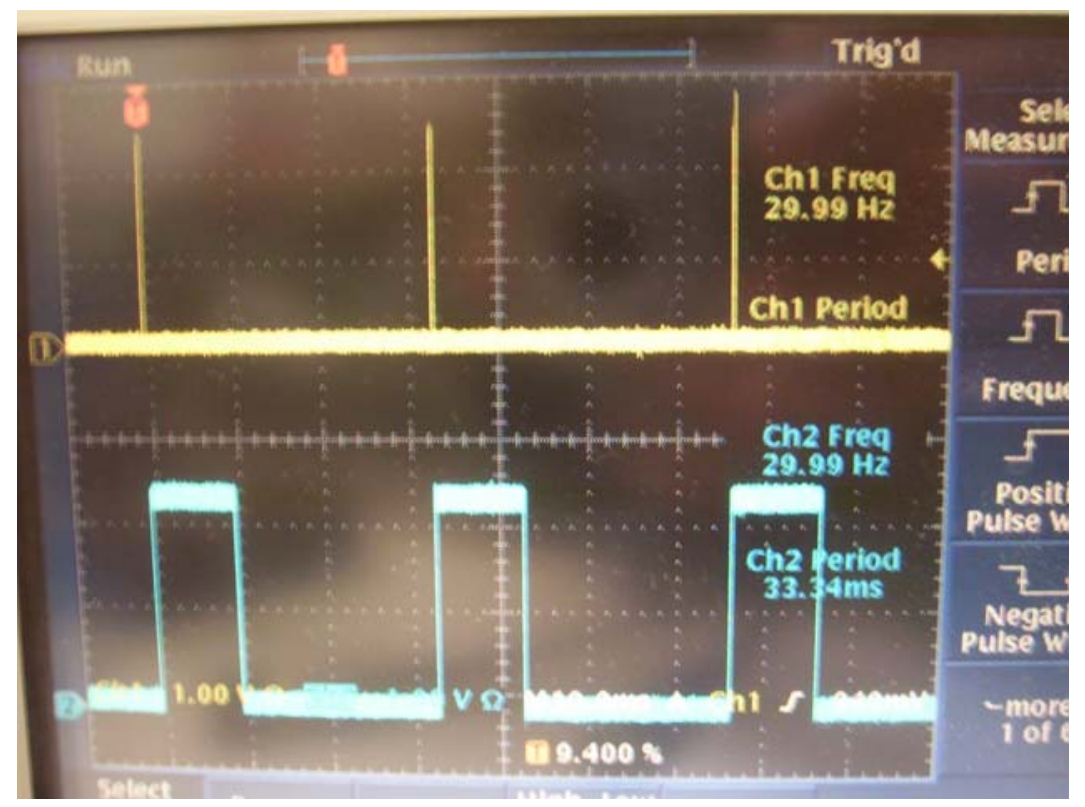

Figure 3: Waveform of one of the triggers.

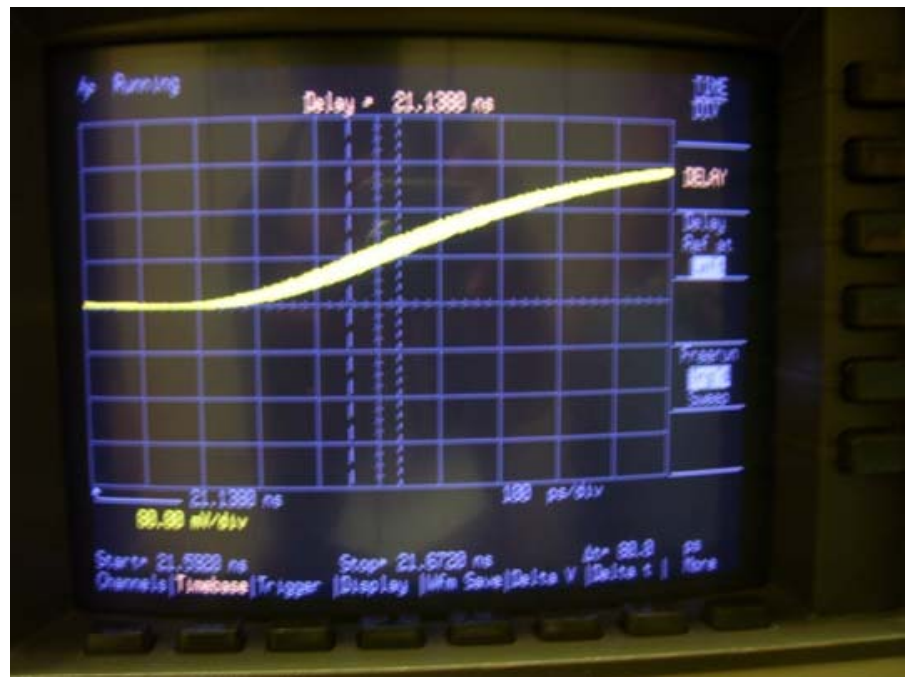

Figure 4: Non-DCM Channel jitter measurement. 


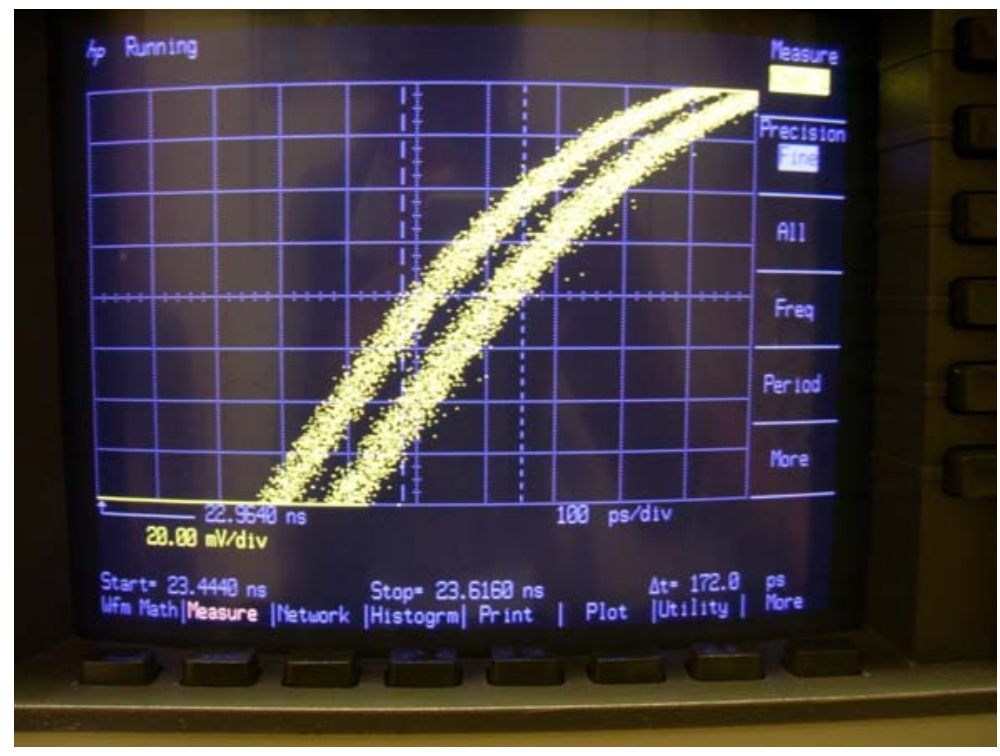

Figure 5: Channel using DCM showing jitter. 


\title{
Fiber Accelerating Structures
}

\author{
Andrew P. Hammond \\ Office of Science, Science Undergraduate Laboratory Internship Program
}

Reed College

Stanford Linear Accelerator Center

Menlo Park, California

August 14, 2009

Prepared in partial fulfillment of the requirement of the Office of Science, Department of Energy's Science Undergraduate Laboratory Internship under the direction of James E. Spencer in the Advanced Accelerator Research Department at the Stanford Linear Accelerator Center.

Participant:

Signature

Research Advisor:

Signature 


\section{Contents}

1 Introduction $\quad 1$

2 Materials and Methods $\quad 4$

$\begin{array}{lll}3 & \text { Results } & 5\end{array}$

3.1 Simple Fiber Maximization . . . . . . . . . . . . . . . . 5

3.2 Double Defect Modes . . . . . . . . . . . . . . . . . . . . . . . . 7

3.3 Perturbed Periodic Lattice Fibers . . . . . . . . . . . . . . . . . . 9

4 Conclusion $\quad 10$ 


\begin{abstract}
One of the options for future particle accelerators are photonic band gap (PBG) fiber accelerators. PBG fibers are specially designed optical fibers that use lasers to excite an electric field that is used to accelerate electrons. To improve PBG accelerators, the basic parameters of the fiber were tested to maximize defect size and acceleration. Using the program CUDOS, several accelerating modes were found that maximized these parameters for several wavelengths. The design of multiple defects, similar to having closely bound fibers, was studied to find possible coupling or the change of modes. The amount of coupling was found to be dependent on distance separated. For certain distances accelerating coupled modes were found and examined. In addition, several non-periodic fiber structures were examined using CUDOS. The non-periodic fibers produced several interesting results and promised more modes given time to study them in more detail.
\end{abstract}




\section{Introduction}

Currently, linear accelerators use high intensity microwaves to drive the electron bunches through metal radio frequency cavities. Unfortunately, this system is very inefficient. Therefore new systems to better accelerate electrons are being developed. One system that might become a future method for electron acceleration is photonic bandgap (PBG) fibers. It has been shown that PBG fibers could form much better accelerating fibers, if several challenges can be overcome.

Photonic crystals are periodic macroscopic structures that have properties of light propagation similar to those associated with electrical propagation in semiconductors. (Joannopoulos et. all, pg. 4, [3]) One of the key properties for light propagation in photonic crystal is the existence of the photonic bandgap. It is possible using the proper lattice of two dielectric materials periodically spaced to create a band gap in the crystal through which light with frequencies in the bandgap cannot propagate. The theoretical basis for the photonic bandgaps is given by Maxwell's equations of electromagnetism in cgs units. (Joannopoulos et. al, pg. 8, [3])

$$
\begin{array}{r}
\nabla \cdot \mathbf{B}=0 \\
\nabla \cdot \mathbf{D}=4 \pi \rho \\
\nabla \times \mathbf{E}=-\frac{1}{c} \frac{\partial \mathbf{B}}{\partial t} \\
\nabla \times \mathbf{H}=\frac{4 \pi}{c} \mathbf{J}+\frac{1}{c} \frac{\partial \mathbf{D}}{\partial t}
\end{array}
$$

Now, we assume that $\mathbf{D}$ is linearly related to $\mathbf{E}$ by the permitivity $\epsilon(\mathbf{r}, \omega)$ and the permeability is unity so that $\mathbf{B}=\mathbf{H}$. In order to separate the equations from the time domain we assume the solutions take the form

$$
\begin{aligned}
& \mathbf{H}(\mathbf{r}, t)=\mathbf{H}(\mathbf{r}) e^{i \omega t} \\
& \mathbf{E}(\mathbf{r}, t)=\mathbf{E}(\mathbf{r}) e^{i \omega t}
\end{aligned}
$$

This can be done without ignoring solutions because the equations are linear so, Fourier Analysis can be used. In a photonic crystal without free charges or currents, the propagation 
of light is given by the wave equation for dielectrics.

$$
\begin{array}{r}
\nabla \times\left(\frac{1}{\epsilon(\mathbf{r})} \nabla \times \mathbf{H}(\mathbf{r})\right)=\left(\frac{\omega}{c}\right)^{2} \mathbf{H}(\mathbf{r}) \\
\mathbf{E}(\mathbf{r})=\left(\frac{-i c}{\omega \epsilon(\mathbf{r})}\right) \nabla \times \mathbf{H}(\mathbf{r})
\end{array}
$$

Combined with the first two Maxewell equations, this provides the necessary conditions for an electromagnetic wave. In a periodic lattice of dielectrics, there exists the condition of translational symmetry within the problem. If one imagines the dielectric as a series of blocks with period a, then the electric and magnetic fields should be the same a distance $a$ away. Combined with the wave equation the general solution for $\mathbf{H}(\mathbf{r})$ is: (Joannopoulos et. all, pg. 31, [3])

$$
\mathbf{H}(\mathbf{r})=\mathbf{u}_{\mathbf{k}}(\mathbf{r}) e^{i(\mathbf{k} \cdot \mathbf{r})}
$$

This in turn translates to a series of oscillatory modes that light must satisfy to propagate through the structure. However, because the frequency is related to the k-value of light, which defines the wavelength, the light will fluctuate through a series of k-terms as frequency changes to remain within the oscillatory mode. Sometimes, for certain wavelengths the kterm must be imaginary to remain in an oscillatory mode. When the $\mathrm{k}$ is imaginary, it implies light at that frequency exponentially decays due to absorption. In short, light with an imaginary k- term cannot propagate through the crystal.

A useful property of the photonic bandgap is the defect mode. Because the bandgap is caused by the periodic structure of the crystal, when that structure is changed in a localized section, the defect, the allowed modes change as well. In the area surrounding the defect it is possible, depending on the geometry of the defect, for light to propagate even if it has a frequency in the bandgap. However, the defect modes are spatially confined in or near the defect and will not propagate in the rest of the crystal because elsewhere there is still the decay term.

These properties of the PBG crystal can be taken advantage of to accelerate electrons using a PBG fiber. A PBG fiber is an optical fiber consisting of a dielectric material, ideally silicon or silica, with periodic columns of vacuum inside. The fiber used here consists of circular columns used to make a hexagonal fiber. In the fiber the defect is a column with a 
greater radius than the others. The defect column would also serve as the tube through which the electron bunch moves. Lasers would be coupled into the fiber to create electromagnetic waves inside the defect which would accelerate the electrons.

Now, this sort of lattice allows at least two kinds of defect mode: a confined mode and a surface mode (Noble et. all, [7]). In the confined mode the electromagnetic field is confined almost entirely inside the defect column. This mode cannot be used to accelerate particles, but can be used to transmit information i.e. increase longitudinal momentum, because it does not form a uniform axial electric field in the column. Because this mode can transmit information and makes few requirements about precision, it is used by the telecom industry. A surface mode is where the light propagates in the central defect and in the area around the defect where the geometry has changed. In this mode the Poynting vector is concentrated around the surface of the defect column. A surface Poynting vector is shown in Fig. 1; notice the "'hot spots"' and their symmetries in the lattice. Inside of the defect, the surface modes can be uniform, perfect for uniformly accelerating a bunch of electrons. However, surface modes also have other useful field patterns, like the creation of a magnetic quadrupole, or electric lens that could be used to confine the electrons to a tight bunch. Unfortunately, because the electric field exists outside of vacuum, eventually the breakdown field will be reached setting a maximum level on the gradient of the electric field inside of the fiber defect. Therefore, when measuring the electric field a program computes inside a fiber the relevant number is the ratio of the accelerating field to the maximum electric field in the lattice material. Despite this limitation, a PBG accelerator should be several orders of magnitude better than current technology.

In order for these new types of accelerators to be built, several hurdles need to be overcome. Currently the telecom industry uses PBG fibers that support core modes. These fibers are similar in that they come in a lattice and have a defect to those that might be used in an accelerator.(Temelkuran et. all [8]) However, the manufacturers have yet to make a defect mode similar to the one that current designs require. the manufacturers also lack the precision to make the specific modes with a more exact lattice. Also, many of the fiber producers are disinterested in making experimental length PBG fibers, which SLAC would like to test out before ordering longer examples. (Spencer) Fortunately, the Research group 
at SLAC is working with a new company, Incom Inc., to create the necessary fibers. However, they have yet to start using high-purity silica or silicon, or added a defect. The fibers currently possible also require a higher wavelength laser, but such lasers do not exist at all let alone at sufficient power. It is also difficult to find a way to get both the electrons and the laser light coupled into the fiber.

\section{Materials and Methods}

In order to study and understand the electric fields resulting from different PBG geometries, several computer programs were used. The program most used was CUDOS. In addition, Mathematica was used for data analysis, and geometry calculations.

The program CUDOS was built and designed by the Physics department at the University of Sydney in Australia. (Kuhlmey, [5]) CUDOS takes a given geometry and returns the magnetic and electric propagation fields, along with the Poynting vector field. The program uses only a $2 \mathrm{D}$ cross section of the fiber making the field calculation assumption that the fiber has infinite length in the third dimension. The software is based on a method which uses a multipole expansion centered on each cylindrical hole of a fiber to enforce boundary conditions and then matches the local and global expansions to determine the expansion coefficients. (Noble, [6]) Once the various fields have been calculated CUDOS displays a gradient mapping the relative field strength. For a more exact look at the relative fields the raw data was imported to Mathematica.

Once suitable electric field plots were found for various geometries, Mathematica was used to get a more in depth picture of the electric field. While CUDOS does create a relative mapping, the system does not examine the field in a quantitative manner. To look at the subtle field changes, as well as scaling to break-down fields, the plots of the raw data were created using Mathematica.

Mathematica has also been used as a method of calculating the geometries of the lattice. The lattice is arranged in a hexagon with all of the cylinder's centers separated by the same distance from the other. The layers of more hexagons need to be calculated with the position coordinates in the polar system. An example of the cylinder geometry of a CUDOS file is 
found in Fig. 1.

\section{Results}

Several new designs for PBG fibers have been created that are covered in several different sections. One important area is the creation of the best simple fiber by changing the pitch, defect radius, and normal cylinder radius. Also the possible coupling of two defect modes propagating together has been examined. The third area worked on was the effect of nonperiodic symmetry in a PBG fiber.

\subsection{Simple Fiber Maximization}

As mentioned above, the company Incom Inc. has been working with SLAC to create an accelerating PBG fiber. They are currently in the process of creating fibers with defect modes. However, when these new fibers are built they need to be at the proper sizes so that they will accelerate for the lasers that are available. Using the simple designs created by previous students, Eddie Lin, Elliot Johnson, and Sara Campbell, as a starting position; the basic parameters of the hexagonal lattice were modified to find the best simple accelerating fiber. The basic parameters that were modified are the pitch, defect radius, and normal cylinder radius. In this field, the goal was to maximize two parameters, the electric field gradient, and make the defect radius large. It is important to have a high electric field gradient so that the electrons undergo a greater acceleration over a certain distance. It is important to maximize the defect radius so that a larger number of electrons can be fit into the defect radius. As these modes are considered it is important to maintain a uniform field across the defect.

The simple PBG fibers were supposed to be designed for accelerating modes at defect wavelengths of 2, 4, 6, 8, and 16 microns. Now, according to the linear model discussed in the introduction, the wave equation solutions allow a simple scaling of the photonic fiber to increase wavelength. Basically, in a PBG fiber it should be possible to take a fiber with a 2 micron accelerating mode and multiply the basic parameters by 2 to make a 4 micron accelerating mode. (Joannopoulos et. al, pg. 19, [3]) Unfortunately, because the refractive 
index changes a significant amount over the range from 2 to 16 microns, this theory requires parameter adjustment. The refractive index changes as one encounters different energies in which silica is easily excited implying a high level of absorbance. Due to time constraints, only the 2 micron case, which was studied by Sara Campbell and is near the wavelength of the powerful YAG laser, and the 16 micron case, which can already be manufactured, were considered.(Spencer, [1])

The study of the 2 micron PBG fiber started from the fiber design of previous SULI student, Sara Campbell. This design was created by scaling a previous design for a 2.016 micron fiber, as such it had a lerge number of significant figures which I pared down to $10^{-2}$ microns. Once the design was simplified, it was experimented with by making small changes to the pitch, the cylinder center to cylinder center distance, of the fiber. However, this quickly caused the defect mode to stop supporting an accelerating mode at 2 microns. Next, the possible changes that could be made in the normal cylinder radius were examined. The higher the radius of the normal cylinders corresponds to a higher electric field gradient as shown in Fig. 2. Finally the radius of the defect was varied. Unfortunately, the electric field gradient is inversely proportional to the size of the defect mode as shown in Fig. 3. Now in an effort to increase the defect radius regardless of the gradient, the defect radius was increased while lowering the normal radius. Using this approach the maximum defect radius before exiting the bandgap was found to be 1.43 microns. However the field gradient continued to decrease at an even sharper rate, shown in Fig. 4. Unfortunately, the simulation does not work to increase the gradient while decreasing the defect radius and increasing the normal radius.

Now, Mathematica was used to insure that the two fiber designs with maximized defect radius or maximized gradient had a constant longitudinal electric field in the defect. The relevant data for these designs is in Table 1. To make sure the electric field was uniform inside the defect, a graph of the longitudinal electric field along the $\mathrm{x}$-axis and the $\mathrm{y}$-axis was used. The graph was formed from a series of data points output from CUDOS. The graphs and the CUDOS mappings are shown in Fig's. 5, 6, 7, 8, 9 and 10. It is interesting to note that the electric field changes its highest point in the lattice inward as the field gradient becomes larger. The largest defect mode was calculated to have a $3.2 \%$ uniformity 
over the defect, and a $1.9 \%$ uniformity over $80 \%$ of the defect. The defect uniformity over the maximum gradient is $9.8 \%$ and over $80 \%$ of the defect it has a uniformity of $7.7 \%$. The uniformity was calculated by taking the difference between the field at the center of the defect and the maximum field in the defect, and then dividing by the field at the center of the defect. Clearly, although neither plot is completely uniform, the large defect mode is more uniform, possibly because the first high points in the larege defect mode have moved inside of the defect for a smaller defect radius. The lack of uniformity light also imply that several modes are mixed into the calculation.

In addition to the 2 micron defect, a design for a 16 micron wavelength defect was concentrated on. This wavelength was chosen because Incom could make the fibers well, and the index of refraction only changed a small amount from the 2 micron case, allowing the design to be mostly scaled up. When the refractive index changes, the most important change is the size of the defect radius. The change in index most changes the defect radius so with a small change I could concentrate only on this small part of the fiber that is important. The index of refraction increased only by 0.025 from 1.459 to 1.484 (Kitamura, [4]). Using CUDOS, I found the different defect radii that supported acceleration with the new index of refraction. The lowest defect radius value, 11.00 microns is 0.78 microns larger than that scaled from the 2 micron case. This proves the basic point that the defect radius increases for higher index. This result seems to preclude the 8 micron case because its index of 0.407 is so small. However, it might be possible to instead use the small defect to increase the defect size past the first hexagonal ring. The calculated values have been organized in Table 1.

\subsection{Double Defect Modes}

Also the effect that a double defect mode might have on a PBG fiber has been examined. A double defect could have several useful qualities. First, it might be possible to accelerate the electrons in one defect using a coupled defect that is excited by a laser. Also, it could be useful to have several defects inside a single fiber all of which accelerate electrons. This could make up for the fact that only a small number of electrons could fit into the defect cylinder. When examining the multiple defect, only a double defect was examined where 
CUDOS had just two defects. Hopefully, this would reveal any useful coupling or changes in the electric field while not perturbing the coupling by involving multiple defects. In order to concentrate on just coupling the 2 micron accelerating case discussed in the section above was used to specify the basic parameters. However, instead of a single defect cylinder, two defect cylinders were added that had several normal cylinders separating them from one another. The number of cylinders in a straight line between defects was used as the independent parameter specifying the different runs. The defect modes were spaced a range of normal cylinders away from 1 to 9 . Outside this range the number of cylinders lead to leakage of the mode and more could not be added without exceeding the memory capacity of the computer being used.

If the two defects are 1 to 3 cylinders away, the accelerating mode disappears. This is probably because the proximity effects the structure of the bandgap and allows coupling between the defect modes. However, the coupling does not show a useful accelerating mode. For larger distances away, 5 and 7 , the two defects only showed the minor coupling in Fig. 11. This map shows a clear shift in the basic single defect field as shown in Fig. 10. First, even modes that appeared only weakly coupled shifted the points of greatest electric field towards each other as shown in Fig. 11. This might show either coupling or a lack of enough outer cylinders to confine the mode at the outer boundary. When looking a the longitudinal field of the double defect, Fig. 12, the defect field falls off sharply as the distance from the center of the fiber increases. This seems to confirm at least partial loss from the lack of layers. However, by examining the longitudinal field with both 6 and 3 layers of hexagons surrounding the single defect, Fig's. 13 and 14, it is clear that the field is not just the two single modes added together. Even if the modes are coupled, the extent that a beam in one defect could be used to drive a second defect is unknown. Unfortunately, CUDOS does not test this coupling possibility and a more advanced program is necessary. However, it seems worthy of more attention considering the difficulty that the laser coupling already faces. If the two defects were farther apart they lose the accelerating mode and begin to stop holding a mode. However, I suspect this is due to the lack of enclosure by outer cylinders. 


\subsection{Perturbed Periodic Lattice Fibers}

As noted above, the simplest and easiest to construct fibers are those with a single defect and the rest of the cylinders all the same. However, by violating this uniformity it is possible to modify the electric field to accomplish some useful goals. In the attempt to eliminate the hot spots of the fiber, two strategies were used. First, small cylinders of pure vacuum were added on top of the hot spots. However, these small cylinders often failed to cover the entire hot spot, as shown in Fig. 15. The lack of coverage only meant that the side of the cylinder would begin to break down first. The cylinders could not be expanded because then the effective index of refraction would change too much making the wave travel significantly slower than the speed of light. An attempt was also made to eliminate high points in the electric field by expanding certain cylinders while contracting others so that the correct index was maintained. Unfortunately, while this did eliminate the largest high point, all that happened was either other equally strong high points were added which needed to be removed through the small hole method as shown in Fig. 16. The large hole could also significantly perturb the band gap creating new modes that were detrimental to the accelerating structure as shown in Fig. 17. None of these modes is satisfactory to eliminating the hot spots. Currently, I believe that a radically different look of the lattice is necessary to remove the field high points within the lattice. While more work should be done looking for these modes, it is necessary to perform more preparatory analysis to find where to look.

While examining the effect of small hole to eliminate the field high points, it was suggested that the small cylinders could be filled with a new material with a closer index of refraction. If filled with the correct substance, the cylinders could become tiny lasers on the side of the fiber that would drive the electromagnetic field. In a side-laser fiber, several cylinders with different index of refraction simulating the lasing index were added to the CUDOS fiber. In the basic design shown in Fig. 10 there are a series of areas where the field is very high. The goal of this experiment was to keep the high points at the same spot but move them inside cylinders that are filled with a lasing source thus theoretically coupling to drive lasers. These side lasers might be able to then drive the fiber accelerator through natural coupling. One expects this should happen over a short distance because of the need to realign electron 
bunches with the electromagnetic driving wave. The model used YAG as the fill material which has a refractive index of $1.82([2])$. The insertion of minor cylinders with this index had almost no effect on the movement of the accelerating modes longitudinal electric field. Ultimately, all high electric field points were covered by YAG cylinders as shown in Fig. 18 . This is a good sign for the possibility of driving the accelerator in this manner, but it would be very difficult to make. Also there is no guarantee that the lasers would produce the accelerating field and not a new mode. These possible effects depend on the mode of the laser. In addition to driving the accelerating field, the cylinders change the effective index. Although the change is not significant, only a difference of 0.01 , this illustrates the principle that the overall effective index can be changed and controlled. As discussed above this changes the movement of the electromagnetic waves, but it could be useful in re-aligning the electron bunch and the accelerating wave because eventually the wave passes the bunch and starts deaccelerating the bunch.

\section{Conclusion}

A photonic band gap fiber is a promising method for accelerating electrons. There are many clear accelerating modes. that can be built and tested in the near future. There also exist a large number of possible modes that could be examined using the relatively simple program CUDOS to solve the problem of coupling the laser into the fiber. The confining lens is also a viable option that needs more testing, but could be a serious possibility for future fibers. However, a PBG fiber also faces difficulties due to its small size, and the difficulty in coupling laser into the defect mode. Therefore, PBG accelerators are a possible future accelerator source that requires more study. 


\section{References}

[1] Eric Colby, Gary Lum, Tomas Plettner, and James Spencer. Gamma radiation studies on optical materials. IEEE Transactions on Nuclear Science, 49(6), 2002.

[2] Northrop Grumman. Undoped [yag].

[3] John D. Joannopoulos, Robert D. Meade, and Joshua N. Winn. Photonic Crystals, Molding the Flow of Light. Princeton University Press, 1995.

[4] R. Kitamura, L. Pilon, and M Jonasz. Optical constants of fused quartz from extreme ultraviolet to far infrared at near room temperatures. Applied Optics, 46(33):8118-8133, 2007.

[5] Boris Kuhlmey. CUDOS MOF Utilities, User's Guide, 2006.

[6] Robert J. Noble. Dispersion information for photonic fiber modes from cudos simulations. $A R D B$ Note 04, SLAC, 2005.

[7] Robert J. Noble, Eric R. Colby, Benjamin Cowan, Christopher M. Sears, Robert H Siemann, and James E. Spencer. Designing photonic bandgap fibers for particle acceleration. In Particle Accelerator Conference, PAC 0\%, SLAC-PUB-12571, June 2007.

[8] Burak Temelkuran, Shandon D. Hart, Gilles Benoit, John D. Joannopoulos, and Yoel Flink. Wavelength-scalable hollow optical fibres with large photonic bandgaps for co2 laser transmission. Nature, 420, 2002. 


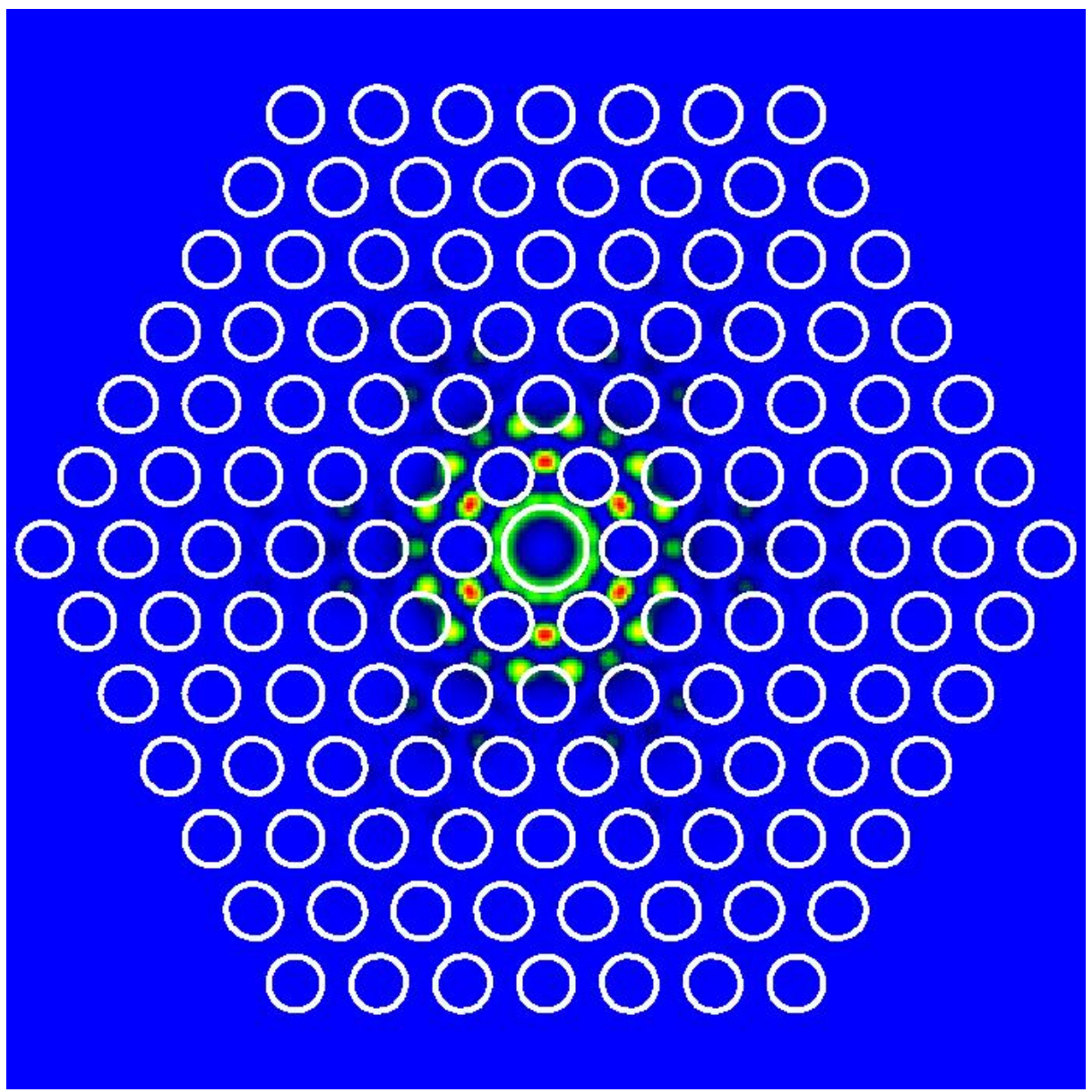

Figure 1: This picture shows the surface mode Poynting vector of a PBG fiber with a defect.

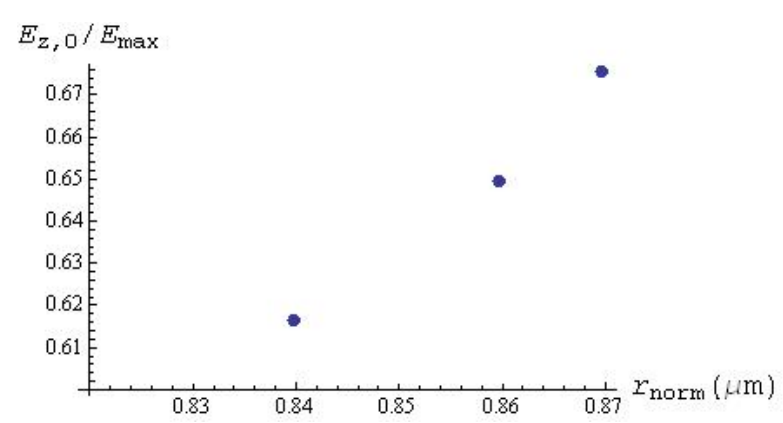

Figure 2: This graph shows the ratio of the longitudinal electric field at the origin to the maximum longitudinal electric field in the fiber(y-axis) compared to the normal cylinder radius(x-axis, measured in microns). The fiber is a 2 micron accelerating fiber the maximum values of which are shown in Table 1 . The ratio is used because it determines the maximum acceleration achievable before the electric field exceeds the fiber's maximum. The plotted points are not all points and there could be smaller radii, the graph just shows the decreasing ratio. 


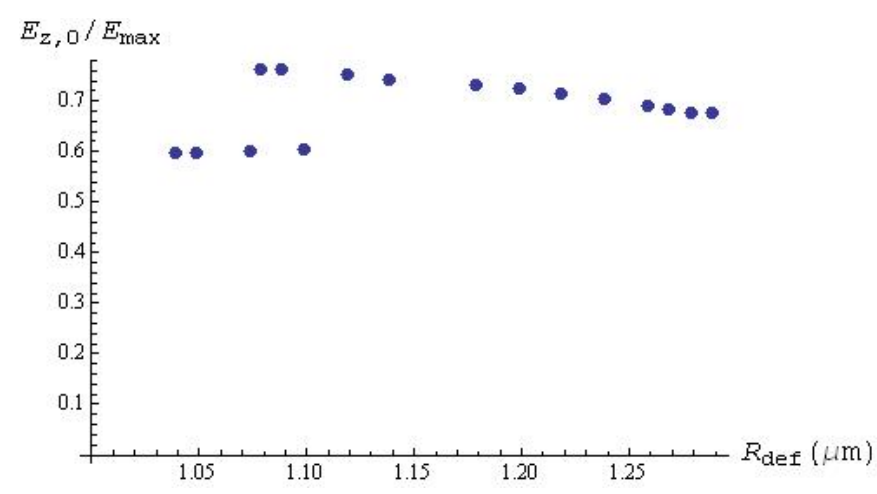

Figure 3: This graph shows the ratio of the longitudinal electric field at the origin to the maximum longitudinal electric field in the fiber(y-axis) compared to the defect cylinder radius(x-axis, measured in microns). The fiber is a 2 micron accelerating fiber the maximum values of which are shown in Table 1 . Near smaller defect radii, the mode begins to switch over into a new ratio regime. This new regime is still an accelerating fiber, but it does not do so as well as the previous regime. This graph shows the entire range of values with a constant normal cylinder radius.

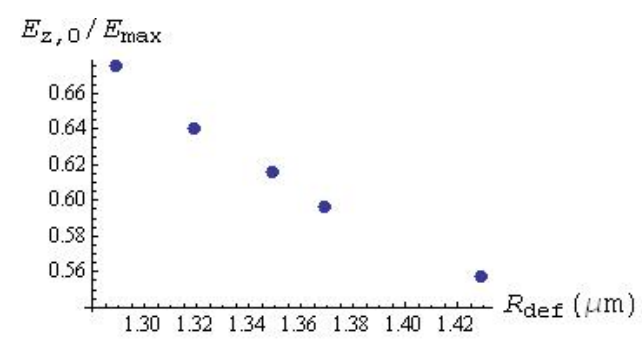

Figure 4: This graph shows the ratio of the longitudinal electric field at the origin to the maximum longitudinal electric field in the fiber(y-axis) compared to the defect cylinder radius(x-axis, measured in microns). The fiber is a 2 micron accelerating fiber the largest defect of which is shown in Table 1. In the graph only the defect radius is shown, but the normal radius is changing as well in order to maintain the accelerating mode. The normal radius each time is as high as it could possibly be consistent with maximum field

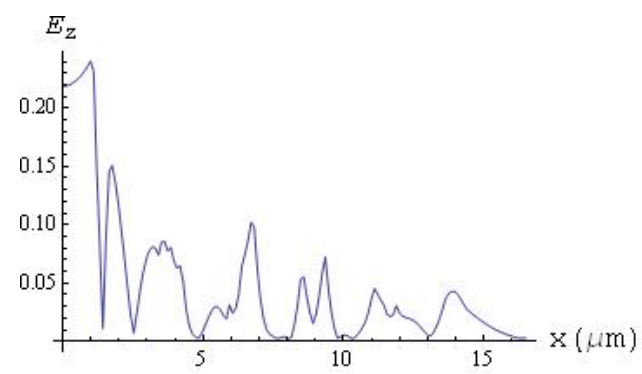

Figure 5: This graph shows the electric field over the x-axis of the highest gradient ratio. The values are shown in row 3 of Table 1. Notice the loss of uniformity around the defect. 


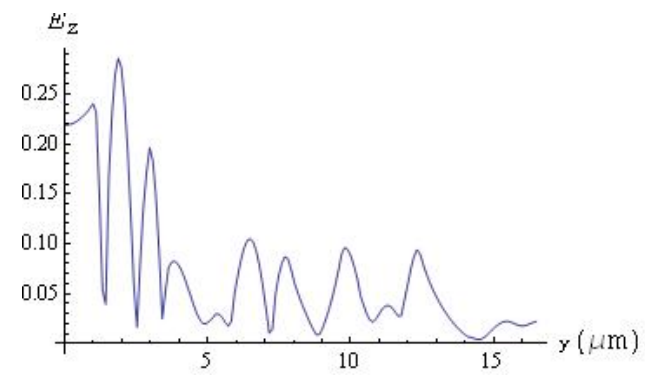

Figure 6: This graph shows the electric field over the y-axis of the highest gradient ratio. The values are shown in row 3 of Table 1. Notice the loss of uniformity around the defect; it is also exactly the same shape inside the defect as in Fig. 5

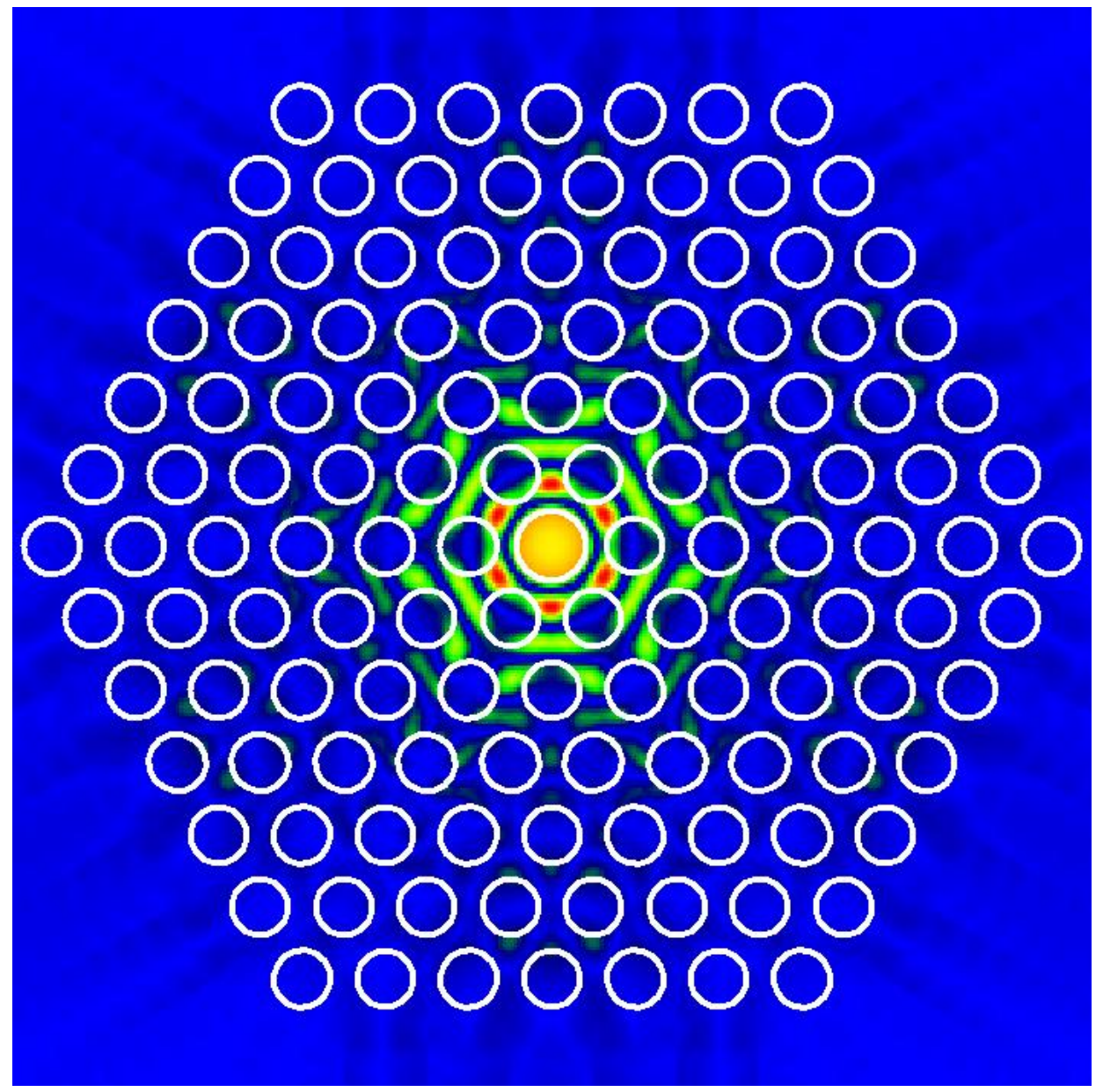

Figure 7: This shows the CUDOS output of the maximum gradient. The values are shown in row 3 of Table 1. 


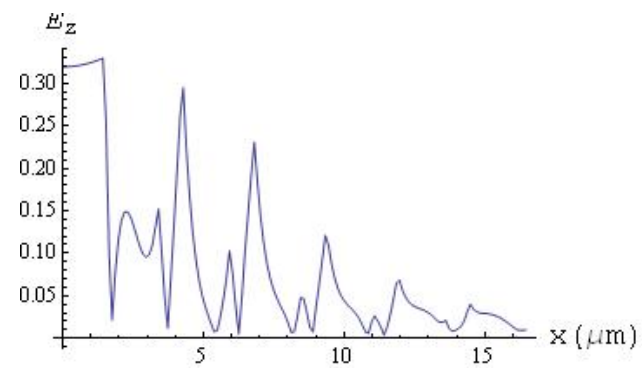

Figure 8: This graph shows the electric field over the x-axis of the highest defect radius. The values are shown in row 2 of Table 1.

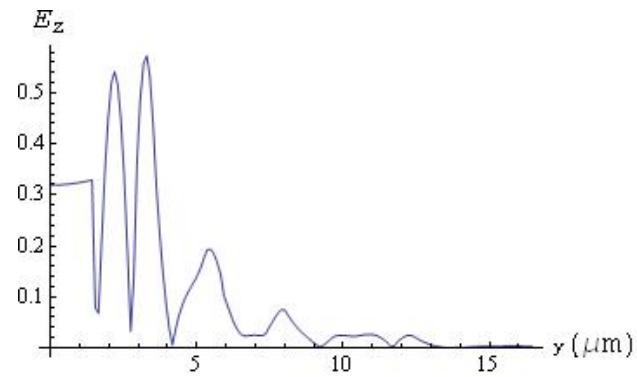

Figure 9: This graph shows the electric field over the y-axis of the highest defect radius. The values are shown in row 2 of Table 1. The defect field here is the same as in Fig. 8.

\begin{tabular}{|c|c|c|c|c|c|c|}
\hline$\lambda(\mu m)$ & $n$ & $n_{\text {eff }}$ & $R(\mu m)$ & $r(\mu m)$ & $P(\mu m)$ & Ratio \\
\hline 2.0 & 1.459 & $1.0017,3.22 \mathrm{E}-4$ & 1.43 & 0.84 & 2.55 & 0.5570 \\
2.0 & 1.459 & $1.0196,1.11 \mathrm{E}-3$ & 1.08 & 0.87 & 2.55 & 0.7639 \\
16.0 & 1.484 & $1.0018,3.67 \mathrm{E}-4$ & 12.50 & 6.92 & 20.40 & 0.4280 \\
16.0 & 1.484 & $1.0192,2.72 \mathrm{E}-4$ & 11.00 & 6.92 & 20.40 & 0.4938 \\
\hline
\end{tabular}

Table 1: This table shows the values of the important variables for the simple lattice structure maximized cases. In the table, $\lambda$ is the wavelength in microns, $n$ is the index of refraction of the matrix, $n_{\text {eff }}$ is the effective index of refraction, a sort of combined refraction for the whole fiber. $R$ is the radius of the defect in microns, $r$ is the normal radius in microns, $P$ is the pitch, the center to center distance, and Ratio is the ratio of the maximum electric field in the lattice to the electric field at the origin. The 2 micron highest gradient case is not the highest tested case because the highest case is in a mixture where two regimes collide. The higher mode might easily be perturbed into a lower gradient mode so a more safe option was chosen. The 16 micron case has also not been tested using a variation of normal cylinder radius, which might improve the maximum values here. 


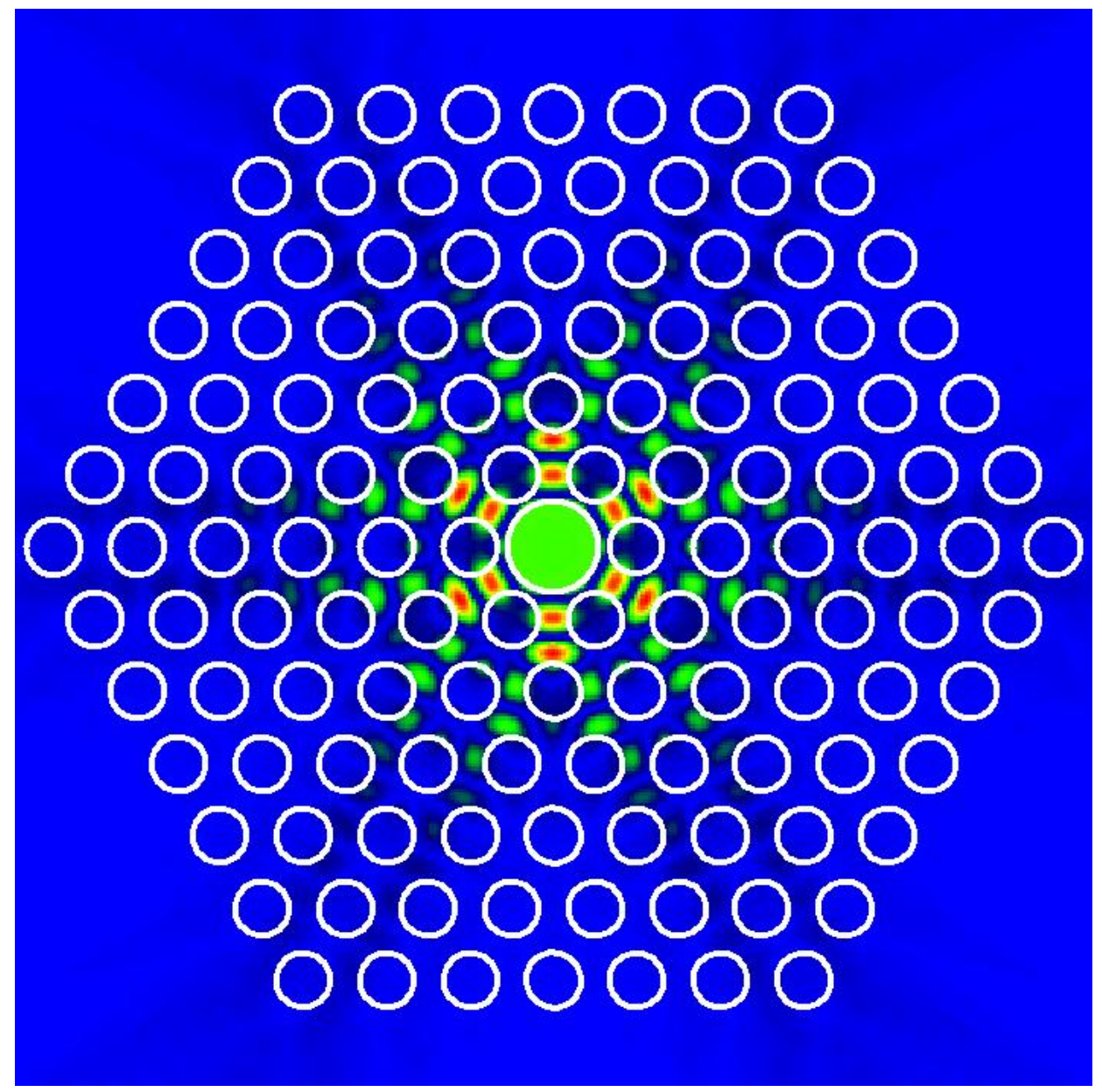

Figure 10: This shows the CUDOS output of the maximum single defect radius. The values are shown in row 2 of Table 1. 


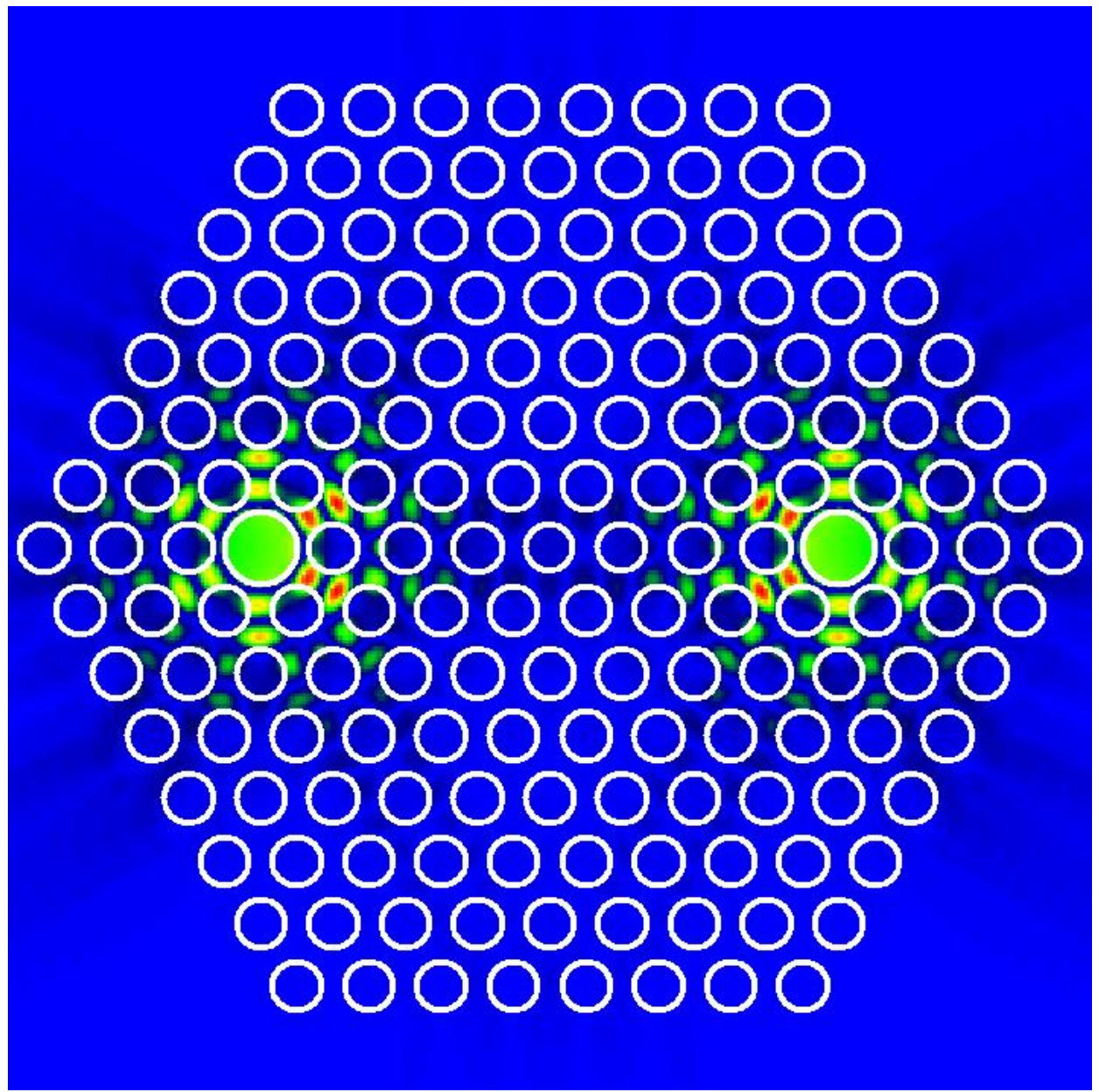

Figure 11: This shows a double defect CUDOS simulation. The values are shown in row 2 of Table 1 except there are two defect radii. Notice the slight coupling along the line of normal cylinders.

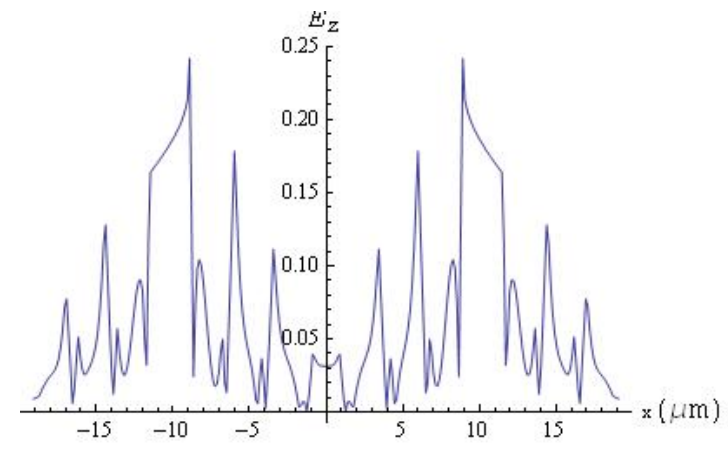

Figure 12: This graph shows the longitudinal electric field along the x-axis. Notice the steep drop off between the two defects extending from \pm 9.65 to \pm 12.19 . However, neither peak is as large as that for a single defect, so perhaps some coupling is occurring. 


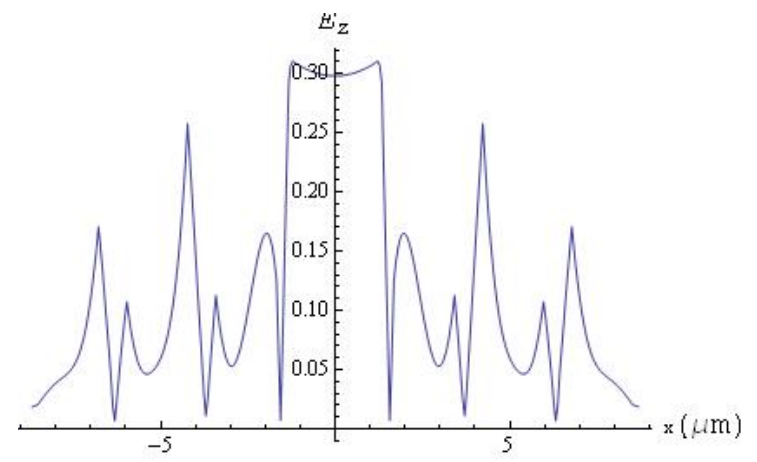

Figure 13: This graph shows the longitudinal electric field for a single defect with only 3 hexagon layers surrounding the defect. The parameters are the same as those for the double defect case, and show a 2 micron accelerating fiber.

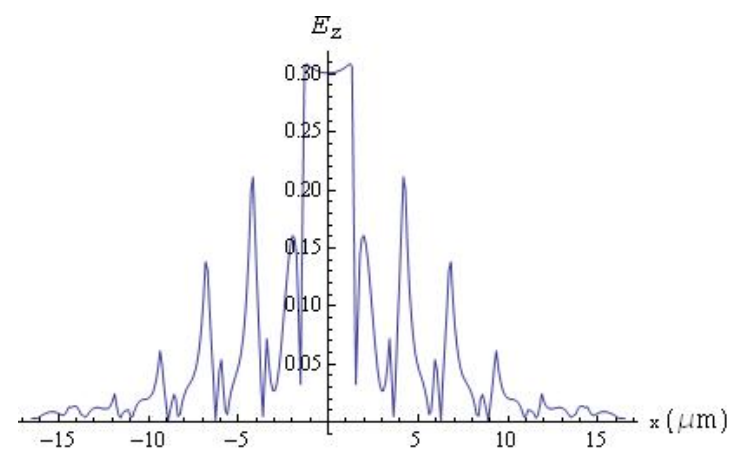

Figure 14: This graph shows the longitudinal electric field for a single defect with 6 hexagon layers surrounding the defect. The parameters are the same as those for the double defect case, and show a 2 micron accelerating fiber. 


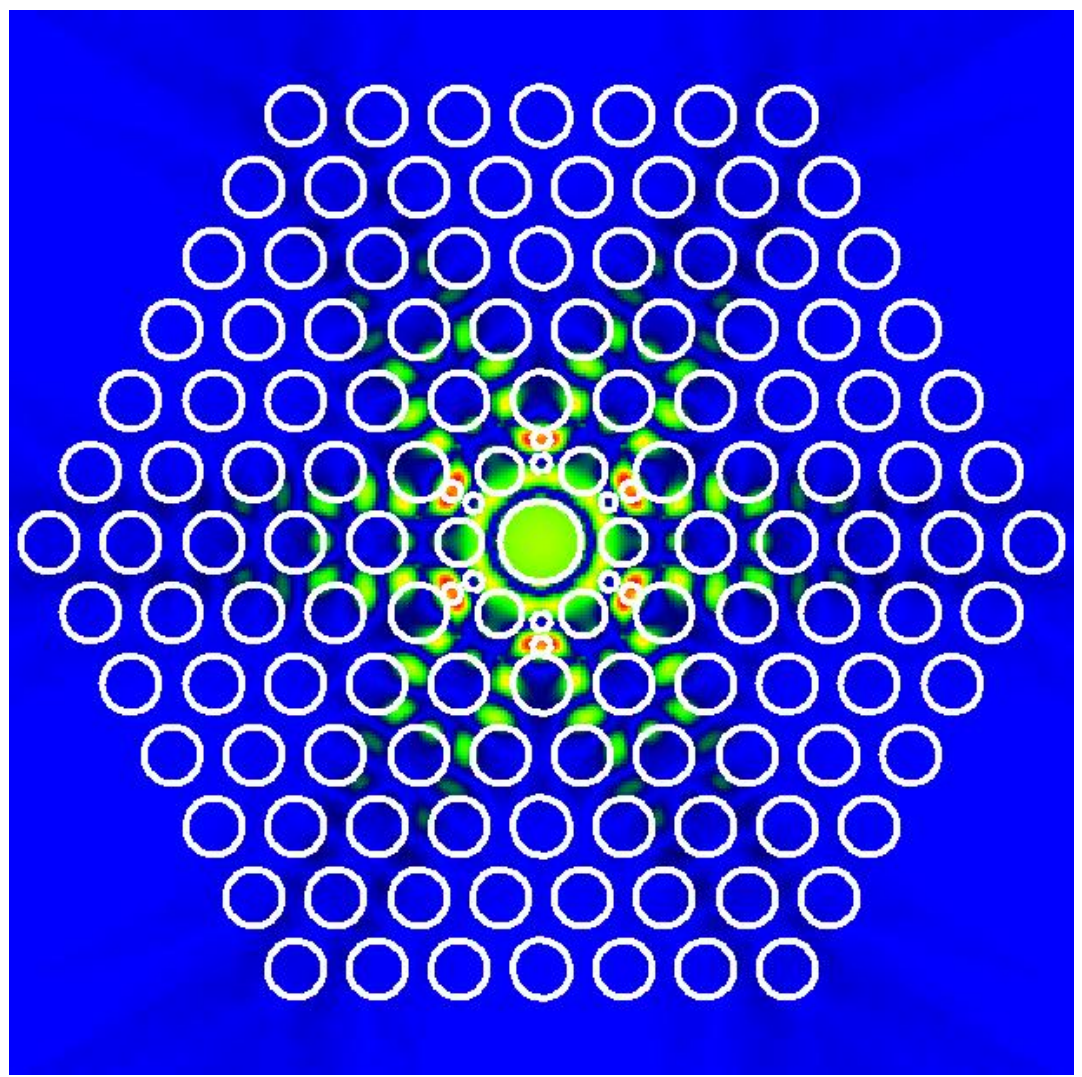

Figure 15: This figure shows the CUDOS map of the longitudinal electric field with small cylinders added to eliminate the hot spots. Notice how the hot spots appear to have expanded from those in Fig. 10. 


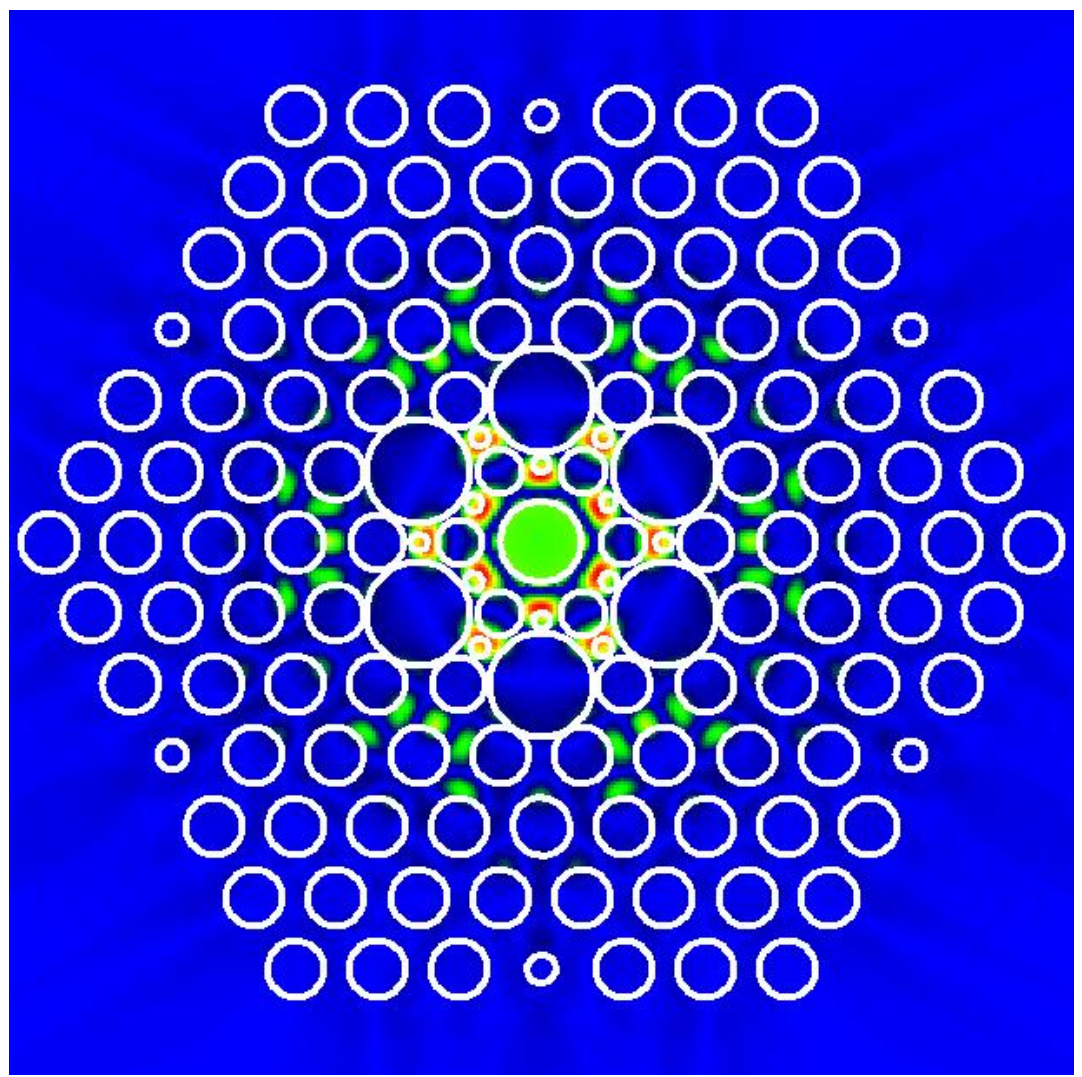

Figure 16: This figure shows the CUDOS map of the longitudinal electric field with a normal cylinder of increased radius to eliminate hot spots. Observe the new hot spots that have been created and covered by small cylinders. 


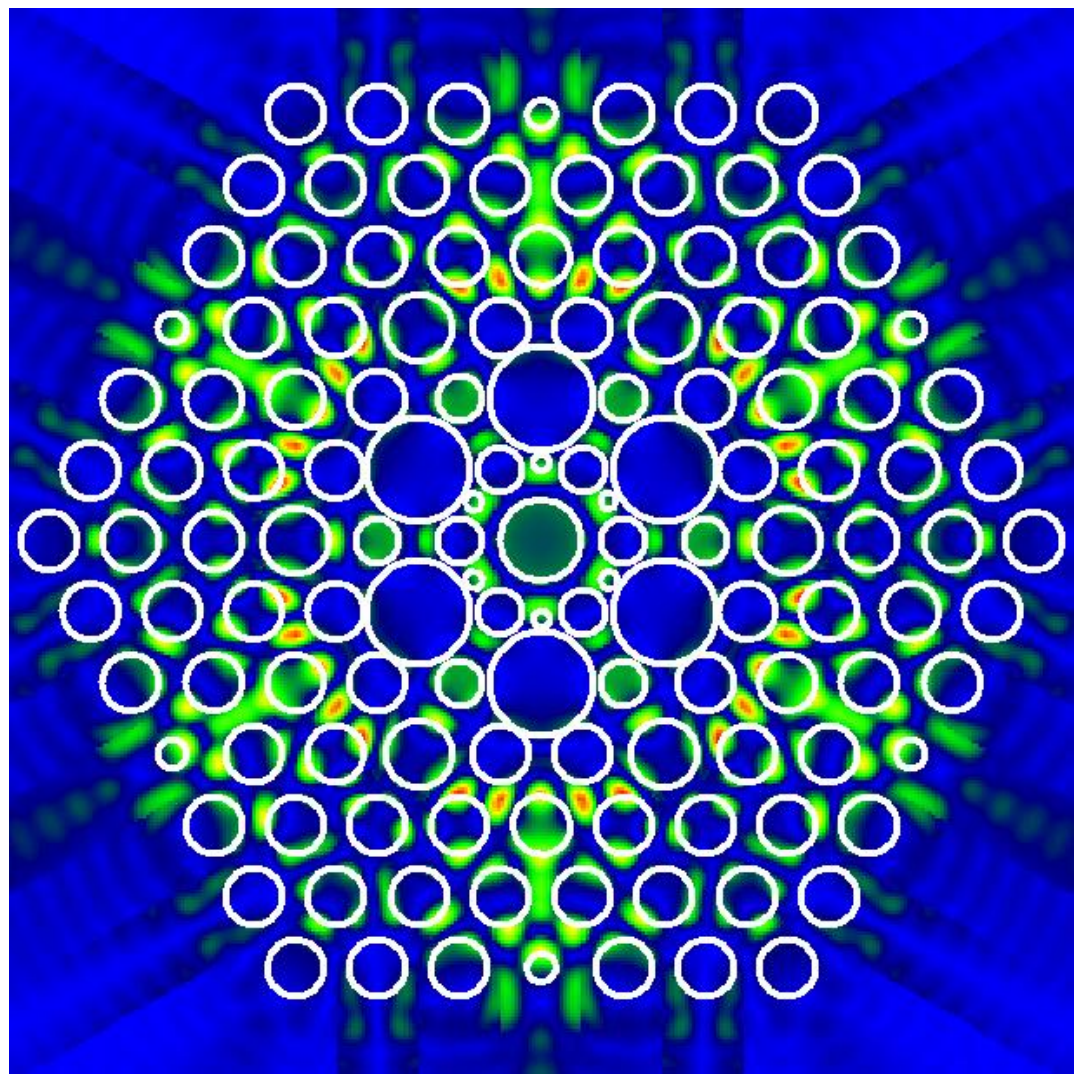

Figure 17: This figure shows the CUDOS map of the longitudinal electric field with a normal cylinder of increased radius to eliminate hot spots. However, the accelerating mode has mostly disappeared and new hot spots have appeared outside of the first hexagons. One now needs to add the missing material back into the lattice to restore the mode. 


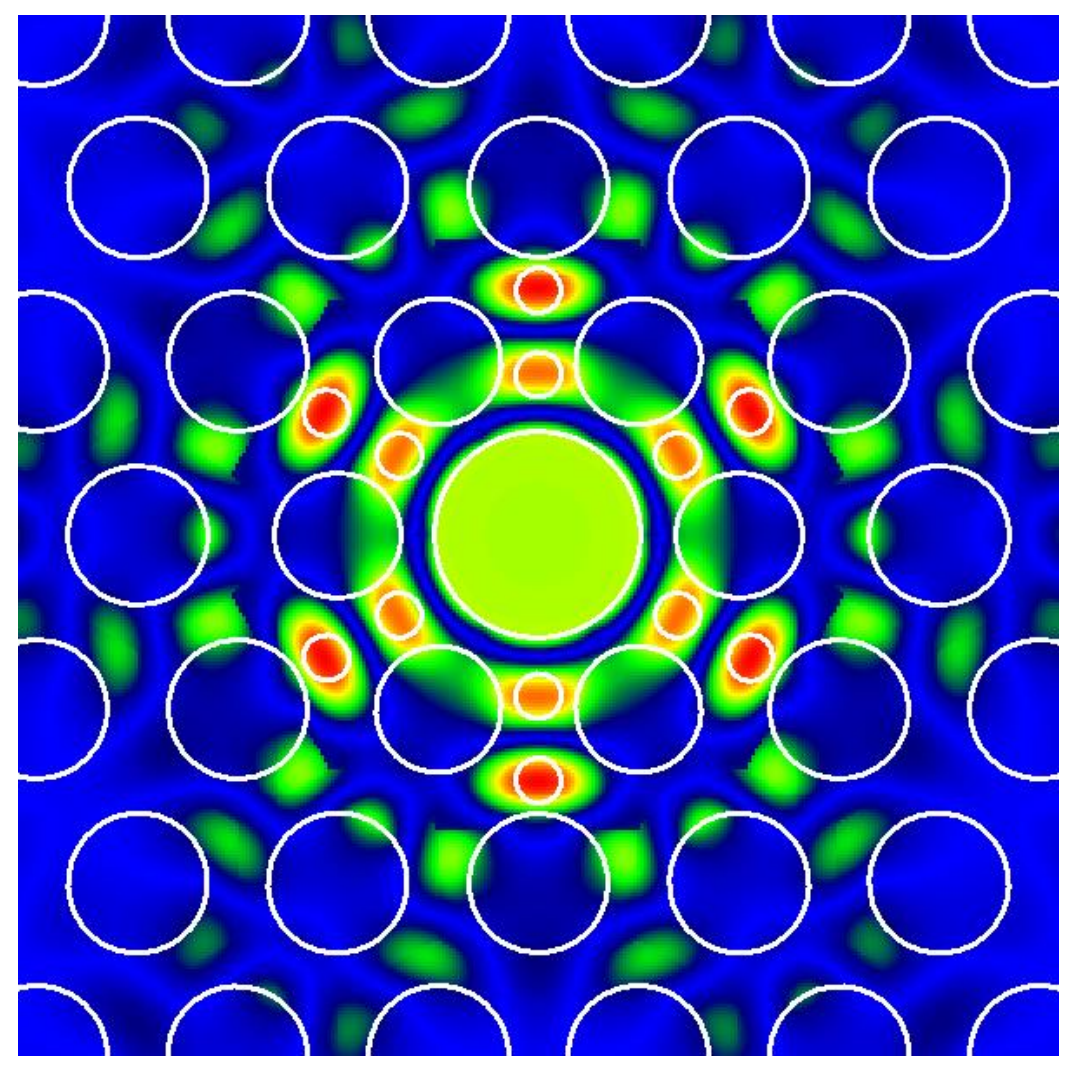

Figure 18: This is a CUDOS file of the longitudinal electric field. I have zoomed in on the central defect to make the smaller cylinders more apparent. The small cylinders have a refractive index equal to that of YAG to make them possible side lasers to drive the electron bunches. Notice that the outer hot spots are strongest. 


\title{
Construction of a Novel X Ray Generator
}

\author{
James Hostetter
}

Office of Science, Science Undergraduate Laboratory Internship (SULI)

Louisiana State University

SLAC National Accelerator Laboratory

Stanford, CA

August 14, 2009

Prepared in partial fulfillment of the requirements of the Office of Science, Department of Energy's Science Undergraduate Laboratory Internship under the direction of David Reis at the PULSE Institute, SLAC National Accelerator Laboratory.

Participant:

Signature

Research Advisor:

Signature 


\section{TABLE OF CONTENTS}

$\begin{array}{ll}\text { Abstract } & \text { ii }\end{array}$

$\begin{array}{ll}\text { Introduction } & 1\end{array}$

Materials and Methods 2

$\begin{array}{lr}\text { Results } & 6\end{array}$

$\begin{array}{ll}\text { Discussion and Conclusions } & 7\end{array}$

$\begin{array}{lr}\text { Acknowledgments } & 7\end{array}$

$\begin{array}{ll}\text { References } & 7\end{array}$ 


\begin{abstract}
Construction of a Novel X Ray Generator. JAMES HOSTETTER (Louisiana State University, Baton Rouge,LA 70820) DAVID REIS (PULSE Institute, SLAC National Accelerator Laboratory, Stanford, CA 94025)

We discuss the designs and procedures for a novel tabletop x-ray generator. The generator is a modification of well-known x-ray generating techniques. Instead of a thermionic cathode simply ejecting electrons into an anode, a short laser pulse ejects electrons from a photocathode and an RF cavity will be incorporated to compress the electron bunches. The generator will produce high flux, short pulse x-rays to complement the LCLS. Special consideration is given to the preparation and behavior of the photocathode.
\end{abstract}




\section{INTRODUCTION}

Since their discovery over a century ago, x-rays have been instrumental in studying materials. We have since developed many different types of x-ray generators to serve our particular needs, from the classic Röntgen design all the way to the LCLS. There are many parameters that go into an x-ray generator: pulse length, brightness, coherence, cost, etc. Our project is aimed towards filling a niche in the generating world. It will, upon completion, create sub-picosecond x-ray pulses at $8 \mathrm{keV}$. These x-ray pulses will be of short enough duration and wavelength to be able to study very small, very fast events such as atomic vibrations or molecular bond breaking.

The LCLS also provides these capabilities. However, many experiments may not require the high flux that comes with the LCLS [1] and may instead prefer the high repetition rate offered by our design for a high signal-to-noise ratio. Also, as the LCLS will be in very high demand, this source will provide a machine to test ideas and train students and post-docs. The machine, while providing similar average flux typically used in $\mathrm{kHz}$ laser experiments on third generation synchrotron sources (but at much higher repetition rates), will be small enough to fit on a tabletop and relatively inexpensive to build. The basic design is very similar in principle to Röntgen's original setup.

We will produce our electrons with a gallium arsenide (GaAs) photocathode that has been coated with cesium[2]. We will excite the photocathode with a $515 \mathrm{~nm}$ laser pulse at $119 \mathrm{MHz}$. This will cause bursts of electrons to be released from the cathode via the photoelectric effect[3]. The freed electron bunches will then be accelerated by a potential difference towards a copper anode, where the electrons will be stopped and emit x-ray radiation via Bremsstrahlung and line emission. These are the fundamentals of the project that have been repeated many times over.

Our electron gun, however, will also feature an RF cavity. This cavity will cause the elec- 
trons in the gun to bunch much more compactly. When the bunches strike the anode target, they will become a source of x-rays that is both brighter and shorter in pulse length. The x-rays will be particularly useful to PULSE scientists for studying areas such as phonon dynamics, molecular alignment, chemical dynamics, thermal transport, and energy conversion in condensed phase.

\section{MATERIALS AND METHODS}

Our x-ray generator will utilize a $50 \mathrm{kV}, 1 \mathrm{~mA}$ electron gun to produce its x-rays. The very basic principles of this, elaborated upon in the introduction of the paper, are extremely well-known and have been used for over 100 years now.

The design of our generator must include a preparation area specifically for the photocathode. For the best electron gun, we need the quantum efficiency of the cathode to be as high as possible. The quantum efficiency is defined as the number of electrons produced per photon. The simplest way to calculate this is with the formula

$$
Q E=\frac{I \times e}{P \times h \times \nu}
$$

where $\mathrm{I}$ is the current, e is the elementary charge, $\mathrm{P}$ is the power of the laser, $\mathrm{h}$ is Planck's constant, and $\nu$ is the laser frequency. The preparation process to achieve this is very delicate and has many requirements if it is to be done successfully.

An ultra-high vacuum (UHV) $\left(<10^{-11}\right.$ Torr $)$ environment is required to prepare and preserve a GaAs target. We want this vacuum to be not only extremely low pressure but also clean, meaning that we must use multiple types of pumps to meet the requirements. In the main vacuum chamber, we need both an ion pump and a non-evaporable getter pump. The ion pump ionizes the gases in the vacuum and then uses a potential difference to accelerate them towards an anode to remove them from the chamber. Because it has no moving parts, 
there is no lubrication needed and the entire process is very clean. Ion pumps are also capable of reaching UHV pressures. The getter pump is housed in the ion pump and acts as a sort of sponge that can very efficiently remove certain chemicals, particularly hydrogen, from the vacuum when bundled with the ion pump. It also has no moving parts and requires no power source, making it a very clean way to create a vacuum. This pump removes the need for a titanium sublimation pump, which requires that a surface be surrendered to depositing the titanium that cleans the vacuum.

Reaching UHV pressures requires a very, very long time to pump (on the order of weeks), so we prefer not to break the vacuum every time we need to switch samples. This means that we require a loadlock system to load the samples. A loadlock is very similar to an airlock. It is a very small chamber that we may isolate from the main vacuum via gate valve, bring up to atmospheric pressure, load in a new, chemically cleaned sample, and then evacuate back to UHV pressures to drastically reduce the amount of time pumping is needed.

After the sample has been loaded successfully, the gate valve to the loadlock system is opened and the sample is moved from the loadlock system into the main vacuum chamber, where it is heat cleaned on a molybdenum surface. The sample is brought to $500-550 \mathrm{C}$ to remove any impurities on the sample. To prevent damage to the sample transporter, it is designed as a fork. This allows the heater to be raised from below the sample to support it and the transporter to withdraw from the preparation chamber during activation.

Because of the vacuum and high temperatures, great care must be taken choosing materials in the system to lessen contamination from outgassing, or the creation of embedded gases in a material when the material is exposed to a vacuum. This is why the GaAs must be seated on molybdenum instead of stainless steel. It also has implications in the types of seals that can be used. All valves must be $100 \%$ metal. Many valves use a Viton seal that is unacceptable because of the high amounts of organic molecules that outgas from it.

Once the sample has been heat cleaned, it is time to cesiate it. Cesiation creates a bias 
within the photocathode that allows the electrons to be brought closer to the surface and more easily freed through the photoelectric effect. To do this, we run a current through a specially designed Alkali Metal Dispenser (AMD) that disperses cesium onto the sample while shining both a lamp and a laser on the sample. We must shine light on the GaAs to make sure that the cesiating is actually happening. The lamp is necessary because it takes several minutes for the cesium to build up enough to show a change due to the laser. When the advantages gained due to pure cesium begin to wane, $\mathrm{NF}_{3}$ is added, which further increases the QE. After the sample has gone through this entire process, the QE begins to decrease with time.

Once we have reached the maximum possible QE, we are ready to load the target into the electron gun. We will transfer it into a second chamber isolated from the preparation chamber (to prevent contamination from the $\mathrm{NF}_{3}$ or cesium). It is at this point that several design details must be considered. We want this chamber to be the source of electrons for the x-ray generator. We also want the x-rays to move parallel to the tabletop. There are three possible ways to achieve this goal.

First, we could leave the sample horizontal and fire the laser vertically, creating a vertical stream of electrons. We could then use a large dipole magnet to bend the electrons $90^{\circ}$ to horizontal (Fig. 1c). However, this adds dispersion that can be difficult to compensate when trying to generate short pulses.

We could also rotate the sample to the vertical position, leaving us with two ways to produce electrons. We could fire the laser into the GaAs, creating a stream of electrons along the same path that the laser and its reflection follow. We would then need to find some way to separate the laser from the electrons. We could either send the laser in at a small deviation from the normal or use a small bending magnet to produce some divergence of the electrons from the laser (Fig. 1a).

The last option is more difficult materially but less complicated in the setup. If the thin 
GaAs sample is grown on Gallium Phosphide (GaP) substrate, the laser can be fired into the sample with the electrons emitting from the opposite side of the sample. The GaP is transparent to the laser, but will prevent the electrons from escaping on the less desirable side of the sample. This way, the whole setup can be done in a line with no bending or angles (Fig. 1b).

We are sure that we will be using one of the last two methods. Thus, we must design a way to move the sample from the horizontal to vertical position. Our sample transporter will be a magnetically driven linear transporter with rotation capabilities around one axis parallel to transport. The photocathode must end up in contact with a negative high voltage supply in order to set up the correct bias for acceleration to the anode and RF cavity. This means that there must be no electrical contact between the photocathode and the chamber. If there was such contact, the entire chamber would become negatively biased, no acceleration would occur, and a potentially dangerous situation would be created.

After the photocathode has been prepared, we can measure its QE. The QE decays over time. Because time was limited to measure this decay, we approximate the results by taking an approximation

$$
Q E=Q E_{0} e^{-\frac{t}{\tau}} \approx Q E_{0}\left(1-\frac{t}{\tau}\right) .
$$

This will allow us to do a linear fit of the decay data as long as $t<<\tau$ and come up with an approximate lifetime. We may also put filters in front of the laser to measure the dependence of the photocurrent (and QE) on laser power. The current was measured instantaneously on a digital nanoammeter and over time with an analog voltmeter. 


\section{RESULTS}

Over the summer, we have specified the vast majority of parts for the first stages of the x-ray generator described in this paper. The loadlock, preparation chamber, and electron source have been designed with only minor details remaining (Fig. 2). The details of the chamber are fairly standard.

We have been allowed access to a similar design, where we were prepared a cathode and measure its quantum efficiency to be 13\%, sufficient for production of $1 \mathrm{~mA}$ current given the laser we intend to use (assuming that there is no saturation at higher powers). Further tests have determined that the dependence of the photocurrent with the power of the laser is roughly linear, indicating that the $\mathrm{QE}$ is proportional to the laser power. How exactly this dependence goes is unclear at the time; the photocurrent oscillates on the order of minutes, incorporating a systematic error into any series of measurements that naturally took several minutes to complete and introducing lots of scatter to the data (Fig. 3). An interesting observation of the data can be easily seen by paying special attention to the three lines that were measured up to full power. The general trend of the other lines is that the later the measurement was taken, the shallower the slope of the line was. This decay occurred much faster than the observed decay when the laser was heavily filtered (1\% transmission). However, the three lines mentioned earlier were measured several hours after the others. The photocurrent obviously recovered and then degraded again upon observation. This may be due to faster decay under higher intensity lasers. The decay of the photocurrent under $1 \%$ intensity has a half-life of approximately 2.63 days (Fig. 4). More measurements are in

progress with higher intensities. The cause of the periodicity of the photocurrent is also of interest. 


\section{DISCUSSION AND CONCLUSIONS}

Because this portion of the x-ray generator is so similar to the design we have been allowed to practice on, there is a high probability that this part of the generator will function well and we have high confidence that it will produce the required current for the generator. Several challenges have been overcome, the project is falling within the expected price range, our test chamber is performing as expected, and we expect the project to be a success and a valuable tool for PULSE in the future.

We have also uncovered several properties that will become very important, such as the long- and short-term behavior of the photocurrent and the basic dependence of the photocurrent on laser power. These studies have also unveiled several interesting behaviors to analyze with more sophisticated equipment and techniques that simply were not available in the time frame of the initial studies.

\section{ACKNOWLEDGMENTS}

I'd like to thank David Reis, Shambhu Ghimire, and Takashi Maruyama for their considerable help during this project. Funding and resources were provided by the Department of Energy and the Science Undergraduate Laboratory Internship Program. All research was conducted at the SLAC National Accelerator Laboratory in Menlo Park, CA.

\section{REFERENCES}

[1] "SLAC Linac Coherent Light Source", accessed 14 Aug, 2009. < http : //lcls.slac.stanford.edu/ >

[2] G.A. Mulhollan et. al., "Photovoltage effects in photoemission from thin GaAs layers",

Physics Letters A, Vol. 282, pp. 309-318, 2001. [3]Einstein, Albert. "Über einen die Erzeugung und Verwandlung des Lichtes betreffenden heuristischen Gesichtspunkt", Annalen der 
Physik, Vol. 17, pp. 132-148, 1905. 


\section{FIGURES}

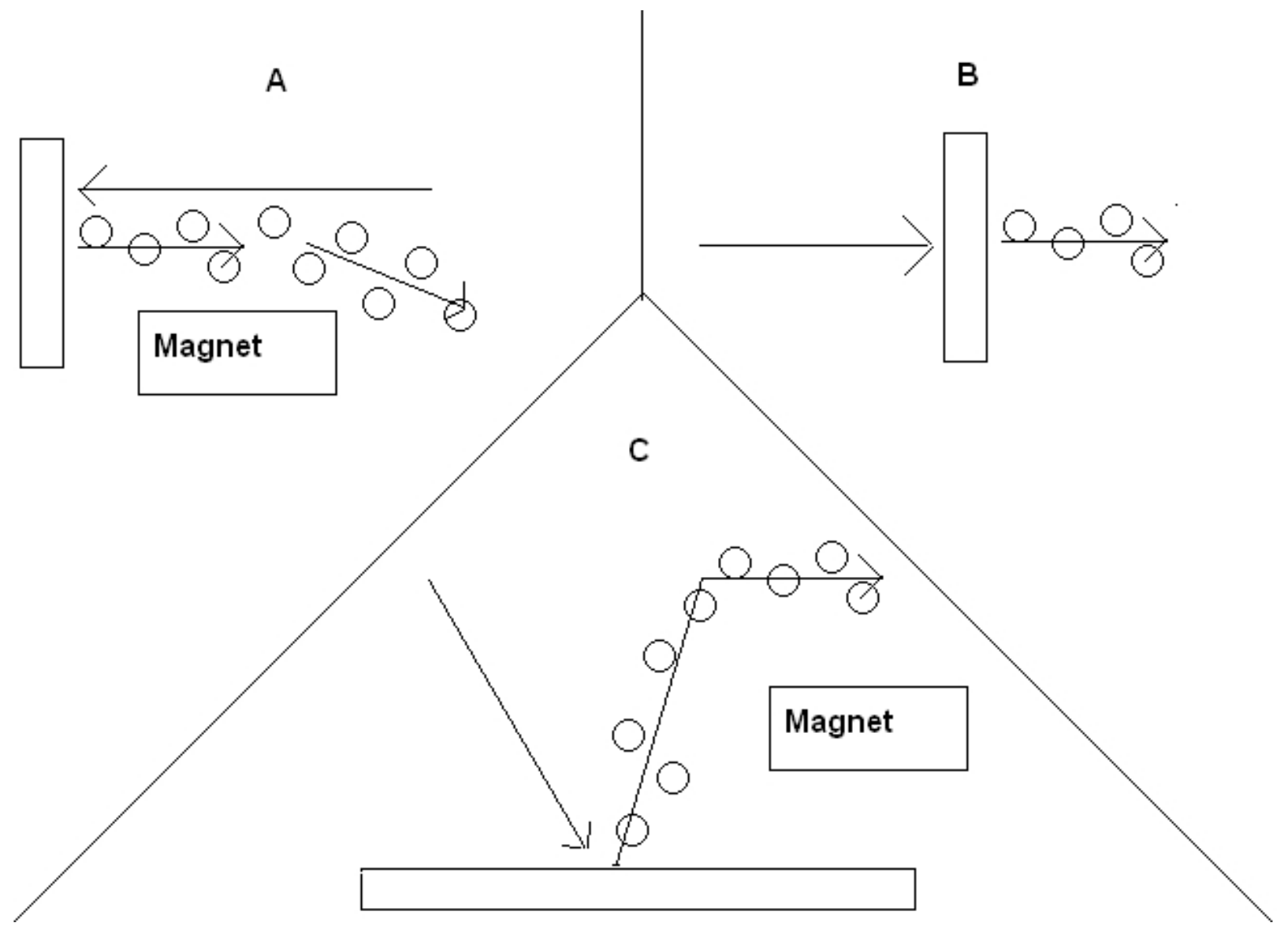

Figure 1: Different setups for the electron gun. 


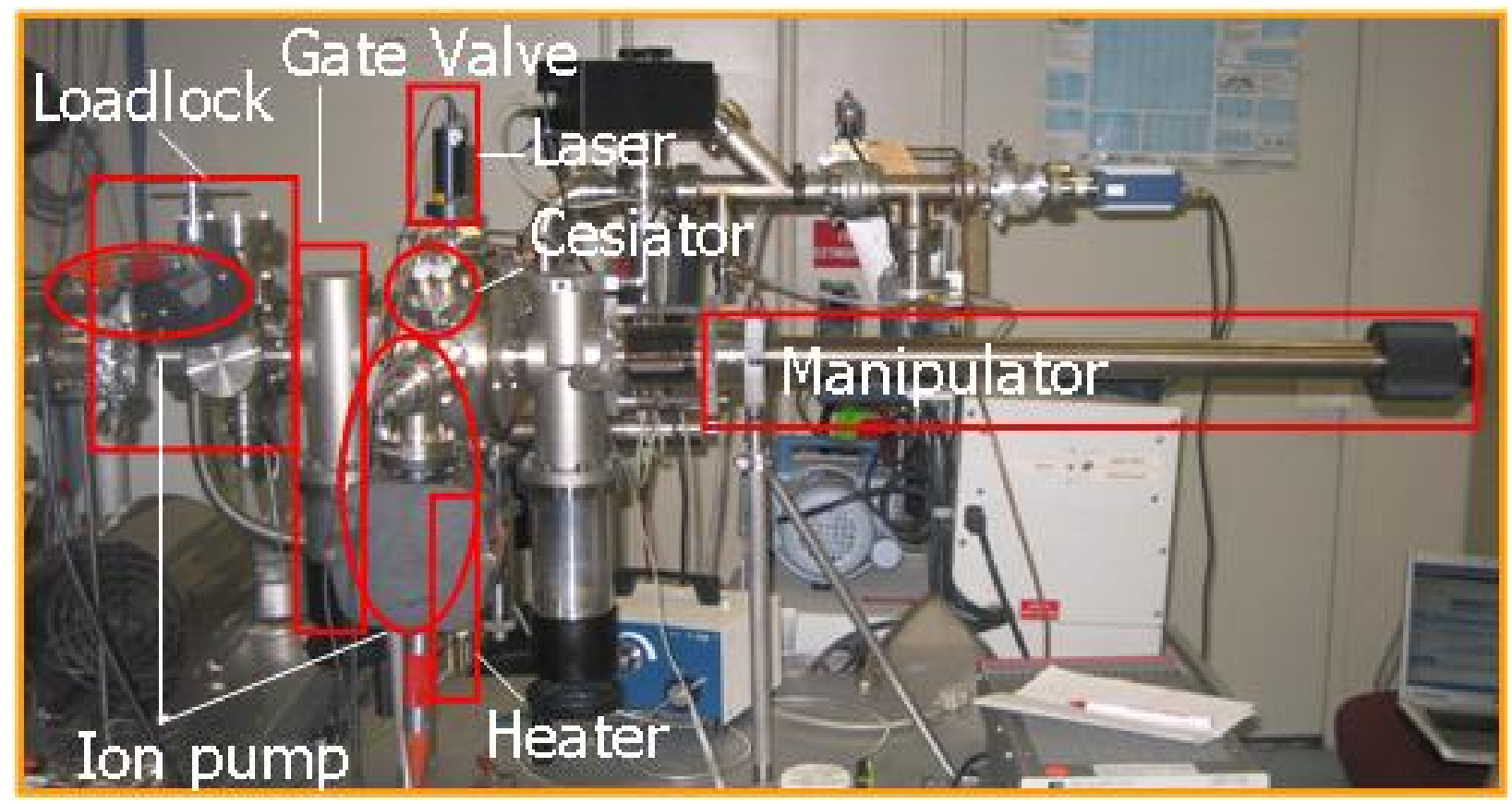

Figure 2: Basic design of a preparation chamber 


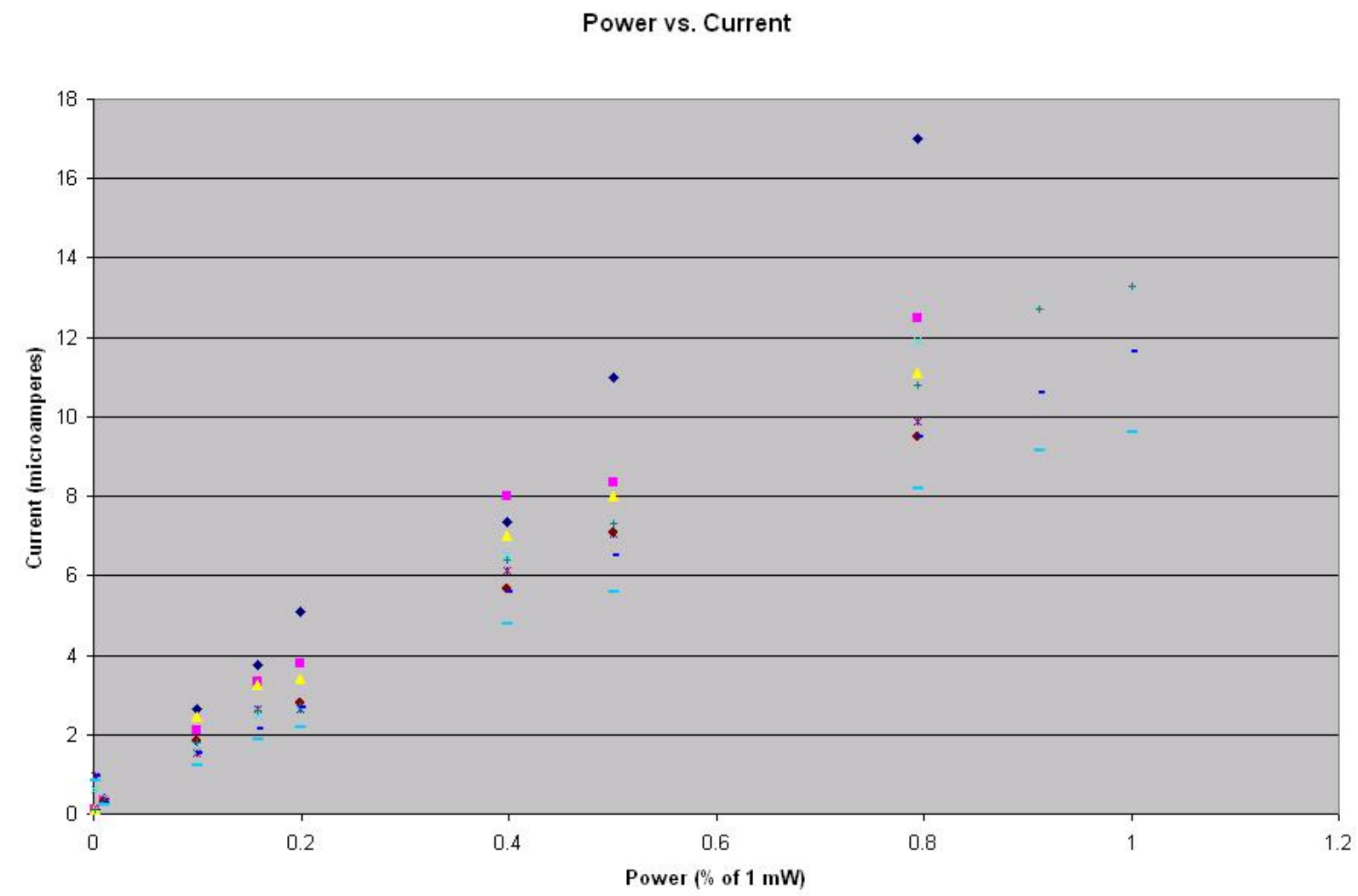

Figure 3: Photocurrent vs. $\%$ transmission of a $1 \mathrm{~mW}$ laser. 
Time vs. Current

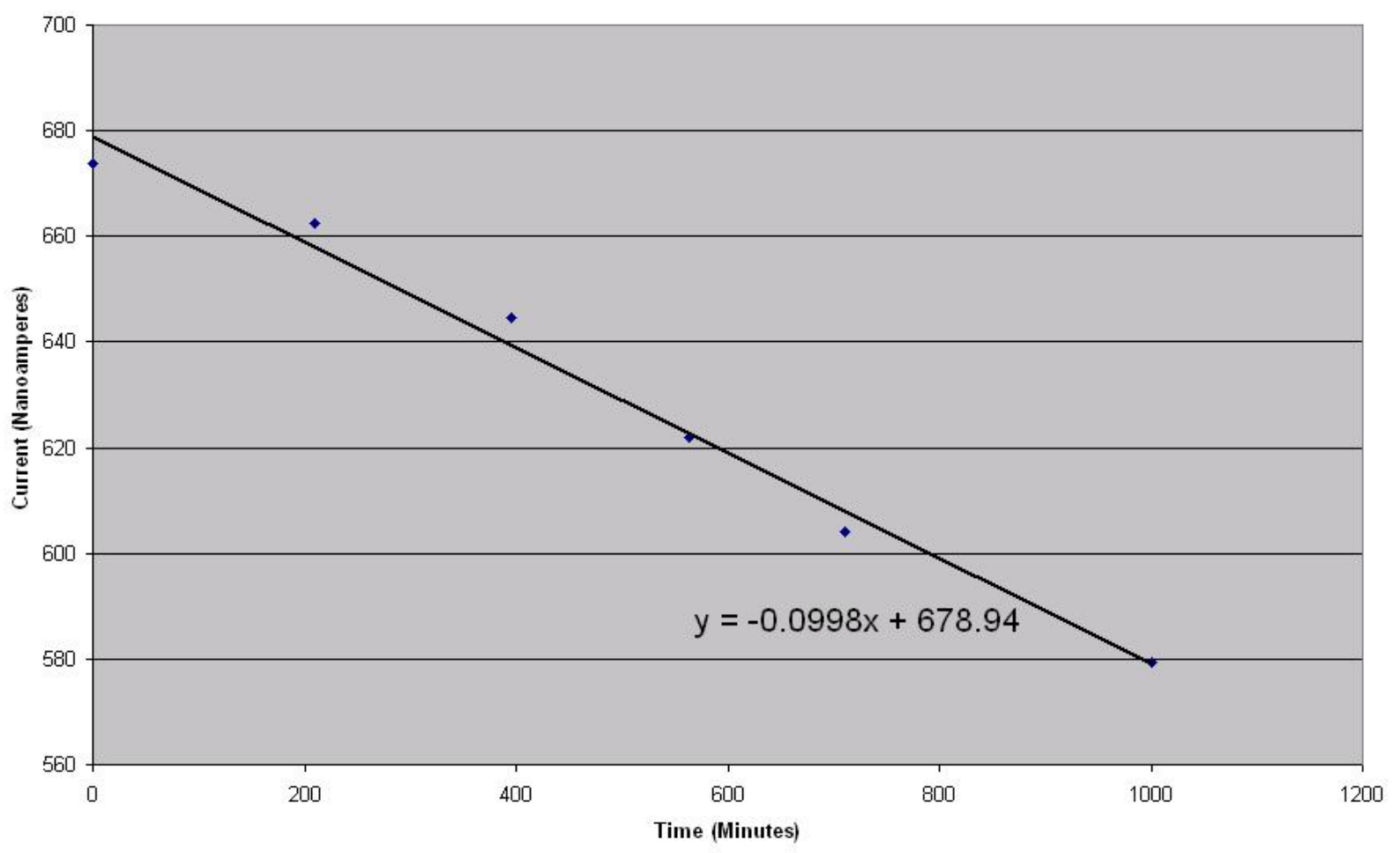

Figure 4: Decay of a photocurrent at $1 \%$ tranmission of a $1 \mathrm{~mW}$ laser. 


\title{
Calculation of Stop Production in Proton-Proton Collisions
}

\author{
Andrea J. Linville \\ Office of Science, Science Undergraduate Laboratory Internship Program
}

Washington University, St. Louis, MO

SLAC National Accelerator Laboratory

Menlo Park, CA

August 13, 2009

Prepared in fulfillment of the requirement of the Office of Science, Department of Energy's Science Undergraduate Laboratory Internship under the direction of JoAnne Hewett in the Theoretical Physics division at SLAC National Accelerator Laboratory.

Participant:

Signature

Research Advisor:

Signature 


\section{Table of Contents}

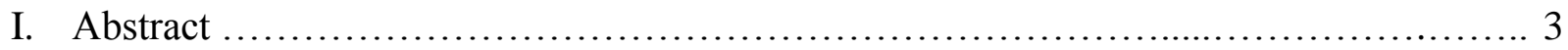

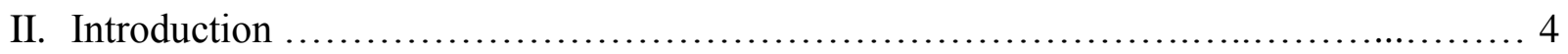

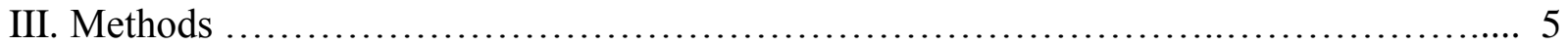

IV. Results ................................................................ 10

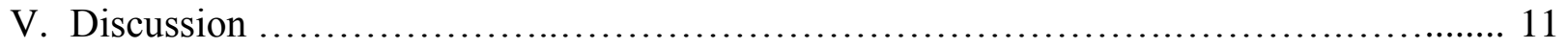

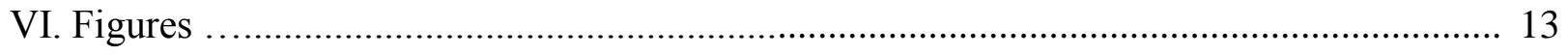

VII Acknowledgements ...................................................... 17

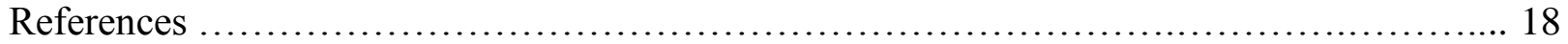




\section{Abstract}

Calculation of Stop Squark Production in Proton-Proton Collisions. ANDREA J. LINVILLE (Washington University, St. Louis, MO 63105) JOANNE HEWETT (SLAC National Accelerator Laboratory, Menlo Park, CA 94025)

Though the Standard Model of particle physics is an elegant theory which has been studied extensively for decades, it leaves many fundamental questions unanswered and is thus widely believed to be incomplete. Possible extensions to the Standard Model (SM) have been postulated and are in the process of being investigated experimentally. The most promising extension is the Minimal Supersymmetric Model (MSSM) which relates every SM particle to a superpartner that differs by $1 / 2$ unit of spin. The lightest supersymmetric quark, or squark, is expected to be the stop, and the search for this particle is an important experimental task. In this analysis, we use parton-model methods to predict the stop production cross section in protonproton collisions at LHC energies. 


\section{Introduction}

The Standard Model of particle physics provides a remarkably accurate description of matter at its most elementary level. It explains three of the four known fundamental interactions and the particles that take part in those interactions. Although the Standard Model (SM) is satisfying in its simplicity, it leaves several fundamental questions unanswered and thus it is widely believed that SM is incomplete.

Let us first summarize the SM before discussing extensions to it. In the SM, twelve spin $1 / 2$ fermions (depicted in Figure 1) are the building blocks of all visible matter. The interactions between these fermions are explained as the result of the exchange of force-mediating bosons, which carry integer spin. The different types of bosons mediate different forces: photons, gluons, and the $\mathrm{W}$ and $\mathrm{Z}$ bosons respectively carry the electromagnetic force, the strong nuclear force, and the weak nuclear force.

Unfortunately, the SM is inadequate in several respects. First of all, it does not explain why gravity is so weak compared to the other forces. In addition, the Higgs boson, which is predicted by the SM and generates the masses of the fermions and the $\mathrm{W}$ and $\mathrm{Z}$ bosons, has not yet been observed experimentally. Other shortfalls of the SM are its inability to predict the nature of dark matter and its lack of an explanation for the imbalance between matter and antimatter in the universe. Furthermore, the SM suffers from a number of adjustable parameters, and one would hope that nature could be described without these free parameters.

A widely studied extension of the SM is known as the Minimal Supersymmetric Model (MSSM). Supersymmetry (SUSY) relates each SM elementary particle to a superpartner, or sparticle, which differs from the original particle by a half unit of spin, but otherwise carries identical quantum numbers. Thus, for every fermion in the standard model, there exists a corresponding supersymmetric boson, and vice versa. 
SUSY, however, is not a perfect symmetry. If it were exact, each SM particle would be mass-degenerate with its superpartner. Since no superpartners have yet been observed, this implies that if SUSY exists, it must be a broken symmetry in order to allow the sparticles to be relatively heavy. Unfortunately, the masses of the superpartners are highly dependent upon the specific model of SUSY breaking, which is presently unknown.

Any experimental evidence of supersymmetry would have a profound influence on SUSY theory. Towards this end, an extensive search for superpartners is soon to be undertaken at the LHC. Although superpartners have not yet been observed, projections have been made for the production rates of the superpartners of quarks and gluons.

Our objective is to calculate the cross section for the production of the supersymmetric partner of the top quark - the stop - in proton-proton collisions, for stop masses ranging from 200 $\mathrm{GeV}$ to $2 \mathrm{TeV}$.

\section{Methods}

\section{i. Calculation of $e^{+} e^{-} \rightarrow \mu^{+} \mu$ cross-section}

Several preliminary steps were taken before calculating the production rate for the stop squark. These initial computations were performed primarily to check our evaluations of familiar quantities before proceeding to calculate unknown values. First, a program was written (in $\mathrm{C}++$ ) to calculate the cross-section of the annihilation of a positron and electron to a muon and antimuon. This SM process involves the s-channel exchange of either a photon or neutral $\mathrm{Z}$ boson (see Figure 2), and both contributions were accounted for. The cross-section is yielded by

$\sigma\left(e^{+} e^{-} \rightarrow \mu^{+} \mu^{-}\right)=Q_{\mu}^{2} Q_{B}^{2} \frac{4 \pi \alpha^{2}}{3 s}+\frac{4 \alpha}{3} \frac{G_{F} Q_{\mu} Q_{B}}{\sqrt{2}}\left(g_{L}+g_{R}\right)^{2} \operatorname{Re}(R)+\frac{4 s}{3 \pi}\left(\frac{G_{F}}{\sqrt{2}}\right)^{2}\left(g_{L}^{2}+g_{R}^{2}\right)^{2}|R|^{2}$ 
The first term corresponds to the photon contribution, the second term is a mixed photon- $Z$ boson component, and the third term is a contribution just from the $\mathrm{Z}$ boson. $\mathrm{Q}_{\mu}$ and $\mathrm{Q}_{\mathrm{e}}$ refer to the muon and electron charges, respectively; $\alpha$ is the fine structure constant; $\sqrt{s}_{\mathrm{s}}$ is the invariant energy of the subprocess; $\mathrm{G}_{\mathrm{F}}$ is Fermi's constant; and $\mathrm{g}_{\mathrm{L}}, \mathrm{g}_{\mathrm{R}}$, and $\mathrm{R}$ are defined as follows:

$$
\begin{aligned}
& g_{L}=x_{w}-1 / 2 \\
& g_{R}=x_{w}
\end{aligned}
$$

and

$$
R(s)=\frac{M_{Z}^{n}}{s-M_{Z}^{n}+i M_{Z} \Gamma_{Z}}
$$

In these equations, $x_{w}$ is the weak mixing angle, $M_{Z}$ is the mass of the $Z$ boson, and $\Gamma_{Z}$ is the decay width of the $\mathrm{Z}$ boson. [3]

\section{ii. Modification of equations to calculate $\sigma\left(q \bar{q} \rightarrow \mu^{+} \mu ;\right.$}

The original program was then modified to calculate the cross-section of the annihilation of a quark and antiquark to a muon and antimuon, which is known as the Drell-Yan process. This task was accomplished simply by substituting quark charge in place of electron charge and summing over the different types of quarks. Separate cross-section components were set up for the quarks with $+2 / 3$ charge (up, charm, and top) and those with $-1 / 3$ charge (down, strange, and bottom).

\section{iii. Inclusion of Parton Distribution Functions}

In order to generalize the program to obtain the hadronic cross-section $\sigma\left(p p \rightarrow \mu^{+} \mu^{-}\right)$, the quark cross-sections were combined with parton distribution functions (PDFs). The parton model assumes that a hadron can be described in terms of quasi-free pointlike structures called partons.[3] Therefore, high-energy proton collisions can instead be envisioned as collisions of the quarks, antiquarks, and gluons within the protons. PDFs describe the probability density for 
finding a parton with a certain momentum fraction, $\mathrm{x}$, of the momentum, $\mathrm{Q}^{2}$, that is transferred between the colliding hadrons. The appropriate cross-sections were then multiplied times each parton's PDF and integrated over all momentum fractions:

$$
\sigma=\int_{0}^{1} \mathrm{dx}_{\mathrm{a}} \int_{0}^{1} \mathrm{dx} \mathrm{b} \hat{\sigma} \sum_{f}\left[\mathrm{f}_{\mathrm{A}}\left(\mathrm{x}_{\mathrm{a}}\right) \mathrm{f}_{\mathrm{B}}\left(\mathrm{x}_{\mathrm{b}}\right)+\mathrm{f}_{\mathrm{A}}\left(\mathrm{x}_{\mathrm{b}}\right) \mathrm{f}_{\mathrm{B}}\left(\mathrm{x}_{\mathrm{a}}\right)\right]
$$

Here, $\mathrm{x}_{\mathrm{a}}$ and $\mathrm{x}_{\mathrm{b}}$ are the momentum fractions of parton $\mathrm{a}$ and parton $\mathrm{b}$, and the partons originate from proton A or B. The parton-level cross section is represented as $\hat{\sigma}$. The following convenient definition can also be made:

$$
\tau \stackrel{\text { deff }}{=} \mathrm{X}_{\mathrm{XXb}}
$$

This is equivalent to

$$
\hat{s}=\tau s
$$

where $\sqrt{\hat{s}}=Q$ is called the scattering energy or the invariant mass, and $\sqrt{s}$ is known as the proton center-of-mass energy or "machine" energy. Equation 5 can subsequently be written as

$$
\sigma=\int_{0}^{1} \mathrm{~d} \tau \int_{\tau}^{1} \mathrm{dx}_{\mathrm{a}} \hat{\sigma} \sum_{f}\left[\mathrm{f}_{\mathrm{A}}\left(\mathrm{x}_{\mathrm{a}}\right) \mathrm{f}_{\mathrm{B}}\left(\tau / \mathrm{x}_{\mathrm{a}}\right)+\mathrm{f}_{\mathrm{A}}\left(\tau / \mathrm{x}_{\mathrm{a}}\right) \mathrm{f}_{\mathrm{B}}\left(\mathrm{x}_{\mathrm{a}}\right)\right]
$$

Calculations were performed by utilizing the PDF set CTEQ6, from the Coordinated TheoreticalExperimental QCD collaboration, and all integrals were evaluated with the VEGAS integration routine.[6] VEGAS is a form of Monte Carlo integration which estimates the value of a multidimensional definite integral by evaluating the integrand at randomly chosen points.

These calculations enabled us to plot the total muon production cross-section as a function of Q (the invariant muon mass). The proton center-of-mass energy was taken to be 14 $\mathrm{TeV}$, which is the projected eventual energy of the LHC, and the integrals were evaluated for Q between $50 \mathrm{GeV}$ to $2 \mathrm{TeV}$, in $1 \mathrm{GeV}$ increments. 


\section{iv. Setup of $\sigma(p p \rightarrow \tilde{q} \overline{\tilde{q}})$ calculation}

The next step was to replace the quark to muon cross-sections with those for the various channels which produce a squark and an antisquark. Three different initial states can result in squark pair production from proton-proton collision: $q q \rightarrow \tilde{q} \tilde{q}, q \bar{q} \rightarrow \tilde{q} \overline{\tilde{q}}$, and $g g \rightarrow \tilde{q} \overline{\tilde{q}}$. Before calculating the cross-section for each case, it is convenient to define the quantities [3]

$$
\begin{aligned}
& \mathscr{S}=\left[s-\left(m_{1}+m_{2}\right)^{2}\right]^{1 / 2}\left[s-\left(m_{1}-m_{2}\right)^{2}\right]^{1 / 2}, \\
& \Delta_{a i}=M_{a}{ }^{2}-m_{i}{ }^{2},
\end{aligned}
$$

and

$$
\Lambda_{a}=\ln \left(\frac{s+\Delta_{a 1}+\Delta_{a 2}-\mathscr{S}}{s+\Delta_{a 1}+\Delta_{a 2}+\mathscr{S}}\right)
$$

where $m_{1}$ and $m_{2}$ are the masses of the produced squarks, and $M_{a}$ refers to the mass of the particle exchanged in either the t- or u-channel, depending upon which Feynman diagram we are examining. With these definitions, the cross-sections for the aforementioned annihilations are:

$$
\begin{aligned}
& \left.\sigma\left(q_{i} q_{j} \rightarrow \widetilde{q}_{i} \widetilde{q}_{j}\right)=\frac{4 \pi \alpha_{s}^{2}}{9 s^{2}}\left[\mid-2 \mathscr{S}-\left(s+\Delta_{t i}+\Delta_{t j}\right) \Lambda_{t}+\frac{1}{1+\delta_{i j}} \frac{\mathscr{S}_{s} m_{\widetilde{8}}^{2}}{\Delta_{t i} \Delta_{t j}+s m_{\widetilde{g}}^{2}}+\frac{1}{3} \delta_{i j} \frac{s m_{\widetilde{g}}^{2}}{s+\Delta_{t i}+\Delta_{t j}} \Lambda_{t}\right]+\delta_{i j}(t \rightarrow u)\right] \\
& \sigma\left(q_{i} \bar{q}_{j} \rightarrow \widetilde{q}_{i} \widetilde{q}_{j}^{*}\right)=\frac{4 \pi \alpha_{s}^{2}}{27 s^{2}}\left[\delta_{i j}\left(\frac{\mathscr{S}^{3}}{s^{2}}+\frac{\mathscr{S}\left(s+\Delta_{t i}+\Delta_{t j}\right)}{s}+\frac{2\left(\Delta_{t i} \Delta_{t j}+m_{\widetilde{g}^{2}}{ }^{2}\right)}{s} \Lambda_{t}\right]\right. \\
& \left.+3\left[-2 \mathscr{S}-\left(s+\Delta_{t i}+\Delta_{t j}\right) \Lambda_{t}+\frac{\mathscr{S} s m_{\tilde{g}}^{2}}{s m_{\tilde{g}}^{2}+\Delta_{t i} \Delta_{t j}}\right]\right]
\end{aligned}
$$

and

$$
\sigma\left(g g \rightarrow \widetilde{q}_{i} \widetilde{q}_{i}^{*}\right)=\frac{\pi \alpha_{s}{ }^{2}}{3 s^{2}}\left[\left(\frac{5}{8}+\frac{31}{4} \frac{m^{2}}{s}\right) \mathscr{S}+\left(4+\frac{m^{2}}{s}\right) m^{2} \ln \left(\frac{s-\mathscr{S}}{s+\mathscr{S}}\right)\right]
$$




\section{v. Calculation of $\sigma(p p \rightarrow \tilde{t} \overline{\bar{t}})$}

Fortunately, equations 12-14 simplify considerably since we are presently only interested in processes resulting in a stop and anti-stop, rather than any squark pair. If one were to look at the production of any squark and antisquark, all the processes shown in the Feynman diagrams in Figure 3 would be possible. However, the Feynman rules that require conservation of charge and conservation of quark flavor at each vertex reduce the number of channels available for the production of a stop and anti-stop. For example, in the quark-antiquark t-channel, flavor conservation requires that the initial quark and antiquark must be a top and anti-top. Since top quarks carry such a tiny fraction of a proton's momentum, this particular channel has essentially no probability of actually occurring. For the same reasons, both the t- and u- channels of quarkquark annihilation are not present in the top quark scenario. So, one need only calculate crosssections for the processes shown in Figure 4.

Thus, equation 12 is not required here (and hence equations 10 or 11 are not needed either!), and equation 13 reduces to

$$
\sigma\left(q_{i} \bar{q}_{i} \rightarrow \widetilde{q}_{j} \widetilde{q}_{j}^{*}\right)=\frac{4 \pi \alpha_{s}{ }^{2}}{27 s^{4}} \mathscr{S}^{3}
$$

Equation 8 is then utilized again, with the incorporation of the applicable parton cross-sections and the corresponding PDFs, and the modification of the lower integration limit to ensure that enough energy is present to produce the stop quark pair. Finally, the total cross-section for stop production following proton-proton collision can be calculated. 


\section{Results}

i. Calculation of $\sigma\left(e^{+} e^{-} \rightarrow \mu^{+} \mu\right)$

The cross-section for $\mathrm{e}^{+} \mathrm{e}^{-} \rightarrow \gamma, Z \rightarrow \mu^{+} \mu^{-}$was calculated to be $61.6 \mathrm{fb}$.

\section{ii. Parton Distributions}

Parton densities for the $\mathrm{u}, \mathrm{d}, \mathrm{c}$, and s quarks and anti-quarks are graphed in Figures 5-7 for $\mathrm{Q}=\sqrt{2}, \sqrt{20}$, and $\sqrt{200} \mathrm{GeV}$, respectively. The densities for $\mathrm{b}$ and $\mathrm{t}$ quarks were negligible at these scattering energies and are therefore omitted from the plots.

iii. Calculation of $\sigma\left(p p \rightarrow \mu^{+} \mu^{-}\right)$

Figure 8 shows the $p p \rightarrow \mu^{+} \mu^{-}$cross-section for invariant muon masses from $50 \mathrm{GeV}$ to $2 \mathrm{TeV}$. A peak in the cross-section was observed at $91 \mathrm{GeV} \pm 0.5$, where it has a maximum value of $0.88 \mathrm{nb}$. When $\mathrm{M}=500 \mathrm{GeV}$, the cross-section has a value of $0.96 \mathrm{fb}$. At the upper mass limit, the cross-section trails off to $0.731 \mathrm{ab}$ at $2 \mathrm{TeV}$.

\section{iv. Calculation of $\sigma(p p \rightarrow \tilde{t} \overline{\tilde{t}})$}

In Figure 9, the $p p \rightarrow \tilde{t} \overline{\tilde{t}}$ cross-section is shown for stop masses between $200 \mathrm{GeV}$ and 2 $\mathrm{TeV}$. The maximum cross-section occurs at $200 \mathrm{GeV}$, with a value of $271 \mathrm{pb}$ and a standard deviation of $1.03 \mathrm{pb}$. This standard deviation results from the accuracy of the VEGAS integration routine, which could be increased by setting the program to calculate the value of the integrand at a greater number of points. ${ }^{1}$ The cross-section decreases steadily to $227 \mathrm{ab}( \pm 0.431 \mathrm{ab})$ at 2 $\mathrm{TeV}$.

\footnotetext{
${ }^{1}$ The primary drawback to evaluating the integrals at more points is the increased CPU usage that is required.
} 


\section{Discussion}

\section{i. Calculation of $\sigma\left(e^{+} e^{-} \rightarrow \mu^{+} \mu\right)$}

The electron-positron annihilation cross section is well known, and our value agrees well with previous data. [7]

\section{ii. Parton Distributions}

The parton distribution results also closely match previous work, ${ }^{2}$ though the correspondence is not exact. Discrepancies most likely result from utilizing different parameterizations of the parton densities. Modifications to PDFs have been made frequently, with different estimates of strong-interaction corrections to elementary parton distributions. While the most recent parameterization, CTEQ6, was applied in this study, many other versions have been used previously. The uncertainty due to these differences is less than 10\% at the LHC [4]. Nevertheless, the general trends of the distributions match expectations. At lower scattering energies, the up and down quarks tend to have the greatest probability of interacting. This is logical since the up and down quarks are the only so-called "valence" quarks within the proton. Valence quarks are the only ones that contribute to a hadron's quantum numbers. The other quarks and the antiquarks are still present in protons as highly unstable "sea" quarks, virtual quark-antiquark pairs formed from the splitting of gluons within the proton. At low scattering energies, sea quarks are confined within hadrons and have minimal contributions to the overall cross-sections. Much more scattering energy is required to enable the sea quarks to collide with partons from the other proton. As can be seen in Figures 5-7, the sea quarks play a more substantial role at higher scattering energies, though the bottom and top quark contributions remain negligible for all the energies plotted.

\footnotetext{
${ }^{2}$ Conducted by Michael Peskin, SLAC
} 


\section{iii. Calculation of $\sigma\left(p p \rightarrow \mu^{+} \mu^{-}\right)$}

The muon production cross-section also demonstrates the expected trend. The resonance in the cross-section at a dimuon mass of $91 \mathrm{GeV}$ corresponds to the mass of the $\mathrm{Z}$ boson. An invariant mass of $91 \mathrm{GeV}$ enables the production of a $\mathrm{Z}$ boson, which subsequently decays into two highly energetic muons.

Literature states that the expected differential cross-section at a dimuon mass of $500 \mathrm{GeV}$ is $\sim 1 \mathrm{fb},[5]$ quite close to the calculated value of $0.96 \mathrm{fb}$. Some error results from the utilization of different parton distributions in these calculations. Additionally, taking data at finer increments would have obviously yielded increased accuracy.

$$
\text { iv. Calculation of } \sigma(p p \rightarrow \tilde{t \tilde{t}})
$$

Finally, the stop cross-section agrees well, though not perfectly, with that determined by Dawson, et.al.[3]. The values differ because we examine only the leading order (LO) Feynman diagrams, while others have incorporated next-to-leading order (NLO). If NLO corrections were to be included, the cross-sections would be increased to values above the LO predictions.

The stop production cross section depends essentially only on the masses of the produced stops. So, after experimentally establishing bounds on the stop production cross-section, the cross-section can be used to ascertain a lower bound on the stop mass. Similarly, if stops are observed, the cross-section can subsequently be employed to determine the masses of the stop particles, which would be of use in pinpointing the specific model of SUSY breaking.[4]

Thus, our projections of the production rates of stop squarks in high-energy collisions are of value both in interpreting data and in preparing new experimental searches for supersymmetry. 


\section{Figures}

\section{Elementary}

\section{Particles}

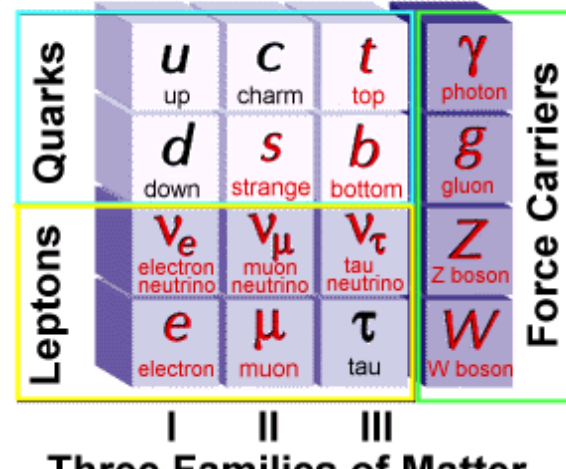

\section{Three Families of Matter}

Figure 1 - Summary of Standard Model Elementary Particles [1]

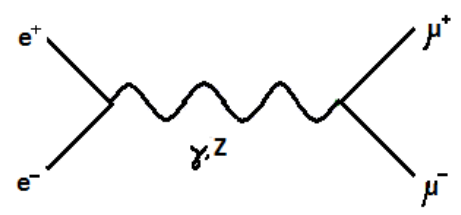

Figure 2 - Feynman diagram of electron-positron annihilation 


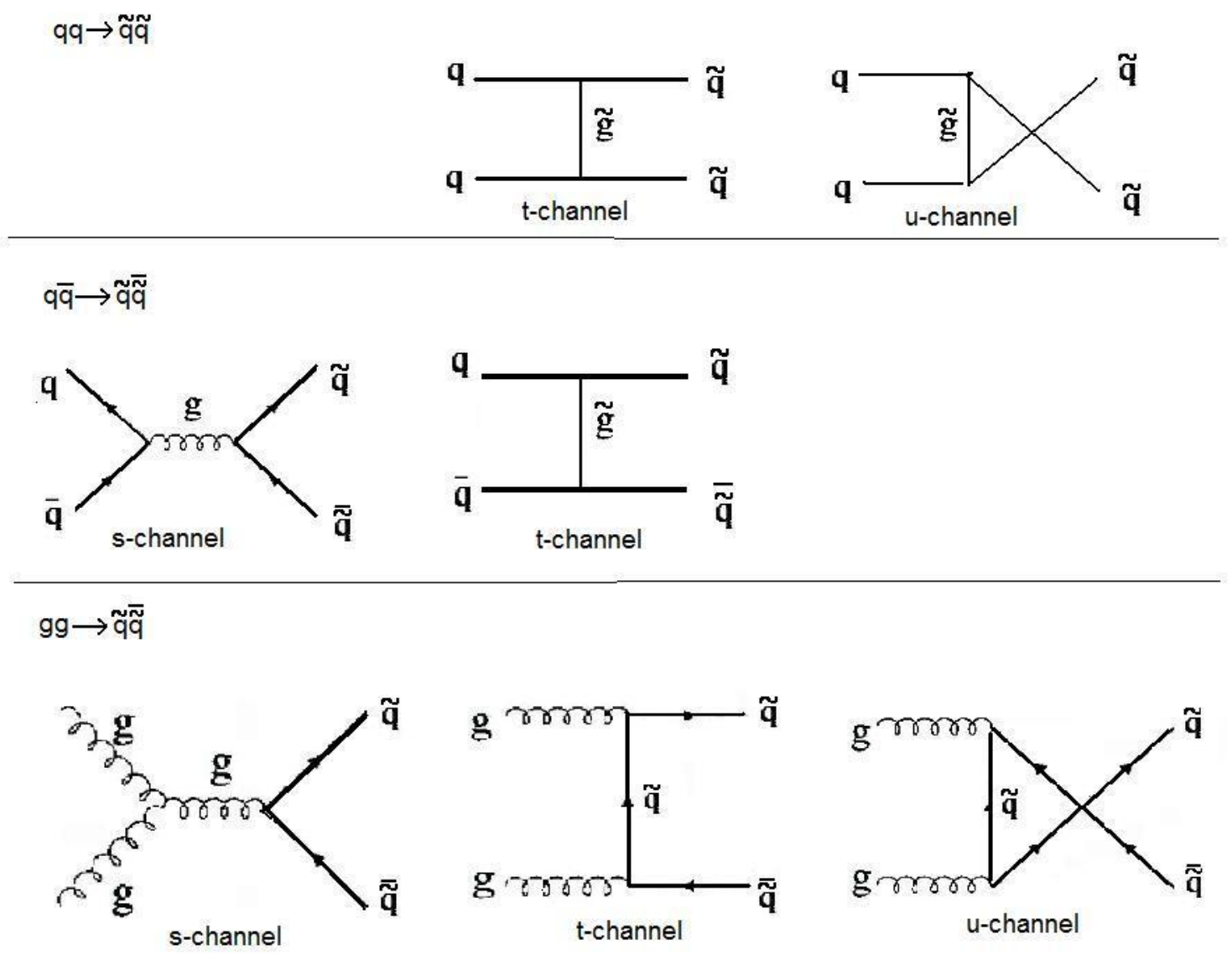

Figure 3 - Feynman diagrams for all processes producing a squark and anti-squark [4]
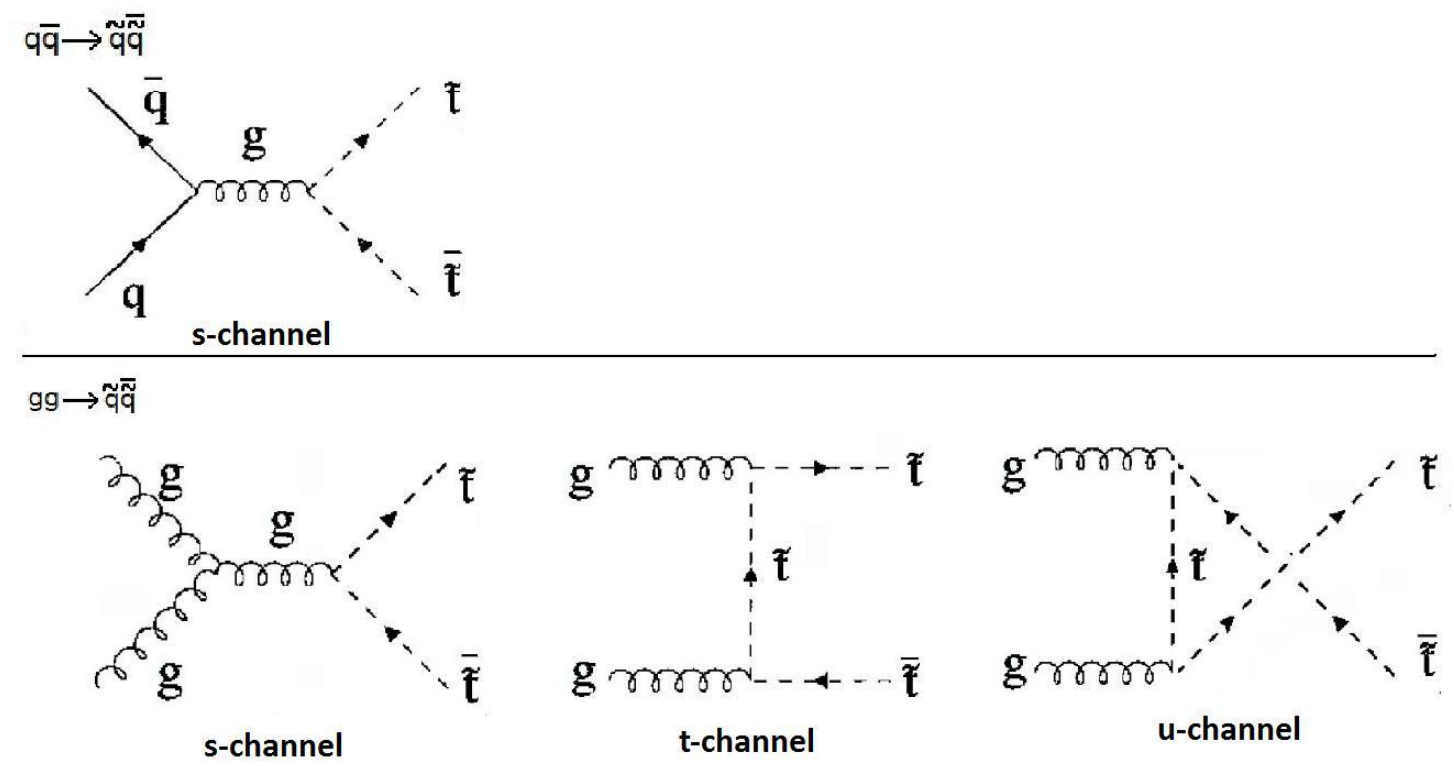

Figure 4 - Feynman diagrams for production of a stop and anti-stop 


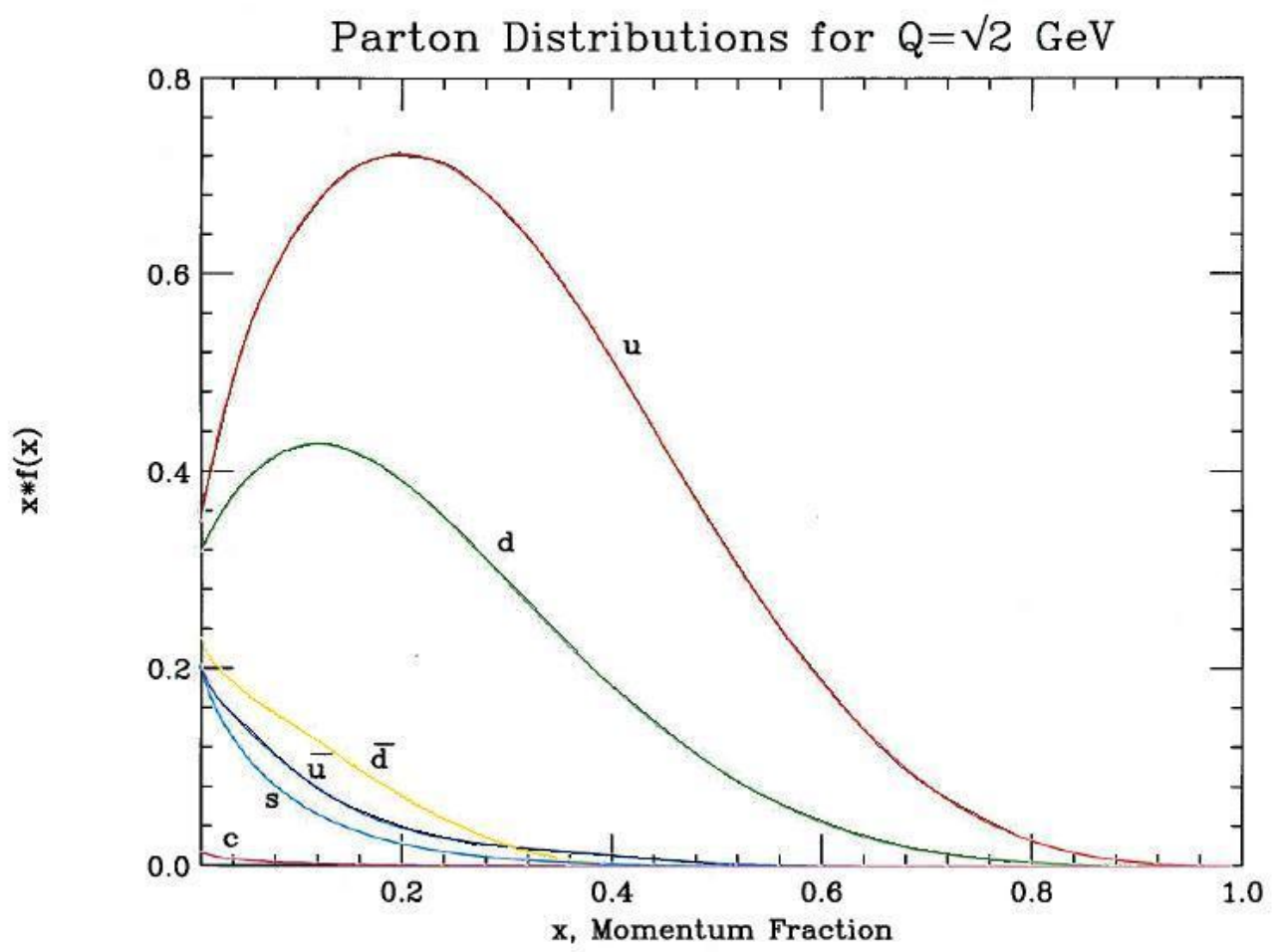

Figure 5 - Parton densities calculated using CTEQ6 PDFs, with scattering energy $Q=\sqrt{ } 2$ GeV

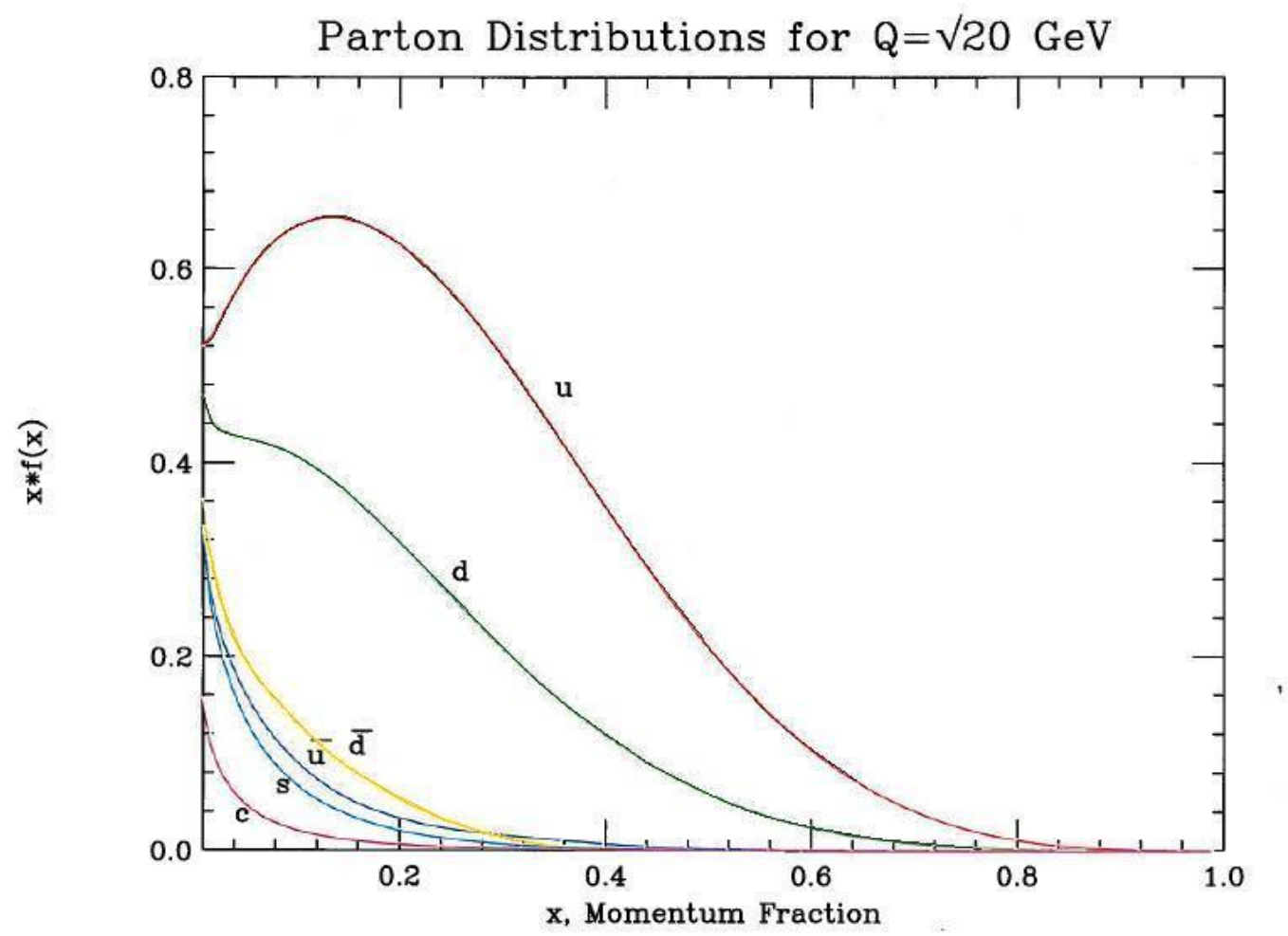

Figure 6 - Parton densities for $Q=\sqrt{ } 20 \mathrm{GeV}$ 


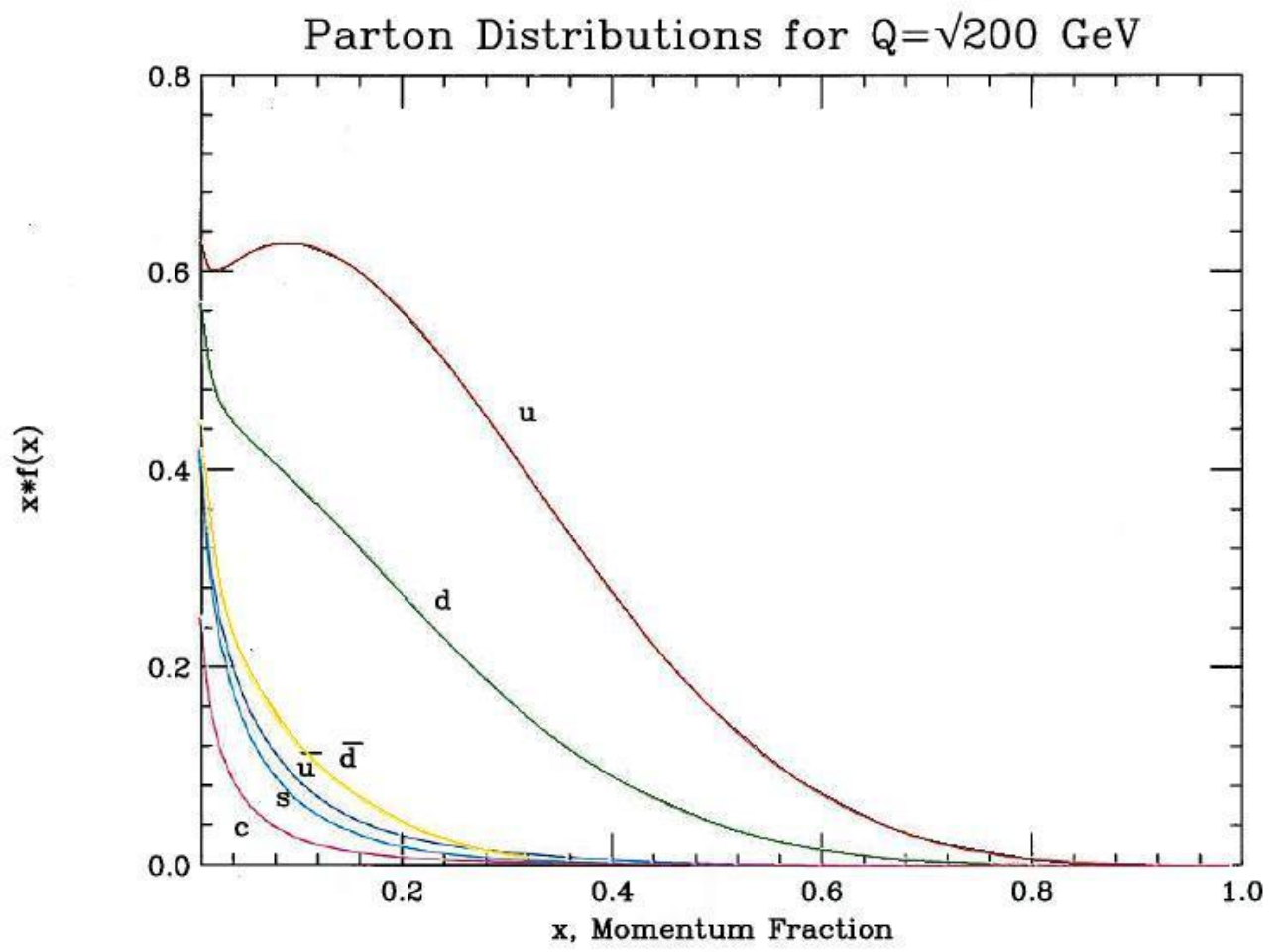

Figure 7 - Parton densities for $Q=\sqrt{ } 200 \mathrm{GeV}$

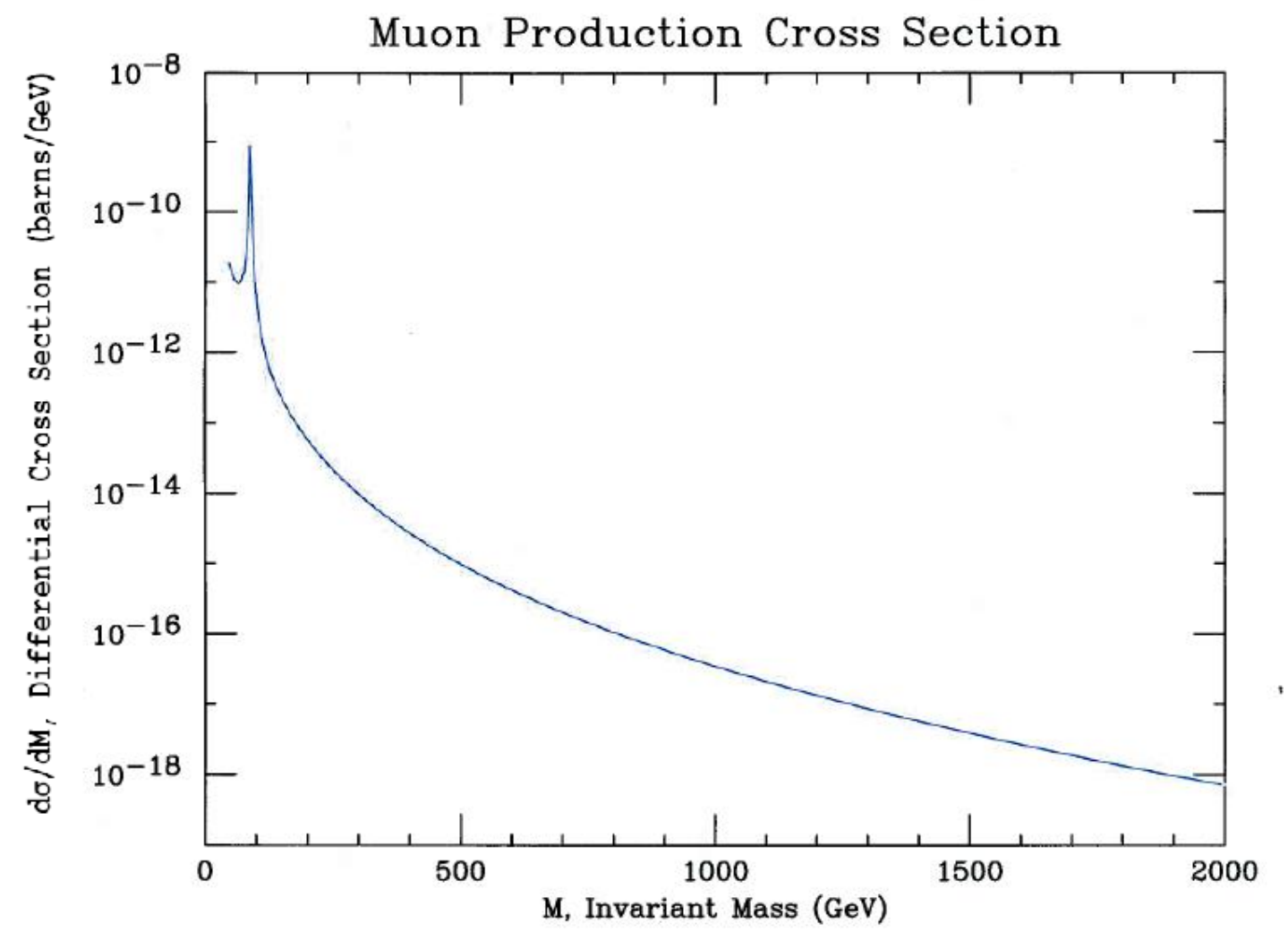

Figure 8 - Differential cross-section for p-p annihilation to muons, at center of mass energy $=14 \mathrm{TeV}$ 


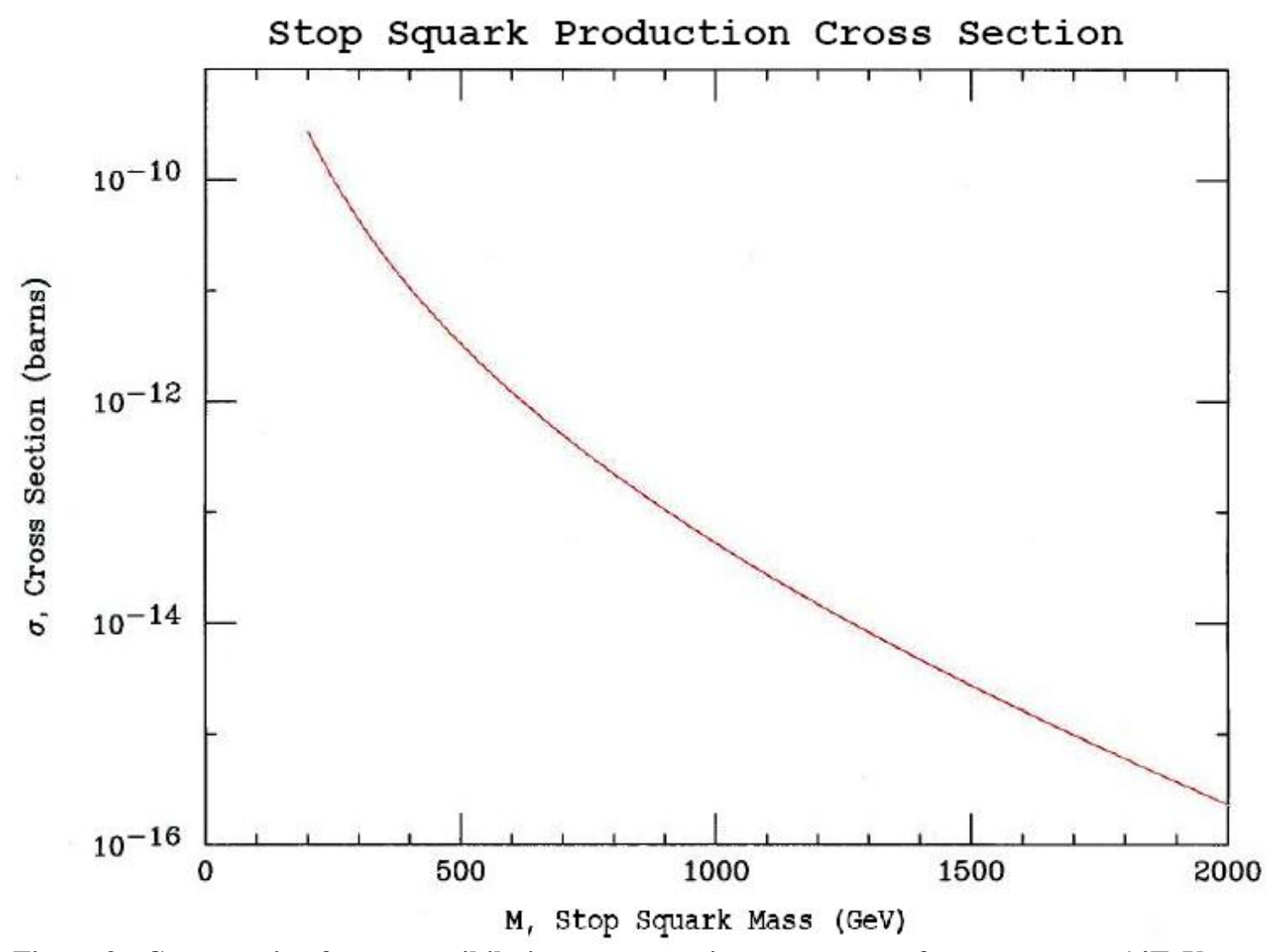

Figure 9 - Cross-section for $p-p$ annihilation to stop-antistop, at center of mass energy $=14 \mathrm{TeV}$

\section{Acknowledgements}

I would like to thank my mentors, JoAnne Hewett and Tom Rizzo, for all their time and assistance. Also, many thanks to Michael Peskin for providing me with the VEGAS integration programs. Lastly, I would like to sincerely thank John Conley, Randel Cotta, and James Gainer for their programming help and their patience with all my questions. 


\section{References}

[1] C.M.S. Collaboration, "The CMS Physics Technical Design Report, Volume 2," CERN/LHCC, vol. 2006/021, no. CMS TDR 8.2.

[2] M. Woods. "Standard Model of Particle Physics." http://www-

sldnt.slac.stanford.edu/alr/standard_model.htm, April 9, 2001 [July 17, 2009]

[3] S. Dawson, E. Eichten, and C. Quigg. "Search for supersymmetric particles in hadron-hadron collisions," Physical Review D, vol. 31, no. 7, pp. 1581-1637, 1 April 1985.

[4] W. Beenakker, M. Krämer, T. Plehn, M. Spira, and P.M. Zerwas. "Stop Production at Hadron Colliders," Nuclear Physics B, vol. 515, pp. 3-14, 1998.

[5] M. Schott. "Z Boson Production at LHC with First Data," The 2007 Europhysics Conference on High Energy Physics Journal of Physics, Conference Series 110, 2008.

[6] P. Nadolsky “CTEQ6 Parton Distributions.” http://hep.pa.msu.edu/cteq/public/cteq6.html Jan 28, 2009 [July 2009]

[7] Elsevier publishing, Physics Letters B, vol. 667, Issues 1-5, 18 September 2008. 
Design and Implementation of a Thermal and Acoustic X-ray Detector to Measure the LCLS Beam Energy

Jennifer L. Loos

Office of Science, Science Undergraduate Laboratory Internship Program

San Jose State University, San Jose, CA

SLAC National Accelerator Laboratory

Menlo Park, California

August 14, 2009

Prepared in partial fulfillment of the requirement of the Office of Science, Department of Energy's Science Undergraduate Laboratory Internship under the direction of Joe Frisch in the Linac Coherent Light Source group of the SLAC National Accelerator Laboratory.

Participant:

Signature

Research Advisor:

Signature 
Table of Contents

$\underline{\text { Page }}$

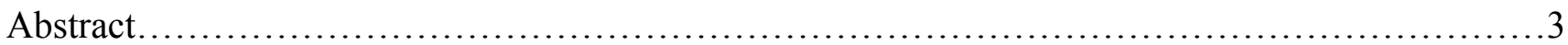

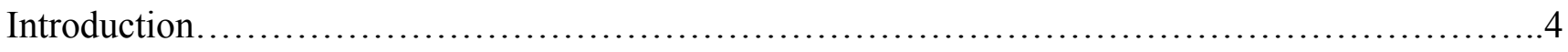

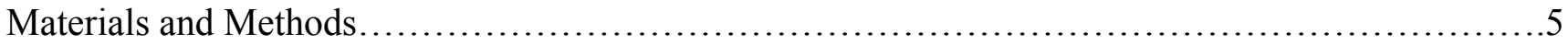

Results............................................................................

Discussion and Conclusion.......................................................

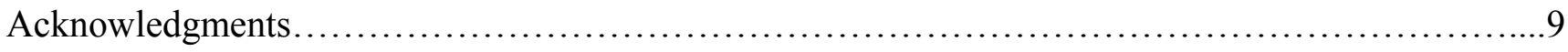

References......................................................................... 10

Figures and Tables.......................................................................... 


\section{ABSTRACT}

Design and Implementation of a Thermal and Acoustic X-ray Detector to Measure the LCLS Beam Energy. JENNIFER L. LOOS (San Jose State University, San Jose, CA 95192) JOE FRISCH (SLAC National Accelerator Laboratory, Menlo Park, CA 94025).

On April 11, 2009, first light was seen from LCLS. The present apparatus being used to measure the x-ray beam energy is the Total Energy Sensor which uses a suite of thermal sensors. Another device is needed to cross-check the energy measurements. This new diagnostic tool utilizes radiation acoustic phenomena to determine the x-ray beam energy. A target is hit by the $\mathrm{x}$-rays from the beam, and a voltage is generated in two piezoelectric sensors attached to the target in response to the consequent deformation. Once the voltage is known, the power can be obtained. Thermal sensors will also be attached to the target for calibration purposes. Material selection and design were based on: durability, ultra-high vacuum compatibility, safety and thermal properties. The target material was also chosen for its acoustic properties which were determined from tests using a frequency generator and laser. Initial tests suggest the device will function as anticipated. 


\section{INTRODUCTION}

In April of 2009, the LCLS became fully operational with an x-ray energy range from $800 \mathrm{eV}$ to $8 \mathrm{keV}$ and a pulse energy around $1 \mathrm{~mJ}$. The present device being used to measure the x-ray beam energy is a Total Energy Sensor [1] which is comprised of a suite of thermal sensor arrays. A Yttrium Aluminum Garnet (YAG) crystal which is part of the Direct Imager [2] is also being used for calibration and imaging purposes. Because additional measurements are needed for comparison and greater precision, the Thermal and Acoustic X-ray Detector will serve as a complementary diagnostic tool in determining the beam energy using acoustic and thermal detectors along with a YAG screen. Piezoelectric sensors will be used for the acoustic measurement and RTDs (resistive thermal detectors) will be used for the thermal measurement. This device will be placed in the beam dump area immediately after the electron beam dump in vacuum chamber ST0 upstream of the current FEE (front end enclosure) diagnostics.

The overall concept of the detector consists of a target being hit by the FEL light of the beam with the incoherent light being blocked by a shield. Attached to the target will be two piezoelectric sensors and two RTDs. This arrangement will rest on an aluminum bar which serves as both a platform for the target and shield as well as a heat sink. A clamp holding the shield in place will also function as a platform to which the YAG screen will be attached.

Although the piezoelectric effect has been applied to many areas of research, the use of acoustic devices for energy measurement is a relatively new technique. "Radiation acoustics is a field of physics in which sound phenomena arising under radiation interacting with matter are studied [3]." In this case, the shock wave from the x-ray beam will create vibrations in the target causing deformations in the target and the acoustic sensors which then generate a voltage in the latter. The power of the beam can then be determined from the measured voltage. "It was found that in tests with a fixed beam 
and a target material in a fixed geometry, the probe output voltage was directly proportional to the peak power of incident beam [3]."

\section{MATERIALS AND METHODS}

Because this instrument will be placed in an ultra-high vacuum environment, careful consideration was given to the building materials that would be appropriate and most reliable. To insure safe and effective functioning of the device in the vacuum chamber, initial calculations were performed to estimate the amounts of ambient heating and the cooling time constants of all materials to be used. The thermal analysis of the materials to be used was done using the relationships:

$$
\mathrm{P}=\mathrm{A}_{1} \sigma\left(\mathrm{T}_{1}{ }^{4}-\mathrm{T}_{2}{ }^{4}\right) /\left[1 / \varepsilon_{1}+\left(\left(1-\varepsilon_{2}\right) \mathrm{A}_{1} / \varepsilon_{2} \mathrm{~A}_{2}\right)\right]
$$

where $P$ is the radiative power transfer from a surface area $A_{1}$ with emissivity $\varepsilon_{1}$ at temperature $T_{1}$ to another surface area $A_{2}$ with emissivity $\varepsilon_{2}$ at temperature $T_{2}$, and $\sigma$ is the Stefan-Boltzmann constant $5.67 \times 10^{-8} \mathrm{~W} / \mathrm{m}^{2} \mathrm{~K}[2]$, and $\mathrm{h}=(\mathrm{kA}) / \mathrm{d}$ where $\mathrm{k}$ is the thermal conductivity of the material, $\mathrm{A}$ is the surface area and $\mathrm{d}$ is the diameter. Then using: $\tau=\mathrm{C}_{\mathrm{v}} / \mathrm{h}$, where $\mathrm{C}_{\mathrm{v}}$ is the specific heat and $\mathrm{h}$ is the sum of the thermal conductivities of the materials divided by the thickness of the materials, the time constants were determined. (Table 1)

Platinum RTDs in a ceramic substrate were selected as ceramic materials are vacuum compatible and can be baked at high temperatures. Ceramic piezoelectric actuators were selected for their high Curie temperature and low out-gassing rate. For the shield, a layer of boron carbide $\left(\mathrm{B}_{4} \mathrm{C}\right.$, another ceramic material $)(2.5 \times .5 \times 1.24 \mathrm{~cm})$ was chosen for the front with a thicker layer of tungsten $(2.5 \times 1.5 \times 1.24 \mathrm{~cm})$ backing it. The shield materials were chosen not only for their vacuum compatibility, but also for their ability to effectively block the spontaneous (incoherent) radiation from the beam allowing only the coherent $150 \mu \mathrm{m}$ beam of x-rays to pass through a small collimator $(3.25$ $\mathrm{mm}$ hole) in the shield to the target. Because the target will absorb the x-ray beam, the very hard 
materials $\mathrm{B}_{4} \mathrm{C}$ (9.3 on Mohs hardness scale) and beryllium (5.5 on Mohs scale) were initially considered as the target material, with $\mathrm{B}_{4} \mathrm{C}$ being the preferred material as beryllium is toxic. Another consideration in choosing the target material was to make sure it was one with a low atomic weight to ensure the material was not easily heated and had low x-ray absorption. Beryllium was considered as it gives a clear 'ringing' signal in response to interactions with ultra-sonic waves. Very little information was available about the acoustic properties of $\mathrm{B}_{4} \mathrm{C}$, so tests were performed to determine its viability as a target material.

To test the $\mathrm{B}_{4} \mathrm{C}$, two piezoelectric sensors $(3 \times 3 \times 2 \mathrm{~mm})$ were clamped on opposite sides of a small piece of $\mathrm{B}_{4} \mathrm{C}$ (approximately $2.54 \times 1.27 \times .5 \mathrm{~cm}$ ). Using a frequency generator, one of the piezos was driven, and the response of the second piezo was measured. From this, the resonances within the piezos were plotted, and a signal with a significant ringing time of about $200 \mu$ s was observed (Figure 1). The next test was to place the $\mathrm{B}_{4} \mathrm{C}$ piece with the piezos casually clamped on to it in a laser beam at $1 \mathrm{~mJ}$ then $2 \mathrm{~mJ}$ to better simulate the x-ray beam. The response signal from the second piezo was about $100 \mu \mathrm{V}$. This was lower than expected, and it was thought that perhaps the shape of the $\mathrm{B}_{4} \mathrm{C}$ (and informality of the test) caused shearing modes that were not foreseen and weakened the signal. More formal tests were later performed to improve the signal.

Aluminum was selected for the heat sink and shield clamps for its efficient cooling properties and thus, relatively short time constant. (A short time constant is preferred so the temperature of the heat sink quickly reaches equilibrium with the outside and does not accumulate heat as the target is repeatedly interfacing with the beam). The aluminum bar will be attached to a translator positioned outside of the vacuum chamber that will move the bar up or down depending on which measurement (from the $\mathrm{B}_{4} \mathrm{C}$ target or the YAG screen) is desired (Figure 3). A Thermionics Z-450 Series Translator was deemed best for this purpose for its stable motion. A close coupler will then be attached to the translator, and spherical octagon will be attached on top of the coupler. Feedthroughs will attach to the octagon. The wires from the RTDs and piezoelectric actuators will connect to the feedthroughs which 
will then be connected to data acquisition electronics including a preamp (BJT Preamp SIM911) and an RTD monitor (SIM 923A). ${ }^{1}$ The preamp was chosen for its especially low noise of $1.8 \mathrm{nV} / \sqrt{ } \mathrm{Hz}$ which was calculated using: $\mathrm{N}_{\text {total }}=\mathrm{V}_{\mathrm{n}}+\mathrm{I}_{\mathrm{n}} \mathrm{Z}$ where $\mathrm{V}_{\mathrm{n}}$ is the voltage noise, $\mathrm{I}_{\mathrm{n}}$ is the current noise $\left(1.2 \times 10^{-12}\right.$ $\mathrm{A} / \sqrt{\mathrm{Hz}}$ ) and $\mathrm{Z}$ is the impedance. The impedance was determined using the relationship: $\mathrm{Z}=1 /[2 \pi \omega \mathrm{C}]$ where $\omega$ is the resonant frequency of the piezos and $\mathrm{C}$ is the capacitance. The total calculated noise was significantly less than the signal from the piezos, so this amplifier was considered a good choice.

\section{RESULTS}

For the second laser proof-of-concept test, to improve the signal, a cubic centimeter of $\mathrm{B}_{4} \mathrm{C}$ was machined $(1.25 \times .8 \times 1 \mathrm{~cm})$ to be tested in the same fashion as the first laser test. ${ }^{2}$ To improve the surface area contact between the piezos and the $\mathrm{B}_{4} \mathrm{C}$, the piezos were epoxied to the top and bottom of the cube. To further improve the signal, each piezo was also connected to four-to-one transformers and an amplifier set to a gain of 100 . With the transformers, more symmetric $\mathrm{B}_{4} \mathrm{C}$ shape and better experimental controls, much stronger signals were observed. Initially, the laser spot size was about the size of the target $(\sim 1 \mathrm{~cm})$. With a laser energy of $1 \mathrm{~mJ}$, and spot size of about $1 \mathrm{~cm}$, the response signal was about $240 \mathrm{mV}$. (Figure 5) There was some increase in the signal when the laser beam spot size was minimized to approximately half the size of the surface of the target $(268 \mathrm{mV})$. It was not expected that the spot size should make much difference in the signal response as long as the full diameter of the beam was in contact with the surface of the $\mathrm{B}_{4} \mathrm{C}$ block. Because there was an increase in the signal as the result of the smaller spot size, it is thought that perhaps some of the beam was missing the target in the first test, but further tests are needed to better understand this.

\footnotetext{
1 Preliminary visualizations of the device were done using Open Office Draw, and after further iterations of the overall design, technical drawings were completed using Solid Edge. (Figures 2, 3 and 4)

2 Two such cubes of $\mathrm{B}_{4} \mathrm{C}$ were machined: one for testing purposes, one for use in vacuum.
} 
With the smaller spot size, tests were then done at a laser power of $1 \mathrm{~mJ}, 2 \mathrm{~mJ}$ and $2.6 \mathrm{~mJ}$. At 1 $\mathrm{mJ}$, the signal from channel 1, corresponding to one piezo, was about $268 \mathrm{mV}$, and from channel 2, corresponding to the other piezo, was about $236 \mathrm{mV}$ (Figure 6). At $2 \mathrm{~mJ}$ of laser power, the signal was about $456 \mathrm{mV}$ on channel 1 and $416 \mathrm{mV}$ on channel 2 (Figure 7). Increasing the laser power from 1 to $2 \mathrm{~mJ}$ roughly doubled the signal, increasing it by about $180 \mathrm{mV}$. This fairly linear response was not seen when the power was increased from 2 to $2.6 \mathrm{~mJ}$. At $2.6 \mathrm{~mJ}$, the signal was $520 \mathrm{mV}$ on channel 1 and $456 \mathrm{mV}$ on channel 2 (Figure 8). (The signal only increased by about $55 \mathrm{mV}$ ). (Table 2, Figure 9) It was determined that further data would be required to obtain a more complete graph of response signal to beam power and better understand their relationship.

A third laser test was conducted to collect more comprehensive data and to possibly discover the cause of the non-linearity of the relationship between the laser beam power and the piezo response. For this test, the device was partially assembled, and the $\mathrm{B}_{4} \mathrm{C}$ cube was epoxied to the aluminum bar. $\mathrm{A}$ low pass filter $(50 \Omega, 1.9 \mathrm{MHz})$ was placed before the amplifier for each channel. The addition of the filter caused an increase in the signal by a factor two (it is not fully understood why this is so). Measurements were taken at energies between $.83 \mathrm{~mJ}$ and $2.67 \mathrm{~mJ}$ for gain settings of 100, 10 and 1 . As in the second laser test, the roughly linear response from lower laser energy to around $2.3 \mathrm{~mJ}$ was observed. However, above $2.3 \mathrm{~mJ}$, the non-linear relationship seen in the previous test occurred. It was thought that perhaps this was due to resonances within the amplifier, but this relationship was apparent even when there was no amplification (Figure 10, 11 and 12).

Another possible reason for the non-linearity is that there may be a saturation point for the piezo response at a certain laser energy, but further tests are required to study this. New tests will include further decreasing the spot size to better simulate the FEL beam to determine if the non-linearity is an issue of beam intensity; if the same relationship is observed with smaller and smaller spot sizes, it is likely due to a saturation point within the piezos. 


\section{DISCUSSION AND CONCLUSION}

From preliminary tests, it appears that this device will be useful in determining the energy of the LCLS x-ray beam. Using results from the laser lab (once they are more comprehensive), it will be possible to obtain a value for the x-ray beam energy from the measured voltage induced in the piezoelectric sensors and the temperature change in the RTDs. By comparing these values with those of the Total Energy Sensor already in place, the power of the beam can be known with greater accuracy.

It is still not clear why there is not a linear relationship between the laser power and the piezo response at energies higher than $\sim 2.3 \mathrm{~mJ}$. The next tests done will be to determine whether or not the piezo electric sensors have a limiting voltage that is being exceeded at higher energies, or whether there are other material effects causing this relationship.

The materials selected for the device seem to be appropriate and effective. Design issues such as how to mount the various pieces to the aluminum bar to ensure stability and proper functioning in vacuum (leak prevention, etc.) have largely been remedied. However, final testing of the device will reveal any areas requiring further attention or improvement.

\section{ACKNOWLEDGMENTS}

Many thanks go to The Department of Energy Office of Science, SLAC National Accelerator Laboratory and the SULI program for providing this rewarding and very educational opportunity. Special thanks also go to my mentor Joe Frisch, Tonee Smith, Mark Petree, Phil Cutino, Dave Shelley and the machinists in Light Fabrication who lent their extensive expertise to this effort. Thanks also to fellow SULI student Greg Bentsen. 


\section{REFERENCES}

[1] S. Friedrich et al, "Design of a bolometer for total-energy measurement of the linear coherent light source pulsed X-ray laser,” Nucl. Instr. Methods in Phys. Res. A ,vol. 599, pp.772-774, 2006.

[2] Richard M. Bionta, "X-ray Transport Optics and Diagnostics Commissioning Report," Lawrence Livermore National Laboratory Report UCRL-PROC-207494, October 23, 2004.

[3] A.I. Kalinichenko, V.T. Lazurik, and I.I. Zalyubovsky, The Physics and Technology of Particle and Photon Beams Volume 9: Introduction to Radiation Acoustics. The Netherlands: Harwood Academic Publishers, 2001.

[4] Alexander Wu Chao, Ed. and Maury Tigner, Ed., Handbook of Accelerator Physics and Engineering. Singapore: World Scientific Publishing Co. Pte. Ltd., 1999.

[5] http://www.physikinstrumente.com/en/products/piezo tutorial.php [Online]. [Accessed: June 2, 2009].

[6] James P. Wolfe, Imaging Phonons: Acoustic Wave Propagation in Solids. United Kingdom: Cambridge University Press, 1998.

[7] "Piezoelectricity," July 30, 2009. [Online]. Available: http://en.wikipedia.org/wiki/Piezoelectricity. [Accessed: June 3, 2009]. 
FIGURES AND TABLES

\section{Boron Carbide (block)}

Specific Heat $\left(\mathrm{J} / \mathrm{kg}^{*} \mathrm{~K}\right)$

Density $\left(\mathrm{kg} / \mathrm{m}^{\wedge} 3\right)$

Molar Mass $(\mathrm{g} / \mathrm{mol})$

Size $(\mathrm{cm})$

Thermal Conductivity (W/m*K)

Thermal Expansion Coeff

(um/m*K)

Speed of Sound $(\mathrm{m} / \mathrm{s})$

Atten Length (um)

Emissivity (1)

Mass (kg)

Target Specific Heat $(\mathrm{J} / \mathrm{K})$

\section{Coefficients (h)}

piezoelectric

wires

air

shield

container

$5.200 \mathrm{E}+00$

$1.090 \mathrm{E}+04$

$2.891 \mathrm{E}+00$

$1.000 \mathrm{E}+00$

2.520E-03

4.353E-02
Time constant for target (s)

295.95

Time constant for top piezo (s)

49.1

388.8

Time Constant for
J. Loos G. Bentsen

4.950E-03 4.950E-03

$2.010 \mathrm{E}-04 \quad 2.010 \mathrm{E}-04$

$2.600 \mathrm{E}-04 \quad 1.600 \mathrm{E}-04$

$5.640 \mathrm{E}-05 \quad 8.700 \mathrm{E}-05$

$2.600 \mathrm{E}-03 \quad 3.000 \mathrm{E}-03$ Aluminum Bar (s)
293.1

48.9

\section{Tungsten (material for shield)}

Min Mass Attenuation Coeff.

$\left(\mathrm{cm}^{\wedge} 2 / \mathrm{g}\right)$

1.378E-01

Density $\left(\mathrm{kg} / \mathrm{m}^{\wedge} 3\right)$

$1.925 \mathrm{E}+04$

Attenuation Length $(\mathrm{cm})$

3.770E-04

95\% Length (cm)

$1.131 \mathrm{E}-03$

Specific Heat $\left(\mathrm{J} / \mathrm{kg}^{*} \mathrm{~K}\right)$

$1.300 \mathrm{E}+02$

Molar Mass (g/mol)

$1.838 \mathrm{E}+02$

Emissivity (1)

4.000E-02

Shield Width $(\mathrm{cm})$

$2.500 \mathrm{E}+00$

Shield Height $(\mathrm{cm})$

$2.000 \mathrm{E}+00$

Shield Mass (g)

$0.000 \mathrm{E}+00$

Shield Specific Heat (J/K)

\section{Piezo}

Piezo Thermal Conductivity

(W/m*K)

$2.427 \mathrm{E}+01$

Piezo Thickness (mm)

$1.100 \mathrm{E}+00$

Piezo Deformation Coeff $(\mathrm{pm} / \mathrm{V})$

Piezo Width (mm)

$4.000 \mathrm{E}+02$

$3.000 \mathrm{E}+00$

Piezo Capacitance (C/V)

$2.500 \mathrm{E}-08$

Table 1: Heat and time constant calculations. Data calculated and compiled by G. Bentsen and J. Loos. 
Piezos with B4C in between

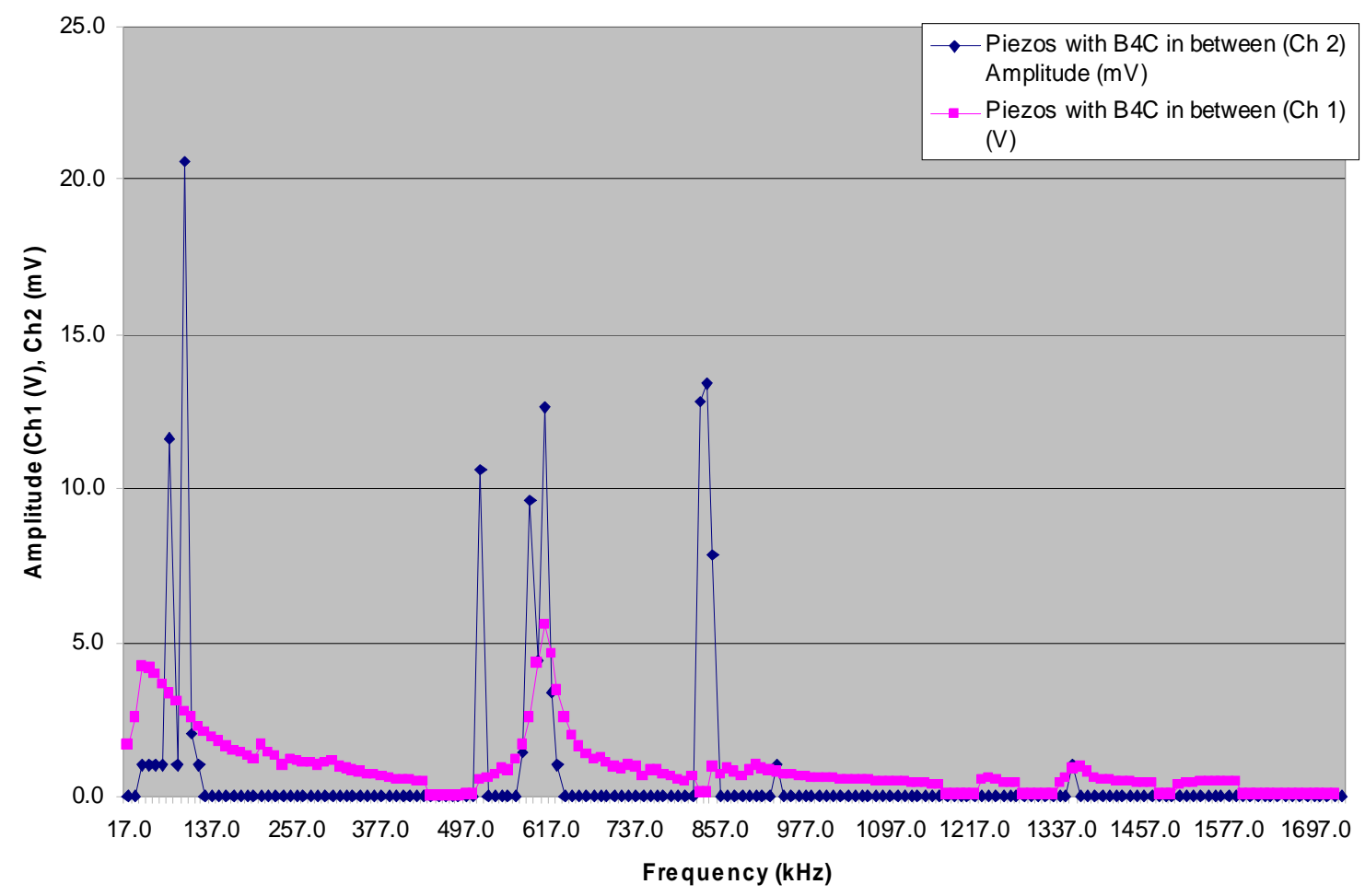

Figure 1: A signal is seen from an initial test of an irregularly-shaped piece of $\mathrm{B}_{4} \mathrm{C}$ using a frequency generator. Ch 1 (pink) is the driving signal in volts, $\mathrm{Ch} 2$ (blue) is the response signal from the second piezo in $\mathrm{mV}$.

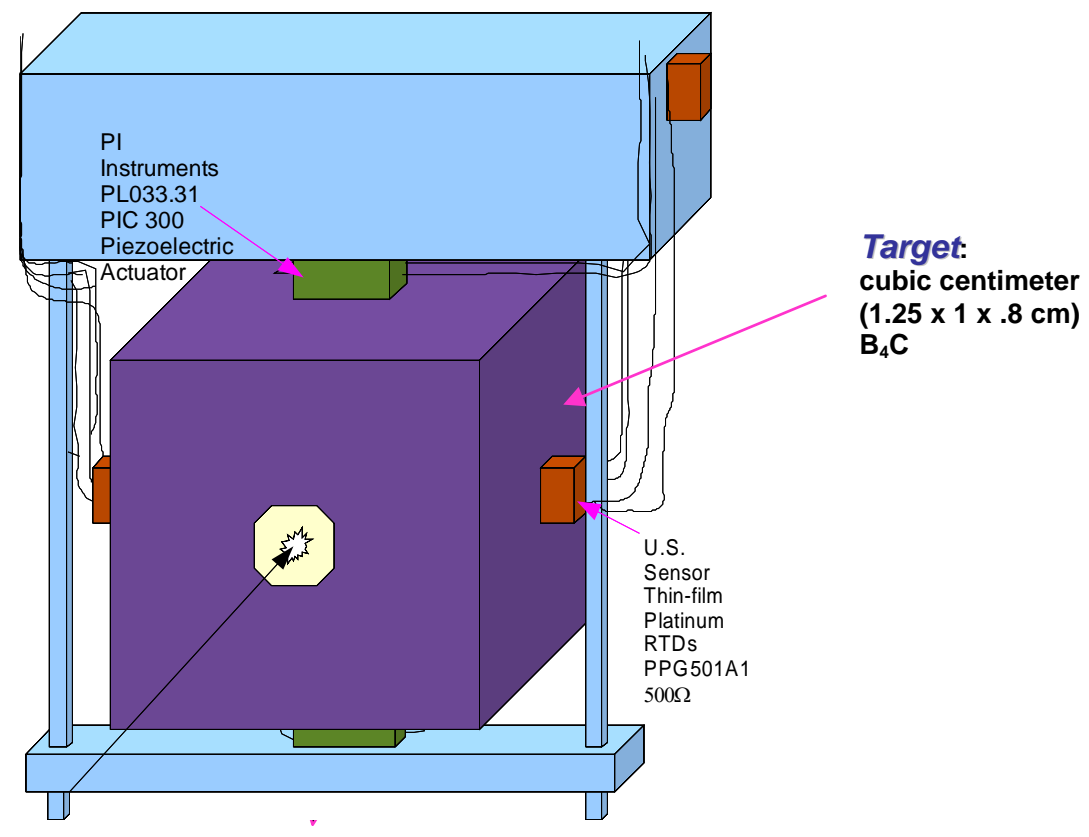

Figure 2: This is an early conceptual drawing of target with piezoelectric sensors and RTDs (J. Loos, June 2009). 


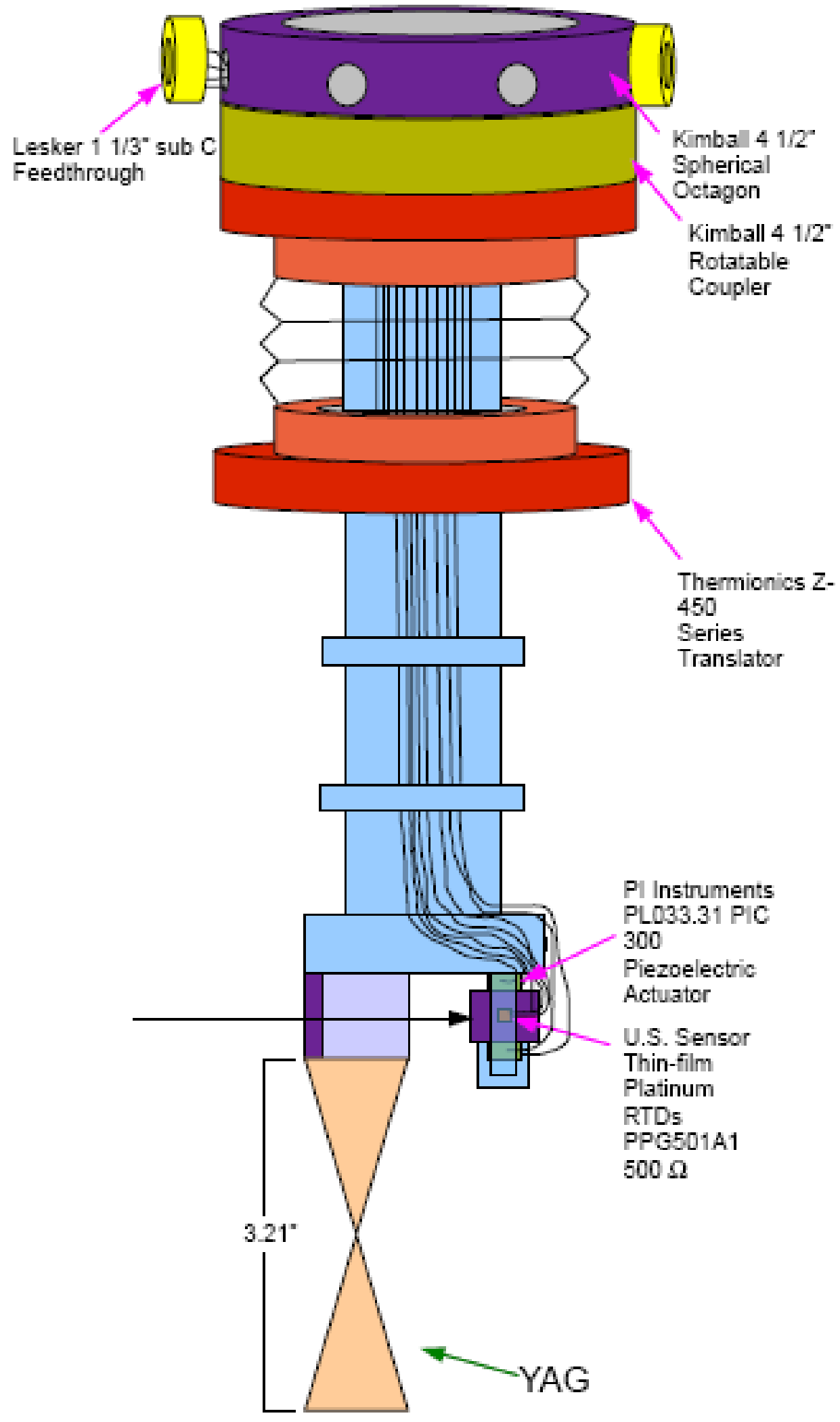

Figure 3: This is an early conceptual drawing for the device created in Open Office Draw (J. Loos, June 2009). In the final design, $100 \Omega$ RTDs and a non-rotatable coupler were chosen. 


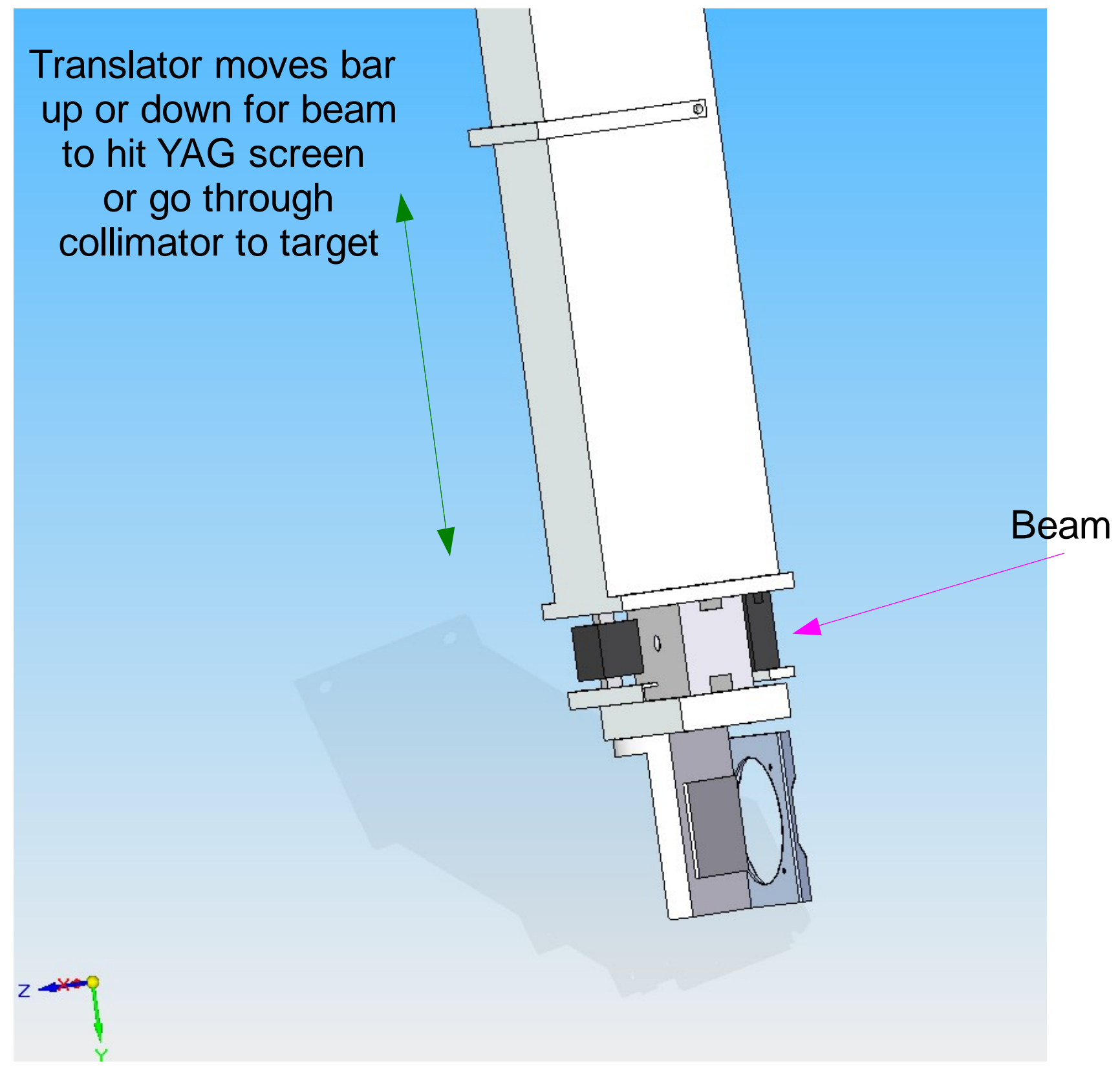

Figure 4: This is the lower half of the assembly with the $\mathrm{B}_{4} \mathrm{C}$ cube and clamp, tungsten and $\mathrm{B}_{4} \mathrm{C}$ shielding with clamps, YAG assembly, aluminum bar heat sink and wire clamp. (J. Loos, July 2009, Solid Edge). 


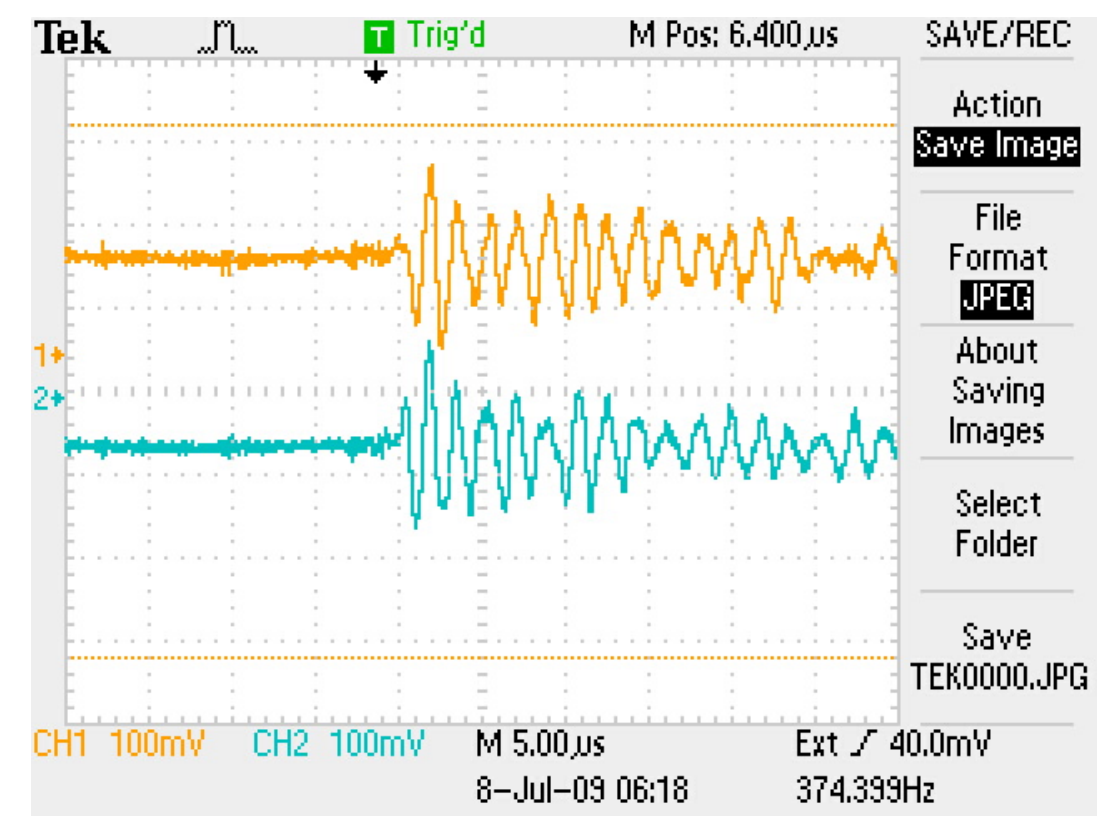

Figure 5: Second laser test with cubic centimeter of B4C, beam energy at $1 \mathrm{~mJ}$, spot size about $1 \mathrm{~cm}$, amplifier x100, signal (ch 1) of about $240 \mathrm{mV}$.

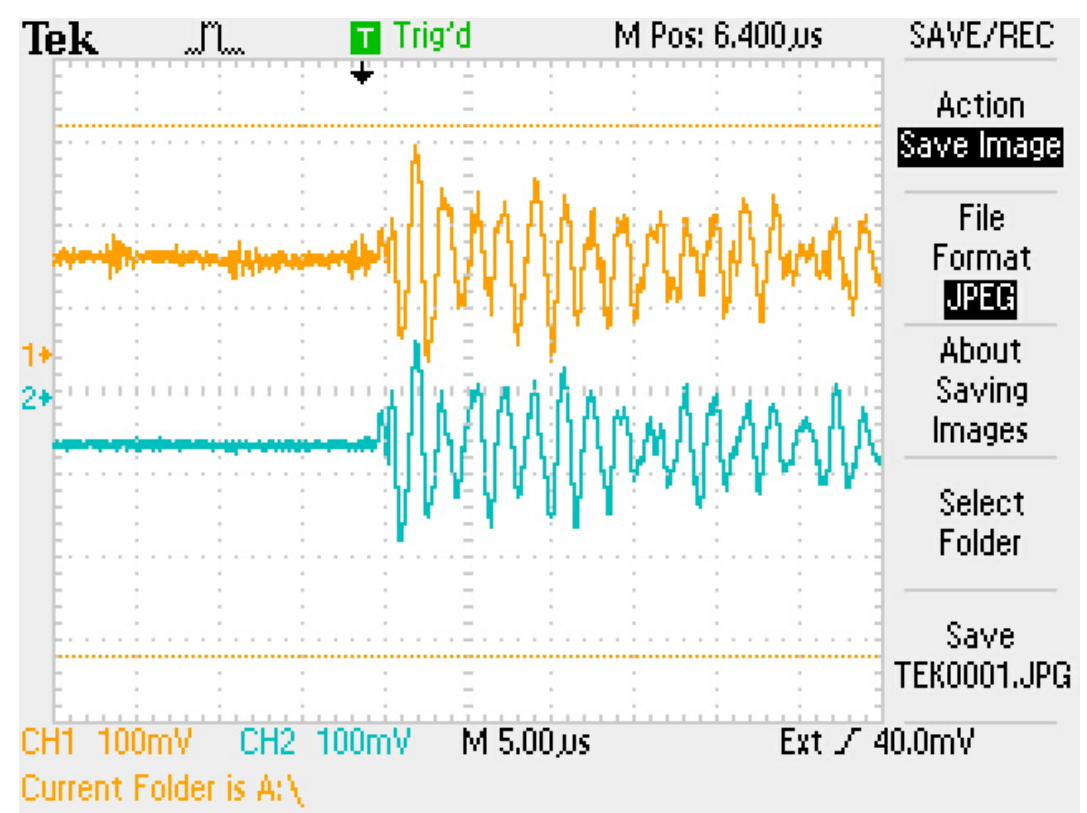

Figure 6: Second laser test with cubic centimeter of B4C, beam energy at $\sim 1 \mathrm{~mJ}$, spot size about $.5 \mathrm{~cm}$, amplifier x100, signal (ch 1) of about $268 \mathrm{mV}$, (ch 2) of about $236 \mathrm{mV}$. 


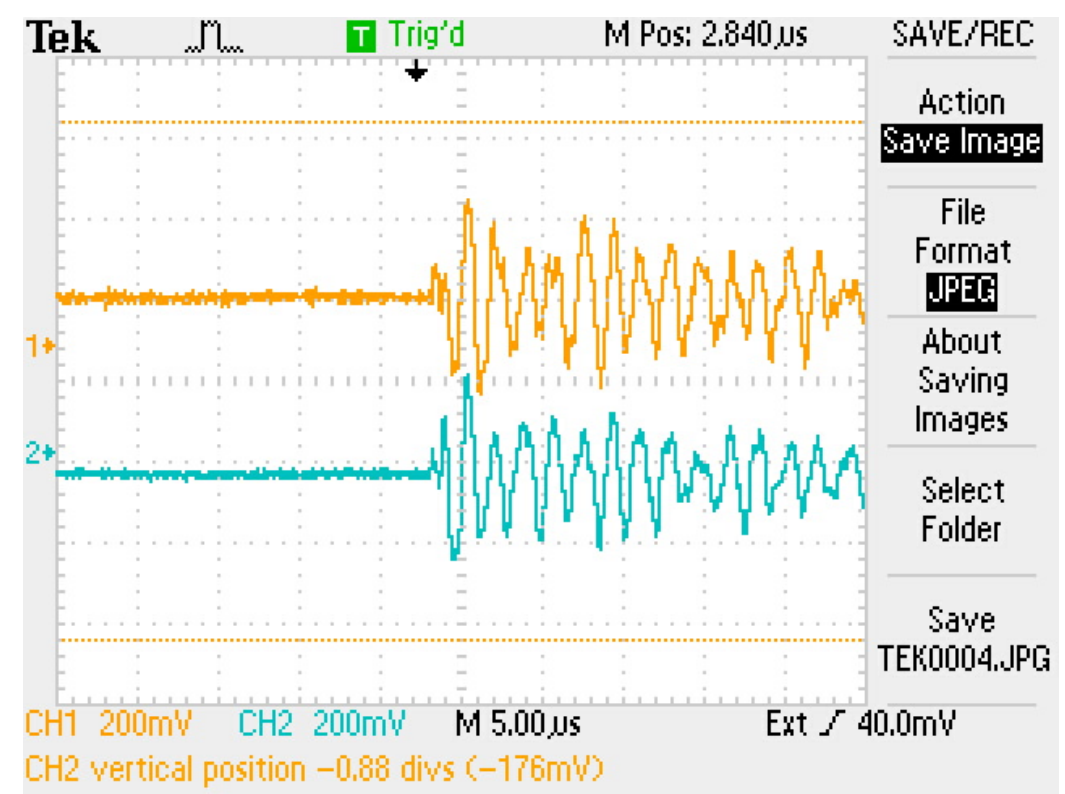

Figure 7: Second laser test with cubic centimeter of B4C, beam energy at $\sim 2 \mathrm{~mJ}$, spot size about $.5 \mathrm{~cm}$, amplifier x100, signal (ch 1) of about $456 \mathrm{mV}$, (ch 2) $416 \mathrm{mV}$.

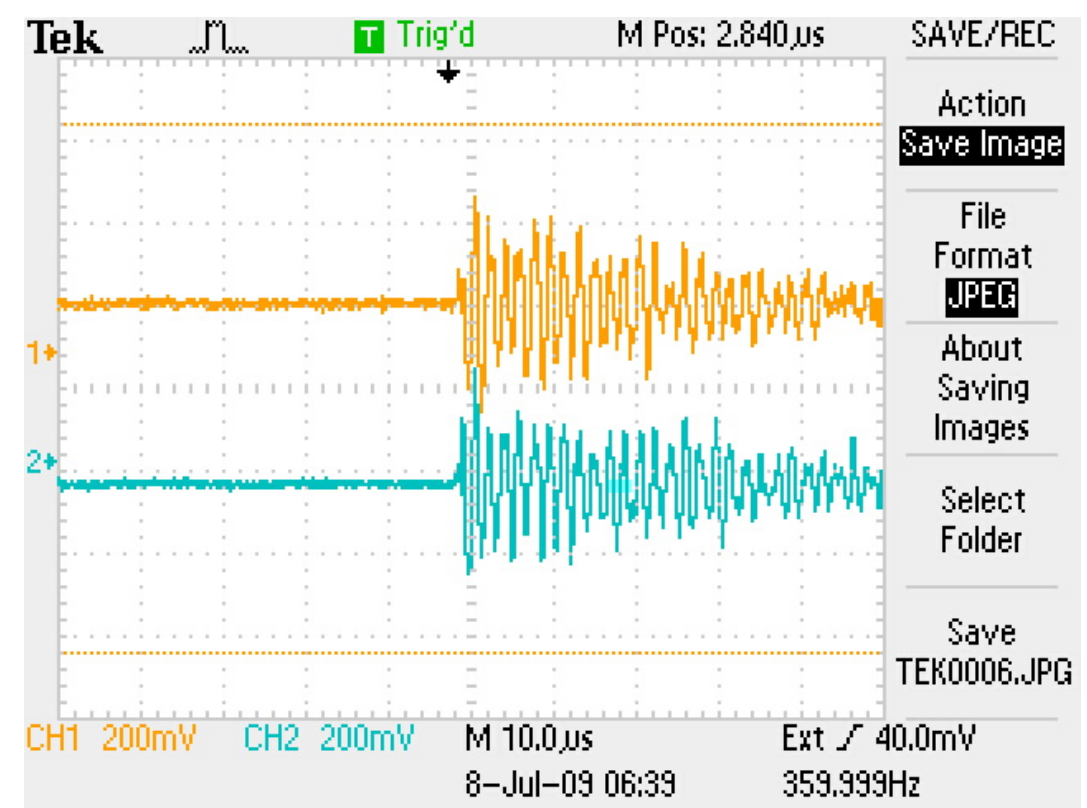

Figure 8: Beam energy at $\sim 2.6 \mathrm{~mJ}$, spot size about $.5 \mathrm{~cm}$, amplifier $\mathrm{x} 100$, signal (ch 1) of about 520 $\mathrm{mV}$, (ch 2) $456 \mathrm{mV}$. 


\begin{tabular}{|c|c|c|c|}
\hline Laser Power $(\mathrm{mJ})$ & Spot size $(\mathrm{cm})$ & Channel 1 $(\mathrm{mV})$ & Channel 2 $(\mathrm{mV})$ \\
\hline 1 & $\sim 1$ & 240 & 220 \\
\hline 1 & $\sim .5$ & 268 & 236 \\
\hline 2 & $\sim .5$ & 456 & 416 \\
\hline 2.6 & $\sim .5$ & 520 & 456 \\
\hline
\end{tabular}

Table 2: Measurements of the response signal $(\mathrm{mV})$ from the piezos as a result of the laser power $(\mathrm{mJ})$ and spot size, second laser test.

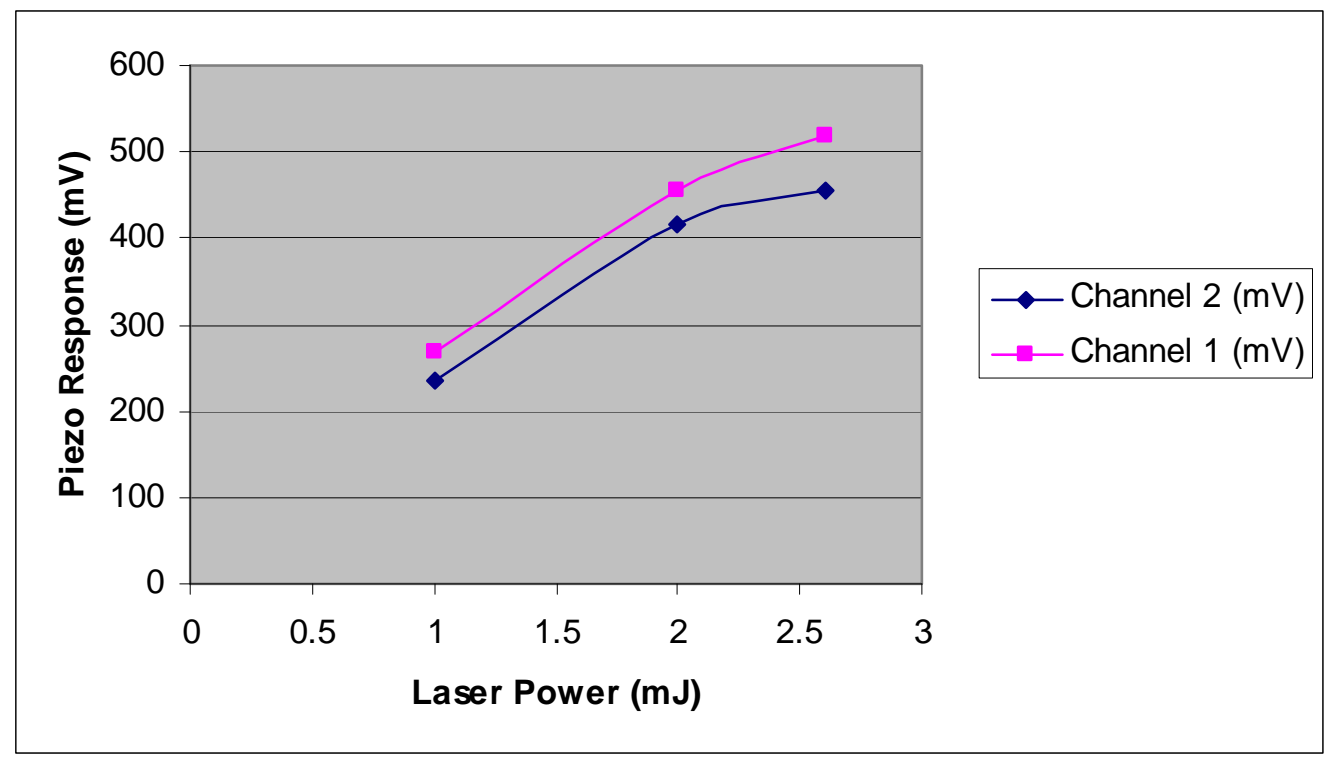

Figure 9: Plot of laser power versus the piezo response showing a non-linear relationship, second laser test. 


\begin{tabular}{|c|c|c|c|c|c|c|}
\hline $\begin{array}{l}\text { Laser Power } \\
\qquad(\mathrm{mJ})\end{array}$ & $\begin{array}{c}\text { Channel } 1(\mathrm{x} 100) \\
(\mathrm{mV})\end{array}$ & $\begin{array}{c}\text { Channel } 2(\mathrm{x} 100) \\
(\mathrm{mV})\end{array}$ & $\begin{array}{c}\text { Channel } 1(\mathrm{x} 10) \\
(\mathrm{mV})\end{array}$ & $\begin{array}{c}\text { Channel } 2(\mathrm{x} 10) \\
(\mathrm{mV})\end{array}$ & $\begin{array}{c}\text { Channel } 1 \text { (x1) } \\
(\mathrm{mV})\end{array}$ & $\begin{array}{c}\text { Channel } 2 \text { (x1) } \\
(\mathrm{mV})\end{array}$ \\
\hline 2.67 & 800.1 & 811.6 & 91.3 & 79.2 & 8.86 & 7.74 \\
\hline 2.5 & & & 87.2 & 75.8 & 8.75 & 7.6 \\
\hline 2.58 & 800.8 & 805.2 & 90.42 & 78.18 & & \\
\hline 2.33 & 787.1 & 741.4 & 81.9 & 70.94 & 8.18 & 7.11 \\
\hline 2.17 & & & 77.3 & 66.2 & & \\
\hline 2 & 728.3 & 659.3 & 73.01 & 63.01 & & \\
\hline 1.67 & 629.6 & 551.8 & 62.3 & 54.07 & & \\
\hline 1.33 & 512.1 & 444.8 & 50.3 & 43.5 & & \\
\hline 1 & 390.4 & 337.1 & 38.51 & 33.4 & & \\
\hline 0.83 & 319.2 & 281.1 & 32.02 & 27.74 & & \\
\hline
\end{tabular}

Table 3: Third laser test data.

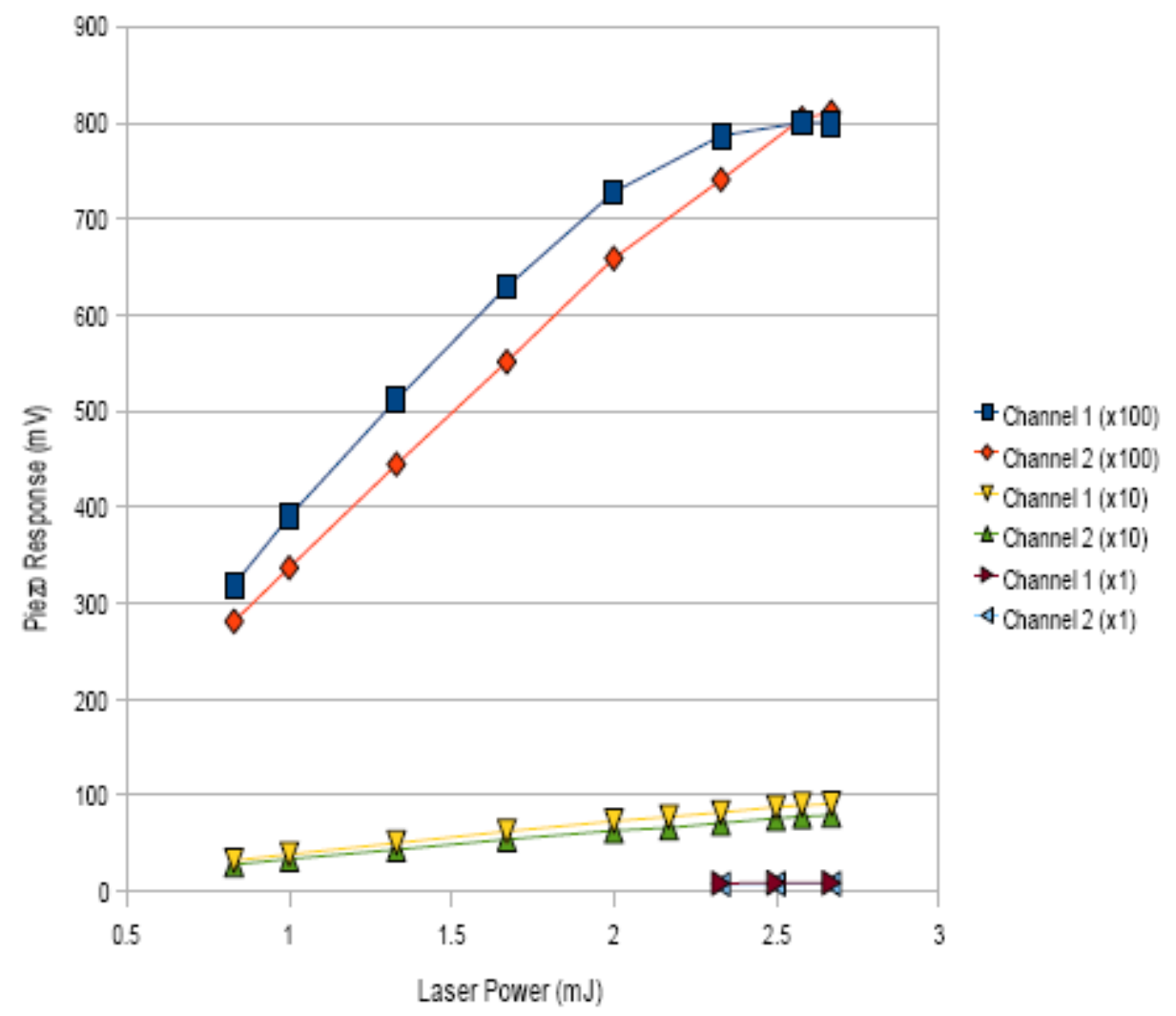

[Figure 10] Third laser test at gain of 100, 10 and 1. 


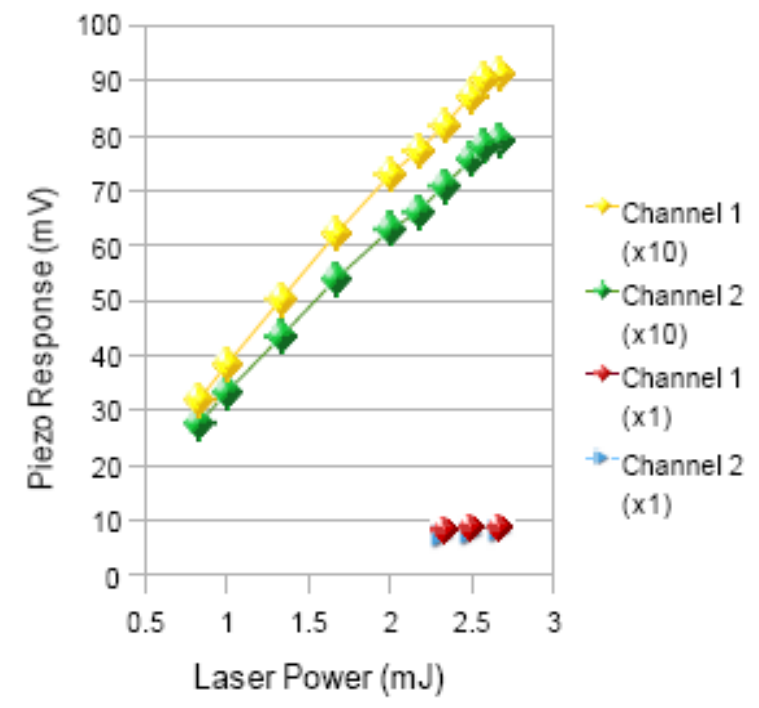

[Figure 11] Third laser test at gain of 10 and 1.

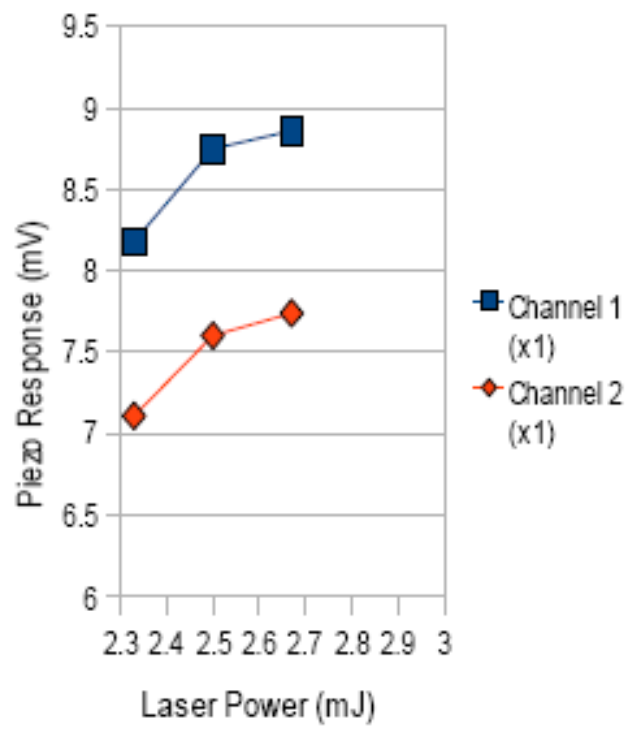

[Figure 12] Third laser test, no amplification. 


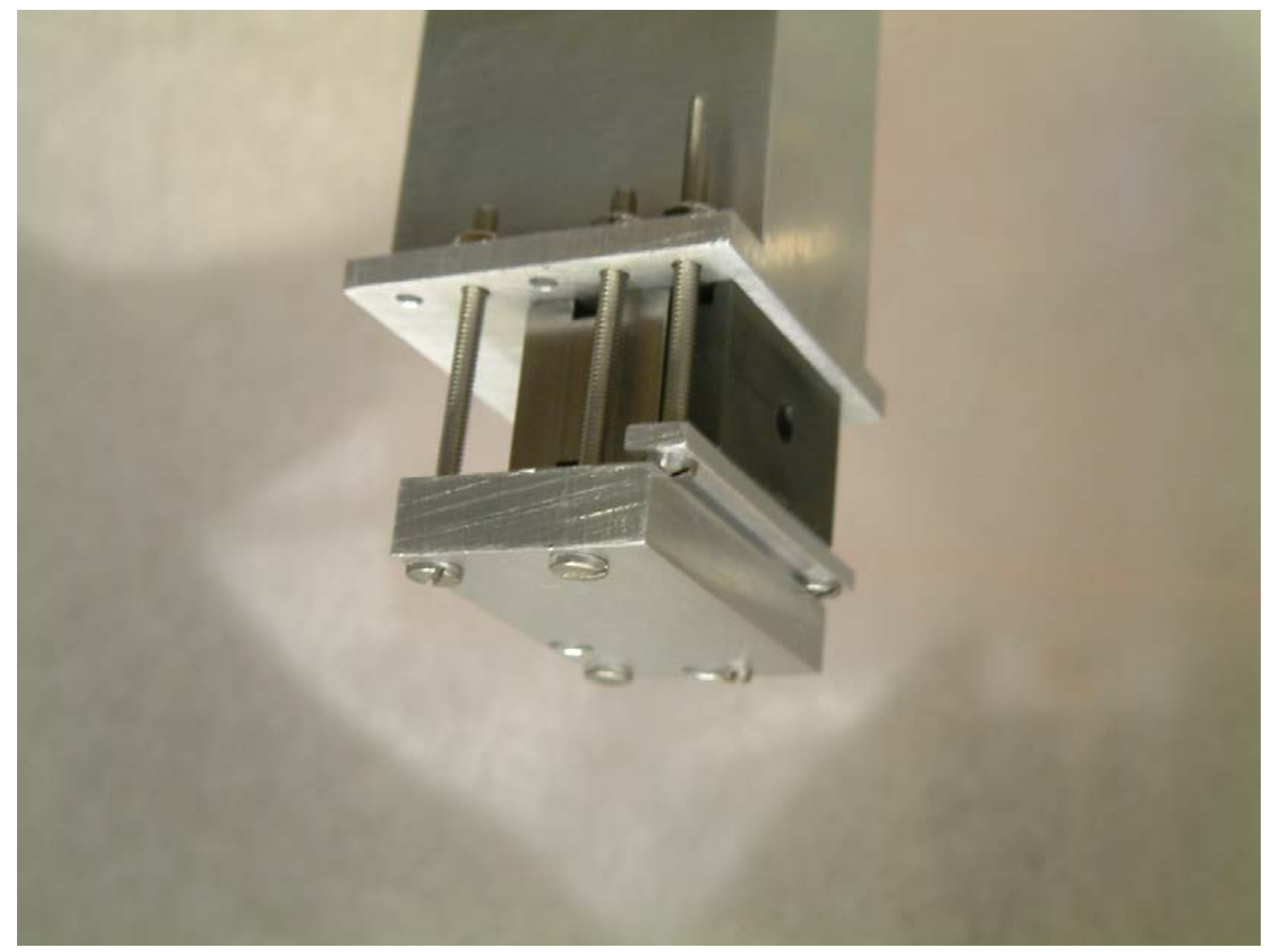

[Figure 13] Partial assembly of detector with shielding and aluminum bar.

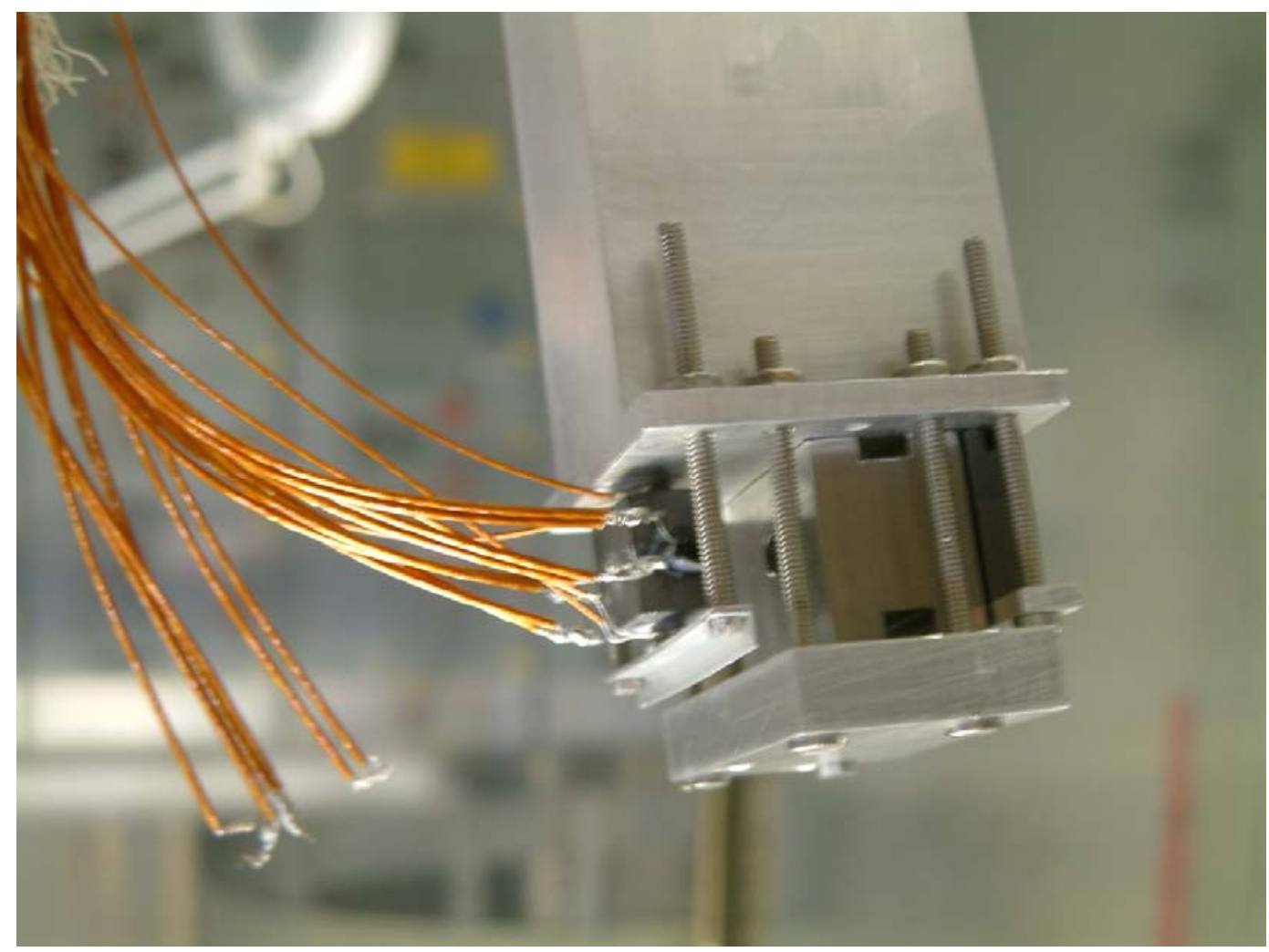

[Figure 14] Partial assembly of detector with shielding, $\mathrm{B}_{4} \mathrm{C}$ cube, RTDs, piezos and aluminum bar. 


\title{
Optimizing Cosmic-Ray Simulations using FGST Observations
}

\author{
Andrew McLeod \\ University of California, San Diego \\ Office of Science, Science Undergraduate Laboratory Internship (SULI) \\ Stanford Linear Accelerator Center \\ Stanford, CA
}

August 14, 2009

Prepared in partial fulfillment of the requirements of the Office of Science, Department of Energy's Science Undergraduate Laboratory Internship under the direction of Markus Ackermann at the Kavli Institute for Particle Astrophysics and Cosmology at the SLAC National Accelerator Laboratory.

Participant:

Signature

Research Advisor:

\author{
Signature
}




\section{TABLE OF CONTENTS}

$\begin{array}{ll}\text { Abstract } & \text { ii }\end{array}$

$\begin{array}{ll}\text { Introduction } & 1\end{array}$

$\begin{array}{lr}\text { Materials and Methods } & 2\end{array}$

$\begin{array}{lr}\text { Results } & 6\end{array}$

$\begin{array}{lr}\text { Discussion and Conclusions } & 8\end{array}$

$\begin{array}{ll}\text { Acknowledgments } & 10\end{array}$

Appendix A: The MAn Software $\quad 11$

$\begin{array}{ll}\text { References } & 13\end{array}$

$\begin{array}{lr}\text { Tables and Figures } & 14\end{array}$ 


\begin{abstract}
Optimizing Cosmic-Ray Simulations using FGST Observations. ANDREW MCLEOD (University of California, San Diego, La Jolla, CA 92093) MARKUS ACKERMANN (Kavli Institute for Particle Astrophysics and Cosmology at the SLAC National Accelerator Laboratory, Stanford, CA 94025)
\end{abstract}

A number of important parameters of cosmic-ray distribution, propagation, and interaction in the Milky Way can be predicted through the comparison of measured cosmic-ray and gamma-ray spectra to the spectra of simulated Galaxies. These predictions are made by altering the physical parameters governing Galaxy simulations until a best-fit set of parameters is found. Since the accuracy of this method is limited by the quality of available data, the exceptional precision of recent FGST gamma-ray measurements makes unprecedented galactic model refinement possible. Consequently, this data was used as a benchmark in optimizing galactic models derived by GALPROP. An in-depth investigation was performed on a wide range of galactic models, and improvement or degeneration in each was gauged through specialized analysis using novel GaDGET software. By analyzing individual galactic parameters and their effects on observed spectra, a new optimized set of parameters was found that better fits the Fermi data than previous GALPROP models. This result is especially important in refining previous estimates of galactic parameters that cannot be measured directly. It also provides an important check on known galactic parameters and enhances GALPROPs value as a high-level modeling tool. 


\section{INTRODUCTION}

In only one year, the Fermi Gamma-ray Space Telescope (FGST) has measured the diffuse cosmic gamma-ray sky with more precision and confidence than any of its predecessors. This success comes as a product of both the enhanced spatial and energy resolution and large field of view of the Large Area Telescope (LAT), the onboard instrument responsible for making these gamma-ray measurements. The energy range accessible to the LAT also greatly exceeds that of previous gamma-ray telescopes, most notably the Energetic Gamma-Ray Experiment Telescope (EGRET) that made the first all-sky gamma-ray surveys in the early 1990s [1]. The Fermi data now available is thus not only more precise but more comprehensive than that which preceded it [2].

This newfound precision imposes new constraints on the as yet unknown parameters that govern gamma-ray and cosmic-ray production and propagation within our Galaxy. These constraints can be determined by modeling the Milky Way with differing physical parameters and comparing the simulated gamma-ray spectra to the observations made by the FGST. As the sophistication in galactic models has increased, however, their number of adjustable parameters has correspondingly grown. There currently exist over 40 parameters, many of which are interdependent, that remain only weakly constrained by the local cosmic-ray spectrum. Due to the fact that the relations between these parameters are nonlinear and not well understood, this significantly complicates efforts to constrain and optimize galactic models. Specifically, due to the size of the relevant parameter space, computerized optimization does not present a viable option. These parameters must therefore be adjusted manually until their contribution to the gamma-ray spectrum is understood, after which they can be fitted to the empirical data gathered by the FGST. Due to the ample spatial and spectral features

of the gamma-ray sky, reaching such an agreement with the Fermi data can constrain many important Milky Way parameters such as its interstellar gas distribution, cosmic-ray injection spectra, and propagation and interaction parameters. This project aims to optimize 
these galactic model parameters and to determine the range of conditions under which such models can be reconciled with the observed gamma-ray sky.

\section{MATERIALS AND METHODS}

To optimize and constrain galactic model parameters, a wide range of Galaxy simulations are generated and compared to the observations made by the FGST. These galactic models are derived using GALPROP, software designed to predict the diffuse gamma-ray sky by calculating the gamma-ray spectra generated by a multitude of individual processes and then summing over these contributions. To do this, it first generates a density field of cosmic-rays (given a cosmic-ray source distribution as initial conditions) by solving a set of transport equations for the different cosmic-ray species, after which it computes their interaction with the interstellar medium. The dominant gamma-ray producing processes in the LAT energy range of $100 \mathrm{MeV}$ to $300 \mathrm{GeV}$ are Bremsstrahlung, Inverse-Compton Scattering, and neutral pion decay, which depend on the Galaxy-wide spectrum and distribution of cosmic-rays as well as the interstellar gas and radiation field [3]. All model parameters are defined in a specification file where the user can alter them from the currently used template. In this file the user also defines the desired complexity of simulation, which is set so as to produce sky maps with approximately $0.5^{\circ}$ resolution, corresponding to the limiting angular resolution of underlying surveys of the interstellar medium.

Another program, GaDGET, is used to compare the predictions of these simulated galaxies with the observed gamma-ray spectrum. GaDGET optimizes the gamma-ray spectrum predicted by GALPROP by assigning renormalizing fit-weights to the different contributing processes in incremental energy bins such that the predictions best fit the data gathered by the FGST. These fit-weights provide an indication of whether a model is over-predicting or under-predicting the gamma-ray contribution from a given process, allowing the user to incrementally adjust model parameters in the aim of producing an optimized set of parameters 
that will constitute a best estimate for the parameters governing the Milky Way. GaDGET also calculates the statistical likelihood and residual difference of the resulting fit-weight adjusted sky maps (per pixel); these quantities help assess how feasible the physical parameters used by a given model are in light of the new, more precise Fermi data.

Two additional measures, component susceptibility and spectrum deviation, are used to complement those employed by GaDGET. Component susceptibility quantifies the change in a component's overall contribution to the gamma-ray spectrum when some model parameter is varied; specifically, it calculates the difference between a component's fit-weights in models immediately adjacent in parameter space and then sums this value over the sequence of models under study. It thus provides an indication of both the magnitude and direction of each components variability. Conversely, spectrum deviation measures the overall difference between a given model's spectrum and the corresponding spectrum measured by experiment. Divergence in the spectrum deviation of the Boron-Carbon ratio in particular (which provides an indication of the fraction of primary to secondary cosmic-rays) helps constrain many model parameters from both above and below. This measure is also computed for the proton spectrum, electron spectrum, and Beryllium 9-Beryllium 10 ratio (which indicates of the relative abundance of radioactive secondary cosmic-rays), and is minimized for each.

The Model Analysis (MAn) software developed for this project uses these various measures to identify preferential galactic models. It accomplishes this by producing a set of thirty-five plots, from which the user can identify relevant trends and select favorable traits. As an example, consider Figure 1, in which the predicted Boron-Carbon ratio of models with a differing Diffusion Coefficient Index are compared to the observations of the High Energy Astronomy Observatory (HEAO-3) [4]. The Diffusion Coefficient Index governs the momentum dependence of the transport of cosmic-rays through the interstellar medium by

$$
D=\frac{v}{c} D_{0}\left(\frac{\rho}{D_{r}}\right)^{D_{i}},
$$


where $D_{0}$ is the diffusion coefficient at the reference rigidity $D_{r}, v$ is the velocity of a cosmicray being accelerated by a magnetic field such that its radius of curvature is $\rho$, and $D_{i}$ is the Diffusion Coefficient Index. It can easily be seen from this graph that an optimized galactic model would have a diffusion coefficient index between .33 and .48 (if all other parameters were to remain the same).

Conversely, Figure 2 plots the Inverse-Compton Scattering fit-weights of models in which Source Parameters 1 and 2 were varied simultaneously as a function of energy. These parameters define the radial distribution of cosmic-ray sources in the simulated Galaxy by the function

$$
R^{\alpha} e^{-\beta R}
$$

where $\mathrm{R}$ is the radial coordinate (in units of the earth's distance from the galactic center), $\alpha$ is Source Parameter 1 , and $\beta$ is Source Parameter 2. This function peaks at $\alpha / \beta$, which is why there is interest in varying both simultaneously. The significance of this plot is twofold: the average fit-weight of each model indicates whether a model is predicting the correct gamma-ray flux, and the energy-dependence of each model's fit-weights indicates whether a model is predicting the correct spectral shape. If the predicted spectrum shape is correct, the same fit-weight would be assigned to each energy bin and no energy dependence would be observed. As such, this plot suggests that Source Parameter 2 should be set near 5.34, since this model seems to have fit-weights near 1 and low energy dependece (and Source Parameter 1 should be set to its corresponding value).

Figure 3 plots the susceptibility of each component of the gamma-ray sky to variation in interstellar gas density. Black data points indicate that a component shifted in only one direction (either positive or negative), while blue points quantify the total amount of variation in a component that shifted in both directions. The Molecular and Atomic Hydrogen contributions to the gamma-ray sky are each separated into three galactic regions; the Inner component ranges from 0 to $7.5 \mathrm{kpc}$, the Local from 7.5 to $9.5 \mathrm{kpc}$, and the Outer from 9.5 
to $20 \mathrm{kpc}$. From this plot, one can infer that the Extragalactic Diffuse contribution to the gamma-ray sky remains unaffected by Galaxy Width, but that the Outer Atomic Hydrogen component changes rapidly. Component susceptibility is thus useful in deciding which galactic parameters to changed if an increase or decrease in the flux of a given gamma-ray component is desired.

Finally, Figure 4 shows the negative log of the statistical likelihood that models with increasing Galactic Width match the Fermi data. The statistical likelihood represents the probability that the Fermi results would be obtained in a Galaxy similar to the model under study; thus, it is a quantity that should be maximized. Since the negative log of this value is plotted, however, the resulting trend should be minimized. This plot effectively constrains galactic models with these parameters to Galactic Disk Widths above 7 kpc.

As illustrated by these examples, the MAn software makes possible two types of investigation: coarse filtering to determine acceptable parameter ranges and refined optimization to find the best-fit set of parameters. This project primarily concerned itself with the latter of these goals, mainly because the optimization of GALPROP enhances the software's value as a high-level modeling tool in studying diverse astrophysical phenomena such as the extragalactic gamma-ray spectrum (through accurate subtraction of the galactic component) above and beyond the prediction of Milky Way parameters. Also, due to the interdependence of many of the parameters, any substantial attempt to constrain even a single parameter would necessarily involve a many-dimensional parameter space. This quickly becomes complicated for the same reason that computerized optimization was rejected; our understanding of these dependencies is still too limited. Conversely, the existence of an acceptable template of parameters simplifies the process of optimization, making it a practical endeavor.

This endeavor is enacted in two stages. First, each parameter is varied individually to determine its effect on observable features such as spectral shapes and fluxes. Since the previous model parameters were established using EGRET sky-maps, they are thought to be reasonably close to their counterpart Milky Way values. As such, it can be assumed that 
the trends observed in parameter space around the current parameter set are valid for the entire parameter space relevant to this investigation. Deliberate improvements can thus be made to the current model's parameters in the second stage of optimization, in which many parameters are varied at once. By means of these deliberate modifications and iterative single-parameter optimizations, considerable agreement between galactic models and the Fermi data should be attainable.

\section{RESULTS}

Eleven galactic model parameters were studied in detail using the MAn software. These parameters included galactic width and radius, interstellar gas distribution, the diffusion coefficient governing the transport of cosmic-rays through the interstellar medium (and its dependence on momentum), breaks in the electron injection spectrum, the Alfven velocity defining a particular type of magneto-hydrodynamic wave's speed, and several constants determining the galactic source distribution. The results of this survey, over five hundred plots, were compiled into an online database from which they can be viewed simultaneously. Some of the investigated parameters, such as the interstellar gas density and galactic radius, were found to have little effect on the gamma-ray and cosmic-ray spectra predicted by resulting galactic models. Others, such as the break in the electron injection spectrum, had too great an effect to be varied without immediately contradicting measurements. The majority, however, displayed complex yet distinct trends. As an example, Figures 5 and 6 display some of the key plots from the Alfven velocity survey. Clear trends can be found in each plot, making possible deliberate parameter modifications when optimizing galactic models; however, the simultaneous optimization of thirty-five of these trends quickly becomes a challenge. In practice, some measures must be given priority over others and the scale over which each measure varies must be taken into account.

Once the individual parameter trends were mapped out, larger scale optimization could 
be attempted. This was accomplished by identifying several intermediate objectives, which included predicting the Boron-Carbon ratio with greater precision, increasing the model statistical likelihood, and improving the component-wise fit-weights by shifting their mean closer to 1 and minimizing their energy dependence. Special attention was paid to the Inverse-Compton Scattering and the Local and Outer Hydrogen components, because these components should be more easily separated from the overall gamma-ray spectrum due to their spectral shape and direction in the sky.

Significant model improvement was achieved by shifting the distribution of cosmic-ray sources towards the inner Galaxy, as shown in Figure 7. The drawback of this modification is that it increases the Boron-Carbon Ratio by approximately $11 \%$ and decreases the Beryllium 10-Beryllium 9 ratio by almost $22 \%$. These effects can be combatted by increasing the diffusion coefficient from $5.8 \times 10^{28}$ to $8.0 \times 10^{28}$ while increasing the Galactic Disk Width from 4 to $5 \mathrm{kpc}$, which has the additional advantage of improving the electron and proton spectra around energies of $10 \mathrm{GeV}$. The total electron and proton injection fluxes were also decreased to compensate for the overproduction of each in the previous model, and the predicted Boron-Carbon Ratio was fine-tuned by altering the Diffusion Coefficient Index (see equation 1) to match the slope of the observed ratio. Finally, the Alfven velocity was raised to increase the Beryllium 10-Beryllium 9 ratio.

The resulting optimized set of parameters is found in Table 1 alongside the initial parameter values, and an analysis of the initial and newly optimized model is found in Figures 8, 9, and 10. Figure 8 shows significant refinement in the Boron-Carbon ratio and the Electron Spectrum and a slight improvement in the Proton Spectrum. Conversely, the Beryllium 10-Beryllium 9 ratio of the new model significantly deviates from measured values. This under-prediction is a common feature of all current GALPROP models (to varying degrees), and indicates that further optimization is needed. Five of the gamma-ray sky components, those plotted in Figures 9a, 9d, 10a, 10c, and 10d, show improvement across all energies, while the fit-weights of components in 9b, 9c, and 10b shifted away from the optimal value of 
1. The statistical likelihood of the new model also showed marked improvement; its negative $\log$ is 0.40321 , compared to the template model's 0.40675 .

\section{DISCUSSION AND CONCLUSIONS}

While the galactic model parameters used by previous GALPROP models are physically feasible, they can be improved. This is apparent in Figures 8, 9, and 10, in which significant improvement has already been made. Importantly, these improvements remain consistent with the parameters of previous models in that they were accomplished through small parameter alterations rather than the introduction of a completely new set of values. Consequently, these results enhance, rather than revise, our understanding of galactic processes and quantities.

Since this galactic parameter optimization was performed with the use of novel data, the resulting parameter optimizations should be given physical significance. This is especially true in the case of parameters that cannot be measured or inferred using other methods, such as the shape of specific cosmic-ray injection spectra and the temperature of interstellar gas. The injection spectrum index and normalization were altered for both electrons and protons in order to maintain consistency with direct observations, disturbed by the increased diffusion coefficient of the optimized model. These predictions can be compared to the theoretical spectra of these types of sources to help constrain the properties of such objects. Similarly, the average temperature of interstellar gas was indirectly predicted through the selection of an optimized gas map. This is because the relative density of the tested gas maps was based on an assumed ratio of atomic hydrogen with parallel and anti-parellel electron spin (relative to the nuclei), known to be dependent on gas temperature. Although the gas density parameter was not changed, important information was learned through the interstellar gas density survey that will be taken into account in future GALPROP models. Specifically, decreasing gas temperature was observed to bring down the Local 
and Outer Atomic Hydrogen fit-weights. This indicates that the gas maps currently used by GALPROP, which assume a uniform gas temperature across the entire galaxy, are not sophisticated enough to be reconciled with the Fermi data. A gas map made using a radial temperature gradient is needed in future studies to reconcile the fit-weights of these two components.

The alteration of the cosmic-ray source distribution also constitutes an interesting result, primarily because this prediction has the possibility of direct confirmation. Such confirmation depends on the resolution of a statistically significant number of sources and on the fraction of sources at each galactic radius that can be resolved. It should be noted that the new source distribution peaks at the same galactic radius as the old distribution, meaning it remains consistent with the measured pulsar population in the Milky Way, which is known to make an important contribution to the galactic cosmic-ray population.

The optimization of galactic models has two further applications beyond making predictions about the constituents of the Milky Way. The first is in the study of the extragalactic gamma-ray sky, which is limited by the precision with which the galactic component can be modeled and subtracted from the overall gamma-ray sky. This process is complicated by both the anisotropy of the galactic gamma-ray sky and its overall flux, which greatly exceeds that of the extragalactic component. The second application is in using GALPROP as a comparison tool for measurements made by the FGST. Because of the nature of the radiation it studies, the point spread function of the FGST is not precise enough to confidently exclude gamma-ray point sources when measuring the gamma-ray background. This is an issue for certain types of data analysis, because it is only by accurately measuring this background that weaker gamma-ray sources can be identified with statistical significance. The anisotropy of the gamma-ray spectrum also complicates this problem, since a background measured at high galactic latitudes cannot be used to study sources near the galactic center. This problem can be solved by using GALPROP simulations, along with the GaDGET fit-weights, as a substitute or a supplement for the observed gamma-ray background in identifying such 
sources. The success of such analyses thus depend greatly on the precision of GALPROP simulations.

While significant improvements have been made to galaxy simulations using the Fermi data, more remains to be done. Only eleven of over forty adjustable parameters have been investigated, and these parameters can be better investigated with increased computing power. The optimized model presented in this paper represents the culmination of only two weeks of investigation following the development of the MAn software. Thus, future studies of larger scope are sure to make further model refinement. Such improvement will in turn enhance the value of the FGST data that made this optimization possible.

\section{ACKNOWLEDGMENTS}

This work was done at the Kavli Institute for Particle Astrophysics and Cosmology at the Stanford Linear Accelerator Center under the direction of the U.S. Department of Energy. I would like to express my sincere thanks to the Department of Energy, Office of Science, and SULI program administrators for providing me with the opportunity to be a part of such an exciting and cutting-edge research project. In addition, I would like to thank my mentor Markus Ackermann, as well as coworkers Josh Lande and Keith Bechtol, for their invaluable assistance during various stages of my appointment. 


\section{APPENDIX A: THE MAN SOFTWARE}

The Model Analysis (MAn) software allows preferential galactic models to be identified by means of a set of quantitative comparisons. Initially developed within the confines of this project, it has since been generalized and can compare any set of user-specified galactic models, making it an ideal tool for further model refinement. For this reason, an introduction to MAns operation follows for those readers who may benefit from its use.

The MAn software is designed for use with a specification file, in which the user defines the scope of their analysis. Specification files can have any name, but each must be placed in a directory named specification_files residing in the same folder as the MAn source code. A template specification file can be found, along with the MAn source code, at the SLAC Confluence website (search for GALPROP Parameter Analysis, or find complete site name below). Once this template file has been retrieved, the first variables to define are those that set the directory paths. Three paths must be set: one to the directory containing the GALPROP output files (galprop_model_directory), another to the directory containing the GaDGET output files (gadget_fits_directory), and a third to the directory in which MAn output files should be saved (directory). The extension of the output images can also be set here in file_type.

Next, the models to be compared can be identified in the files array by model number (the number in the model's GALDEF file name) For example, a model whose parameters are defined in galdef_54_77XvMM7A should be identified as 54_77XvMM7A. Furthermore, each model's output files should reside in a folder of this same name (the model number) within the respective output directories (GALPROP and GaDGET). So, for instance, in the GALPROP output directory there should exist a folder 54-77XvMM7A that contains the galprop and p6_v3_diffuse folders created by running GALPROP with galdef_54_r7XvMM7A. Similarly, the GaDGET output directory should contain a folder 54-77XvMM7A in which the five FITS files created by running GaDGET on the same model can be found. 
Once these models are properly specified, some parameter must be identified that varies between them (even if it is merely model number). This parameter should be specified in parameter and parameter_label, where the latter should include units if applicable. The value of this parameter in each model should also be identified in the labels array, and entered as float variables into the parameter_values array (in increasing order). These parameter values will be identified with a given model by virtue of their position in the array, so care should be taken in defining these variables.

Thirty-five differing comparisons are now available within the specification file. Each can be turned on or off by setting the (comparison_type)_output variable to 0 (off), 1 (save to file), or 2 (display upon software execution). If a specific view is desired, the axis limits of a plot can be defined by the user in (comparison_type)_view. Specifying two numbers will define the $\mathrm{x}$-axis limits, specifying four numbers will define the $\mathrm{x}$-axis limits and then the y-axis limits, and specifying no numbers will revert to the default setting, in which the plot limits will be chosen to best accommodate all data points. A description of each of these comparisons is given in detail on the Confluence page, alongside a number of sample plots. Complete documentation of the source code can also be found on this website if needed.

https://confluence.slac.stanford.edu/display/SCIGRPS/GALPROP+parameter+analysis 


\section{REFERENCES}

[1] C. Fichtel et al., "The first energetic gamma-ray experiment telescope (EGRET) source catalog," The Astrophysical Journal Supplement Series, vol. 94, pp. 551-581, Oct. 1994.

[2] W. B. Atwood et al., "The Large Area Telescope on the Fermi Gamma-ray Space Telescope Mission," Astrophys. J., vol. 697, pp. 1071-1102, 2009.

[3] I. Moskalenko et al., "GALPROP C++ v.50: Explanatory Supplement," GALPROP Manual, 2006. [Online]. Available: http://galprop.stanford.edu/manuals/manual.pdf [Accessed: August 3, 2009].

[4] J. J. Engelmann, P. Ferrando, A. Soutoul, P. Goret, and E. Juliusson, "Charge composition and energy spectra of cosmic-ray nuclei for elements from Be to NI - Results from HEAO-3-C2," Astronomy and Astrophysics, vol. 233, pp. 96-111, Jul. 1990.

[5] A. W. Strong, I. V. Moskalenko, and V. S. Ptuskin, "Cosmic-ray propagation and interactions in the galaxy," 2007. [Online]. Available: doi:10.1146/annurev.nucl.57. 090506.123011 


\section{TABLES AND FIGURES}

Table 1: A comparison of the previous and optimized set of parameters.

\begin{tabular}{ccc} 
Parameter & Template Model & New Model \\
\hline \hline Diffusion Coefficient & $5.8 \times 10^{28}$ & $8.0 \times 10^{28}$ \\
\hline Diffusion Coefficient Index & 0.33 & 0.42 \\
\hline Galactic Width & $4 \mathrm{kpc}$ & $5 \mathrm{kpc}$ \\
\hline Source Parameter 1 & 1.25 & 2.0 \\
\hline Source Parameter 2 & 3.56 & 6.0 \\
\hline Alfven Velocity & 30 & 39 \\
\hline Electron Injection Normalization & $0.56 \times 10^{-9}$ & $0.32 \times 10^{-9}$ \\
\hline Electron Injection Index (low energies) & 1.6 & 1.5 \\
\hline Plectron Injection Index (high energies) & 2.42 & 2.32 \\
\hline Proton Injection Normalization & $5.75 \times 10^{-9}$ & $5.0 \times 10^{-9}$ \\
\hline Proton Injection Index (low energies) & 1.98 & 1.86 \\
\hline
\end{tabular}




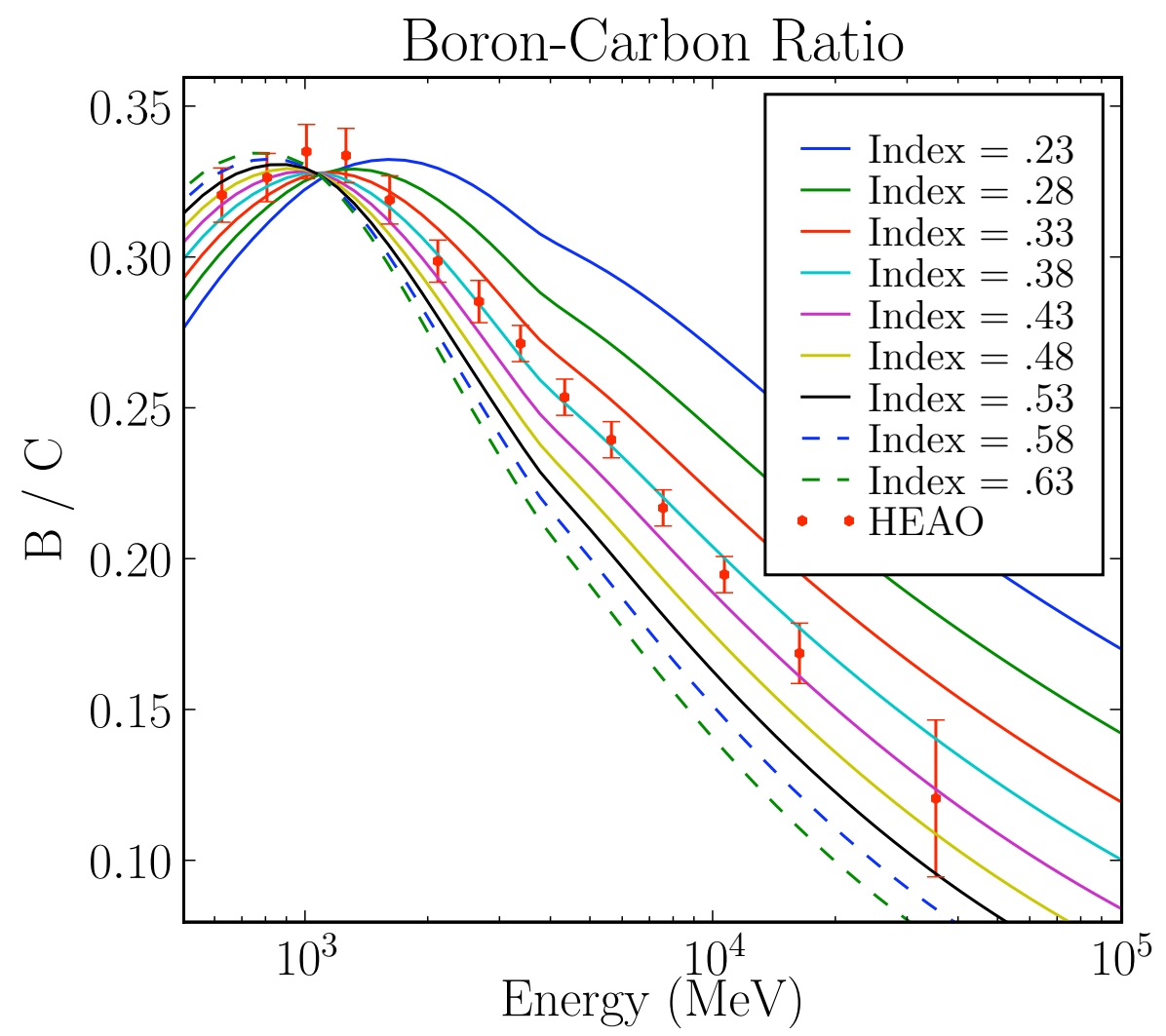

Figure 1: The predicted Boron-Carbon Ratio as a function of energy in models with Increasing Diffusion Coefficient Index. These predictions are compared to measurements made by the third High Energy Astronomy Observatory (HEAO) in 1979-1981 [4]. The Diffusion Coefficient Index determines the dependence of cosmic-ray diffusion on momentum, as seen in Equation (1). 


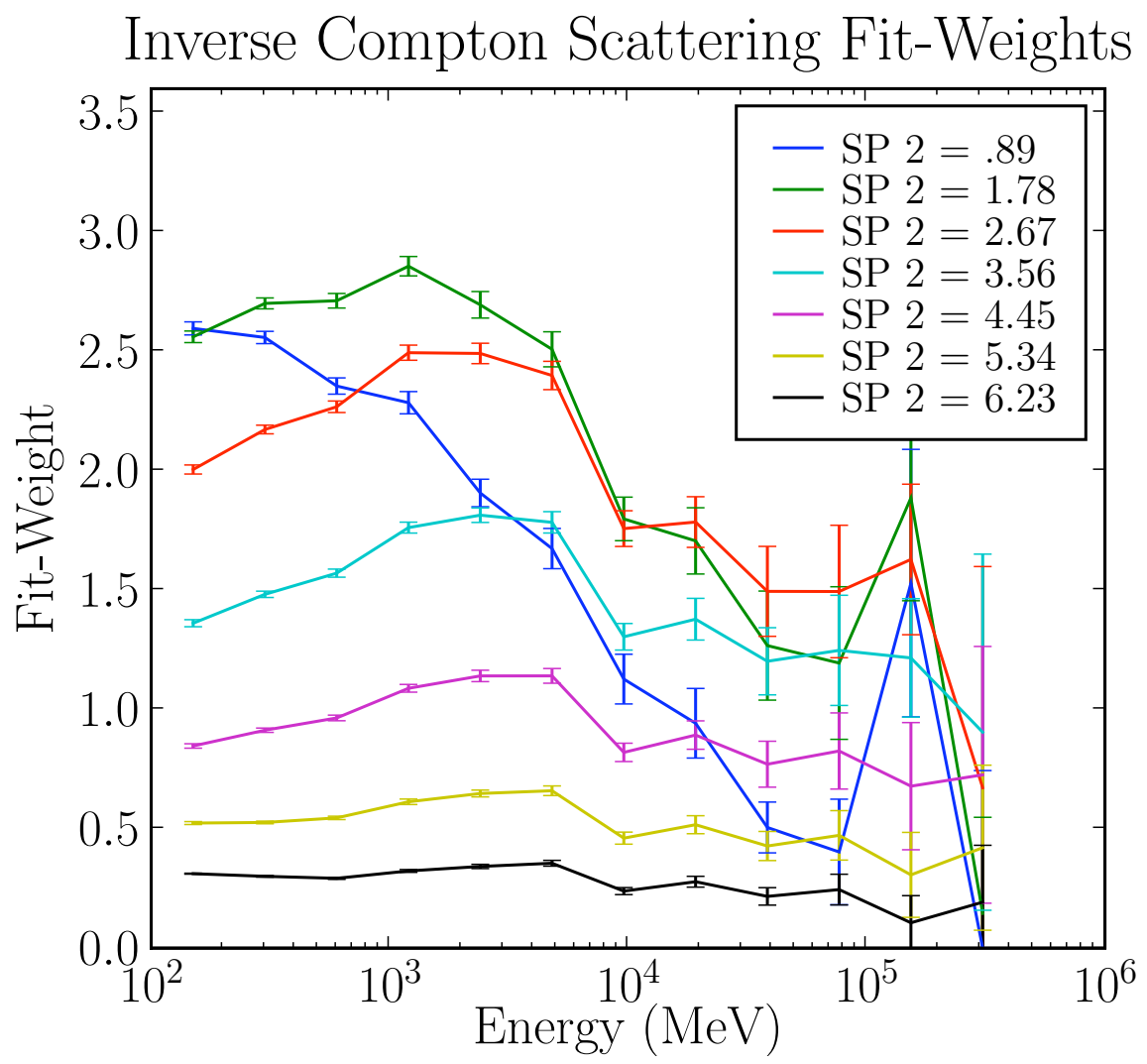

Figure 2: Fit-Weights of the Inverse-Compton Scattering component of the gamma-ray sky in models with differing source distribution as a function of energy. These fit-weights indicate the factor by which a given energy bin should by normalized to best fit Fermi observations. An optimized model would therefore have fit-weights close to 1 and would display no energy dependence. 


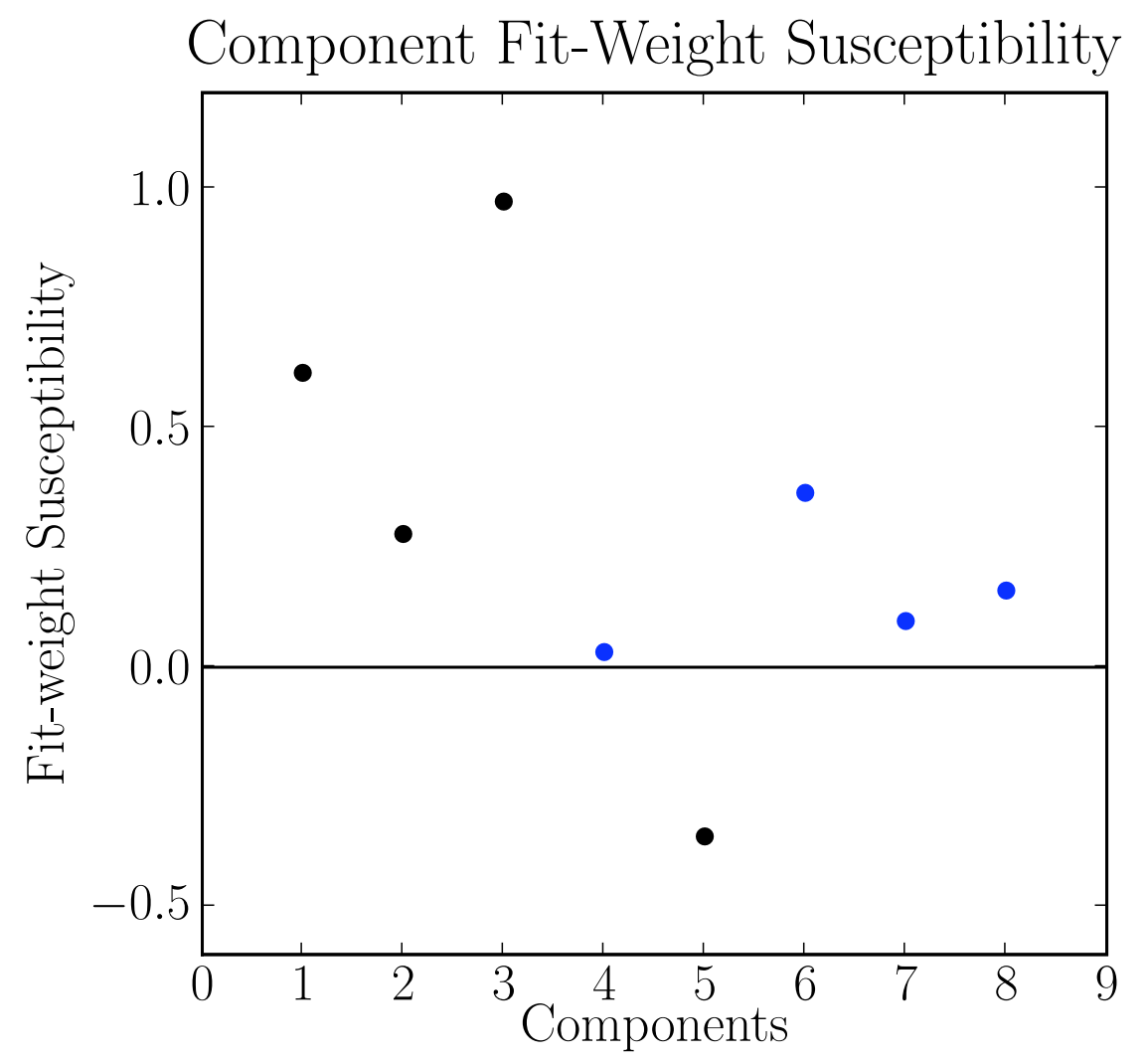

Figure 3: Component fit-weight susceptibility in models with decreasing interstellar gas density. The component numbers are as follows: 1-Atomic Hydrogen (Inner), 2-Atomic Hydrogen (Local), 3-Atomic Hydrogen (Outer), 4-Extragalactic Diffuse 5-Inverse-Compton Scattering, 5-Molecular Hydrogen (Inner), 6-Molecular Hydrogen (Local), 7-Molecular Hydrogen (Outer). Black data points indicate that the component changed in only the positive or negative direction when subjected to gas density variation, and blue data points indicate that the component both increased and decreased between models, and they represent the absolute value of the component variation. 


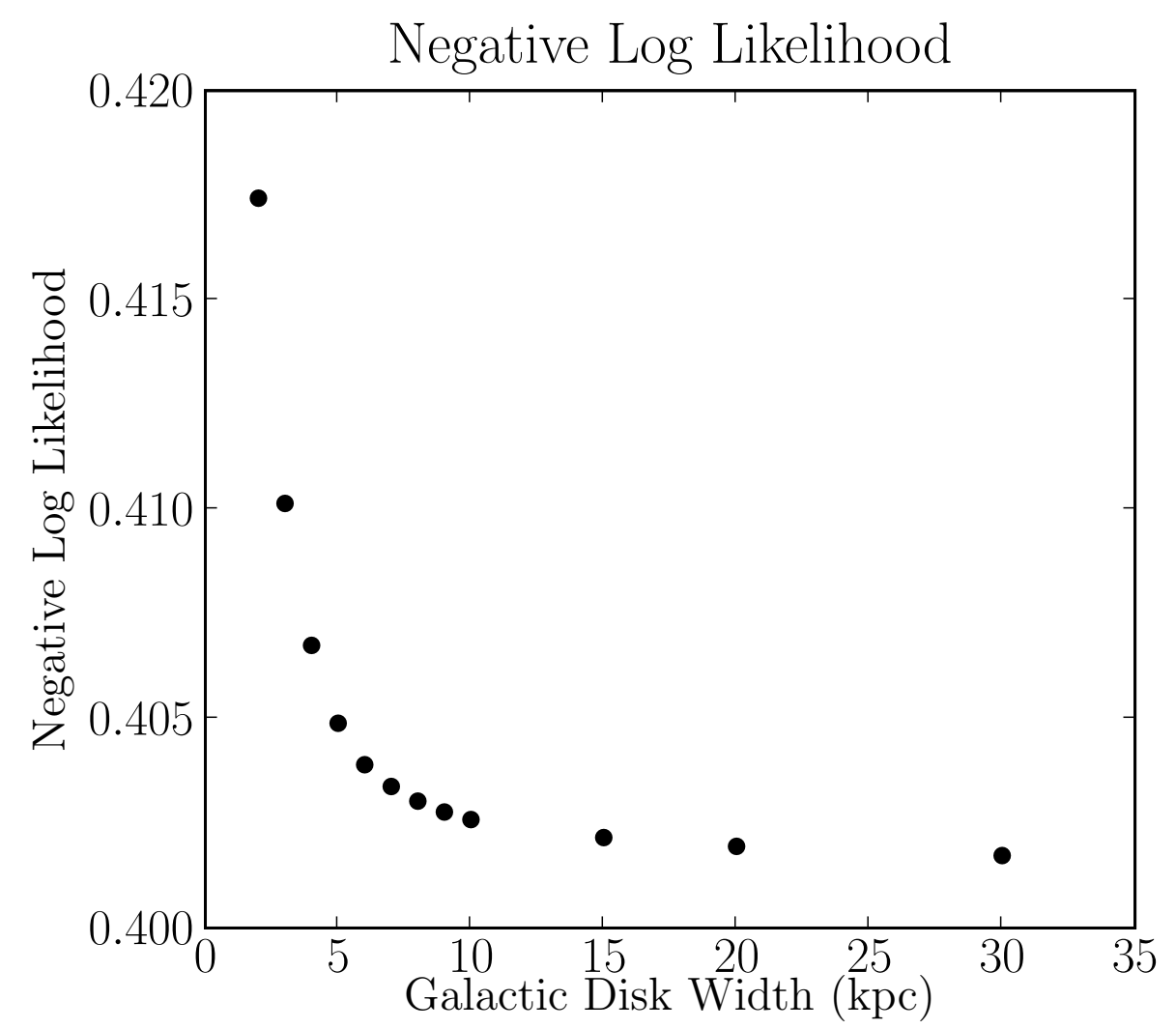

Figure 4: Negative Log of the Likelihood of Models with Increasing Galactic Disk Width. 


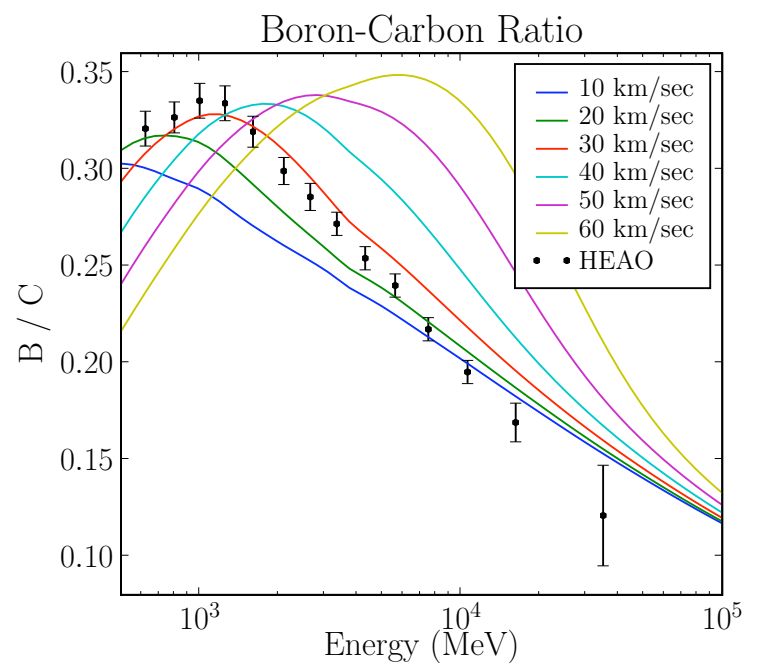

(a)

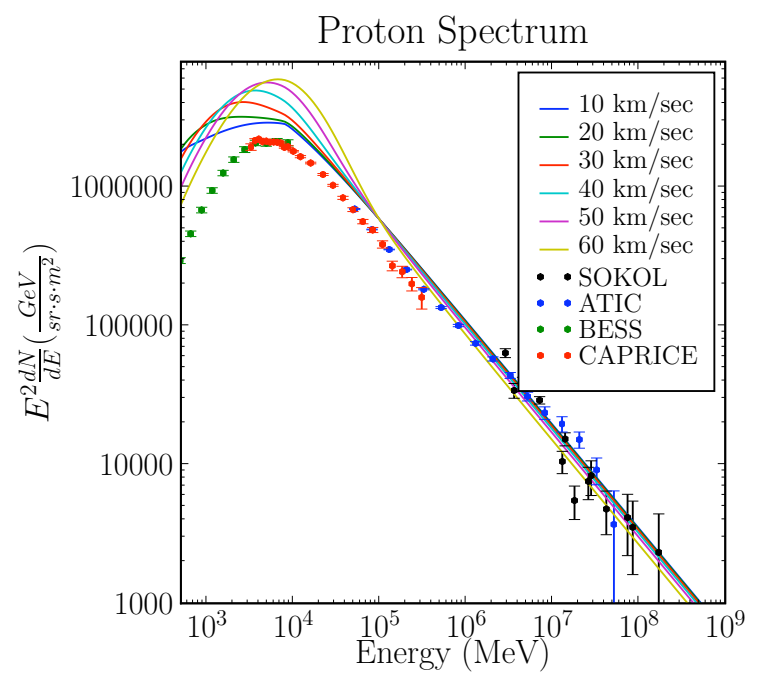

(c)

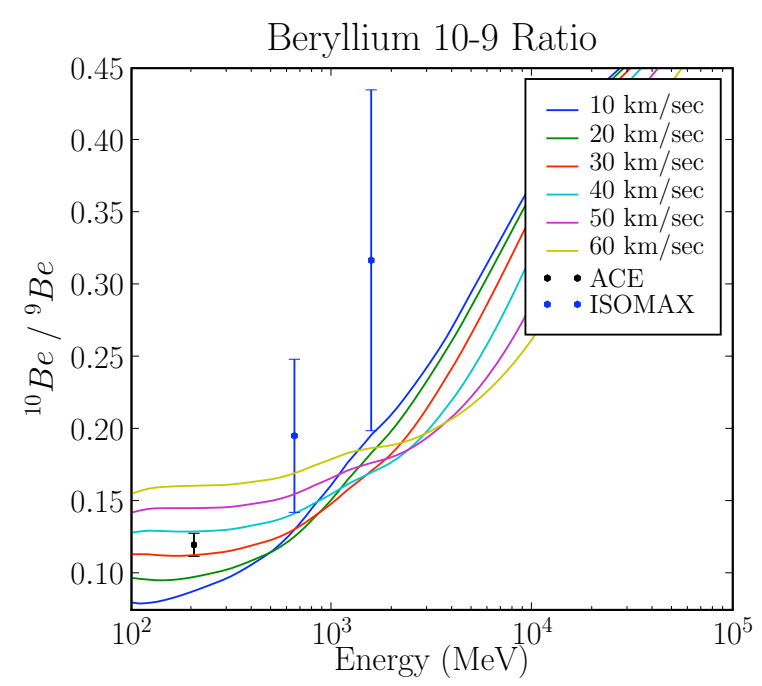

(b)

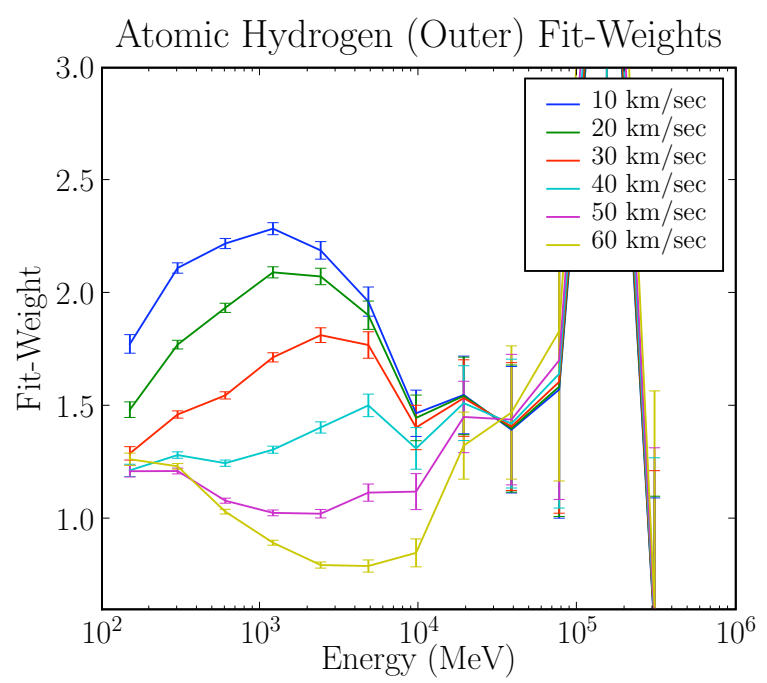

(d)

Figure 5: Various plots comparing the predictions of GALPROP models with increasing Alfven Velocity to observations. For a description of 5a and 5d see Figures 1 and 2. Figure 5b plots ${ }^{10} \mathrm{Be} /{ }^{9} \mathrm{Be}$ model predictions as a function of energy next to data from the Atmospheric Chemistry Experiment (ACE) and the Isotope Magnet Experiment (ISOMAX) [5]. Similarly, Figure 5c plots the predicted model proton spectra, which it then compares to measurements made by a range of experiments including the Advance Thin Ionization Calorimeter (ATIC) and the Cosmic AntiParticle Ring Imaging Cherenkov Experiment (CAPRICE) [5]. 


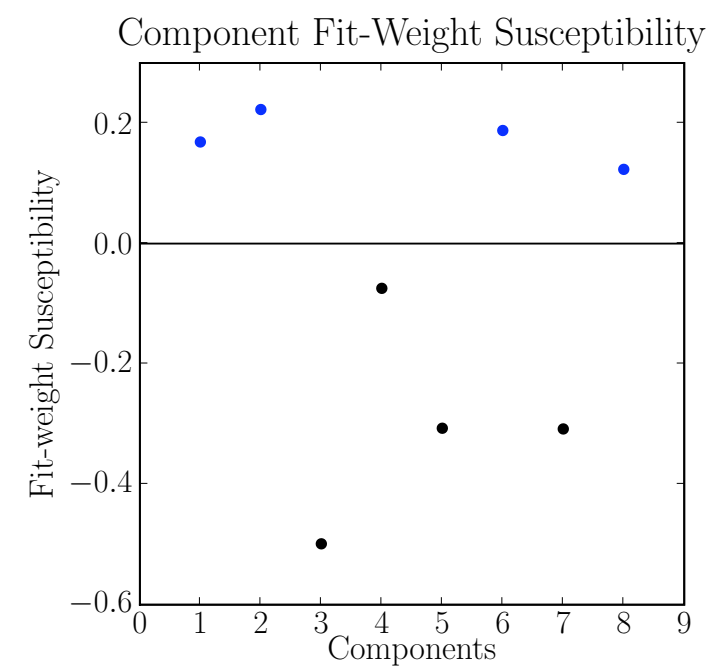

(a)

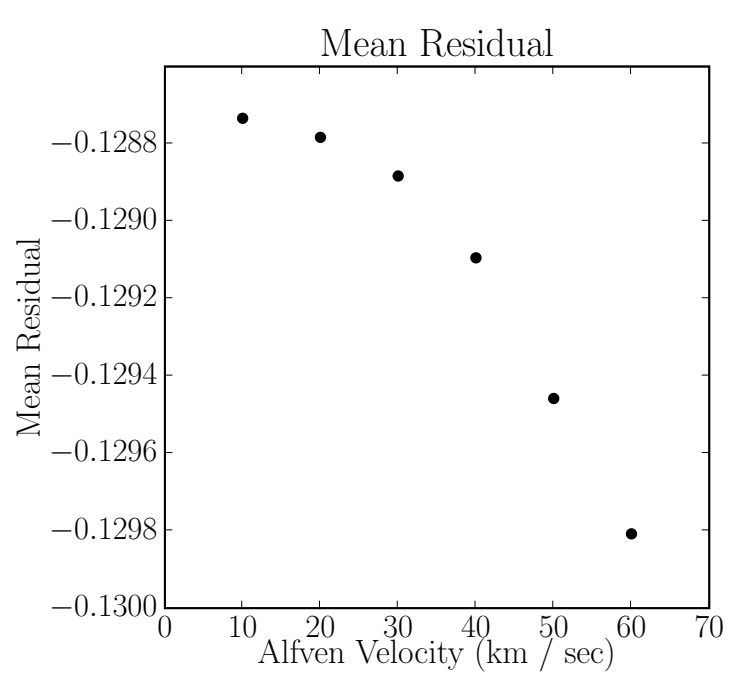

(c)

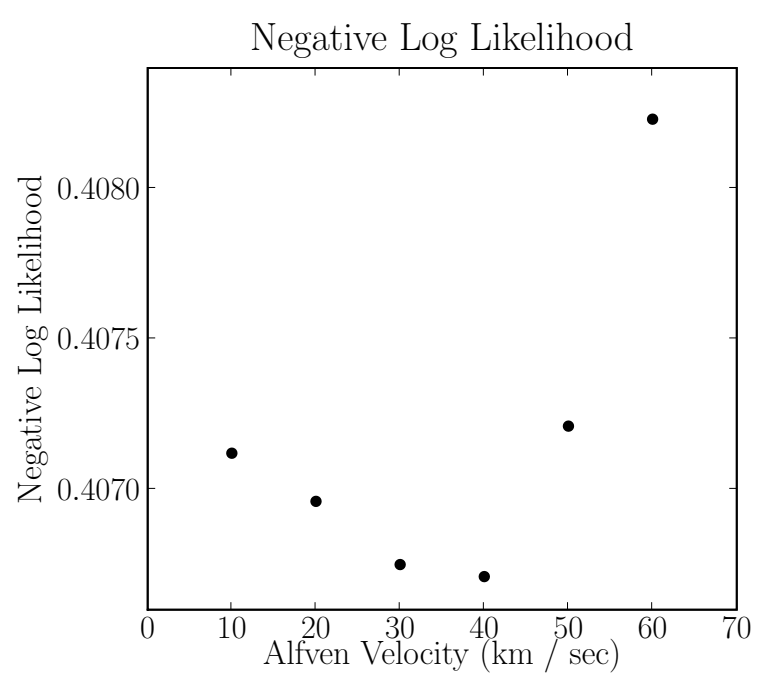

(b)

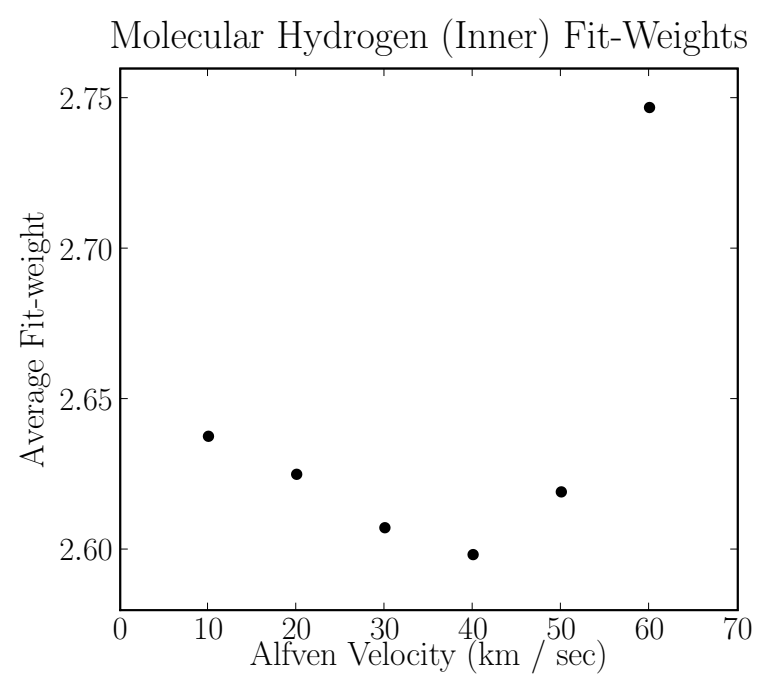

(d)

Figure 6: Additional plots comparing models with increasing Alfven Velocity. Descriptions of $6 \mathrm{a}$ and $6 \mathrm{~b}$ can be found under Figures 3 and 4 . Figure $6 \mathrm{c}$ plots the mean per-pixel residual for each model, where the residual is defined as the difference between the gamma-ray flux of the fit-weight adjusted sky map and the Fermi measurements. Since the predicted flux is as likely to be above the measured flux as below, the average of these values is expected to be near zero. Figure 6d displays the average fit-weights for the Molecular Hydrogen(Inner) component; as discussed in Figure 2, this value is optimized near zero. This type of plot makes the optimization of average fit-weight value more precise than plots such as 2 and $5 \mathrm{~d}$, and they are therefore used in conjunction. 


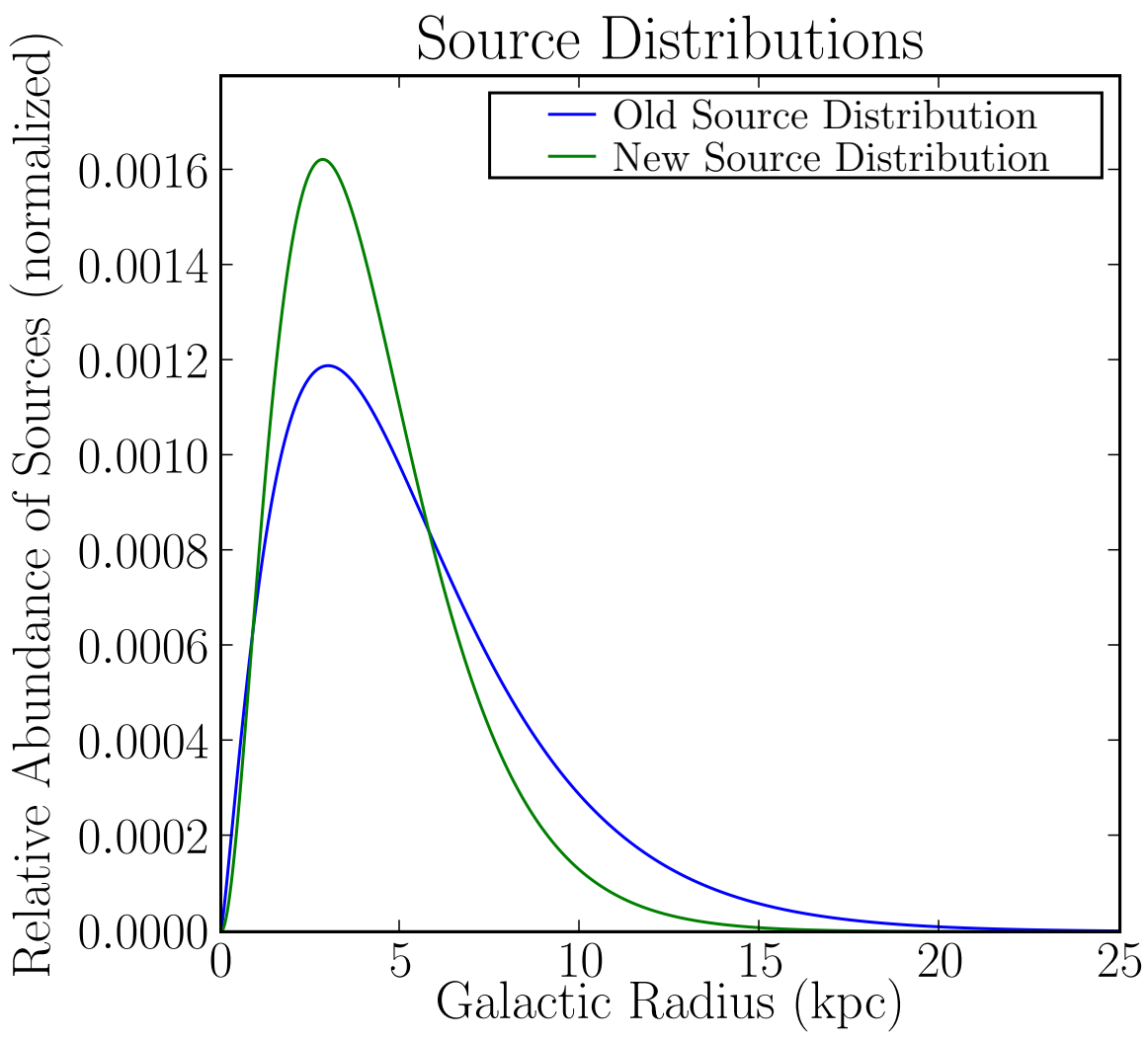

Figure 7: The relative cosmic-ray source distributions used by the template and optimized models. 


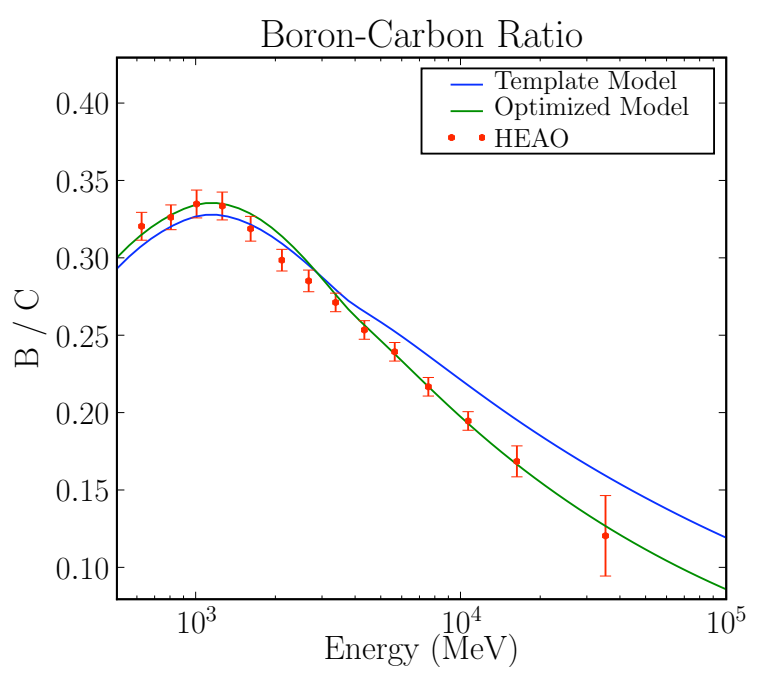

(a)

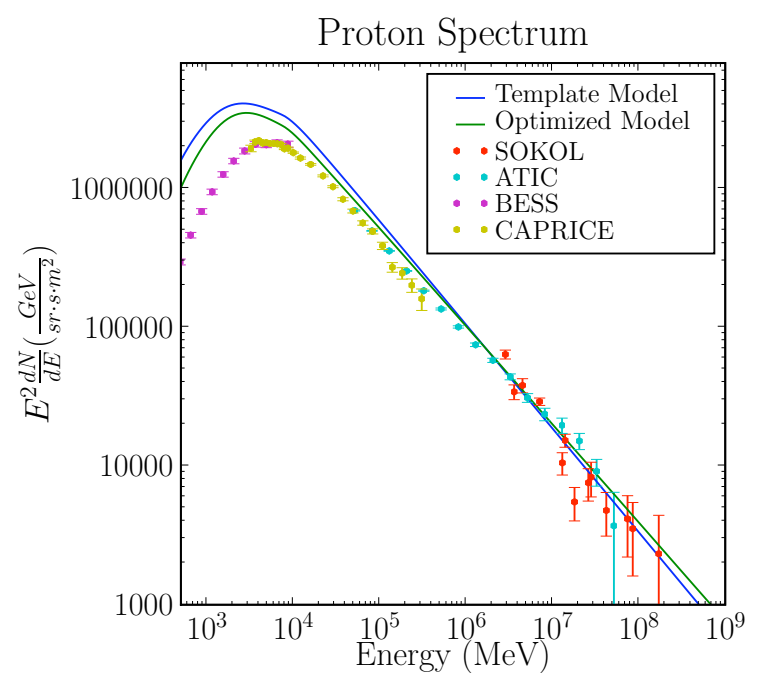

(c)

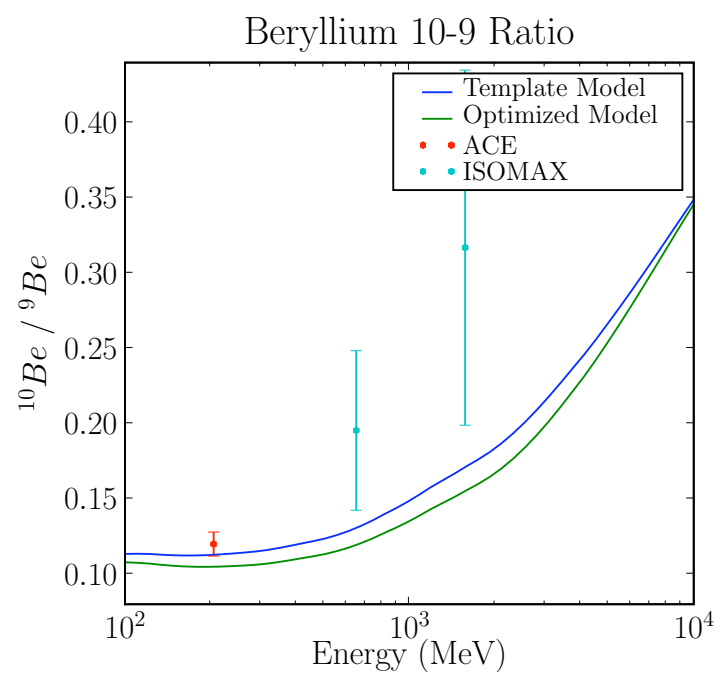

(b)

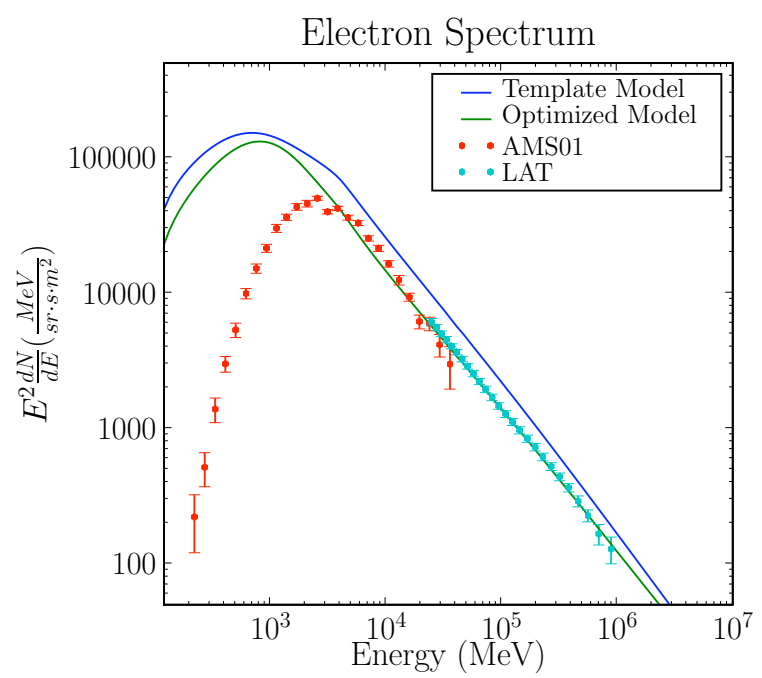

(d)

Figure 8: The predicted cosmic-ray spectra of the initial and newly optimized model, compared to measured values [5]. 


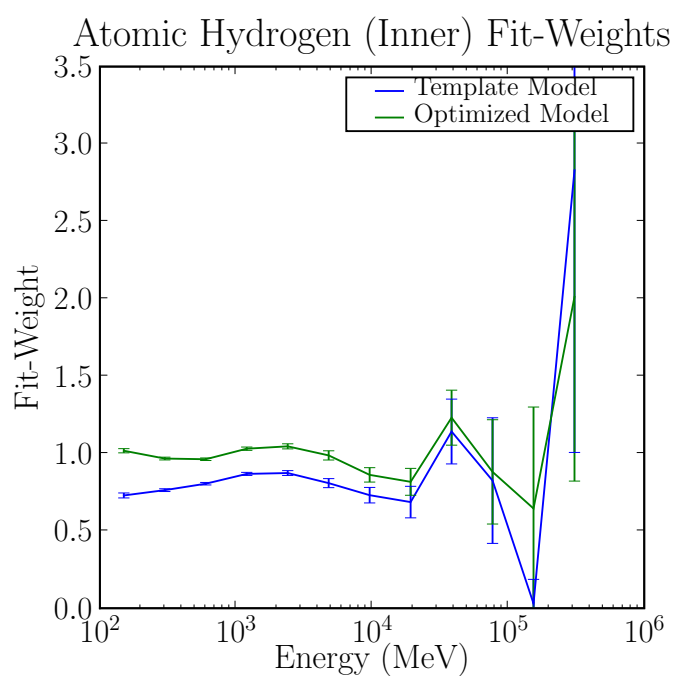

(a)

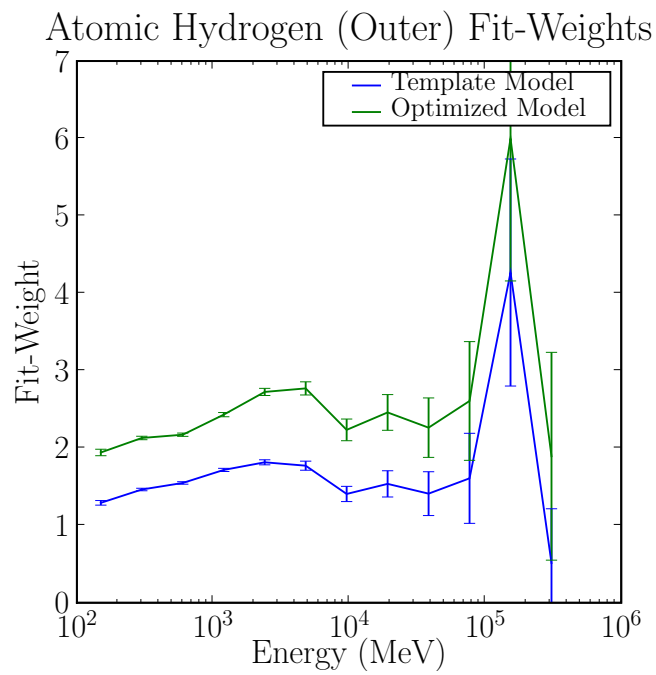

(c)

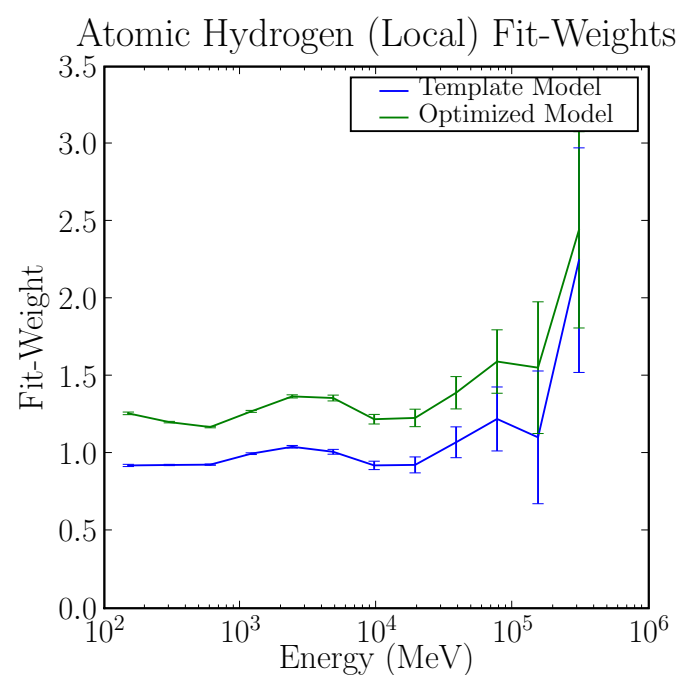

(b)

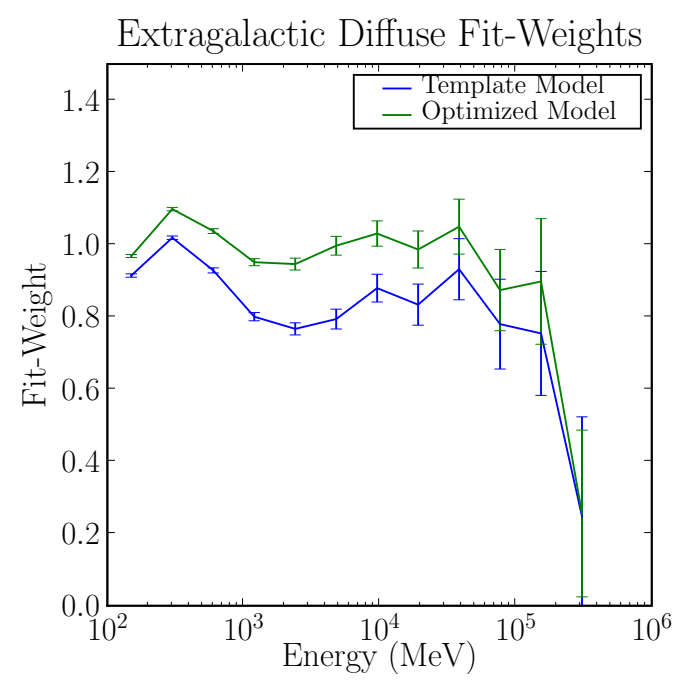

(d)

Figure 9: The Atomic Hydrogen and Extragalactic Diffuse fit-weights as a function of energy in the initial and newly optimized model. 


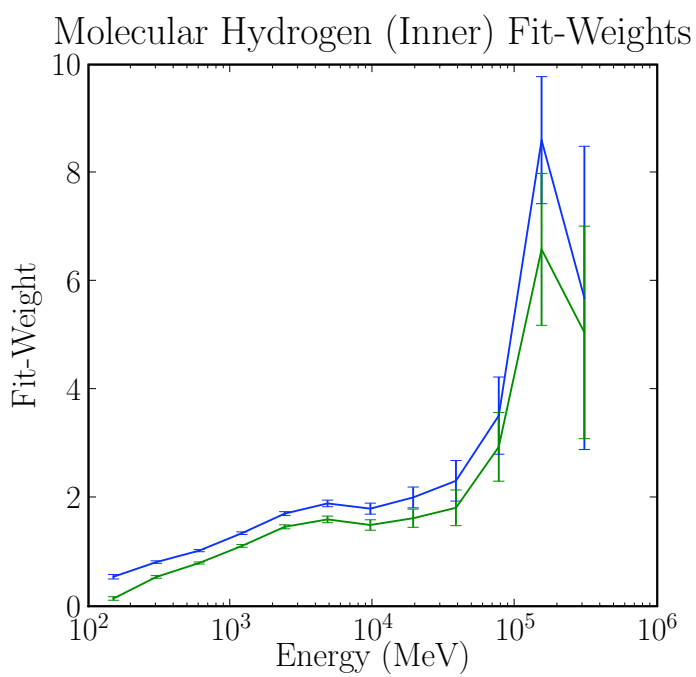

(a)

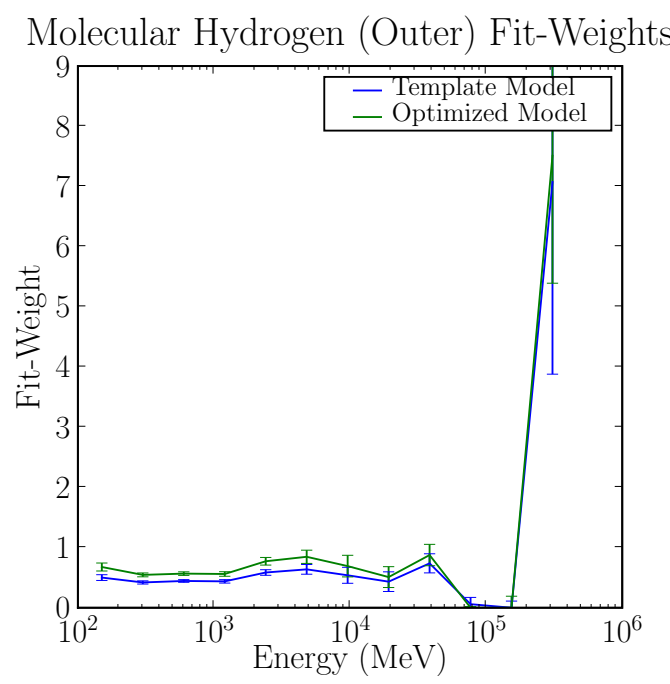

(c)

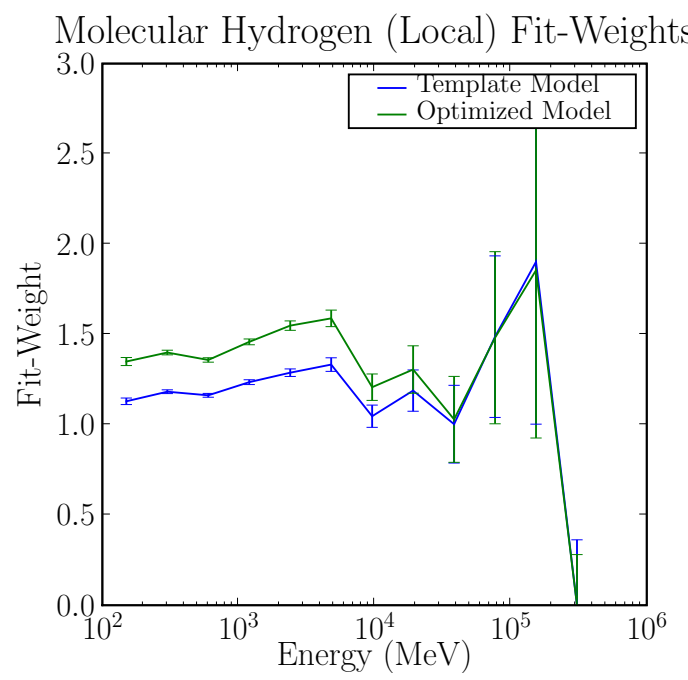

(b)

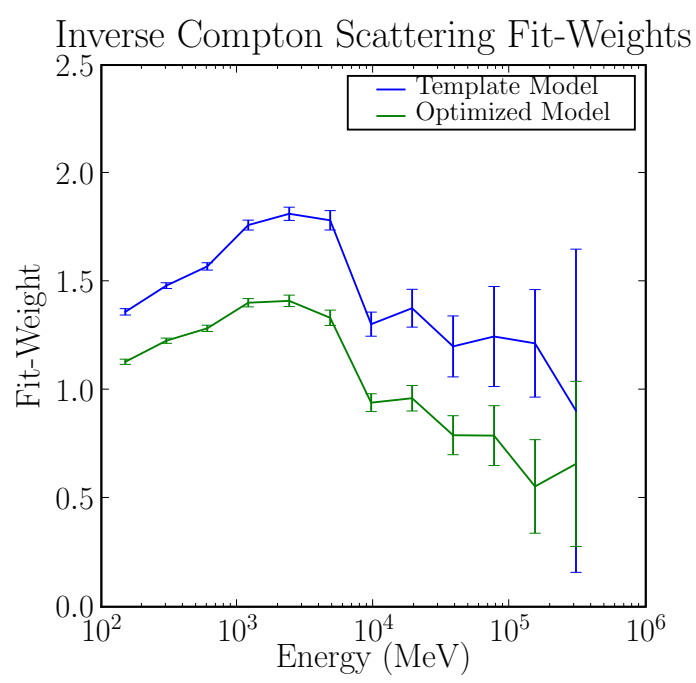

(d)

Figure 10: The Molecular Hydrogen and Inverse-Compton Scattering fit-weights as a function of energy in the initial and newly optimized model. 


\section{A Study of the Transverse Coherence Properties of the LCLS Samuel Ocko}

\section{Advisors: Zhirong Huang and Yuantao Ding}

(Dated: August 14, 2009) 


\begin{abstract}
The LINAC Coherent Light Source (LCLS), an X-Ray free-electron laser(FEL) based on the self amplified spontaneous emission principle, has recently come on-line. For many users it is desirable to have an idea of the level of transverse coherence of the X-Ray beam produced. In this paper, we analyze the output of GENESIS simulations of electrons traveling through the FEL. We first test the validity of an approach that ignores the details of how the beam was produced, and instead, by assuming a Gaussian-Schell model of transverse coherence, predicts the level of transverse coherence simply through looking at the beam radius at several longitudinal slices. We then develop a Markov chain Monte Carlo approach to calculating the degree of transverse coherence, which offers a $\sim 100$-fold speedup compared to the brute-force algorithm previously in use. We find the beam highly coherent. Using a similar Markov chain Monte Carlo approach, we estimate the reasonability of assuming the beam to have a Gaussian-Schell model of transverse coherence, with inconclusive results.
\end{abstract}




\section{Contents}

$\begin{array}{ll}\text { Abstract } & 2\end{array}$

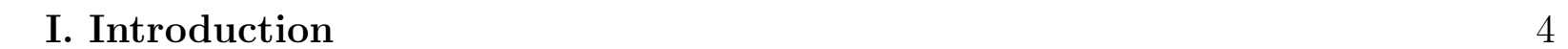

\begin{tabular}{|l|l}
\hline A. Transverse Coherence & 4
\end{tabular}

\begin{tabular}{ll}
\hline B. Mathematical Formulation & 5
\end{tabular}

\begin{tabular}{ll}
\hline C. The Gaussian-Schell model & 6
\end{tabular}

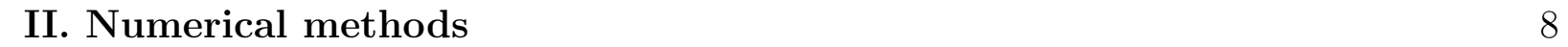

\begin{tabular}{ll}
\hline A. Curve Fitting & 8
\end{tabular}

1. Finding the beam radius 8

2. Fitting beam radius to the GSM

B. Calculating the Degree of Transverse Coherence 9

C. Estimating the Similarity of the Emitted Radiation to the GSM 10

1. Monte Carlo error estimation 11

\begin{tabular}{ll}
\hline D. Implementation & 12 \\
\hline
\end{tabular}

$\begin{array}{ll}\text { III. Results and Discussion } & 12\end{array}$

\begin{tabular}{ll}
\hline A. Curve Fitting & 12
\end{tabular}

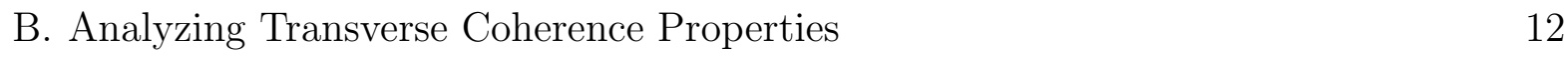

\begin{tabular}{ll}
\hline IV. Conclusion & 13
\end{tabular}

$\begin{array}{ll}\text { Acknowledgments } & 14\end{array}$

$\begin{array}{ll}\text { V. Figures } & 15\end{array}$

A. Fitting for Simulated FEL Having 84 Meters of Undulators 15

\begin{tabular}{|l|l}
\hline B. Analysis of Fields for FEL with 100 meters of undulators & 16
\end{tabular}

$\begin{array}{ll}\text { References } & 20\end{array}$ 


\section{INTRODUCTION}

The LINAC Coherent Light Source (LCLS) has recently come on-line. The LCLS is a free-electron laser (FEL) that works based on the self amplified spontaneous emission(SASE) principle! ${ }^{1}$ The LCLS can create coherent X-Ray radiation, which will be able to be used for imaging very small objects, such as single proteins. Since the radiation produced by the LCLS is highly coherent, objects can be imaged through looking at the interference patterns produced. This is often more powerful than simply looking at absorption patterns as in the case, for example, with a conventional microscope.

The level of transverse coherence of the LCLS tells us a lot about its imaging capabilities. Therefore, it is desirable to have an accurate idea of the level of transverse coherence produced by the LCLS. Many diagnostics are being proposed and run to measure the properties of the beam produced. Traditionally, transverse coherence properties can be measured through setting up a double slit experiment and measuring the position and visibility of fringes produced. However, the LCLS is producing X-Rays, with wavelengths of $\sim 1.5$ Angstroms, roughly the size an atom, so transverse coherence properties can not be easily measured through these standard techniques.

\section{A. Transverse Coherence}

Consider a flat surface of many light sources emitting monochromatic light of a wavelength much smaller than the size of the surface. We want to find how the light emitted depends on the level of coherence between the different light sources.

When the sources are in-phase and completely coherent, they will interfere to form a fairly straight beam pointed orthogonal to the surface. This is because when an observer is far away from the surface in the orthogonal direction, all of the sources are about the same distance apart and are in-phase. Therefore all sources positively interfere to create a high intensity orthogonal to the surface. However, if an observer is far away from the surface in a non-orthogonal direction, the observer is at a different distance from each source, therefore, most of the sources will destructively interfere. Therefore, the vast majority of the intensity will be concentrated in a coherent beam orthogonal to the surface, which will only be slightly divergent. 
When the multiple sources are completely incoherent, the different sources have a random phase relative to each other. Therefore, the light produced by each source will not interfere with the light produced by other sources, as the random phase difference between sources remains a random phase difference regardless of the phase factor from differing distances between the sources and the observer. Therefore, light will emanate from our set of sources equally in all directions. Note that this is just a fancy way of describing something very intuitive-light produced by bulbs arranged on a surface will not spontaneously interfere to create a coherent beam.

We see that a completely coherent set of in-phase sources on a plane produces a beam that diverges very little. However, when the sources are made completely incoherent, they produce no beam at all, instead radiating equally in all directions. Viewing a continuous source as an infinite array of infinitesimally small point sources, we see that the level of transverse coherence will determine how quickly the resultant beam will diverge. A highly coherent source will produce a slightly divergent beam; a low-coherence source will produce a much more divergent beam. Armed with this notion, we now get a little more mathematical.

\section{B. Mathematical Formulation}

Many of the statistical properties of light can be described by the mutual coherence function.2[3

$$
\Gamma\left(\mathbf{r}_{1}, \mathbf{r}_{2}, \tau\right)=\left\langle E\left(\mathbf{r}_{1}, t\right) E^{*}\left(\mathbf{r}_{2}, t+\tau\right)\right\rangle
$$

Where $\mathbf{r}_{1}$ and $\mathbf{r}_{2}$ are positions, and $E(\mathbf{r}, t)$ is the complex-valued electric field at position $\mathbf{r}$ and time $t$.

We can also consider the coherency between two points limited to a single frequency basis, which is called the cross-spectral density function.

$$
W\left(\mathbf{r}_{1}, \mathbf{r}_{2}, \omega\right)=\int_{-\infty}^{\infty} \Gamma\left(\mathbf{r}_{1}, \mathbf{r}_{2}, \tau\right) e^{-i \omega \tau} \delta \tau
$$

We also care about the mutual intensity function

$$
J\left(\mathbf{r}_{1}, \mathbf{r}_{2}\right)=\Gamma\left(\mathbf{r}_{1}, \mathbf{r}_{2}, 0\right)
$$


When we are dealing with light that is close to monochromatic, we can assume that all of the light is close to the frequency $\omega_{0}$, and we make the assumption:

$$
J\left(\mathbf{r}_{1}, \mathbf{r}_{2}\right) \propto W\left(\mathbf{r}_{1}, \mathbf{r}_{2}, \omega_{0}\right)
$$

\section{The Gaussian-Schell model}

The Gaussian-Schell model is a model of transverse coherence of a beam moving along the $\mathrm{z}$-axis. At $z=0$, the source point of the beam,

$$
W\left(\mathbf{r}_{1}, \mathbf{r}_{2}, z=0, \omega=\omega_{0}\right)=A^{2} \exp \left[-\frac{\left(\mathbf{r}_{1}^{2}+\mathbf{r}_{2}^{2}\right)}{4 \sigma_{S}^{2}}\right] \exp \left[-\frac{\left(\mathbf{r}_{1}-\mathbf{r}_{2}\right)^{2}}{2 \sigma_{G}^{2}}\right]
$$

$A$ is the amplitude

$\mathbf{r}_{2}, \mathbf{r}_{2}$ are two-dimensional vectors representing transverse positions

$\sigma_{S}$ is equal to the beam radius at the source, $\sigma_{G}$ is equal to the transverse coherence length at the source

We are making a quasi-monocromatic approximation, $J \propto W\left(\omega_{0}\right)$ so we can say:

$$
J\left(\mathbf{r}_{1}, \mathbf{r}_{2}, z=0\right) \propto \exp \left[-\frac{\left(\mathbf{r}_{1}^{2}+\mathbf{r}_{2}^{2}\right)}{4 \sigma_{S}^{2}}\right] \exp \left[-\frac{\left(\mathbf{r}_{1}-\mathbf{r}_{2}\right)^{2}}{2 \sigma_{G}^{2}}\right]
$$

We can see that the intensity at the source, $I \propto J(\mathbf{r}, \mathbf{r}, z=0) \propto \exp \left[\frac{-\mathbf{r}^{2}}{2 \sigma_{S}}\right]$, so at the source, there is a beam of Gaussian intensity, where $\sigma_{S}$ is the beam radius.

The correlation coefficient between the field at any two points is

$$
\frac{J\left(\mathbf{r}_{1}, \mathbf{r}_{2}\right)}{\left(\sqrt{J\left(\mathbf{r}_{1}, \mathbf{r}_{1}\right) J\left(\mathbf{r}_{2}, \mathbf{r}_{2}\right)}\right)}=\exp \left[-\frac{\left(\mathbf{r}_{1}-\mathbf{r}_{2}\right)^{2}}{2 \sigma_{G}}\right]
$$

We can see that the correlation coefficient decays as a Gaussian with the distance between $\mathbf{r}_{1}$ and $\mathbf{r}_{2}$, where $\sigma_{G}$ is the transverse coherence length

Now we want to see what the properties of the beam are away from the source point. At points away from the source, $z>0$, the beam propagates as:

$$
J\left(\mathbf{r}_{1}, \mathbf{r}_{2}, z\right) \propto \frac{1}{\Delta(z)^{2}} \exp \left[-\frac{\left(\mathbf{r}_{1}^{2}+\mathbf{r}_{2}^{2}\right)}{4 \sigma_{S}^{2}[\Delta(z)]^{2}}\right] \exp \left[-\frac{\left(\mathbf{r}_{1}-\mathbf{r}_{2}\right)^{2}}{2 \sigma_{G}^{2}[\Delta(z)]^{2}}\right] \exp \left[-\frac{i k\left(\mathbf{r}_{1}^{2}-\mathbf{r}_{2}^{2}\right)}{2 R(z)}\right]
$$

where $\Delta(z)$ is the expansion coefficient

$$
\Delta(z)=\sqrt{1+\left(z / k \sigma_{S} \delta\right)^{2}}
$$




$$
\begin{aligned}
\frac{1}{\delta^{2}} & =\frac{1}{\left(2 \sigma_{S}\right)^{2}}+\frac{1}{\sigma_{G}^{2}} \\
R(z) & =z\left[1+\left(\frac{k \sigma_{S} \delta}{z}\right)^{2}\right]
\end{aligned}
$$

where $k$ is the wavenumber of the frequency $\omega_{0}$.

The first exponential term is related to the beam radius, as it is the only term that doesn't go to 1 as $\mathbf{r}_{1} \rightarrow \mathbf{r}_{2}$. The second term is related to the transverse coherence length, whose magnitude scales as a Gaussian of the distance between $\mathbf{r}_{1}$ and $\mathbf{r}_{2}$. The third term is a phase difference due to the slightly different lengths of $\mathbf{r}_{1}$ and $\mathbf{r}_{2}$ from the source. We can see that the both beam radius and the transverse coherence length increase proportionally to the expansion coefficient, $\Delta(z)$.

The degree of transverse coherence is:

$$
\begin{gathered}
\zeta(z)=\frac{\left.\int\left|J_{12}\right|^{2} \delta \mathbf{r}_{1} \delta \mathbf{r}_{2}\right)}{\left(\int J_{11} \delta \mathbf{r}_{1}\right)^{2}} \\
J\left(\mathbf{r}_{1}, \mathbf{r}_{2}, z\right)=\left\langle E\left(\mathbf{r}_{1}, z\right) E^{*}\left(\mathbf{r}_{2}, z\right)\right\rangle
\end{gathered}
$$

Where $J_{12}=J\left(\mathbf{r}_{1}, \mathbf{r}_{2}, z\right)$, and $J_{11}=J\left(\mathbf{r}_{1}, \mathbf{r}_{1}, z\right)$

For the GSM, evaluating the degree of transverse coherence analytically shows that it depends on the ratio between the beam radius and the transverse coherence length, and is independent of $z$

$$
\zeta_{G S M}=\frac{\sigma_{G}^{2}}{\sigma_{G}^{2}+\left(2 \sigma_{S}\right)^{2}}
$$

The appeal of assuming a GSM is that we can predict all coherence properties through simply measuring the beam radius for several values of z (Possible experimentally or through a computer simulation) without ever considering the details of how the beam was actually produced. Then, through knowing the expansion coefficient, we can calculate all transverse coherence properties of the beam produced. The question is, of course, whether the actual beam properties are close enough to the GSM to make this approximation reasonable. Another issue when assuming a GSM for an FEL is where to assume the beam source to be; unlike in a conventional source, the radiation from a FEL originates at all points within the undulators. 


\section{NUMERICAL METHODS}

\section{A. Curve Fitting}

\section{Finding the beam radius}

One way we can find the beam radius is by finding the root-mean-square of the beam intensity.

$$
\text { Beam Width }^{2}=\frac{1}{2} \frac{\int J(\mathbf{r}, \mathbf{r}) \mathbf{r}^{2}}{\text { Power }^{2}}
$$

The factor of $\frac{1}{2}$ is because we are integrating over a two-dimensional vector.

Another way we can find the beam radius is through fitting. Since both the simulated beam intensity profile and a Gaussian beam intensity profile are functions over the same space, we can treat them as vectors, and try to minimize the angle between them.

$$
\cos (\theta)=\frac{I_{\text {Measured }} \cdot I_{\text {Gaussian }}}{\left|I_{\text {Measured }}\right|\left|I_{\text {Gaussian }}\right|}
$$

This is equivalent to maximizing the quantity,

$$
\frac{\left(I_{\text {Measured }} \cdot I_{\text {Gaussian }}\right)^{2}}{\left|I_{\text {Measured }}\right|^{2}\left|I_{\text {Gaussian }}\right|^{2}}
$$

\section{Fitting beam radius to the GSM}

If we know the location of the beam source, then all we need to know is $\delta$ and $\sigma_{S}$ to know all of the properties of the beam. Therefore, we assume a source point, which is a somewhat unsafe assumption, as the radiation produced comes from the total length of the undulators. Having assumed a source point, we assume $\sigma_{S}$ to be the beam radius at the source point. We can then simply fit $\delta$ with a least-squares fit. This approach has the advantage in that it can be done simply through looking at the beam radius at various points without ever considering the details of how the beam was produced. The same approach was used to model the properties of FLASH(Free electron LASer at Hamburg), a FEL at DESY operating under the SASE principle. 4 


\section{B. Calculating the Degree of Transverse Coherence}

Using data produced by the GENESIS $\operatorname{cod} e^{5}$, which performs simulations of electrons traveling through the undulators, we can measure the electric field at all points in time at every position along the z-axis. Since in a simulation, unlike in experiment, we can observe everything, we can make calculations we could not otherwise make.

One quantity of interest is the degree of transverse coherence, which is

$$
\zeta(z)=\frac{\iint\left|J_{12}\right|^{2} \delta \mathbf{r}_{1} \delta \mathbf{r}_{2}}{\left(\int J_{11} \delta \mathbf{r}_{1}\right)^{2}}
$$

where $J_{12}=J\left(\mathbf{r}_{1}, \mathbf{r}_{2}, z\right), J_{11}=J\left(\mathbf{r}_{1}, \mathbf{r}_{1}, z\right)$.

Note that $J_{11}$ is equal to the intensity at $\mathbf{r}_{1}$; Therefore, $\int J_{11} \delta \mathbf{r}_{1} \propto$ Beam Power. We can replace the term in the denominator with the total power squared, giving us:

$$
\zeta=\frac{\iint\left|J_{12}\right|^{2} \delta \mathbf{r}_{1} \delta \mathbf{r}_{2}}{\text { Power }^{2}}
$$

$\zeta$ is difficult to calculate explicitly, as the integration area is very large. To do this, we need to integrate over all pairs of points $\left(\mathbf{r}_{1}, \mathbf{r}_{2}\right)$ along the axis. For example, if your electric field is 100 cells wide, then you have 10,000 values of $\mathbf{r}$. Therefore, you have to calculate $10,000^{2}=100$ million quantities for $J_{12}$ (One for each pair $\left.\left(\mathbf{r}_{1}, \mathbf{r}_{2}\right)\right)$ : Note-You can get it down to 50 million using the Hermitian propertiy of the $J$ matrix, as $J\left(\mathbf{r}_{1}, \mathbf{r}_{2}\right)=J\left(\mathbf{r}_{2}, \mathbf{r}_{1}\right)^{*}$, but it's still a fairly unwieldy calculation.

Fortunately, we can make the degree of transverse coherence susceptible to Markov chain Monte Carlo techniques by rewriting the degree of transverse coherence as:

$$
\zeta(z)=\int \frac{\left|J_{12}\right|^{2}}{\text { Power }^{2}}=\int \frac{\left|J_{12}\right|^{2}}{J_{11} J_{22}} \frac{J_{11} J_{22}}{\text { Power }^{2}}=\int f\left(\mathbf{r}_{1}, \mathbf{r}_{2}\right) P\left(\mathbf{r}_{1}, \mathbf{r}_{2}\right)
$$

Where $P\left(\mathbf{r}_{1}, \mathbf{r}_{2}\right)=\frac{J_{11} J_{22}}{\text { Power }^{2}}$ and

$f\left(\mathbf{r}_{1}, \mathbf{r}_{2}\right)=\frac{\left|J_{12}\right|^{2}}{J_{11} J_{22}}$

We can treat $P\left(\mathbf{r}_{1}, \mathbf{r}_{2}\right)$ as a probability, as $\int\left(P\left(\mathbf{r}_{1}, \mathbf{r}_{2}\right)\right)=\frac{\int J_{11} \int J_{22}}{\text { Power }^{2}}=\frac{\text { Power }^{2}}{\text { Power }^{2}}=1$. Therefore, we can estimate it by sampling $f\left(\mathbf{r}_{1}, \mathbf{r}_{2}\right)$ with probability $P\left(\mathbf{r}_{1}, \mathbf{r}_{2}\right)$, which we can do through using a random weighted walk through the state space. In this case, a state $\alpha$ represents a pair of transverse points $\left(\mathbf{r}_{1}, \mathbf{r}_{2}\right)$. 
We use a weighted random walk through the state space. Each state $\alpha$ has a certain probability distribution of states $\alpha^{\prime}$ it can transition to during each iteration. The pseudocode for the algorithm is straightforward and is shown below:

$$
\begin{aligned}
& \text { State } \alpha \\
& \text { State } \alpha^{\prime}=\operatorname{randomNeighboringState}(\alpha) \\
& \text { if }\left(\operatorname{rand}()<\frac{\operatorname{Probability}\left(\alpha^{\prime}\right)}{\operatorname{Probability}(\alpha)}\right) \\
& \quad \alpha \leftarrow \alpha^{\prime}
\end{aligned}
$$$$
\text { Sample } \mathrm{f}(\alpha)
$$

where rand() returns a random number from zero to 1.

It can be proven that, as long as, for all $\left(\alpha_{1}, \alpha_{2}\right)$, the probability of $\alpha_{2}$ being proposed as a transition from $\alpha_{1}$ is equal to the probability of the opposite, the correct probability distribution is generated by this set of transition probabilities. Markov chain Monte Carlo algorithms have the significant advantage over other Monte Carlo algorithms in that most of the time is spent sampling from the most important parts of the summation. This makes a Monte Carlo approach faster.

Initial states of the random walk are determined through rejection sampling, where we propose states with uniform probability, and then only accept them with probability proportional to $P(\alpha)$. This algorithm will return the correct distribution of $\alpha$. Since for our implementation, $P(\alpha)=P\left(\mathbf{r}_{1}\right) P\left(\mathbf{r}_{2}\right)$, values of $\mathbf{r}_{1}$ and $\mathbf{r}_{2}$ can be generated independently from each other, speeding up the algorithm significantly.

\section{Estimating the Similarity of the Emitted Radiation to the GSM}

One way to see how well our model fits is to estimate the difference between $J(\alpha)$ and $G(\alpha)$. In doing so, we neglect the phase term of the GSM, as it depends on the source point, which we don't exactly know. This might not be a safe assumption.

Since both $J(\alpha)$ (measured from simulation data) and $G(\alpha)$ (our GSM based on the cal-

culated beam radius and measured degree of transverse coherence) are functions over the same space, we can treat them both as vectors and find the "angle" between the two. 


$$
\begin{gathered}
\cos (\theta)=\frac{J \cdot G}{|J||G|} \\
\cos (\theta)^{2}=\frac{(J \cdot G)^{2}}{(J \cdot J)(G \cdot G)} \\
J \cdot G=\int_{\alpha} J(\alpha) G^{*}(\alpha) \\
J \cdot G=\int_{\alpha} \frac{J(\alpha) G^{*}(\alpha)}{J_{11}(\alpha) J_{22}(\alpha)} J_{11}(\alpha) J_{22}(\alpha)=\operatorname{Power}^{2} \int_{\alpha} \frac{J(\alpha) G^{*}(\alpha)}{J_{11}(\alpha) J_{22}(\alpha)} \frac{J_{11}(\alpha) J_{22}(\alpha)}{\operatorname{Power}^{2}} \\
=\operatorname{Power}^{2} \int_{\alpha} f_{J G}(\alpha) P(\alpha)
\end{gathered}
$$

Where $f_{J G}(\alpha)=\frac{J(\alpha) G^{*}(\alpha)}{J_{11}(\alpha) J_{22}(\alpha)}$, and $P(\alpha)$ is still $\frac{J_{11}(\alpha) J_{22}(\alpha)}{\text { Power }^{2}}$

We use the same scheme for $J \cdot J$ and $G \cdot G$ :

$$
\begin{gathered}
J \cdot J=\text { Power }^{2} \int_{\alpha} P(\alpha) f_{J J}(\alpha), \text { where } f_{J J}(\alpha)=\frac{|J(\alpha)|^{2}}{J_{11}(\alpha) J_{22}(\alpha)} \\
G \cdot G=\text { Power }^{2} \int_{\alpha} P(\alpha) f_{G G}(\alpha), \text { where } f_{G G}(\alpha)=\frac{|G(\alpha)|^{2}}{J_{11}(\alpha) J_{22}(\alpha)} \\
\cos (\theta)^{2}=\frac{(J \cdot G)^{2}}{(J \cdot J)(G \cdot G)}=\frac{\left(\text { Power }^{2} \int_{\alpha} P(\alpha) f_{J G}(\alpha)\right)^{2}}{\text { Power }^{2} \int_{\alpha} P(\alpha) f_{J J}(\alpha) \operatorname{Power}^{2} \int_{\alpha} P(\alpha) f_{G G}(\alpha)} \\
=\frac{\left(\int_{\alpha} P(\alpha) f_{J G}(\alpha)\right)^{2}}{\int_{\alpha} P(\alpha) f_{J J}(\alpha) \int_{\alpha} P(\alpha) f_{G G}(\alpha)}
\end{gathered}
$$

We estimate each of the three quantities $(J \cdot G, J \cdot J$ and $G \cdot G)$ using the same Markov chain. By doing this, we cause the uncertainties in each measurement to be correlated to each other, significantly reducing the error.

\section{Monte Carlo error estimation}

We estimate $J \cdot G, J \cdot J$ and $G \cdot G$ using the same set of statistically independent Markov Chains. We have enough statistically independent branches (400) to provide a good estimate of the distribution of measured $(J \cdot G, J \cdot J, G \cdot G)$. Therefore, what we do is randomly select $\mathrm{N}$ estimations out of our N Markov chains (Some being selected multiple times and some not at all). This will give us an estimate of the distribution of calculations we were 
likely to make. Therefore, from doing this multiple times, we can find the error bar of our calculation. This is a much more straightforward method than explicitly calculating all of the standard deviations and correlations between the three quantities estimated.

\section{Implementation}

I implemented the algorithms in MATLAB. All random walks were executed in parallel using MATLAB's array functionality.

\section{RESULTS AND DISCUSSION}

\section{A. Curve Fitting}

Having 84 meters of undulators, we first naively assume the source of the GSM to be at the end of the undulators, and fit the measured growth of the beam radius to that predicted by the GSM. [Fig. 1]

This doesn't work very well at all. The expansion coefficient of the GSM starts off with a quadratic regime and then goes to a linear regime. However, the beam radius increases fairly linearly, which causes a poor fit. We therefore assume the source to be located inside the undulators at 64 meters, which is approximately where saturation is reached and the beam power plateaus. [Fig. 2]]. This gives us somewhat better results, but still leaves much to be desired.

\section{B. Analyzing Transverse Coherence Properties}

The Markov chain Monte Carlo approach works about 100 times faster than the brute force algorithm, and converges to within a reasonable error bar within 45 seconds. This is much better than the hour and a half runtime that the brute-force algorithm takes[Fig. 4] , and will be a very useful for analyzing future simulations.

Analyzing the degree of transverse coherence[Fig. 5], we can see that the degree of transverse coherence peaks at approximately 70 meters, which is about the same point where the beam power plateaus. Transverse coherence peaks at almost $90 \%$, which is very 
good. We can either estimate the beam radius as the (root mean square) rms of intensity, or by fitting the intensity profile to a Gaussian. We compare the agreement between these two approaches. [Fig. 6] We see that we get a somewhat better agreement when we find the beam radius through fitting rather than through a rms calculation. Finding a value of the beam radius through fitting gives a somewhat smaller value [Fig. 7]. This is because the intensity profile is mostly Gaussian, but has a non-Gaussian "Halo", which "contaminates" the rms calculation of the beam radius. Knowing the beam radius (both found through rms and fitting) and the degree of transverse coherence, we can propose a GSM and see how well the mutual intensity function $J$ fits the predictions of the GSM. We ignore the phase factor of the GSM, as it depends on the location of the source. [Fig. 8].

This is somewhat close, but certainly not close enough to consider the GSM to be the end-all model for transverse coherence of the LCLS. To see how much better the agreement might be if we were to have picked the right source point which produced the right phase factor, we test the agreement between the magnitude of the GSM and the magnitude of the mutual intensity function [Fig. 9] ]. This provides an upper-bound on any possible agreement, as different phase factors between the two can only make the agreement worse. The agreement in this case is extremely good at some points, which suggests that the GSM might still be the correct model of transverse coherence if we chose a reasonable source point.

\section{CONCLUSION}

I have devised and written a Markov Chain Monte Approach which will quickly analyze the transverse coherence, performing $~ 100$ times faster than the brute force algorithm previously in use. I have found the GENESIS simulation code to predict a very high level of transverse coherence for the LCLS, which is encouraging. One interesting result I have found is that the transverse coherence declines significantly after saturation, which suggests that users who want a high degree of coherence might be better off using the LCLS without the full length of the undulators, sacrificing some intensity in exchange for greater coherence. A comparison of the emitted radiation and beam divergence to that predicted by the GSM is somewhat inconclusive. Certainly one problem of the GSM model of transverse coherence is that it requires a single source point, which is not the case for an FEL. The LCLS has only recently come online, and the prediction of it's properties is ongoing work. 
My work will provide a useful tool for predicting the degree of transverse coherence, and I have made my code easily extensible to predict the accuracy of any proposed models of transverse coherence.

\section{Acknowledgments}

I'd like to thank my advisor, Zhirong Huang for all his help, support, and teaching over the last couple of months. I'd also like to thank Yuantao Ding for his help in teaching in explaining the basic principles behind FELs. I'd also like to thank Steve Rock for helping to organize the SULI program and making it a great experience. I'd also like to thank the SULI program of the Department of Energy, and SLAC National Accelerator Laboratory for giving me this opportunity. 
TABLE I: Parameters of the Electrons Entering the Undulators

Slice Emittance : $0.4 \mu \mathrm{m}$

Peak Current: $3 \mathrm{kA}$

Slice Energy Spread: $0.01 \%$

\section{FIGURES}

\section{A. Fitting for Simulated FEL Having 84 Meters of Undulators}

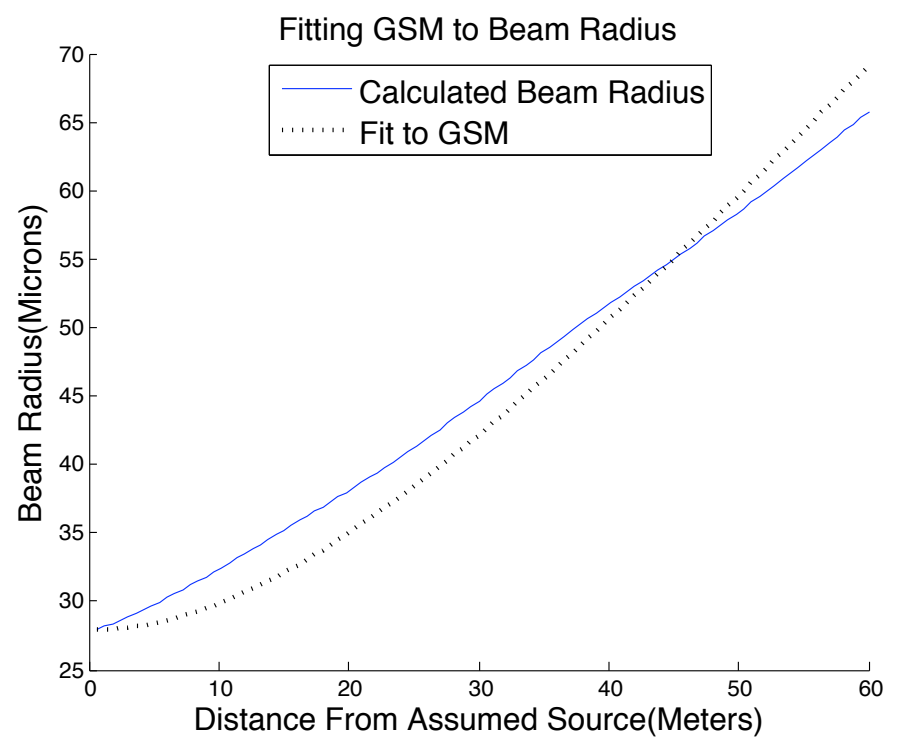

FIG. 1: Curve fit of beam radius to GSM assuming a point source at 84 meters(end of the last undulator). 


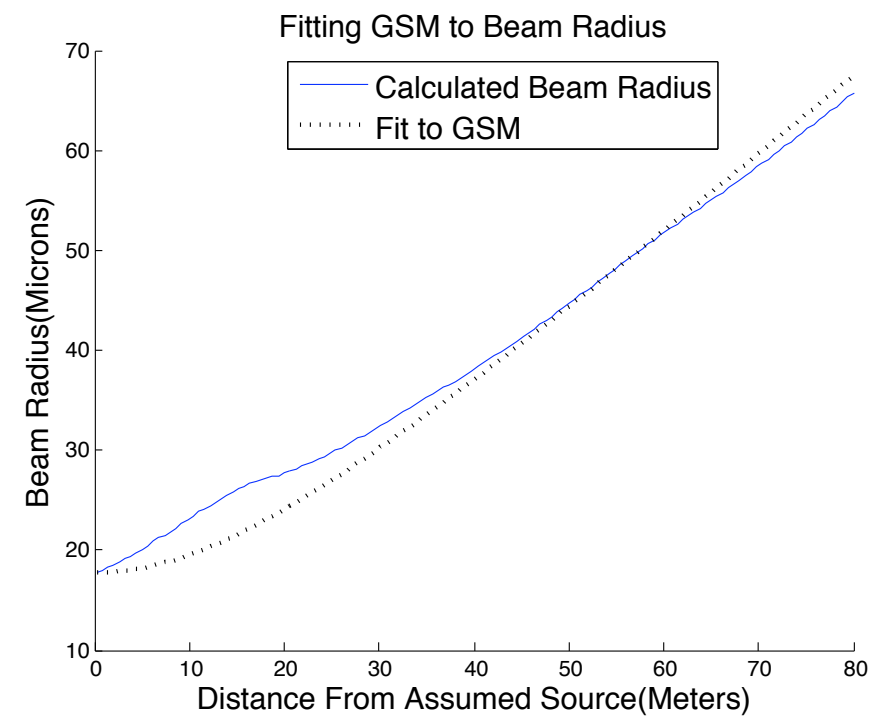

FIG. 2: Curve fit of beam radius to GSM assuming a point source at 64 meters(around the saturation point).

\section{B. Analysis of Fields for FEL with 100 meters of undulators}

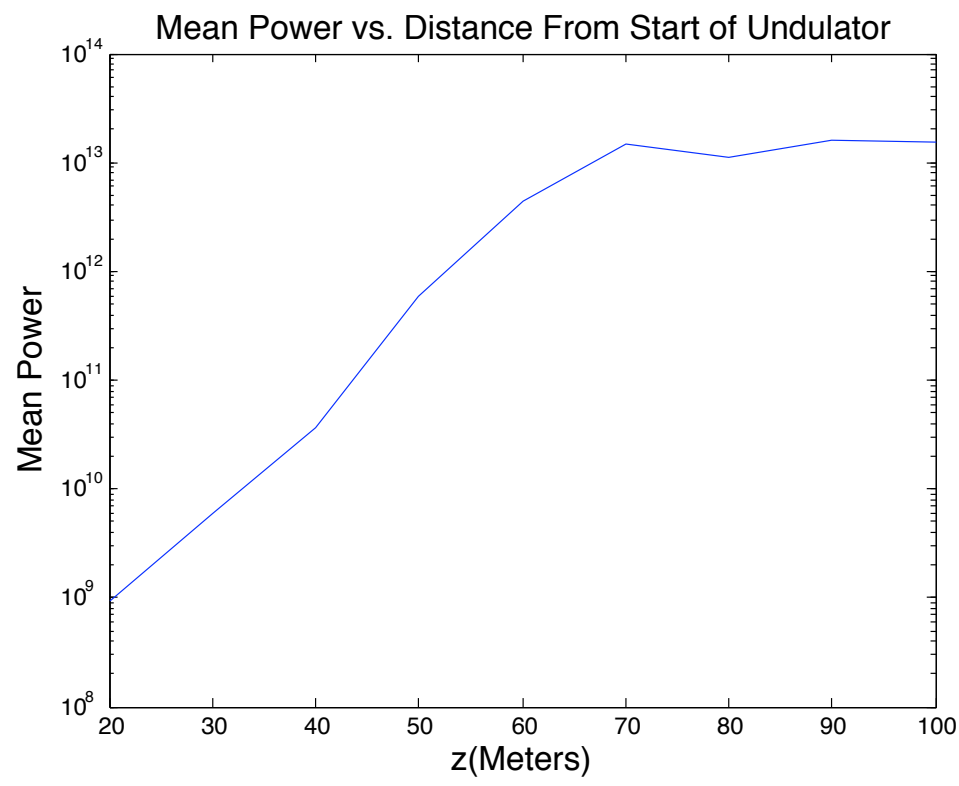

FIG. 3: Beam power, note that saturation occurs at about 64 meters. 


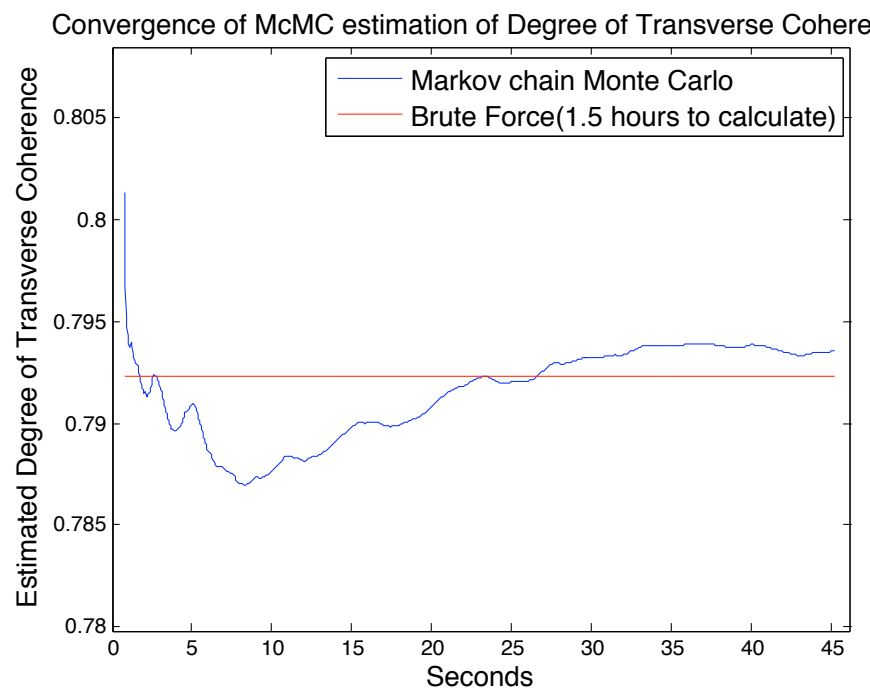

FIG. 4: The Markov chain Monte Carlo algorithm quickly converges to the correct value.

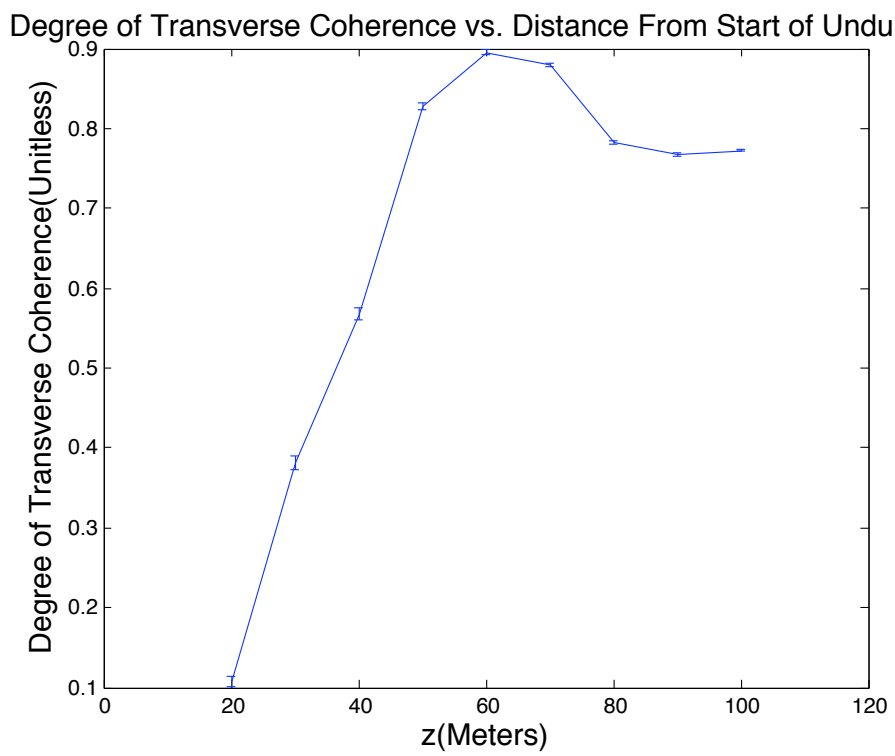

FIG. 5: Degree of transverse coherence as a function of $z$. 


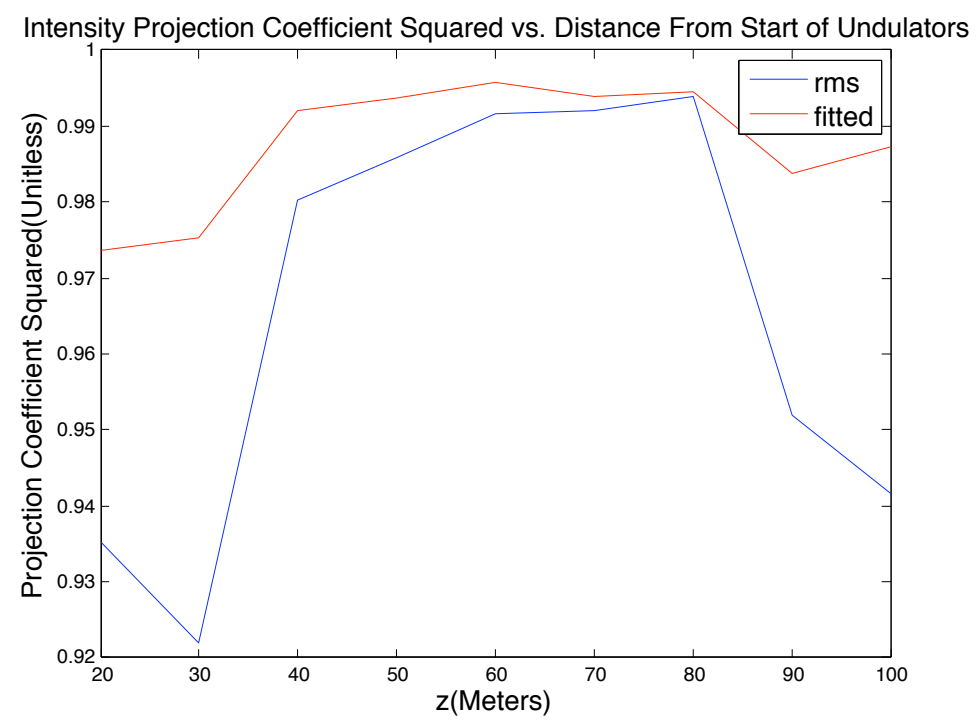

FIG. 6: Agreement of measured intensity with Gaussian model.

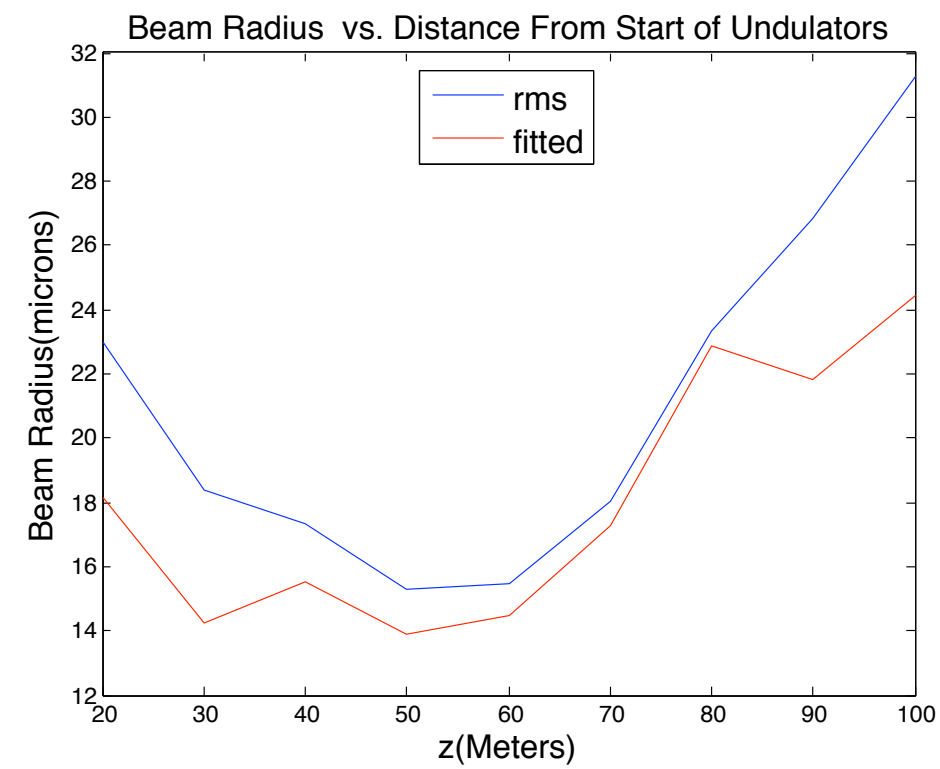

FIG. 7: Beam radius, both found by rms calculation and fitting. 


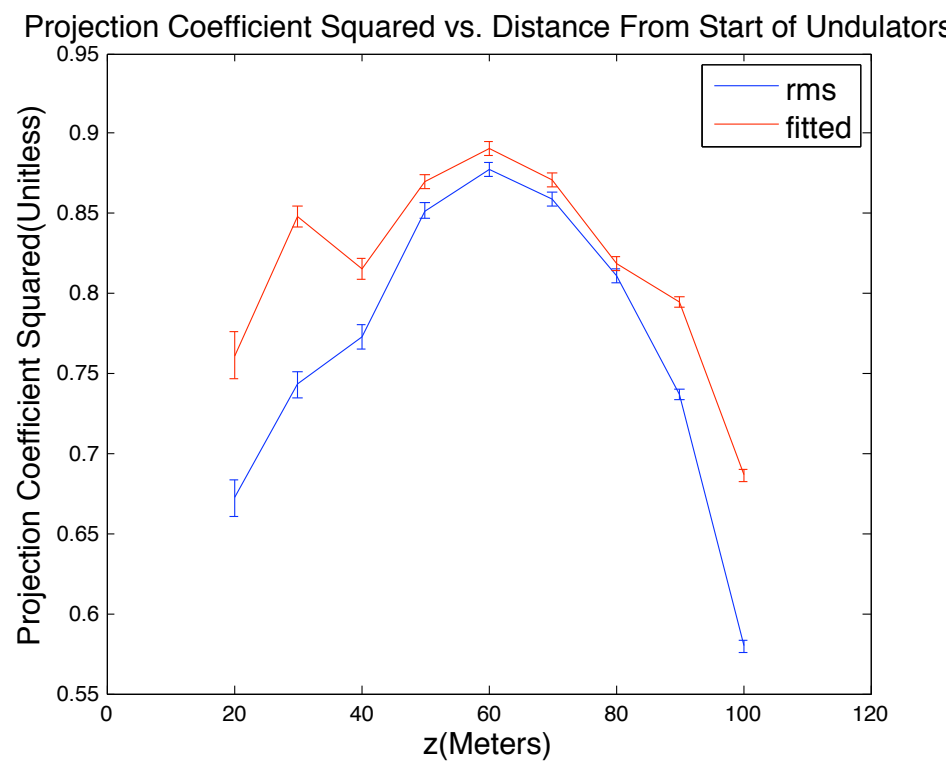

FIG. 8: Projection of mutual intensity function onto phase-less GSM model.

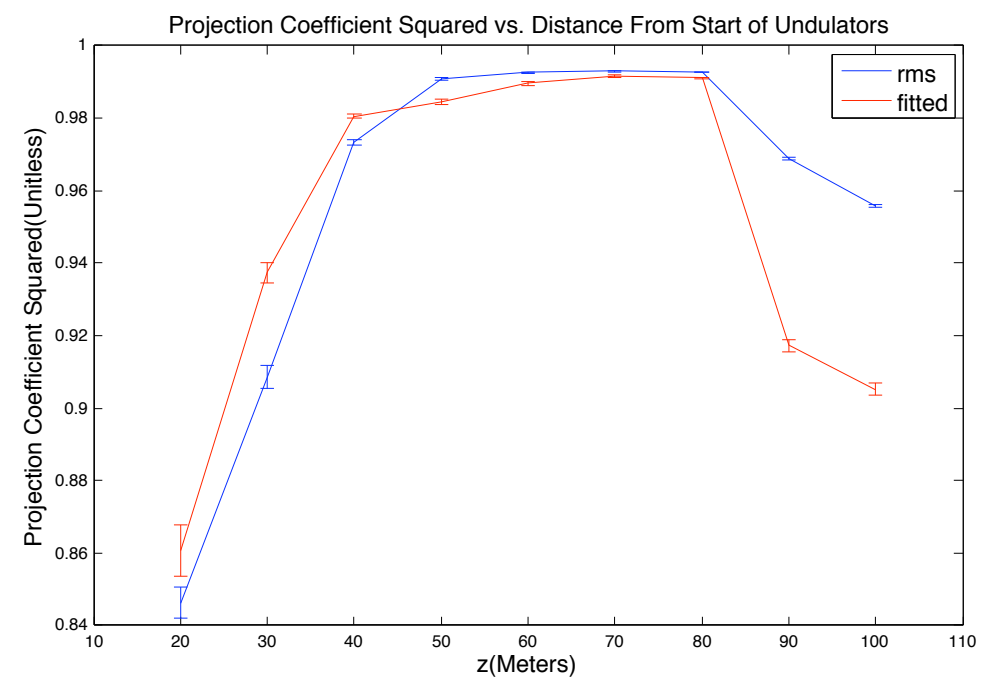

FIG. 9: Projection of magnitude of mutual intensity function onto GSM model, neglecting phase differences. 
1 Zhirong Huang and Kwang-Je Kim, "Review of x-ray free-electron laser theory", Phys. Rev. ST Accel. Beams, vol. 10, no. 3, pp. 034801, Mar 2007.

2 J.W. Goodman, "Statistical optics", Wiley, 1985.

3 I L. Mandel and E. Wolf, "Optical coherence and quantum optics", Cambridge University Press, Cambridge, England,, vol. 100, pp. 077203, 1995.

4 A. Singer, I. A. Vartanyants, M. Kuhlmann, S. Duesterer, R. Treusch, and J. Feldhaus, "Transverse-coherence properties of the free-electron-laser flash at desy", Physical Review Letters, vol. 101, no. 25, pp. 254801, 2008.

5 Sect. S. Reiche et al, "Genesis 1.3: a fully 3d time-dependent fel simulation code", Nucl. Instrum. Methods Phys. Res. Sect A, vol. 429, no. 243-248, 1999. 


\title{
An Implementation of a Texture Analysis System
}

\author{
Ronald Pandolfi \\ Office of Science, Science Undergraduate Laboratory Internship \\ Program
}

University of California, Merced

Stanford Synchrotron Radiation Lightsource
Stanford, California

August 6, 2009

Prepared in partial fulfillment of the requirement of the Office of Science, Department of Energy's Science Undergraduate Laboratory Internship under the direction of Apurva Mehta in Stanford Synchrotron Radiaion Lightsource division of the Stanford Linear Accelerator Center.

Participant:

Signature

Research Advisor:

Signature 


\section{Contents}

1 Introduction $\quad 4$

2 Diffraction Geometry $\quad 5$

3 Methods $\quad 5$

4 Conclusions $r$

5 Acknowledgements $\quad 9$ 


\begin{abstract}
An Implementation of a Texture Analysis System. RONALD PANDOLFI (University of California, Merced 95243) APURVA MEHTA (Stanford Synchrotron Radiation Lightsource, Stanford, CA 94025) MATT BIBEE (Stanford Synchrotron Radiation Lightsource, Stanford, CA 94025) JAMES (RANDY) GROVES (Stanford Synchrotron Radiation Lightsource, Stanford, CA 94025).
\end{abstract}

From analysis of the crystalline texture (the distribution of crystalline orientations in a polycrystalline substance) of target samples, some critical material properties of those samples may be deduced. Texture typically results in a specimen from the conditions by which its substance is formed or engineered, and may be used to quantify its qualities. This project aimed to produce a full process for texture analysis of target samples. It was intended that this process be implemented for CCD or imaging plate diffraction data recorded in the Stanford Synchrotron Radiation Lightsource Beamline 11-3. A process was implemented for smoothly analyzing diffraction images and producing full pole figures. The MATLAB package MTEX formed a base for the implementation of this process. This process was applied to diffraction data from a rolled $\mathrm{Cu}$ specimen. The resulting raw-interpolated and completed pole figures did not sufficiently match to indicate a correct approximation of the orientation distribution function (ODF) for that specimen. A comparative analysis of MTEX and other pole figure inversion algorithms, as applied to this $\mathrm{Cu}$ data, may reveal that another method better approximates the ODF. 


\section{INTRODUCTION}

The texture of a polycrystalline sample is its distribution of crystalline grain orientation in a given direction. Typically, texture results from the conditions of the formation of a sample (either naturally or thorugh an engineered process). Texture of a sample governs some critical material properties of that sample may be deduced. In crystalline samples, most physical properties of interest are anisotropic (have preferred orientations). Additionally, most substances are polycrystalline, making texture analysis frequently necessary [4].

A major motivation for texture analysis is the study of biological structures such as tooth enamel and nacre (see Figure 1). These samples each have significant texture which effects their mechanical properties. The texture of these organically produced structures may be such that it enhances their mechanical properties in directions of importance, such as a chewing direction.

The diffraction of x-rays through a target sample is affected by the texture of that sample. An x-ray source can therefore be used as an illumination source for texture analysis. At the Stanford Synchrotron Radiation Lightsource (SSRL), high intensity x-ray beams provide a suitable source of illumination for x-ray diffraction with goniometers and texture analysis. SSRL beamlines have a setup that would be compatible with a texture analysis system. Experimentation on these beamlines typically involves the illumination of a target sample which may be rotated such that diffraction data can be recorded for various specimen orientations using a CCD or an image plate detector. These area detectors alow quick scanning of the diffraction image. Both current and past SSRL experiments would benefit from a system for texture analysis. A process for texture analysis was thus implemented for this purpose.

\section{DIFFRACTION GEOMETRY}

A variety of notations and formalisms are used to describe diffraction geometry, so a clarification of the geometry in use here is provided. In our case, the target sample is held 
by a goniometer which allows rotation of the sample about a horizontal axis perpendicular to the beam line $(\Delta)$. The diffracted $\mathrm{x}$-rays are visible at the detector as Debye rings corresponding to the intersections of the reciprocal sphere with the Ewald sphere, as shown in Figure 2. These rings each then correspond to diffraction from separate Miller indices. The coordinate system for diffracted points is defined by two coordinates for the location on the Ewald sphere, and a third coordinate for the rotation angle of the sample. These anlges are respectively represented as $\chi, \theta$, and $\Delta$ ) as shown in Figure 3. An equal area projection of the diffraction rings was used, as it is the most suitable for Texture analysis [4], as shown in Figure 6. The resulting planar projections of the Ewald sphere are pole figures. Intensities on pole figures are relative to the density of diffraction points along the intersection of the Ewald and reciprocal spheres. Pole figures are therefore projections of the distribution of crystallite orientations. This distribution of crystallite orientations for a sample is defined as the orientation distribution function (ODF).

\section{METHODS}

The process of analyzing diffraction data to represent it as completed pole figures involves five primary steps, as outlined below [3]:

1. Calibration of the diffraction pattern to account for imaging distortions and extraction of relative intensity peaks from the diffraction image

2. Converting diffraction data into partial and partial-interpolated pole figures

3. Calculation of the ODF from partial pole-figures

4. Plotting of completed pole figures from the ODF

5. Comparison of completed pole figure to the partial pole figure to assess the accuracy of the odf.

3.1 Calibration and the extraction of peaks 
A suitable impelementation of this first step was available, leaving the final four steps to be implemented. This first step can be accomplished using the open-source project Area Diffraction Machine. This program reads a diffraction image, such as in Figure 7, and records peaks along the diffraction rings. These intensity peaks are written to ASCII files with their corresponding $\Delta, \chi, 2 \theta$, and relative intensity values.

\subsection{Converting diffraction data into pole figures}

A process was implemented in MATLAB to convert these peaklist values to Euler angles [5]. The following relations hold for the diffraction geometry described in Section 2.

The spherical law of cosines [2] is applied to the geometry in Figure 3,

$$
\begin{gathered}
\cos (\psi)=\cos \left(\frac{\pi}{2}-\theta\right) \cos (\Delta)+\sin \left(\frac{\pi}{2}-\theta\right) \sin (\Delta) \cos \left(\chi-\frac{\pi}{2}\right) \\
\cos (\psi)=\sin (\theta) \cos (\Delta)+\cos (\theta) \sin (\Delta) \sin (\chi)
\end{gathered}
$$

From Equation 2, the Euler angle $\psi$ can then be calculated. The spherical law of sines [2] is applied to the same geometry,

$$
\begin{aligned}
& \frac{\sin (\pi-\phi)}{\sin \left(\frac{\pi}{2}-\theta\right)}=\frac{\sin \left(\chi-\frac{\pi}{2}\right)}{\sin (\psi)} \\
& \sin (\phi)=-\frac{\cos (\theta) \cos (\chi)}{\sin (\psi)}
\end{aligned}
$$

For the special case when $\Delta=0$, Equations 1 and 2 reduce as expected,

$$
\begin{gathered}
\cos (\psi)=\sin (\theta) \cos (0)+\sin (\theta) \sin (0) \sin (\chi) \\
\cos (\psi)=\sin (\theta) \\
\sin (\phi)=-\frac{\cos (\theta) \cos (\chi)}{\sin \left(\frac{\pi}{2}-\theta\right)}
\end{gathered}
$$




$$
\sin (\phi)=-\cos (\chi)
$$

The angles $\phi$ and $\psi$ are real-valued. Since the range of $\operatorname{asin}$ is limited to $\left[-\frac{\pi}{2}, \frac{\pi}{2}\right], \phi$ is then defined as a piecewise function to ensure full range, with the critical value separating its quadrants defined as $\chi_{0}$,

$$
\cos \left(\chi_{0}\right)=\frac{\sin \left(\operatorname{acos}\left(\frac{\sin (\theta)}{\cos (\Delta)}\right)\right)}{\cos (\theta)}
$$

The relation between $\chi$ and $\chi_{0}$ can then be used ensure that $\phi$ is in the correct quadrant,

$\phi^{\prime}= \begin{cases}\phi & \left.\left(\chi_{0} \leq \chi<\pi-\chi_{0} \text { and } 0 \leq \Delta\right) \text { or }\left(\left(0 \leq \chi<\pi+\chi_{0}\right) \text { or }\left(\chi>2 \pi-\chi_{0}\right)\right) \text { and } \Delta<0\right) \\ \pi-\phi & \left(\left(\left(\pi-\chi_{0} \leq \chi<2 \pi\right) \text { or } \chi<\chi_{0}\right) \text { and } 0 \leq \Delta\right) \text { or }\left(\left(\pi+\chi_{0} \leq \chi<2 \pi-\chi_{0}\right) \text { and } \Delta<0\right)\end{cases}$

An equal-area projection onto a planar surface tangent to the diffraction sphere (as represented in Figure 6) is then applied to the Euler angles, defined by [4]

$$
\sin \left(\psi^{\prime}\right)=\sqrt{2} \sin \left(\frac{\psi}{2}\right)
$$

\subsection{Calculation of the $O D F$}

The calculation of the ODF is a complex mathematical process. To accomplish this, sets of pole figures must be together inverted to construct an approximation of the ODF. This inversion process is analagous to tomography, in that a 3D structure must be reconstructed from its 2D projections. As such, the solutions are not unique, although algorithms for this inversion process may well approximate the ODF with sufficient data.

The threetuples (Intensity, $\phi^{\prime}$, and $\psi^{\prime}$ ) resulting from the end of Section 3.2 are binned into user-defined polar area segments (a typical size being $1^{\circ} \times 1^{\circ}$ ) with similar-binned points being averaged together. The binned data is written as a raw pole 
figure file in Philips diffractometer format. This file can then be read in as needed to display a pole figure, or combined with other pole figures and further processed to produce an ODF. Each pole figure corresponds to a separate Miller index. These pole figures are 'raw' and incomplete due to the limitations of data coverage. Since the range of sample rotation is limited to approximately $\left[-50^{\circ}, 50^{\circ}\right]$ in our case, some regions of these pole figures cannot be scanned. The resulting data coverage fills an area as shown in Figure 8.

The raw pole figure data is read into MATLAB using functionality provided by the software package MTEX [1] (a set of MATLAB functions). The MTEX algorithm is a recently developed algorithm that was implemented in a set of open-source MATLAB functions. The general structure of this algorithm begins with the selection of a radial basis function on $\mathrm{SO}(3) \rightarrow \mathbb{R}$. An equidribution of crystal orientations is then determined. The unknown ODF is then approximated by a finite linear combination of radially symmetric unimodal ODFs. The coefficients of the linear combination and normalization coefficients are then determined as a minimization problem by a mean square like function with the additional constraints that the resulting ODF is non-negative and smooth. Additionally, this method allows for an arbitrary crystal and diffraction geometry as well. For a more detailed description of the MTEX algorithm, see the paper by Hielscher and Schaeben [1]. An estimated ODF is then calculated using the MTEX pole-figure inversion algorithm.

\subsection{Plotting pole figures}

The 'completed' pole figures can then be calculated and plotted from the ODF. The incomplete-interpolated pole figures are also plotted from the raw pole figure files for comparison. Plotting these pole figures is simply accomplished through the use of MTEX's functions.

\subsection{Comparison of the pole figures as a check of accuracy}

Comparison of completed pole figure to the partial pole figure to assess the accuracy of the odf. An iterative path may be taken to produce a series of better 'complete' pole figures based on a comparison between the interpolated and incomplete pole figures, either 
programmatically or by user query. If, either computationally or by user inspection, the completed and incomplete-interpolated pole figures do not sufficiently correlate, the ODF may be recalculated with higher resolution for a better approximation.

\subsection{Experimental evaluation of this process}

In order to evaluate the correctness of this process and the MTEX algorithm, diffraction data was taken for $\Delta$ values between $-50^{\circ}$ and $50^{\circ}$ in increments of $1^{\circ}$ for a well textured (assumedly rolled) $\mathrm{Cu}$ specimen. This data was taken on SSRL Beam Line 11-3 (shown in Figures 4 and 5) using a Mar345 imaging plate detector. For specifications of the experimental setup, see Table. The Cu diffraction data was plotted in pole figures (see Figures 9-12) and interpolated over constant $\psi$ values (see Figures 13-16). The MTEX pole

figure inversion method was applied to the raw pole figures, resulting in the ODF shown in Figure 21. From the computed ODF, completed pole figures (see Figures 17-20) were plotted and compared with the raw pole figures as a check.

\section{RESULTS}

In comparing the interpolated raw and completed pole figures, the $\{111\}$ (Figures 13 and 17) and \{220\} (Figures 15 and 19) pole figures exhibited moderate similarity. The $\{200\}$ (Figures 14 and 18) and \{311\} (Figures 16 and 20) pole figures showed little similarity. The completed pole figures only slightly resemble their corresponding raw pole figures, indicating that the MTEX algorithm did not produce a sufficiently accurate ODF for the $\mathrm{Cu}$ specimen.

\section{CONCLUSIONS}

From the apparent inconsistencies between the raw-interpolated and completed pole figures, it is clear that the MTEX algortihm did not produce a sufficient approximation of the specimen ODF. In future work, the MTEX algorithm may be replaced with the older WIMV algorithm, which is known to be robust in obtaining the ODF from incomplete pole figure data. A comparative analysis of the results from these and other algorithms would provide a better benchmark for the selection of a pole figure inversion method. 
Additionally, a complete automation of this procedure would be desired.

\section{ACKNOWLEDGEMENTS}

I much appreciate the continued guidance and support from my mentor, Apurva Mehta. Additional thanks go to Matthew Bibee, James (Randy) Groves, Marc Davidson, and Karen Magid. I also thank the DOE, SULI Program, the Stanford Linear Accelerator Center, and the Stanford Synchrotron Radiation Lightsource laboratory for cooperating to provide myself and others with engaging undergraduate research opportunities.

\section{REFERENCES}

[1] Journal of Applied Crystallography, Vol. 41, No. 6. (December 2008), pp. 1024-1037.

[2] Weisstein, Eric W. S̈pherical Trigonometry.ت̈rom MathWorld-A Wolfram Web Resource. http://mathworld.wolfram.com/SphericalTrigonometry.html

[3] H.-R. Wenk and S. Grigull (2003). Synchrotron texture analysis with area detectors. J. Appl. Cryst. 36, 1040-1049.

[4] U.F. Kocks and J.S. Kallend. In: U.F. Kocks, C.N. Tom and H.-R. Wenk, Editors, Texture and Anisotropy, Cambridge University Press, Cambridge, UK (1998), p. 54, 104-124.

[5] Weisstein, Eric W. "Euler Angles." From MathWorld-A Wolfram Web Resource. http://mathworld.wolfram.com/EulerAngles.html 
Figures:

\begin{tabular}{|c|c|c|c|}
\hline & Harmonic & MTEX & WIMV/APC \\
\hline \hline Flexibility of Implementation & $\times[4]$ & $\checkmark[1]$ & $\checkmark[4]$ \\
\hline Ghost Correction & $\times[4]$ & & $\checkmark[4]$ \\
\hline Incomplete Pole Figures & $\times[4]$ & & $\checkmark[4]$ \\
\hline Low Symmetry Samples & $\times[4]$ & & $\checkmark[4]$ \\
\hline Complexity of Implementation & $\times[4]$ & $\checkmark[1]$ & \\
\hline Economy of Implementation & $\checkmark[4]$ & $\checkmark[1]$ & $\times[4]$ \\
\hline Poor Quality Diffraction Data & $\checkmark[4]$ & & $\times[4]$ \\
\hline Susceptibility to Noise & $\checkmark[4]$ & & $\times[4]$ \\
\hline Area Detectors & & $\checkmark[1]$ & \\
\hline Sharp Textures & & $\checkmark[1]$ & \\
\hline High Resolution Pole Figures & & $\checkmark[1]$ & \\
\hline
\end{tabular}

istics of pole figure inversion algorithms. Checks indicate a favorable characteristic, and crosses indicate unfavorable characteristics. The adjacent bracketed numbers are the related references.

\begin{tabular}{|c|c|}
\hline & Specifications for $\mathrm{Cu}$ diffraction experiment \\
\hline \hline Beam Energy & $12.7 \mathrm{keV}$ fixed \\
\hline Beam Wavelength & $.97 \AA$ \\
\hline Spot Size & $150 \times 150 \mu \mathrm{m}$ \\
\hline$\Delta$ Range & $-50^{\circ}$ to $50^{\circ}$ \\
\hline$\Delta$ Step Size & 1 \\
\hline Sample Type & Thin $\mathrm{Cu}$ foil \\
\hline
\end{tabular}

Table 2: Specifications

for the evaluational diffraction experiment. 


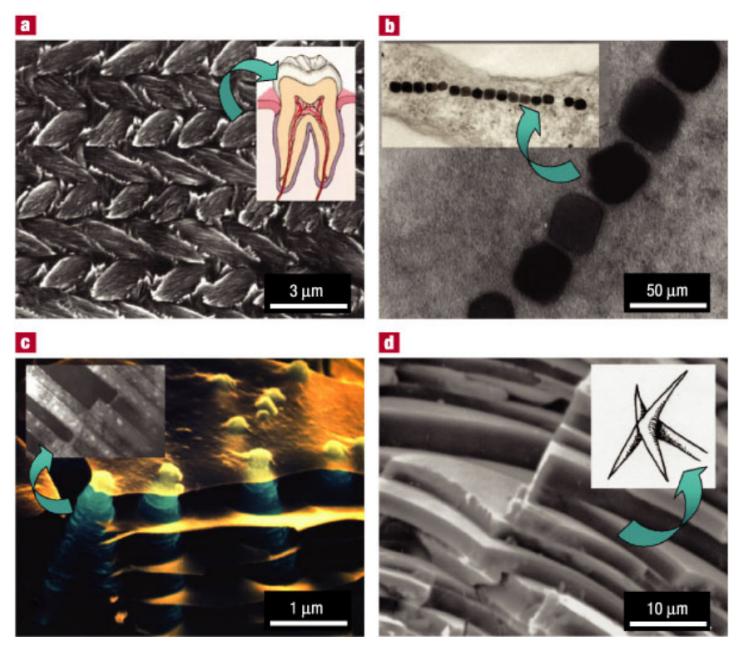

Figure 1: Mouse enamel, magnetotactic bacterium, abalone aragonite platelets, and sponge spicule each have texture.

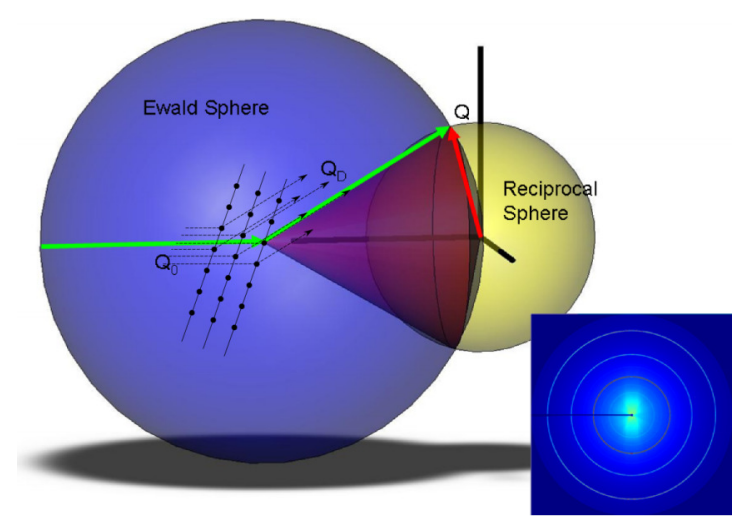

Figure 2: The ewald and reciprocal spheres. The intersection of the surfaces of these spheres is a ring. Diffraction data therefore appears as a series of concentric rings on the detector plane. 


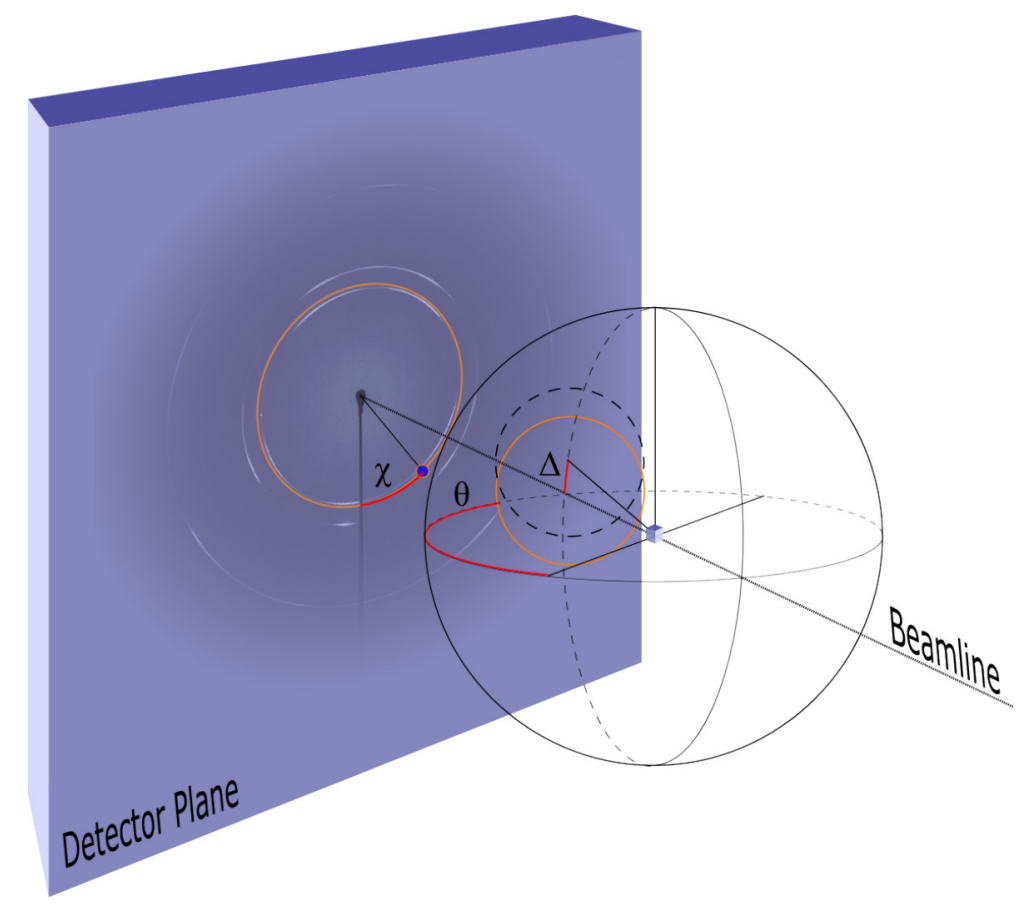

Figure 3: The diffraction geometry coordinate system used for the peak list data sets. The sample is rotated along the $\Delta$ angle axis.

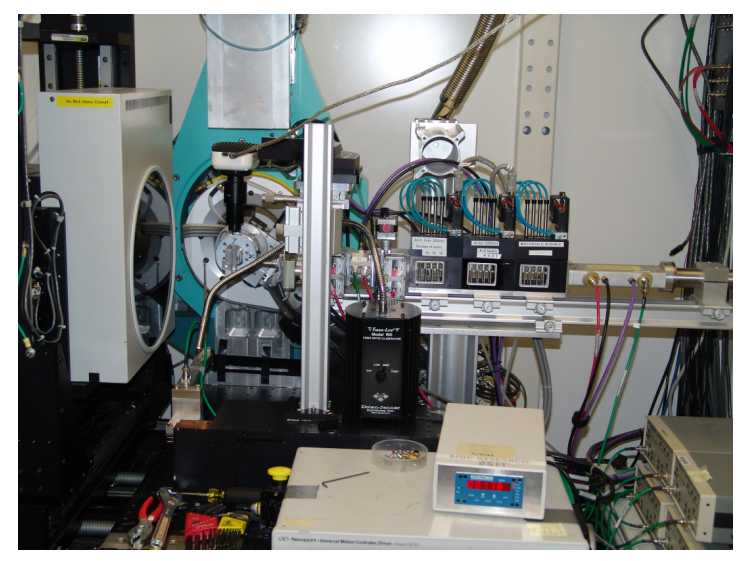

Figure 4: A view of Beamline 11-3 from the hutch door. The x-ray beam comes from right to left. 


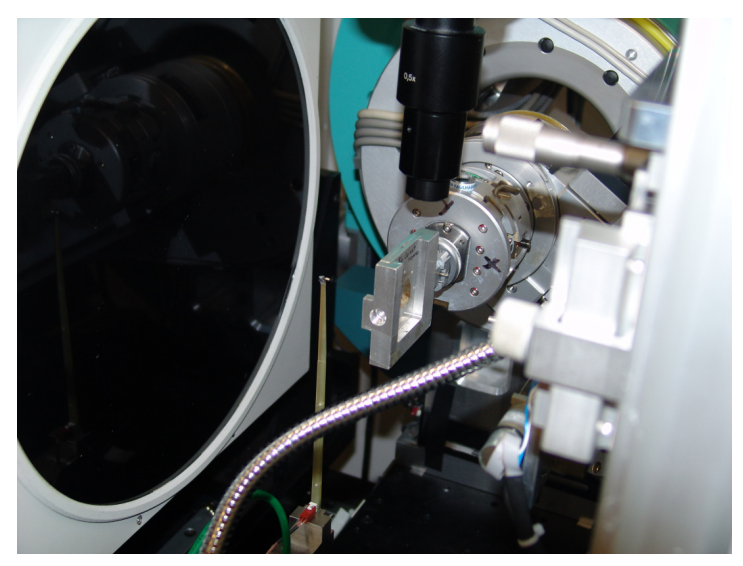

Figure 5: A view of the $\mathrm{Cu}$ sample and specimen holder. The goniometer arm rotates the sample in place. 


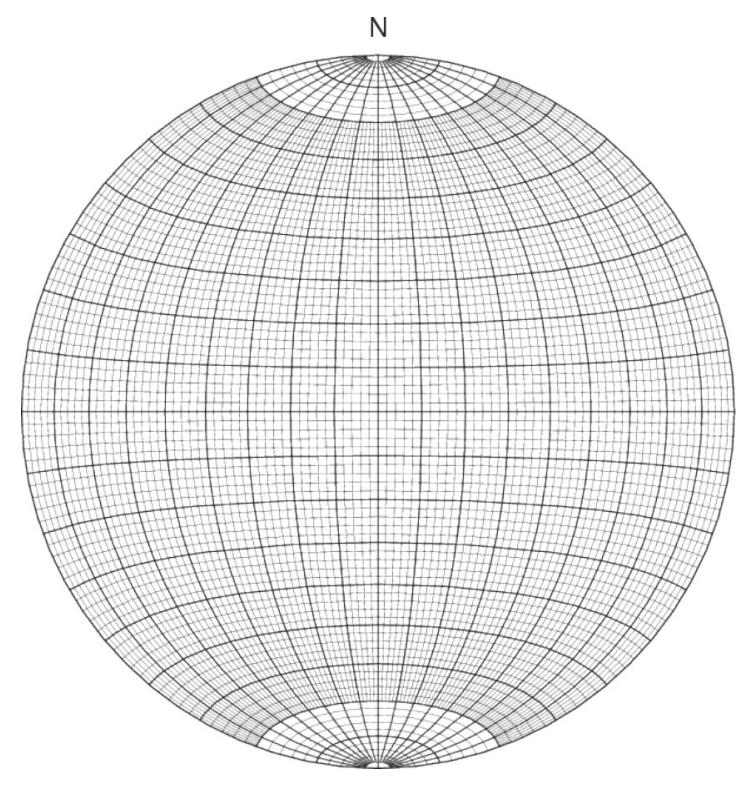

Figure 6: An equal area net on a sphere. A flattened hemisphere of the equal area net is the equal area projection on a planar surface. 


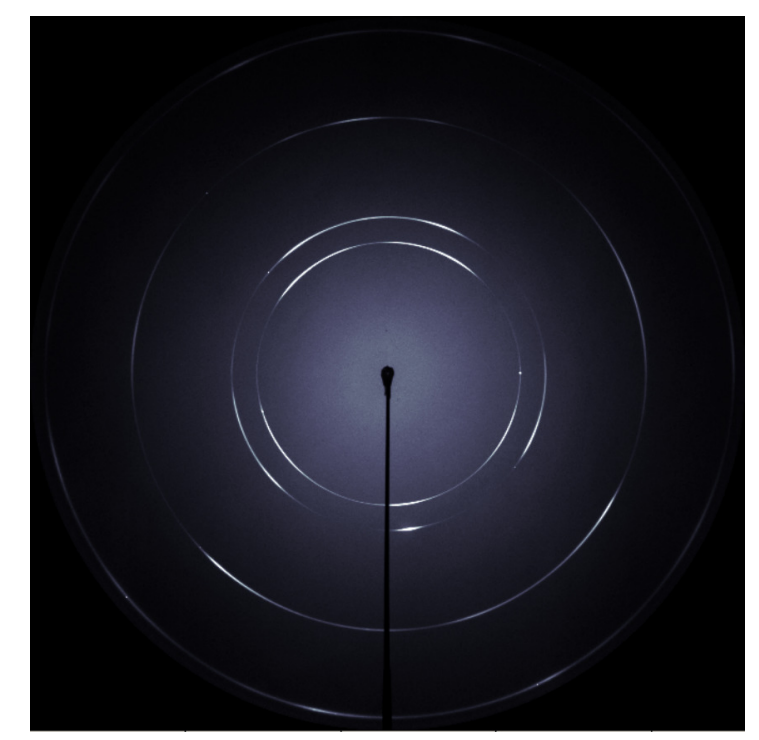

Figure 7: An example of a diffraction image recorded from a Mar345 imaging plate detector, displayed in Area Diffraction Machine.

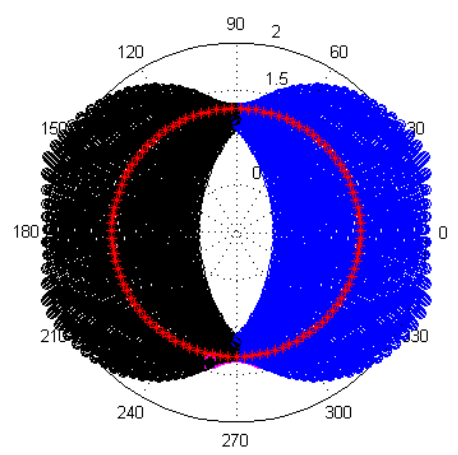

Figure 8: An example of the range of coverage of diffraction data for this experimental setup. 


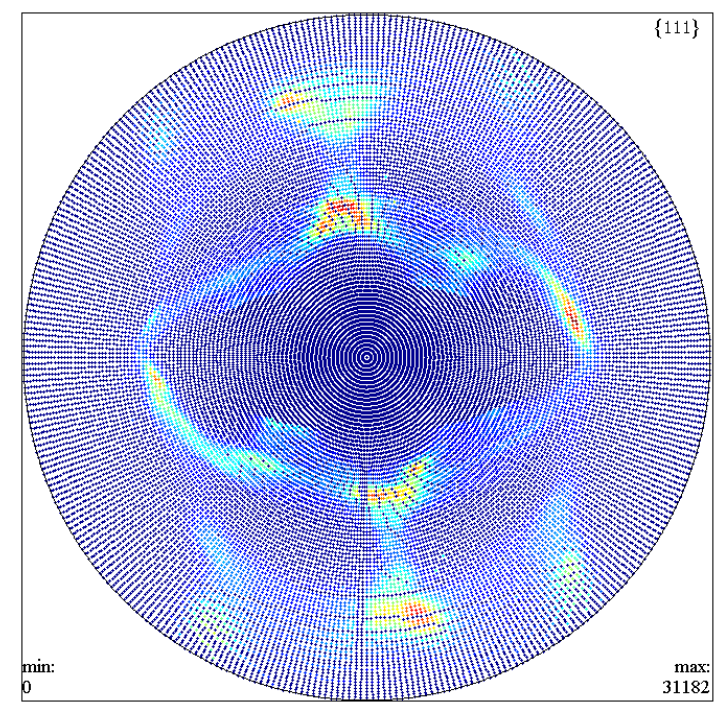

Figure 9: The (111) pole figure for the $\mathrm{Cu}$ diffraction data set.

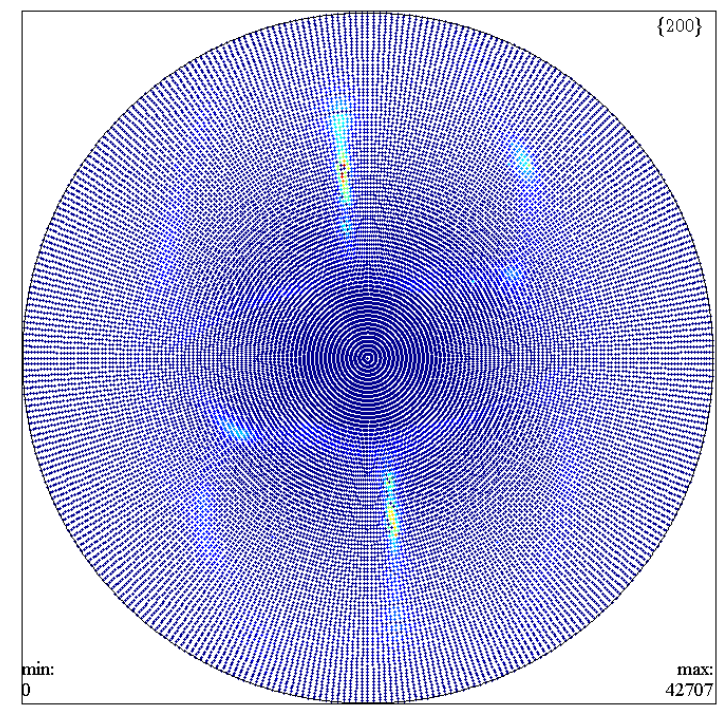

Figure 10: The (200) pole figure for the $\mathrm{Cu}$ diffraction data set. 


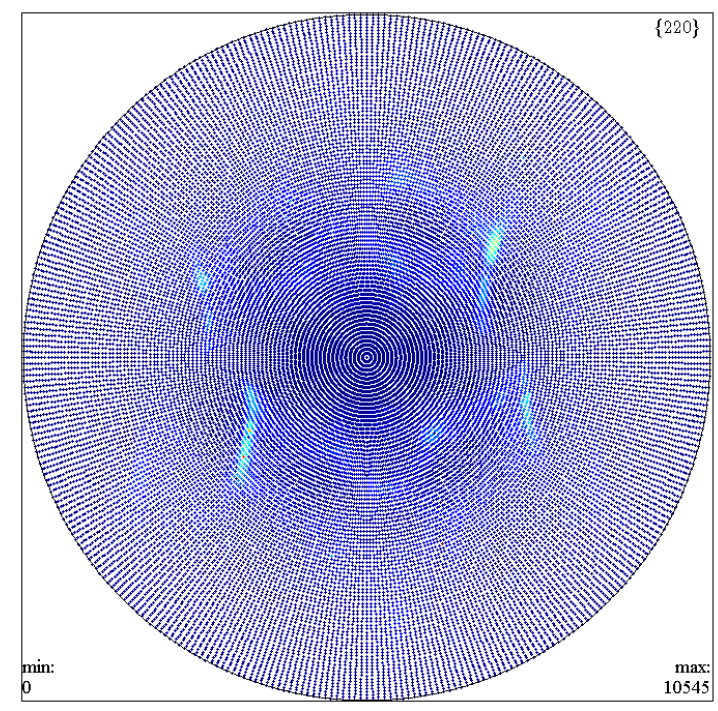

Figure 11: The (220) pole figure for the $\mathrm{Cu}$ diffraction data set.

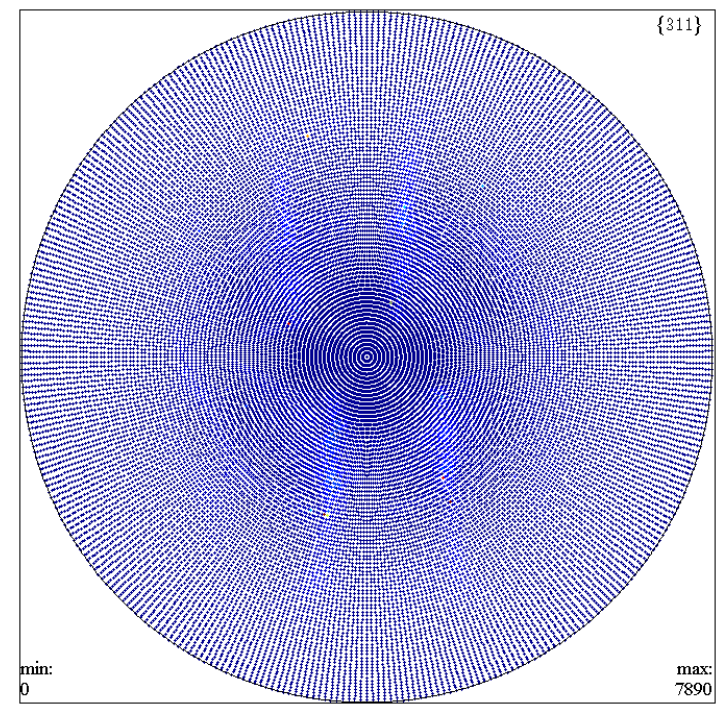

Figure 12: The (311) pole figure for the $\mathrm{Cu}$ diffraction data set. 


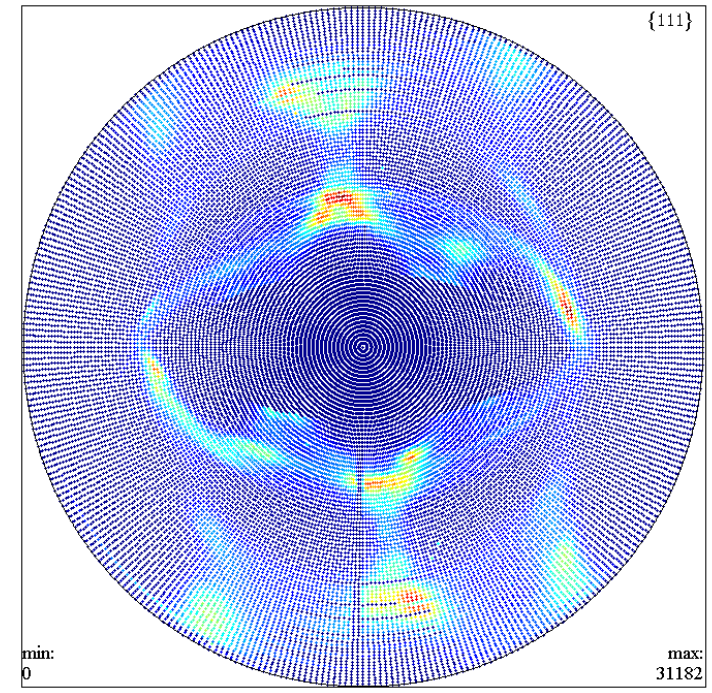

Figure 13: The (111) interpolated pole figure for the $\mathrm{Cu}$ diffraction data set.

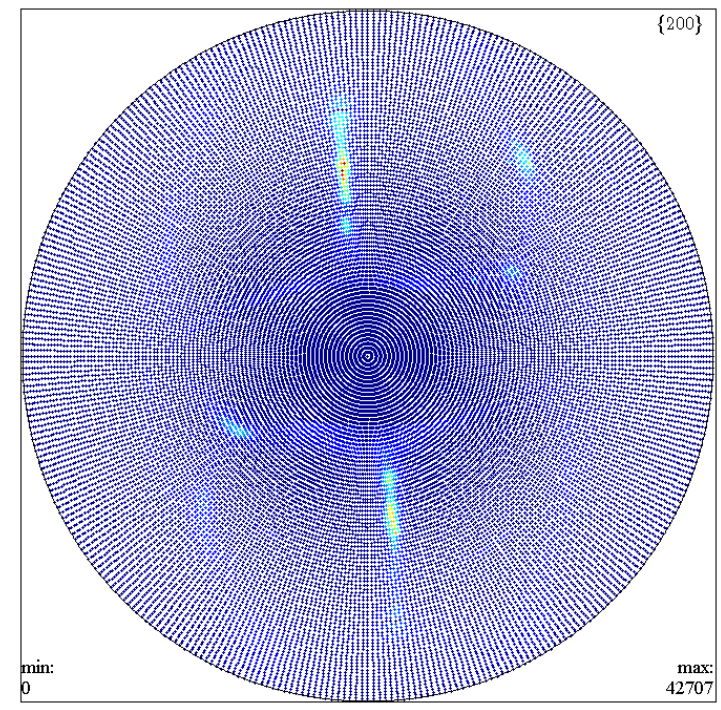

Figure 14: The (200) interpolated pole figure for the $\mathrm{Cu}$ diffraction data set. 


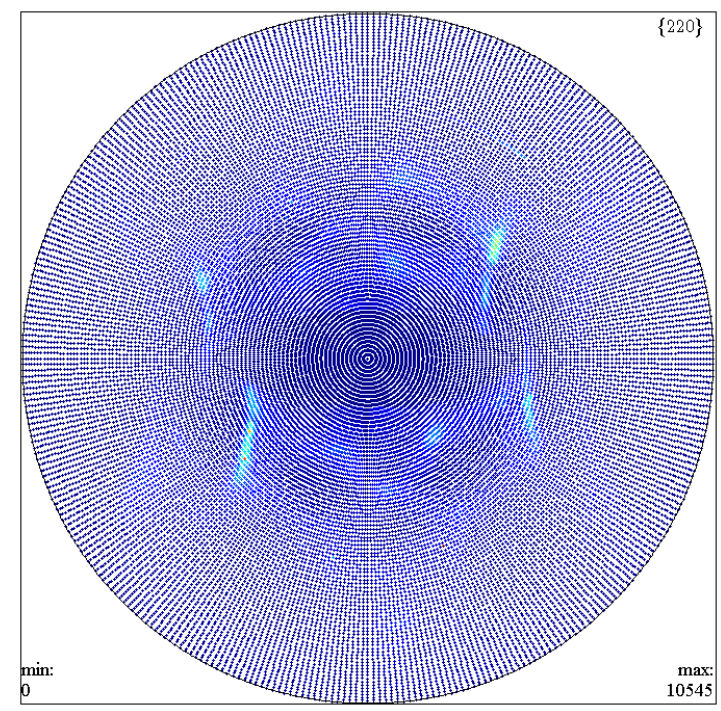

Figure 15: The (220) interpolated pole figure for the $\mathrm{Cu}$ diffraction data set.

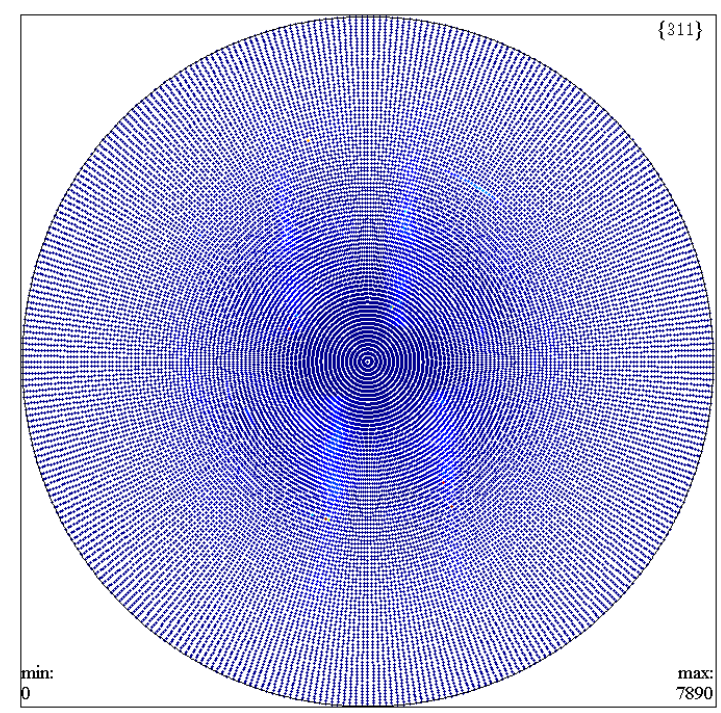

Figure 16: The (311) interpolated pole figure for the $\mathrm{Cu}$ diffraction data set. 


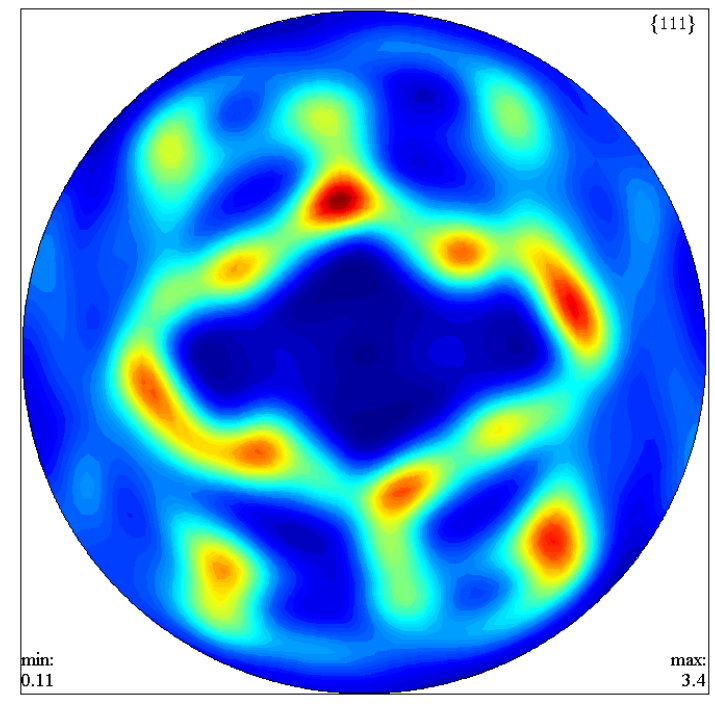

Figure 17: The (111) completed pole figure for the $\mathrm{Cu}$ diffraction data set. 


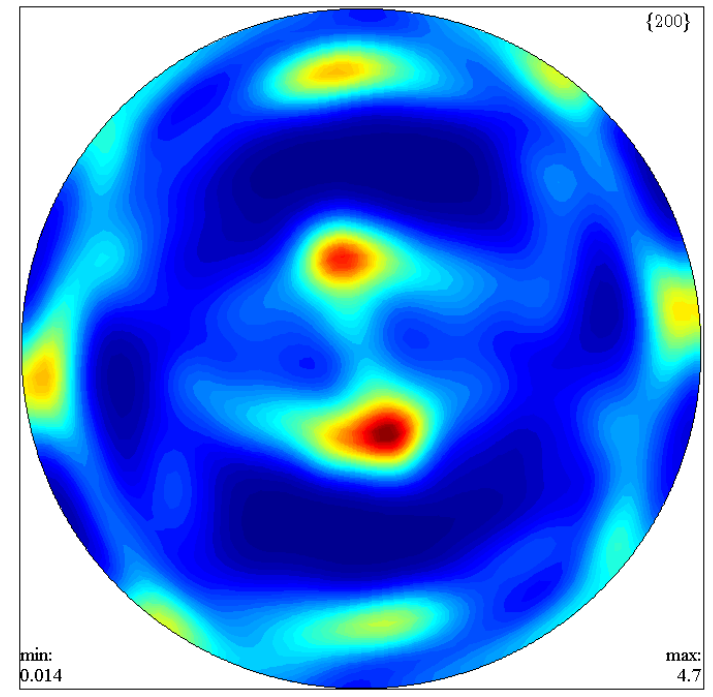

Figure 18: The (200) completed pole figure for the $\mathrm{Cu}$ diffraction data set.

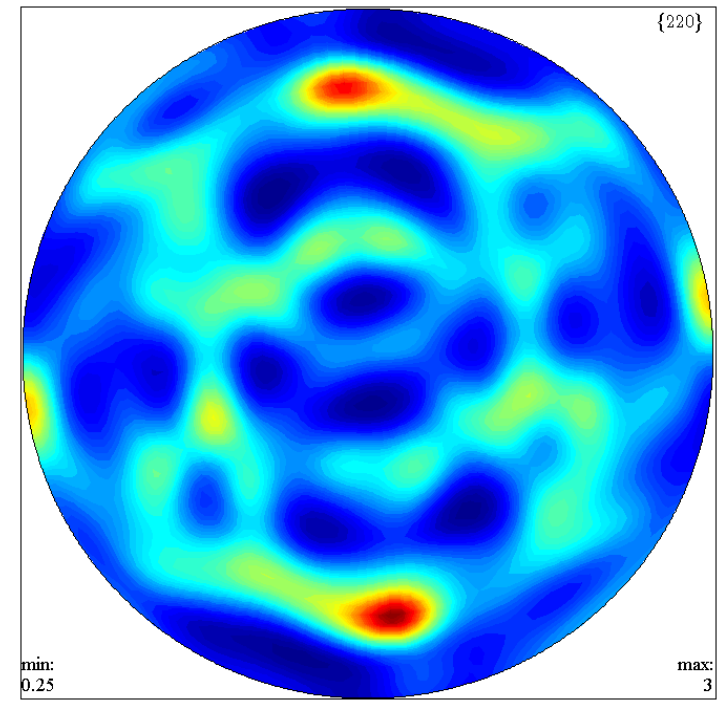

Figure 19: The (220) completed pole figure for the $\mathrm{Cu}$ diffraction data set. 


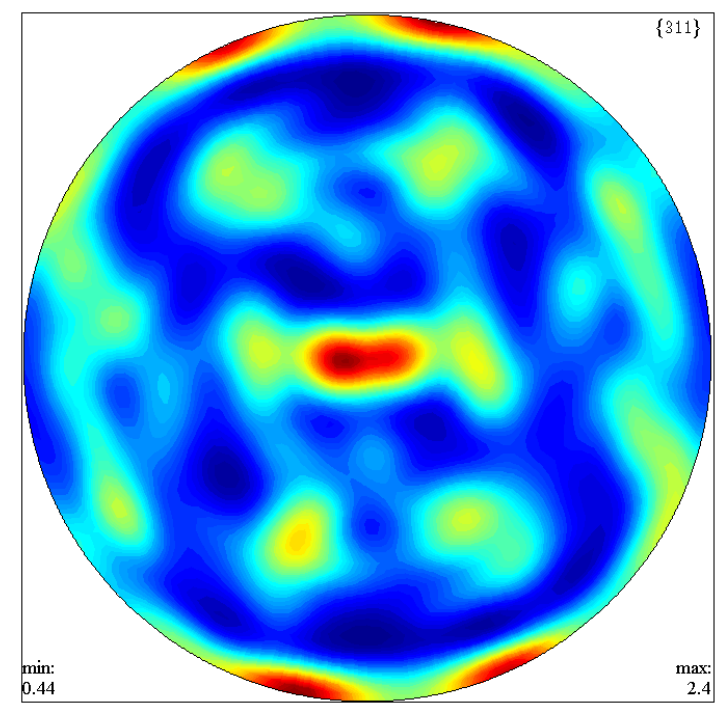

Figure 20: The (311) completed pole figure for the $\mathrm{Cu}$ diffraction data set.

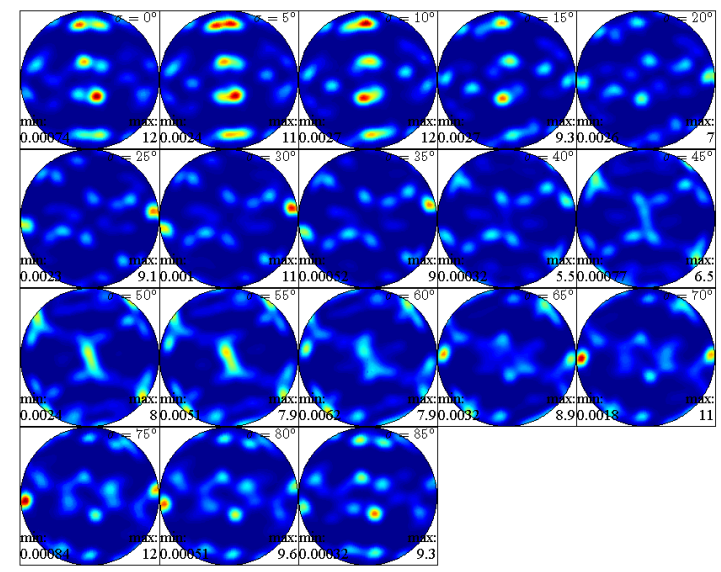

Figure 21: The ODF for the $\mathrm{Cu}$ diffraction data set. 


\title{
X-Ray Tomographic Reconstruction
}

\author{
Bonnie Schmittberger \\ Science Undergraduate Laboratory Internship Program
}

Bryn Mawr College

SLAC National Accelerator Laboratory

Menlo Park, California

August 14, 2009

Prepared in partial fulfillment of the requirement of the Department of Energy's Science Undergraduate Laboratory Internship program under the direction of Samuel Webb at the Stanford Synchrotron Radiation Lightsource at SLAC National Accelerator Laboratory.

Participant:

\section{Signature}

Project Advisor:

\author{
Signature
}




\section{Table of Contents}

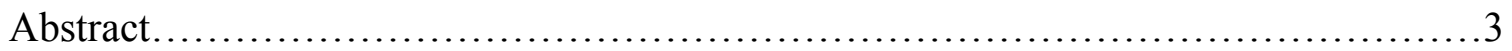

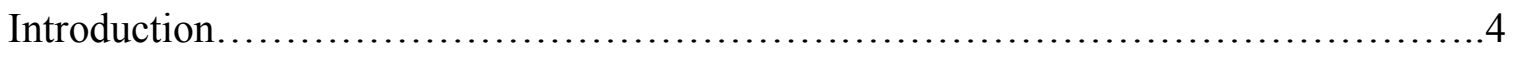

Materials and Methods.......................................................

Results............................................................... 10

Discussion.............................................................. 10

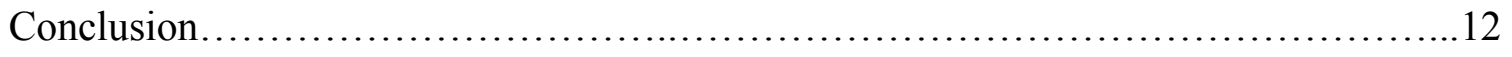

Acknowledgments...................................................... 12

References............................................................. 13

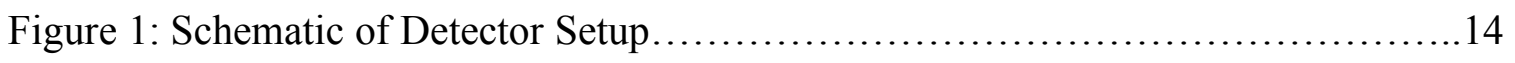

Figure 2: Calcium and Iron Tomographic Scans.................................. 15

Figure 3: Schematic of Axis Setup .......................................... 16

Figure 4: Tomographic Scan and Reconstruction of Arabidopsis Thaliana..............17

Appendix: Copy of Reconstruction Code......................................... 18 


\begin{abstract}
X-Ray Tomographic Reconstruction. BONNIE SCHMITTBERGER (Bryn Mawr College, Bryn Mawr, PA, 19010) DR. SAMUEL WEBB (Stanford Synchrotron Radiation Laboratory at SLAC National Acceleratory Laboratory, Menlo Park, CA 94025)
\end{abstract}

Tomographic scans have revolutionized imaging techniques used in medical and biological research by resolving individual sample slices instead of several superimposed images that are obtained from regular X-ray scans. X-Ray fluorescence computed tomography, a more specific tomography technique, bombards the sample with synchrotron x-rays and detects the fluorescent photons emitted from the sample. However, since $\mathrm{x}$-rays are attenuated as they pass through the sample, tomographic scans often produce images with erroneous low densities in areas where the x-rays have already passed through most of the sample. To correct for this and correctly reconstruct the data in order to obtain the most accurate images, a program employing iterative methods based on the inverse Radon transform was written. Applying this reconstruction method to a tomographic image recovered some of the lost densities, providing a more accurate image from which element concentrations and internal structure can be determined. 


\section{INTRODUCTION}

$\mathrm{X}$-Ray fluorescence computed tomography (XFCT) is a synchrotron-based imaging technique used for mapping the distribution of elements within a sample. In $\mathrm{XFCT}$, a sample is bombarded with $\mathrm{x}$-rays that excite k-shell electrons. When these atoms return to their stable state, they emit fluorescent x-rays at energies characteristic of the element. These photons are collected by a solid state silicon detector that records multiple energies simultaneously. The total number of photons recorded is a function of the sum of the various element concentrations along the line of the incident beam. By rotating the object and compiling horizontal scans, it is possible to obtain a complete tomographic reconstruction of the distribution of the elements within a sample.

Since the incident beam is attenuated through the sample and part of the emission is absorbed by the sample, attenuation correction is necessary in order to obtain accurate results. If reconstruction techniques are not employed, the image of the center of the sample is blurred, and its density, as recorded by the scan, is much lower than its true density. Previous reconstruction techniques required a known attenuation at each fluorescence energy, which necessitated the time-consuming process of rescanning the sample at all the relevant energies [1]. Tomographic reconstruction is also possible by a series of mathematical corrections based on the inverse Radon transform, which is a faster and simpler method.

These reconstruction techniques have attracted numerous scientific disciplines to XFCT. In particular, the high sensitivity and sub-micrometer resolution of this method is useful in medicine [2]. The presence of metals and other trace elements drastically affect intracellular processes in any organism [3]. XFCT is the only sub-micrometer technique 
that can map these elements within cells and search for abnormal quantities and distributions accompanying the development of certain diseases [3].

\section{MATERIALS AND METHODS}

\section{i. Data Collection}

Once the code was completed, data was collected at the Stanford Synchrotron Radiation Lightsource. X-Rays obtained from the synchrotron are sent through an ion chamber to measure the energy of the incident beam. The x-rays are then directed into a helium-purged chamber where they are focused down to a $2 \mu \mathrm{m}$ diameter by two elliptical mirrors. This focused beam is then sent out to the sample, which is scanned by moving completely across the incident beam, then rotating by a certain small angle, typically 1 to 3 degrees, and repeating until 180 degrees are covered. This is called a full translation, half rotation tomographic scan. If the scan were to cover a full rotation, the amount of attenuation correction would be minimized because the image would only contain artifacts towards the center of the sample, but that process doubles the scanning time, generally requiring three to four extra hours.

The detector is placed behind the sample to collect the transmitted x-rays, and another is placed at 90 degrees to the incident beam to collect the fluorescent photons, as depicted in Figure 1. A uniform fluorescence around the sample is assumed, so that one fluorescent photon detector is sufficient. Because elements have signature fluorescence energies, a fluorescent photon detector that can distinguish different photon energy levels is used. This detector counts the number of photons that it receives at each energy level, so the concentrations of different elements in the sample can be determined. 


\section{ii. Basic Reconstruction Technique}

The data from a tomographic scan is formatted into a two-dimensional matrix composed of pixel intensities. Assuming uniform attenuation throughout the sample, a simple reconstruction method employs the inverse Radon transform.

The inverse Radon transform is the fundamental method for image reconstruction because the Radon Transform acts as a continuous mathematical model for exponentially decreasing density based on a coefficient. A line integral represents the total attenuation of an x-ray as it travels in a straight line through the sample. A tomographic image can be defined by $f(x, y)$, and the attenuation is therefore represented by

$$
P_{\theta}(t)=\int_{(\theta, t)} f(x, y) d s,(1)
$$

where $(\theta, t)$ are the line integral parameters. This can be rewritten as

$$
P_{\theta}(t)=\int_{-\infty}^{\infty} \int_{-\infty}^{\infty} f(x, y) \delta(x \cos \theta+y \sin \theta-t) d x d y,(2)
$$

which is the definition of the Radon transform [4].

To understand the effects of attenuation in XFCT, a Radon transform can be applied to simulated data to create an approximate attenuated image. Figure 2(a) shows a simulated sample of iron without correction for attenuation. Applying an inverse Radon transform with the proper attenuation coefficient produces Figure 2(b), the reconstructed image. Figure 2(c) and 2(d) are analogous images of a calcium sample. The calcium sample is originally more attenuated than the iron sample because the fluorescent photon energies of calcium are lower than those of iron, which makes it harder for them to escape the sample.

A simple reconstruction method takes the inverse Radon transform of the data, and creates a second matrix that defines the shape of the sample, having a "1" wherever 
the object is, and a " 0 " everywhere else. The absorption effect can be estimated by taking the Radon Transform of the shape, which is analogous to the survival probability of a photon reaching a point in the sample. Defining this transform as $f(\theta, s, u)$, it can then be estimated that

$$
f(\theta, s, u)=\exp \left(-\int_{-\infty}^{u} \mu\left(s, u^{\prime}\right) d u^{\prime}\right)
$$

where $\mu$ is the attenuation coefficient, and $(s, u)$ is a point in the matrix as shown in Figure 3, where the s and $\mathrm{u}$ axes are parallel to the translation direction and the beam, respectively [5]. The exponential in Equation 1 is therefore the absorption effect. Dividing the initial data by the absorption effect will give the corrected data, and taking the inverse Radon transform of that will give the final corrected image.

This method is useful for ideal samples with uniform attenuation. Otherwise, this technique only works for very small attenuation coefficients on the order of $\mu=0.01$. For realistic situations, a much more comprehensive method is needed. It must be able to integrate over varying densities with different attenuation coefficients. A successful technique is described in Part II; it employs iterative methods for image reconstruction.

\section{iii. Algorithm Theory}

The number of emitted photons detected from a given point in the sample can be defined as

$$
d(t, \theta)=\mathbf{R}_{W} f(t, \theta)=\int_{x \cdot \xi=t} W(x, \theta) f(x) d x,
$$

where $t$ and $\theta$ are parameters defining the line, $\xi=(\cos \theta, \sin \theta)[6]$. The first integrated function, 


$$
W(x, \theta)=\exp [-\mathrm{D} \lambda(x, \theta+\pi)] \int_{\gamma_{1}}^{\gamma_{2}} \exp [-\mathrm{D} \mu(x, \theta+\gamma) d \gamma],
$$

accounts for the different fluorescence energies of elements and gives more weight to elements whose fluorescence energies are lower [6]. The limits of integration refer to the angle range $\left[\gamma_{1}, \gamma_{2}\right]$ at each emission point [6]. The operator $\mathbf{D}$ is the divergent beam transform defined by

$$
\mathrm{D} \mu(x, \theta)=\int_{0}^{\infty} \mu\left(x+q \xi^{\perp}\right) d q,(6)
$$

where $\mu$ is the estimated fluorescence attenuation coefficient and $\lambda$ is the known transmission coefficient [6]. When $\mu=\lambda=0, \mathbf{R}_{W}$ is the general Radon transform [6]. Therefore, similar to the method used in Part I, the attenuation problem can be solved by inverting the operator $\mathbf{R}_{W}[6]$.

When $\mu=0$, the density of a sample can be approximated as

$$
f_{c}(x)=\frac{1}{2 \pi} \frac{\mathbf{R}^{-1}\{d\}(x)}{a(x)},(7)
$$

where

$$
a(x)=\frac{1}{2 \pi} \int_{0}^{2 \pi} W(x, \theta) d \theta
$$

is the correction factor [6]. Miqueles and De Pierro [4] discovered that this general formula is actually the first iteration of an iterative method, where each iteration consists of an inverse Radon Transform $\mathbf{R}^{-1}$. They define the sequence

$$
f^{(k+1)}=f^{(k)}+\alpha_{k} e^{(k)},
$$

where $\alpha_{k}$ is the positive relaxation factor and $e^{(k)}$ is defined as

$$
e^{k}=\frac{\mathbf{R}^{-1}\left(d-\mathbf{R}_{W} f^{(k)}\right)}{a}[6]
$$


Therefore the complete reconstruction algorithm by iteration is

$$
f^{(k+1)}(x)=f^{(k)}(x) \frac{\mathbf{B}_{W} d^{(k)}(x)}{\mathbf{B}_{W} e(x)}
$$

where $e(t, \theta)=1$, and

$$
d^{(k)}(t, \theta)=\frac{d(t, \theta)}{\mathbf{R}_{W} f^{(k)}(t, \theta)}[4] .
$$

$\mathbf{B}_{W}$ is the attenuated backprojection operator, defined as

$$
\mathbf{B}_{W} d(x)=\int_{0}^{2 \pi} W(x, \theta) d(x \cdot \xi, \theta) d \theta,
$$

and it can be shown that it is the adjoint operator of $\mathbf{R}_{W}[6]$.

This method was translated into a code that processes images from tomographic scans. The matrix data was corrected so that each pixel was given a size of 1 . This method was then applied, treating each element of the matrix as a point in space.

In order to translate this into a working code, the algorithm from Equation 9 was applied to every point in the tomographic image for both a beam attenuation correction, which accounts for any attenuation from the source to a point in the sample, and for a fluorescence attenuation correction, which accounts for any attenuation from that point in the sample to the detector. From both sets of attenuation, a matrix of absorption corrections was constructed. A filter was applied to the initial data, which distinguished the areas where the most attenuation occurred and where the least attenuation occurred. In order to get the final image, the filtered image was divided by the absorption corrections at each pixel. 


\section{RESULTS}

This method was applied to a tomographic scan of a stem of the plant Arabidopsis thaliana with an estimated attenuation coefficient of 0.08 . The initial scan is shown in Figure 4a, and its inverse Radon transform is shown in Figure 4b. The tomographic image with the applied reconstruction is shown in Figure 4c, and while the elimination of attenuation artifacts is not immediately noticed, data analysis showed an improvement.

In order to quantitatively measure the effect of the reconstruction program, the original data was subtracted from the corrected data, providing a matrix of absorption corrections that had been made. The maximum attenuation occurred in the back of the sample, opposite from the incident x-rays, which is expected from a half rotation, full translation scan. The pixels in the back of the sample had lost at least $16 \%$ of their original density, which was added back during the reconstruction.

Because there are still attenuation artifacts present in the corrected image, the inputted attenuation coefficient was not high enough. Inputting coefficients that are too low barely affect the attenuated data, and inputting coefficients that are too high add too much density back to the image and results in a loss of real data. A solution to this problem is discussed further in Section $4 i$.

\section{DISCUSSION}

\section{i. Method Discussion}

The proposed method yields accurate images that provide more detailed information about the sample. Although running the program requires approximately an 
hour, changing with the size of the sample, it is much faster than the time that would need to be allotted to take a full translation, full rotation scan.

A potential disadvantage of the method is the required estimation for the minimum and maximum attenuation coefficients. An ideal attenuation coefficient input is a matrix of varying attenuation coefficients that can be constructed from the density map of the sample obtained from the transmission photon detector. Once an estimated matrix is entered, the iterative method from Equation 11 can be applied to adjust the coefficients until an ideal image is obtained. Miqueles and De Pierro [4] found that using several iterations will limit the attenuation coefficients and further improve the data.

The reconstruction code is given as an appendix. The filtering functions and the mean correlated matrix function perform the reconstruction and create a two-dimensional array of self-absorption corrections. These functions can be added to any module that processes tomographic scans. Dividing the inverse Radon transform of the original scan by this matrix will produce the corrected tomographic image; a simple version of this is given in the fluorescent matrix function, but most modules already include an inverse Radon transform code.

\section{ii. Sample Discussion}

The sample used was brought to SSRL by Tracy Punshon from Dartmouth College. The plant Arabidopsis thaliana is of interest because it is the first plant to have its entire genome sequenced, thus being a model organism for understanding plant biology and plant traits. This sample was the wild type Arabidopsis, and other measurements consisted of plants that had a gene removed for iron transport; the intention was to study how the distribution of iron changes without the gene for iron 
transport.

This is significant for the current interest in trying to figure out ways to encourage iron transport in other plants, such as rice. If the process can be encouraged, rice could be infused with iron, which could potentially solve the growing iron deficiency problems in developing countries.

\section{CONCLUSION}

XFCT has the potential to provide incredibly informative images once a reconstruction has been applied to the data. An effective reconstruction technique employs the inverse Radon transform and accounts for changing attenuation coefficients and the variance of fluorescence energies. The attenuation coefficient map is the key input for a successful reconstruction, and its creation can be perfected by using iterative methods. Using a reconstruction method can provide accurate results despite the large attenuation effects accumulated from a half rotation scan. By correcting for the attenuation effects, the inaccuracies associated with XFCT become nearly irrelevant, which makes it very attractive, especially to the biological and medical communities. This method has become vital for the analysis and mapping of elemental content in cells and tissues.

\section{ACKNOWLEDGEMENTS}

This data was obtained at the Stanford Synchrotron Radiation Lightsource at SLAC National Accelerator Laboratory. I would like to thank my mentor, Samuel Webb, for his help and guidance, as well as his persistent positive encouragement throughout 
this program. I would like to thank Tracy Punshon for providing interesting samples, and Apurva Mehta for all his feedback. I would also like to thank Stephen Rock, SueVon Gee, Elizabeth Smith, and Vivian Lee for their support of the SULI Program. Finally, I would like to thank the Department of Energy and Stanford University for the opportunity to participate in this internship.

\section{REFERENCES}

[1] P. La Rivière, D. Billmire, P. Vargas, M. Rivers, S. Sutton, Penalized-likelihood image reconstruction $x$-ray fluorescence computed tomography, Optical Engineering, Vol. 45(7). (2006)

[2] C. Schroer, Reconstructing $x$-ray fluorescence microtomograms, Applied Physics Letters, Vol. 79, Number 12. (2001)

[3] T. Paunesku, S. Vogt, J. Maser, B. Lai, G. Woloschak, X-Ray Fluorescence Microprobe Imaging in Biology and Medicine, Journal of Cellular Biochemistry 99, 1489-1502. (2006)

[4] A. Kak, M. Slaney, Principles of Computerized Tomographic Imaging, Society for Industrial and Applied Mathematics, IEEE Press, New York. (2001)

[5] A. Brunetti, B. Golosio, Software for X-Ray fluorescence and scattering tomographic reconstruction, Computer Physics Communications 141, 412-425. (2001)

[6] E. Miqueles, A. De Pierro, Fluorescence Tomography: Reconstruction By Iterative Methods, Applied Mathematics Department, State University of Campinas, Brazil. (2008) 


\section{FIGURES}

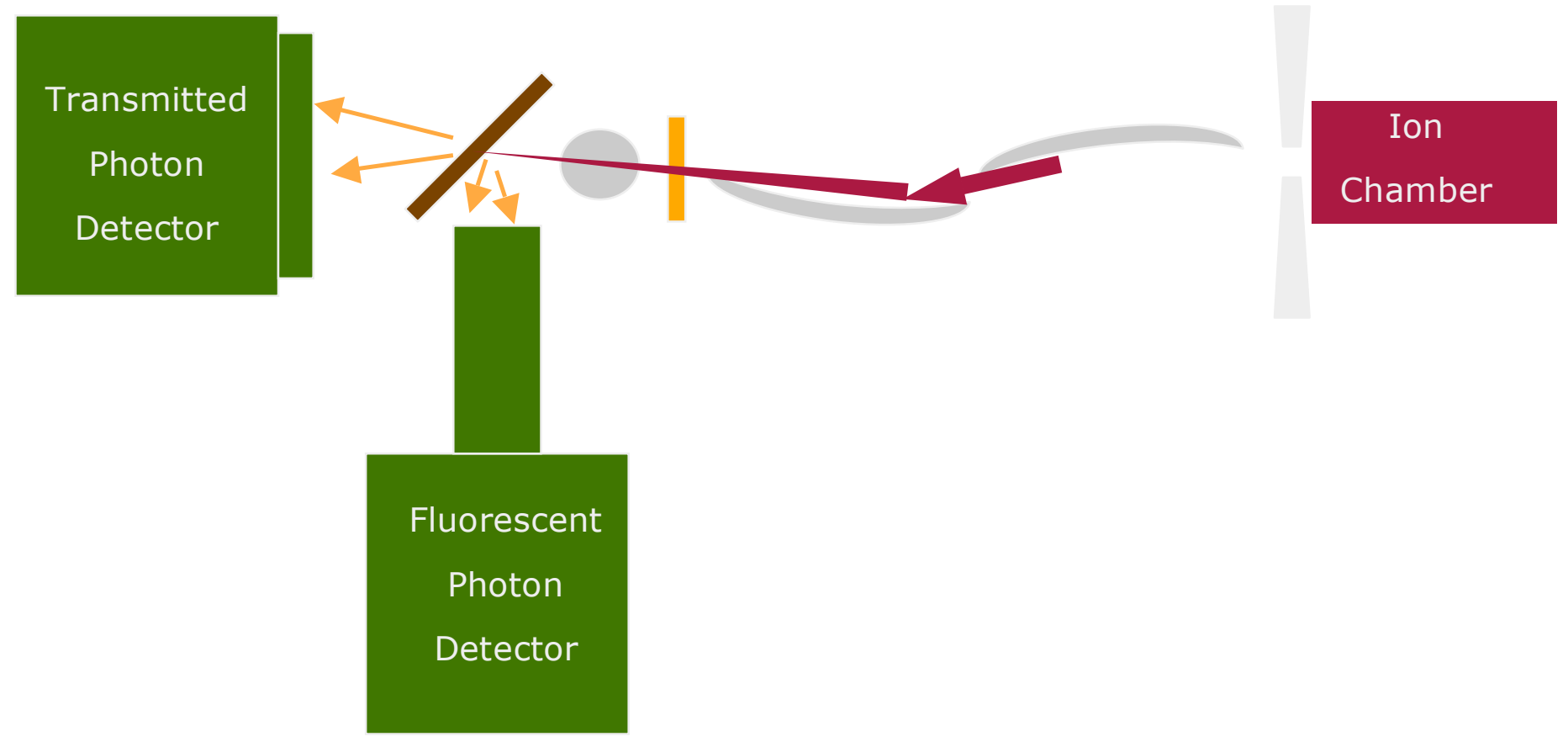

Figure 1: Schematic of experimental setup. 


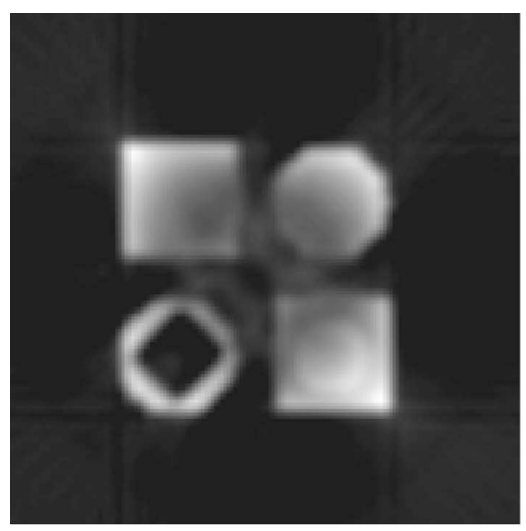

(a)

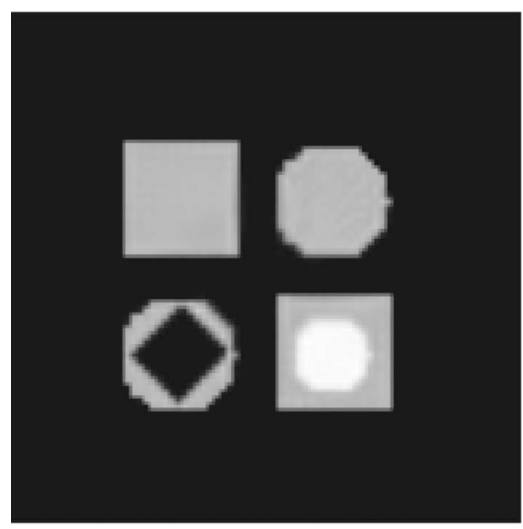

(b)

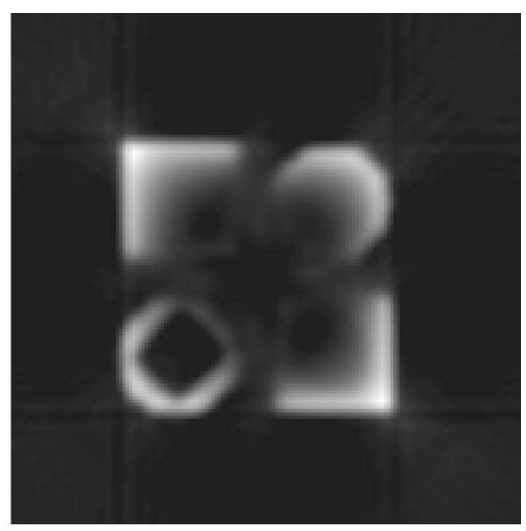

(c)

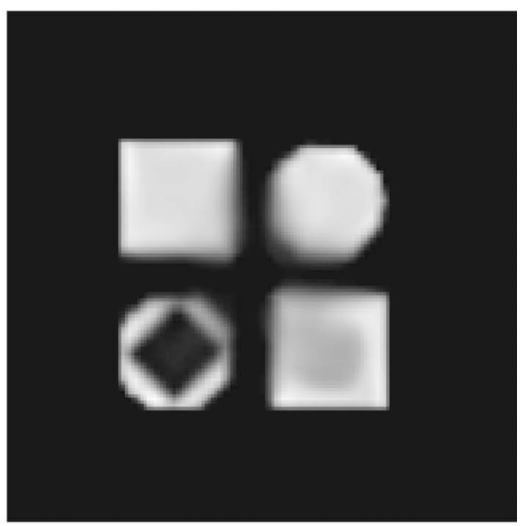

(d)

Figure 2: Simulated iron and calcium distributions. (a) Iron without correction for attenuation. (b) Iron with correction for attenuation. (c) Calcium without correction for attenuation. (d) Calcium with correction for attenuation. [1] 


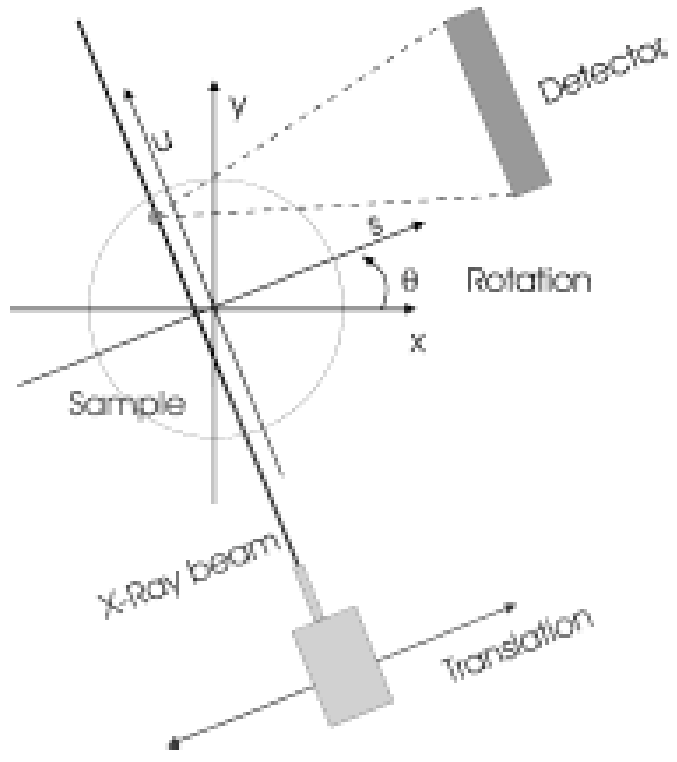

Figure 3: Diagram of fluorescence tomography [5]. 


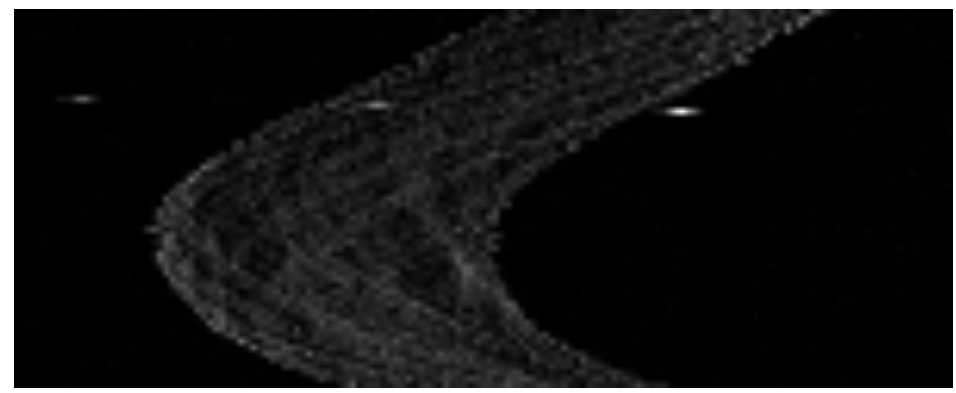

(a)

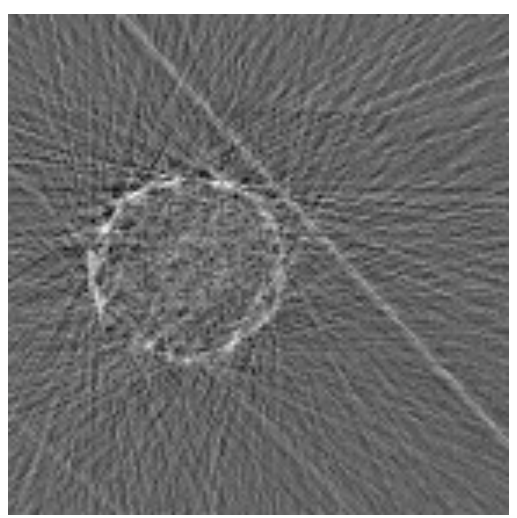

(b)

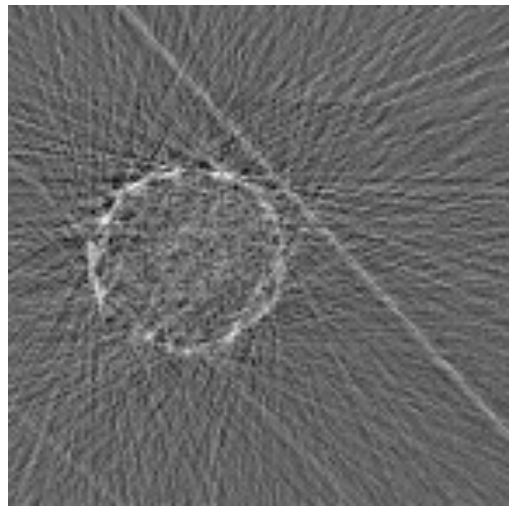

(c)

Figure 4: Tomographic scan and reconstruction of Arabidopsis thaliana. (a) The initial scan. (b) The inverse Radon transform of the scan, producing the tomographic image. (c) The fluorescence corrected image. 


\section{APPENDIX}

Copy of Reconstruction Code:

from math import *

from numpy import *

class Mkcorralgo:

def__init_(self, x, y, Dim, Dim2, PhiMin, PhiMax, PhiStep, DetPos, DetPixelNum, DetArea, data_file, AttLowVal, AttHiVal, AttLowSize, AttHiSize, CorrMatrixSize,

Alpha, SLeft, SRight, Scan):

self. $\mathrm{x}=\mathrm{x} \#$ used as spatial $\mathrm{x}$-coordinate of the pixel

self. $y=y \#$ used as spatial $y$-coordinate of the pixel

self.xIdx $=0$

self.yIdx $=0$

self.Dim $=$ Dim \# Number of pixels across one sample axis, assuming square sample

self.Dim2 = Dim2 \# Number of angles covered by sample rotation

self.PhiMin $=$ PhiMin*pi/180.

self.PhiMax $=$ PhiMax*pi/180.

self.PhiStep $=$ PhiStep \# Angle Incrementation

self.DetPos = DetPos \# Distance from center of sample to detector

self.DetPixelNum = DetPixelNum \# Number of pixels across one sample axis

self.DetArea $=$ DetArea \# Area of detector

self.DetLen $=2 *$ math.sqrt(DetArea/pi)

self.DetPixelSize $=$ self.DetLen/self.DetPixelNum

self.data $=$ data_file

self.FluorAbsorptionArray = zeros((self.Dim), float)

\# Integrals of the absorption coefficient at fluorescence energy

self.AttLow $=$ zeros((self.Dim,self.Dim),float $)$

self.AttLow $=$ AttLowVal \# Matrix of absorption coefficients at fluorescent energy

self.AttLowSize $=$ AttLowSize \# Physical size of sample along one axis

self.AttLowDim = self.Dim

self.AttHi $=$ zeros((self.Dim, self.Dim),float $)$

self.AttHi = AttHiVal \# Matrix of absorption coefficients at beam energy

self.AttHiSize $=$ AttHiSize \# Physical size of sample along one axis

self.AttHiDim = self.Dim

self.maxDim $=$ self.Dim

self.SLeft $=$ SLeft \# Parameter of self.Proj

self.SRight $=$ SRight \# Paramter of self.Proj

self.Scan $=$ Scan \# Parameter of self.Proj

self.ProjNum $=\operatorname{int}(($ self.PhiMax - self.PhiMin $) /$ self.PhiStep $)+1$

self.ProjSize $=$ self.SRight - self.SLeft \# for self.Proj

self.ScanNum = int(self.ProjSize/Scan) \# for self.Proj

self.CorrMatrixDim = Dim

self.CorrMatrixSize $=$ CorrMatrixSize

self.FluorMatrixDim $=$ Dim 
self.FluorMatrixSize $=$ self.CorrMatrixSixe*self.Dim

self.Rho2 $=0$ \# Distance from the fluorescence point to detector points

self.FilteredProj $=$ zeros((self.ScanNum, self.ProjNum), float $)$

self.MeanCorrMatrix = zeros((self.Dim, self.Dim $)$, float $)$

self.FluorMatrix = zeros((self.Dim, self.Dim), float) \# Fluor Reconstruction Image

self.Filter $=$ zeros(self.ScanNum, float) \# Filter for backprojection

self.alpha $=$ Alpha

self.FluorAbs $=0$

\# Orthogonal distance from the fluorescence point to the detector

def idxToCoord(self, Idx, Dim, Size):

\# Converts an array index to a spatial coordinate.

return (float(Idx)/float(Dim))*Size

def coordToAngle(self, $\mathrm{x}, \mathrm{y})$ :

\# Finds the polar angle of a point $(x, y)$.

$\mathrm{r}=\operatorname{sqrt}\left(\mathrm{x}^{*} \mathrm{x}+\mathrm{y} * \mathrm{y}\right)$

if $\mathrm{r}<=\mathrm{x}$ :

return 0

Phi $=\operatorname{acos}(\mathrm{x} / \mathrm{r})$

if $\mathrm{y}<0$ :

Phi $=2 *$ pi - Phi

return Phi

else:

return Phi

def fluorAttenuation(self, $x, y$, Phi):

\# Attenuation of the fluorescent photons from point $(\mathrm{x}, \mathrm{y})$ to the detector.

$\mathrm{xDet} 0=$ self.DetPos* $\cos (\mathrm{Phi}) \#$ Coordinates of detector center.

$y \operatorname{Det} 0=$ self.DetPos* $\sin (\mathrm{Phi})$

self.FluorToDetDist $=$ self.DetPos - self. $x * \cos ($ Phi $)-$ self.y*sin $($ Phi $)$

\#The detector surface is sampled.

$\mathrm{xDet}=\mathrm{xDet} 0$

$\mathrm{yDet}=\mathrm{yDet} 0$

$\mathrm{x} 1=\mathrm{xDet}-\mathrm{x}$

$\mathrm{y} 1=\mathrm{yDet}-\mathrm{y}$

self.Rho $2=x 1 * x 1+y 1 * y 1$ \# Square distance from $(x, y)$ to the pixel.

Gamma $=$ self.coordToAngle $(x 1, y 1)$ \# Polar angle from $(x, y)$ to the pixel.

integ $=$ ArrayIntegral $(\mathrm{x}, \mathrm{y}$, Gamma, self.AttLow, 0, 0,

self.AttLowSize/self.AttLowDim, self.AttLowDim, self.AttLowDim, self.maxDim)

\# Integral of the absorption coefficient at fluorescence energy $\backslash$

\# from (x, y) to the pixel.

self.FluorAbs = integ.integral()

return self.planeAttenuation() 
def planeAttenuation(self):

self.Attenuation $=0$

\# Integrates the attenuation from $(\mathrm{x}, \mathrm{y})$ to the detector surface in the $\backslash \backslash$

\# angular range covered by the detector.

for $i$ in range(self.DetPixelNum):

DetIdx $=\mathrm{i}$

DGamma $=$ self.DetPixelSize*self.FluorToDetDist/self.Rho2

self.Attenuation $+=\exp$ (-self.FluorAbs)*DGamma

def beamAttenuation(self, $x, y, P h i)$ :

\# Finds the attenuation of the $\mathrm{x}$-ray beam from the source to $(\mathrm{x}, \mathrm{y})$.

PhiBeam $=$ Phi - pi $/ 2$

integ $=$ ArrayIntegral(x, y, PhiBeam, self.AttHi, 0, 0, self.AttHiSize/self.AttHiDim, self.AttHiDim, self.AttHiDim, self.maxDim)

temp $=$ integ.integral ()

self.beam $=\exp (-$ temp $)$

def makeCorrMatrix(self):

\# Evaluates and stores 2-D array of self absorption corrections.

for $\mathrm{i}$ in range(int(self.ProjNum)):

for $\mathrm{j}$ in range(int(self.CorrMatrixDim)):

for $\mathrm{k}$ in range(int(self.CorrMatrixDim)):

print $\mathrm{i}, \mathrm{j}, \mathrm{k}$

AngleIdx $=\mathrm{i}$

$\mathrm{xIdx}=\mathrm{j}$

$\mathrm{yIdx}=\mathrm{k}$

if AngleIdx $==0$ :

self.MeanCorrMatrix $[\mathrm{xIdx}, \mathrm{yIdx}]=0$

$\mathrm{x}=$ self.idxToCoord(xIdx, self.CorrMatrixDim, self.CorrMatrixSize)

$y=$ self.idxToCoord(yIdx, self.CorrMatrixDim, self.CorrMatrixSize)

Phi $=$ self.PhiMin + AngleIdx*self.PhiStep

self.beamAttenuation(x, y, Phi)

self.fluorAttenuation(x, y, Phi)

self.CorrElem $=$ self.beam $*$ self.Attenuation

self.MeanCorrMatrix[xIdx, yIdx] = self.CorrElem/self.ProjNum

def makeFluorMatrix(self):

self.makeCorrMatrix()

self.tomoFilter(self.data, 1)

\# Evaluates fluorescence image through backprojection algorithm using

\# self absorption corrections data.

Delta $=$ pi/self.ProjNum

for $\mathrm{i}$ in range(self.FluorMatrixDim):

for $\mathrm{j}$ in range(self.FluorMatrixDim): 


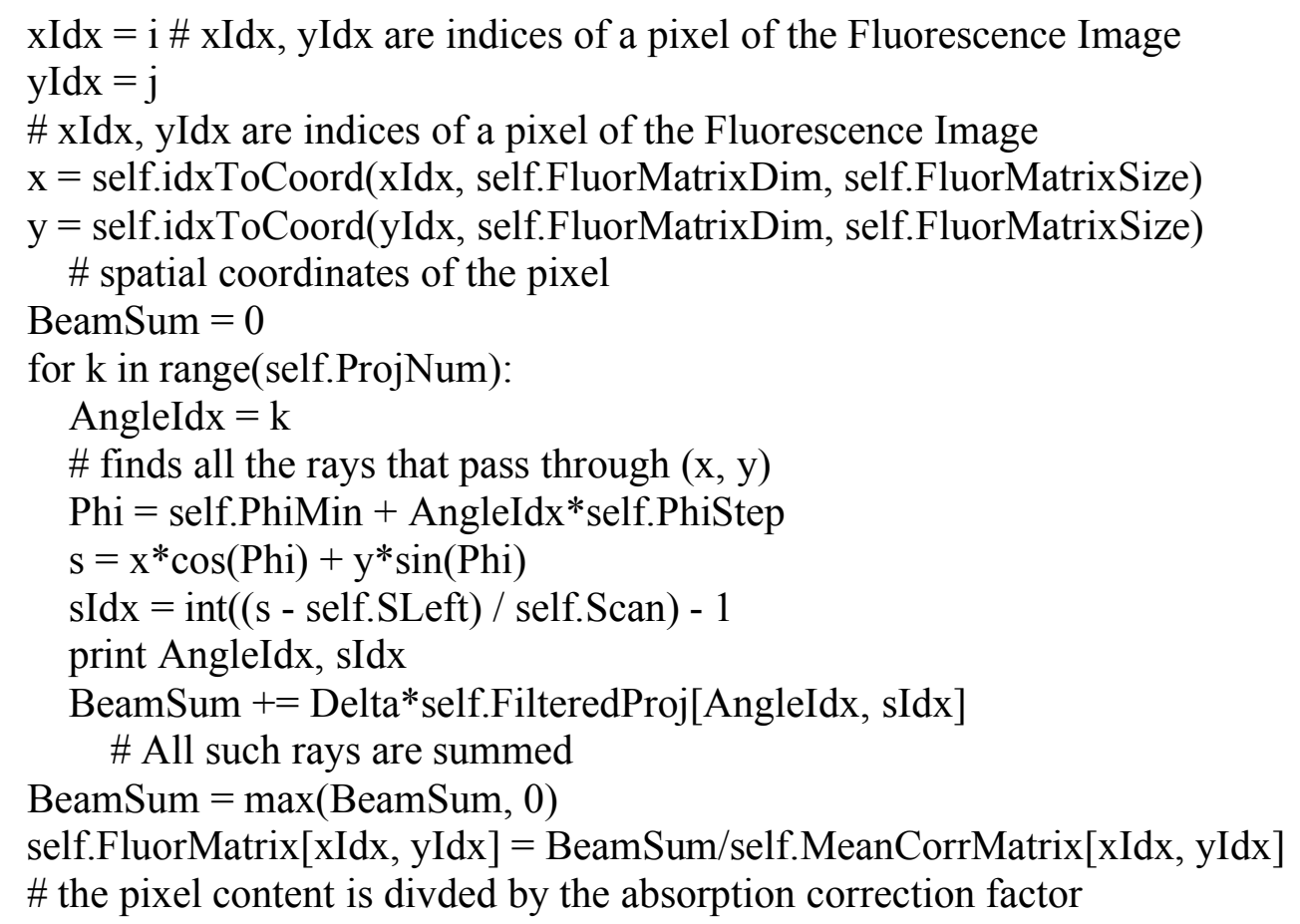

def SLFilter(self, $x, d)$ :

\# if self.alpha $==0$, the Shepp-Logan filter is used

Filter $=\left(\mathrm{pi}^{* * 2}\right) * \mathrm{~d} *(1-4 *(\mathrm{x} * * 2))$

for $i$ in range(len(Filter) - 1):

if $\operatorname{abs}($ Filter $[i])<1 \mathrm{e}-6$ :

Filter $[i]=0.001$

return $2 /$ Filter

def RLFilter(self, $x, d)$ :

\# if self.alpha $>0$, the Ram-Lak Filter calculated and then the GH Filter is used

$\mathrm{q}=\mathrm{x}$

$\mathrm{y}=-(\sin (\mathrm{x} * \mathrm{pi} / 2)) * 2 /((\mathrm{pi} * * 2) *(\mathrm{q} * * 2) * \mathrm{~d})$

for $\mathrm{i}$ in range $(\operatorname{len}(\mathrm{q}))$ :

if $(x[i]-\operatorname{int}(d / 2))==0$ :

$\mathrm{y}[\mathrm{i}]=1 /\left(4 \cdot{ }^{*} \mathrm{~d}\right)$

return y

def GHFilter(self, x, d):

\# the General Hamming Filter

return self.alpha*self.RLFilter $(\mathrm{x}, \mathrm{d})+0.5^{*}(1-$ self.alpha $) *(\operatorname{self} . R L F i l t e r(\mathrm{x}-1, \mathrm{~d})+$ self.RLFilter $(x+1, d))$

def tomoFilter(self, image, d, filter_size $=0$ ):

dims $=$ shape (image)

if not filter_size:

filter_size $=\operatorname{int}(\operatorname{dims}[0] / 4$. $)$ 


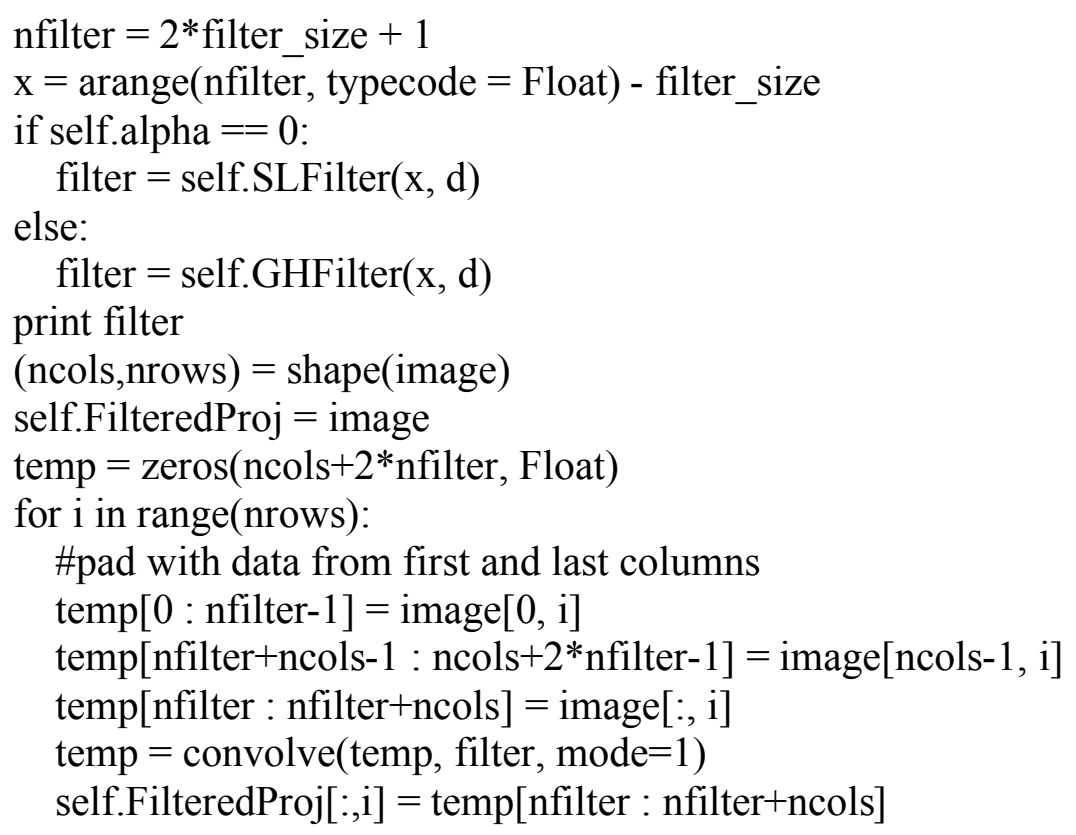

class ArrayIntegral:

def__init_(self, X, Y, Phi, Array, XRect, YRect, PixelSize, Nx, Ny, MAXNY):

self.XRect $=$ XRect \# X-component of corner of the rectangle

self.YRect $=$ YRect \# y-component of corner of the rectangle

self. $\mathrm{Nx}=\mathrm{Nx}$ \# width of the rectangle*PixelSize

self.Ny $=\mathrm{Ny} \#$ height of the rectangle*PixelSize

self.array $=$ Array \# the two-dimensional distribution

self.PixelSize = PixelSize \# ...pretty self-explanatory

self.maxNy = MAXNY \# maximum height of the rectangle

self.Dx $=1$ \# incrementation in $\mathrm{x}$-direction

self.Dy $=1$ \# incrementation in $y$-direction

self.Phi $=$ Phi \# angle from $\mathrm{x}$-axis to point $(\mathrm{x}, \mathrm{y})$

self. $x=(X-X R e c t) /$ PixelSize \# reduced $x$-coordinate

self.y $=(Y-Y R e c t) /$ PixelSize \# reduced y-coordinate

self.IncX $=\{\}$

self.IncY $=\{\}$

self.IncX $[0]=$ self.Dx

self.IncX $[1]=$ self.Dx

self.IncY $[0]=$ self.Dy

self.IncY $[1]=$ self.Dy

self.length $=0$

self.Integral $=0$

self.finalIntegral $=0$

def xMatrixCheck(self):

\# Checks if the starting point $(\mathrm{x}, \mathrm{y})$ is outside the rectangle.

if self. $x<0$ : 
\# If $\mathrm{x}<0$, finds the intersection of the ray with the $\mathrm{x}=0$ axis.

if self. $x+\cos ($ self.Phi) $!=$ self.x:

xAlpha $=$ self.y - self. ${ }^{*} \sin ($ self.Phi) $/ \cos ($ self.Phi)

if $x$ Alpha $>=0$ and $x$ Alpha $<=$ self.Ny:

\# If the intersection lies on the rectangle edge,it is taken

\# as a new starting point for the integral.

self. $x=0$

self.y $=x A l p h a$

elif self. $x>$ self.Nx:

\# If $x>N x$, finds the intersection of the ray with the $x=N x$ axis.

$\mathrm{xDelta}=$ self.Nx - self. $\mathrm{x}$

if $x$ Delta $+\cos ($ self.Phi) $!=x$ Delta:

$x A l p h a=$ self.y $+x$ Delta* $\sin ($ self.Phi $) / \cos ($ self.Phi)

if $x$ Alpha $>=0$ and $x$ Alpha $<=$ self.Ny:

self. $x=$ self.Nx

else:

self. $y=x$ Alpha

\# The $\mathrm{x}$-coordinate is in the rectangle

self. $x=$ self. $x$

def yMatrixCheck(self):

if self.y $<0$ :

\# If $y<0$, finds the intersection of the ray with the $y=0$ axis.

if self.y $+\sin ($ self.Phi) != self.y:

yAlpha $=$ self.x - self. $y * \cos ($ self.Phi $) / \sin ($ self.Phi $)$

if yAlpha $>=0$ and yAlpha $<=$ self.Nx:

self. $y=0$

self. $x=y A l p h a$

elif self.y > self.Ny:

\# If $y>N y$, finds the intersection of the ray with the $y=N y$ axis.

yDelta $=$ self.Ny - self.y

if yDelta $+\sin ($ self.Phi) != yDelta:

yAlpha $=y$ Delta $* \cos ($ self.Phi $) / \sin ($ self.Phi)

if yAlpha $>=0$ and yAlpha $<=$ self.Nx:

self.y $=$ self.Ny

self. $x=y A l p h a$

else:

\# The y-coord is in the rectangle

self.y $=$ self.y

def integral(self):

self.xMatrixCheck

self.yMatrixCheck

self.ix $=$ int(self.x $) \#$ integral part of $x$ 


$$
\begin{aligned}
& \text { self.iy }=\text { int(self.y) \# integral part of y } \\
& \text { self.tx }=\text { self. } x \text { - self.ix \# fractional part of } x \\
& \text { self.ty }=\text { self.y - self.iy \# fractional part of } y \\
& \text { if self.ix }==\text { self. } N x: \\
& \quad \text { self.ix }=\text { self.ix - } 1 \\
& \text { self.tx }=1 \\
& \text { if self.iy }==\text { self.Ny: } \\
& \text { self.iy }=\text { self.iy - } 1 \\
& \text { self.ty }=1
\end{aligned}
$$

\section{\# Angle Restriction}

\# Used to reduce the problem to the case of $0<\mathrm{Phi}<\mathrm{pi} / 4$.

if self.Phi < 0 : \#makes Phi element of [0, 2pi]

while self.Phi $<0$ :

$$
\text { self.Phi }=\text { self.Phi }+2 * \text { pi }
$$

if self.Phi > pi: \#makes Phi element of $[0$, pi]

$$
\text { self.Phi }=2 * \text { pi - self.Phi }
$$

self.Dy $=-1$

self.ty $=1-$ self.ty

if self.Phi > pi/2: \#makes Phi element of $[0, \mathrm{pi} / 2)$

self.Phi $=$ pi - self.Phi

self.Dx $=-1$

self.tx $=1-$ self.tx

\# When the line integral crosses from one pixel to another, only one of the

\# two indices, ix or iy, must be changed, depending on which axis,

\# $\mathrm{y}$ or $\mathrm{x}$, is intersected. The indices 0,1 of the variables IncX, IncY

\# refer to the $\mathrm{x}, \mathrm{y}$ axes, respectively.

if self.Phi > pi/4:

self.Phi $=$ pi $/ 2$ - self.Phi

self.Inc $X[1]=0$

self.Inc $Y[0]=0$

self.swap(self.tx, self.ty)

else:

self.IncX $[0]=0$

self.IncY $[1]=0$

\# Calculates the integral over the first pixel.

$$
\begin{aligned}
& \text { self.ux }=1 \text { - self.tx } \\
& \text { self.uy }=1-\text { self.ty } \\
& \text { self.t }=\text { self.ux } * \tan (\text { self.Phi) } \\
& \text { if self.t }<=\text { self.uy: } \\
& \quad \text { self. } t+=\text { self.ty }
\end{aligned}
$$




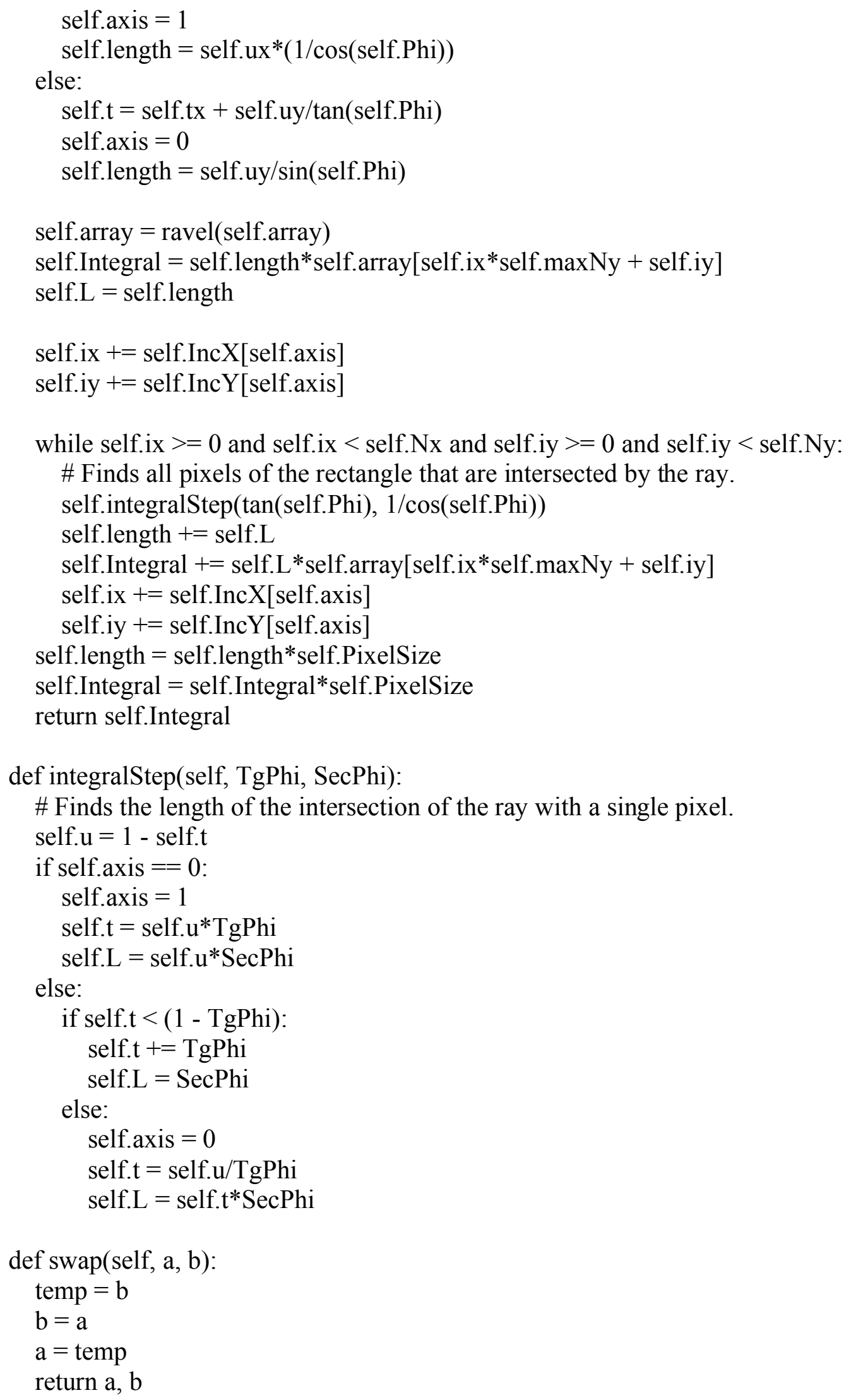




\title{
Calibration of the Nikon D200 for Close Range Photogrammetry
}

\section{Lassana Sheriff}

Office of Science, Science Undergraduate Laboratory Internship Program

\author{
City College of New York, New York NY
}

\section{SLAC National Accelerator Laboratory \\ Menlo Park, California}

August 14, 2009

Prepared in partial fulfillment of the requirement of the Office of Science, Department of Energy's Science Undergraduate Laboratory Internship under the direction of Brian Fuss in the Metrology Department, Alignment Engineering Group of the Stanford Linear Accelerator Center.

Participant:

$$
\text { Signature }
$$

Research Advisor:

\section{Signature}




\section{Table of Contents}

\section{$\underline{\text { Page }}$}

Abstract......................................................................

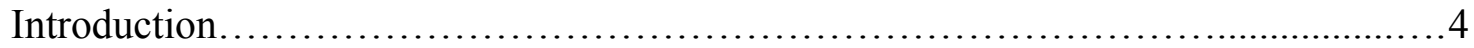

Methods.................................................................. 5

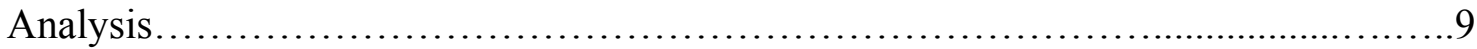

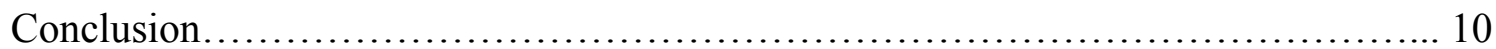

Acknowledgments...................................................... 10

References.................................................................. 11

Figures.............................................................. 


\begin{abstract}
Calibration of the Nikon D200 for Close Range Photogrammetry. LASSANA SHERIFF (City College of New York, New York, New York, 10031) BRIAN FUSS (Stanford Linear Accelerator Center, Menlo Park, CA 94025).
\end{abstract}

The overall objective of this project is to study the stability and reproducibility of the calibration parameters of the Nikon D200 camera with a Nikkor $20 \mathrm{~mm}$ lens for close-range photogrammetric surveys. The well known "central perspective projection" model is used to determine the camera parameters for interior orientation. The Brown model extends it with the introduction of radial distortion and other less critical variables. The calibration process requires a dense network of targets to be photographed at different angles. For faster processing, reflective coded targets are chosen. Two scenarios have been used to check the reproducibility of the parameters. The first one is using a flat $2 \mathrm{D}$ wall with 141 coded targets and 12 custom targets that were previously measured with a laser tracker. The second one is a 3D Unistrut structure with a combination of coded targets and 3D reflective spheres. The study has shown that this setup is only stable during a short period of time. In conclusion, this camera is acceptable when calibrated before each use. Future work should include actual field tests and possible mechanical improvements, such as securing the lens to the camera body. 


\section{INTRODUCTION}

The Alignment Engineering Group (AEG) and Metrology Department are responsible for precisely measuring and aligning various objects and components on the site. This project involves working on a photogrammetric system that they are developing. Photogrammetry is one technique to measure geometrical information on items such as electromagnets which are used in physics experiments. A photogrammetry software package called Australis is being used where I have to photograph and process images to measure $3 \mathrm{D}$ objects. This program generates parameters that can then be used to significantly improve the accuracy of the system.

Photogrammetry stands for "photographic metrology [5]." The technique works in much the same way as human stereo vision, where depth is perceived partially as a function of the angle of two intersecting light rays running from the point of interest to your two eyes. With photogrammetry the intersection of all light rays from several images yields the required XYZ coordinates for a point via a mathematical reconstruction of 3-D shape from the multiple 2-D images. Therefore the model requires a calibrated camera in order to accurately compare these coordinates.

There are two types of photogrammetry; one of which is close-range that I have been investigating. The other one is aerial or space borne. Close-range photogrammetry is used to describe the technique when the extent of the object to be measured is less that about 100 meters and cameras are positioned close to it [3]. Photos of specially coded retro-reflective targets from different angles are made and then these photos are loaded [1] into Australis for processing. The results include a well known 10 parameter correction model defined as follows:

- Camera interior orientation: $c, x \mathrm{p}, y \mathrm{p}$

- Radial distortion parameters: $k 1, k 2, k 3$

- Decentering (aka tangential) distortion parameters: $p 1, p 2$

- Linear distortion (aka shearing) parameters: $b 1, b 2$ 
These are corrections that simulate the ideal "central perspective model" discussed in section 2.

Upon using the Australis program [2] to perform fully automatic measurements, certain requirements need to be met. Every targeted object point must appear in two or more images that provide good ray intersection geometry. There needs to be a sufficient number of coded targets and the camera must be rotated or rolled approximately ninety degrees between images to distribute where the target image falls on the CCD. Once these requirements are met and the images are loaded into the project, the network of coded targets is automatically measured to provide the camera self-calibration.

A stable relatively flat wall is being used in the Alignment Engineering Group's Sector 10 Calibration Laboratory. A pattern of coded retro-reflective targets is used to study the effect of the camera and target position and the effect of the addition of a scale bar to the model. A 3D structure was added to the project to compare with the flat wall. The results of these two calibration techniques are compared.

\section{METHODS}

The camera used in this project is a commercially available Nikon D200 SLR with a regular lens and built-in flash. The camera is a DX format of $23.7 \mathrm{~mm} \times 15.7 \mathrm{~mm}$. It is about 10.2 megapixels (3872 x 2592); and the lens is a wide angle Nikkor of $20 \mathrm{~mm}$. The pictures are taken with "Manual" mode option and the settings in Table 1. 


\begin{tabular}{|l|l|}
\hline \multicolumn{2}{|c|}{ Nikon D200 SLC Camera Settings } \\
\hline Option & Setting \\
\hline Aperture & $\mathrm{f}=11$ \\
\hline Distance & Between $2 \mathrm{~m}$ and $\infty$ \\
\hline ISO & 100 \\
\hline Speed & $1 / 250$ \\
\hline Flash Exposure & Compensated to -3.0 (and paper filter) \\
\hline
\end{tabular}

Table 1. Camera Settings

\section{Software Targets:}

Australis is a photogrametry program used to make automatic 3D measurements of an array of specially coded or uncoded targets placed on or an object of interest. Basic targets are flat circular objects made of gray retro-reflective material. They come in different diameters usually being at least $1 / 100^{\text {th }}$ of the object size. They start losing their reflective property when viewed from an angle greater than 60 degrees off-axis. Coded targets are automatically detected, identified and measured by the software. For Australis, the coded targets are made of an arrangement of dots. The central dot gives the position of the target and the pattern of dots is the key to the target's identification. The spacing of the dots can also be used for scale determination. Retro-reflective targets can be mounted onto spheres and these may be interchanged with other spheres that survey instruments such as laser trackers can measure.

\section{Scale Bar:}

To scale a photogrammetric measurement, at least one known distance is needed. If the actual coordinates of some targeted points are known beforehand, the distances between these points is computed and used to scale the measurement. Another possibility is to use a fixture 
such as a bar with targets on it and measure this along with the object. The distance between the targets on the bar is known (it had been measured on a Coordinate Measuring Machine) and can be used to scale the measurement. Such fixtures are commonly called scale bars [3].

\section{Camera Parameters and Model:}

Data for the focal length and the principal point of the camera: $c, x p$, and $y p$, radial distortion, $k 1, k 2$, and $k 3$ will be analyzed. These parameters are compared in both 2D and 3D targets set-ups (figures 11 and 12). By analyzing these six key parameters with a statically significant number of samples, an assessment of the camera's stability can be determined. Figure 1 illustrates an ideal basic photogrammetry model. This is known as the "central perspective projection". Geometrically it is a 3D similarity transformation between 2 coordinate systems. The first coordinate system is positioned arbitrarily and is generally characteristic of an object being measured. It is noted: $(\mathrm{X}, \mathrm{Y}, \mathrm{Z})$. The second coordinate system is linked to the camera position: its origin is at the perspective camera center $\mathrm{C}$, its z-axis coincides with the principal axis of the camera. The image plane is at $\mathrm{z}=-c$.

This central perspective model is only an idealization of the real optical geometry of a camera [4]. The major differences come from the stability of the interior parameters, the lens distortion and the Charge Coupled Device (CCD) flatness and regularity. The camera interior orientation is as follows $c, x p$, and $y p$; where $c$ is the focal length of the camera. $x p$ and $y p$ are the coordinates of the principal point and they are often called principal position offsets. These form the camera interior parameters. The camera perspective center $\mathrm{C}$ has the coordinates $(\mathrm{Xc}, \mathrm{Yc}$, $\mathrm{Zc}$ ) in the object system. Let $\mathrm{M}$ be a point in the space and $\mathrm{m}$ its image. The coordinates of $\mathrm{M}$ in the object space are $\left(X_{M}, Y_{M}, Z_{M}\right)$. Its image $m$ has the coordinate $\left(x_{M}, y_{M},-c\right)$ in the camera system. 
The transformation between the 2 systems can be written:

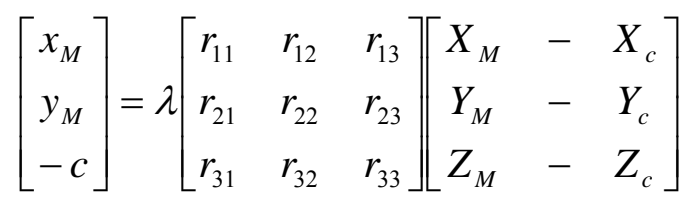

The perspective center the image point and the object point are required to lie along one straight line. The above 3 equations can be combined into 2 to eliminate the scale factor:

$$
\begin{aligned}
x_{M} & =-c \frac{r_{11}\left(X_{M}-X_{c}\right)+r_{12}\left(Y_{M}-Y_{c}\right)+r_{13}\left(Z_{M}-Z_{c}\right)}{r_{31}\left(X_{M}-X_{c}\right)+r_{32}\left(Y_{M}-Y_{M}\right)+r_{33}\left(Z_{M}-Z_{c}\right)} \\
y_{M} & =-c \frac{r_{21}\left(X_{M}-X_{c}\right)+r_{22}\left(Y_{M}-Y_{c}\right)+r_{23}\left(Z_{M}-Z_{c}\right)}{r_{31}\left(X_{M}-X_{c}\right)+r_{32}\left(Y_{M}-Y_{c}\right)+r_{33}\left(Z_{M}-Z_{c}\right)}
\end{aligned}
$$

The equations are known as the collinearity equations.

A 2D coordinate system represents the projection plane of the camera which also is the CCD. Its origin is at the center of the CCD. Components of $\mathrm{r}(d \mathrm{x}$ and $d \mathrm{y})$ are part of the Brown [1] model and are computed as follows:

$$
\begin{aligned}
& d x=\bar{x} d r+p_{1}\left(r^{2}+2 \bar{x}^{2}\right)+2 p_{2} \overline{x y}+b_{1} \bar{x}+b_{2} \bar{y} \\
& d y=\bar{y} d r+p_{2}\left(r^{2}+2 \bar{y}^{2}\right)+2 p_{1} \overline{x y}
\end{aligned}
$$

where:

$$
\begin{array}{ll}
\bar{x}=x_{M}-x_{p} & \bar{y}=y_{M}-y_{p} \\
r^{2}=\bar{x}^{2}+\bar{y}^{2} & d r=k_{1} r^{2}+k_{2} r^{4}+k_{3} r^{6}
\end{array}
$$

These can be added to the basic collinearity equations to give:

$$
\begin{aligned}
& x_{M}=x_{p}-c \frac{r_{11}\left(X_{M}-X_{C}\right)+r_{12}\left(Y_{M}-Y_{C}\right)+r_{13}\left(Z_{M}-Z_{C}\right)}{r_{31}\left(X_{M}-X_{C}\right)+r_{32}\left(Y_{M}-Y_{C}\right)+r_{33}\left(Z_{M}-Z_{C}\right)}+d x \\
& y_{M}=y_{p}-c \frac{r_{21}\left(X_{M}-X_{C}\right)+r_{22}\left(Y_{M}-Y_{C}\right)+r_{23}\left(Z_{M}-Z_{C}\right)}{r_{31}\left(X_{M}-X_{C}\right)+r_{32}\left(Y_{M}-Y_{C}\right)+r_{33}\left(Z_{M}-Z_{C}\right)}+d y
\end{aligned}
$$




\section{ANALYSIS}

To study the interior parameters of the camera two graph types were used, line graphs and histograms. The line graphs have the date on the $\mathrm{x}$-axis and the value of the parameter is on the $\mathrm{y}$-axis. The purpose of this graph is to check for any trends in the data. The histograms are used to study the distribution of the parameters. As the number of observations grows, it is expected that the histogram should look like those with a normal distribution [4].

Looking at the line graphs of the D200's interior orientation (figures 2 through 7), as expected, there seems to be no trend as the data is fairly scattered. For example for $c$, the focal length of the camera, most of the data are within a narrow band, but some points were far off the average for reasons that are likely due to camera transportation and handling. The data shows that photo sets taken within a short period of time showed positive results as the values were close together. The histograms for the principal point positions $x p$ and $y p$ (figures 9 and 10) are almost similar and not normally distributed. The focal length data looks more like a normal distribution but it isn't as good as expected (figure 8).

The data for the 2D wall and 3D structure have been kept separate. The number of observations for the $3 \mathrm{D}$ structure is minimal but they agree with the $2 \mathrm{D}$ data showing the same pattern. Further studies will be needed to evaluate if changing the types of camera angles to the 3D structure will give different results.

For the 2D study, 13 points were precisely measured with a laser tracker. The photogrammetric coordinates were transformed to the laser tracker coordinate system within the Australis program. The quality of the survey can be judged by the global RMS from each transformation. The average global RMS is $0.143 \mathrm{~mm}$ and $0.030 \mathrm{~mm}$ standard deviation. Values less than 100 
micrometers were expected. Some commercial systems can achieve accuracies as good as 50 micrometers [4].

\section{CONCLUSION}

Not all parameters show a normal distribution. This suggests that the camera can be used for photogrammetric measurements only if calibrated before each use. Testing in the future should include field tests and possible mechanical improvement to the camera such as securing the lens to the camera body. Future tests should be also include more observations of the 3D structure with more photos from many more angles to get true 360 degree coverage..

\section{ACKNOWLEDGEMENTS}

The Science Undergraduate Laboratory Internship SULI program wouldn't have been possible without the funding of the Department of Energy DOE here at Stanford Linear Accelerator Center SLAC. Special acknowledgment goes to my mentor BRIAN FUSS for his diligent guidance and support. I would like to acknowledge CATHERINE LECOCQ for her resilient work and direction in the entire project. And finally acknowledgement to BRENDAN DIX for his participation in building the 3D structure; and to the rest of the members of Alignment Engineering Group for their support. 


\section{REFERENCES}

[1] Brown, D.C.,. “Close-Range Camera Calibration”. PE\&RS, Vol. 37(8), (1971): 855-866.

[2] User Manual for Australis:

http://www.photometrix.com.au/downloads/australis/Australis7 Users Guide.pdf

[3] The Basics of Photogrammetry:

http://www.geodetic.com/Downloadfiles/Basics\%20of\%20Photogrammetry.pdf

[4] LeCocq, C. Internal notes. SLAC (2009).

[5] Atkinson, K.B. “Close Range Phptogrammerty and Machine Vision”. (1996) 


\section{FIGURES}

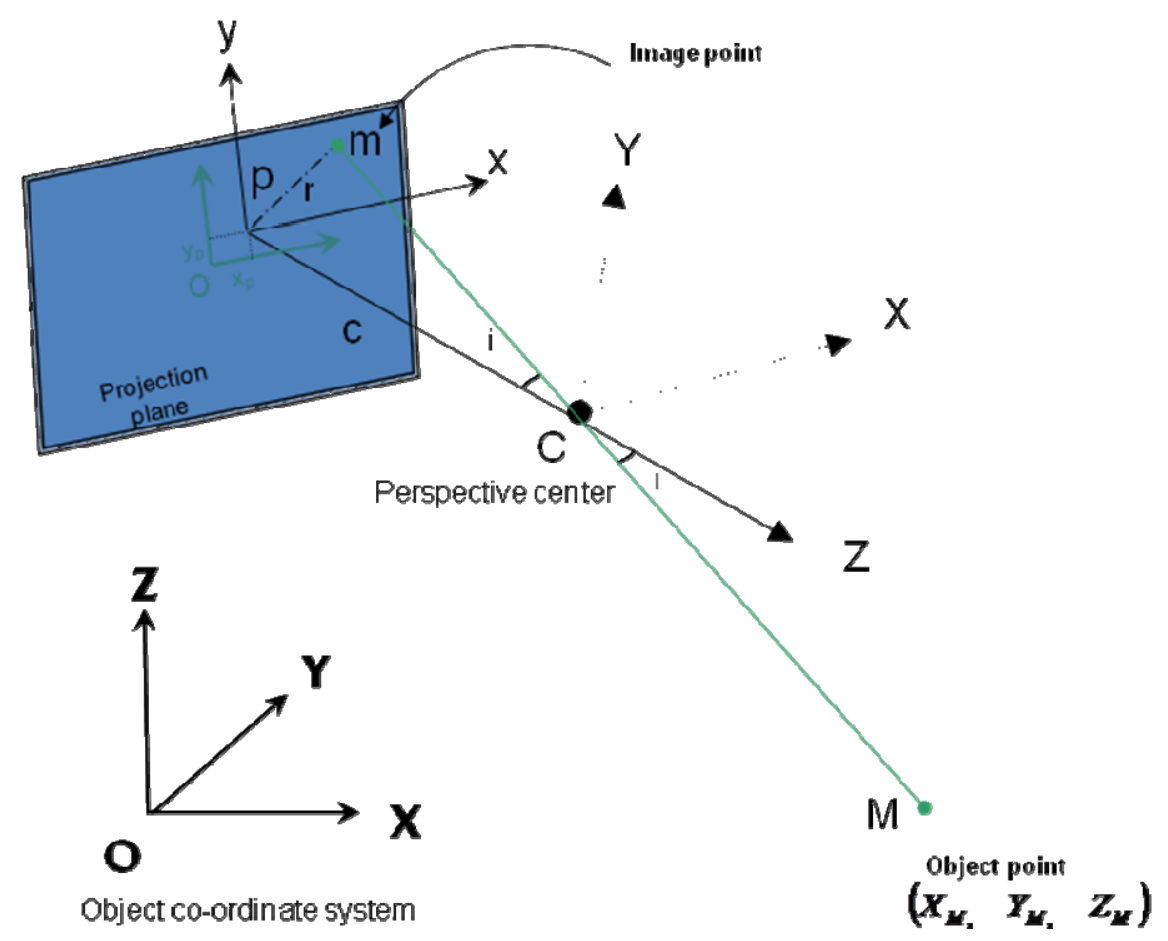

Figure 1. The central perspective model

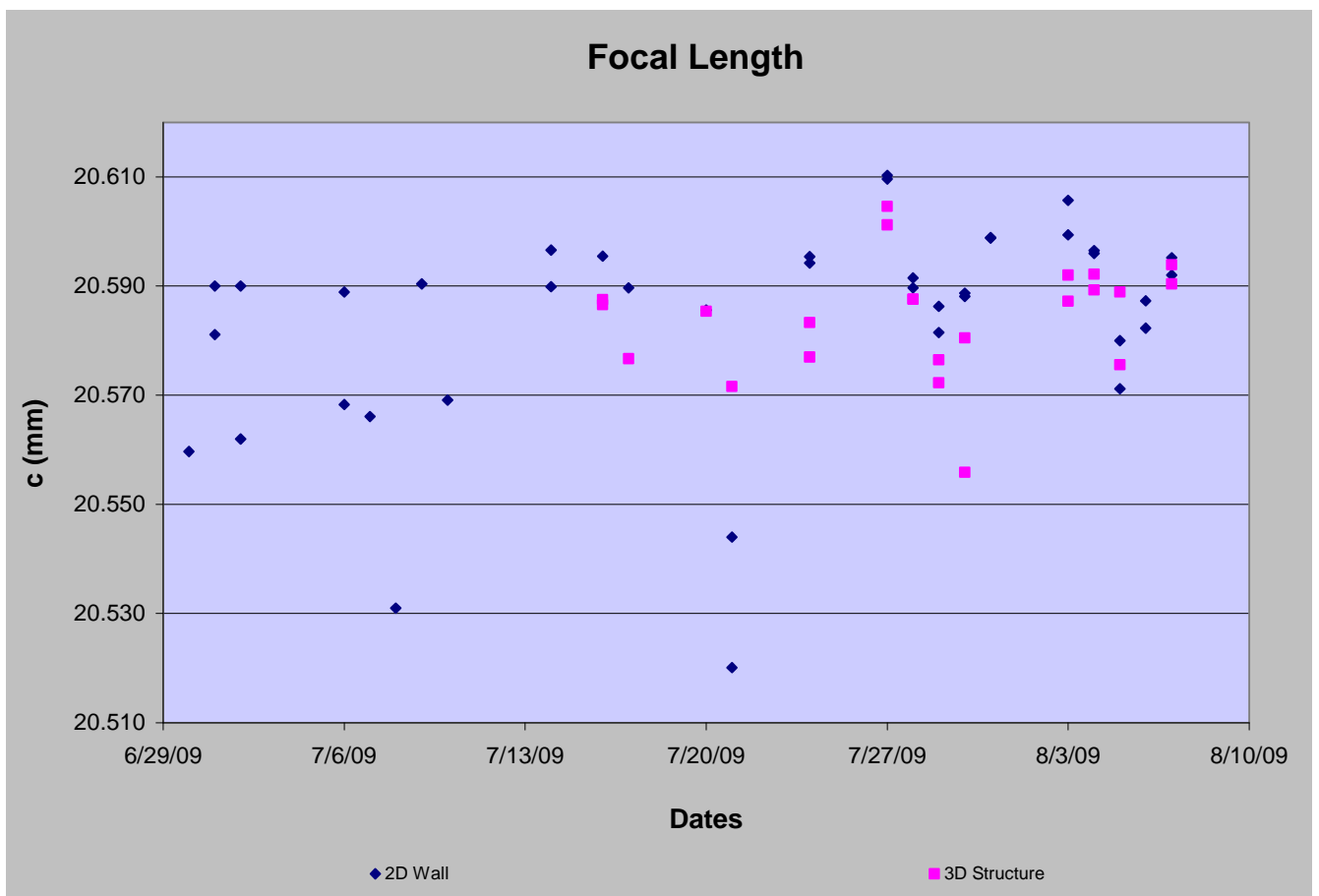

Figure 2. Nikon D200 focal length parameter measured over time 


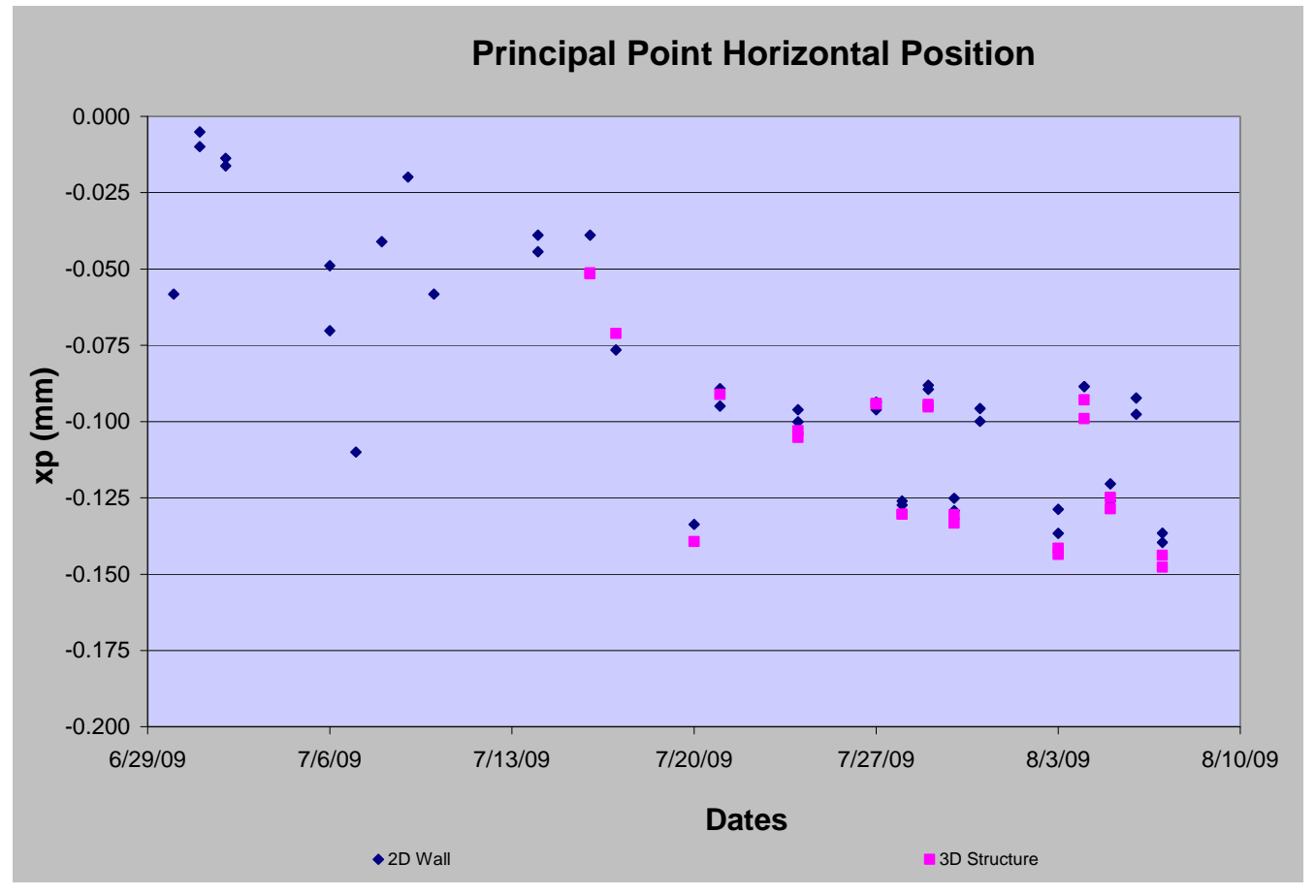

Figure 3. Nikon D200 horizontal position for the principal point

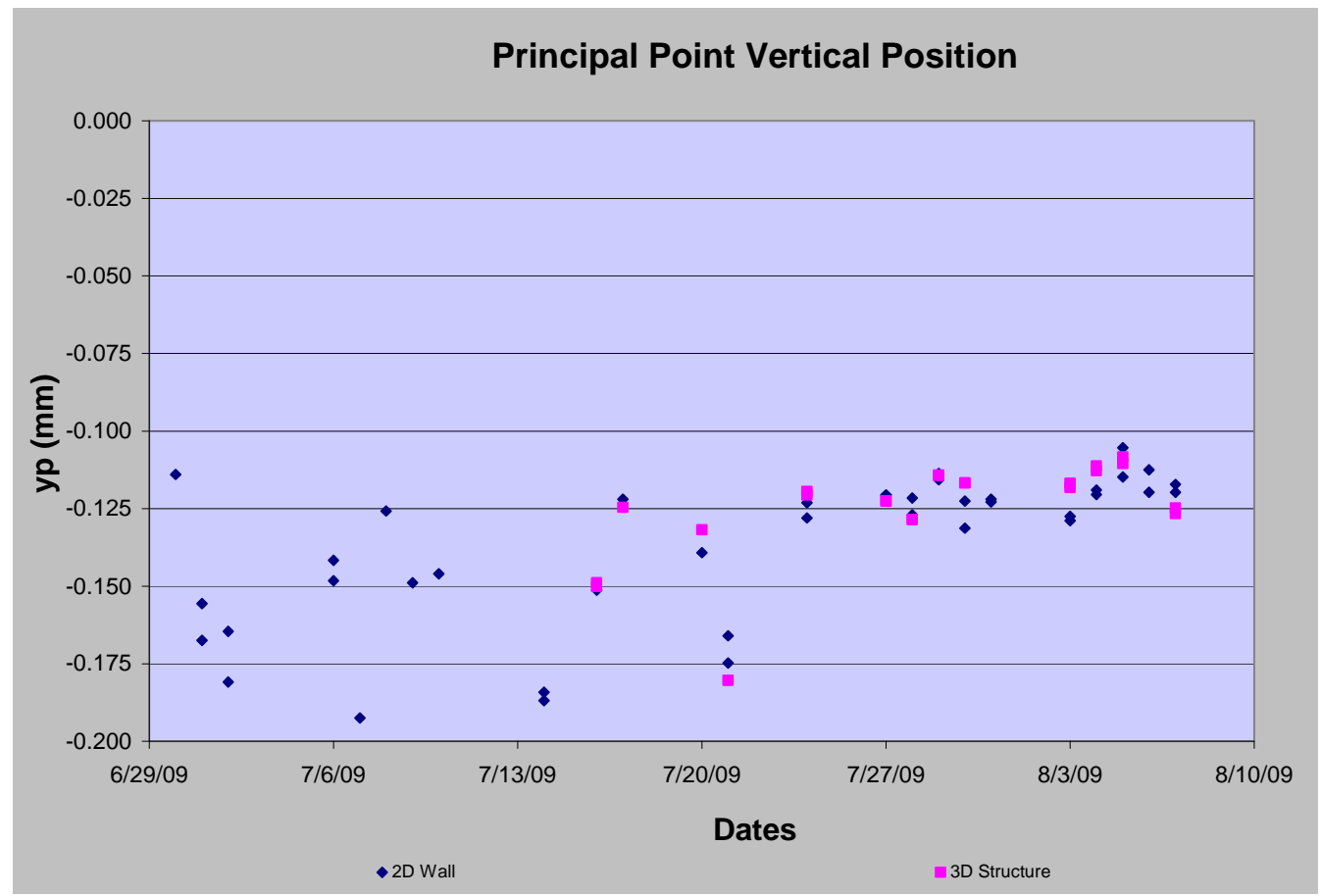

Figure 4. Nikon D200 vertical position for the principal point 


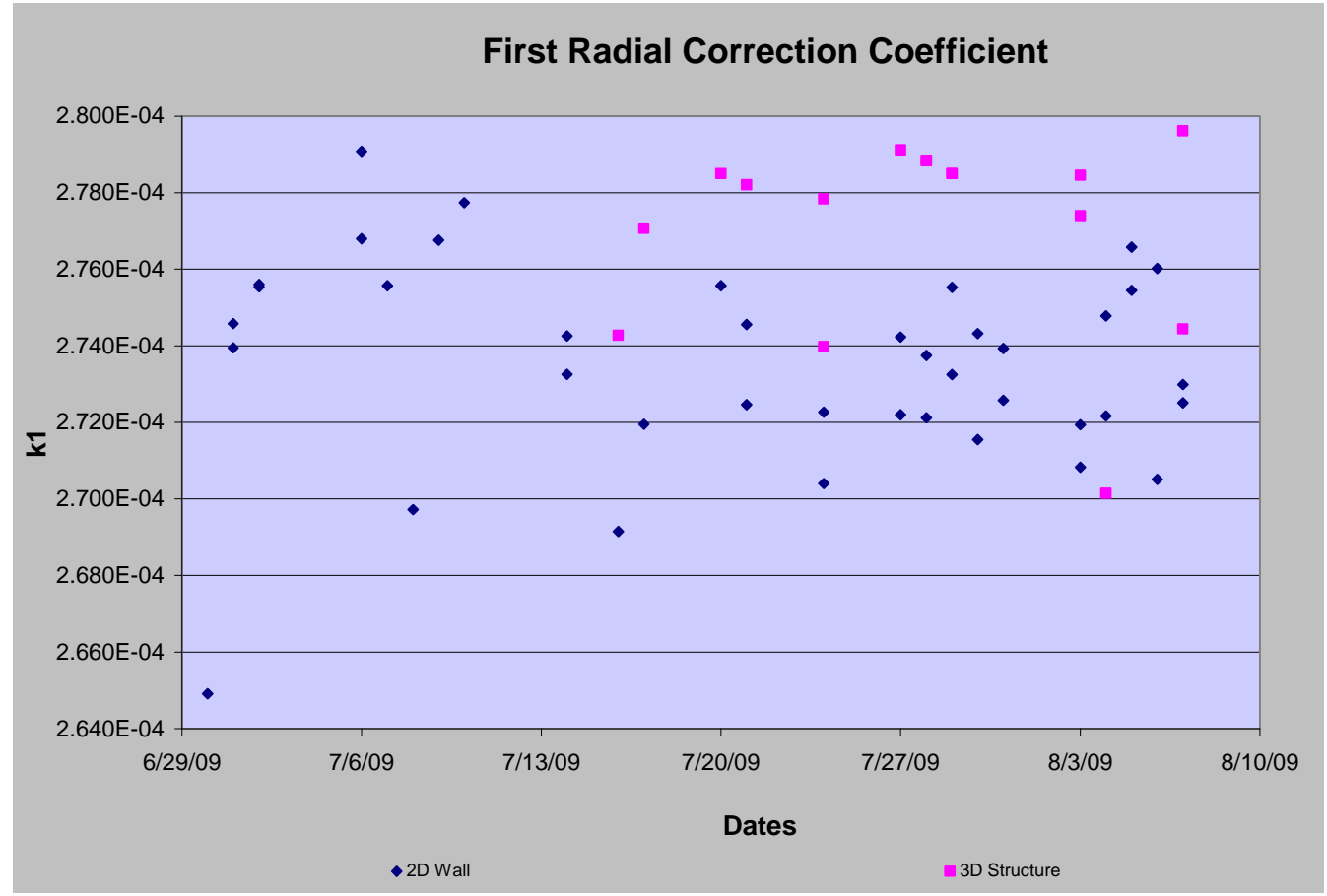

Figure 5. Nikon D200 first radial correction coefficient

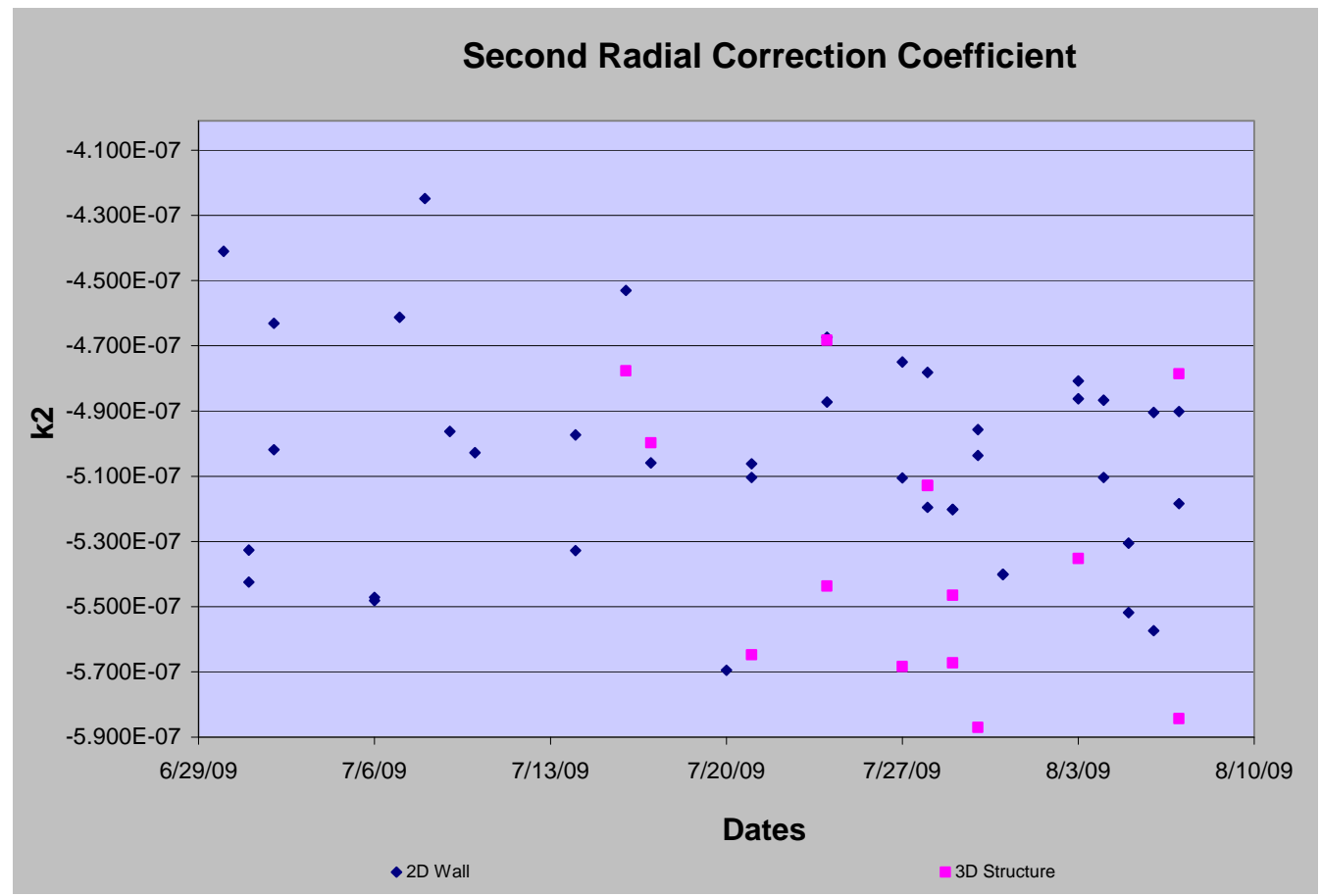

Figure 6. Nikon D200 second radial correction coefficient 


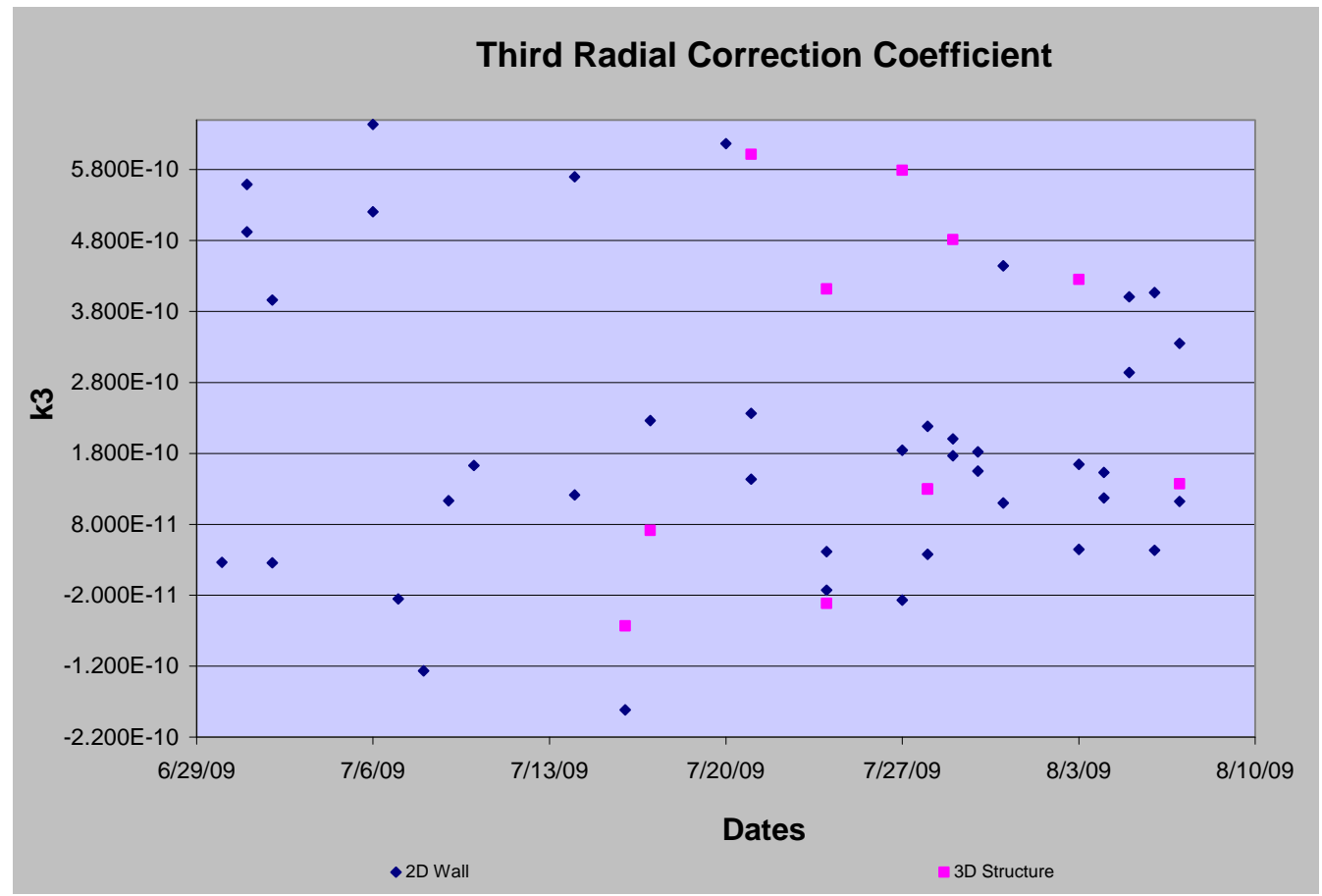

Figure 7. Nikon D200 third radial correction coefficient

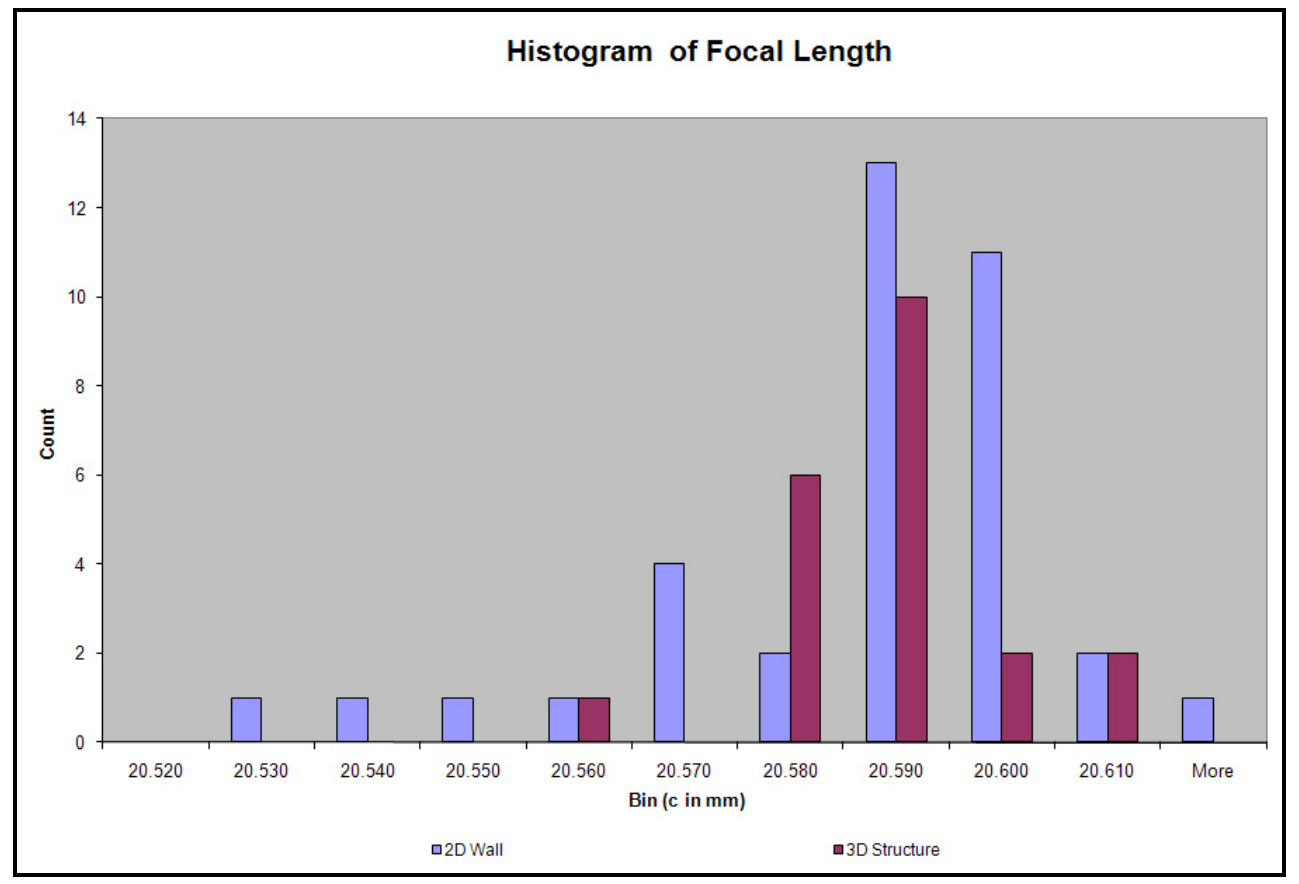

Figure 8. Nikon D200 focal length distribution 


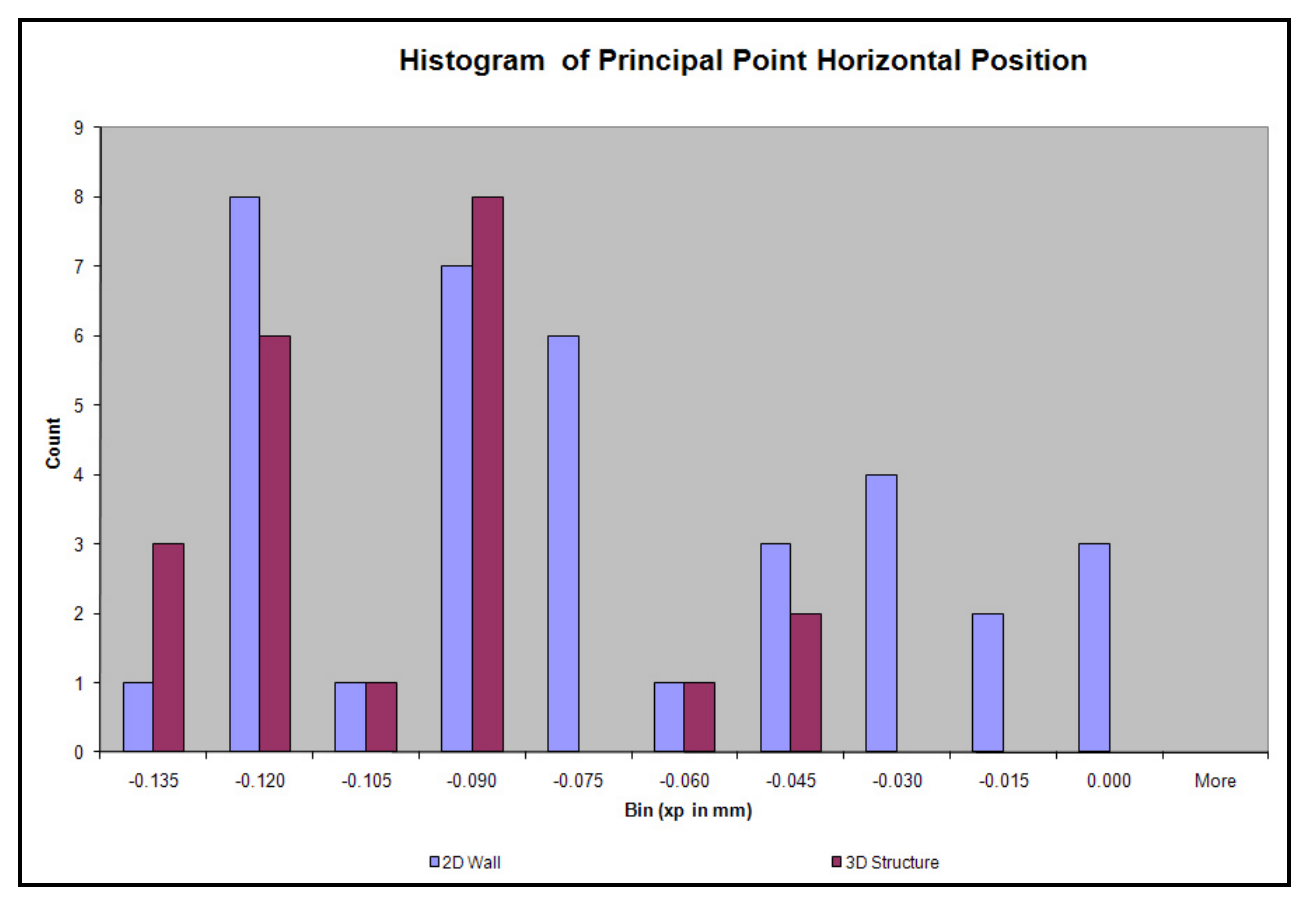

Figure 9. Nikon D200 horizontal principal point distribution

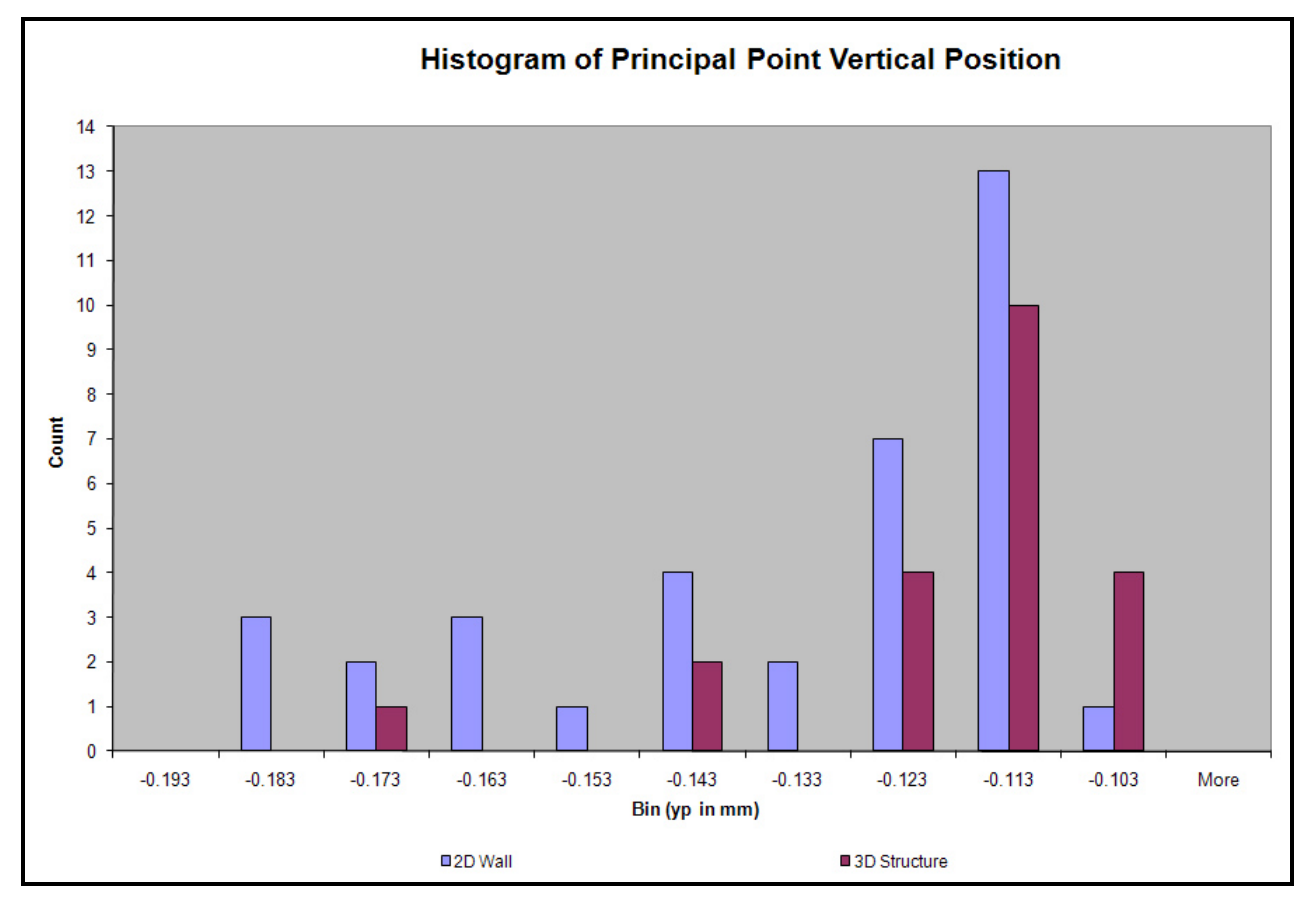

Figure 10. Nikon D200 vertical principal point distribution 


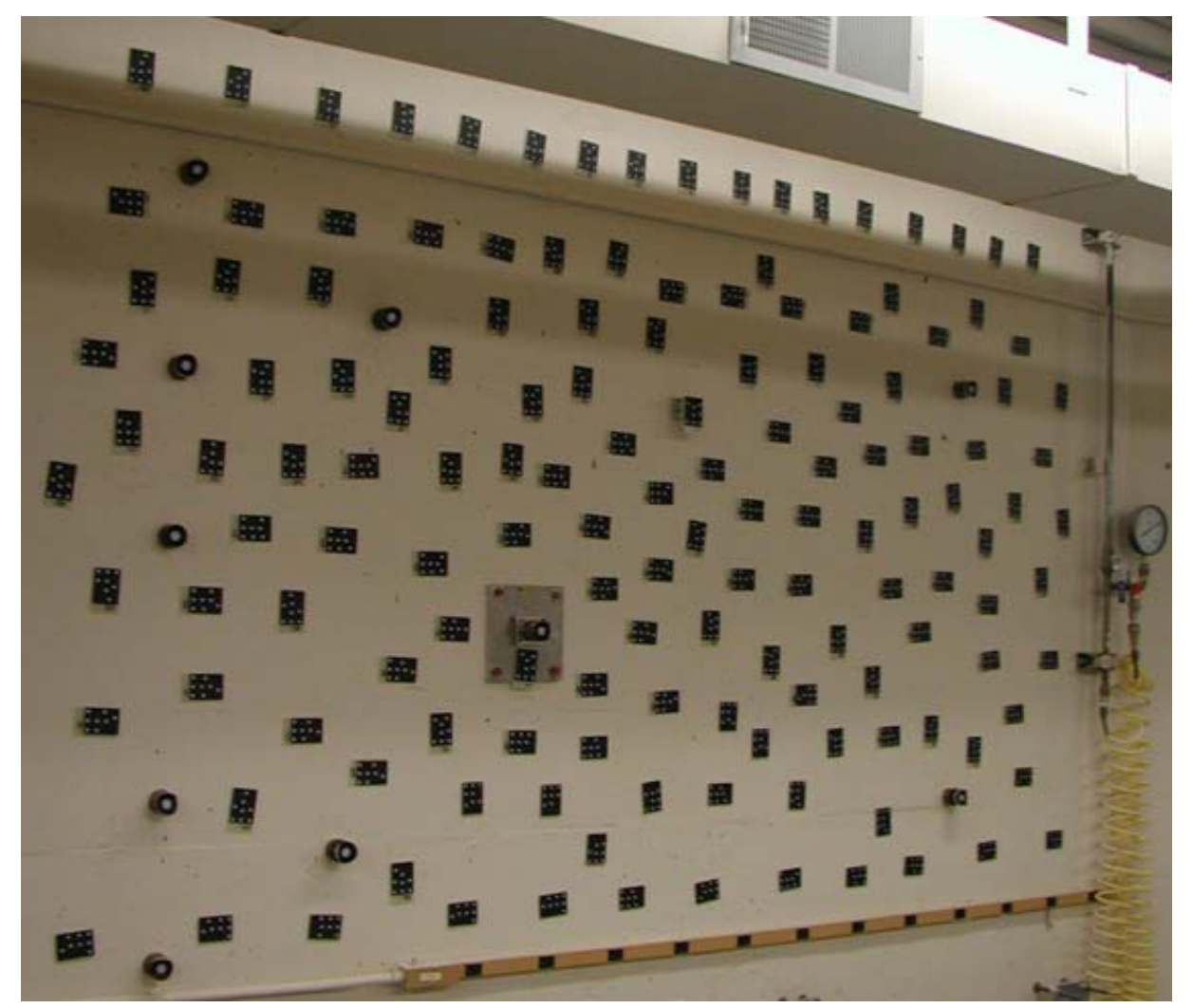

Figure 11. Wall test structure for camera calibration (2D Wall)

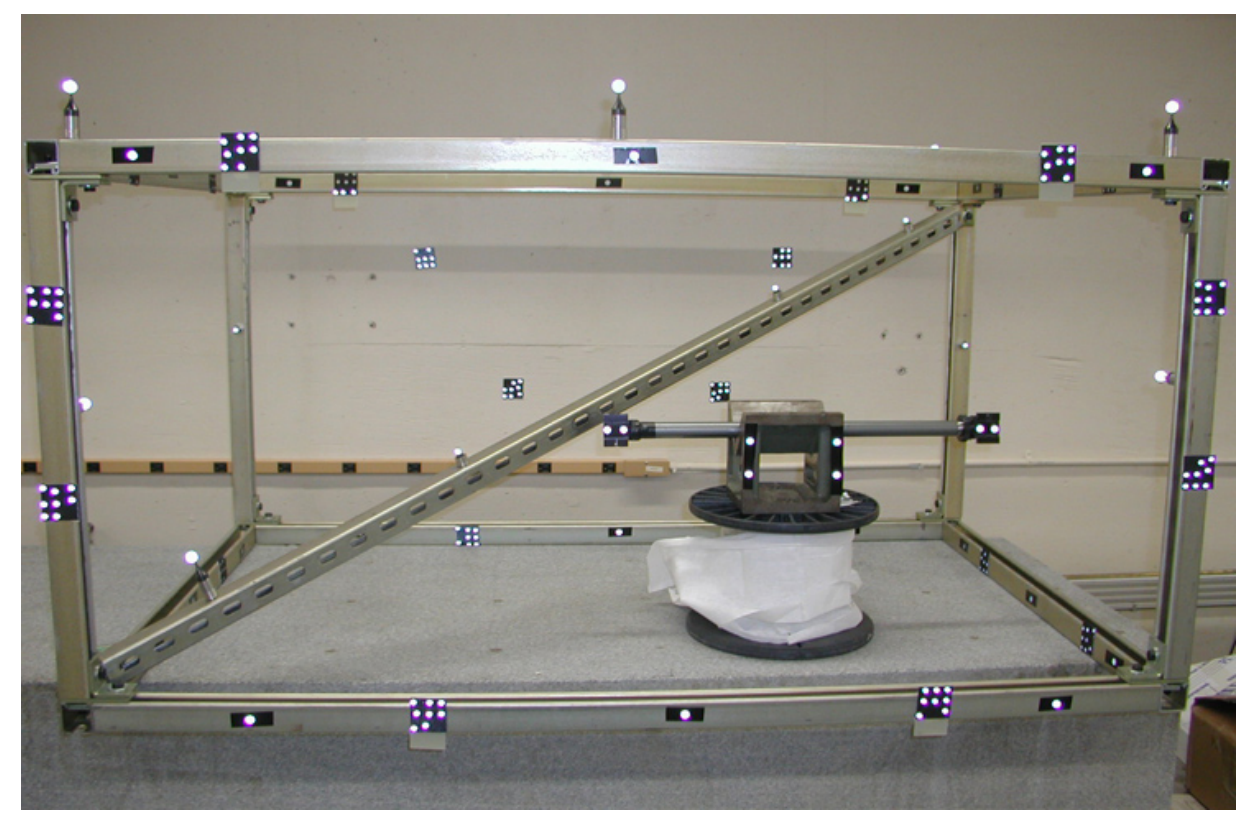

Figure 12. 3D Structure test for camera calibration (with scale bar) 


\title{
Mechanical Design
}

\author{
Richard Shook \\ Office of Science, Science Undergraduate Laboratory Internship
}

Marquette University

SLAC National Accelerator

Menlo Park, California

August 15, 2009

Prepared in partial fulfillment of the requirement of the Science Undergraduate Laboratory Internship Program for the Department of Energy, under the supervision of Rick Tankersley, Paul Stephens and Steve Score in the Mechanical Design Department at SLAC National Accelerator

Participant:

Signature

Research Advisor:

$$
\text { Signature }
$$




\section{Table of Contents}

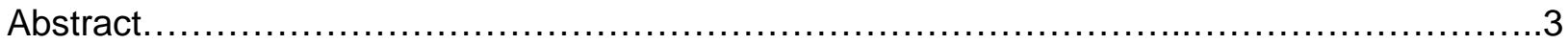

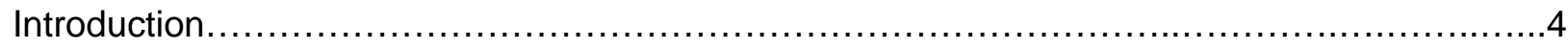

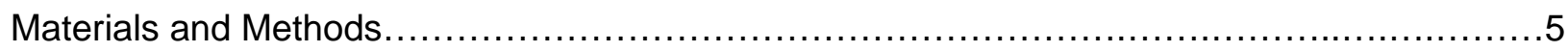

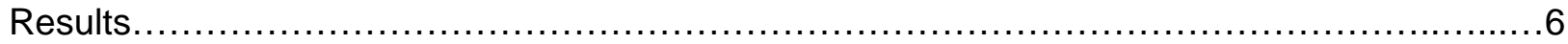

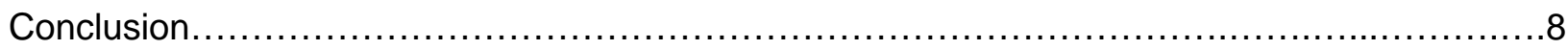

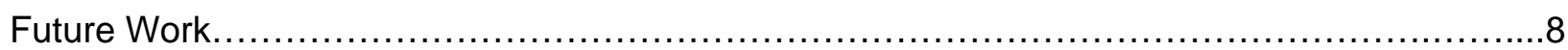

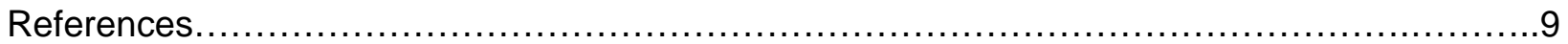

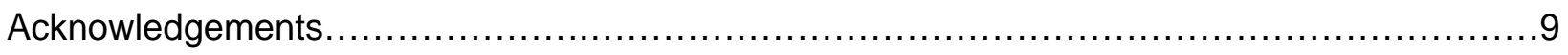

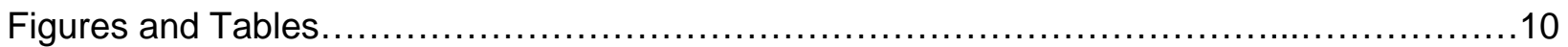




\begin{abstract}
Mechanical Design. RICHARD SHOOK (Marquette University, Milwaukee WI, 53233) RICK TANKERSLEY (SLAC National Accelerator, Menlo Park, CA 94025)
\end{abstract}

The particle beam of the SXR (soft x-ray) beam line in the LCLS (Linac Coherent Light Source) has a high intensity in order to penetrate through samples at the atomic level. However, the intensity is so high that many experiments fail because of severe damage. To correct this issue, attenuators are put into the beam line to reduce this intensity to a level suitable for experimentation. Attenuation is defined as "the gradual loss in intensity of any flux through a medium" by [1].It is found that Beryllium and Boron Carbide can survive the intensity of the beam. At very thin films, both of these materials work very well as filters for reducing the beam intensity. Using a total of 12 filters, the first 9 being made of Beryllium and the rest made of Boron Carbide, the beam's energy range of photons can be attenuated between $800 \mathrm{eV}$ and $9000 \mathrm{eV}$. The design of the filters allows attenuation for different beam intensities so that experiments can obtain different intensities from the beam if desired. The step of attenuation varies, but is relative to the thickness of the filter as a power function of 2 . A relationship for this is $f(n)=x_{0} 2^{n}$ where $n$ is the step of attenuation desired and $x_{0}$ is the initial thickness of the material. To allow for this desired variation, a mechanism must be designed within the test chamber. This is visualized using a 3D computer aided design modeling tool known as Solid Edge. 


\section{Introduction}

Many engineering objectives require the use of Computer-Aided Design (CAD) to further the completion of a design process or project. Several projects will include a Mechanical Design team to develop computer generated representations, or simply models, of the final product. This allows the engineers and scientists to have a visualization of their calculations and a better idea of possible modifications to the overall design. Drafts of the accepted models can also be used as legal documentation between the engineer and the manufacturer if properly dimensioned to the standards of some national or international governing body for geometric dimensioning and tolerancing, such the American Society of Mechanical Engineers (ASME). In many entrepreneurial missions, there is a prototype of the model to have an even better visualization of the design to an actual scale. However, to save resources a three-dimensional CAD model is sufficient.

Recent work with the Mechanical Design department at SLAC deals mostly with modeling and drafting components going in the Linac Coherent Light Source (LCLS) for experimentation; more specifically for the Soft x-ray (SXR) Beam line. Other work includes revising old or incomplete drawings, assisting the LUSI engineering team in designing systems for maintaining its high vacuum environment, and designing other small components as needed by the Mechanical Design department.

One experiment taking place in the SXR Beam line occurs in Section 91-1, where photon tests are placed. Because of the high intensity of the beam many samples are at risk of severe damage, which can compromise the integrity of the results or even destroy the entire experiment. To resolve this filters are placed into the beam line to attenuate the high intensity. 


\section{Materials and Methods}

\section{Materials}

Designing the filters and the other photon experiments required use of a computer aided design tool, Solid Edge. This is a powerful tool that allows a user to make three-dimensional models of a desired product. Another computer tool used is Windows Excel. The materials used for the filters are chosen based on past trial and error analysis. Currently the filters are made of Beryllium or Boron Carbide.

\section{Methods}

There are certain constraints placed on determining acceptable materials used as filters for the photon beam. First the range of photon's energy being attenuated is from $800 \mathrm{eV}$ to $9000 \mathrm{eV}$. This is not so much as a limit as it is a parameter of the beam itself. However, it designates the range for which it must be attenuated. Another constraint is the thickness of the filter. Generally metals are denser than ceramics, so we must keep the metals at a lower thickness. This is due to how tightly packed the metal structure is compared to ceramics. A photon is more likely absorbed if space between each compound decreases, thus a smaller thickness is needed to for the attenuation. The ceramics have a more relaxed constraint. For this design 10 microns is acceptable by a metal acting as a filter; 100 microns is acceptable for a ceramic. It just so happens this correlates perfectly with the attenuation we require, which is the third constraint. If we go anywhere below 10 attenuations, then the material becomes too thin for the beam and would have little to no effect. If we go above 100, then the material is too thick and should no longer be treated as a filter, but more so as a thermodynamic plate. 


\section{Results}

It was determined that a relationship can be used to determine the transmission of the photons traveling through the particles. The distance that a particle travels before its probability of being transmitted decreases by a factor of $1 / e$ is known as the attenuation length or the absorption length [2]. This probability can also be accepted as evidence for determining the depth at which

the particles are absorbed because of the Beer-Lambert Law [6]. Given as: $P(x)=e^{-x / \lambda}$ where $x$ is the depth of the particle, and $\lambda$ is the distance into the material when the probability of being transmitted drops to 1/e. What has been determined from the intensity of the beam at $800 \mathrm{eV}$ can be seen in Table 1. Beryllium at 20 microns successfully attenuates the beam for attenuation factor of 0.01 . Until the attenuation reaches 0.10 , Beryllium will stay successful. Table 1 also shows that Beryllium fails when the beam energy is at approximately $986 \mathrm{eV}$. This is the range at which Beryllium is acceptable at this depth of a filter for the beam. Further ranges of Beryllium are accepted for higher energies as the thickness doubles. This is interesting in that we want attenuations from 10 microns to 100 microns (0.01 and 0.10) and we effectively get a range that is covered by the thickness as a function of $f(x) \propto 2^{x}$, so that any initial thickness that you choose can simply be multiplied by any power of 2 . The expression for this, if we say that our initial thickness is $x_{0}$ and our desired attenuation band is $n$ power of 2, is: $f(n)=x_{0} 2^{n}$. This is still governed by the boundaries of attenuation factors between 0.01 and 0.10 . Once one range fails, another thickness can be chosen using the above expression. This is expressed more clearly in Figure 1. However, there is a point at which materials change and Boron Carbide is used to complete the process. The reason for this is that the thickness of Beryllium becomes too great and it is more beneficial to use another material. Because Boron Carbide is the only other known material to survive the intensity of the beam, we use an additional 3 filters 
of Boron Carbide to finish. The analysis for this is found in Figure 1. The energy range that each filter covers increases because this data was acquired on a log scale.

Designing the holder for all of these filters is another aspect of the design. Because of the thinness, these filters cannot be poked or probed. The other limitation is having a holder that can fit inside the current test chamber without striking the bottom causing the chamber to crack and ruin the vacuum equipment. The last requirement limits the distance the samples have between each other and how many can go in the chamber at once. The holder should be universal for other experiments to take place on the same section of the beam line.

What was done to achieve most of these requirements was to design a two-legged fork with a series of threaded holes. This would allow for washers to hold the filters in place and use the force of the screw to keep them clamped down. The filter strips would be ordered at 1 inch long and $1 / 4$ inch tall. This limits the number of filters that can be placed in the test with another sample. Fortunately, this is not required and all of the filters can be placed. In Figure 2, a better visualization is seen for a better idea of what the fork looks like. The holes holding each filter are \#2-56 UNC 2B threaded holes. Washers are placed on the face of the fork to clamp the filters in place. There are 15 places in total. In figures 3 and 4, the fork is attached to its translator assembly and placed in the test chamber. 


\section{Conclusion}

It is important to savor the integrity of soft x-ray experiments, and any experiment in high-energy physics. Data trials can sometimes only be taken once or twice, so it is necessary for the experiments to be treated with the highest delicacy. Whether or not the research is successful will be proven later on in the summer. It is likely that a more efficient system will be developed and the trials for this method might not ever occur. However, the work done here will be vital in developing new techniques for attenuating high intensity beams.

\section{Future Work}

Once more research and testing is done on these filters, work can begin to reduce the number of filters allowing a greater range of energies attenuated. The issue with using anything other than Beryllium and Boron Carbide is that anything heavier will have too small of an absorption length. The issue here is finding companies willing or able to produce such small thicknesses. Based on current research, many carbide materials should be effective, as they would have an equal number of carbon atoms as the number of heavier metal atoms 


\section{References}

[1] R. S. Figliola, D. E. Beasley, Theory and Design for Mechanical Measurements, 4th ed. Hoboken, NJ: Wiley, 2005

[2] R. K. Bock, "Collision Length" ct.infn.it, April 9, 1998. [Online] Available: http://www.ct.infn.it/ rivel/Glossario/node30.html\#29. [Accessed: July 23, 2009].

[3] G. S. Brady, H. R. Clauser and J. A. Vaccari, Materials Handbook, 14th ed. New York: McGraw-Hill, 1997, pp. 93-94, 112.

[4] R. Eisberg, R. Resnick, Quantum Physics of Atoms, Molecules, Solids, Nuclei, and Particles. Hoboken, NJ: John Wiley and Sons, 1985

[5] E. Gullikson, “X-Ray Attenuation Length" henke.Ibl.gov, 2008. [Online] Available: http://henke.Ibl.gov/optical_constants/atten2.html. [Accessed: July 23, 2009].

[6] "Beers Law". [Online] Available:

http://teaching.shu.ac.uk/hwb/chemistry/tutorials/molspec/beers1.htm. [Accessed: Aug. 8, 2009]

\section{Acknowledgements}

The work performed was granted by the United States Department of Energy, Office of Science, Student Undergraduate Laboratory Institute at SLAC National Accelerator. Special thanks goes to Rick Tankersley, Head of the Mechanical Design department at SLAC and my mentor, for providing the resources to becoming a better engineer. A Grand Special Thanks goes to the entire Mechanical Design department. Without their help and watchful eyes, I would not have gotten where I am today. 
Figures and Tables

\begin{tabular}{|c|c|c|c|c|c|}
\hline MATERIAL: & $\mathrm{Be}$ & & & & \\
\hline & & & THICKNESS: & 20 & microns \\
\hline & PHOTON ENERGY (eV) & ATTN LENGTH (microns) & & & \\
\hline & & & & TRANSMISSION & \\
\hline & 800 & 4.66859 & & \begin{tabular}{|l|}
0.01378811 \\
\end{tabular} & \\
\hline & 803.882 & 4.73582 & & 0.014652659 & \\
\hline & 807.783 & 4.80401 & & 0.01555787 & \\
\hline & 811.703 & 4.87319 & & 0.016505067 & \\
\hline & 815.641 & 4.94338 & & 0.017495443 & \\
\hline & 819.599 & 5.01458 & & 0.018529895 & \\
\hline & 823.576 & 5.08679 & & 0.019609274 & \\
\hline & 827.573 & 5.16006 & & 0.020735171 & \\
\hline & 831.588 & 5.23439 & & 0.021908413 & \\
\hline & 835.624 & 5.30978 & & 0.023129778 & \\
\hline & 839.679 & 5.38626 & & 0.024400501 & \\
\hline & 843.753 & 5.46382 & & 0.025721124 & \\
\hline & 847.847 & 5.54251 & & 0.027093175 & \\
\hline & 851.961 & 5.62233 & & 0.028517308 & \\
\hline & 856.096 & 5.7033 & & 0.029994486 & \\
\hline & 860.25 & 5.78543 & & 0.031525454 & \\
\hline & 864.424 & 5.86875 & & 0.033111298 & \\
\hline & 868.619 & 5.95327 & & 0.034752693 & \\
\hline & 872.834 & 6.039 & & 0.036450264 & \\
\hline & 877.069 & 6.12597 & & 0.038204992 & \\
\hline & 881.325 & 6.21395 & & 0.040012448 & \\
\hline & 885.602 & 6.30293 & & 0.04187244 & \\
\hline & 889.899 & 6.39318 & & 0.043790708 & \\
\hline & 894.217 & 6.48472 & & 0.045767857 & \\
\hline & 898.556 & 6.57756 & & 0.047804227 & \\
\hline & 902.917 & 6.67174 & & 0.049900775 & \\
\hline & 907.298 & 6.76726 & & 0.052057527 & \\
\hline & 911.701 & 6.86416 & & 0.054275356 & \\
\hline & 916.125 & 6.96246 & & 0.056554646 & \\
\hline & 920.57 & 7.06216 & & 0.058895255 & \\
\hline & 925.037 & 7.16328 & & 0.061297448 & \\
\hline & 929.526 & 7.26584 & & 0.063761436 & \\
\hline & 934.036 & 7.36986 & & 0.066287379 & \\
\hline & 938.569 & 7.47572 & & 0.068884259 & \\
\hline & 943.123 & 7.58433 & & 0.071574525 & \\
\hline & 947.7 & 7.69451 & & 0.074328871 & \\
\hline & 952.298 & 7.8063 & & 0.077147722 & \\
\hline & 956.919 & 7.91971 & & 0.080030698 & \\
\hline & 961.563 & 8.03477 & & 0.082977865 & \\
\hline & 966.229 & 8.15149 & & 0.085988719 & \\
\hline & 970.917 & 8.2699 & & 0.089063211 & \\
\hline & 975.629 & 8.39002 & & 0.092200978 & \\
\hline & 980.363 & 8.51189 & & 0.095402125 & \\
\hline & 985.12 & 8.63554 & & 0.098666446 & \\
\hline
\end{tabular}

Table 1: Particle Transmission for Beryllium film at 20 microns thick. The attenuation lengths are calculated based on [5]. 


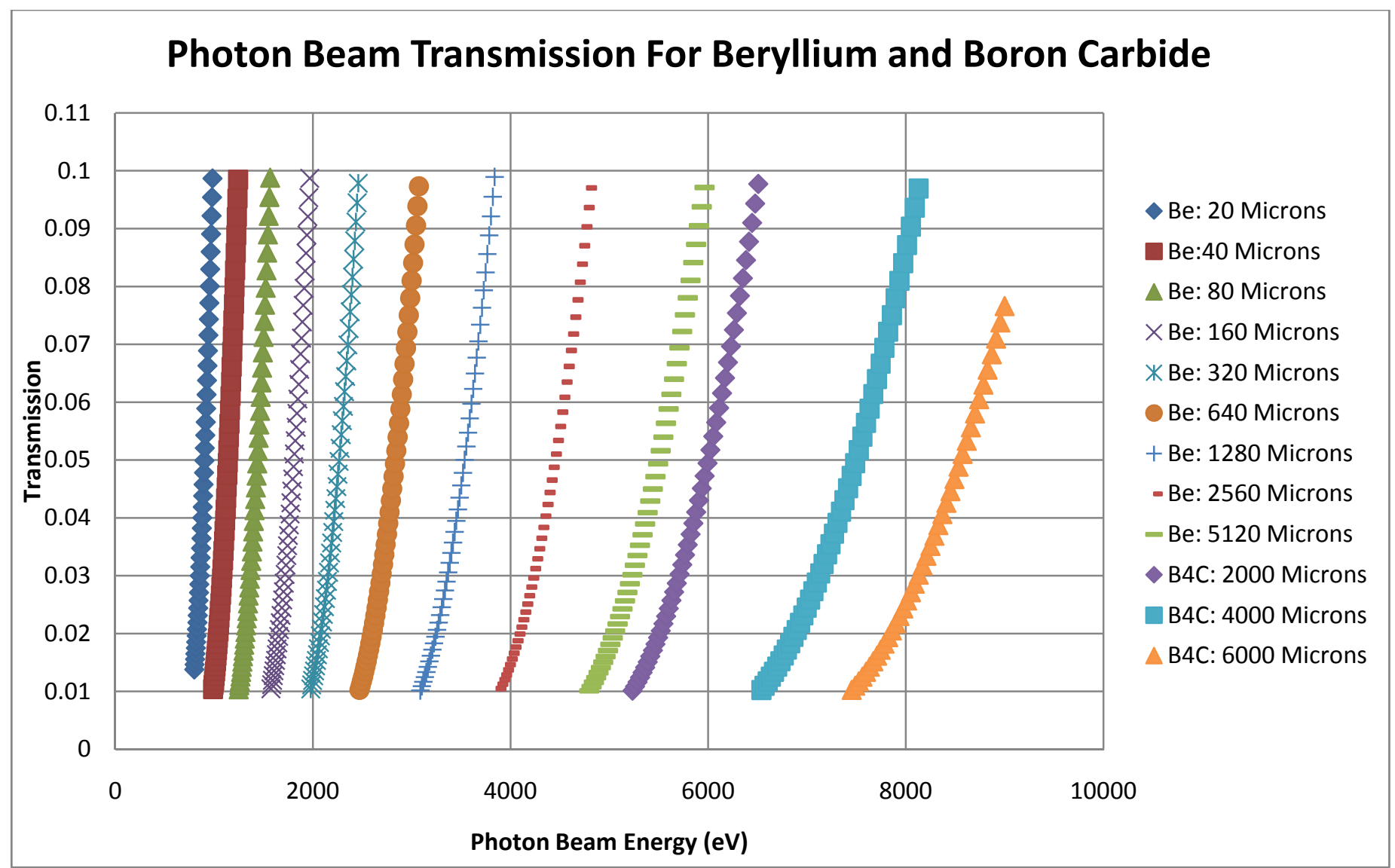

Figure 1: Data Plot of the Photon Transmission for Beryllium and Boron Carbide. Shows the transmission range and the energy ranges for each thickness of filter. 


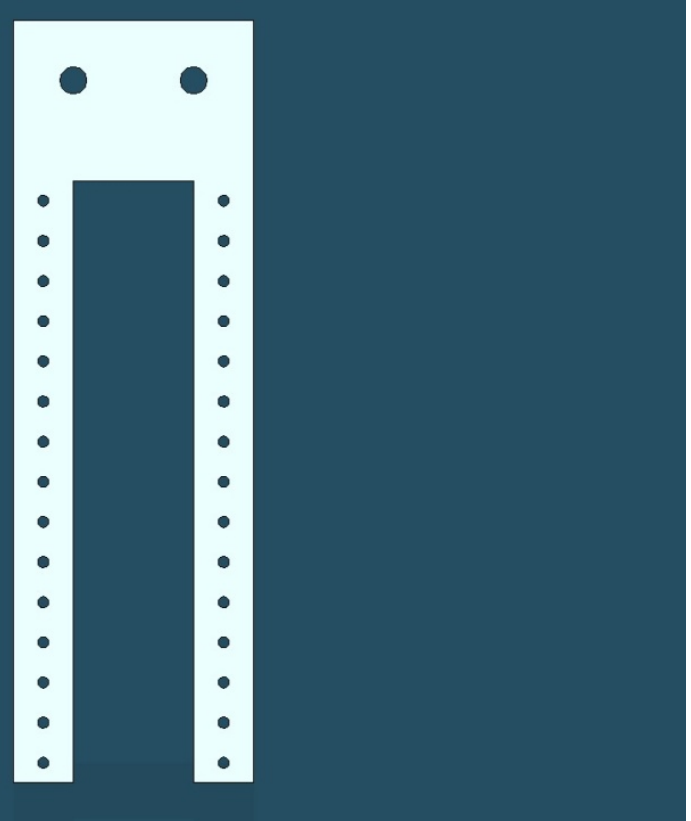

Figure 2: Filter Fork Holder with 15 spaces for \#2-56 UNC threaded screws and washers.

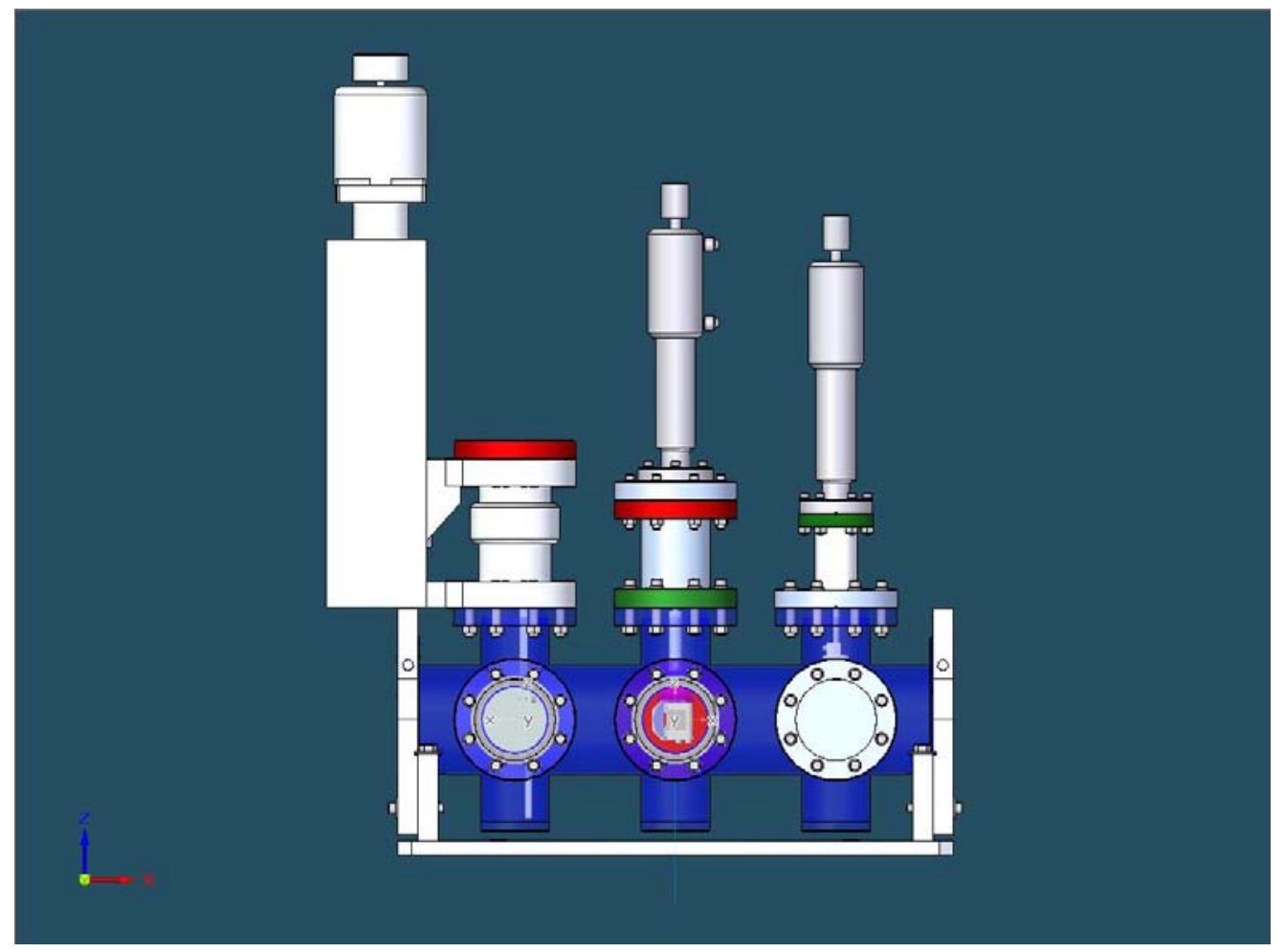

Figure 3: Filter Fork Holder Assembly placed in the ST-0 replacement test chamber. The Fork assembly is located in the chamber 1 , furthest to the left. 


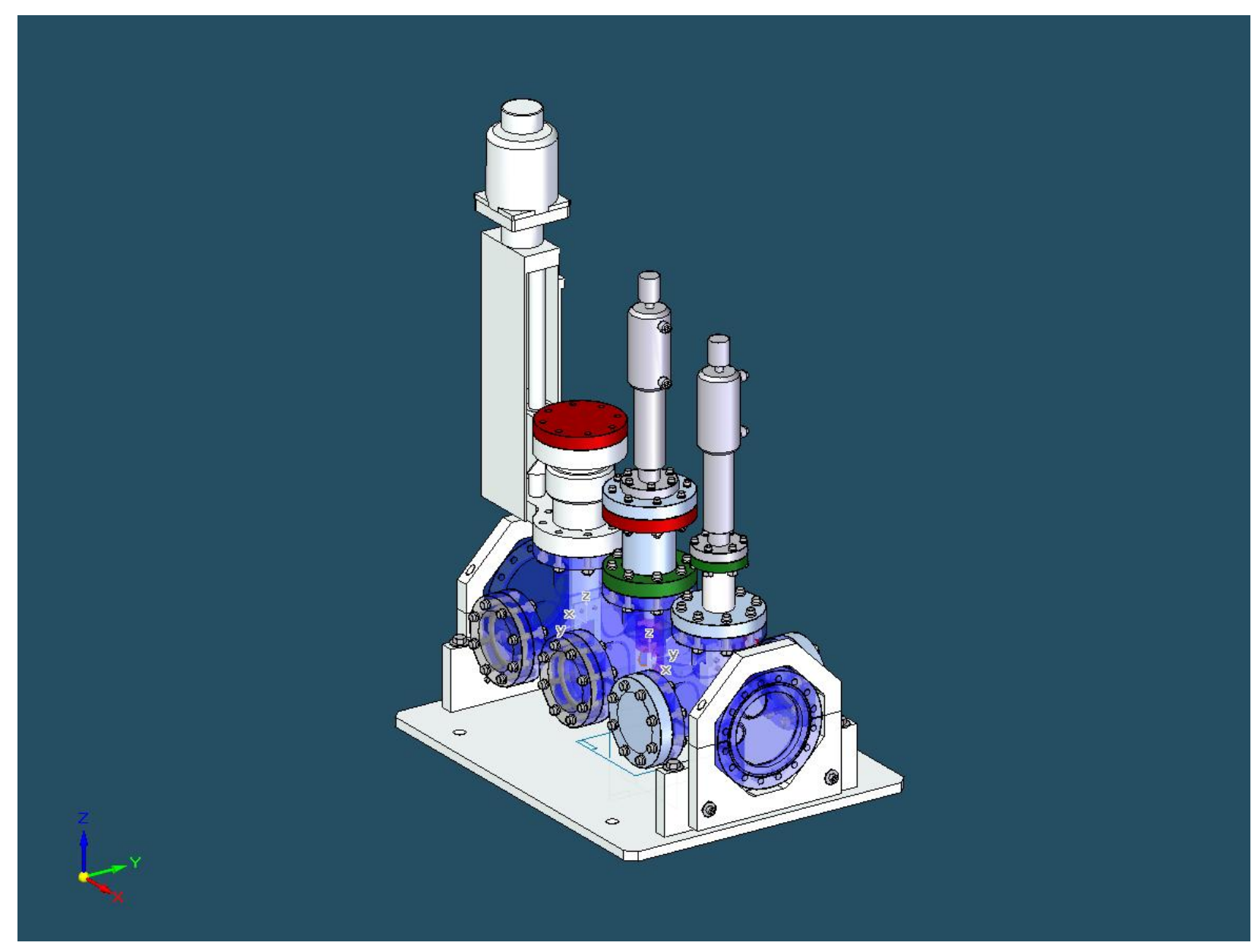

Figure 4: Filter Fork Holder Assembly in an isometric view relative to the other photon tests and the beam line direction. Beam line is along $x$-axis based on the coordinate system to the left. 
Synthesis of Samarium Cobalt Nanoblades

Darren Steele

Office of Science, Science Undergraduate Laboratory Internship Program

California State University, Sacramento

SLAC National Accelerator Laboratory

Menlo Park, CA

Prepared in partial fulfillment of the requirement of the Office of Science, Department of Energy's Science Undergraduate Laboratory Internship under the direction of James Spencer in the Advanced Accelerator Research division at the SLAC National Accelerator Laboratory.

August 13, 2009

Participant:

Signature

Research Advisor:

$$
\text { Signature }
$$




\section{Table of Contents}

$\begin{array}{ll}\text { Abstract } & 3\end{array}$

$\begin{array}{ll}\text { Introduction } & 4\end{array}$

Methods $\quad 8$

$\begin{array}{ll}\text { Results } & 12\end{array}$

Discussion and Conclusion 13

$\begin{array}{ll}\text { References } & 15\end{array}$

$\begin{array}{ll}\text { Acknowledgements } & 15\end{array}$

$\begin{array}{ll}\text { Figures and Tables } & 16\end{array}$ 


\begin{abstract}
Synthesis of Samarium Cobalt Nanoblades. DARREN M. STEELE (Sacramento State University, Sacramento, CA 95819) JAMES E. SPENCER (SLAC National Accelerator Laboratory, Menlo Park, CA 94025).

As new portable particle acceleration technologies become feasible the need for small high performance permanent magnets becomes critical. With particle accelerating cavities of a few microns, the photonic crystal fiber (PCF) candidate demands magnets of comparable size. To address this need, samarium cobalt ( $\mathrm{SmCo}$ ) nanoblades were attempted to be synthesized using the polyol process. Since it is preferable to have blades of 1-2 $\mu \mathrm{m}$ in length, key parameters affecting size and morphology including method of stirring, reaction temperature, reaction time and addition of hydroxide were examined. Nanoparticles consisting of $70-200 \mathrm{~nm}$ spherical clusters with a $3-5 \mathrm{~nm}$ polyvinylpyrrolidone (PVP) coating were synthesized at $285^{\circ} \mathrm{C}$ and found to be ferromagnetic. Nanoblades of $25 \mathrm{~nm}$ in length were observed at the surface of the nanoclusters and appeared to suggest agglomeration was occurring even with PVP employed. Morphology and size were characterized using a transmission electron microscope (TEM). Powder X-Ray Diffraction (XRD) analysis was conducted to determine composition but no supportive evidence for any particular SmCo phase has yet been observed.
\end{abstract}




\section{Introduction:}

\section{i. Demand and Applications of Nano-scale Magnets.}

As modern science and technology progress proceeds through the $21^{\text {st }}$ century, material scientists and engineers will continue to create and take nanotechnology to unimaginable limits. One interesting aspect of nanotechnology is the use of nano-scale permanent magnetic materials. Applications range from high density magnetic recording devices to MRI contrast agents. Magnetic nanoparticles are even being used in Germany as part of an experimental tumor destroying procedure known as magnetic hyperthermia [1]. A very recent suggestion made by Dr. James Spencer of SLAC National Accelerator Laboratory, is to employ magnetic nanoparticles in a future particle accelerator concept based on PCFs.

\section{ii. The involvement of Magnets in Particle Accelerators.}

The very basis for magnets to be employed in particle accelerators goes back to the $19^{\text {th }}$ century in the theory of electricity and magnetism. One of the most important results based on experiment is known as the Lorentz force and is given by,

$$
F=q(E+v x B)
$$

The second term of this equation says that the magnetic force will be orthogonal to both the velocity of the particle and the magnetic field it interacts with. This implies that if one can create just the right configuration of magnets, one can establish a magnetic field that will influence the trajectory of a charged particle beam in just the way one desires. For example, a linear accelerator requires a series of focusing and defocusing magnets that will keep an electron or positron beam from diverging and interacting with the accelerating cavity's walls. A circular accelerator requires bending magnets which direct 
particles into circular motion. Finally, a free electron laser which utilizes a linear accelerator requires a series of alternating dipole magnets known as an undulator to oscillate an electron beam to produce intense coherent photon beams.

\section{iii. A Future Linear Accelerator Based on PCFs.}

The traditional linear accelerator employs long copper cavities and RF power to accelerate electrons and positrons with a gradient of $25 \mathrm{MeV} / \mathrm{m}$. Based on this number, the SLAC LINAC at $3000 \mathrm{~m}$ can boost electrons and positrons to a maximum energy of $75 \mathrm{GeV}$. In order to make new discoveries in high energy physics, this number must be inflated upto a $\mathrm{TeV}$, where one $\mathrm{TeV}=10^{3} \mathrm{GeV}$. With this said, a $1 \mathrm{TeV}$ linear accelerator based on copper RF cavities must be $40 \mathrm{~km}$ long. At this size economic feasibility becomes a real draw back. Accelerator physicists have devised new approaches to reaching higher energies, including the use of PCFs as an accelerating structure. A PCF is a silicon or silica optical fiber with an artificially constructed periodic array of vacuum defects that utilizes a photonic band gap to propagate specific frequencies of radiation. It has high potential as an accelerating structure as it was calculated by Eddie Lin that such a structure could produce an accelerating gradient of $0.38 \mathrm{GeV} / \mathrm{m}$, which is more than 15 times larger than that of SLAC's LINAC [2]. With an acceleration cavity of a few microns, it is desirable to have the previously mentioned magnetic requirements of particle accelerators brought down to the same scale in order for PCF accelerators to become a reality.

iv. Samarium Cobalt Nanoblades.

A possible solution to the previous problem appeared in the summer of 2008 as researchers from Northeastern University and Sandia National Laboratory published a 
paper proposing a new chemical method for producing samarium cobalt nanoblades of $100 \mathrm{~nm}$ by $10 \mathrm{~nm}$ dimension and values of the intrinsic coercivity and magnetization of $6.1 \mathrm{kOe}$ and $40 \mathrm{emu} / \mathrm{g}$ and $8.5 \mathrm{kOe}$ and $44 \mathrm{emu} / \mathrm{g}$, at room temperature and $10 \mathrm{~K}$, respectively [3]. The nanoparticle synthesis employed is commonly referred to as the polyol process.

\section{v. The Polyol Process.}

The polyol process is a chemical process which allows for the production of nanoparticles of a narrow size distribution. This occurs through the control of several parameters, most importantly, reaction time, reaction temperature, heating rate, and surfactant to ion concentration. The process involves the classic redox reaction, where redox is short for reduction-oxidation. This refers to the ability of two chemically distinct species to transform as one species gains electrons and the other loses electrons. In the polyol process, a solvent is chosen which also acts as the reducing agent by reducing dissolved metal cations into metal atoms as it itself is being reduced. The reaction mechanism for formation of SmCo particles involves an initial reduction of $\mathrm{Co}^{2+}$ to $\mathrm{Co}$ and the creation of amorphous $\mathrm{Sm}_{2} \mathrm{O}_{3}$ as an intermediate both of which involving tetraethylene glycol (TrEG). The intermediate $\mathrm{Sm}_{2} \mathrm{O}_{3}$ phase favors a rapid generation of dissolved Co species that are finally reduced to form crystalline SmCo particles [3]. A reaction scheme can be seen in Scheme 1. Once SmCo particles form in nuclei, crystal growth is regulated by the interaction between the metal particles and surfactant. In the paper, polyvinylpyrrolidone (PVP) was employed. Its carbonyl group interacts with the charge distribution at the metal surface resulting in a shift in electron density and electrostatic attraction due to the high electronegativity of the carbonyl oxygen. This 
results in an organic coating of the metal nanoparticles which may function to keep particles from aggregating through steric hindrance (electrostatic repulsion) and to protect from oxidation as nanoparticles are extremely prone to reaction with oxygen gas due to their large surface area to volume ratio [4]. Molecular structures of TrEG and the basic unit of PVP may be seen in Figure 1.

\section{vi. Samarium Cobalt Physical Properties.}

Samarium cobalt is a binary compound that exists as a metal alloy with many phases as seen in the phase diagram found in Figure 2 [5]. SmCo alloy is a rare-earth-transitionmetal (RE-TM) permanent magnetic material, which is characterized by having a sufficiently high Curie temperature, a high remanent magnetization, and a high magnetocrystalline anisotropy [3], [5]. The first two of these properties are provided predominantly by the sublattice of the $3 \mathrm{~d}$ element cobalt, while the third is due mainly to the rare-earth samarium, sublattice [5]. The high Curie temperature allows for the material to remain in a ferromagnetic phase, hence remain spontaneously magnetized which is important for the integration into a particle accelerator. The high remenance magnetization allows for the construction of strong enough magnetic fields to adequately influence charged particle beams in a desired fashion. Finally, the high magnetocrystalline anisotropy helps prevent domain reversal by establishing a crystal easy axis, hence allowing for a better preservation of magnetization. The domains will energetically favor preserving the anisotropy even when external fields are applied, thus particularly dominant anisotropic phases, $1: 5$ and 2:17, will tend to be present each with a high intrinsic coercivity [6]. The 2:17 phase exhibits higher magnetic performance at the 
cost of having a more complex lattice structure. Unit cells for each of these phases are found in Figure 2 [5].

\section{vii. Experimental Purpose.}

In order to implement samarium cobalt magnets into PCFs for accelerator design, they must first be created to the desired scale. Since there is no easy way to synthesize blade-like particles of microns in length, the previously proposed method was attempted to be reproduced with emphasis on the influences of stirring method, reaction temperature, reaction time and addition of sodium hydroxide in optimization of nanoparticle creation and size. The influence of key parameters on magnetic properties would also be critical and would be a later extension of this work. For the 1:5 phase, it can be estimated that the maximum size of a monodomain particle is given by,

$$
\mathrm{d}_{\mathrm{c}}=18 \gamma / \mu_{0} \mathrm{M}_{\mathrm{s}}^{2}=2 \mu \mathrm{m}
$$

where $\gamma$ is the domain wall energy per unit area and $M_{s}$ is the saturation magnetization [7]. Based on this approximation and the PCF cavity size, a narrow size distribution within the 1-2 $\mu \mathrm{m}$ size range of 1:5 phase crystallites would be ideal once a basis of understanding the synthesis process is established.

\section{Methods:}

\section{i. Safety Considerations.}

When working in a wet chemical lab it is imperative to wear proper personal protection equipment (PPE). For the purposes of this experiment, nitrile gloves, complete coverage safety goggles and a lab coat have been worn and maintained at the experimental site. MSDS information reported that none of the required chemicals have 
serious acute toxicity concerns or carcinogenic effects; however cobalt containing compounds are known to be toxic in large doses. Review Table $\mathbf{1}$ for experimental hazard descriptions and hazard controls.

\section{ii. Chemicals and Apparatus.}

99.9\% purity samarium (III) nitrate hexahydrate, 98\% purity cobalt (II) nitrate hexahydrate, 99\% purity tetraethylene glycol, polyvinylpyrrolidone (PVP, molecular weight $=55 \mathrm{k}$ ), ethanol and hexane were obtained from Sigma Aldrich. A series of syntheses were conducted at the Geballe Laboratory of Advanced Materials (GLAM) at Stanford University. The syntheses deviated from the original published work by being conducted using a Schlenk line technique as opposed to the more laborious glove box method. A typical double manifold Schlenk line may be seen in Figure 3 [8]. Please consult references [9] and [10] for more information on Schlenk line procedural details.

\section{iii. Characterization Instrumentation.}

Optical microscopy was performed using an Olympus SZX9 camera. TEM imaging was conducted on a FEI TECNAI TEM using a copper grid for sample placement. XRD was done using a PANalytic X'Pert PRO instrument with a copper target. Both instruments were used at the Stanford University Nanocharacterization Laboratory (SNL).

iv. Synthesis 1: Magnetic Stir Bar Method.

$2.2 \mathrm{mg} \mathrm{Sm}\left(\mathrm{NO}_{3}\right)_{3} \cdot 6 \mathrm{H}_{2} \mathrm{O}, 7.3 \mathrm{mg} \mathrm{Co}\left(\mathrm{NO}_{3}\right)_{2} \cdot 6 \mathrm{H}_{2} \mathrm{O}$, and $564 \mathrm{mg}$ PVP (55k) were dissolved in $15 \mathrm{ml}$ TrEG in a $25 \mathrm{ml}$ three-necked, round-bottom flask that contained a half inch magnetic stirring bar. The central neck of the flask was connected to a condenser and attached to the apparatus. The flask was supported underneath by a heating 
mantle which rested on an elevated magnetic stirring plate. A rubber septum was placed on one side neck and a thermocouple attached to the other. The reaction vessel was evacuated for $15 \mathrm{~min}$ at room temperature and then for 30 minutes at $100^{\circ} \mathrm{C}$. The solution became a pink color due to the dissolved $\mathrm{Co}\left(\mathrm{NO}_{3}\right)_{2} \cdot 6 \mathrm{H}_{2} \mathrm{O}$. The vessel was then filled with nitrogen gas at 4 psi and heated to $200^{\circ} \mathrm{C}$ for one hour. Color changes were rather quick from pink, to orange, to red, to merlot and finally to black. After one hour of heating the reaction vessel was cooled to room temp, the magnetic stir bar removed and the solution centrifuged for 15 minutes at $6500 \mathrm{rpm}$ to reveal no noticeable product. The magnetic stir bar was then looked at under an optical microscope.

\section{v. Synthesis 2: Nitrogenation Method.}

$3.3 \mathrm{mg} \mathrm{Sm}\left(\mathrm{NO}_{3}\right)_{3} \cdot 6 \mathrm{H}_{2} \mathrm{O}, 10.5 \mathrm{mg} \mathrm{Co}\left(\mathrm{NO}_{3}\right)_{2} \cdot 6 \mathrm{H}_{2} \mathrm{O}$ and $554 \mathrm{mg}$ PVP (55k) were dissolved in $15 \mathrm{ml}$ TrEG in a $25 \mathrm{ml}$ three-necked, round-bottom flask. A septum was added to one side neck and a thermocouple to the other. No magnetic stir bar was present. The reaction vessel was evacuated for the same duration as synthesis 1 . Prior to switching to nitrogen, a 16 gauge trans-spinal needle connected to a nitrogen gas source was injected through the septum. The vacuum was switched to nitrogen followed by turning on of the additional nitrogen bubbling source. Heating was conducted at $200^{\circ} \mathrm{C}$ for one hour with the same color changes observed. The final black solution was centrifuged at 7500 for 15 min after adding ethanol with no observable product.

\section{vi. Synthesis 3: Magnetic Stir Bar Method with $\mathrm{SmCo}_{5}$ Magnet.}

$13 \mathrm{mg} \mathrm{Sm}\left(\mathrm{NO}_{3}\right)_{3} \cdot 6 \mathrm{H}_{2} \mathrm{O}, 42 \mathrm{mg} \mathrm{Co}\left(\mathrm{NO}_{3}\right)_{2} \cdot 6 \mathrm{H}_{2} \mathrm{O}$ and $2.22 \mathrm{~g}$ PVP (55k) were disolved in $60 \mathrm{ml}$ TrEG in a custom three-necked flask after adding an approximately inch and a half $\mathrm{SmCo}_{5}$ permanent bar magnet was wrapped in teflon except for a small portion at the 
center of the easy axis face. The vessel was evacuated for 15 minutes at room temperature followed by 30 minutes at $100^{\circ} \mathrm{C}$. Once vacuum was switched to nitrogen, the vessel was heated to $200^{\circ} \mathrm{C}$ and sat there for one hour. After the vessel was cooled similar processes were conducted with solution to reveal no noticeable product.

vii. Synthesis 4: Nitrogenation at Elevated Temperature.

$3.2 \mathrm{mg} \mathrm{Sm}\left(\mathrm{NO}_{3}\right)_{3} \cdot 6 \mathrm{H}_{2} \mathrm{O}, 10.0 \mathrm{mg} \mathrm{Co}\left(\mathrm{NO}_{3}\right)_{2} \cdot 6 \mathrm{H}_{2} \mathrm{O}$ and $550 \mathrm{mg}$ PVP (55k) were disolved in $15 \mathrm{ml} \mathrm{TrEG}$ in a $25 \mathrm{ml}$ three-necked, round-bottom flask with no magnetic stirring bar. Vacuum procedures followed synthesis two and the same 16 gauge transspinal needle was injected through septum following switch from vacuum to nitrogen just as synthesis 2 . The flask was wrapped in aluminum foil and heated to $290^{\circ} \mathrm{C}$ and allowed to sit for one hour after stabilizing at that temperature. A dark black solution was observed. After adding hexane to solution, centrifugation at $8000 \mathrm{rpm}$ for $30 \mathrm{~min}$ resulted in the observation of a dark black powder that was dispersed in ethanol. TEM images were taken as will be discussed in the data section.

viii: Synthesis 5: Nitrogenation at Elevated Temperature for a Longer Time. $43.0 \mathrm{mg} \mathrm{Sm}\left(\mathrm{NO}_{3}\right)_{3} \cdot 6 \mathrm{H}_{2} \mathrm{O}, 134 \mathrm{mg} \mathrm{Co}\left(\mathrm{NO}_{3}\right)_{2} \cdot 6 \mathrm{H}_{2} \mathrm{O}$ and $7.33 \mathrm{~g}$ PVP (55k) were dissolved in $200 \mathrm{ml}$ TrEG in a $250 \mathrm{ml}$ three-necked, round-bottom flask. The vessel was evacuated for $30 \mathrm{~min}$ at room temperature and $45 \mathrm{~min}$ at $100^{\circ} \mathrm{C}$. After switching to nitrogen and adding the bubbling needle, the flask was covered in aluminum foil and the solution was heated at a rate of $7.5-9.0^{\circ} \mathrm{C} / \mathrm{min}$ for $1.25 \mathrm{hrs}$ until $285^{\circ} \mathrm{C}$ was reached. The solution was stabilized at $285^{\circ} \mathrm{C}$ for $1.5 \mathrm{hrs}$, then allowed to cool to room temperature for $1 \mathrm{hr}$. Removal of the foil revealed a dark black solution. Following the same centrifugation procedure as synthesis 4 , a darker black powder was recovered and 
dispersed in ethanol. XRD and TEM techniques were used to characterize particles as will be discussed.

ix. Synthesis 6: Addition of Sodium Hydroxide.

$7.3 \mathrm{mg} \mathrm{Sm}\left(\mathrm{NO}_{3}\right)_{3} \cdot 6 \mathrm{H}_{2} \mathrm{O}, 20.1 \mathrm{mg} \mathrm{Co}\left(\mathrm{NO}_{3}\right)_{2} \cdot 6 \mathrm{H}_{2} \mathrm{O}, 19.3 \mathrm{mg} \mathrm{NaOH}$ and $1.10 \mathrm{~g}$ PVP (55k) were dissolved in $30 \mathrm{ml} \mathrm{TrEG}$ in a $50 \mathrm{ml}$ three-necked round-bottom flask. The vessel was evacuated for $15 \mathrm{~min}$ at room temperature, $10 \mathrm{~min}$ at $50^{\circ} \mathrm{C}$ and $15 \mathrm{~min}$ at $100^{\circ} \mathrm{C}$. At $85^{\circ} \mathrm{C}$, the initial pink solution changed to a light yellow-green then to golf.

The vacuum was switched to nitrogen and a 16 gauge trans-spinal needle was injected for nitrogenation. The solution was heated slowly for $1 \mathrm{hr}$ till it reached $285^{\circ} \mathrm{C}$. Once stabilized, the solution was stirred and maintained at $285^{\circ} \mathrm{C}$ for $2 \mathrm{hrs}$. The solution was cooled to $50^{\circ} \mathrm{C}$ for $30 \mathrm{~min}$ and centrifuged at $8000 \mathrm{rpm}$ for $30 \mathrm{~min}$ after adding toluene to yield a black powder product. The powder was rinsed several times and dispersed in ethanol. XRD and TEM characterization is in progress.

\section{Results:}

Optical microscope imaging of the magnetic stir bar of synthesis 1 revealed what appears to be reflective metallic circular structures on the surface as can be seen in Figure 4. Synthesis 2 produced no noticeable product. Synthesis 3 may have produced similar light depositing as the teflon coated stir bar synthesis 1 , but no significant crystal growth was observed. Synthesis 4 produced $70 \mathrm{~nm}$ polycrystalline nanoclusters as can be seen in Figure 5. A PVP coating of 3-5 nm was observed in Figure 6. Synthesis 5 produced 150-200 nm polycrystalline nanoclusters as can be seen in Figure 7. Nanoblades of $25 \mathrm{~nm}$ were clearly differentiated and appeared amongst the nanoclusters as can be seen in Figure 8. An XRD spectrum was taken but is not reported as it did not 
match a single SmCo or Co phase. When dispersed in ethanol the particles produced in synthesis 6 have a similar optical density and settling behavior as the nanoclusters from syntheses 4 and 5 suggesting agglomeration once again. XRD and TEM characterization have not yet been conducted.

\section{Discussion and Conclusion:}

\section{i. Stirring method.}

The results of syntheses one and three reveal that the use of conventional magnetic stirring is ineffective for producing nanoscale particles capable of being dispersed at least at a reaction temperature of $200^{\circ} \mathrm{C}$. Further investigation on the effects of magnetic stirring at varying temperature would need to be conducted in order to be effective. It does ask the question of whether metal structures may be grown uniformly off a magnetic substrate, which would also require further investigation. The use of a stainless steel needle allowed for stirring through nitrogenation and proved a successful method although the effect of nitrogen flow rate on particle size and morphology is unknown. It seems clear that adequate stirring that perturbs or prevents the settling out of the heavy metal alloy should improve the size and rate of crystal growth.

\section{ii. Reaction temperature.}

It is clear that a reaction temperature of $285-290^{\circ} \mathrm{C}$ which is close to the boiling point of TrEG is preferable for efficiently producing nanoparticles as the products of syntheses 4-6 indicate. A direct study of reaction temperature and particle size distribution at constant reaction time is a future possibility for reaction temperature optimization. 


\section{iii. Reaction time.}

From the TEM images of the products of Syntheses 4 and 5, it is apparent that a longer reaction time resulted in a doubling of particle size. Reaction time could be optimized with further study in conjunction with more optimal methods.

iv. Sodium Hydroxide Addition.

Characterization of the product of Synthesis 6 is currently being done.

\section{v. Other Findings.}

The near transparent coating seen in the TEM images at the nanocluster surfaces suggests PVP has successfully coated the metal particles which may prevent oxidation but may impede crystal growth. The different shades within the particles suggests a polycrystalline composition, but there is no conclusive evidence yet to identify the products as $\mathrm{SmCo}$ or even Co and so further compositional analysis is necessary. It is possible that the coreduction was faulty which lead to addition of $\mathrm{NaOH}$ in Synthesis Six in order to help boost the kinetics of the Samarium ion reduction which is imperative to coalesce with the Cobalt ion reduction in order to successfully form SmCo nuclei. The products of Syntheses 4-6 were found to be ferromagnetic due to their attraction to a permanent magnet and it is unknown whether they are mono or poly domain. As apparent from the very formation of the nanoclusters, agglomeration is an issue preventing monodispersion of nanoblades, which will require greater investigation. Work on this project is to be continued over the course of the next several months at GLAM as well as at California State University, Sacramento and University of California at Davis. 


\section{References:}

[1] Stéphane Mornet, Sébastien Vasseur, Fabien Grasset and Etienne Duguet. "Magnetic nanoparticle design for medical diagnosis and therapy." J. Mater. Chem., vol. 14, pp. 2161-2175, 2004.

[2] Xintian Eddie Lin. "Photonic band gap fiber accelerator." Phys. Rev. STAB, vol .4, 2001.

[3] C. N. Chinnasamy, J. Y. Huang, L. H. Lewis, B. Latha, C. Vittoria, and V. G. Harris. "Direct chemical synthesis of high coercivity air-stable SmCo nanoblades." Appl. Phys. Let, vol..93.

[4] Y. Hou et al. "A Facile Synthesis of SmCo5 Magnets from Core/Shell Co/Sm2O3 Nanoparticles.” Adv. Mater,. vol. 19, pp. 3349-3352, 2007.

[5] Pfeiler, W. Alloy Physics: A Comprehensive Reference, $1^{\text {st }}$ ed., Wiley: New York, 2007; pp 878-879.

[6] Buschow, K. H. J.; De Boer, F. R. Physics of Magnetism and Magnetic Materials, $1^{\text {st }}$ ed., Kluwer Academic/Plenum Publishers: New York, 2003; p 107.

[7] E.M. Kirkpatrick, S.A. Majetech, and M.E. McHenry. "Magnetic properties of single domain samarium cobalt nanoparticles." IEEE Transactions on Magnetics, vol. 32, p. 4502, 1996.

[8] Shriver, D. F.; Drezdzon, M.A. The Manipulation of Air-Sensitive Compounds, $2^{\text {nd }}$ ed., Wiley: New York, 1986.

[9] Girolami, G. S.; Rauchfuss, T. B.; Angelici, R. J. Synthesis and Technique in Inorganic Chemistry, $3^{\text {rd }}$ ed., University Science Books: Sausalito, CA, 1999; p 173.

[10] Kubiak, C. P. Kubiak Lab Manual. http://kubiak.ucsd.edu/manual/schlenktech.php (accessed July 21, 2009).

\section{Acknowledgements:}

Special thanks for the facilities support and training from Steve Connor, Yi Cui, Ben Weil and Wen Zheng at GLAM, the mentoring from Jim Spencer, the experimental details from C.N. Chinnasamy at Northeastern University, and the financial support and organization from SLAC, the Office of Science and the U.S. Dept. of Energy. 


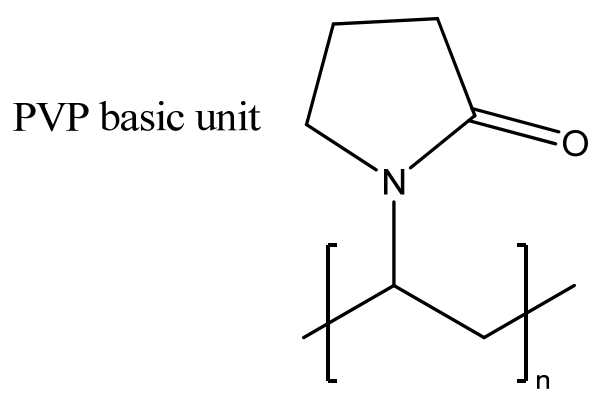

TrEG

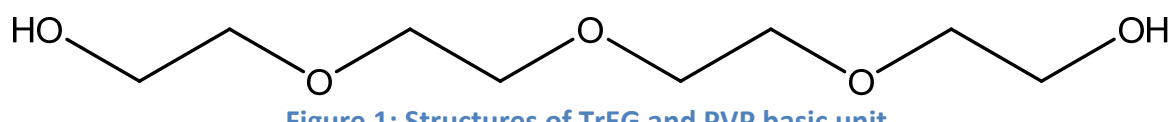

Figure 1: Structures of TrEG and PVP basic unit.

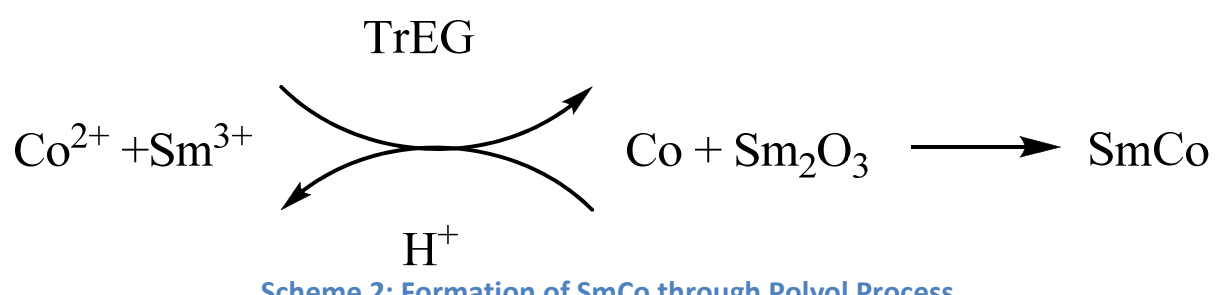

Scheme 2: Formation of SmCo through Polyol Process.

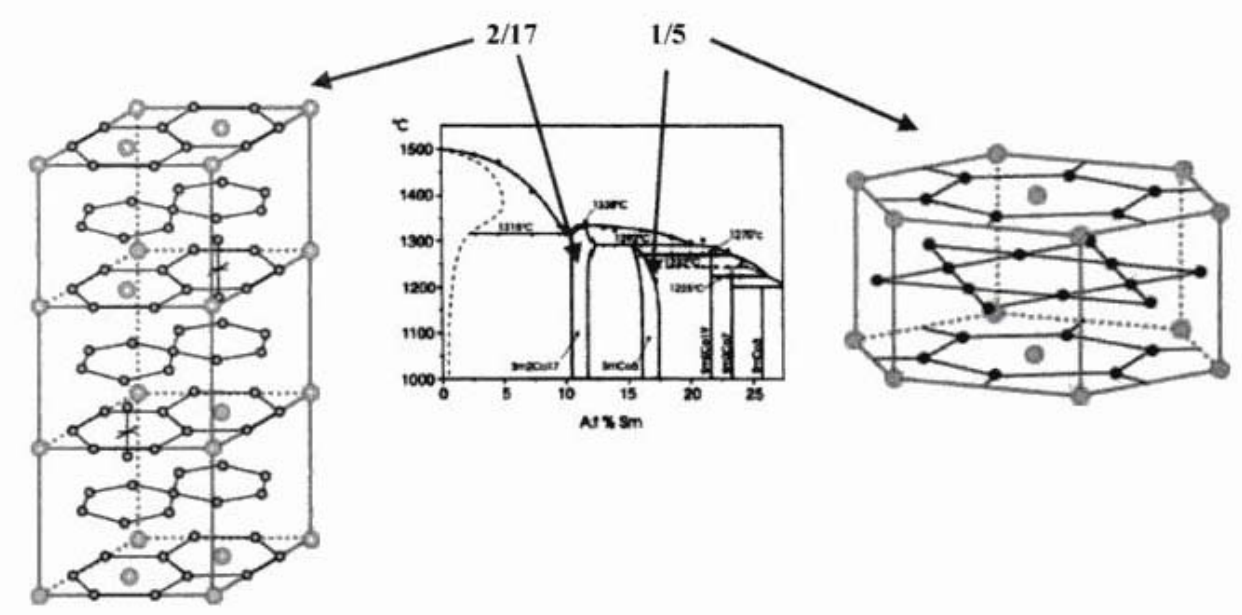

Figure 2: Samarium cobalt alloy phases. $\mathrm{SmCo}_{5}$ is on the right. 


\begin{tabular}{|c|c|c|c|c|}
\hline Hazard & Hazard Description & Probability/Severity & Control & $\begin{array}{l}\text { Hazard Risk with } \\
\text { control implemented }\end{array}$ \\
\hline $\begin{array}{l}\text { Chemical exposure to } \\
\text { skin }\end{array}$ & $\begin{array}{l}\text { Irritation or chemical } \\
\text { absorption through } \\
\text { skin. Acute and chronic } \\
\text { risks found in MSDS. }\end{array}$ & $\begin{array}{l}\text { High probability of skin } \\
\text { contact/ Low severity of } \\
\text { direct external exposure. }\end{array}$ & $\begin{array}{l}\text { Utilization of proper } \\
\text { PPE. Nitrile gloves and } \\
\text { lab coat. Store unused } \\
\text { reagents in respective } \\
\text { chemical cabinets and } \\
\text { chemical waste in } \\
\text { designated waste } \\
\text { containers. }\end{array}$ & Very low. \\
\hline $\begin{array}{l}\text { Chemical exposure to } \\
\text { eyes }\end{array}$ & $\begin{array}{l}\text { Irritation or blindness. } \\
\text { Specific descriptions } \\
\text { found in MSDS. }\end{array}$ & $\begin{array}{l}\text { Moderate probability of } \\
\text { eye contact/ Moderate to } \\
\text { high severity from eye } \\
\text { contact }\end{array}$ & $\begin{array}{l}\text { Utilization of proper } \\
\text { PPE. Complete closure } \\
\text { safety goggles. }\end{array}$ & Very low. \\
\hline $\begin{array}{l}\text { Chemical exposure by } \\
\text { inhalation of ingestion }\end{array}$ & $\begin{array}{l}\text { Specific effects depend } \\
\text { on dose and given in } \\
\text { MSDS. Chronic } \\
\text { toxicity effects are } \\
\text { noted but not fully } \\
\text { evaluated for both } \\
\text { metal nitrates. }\end{array}$ & $\begin{array}{l}\text { Moderate probability/ } \\
\text { Low to moderate severity }\end{array}$ & $\begin{array}{l}\text { Dry chemicals and } \\
\text { liquids should be } \\
\text { handled in fume hood } \\
\text { as much as possible, } \\
\text { storage containers } \\
\text { should be closed after } \\
\text { use, and all spills } \\
\text { should be cleaned up } \\
\text { immediately. }\end{array}$ & Very low. \\
\hline $\begin{array}{l}\text { Direct exposure to } \\
\text { liquid nitrogen }\end{array}$ & $\begin{array}{l}\text { Contact of liquid } \\
\text { nitrogen by skin or } \\
\text { clothes may result in } \\
\text { severe burns and } \\
\text { permanent tissue } \\
\text { damage. }\end{array}$ & $\begin{array}{l}\text { Low probability/ Low to } \\
\text { high severity depending } \\
\text { on circumstance. }\end{array}$ & $\begin{array}{l}\text { Store liquid nitrogen } \\
\text { out of immediate work } \\
\text { area in properly } \\
\text { labeled dewar and } \\
\text { utilize standard liquid } \\
\text { nitrogen PPE including } \\
\text { cryogenic gloves and } \\
\text { safety goggles during } \\
\text { transfer. }\end{array}$ & Moderately low. \\
\hline $\begin{array}{l}\text { Contact of heating } \\
\text { mantle or hot glass }\end{array}$ & $\begin{array}{l}\text { Moderate burns may } \\
\text { result from contacting } \\
\text { heated objects. }\end{array}$ & $\begin{array}{l}\text { Low probability/ Low } \\
\text { severity. }\end{array}$ & $\begin{array}{l}\text { Maintain knowledge of } \\
\text { where heated objects } \\
\text { are located and allow } \\
\text { hot glass to return to } \\
\text { room temperature } \\
\text { touching glass with } \\
\text { back of hand prior to } \\
\text { handling. }\end{array}$ & Very low. \\
\hline $\begin{array}{l}\text { Ignition of samarium } \\
\text { cobalt alloy or } \\
\text { tetraethylene glycol } \\
\text { during reaction phase. }\end{array}$ & $\begin{array}{l}\text { Heating up to } 300^{\circ} \mathrm{C} \\
\text { provides conditions for } \\
\text { combustion of SmCo } \\
\text { metal and glycol vapor, } \\
\text { if oxygen gas and an } \\
\text { ignition source are } \\
\text { present in reaction } \\
\text { vessel. }\end{array}$ & $\begin{array}{l}\text { Low probability/ } \\
\text { Moderate severity }\end{array}$ & $\begin{array}{l}\text { Allow for several } \\
\text { vacuum-nitrogen } \\
\text { cycles prior to heating. } \\
\text { Close fume hood } \\
\text { during reaction in the } \\
\text { unlikely event that a } \\
\text { systematic failure } \\
\text { results in combustion. } \\
\text { Ensure no flames or } \\
\text { source of sparks are } \\
\text { present in fume hood } \\
\text { during reaction. }\end{array}$ & Moderately low. \\
\hline $\begin{array}{l}\text { Condensation of } \\
\text { oxygen in Schenk line } \\
\text { trap }\end{array}$ & $\begin{array}{l}\text { Liquid oxygen is } \\
\text { extremely dangerous } \\
\text { and reacts violently } \\
\text { with organic } \\
\text { compounds. If it } \\
\text { collects in trap and then } \\
\text { evaporates, it may } \\
\text { result in Schenk line } \\
\text { manifold explosion. }\end{array}$ & $\begin{array}{l}\text { Moderate probability/ } \\
\text { Moderate to high } \\
\text { severity depending on } \\
\text { concentrations of organic } \\
\text { compounds in cold trap. }\end{array}$ & $\begin{array}{l}\text { When shutting down } \\
\text { Schlenk line manifold, } \\
\text { remove liquid nitrogen } \\
\text { from trap and allow } \\
\text { glass to return to room } \\
\text { temperature prior to } \\
\text { shutting off vacuum } \\
\text { pump. }\end{array}$ & Very low. \\
\hline
\end{tabular}

Table 1: Experimental Hazards and Controls 


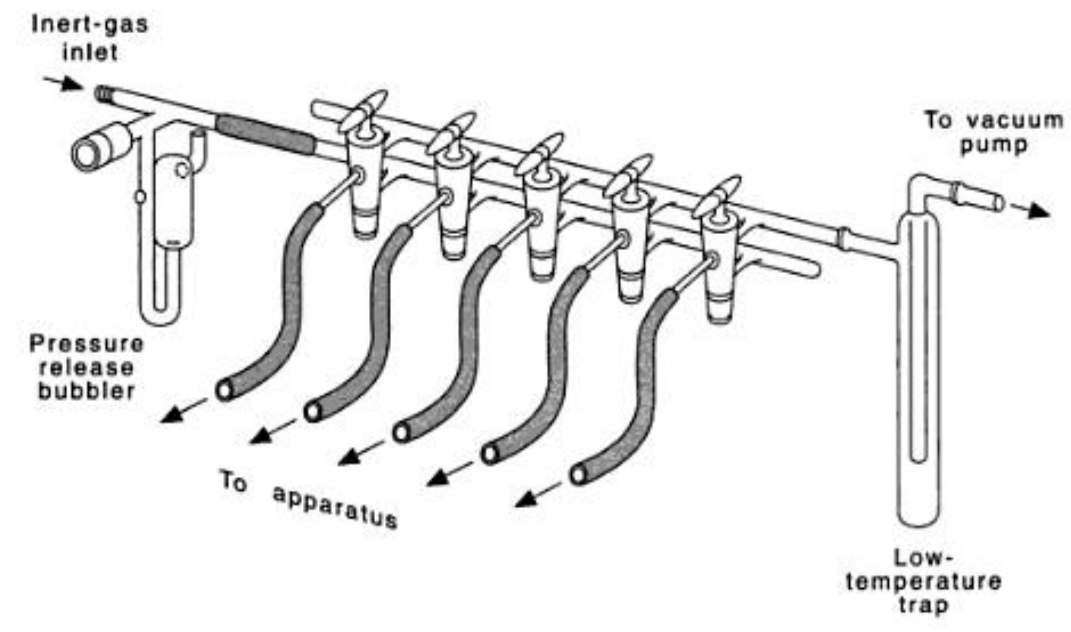

Figure 3: Schlenk line double manifold apparatus.

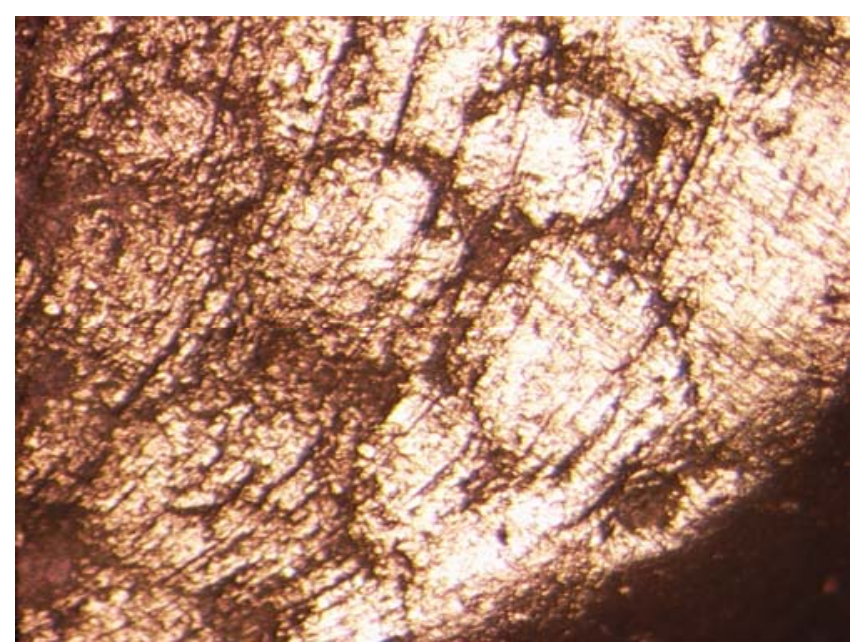

Figure 4: Optical microscope image of the Teflon coated magnetic stir bar surface from synthesis 1 . The metallic coating over the teflon is clearly evident. 


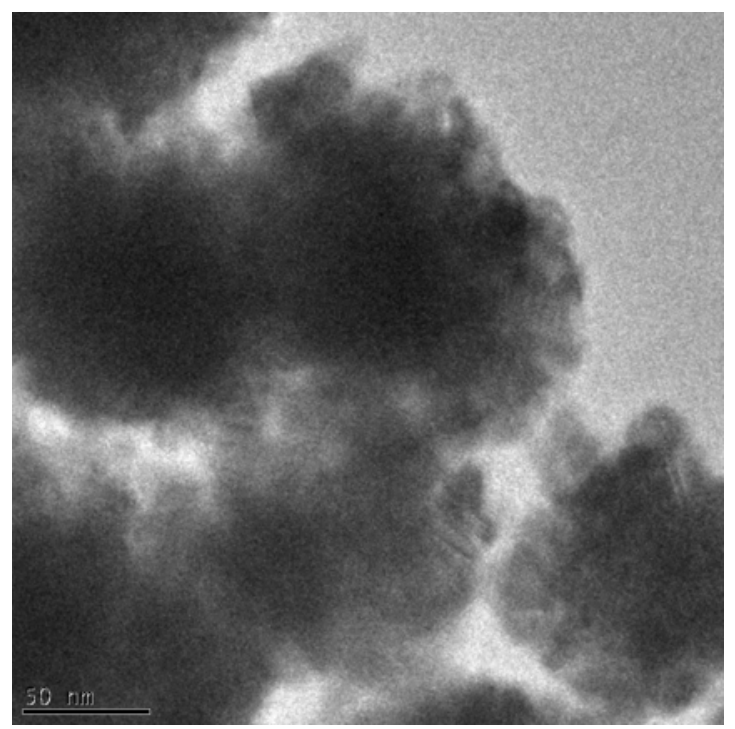

Figure 5: TEM image of nanoclusters from synthesis 4 .

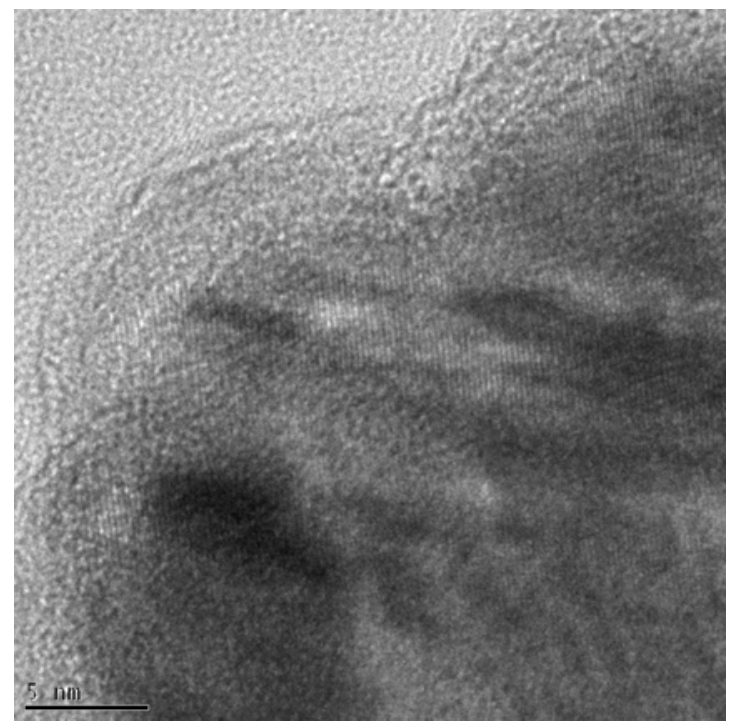

Figure 6: TEM image of nanocluster surface showing PVP coating and polycrystalline composition. 


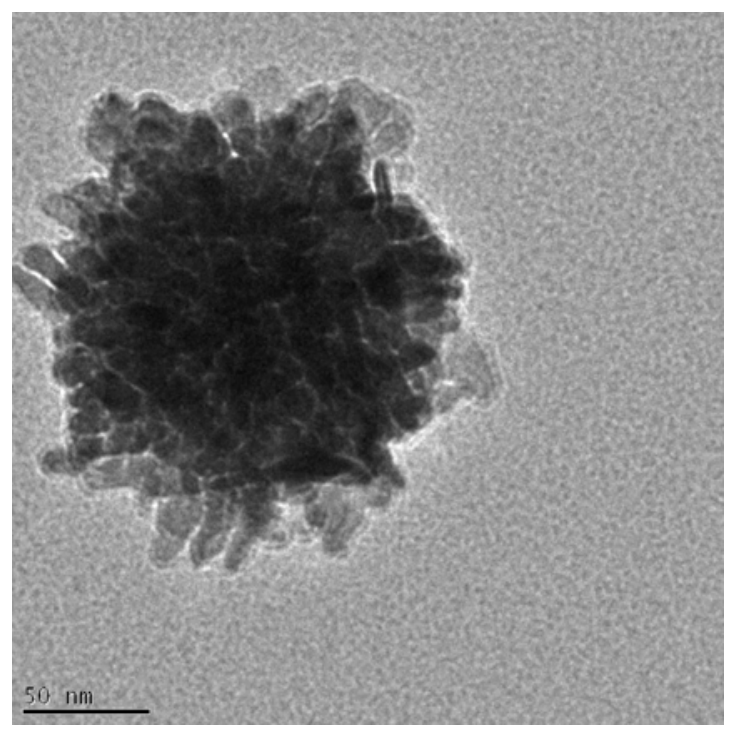

Figure 7: TEM image of larger nanocluster from synthesis 5.

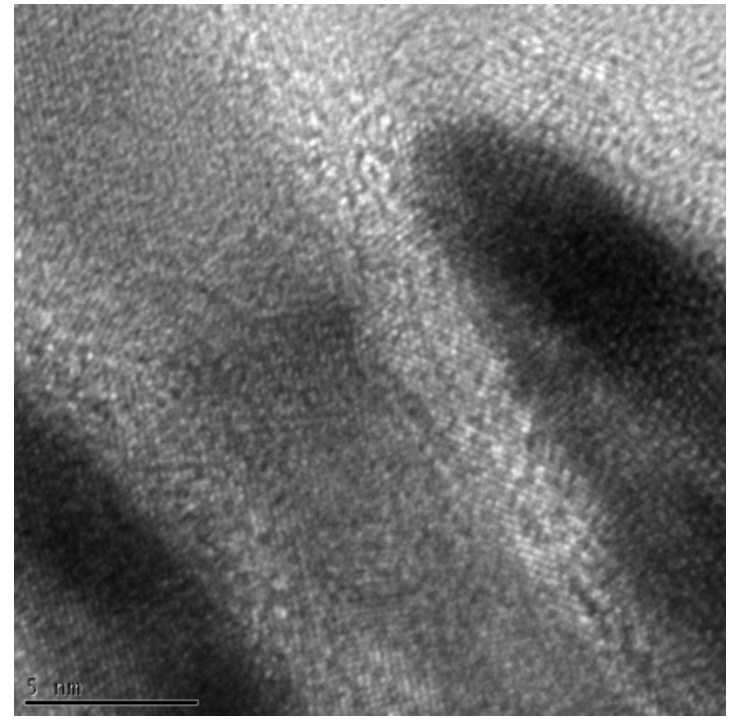

Figure 8: TEM image of nanoblades from surface of nanocluster. 
Development of Methods and Means of Configuration Data Transfer For Use in an FPGA Based Trigger Controller Device

\author{
Kelton D. Stefan \\ Office of Science, Science Undergraduate Laboratory Internship \\ Program \\ Purdue University
}

SLAC National Accelerator Laboratory

Menlo Park, California

August $14^{\text {th }}, 2009$

Prepared in partial fulfillment of the requirement of the Office of Science, Department of Energy's Science Undergraduate Laboratory Internship under the direction of Ronald Akre in the Klystron/Microwave Department at SLAC National Accelerator Laboratory .

Participant:

Signature

Research Adviser:

Signature 


\section{Table of Contents:}

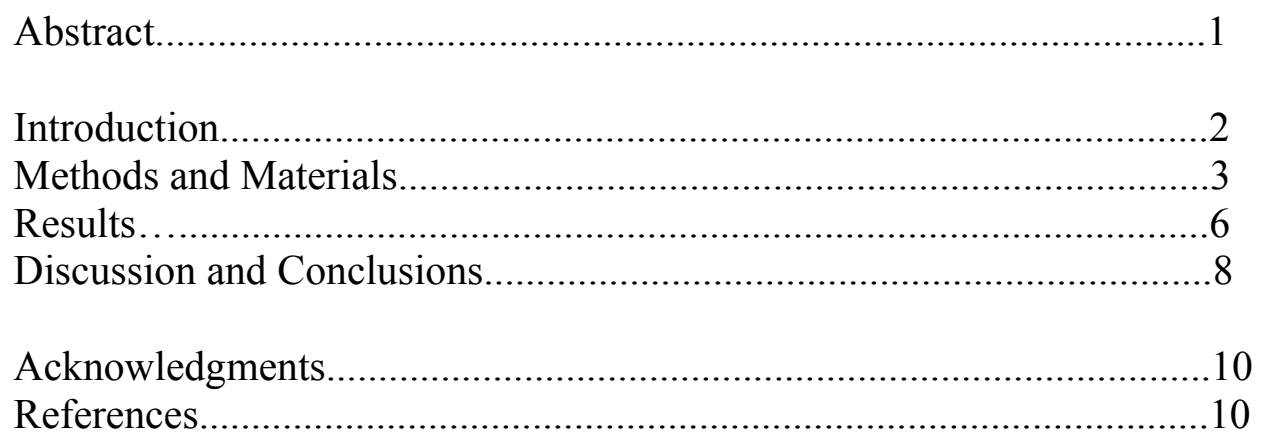

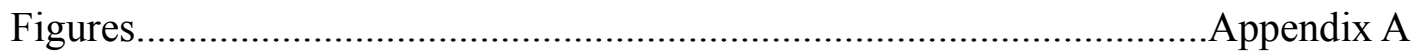


Kelton Stefan, August $14^{\text {th }}, 2009$

\begin{abstract}
:
To determine if klystrons will perform to the specifications of the LCLS (Linac Coherent Light Source) project, a new digital trigger controller is needed for the Klystron/Microwave Department Test Laboratory. The controller needed to be programmed and Windows based user interface software needed to be written to interface with the device over a USB (Universal Serial Bus). Programming the device consisted of writing logic in VHDL (VHSIC (Very High Speed Integrated Circuits) hardware description language), and the Windows interface software was written in $\mathrm{C}++$. Xilinx ISE (Integrated Software Environment) was used to compile the VHDL code and program the device, and Microsoft Visual Studio 2005 was used to compile the C++ based Windows software. The device was programmed in such a way as to easily allow $\mathrm{read} /$ write operations to it using a simple addressing model, and Windows software was developed to interface with the device over a USB connection. A method of setting configuration registers in the trigger device is absolutely necessary to the development of a new triggering system, and the method developed will fulfill this need adequately. More work is needed before the new trigger system is ready for use. The configuration registers in the device need to be fully integrated with the logic that will generate the RF signals, and this system will need to be tested extensively to determine if it meets the requirements for low noise trigger outputs.
\end{abstract}




\section{Introduction:}

Control is a very important part of almost anything going on at the SLAC National Accelerator Laboratory. This certainly holds true for the Klystron/Microwave Department Test Laboratory where new and reworked klystrons are constantly undergoing burn-in and testing routines. In an effort to improve the control system of each station in the test lab, a new digital FPGA (Field Programmable Gate Array) device was built and programmed to replace the old mostly analog systems. This upgrade was to meet the need of determining if klystrons will perform to the specifications of the LCLS project. Precise measurement of phase requires a low noise source. This new FPGA device will fulfill this requirement as well as introduce the possibility of synchronizing multiple test stations so that multiple klystrons can be operated in phase.

The FPGA device will control the various pieces of equipment at a test station using trigger signals. These trigger signals are precisely timed signals that have various parameters (see Figure 2). These parameters include rep rate (repetition rate), pulse delay, pulse width, and time slot. Rep rate is simply the frequency of the trigger pulses measured in Hz. Pulse delay is the time between the start of the signal to the rising edge of the pulse measured in ns. Pulse width is the time between the rising and the falling edges of the signal measured in ns. Finally, time slot is one of several possible clock pulses within a block of the $360 \mathrm{~Hz}$ signal defined by the desired rep rate. For example, if you require a $60 \mathrm{~Hz}$ rep rate, there are $360 / 60=6$ different time slots you have the freedom to operate on.

Central to the new trigger device is a Xilinx Spartan FPGA (Field Programmable Gate Array) [1] that will be programmed to do everything required for triggering. There are four inputs, three of which we have plans of using. There are eight output channels, all of which are used in the design. These eight channels are divided into a six channel group and a two channel 
group. Each group can be operated on a different time slot.

The triggering logic in the FPGA will get its operating parameters from a set of registers. These parameters will be set from a computer. A USB port is used to connect this device to the computer. Computer software was written to interface with the device, so that all operating parameters could easily be adjusted using a GUI (graphical user interface). My assignment was to write the computer software to interface with this device, as well as to write the logic for the FPGA to read and write data through the USB connection and to store and retrieve this data from registers.

The USB interface is facilitated by a small stamp called USBMOD4 [2]. It has an onboard USB interpreter, so that knowledge of the USB protocol is not required. This stamp is bundled with a DLL (Dynamic Linked Library) that can be easily incorporated into a another program. This software had to have several input fields and drop down menus. It allows the user to select the global rep rate, then the time slot for each group of output channels, and finally the delay and width of the pulse trigger for each channel output. When the "Enter" key is pressed, the data in the selected field is sent to the appropriate register. The data is then read back and compared to the original to make sure that there was no corruption during the transfer.

\section{Methods and Materials:}

The device itself (See Figure 1) is constructed on a custom board designed at SLAC. The heart of the device is a Xilinx Spartan XC3S500E FPGA [1]. This chip is relatively low cost, yet has more than enough functionality for our current project. The board has voltage regulators and routs power from the input power jack to each active component. The board also connects the Xilinx chip to twelve BNC (Bayonet Neill-Concelman) connections through buffers. Eight of 
these twelve ports are output ports, and the other four are input ports. Two of these input ports are for RF signals only as they are magnetically coupled whereas the other two are direct connections. Another key to this device and hence the project was the USB interface. This function was performed by the stamp USBMOD4 by ELEXOL [2]. This stamp is based around the FTDI FT245BM USB FIFO (First In First Out) IC (integrated circuit) [3]. This chip handles the USB protocol, and comes with a DLL [4] that can be integrated into one of several commonly used software programming languages. My use of this device and the DLL [4] in my software made the software programming side of things quit simple.

Aside from the device itself, I had an assortment of other resources available to me for my project. I had all of the equipment in the lab of the second floor of building 44 (Klystron/Microwave Department Test Laboratory) available to me. The tools I used regularly from this lab were a soldering iron, a portable oscilloscope with the related probes and cables, a parallel JTAG (Joint Test Action Group) cable, a small 5VDC power supply, and an assortment of small hand tools such as screwdrivers, precision cutting instruments, etc. Some of these items, the oscilloscope for instance, was relocated to my office for convenience. In my office, probably the one object used most to facilitate the completion of this project was the computer workstation assigned to me. Of course it seems natural that this was the case considering the nature of my assignment. The software applications most used were: Microsoft Visual Studio 2005, Xilinx ISE 11 suite, and Mozilla Firefox.

The online references proved to be of the greatest assistance in this project. I very regularly referred to several different internet sources [1-18] for information regarding the programming languages used $(\mathrm{C}++$ and $\mathrm{VHDL})$ as well as for the technical documents required to adequately understand the hardware I was working with. I referenced several data sheets regularly. These included the data sheet for the Xilinx FPGA [1], USBMOD4 stamp [2], and the 
FT245BM USB IC [3]. Aside from the references I used for the hardware pieces I was working with, a reference [4] on the DLL for the USB interface was vital to the rapid development of first console based test software, and then finally to the finished GUI based software.

As mentioned previously, my assignment was to get certain device setting parameters from a GUI on a computer workstation through a USB interface to certain registers on the device and then be able to read these registers back to verify error free data transfer. The methodology of this data movement evolved considerably during the initial work on this project. I considered several options. At first I considered writing all data in one large chunk but finally, I decided that some sort of addressing was the best option. I decided on a method based around five octet words. The first octet would be an address octet and the four octets after would be data octets. At first I was thinking that it would work well to automatically send back to the computer the data that was written to the device. Directly after five octets were written to the device, those same five octets would be written back to the computer without any command from the computer. Although this might work alright, it would not allow the configuration settings to be read back from the FPGA during the software startup. It was decided that the best way to setup the system was to be able to read from or write to any of the four octet registers in the device at any time. To write a register, an address octet and four data octets would be sent to the device. To read from the device, only the address octet would be sent. This idea for the system seemed to be the best at the time, and so I decided to implement it in my design.

To accomplish this required a count selector, an intermediate five octet register, a 128 octet register, and some additional control logic (see Figure 3). Each piece was written in VHDL. The count selector basically just counts to five while selecting different registers to either read from or write to. The intermediate five octet register takes the octet data stream from the USB card and writes it into parallel accessible registers. Finally the big register stores these four octets 
in one of 32 four octet divisions. The control logic simply coordinates steps taken by each of these other pieces. In Figure 3, the control logic has been combined with the count selector and labeled "Timing Controller".

After programming the FPGA to read and write data to the registers in the way described, I wrote the software that the users would use in the test lab on their workstations. This software was written in $\mathrm{C}++$ and used the FLTK (Fast Light Tool Kit) library for the GUI, the FTD2XX DLL for USB communication, and the open-source functions IniReader and IniWriter for working with the INI file that holds configuration data. The GUI (see Figure 5) has several different settings available. There are settings for the rep rate, time slots for two separate groups of channels and the delay and width times. When a user enters the desired settings, the software sends the data to the device and writes it to the INI file for later use. After that, the software sends only the address octets of the previously sent data. The data associated with that address is sent back to the computer for transfer verification. If the received data matches the sent data, the setting entered is displayed to the right of the input box. If the data received does not match what was sent, then an error message will appear in the window warning the user about the fault.

\section{Results:}

The efforts put forth for this particular project have been very well rewarded. Although, the objectives themselves evolved slightly as I became more fully acquainted with the obstacles I was facing, I was able to satisfy most all of the initial design objectives.

Using the VHDL hardware description language I was successfully able to program the FPGA used in this project to interface with the USBMOD4 module. The FPGA accepts incoming data from the USBMOD4 module in the form of five octet words, stores the last four octets in 
one of 32 four octet registers based upon the first (address) octet, and finally can read back the data to the USBMOD4 module after accepting a single octet address. This set of registers has a synchronization input to enable the synchronization of the registers to the $119 \mathrm{MHz}$ clock that clocks the rest of the logic on the FPGA.

I was also able to write the computer software to read and write this register data. I wrote this software in $\mathrm{C}++$ along with the FTD2XX DLL, the FLTK libraries and some open-source INI functions. The code that I wrote myself ended up being $~ 1000$ lines long. It is based around a graphical user interface (see Figures 4 and 5) with several "Widgets" that make up the controls available to the user.

The GUI has a nine option drop-down menu for the rep-rate selection and two value entries for the slot selections for the first and second groups of trigger channels. It has a value entry for the delay and width times of each channel. It even has a channel description text entry for each channel. When values are entered for the delay and width of a channel, these values are converted into start and stop times, rounded to the nearest $8.4 \mathrm{~ns}$ division integer, and written to the FPGA through the USB port. The software then reads back the values for that channel and displays them under the header "Current Operation". When the software first starts up, all of the settings stored in the FPGA are read and displayed under the "Current Operation" heading and all of the previous time values and channel descriptions are read back from an INI file and put in their respective places. If there is any problem opening the USB communications during the software startup process, a message is presented to the user which gives the options "Retry" or "Quit". After the software is first loaded, if there is any problem with USB communications, an error message is presented to the user that states the presence of a USB error, and the necessity of re-opening the software. The only option is for the user to click the button "Close" at which time the software quits. 


\section{Discussion and Conclusions:}

When examining the results of this project and comparing them with the objectives at the outset, I think it is reasonable to conclude that I had much success. Although I was not able to test my design with the trigger generating logic to the extent initially desired, I believe that my contributions to this new triggering system for the klystron test stations, will prove to be very beneficial to SLAC in general, and the Klystron/Microwave Department Test Laboratory in specific. Although the logic and software I wrote were aimed at a very specific purpose, they could be very easily adapted to other applications relating to control using digital logic.

Looking back, I can see now that I would do things differently if given the opportunity. One thing I would probably investigate doing differently is the basic method of USB communication. The way I set up the USB communication was that when the computer sends five octets of data, the FPGA assumes that it is supposed to write it to a register block based on the first octet which is treated as an address. If one octet is sent, the FPGA assumes that it is supposed to read back data from the register block. These assumptions forced me to divide the clock frequency of the read/write logic of the FPGA to $\sim 1 \mathrm{MHz}$ to reduce the chances of a data under-run. If I were to do the same or a similar project again, I would investigate alternative methods of USB control, and would choose one that would eliminate the need for reducing the clock frequency. Also my VHDL structure could definitely use some improvement. Although it performs the job required of it, the structure is in some respects disorganized. In all, I am using seven different .vhd files. Some of these files are several hundred lines of code whereas some of them are under 50 . The logic contained in these files could be more evenly and logically distributed resulting in more maintainable source code.

There are also several improvements that could be made on the computer software that 
enables a user to read and write to the FPGA. One thing would be to have the software write and maintain a log file. This file would simply be a record of the settings being entered into the FPGA according to date and time. This would be useful for the operators to be able to look back and see exactly when they or someone else made a change to the operating parameters. Other improvements could be made in the general area of usability. It would be nice to have a button and keyboard shortcut to load all time entries. It would also be nice if when the user tabbed from one input field to the next, that the field jumped from would be written to the FPGA. The error handling could also be improved. Right now, if something goes wrong during a USB read or write operation, the only option available to the user is to close the software and reopen it. Although this is not that much of annoyance, it could be remedied by writing the error messages in such a way as to allow the user to retry instead of simply exiting.

Once again, with the $\mathrm{C}++$ code that I wrote this software in, many structural improvements could be made. I know that in many ways, it is inefficient and not according to good coding practice. Given more time, this code could be re-factored and made easier to read. I was able to contribute something significant to the Klystron/Microwave Department Test Laboratory. I am confident that my results will be very useful and I am optimistic about this project's future. 


\section{Acknowledgments:}

Special Thanks to :

\section{U. S. Department of Energy \& DOE Office of Science}

For providing the Science Undergraduate Laboratory Internship program.

\section{National Science Foundation}

For funding this internship.

\section{SLAC National Laboratory}

Ron Akre

For hosting my internship.

\section{Jeff Olson}

For electing to be a mentor to me, and for giving assistance and direction.

\section{Greg Dalit}

For assistance in writing the the VHDL code for the FPGA.

\section{Darius Grey}

For soldering components on my device board, and finding parts.

For technical and moral support while acting as my partner in this project.

\section{References:}

[1] Xilinx, Inc., General Products Division (GPD), "Xilinx DS312 Spartan-3E FPGA Family Data Sheet," [Online Document], 2008 Apr 18 (v3.7), [cited 2009 Aug 10], Available HTTP http://www.xilinx.com/support/documentation/data_sheets/ds312.pdf

[2] Jason Stolzenberg, “USBMOD4 Datasheet," [Online Document], 2005 Apr 7 (v1.1), [cited 2009 Aug 10], Available HTTP http://www.elexol.com/Download/documents/USBMOD4DS2.PDF

[3] Fred Dart, "FT245BM USB UART ( USB - Serial) I.C.," [Online Document], 2005 Nov 30(v1.7), [cited 2009 Aug 10], Available HTTP http://www.ftdichip.com/Documents/DataSheets/DS_FT245BM.pdf

[4] Future Technology Devices International Ltd., “D2XX Programmer's Guide,” [Online Document], 2007 Jul 7, [cited 2009 Aug 10], Available HTTP http://www.ftdichip.com/Documents/ProgramGuides/D2XXPG34.pdf

[5] Roger Traylor, "ECE 474 - VLSI System Design,” [Online Source], [cited 2009 Aug 10], Available HTTP http://classes.engr.oregonstate.edu/eecs/winter2007/ece474/

[6] "VHDL reference material," [Online Source], 2009 Feb 5, [cited 2009 Aug 10], Available HTTP http://www.csee.umbc.edu/help/VHDL/

[7] Auburn University Department of Electrical \& Computer Engineering, "VHDL Tutorial," 
[Online Source], [cited 2009 Aug 10], Available HTTP

http://www.eng.auburn.edu/department/ee/mgc/vhdl.html

[8] Sudhakar Yalamanchili, VHDL Starters Guide, $2^{\text {nd }}$ edition [Online Book], Prentice Hall, 2005, [cited 2009 Aug 10], Available HTTP http://users.ece.gatech.edu/ sudha/book/starters-guide/

[9] “Clock divider," [Online Source], 2008 May 10, [cited 2009 Aug 10], Available HTTP http://snipplr.com/view/6173/clock-divider/

[10] “C++ Library Reference," [Online Source], [cited 2009 Aug 10], Available HTTP http://www.cplusplus.com/reference/

[11] Alex, "C++ Tutorial," [Online Source], [cited 2009 Aug 10], Available HTTP http://www.learncpp.com/cpp-tutorial/

[12] F. Costantini, D. Gibson, M. Melcher, A. Schlosser, B. Spitzak and M. Sweet, "FLTK Programming Manual," [Online Manual], 2009, [cited 2009 Aug 10], Available HTTP http://www.fltk.org/doc-1.3/index.html

[13] Robert Arkiletian, "Beginner FLTK Tutorial," [Online Document], 2005 Jan 4, [cited 2009 Aug 10], Available HTTP http://www3.telus.net/public/robark/

[14] "Convert array of char[] to octet[] and vice versa? C++," [Online Forum], 2009 May 18, [cited 2009 Aug 10], Available HTTP http://stackoverflow.com/questions/876213/ convert-array-of-char-to-octet-and-vice-versa-c

[15] "Hexadecimal variable help!," [Online Forum], 2008 Feb 27, [cited 2009 Aug 10], Available HTTP http://www.daniweb.com/forums/thread111263.html\#

[16] Todd A. Gibson, "Tips and tricks for using C++ I/O (input/output)," [Online Document], 2007 Mar 1, [cited 2009 Aug 10], Available HTTP http://augustcouncil.com/ tgibson/tutorial/iotips.html\#prepare

[17] Dean DeFino, "Using Type Casting to "Round Off" a Value in Memory," [Online Document], [cited 2009 Aug 10], Available HTTP http://faculty.salisbury.edu/ dxdefino/RoundOff.htm

[18] Xiangxiong Jian, "A Small Class to Read INI File," [Online C++ Source Code], 2005 Jun 27, [cited 2009 Aug 10], Available HTTP http://www.codeproject.com/KB/cpp/IniReader.aspx 


\section{Tables and Figures:}

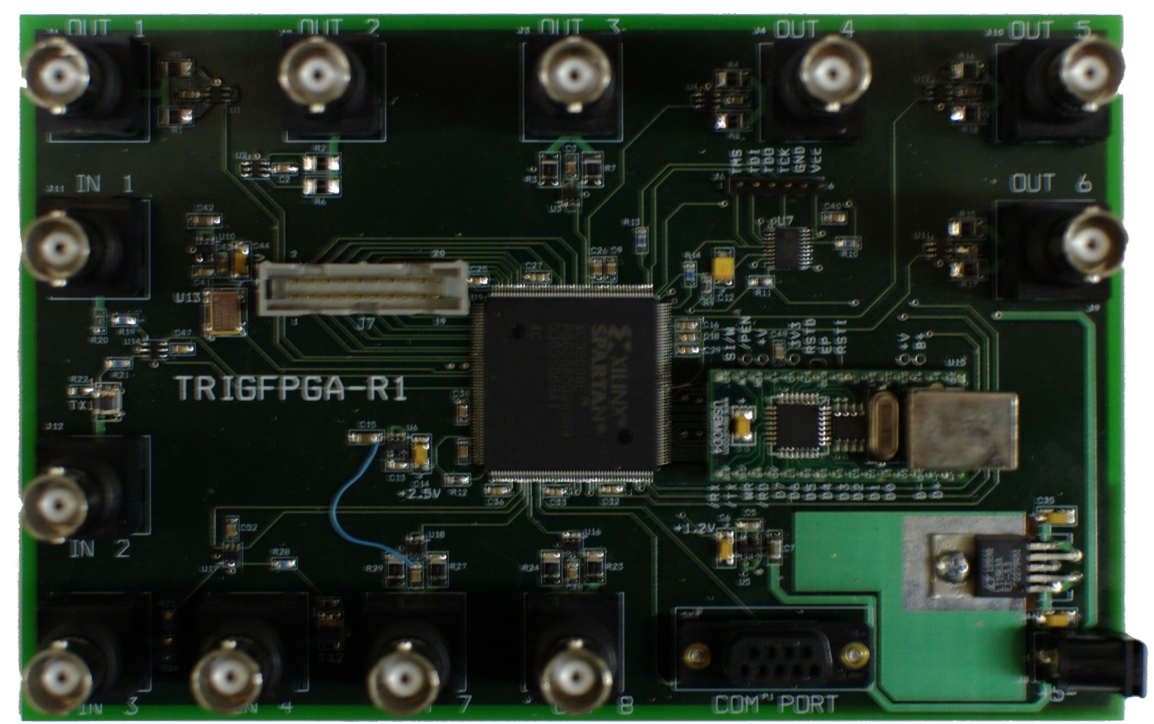

Figure 1: The custom FPGA based trigger device used in this project

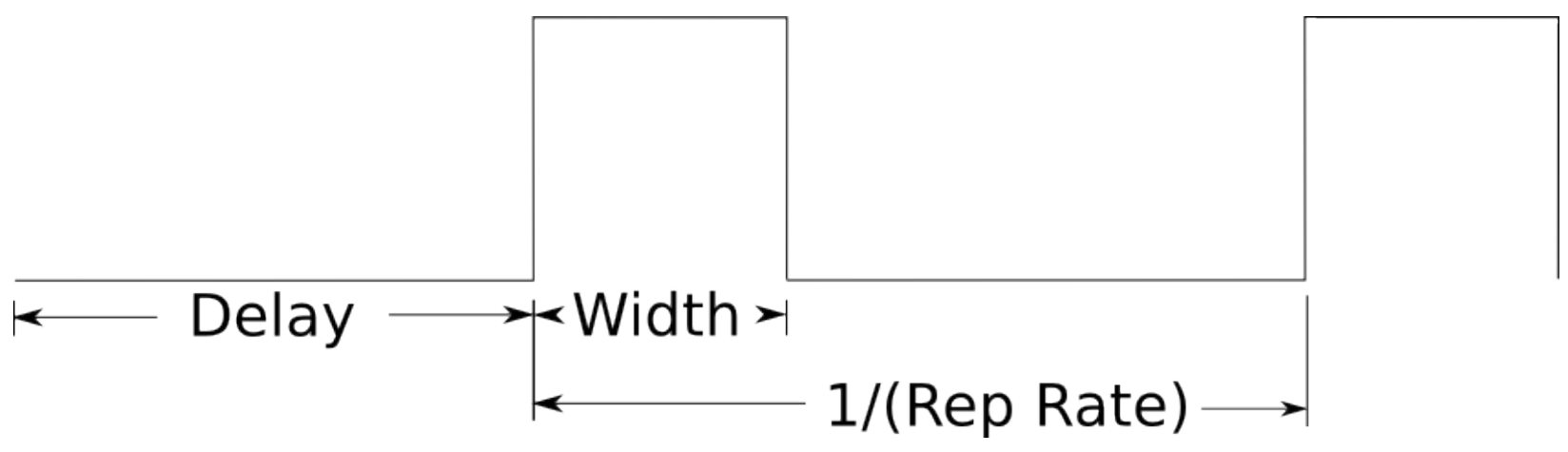

Figure 2: Trigger signal features 


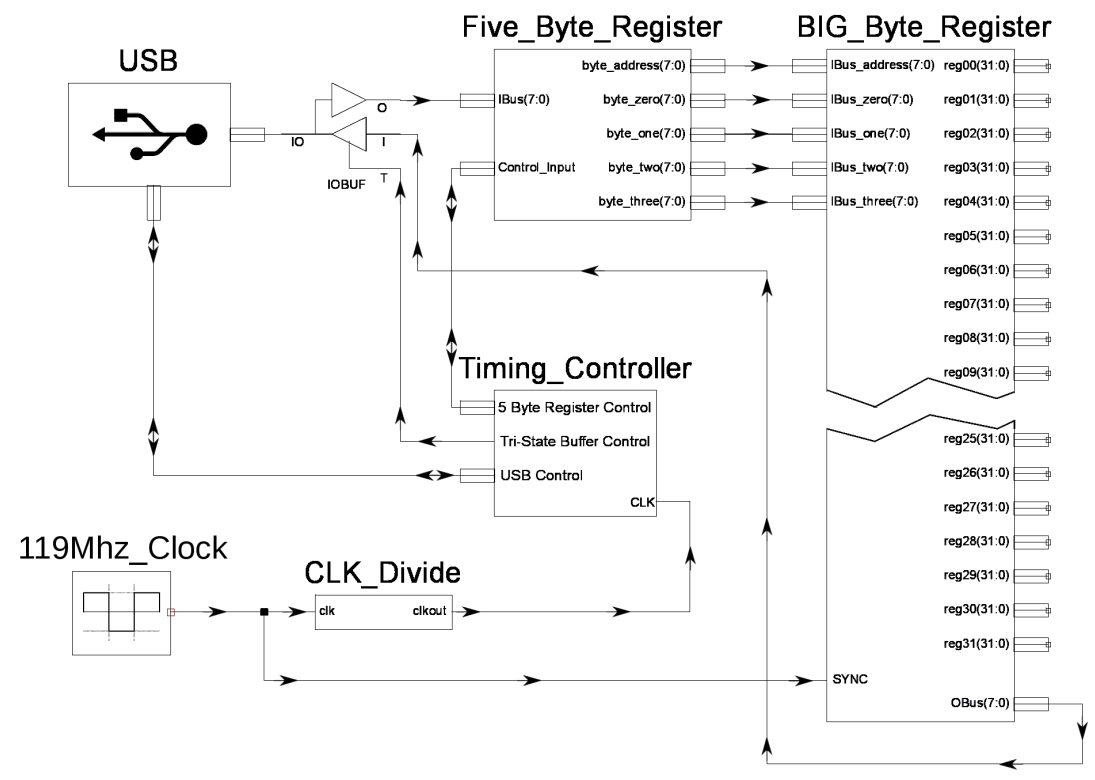

Figure 3: Simplified diagram of data flow from the USB port to the registers

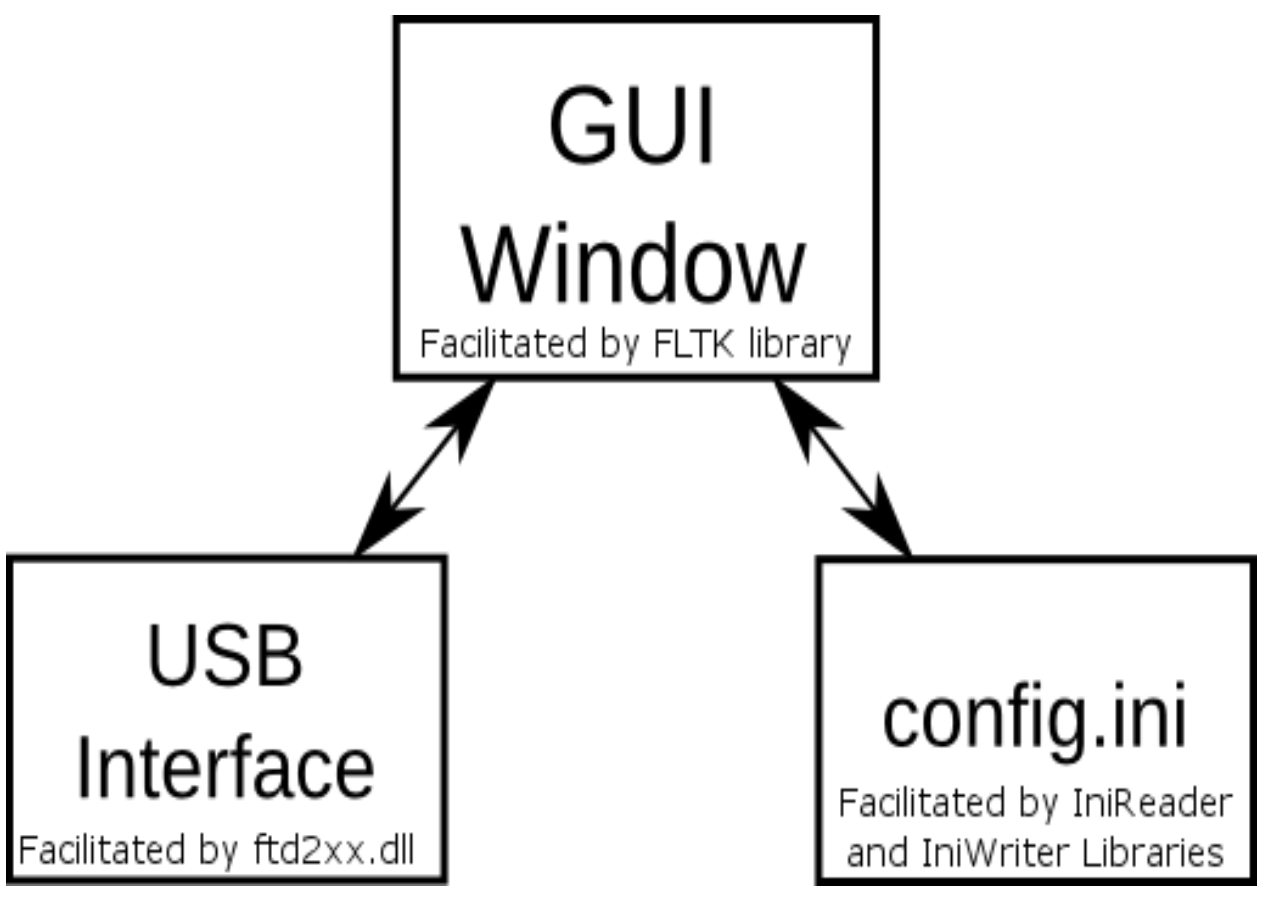

Figure 4: Block diagram showing the features of the Trigger Control software in relation 


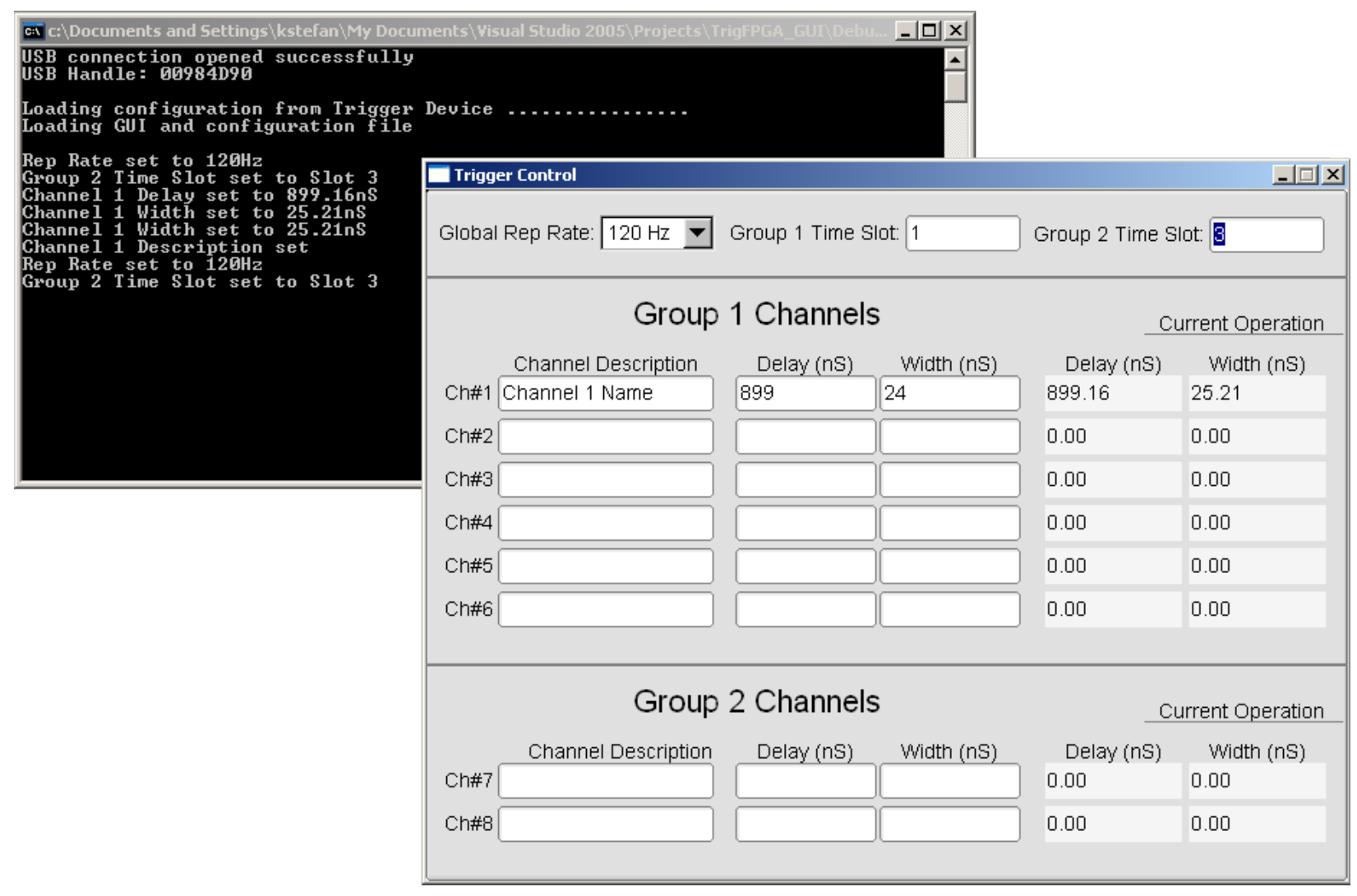

Figure 5: Trigger Control software user interface with the console messages in background 
Identifying Sources of Vertical Floor Motion in the SLAC SSRL Storage Ring Using Spectral Analysis

Nikita Sunilkumar

Science Undergraduate Laboratory Internship Program

University of Southern California, Los Angeles CA

Stanford Linear Accelerator Center

Menlo Park, California

August 7, 2009

Prepared in partial fulfillment of the requirement of the Office of Science, Department of Energy's Science Undergraduate Laboratory Internship under the direction of James Safranek in the Stanford Synchrotron Radiation Laboratory division of the Stanford Linear Accelerator Center.

Participant:

Signature

Research Advisor:

Signature 


\section{Table of Contents}

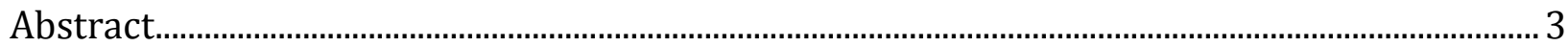

I. Introduction

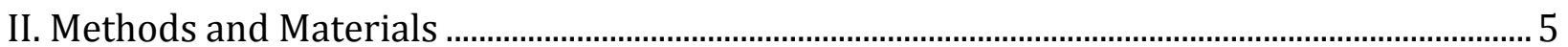

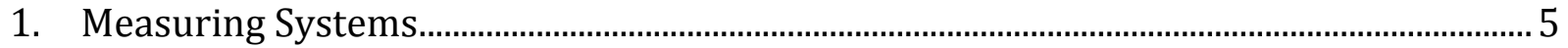

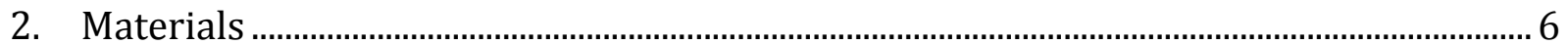

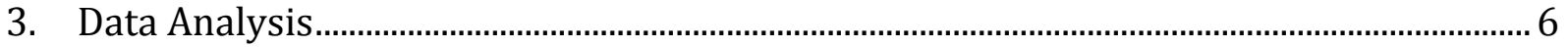

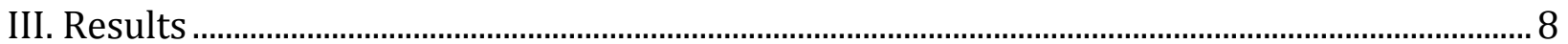

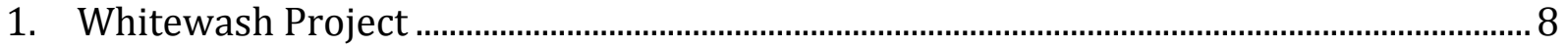

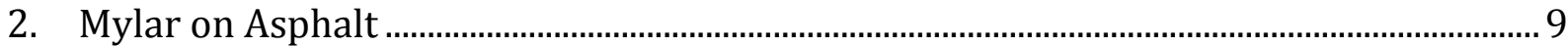

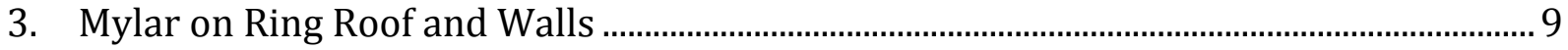

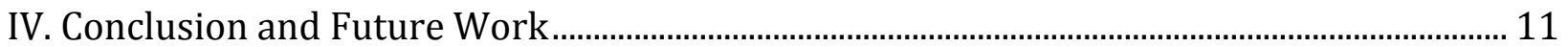

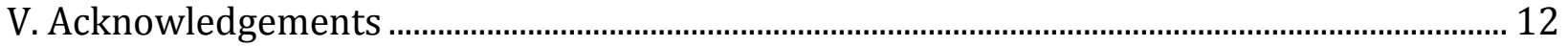

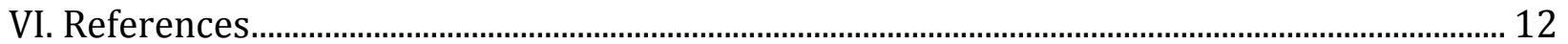

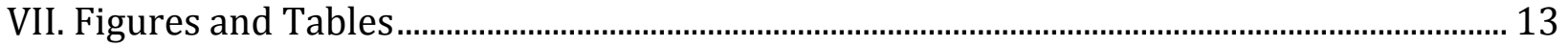




\begin{abstract}
Identifying Sources of Vertical Floor Motion in the SLAC SSRL Storage Ring using Spectral Analysis. NIKITA SUNILKUMAR (University of Southern California, Los Angeles, CA 90007) JAMES SAFRANEK (Stanford Linear Accelerator Center, Menlo Park, CA 94025)

Users of the Stanford Synchrotron Radiation Lightsource (SSRL) are being affected by diurnal motion of the synchrotron's storage ring, which undergoes structural changes due to outdoor temperature fluctuations. In order to minimize the effects of diurnal temperature fluctuations, especially on the vertical motion of the ring floor, scientists at SSRL tried three approaches: painting the storage ring white, covering the asphalt in the middle of the ring with highly reflective Mylar and installing Mylar on a portion of the ring roof and walls. Vertical motion in the storage ring is measured by a Hydrostatic Leveling System (HLS), which calculates the relative height of water in a pipe that extends around the ring. The 24-hr amplitude of the floor motion was determined using spectral analysis of HLS data, and the ratio of this amplitude before and after each experiment was used to quantitatively determine the efficacy of each approach. The results of this analysis showed that the Mylar did not have any significant effect on floor motion, although the whitewash project did yield a reduction in overall HLS variation of 15 percent. However, further analysis showed that the reduction can largely be attributed to a few local changes rather than an overall reduction in floor motion around the ring. Future work will consist of identifying and selectively insulating these local regions in order to find the driving force behind diurnal floor motion in the storage ring.
\end{abstract}




\section{Introduction}

The Stanford Synchrotron Radiation Lightsource (SSRL) provides high energy photon beams to various users working along a 234-m circumference electron beam storage ring (SPEAR). Due to the sensitive nature of the users' experiments, it is essential that the electron beam and photon beam remain stable over long periods of time. However, challenges have arisen in maintaining the vertical stability of the beam due to movements of the ring floor. A Hydrostatic Leveling System (HLS) and Radio-Frequency orbit feedback (RF) have observed the storage ring of the synchrotron expand, contract and shift vertically on the order of hundreds of microns within days. Such movement has led to difficulties for SSRL users who need the incident photon beams to remain stable to better than ten microns. The cause for this instability is unknown, but one hypothesis identifies temperature variations inside, outside and within the concrete surfaces of the ring as being directly correlated to the motion of the tunnel.

In an attempt to stabilize the ring, scientists from the Accelerator Physics division of SSRL have tried three approaches. In the summer of 2008, the roof and walls of the storage ring were painted white. In the June of 2009, highly reflective aluminum Mylar was installed on the asphalt that covers the ground in the middle of the ring. In July, the Mylar was also installed on the roof and walls of a portion of the ring, and fans were placed inside the ring to promote ambient temperature stability. The reasoning behind all three experiments was the same: by finding ways to shield the ring and surrounding structures from cyclical temperature changes, the scientists hoped to prevent the expansion and contraction of the ring due to heat absorption and loss. The white paint, and later Mylar, was intended to protect the concrete walls of the ring from solar radiation, which causes diurnal heating and cooling that can lead to internal buckling or shear forces. The Mylar on the asphalt was intended to prevent outward expansion that would 
cause stress on the inner walls of SPEAR and perhaps translate into vertical or rotational movement of the ring floor.

The efficacy of the three experiments mentioned above may be determined by processing and analyzing the data provided by the HLS and RF systems. In the future, the knowledge gained from these experiments may help scientists at SSRL determine the exact causation and correlation relationship that links temperature variation with ring structure movement.

\section{Methods and Materials}

\section{Measuring Systems}

Any attempt to pinpoint and correct issues of structural instability relies heavily on the systems that are in place to measure it accurately. The two systems in place at SSRL are the HLS and RF systems. The HLS, in brief, consists of a series of tubes half-filled with water placed on the concrete floor around the storage ring. Sensors placed at points around the ring measure the water level in reference to a central sensor. The data coming from one sensor shows the amount of vertical movement occurring at that point in relation to a sensor upstream of the East Pit [1]. SPEAR contains 22 sensors, with pairs of sensors placed upstream and downstream of points where photon beam lines move tangentially off from the electron beam. HLS data is measured in microns, and the sampling rate is every 10 seconds.

The second system in place is the RF system, which provides data regarding the circumference of the beam. The data provided by this system represents the change in the frequency of the radio signal that steers the beam - the delta RF signal is proportionally equal to the change in the circumference of the ring by the following relationship [2]:

$$
\Delta C=-C \cdot \Delta f_{R F} / f_{R F} .
$$


The RF data is in $\mathrm{Hz}$, and the sampling rate is every minute.

Thermocouples (TC) placed at various locations inside and outside the ring provide data on temperature fluctuations taking place over any given period of time. TC data is taken in degrees centigrade, and the sampling rate is every minute.

\section{Materials}

All of the walls and the roof of SPEAR were painted with a white, titanium oxide based paint that also contained a Borosilicate glass additive. The Mylar used to cover one half of the ground in the center of SPEAR, and later one quarter of the South Arc, is a thin polyester film coated with aluminum. The floor of SPEAR is composed of six sections, with four continuous concrete slabs forming the north and south arc, and two concrete blocks anchoring the East and West Pit. Most of the interior wall of the storage ring is composed of discrete concrete blocks 61 $\mathrm{cm}$ thick that are placed on the asphalt that covers the inner region of the ring; however, in some regions, the wall has been cast as a continuous structure that extends 3 feet below the ring floor (See Figure 2).

\section{Data Analysis}

Data from all three experiments was analyzed using one method, which consists of simple filters, a planar extraction and spectral analysis.

The SPEAR schedule during operation consists of 12 day cycles of uninterrupted beam time followed by two days of either Accelerator Physics sessions or maintenance projects. During the Accelerator Physics sessions, which include various tests and changes to the beam itself, the RF signal becomes erratic and noisy. Similarly, when the ring is open to maintenance traffic, occasional bumps and vibrations can disrupt the HLS sensors, and cause faulty or invalid readings. A simple search and replace filter written as a MATLAB function allowed the signal to be cleaned before further analysis. 
The filter consists of two steps: the first involving a moving window within which data points above and below a range of standard deviations were replaced by the MATLAB value $\mathrm{NaN}$ (not a number), and the second which replaced all the NaNs in the vector with interpolated values. Also, some regions of data taken during periods with known disruptions were manually removed and replaced with interpolated values.

In addition to the simple filter, the HLS data was also corrected for overall planar movement. Although each sensor of the HLS provides readings independent of the others, the entire system forms a plane parallel to the ring itself. A portion of the movement recorded by each sensor actually corresponds to the movement of this plane and not just the movement of the region measured by each sensor. A MATLAB function was written to calculate the equation for the best fit plane at each time denomination using the coordinates of each of the sensors and the vertical reading $(\mathrm{Y})$ of each sensor at that time. The coordinate system for the HLS sensors is based on the major and minor axes (labeled X and Z, respectively) of the ellipse formed by SPEAR. The function then subtracted the Y-value calculated from the best fit equation from the actual data point. The function also returned the slopes of the $\mathrm{X}$ and $\mathrm{Z}$ axes from each best fit plane.

The planar movement was subtracted from the HLS data since it represents uniform motion across the ring; however, it is unclear whether the planar behavior of the ring can be extended onto the photon beamline floor. The beamlines extend tangentially from the ring and are placed on foundations that are distinct from the SPEAR arcs. For the purposes of analyzing instability of just the storage ring and not the photon beamlines, the planar correction is not detrimental, but the function written for this analysis may have to be modified before it is applied to photon beamline motion. The sources for the planar motion of the ring are unknown, although certain hypotheses suggest that the planar slopes can be accounted for in part by tidal motion at 
12-hour, 24-hour and 14-day frequencies. In order to calculate the theoretical tidal motion at SPEAR, the program Solid_UTC was used, which generates tidal shifts based on a solid Earth assumption [3]. An initial comparison of the best-fit slopes and theoretical tidal slopes shows that both values are on the same order of magnitude (See Figure 1). The best-fit slopes even approximate the 12-hour amplitude of tidal motion within 14 percent, although the 24-hour and 14-day content does not seem to be correlated (See Table 1).

After processing the data through the simple filter and the planar extraction function, a spectral analysis method was used to determine the characteristic frequencies embedded within each vector of HLS data. As expected, the HLS sensors' readings all contained a noticeable 24hour period and a smaller 12-hour frequency in some cases (See Figure 3). An existing MATLAB function was modified to perform the spectral analysis - this code takes the Fast Fourier Transform (FFT) of the HLS data, multiplies by the conjugate of the FFT and generates a Power Spectrum Density (PSD) plot. On logarithmic axes, the PSD plot shows clear peaks at the 12-hour and 24-hour frequencies where the majority of the power in the signal is concentrated. Then, by Parseval's Theorem, integrating the power spectrum density curve produces an “Integrated Displacement Power Spectrum” plot, which shows the typical amplitude of the signal across a domain of frequencies. By measuring the displacement, or 'step', at the 24-hour frequency before and after an experiment, the change in diurnal variation for each HLS sensor may be determined (See Figure 4).

\section{Results}

\section{Whitewash Project}

The painting of the SPEAR roof and walls during the summer of 2008 did yield significant results, according to analysis of data from May 2008 (before paint) and May 2009 
(after). As shown in the first row of Table 2, the two months had nearly identical daily variations in outdoor temperature. However, the daily temperature fluctuation of the concrete roof was reduced by a factor of two after the addition of white paint, and the internal ambient temperature fluctuation of the ring was reduced by 15 percent. The diurnal HLS variation, calculated as the mean of all sensors' variations, was also reduced by 15 percent, although the greatest contribution for the change came from a few select sensors. The changes in each sensor are shown in Figure 5, with the sensors arranged in order as they would be seen from Girder 3, looking from left to right toward the ring center. Since there was no uniform reduction in HLS variation throughout the ring, it is not likely that the internal ambient temperature is directly correlated to vertical floor motion, though it may make a minor contribution to the dynamics of the entire system.

\section{Mylar on Asphalt}

Row two of Table 2 shows the changes in temperature and HLS after installation of Mylar on the asphalt that covers the center of the ring. The two rows represent different data sets: the first compared data from one week each in May and July 2009, and the second compared 17 days of data taken immediately before and after the Mylar was installed. Both analyses showed that despite a significant reduction in the temperature variation of the asphalt (which was estimated to be by nearly a factor of six), the HLS variation did not improve. Even the RF signal did not improve after the installation of the Mylar, which suggests that the predicted asphalt expansion and ring motion are entirely decoupled.

\section{Mylar on Ring Roof and Walls}

The results of the first two experiments prompted the installation of Mylar on the roof and walls of a section of SPEAR. It was hoped that the Mylar would reduce the temperature fluctuation of the roof by additional factors beyond the reduction caused by white paint alone. 
Due to the relationships demonstrated in the analysis of the roof painting project, it was hoped that the internal ambient variation, and subsequently the HLS motion, would also decrease. In addition to the Mylar, fans were also placed at locations around the ring to increase airflow and minimize temperature gradients between the floor and ceiling of the ring. Although the amount of data recorded at this point is insufficient to conduct a thorough analysis, a partial analysis of the Beam Line 11 (BL11) sensors showed no improvement in floor stability, despite a slight reduction in roof temperature (See Table 2). The reduction in the interior wall temperature variation only amounts to an actual change of 0.05 degrees Centigrade. One week each from July and August were compared for this analysis.

Analysis of the three experiments discussed above has also suggested another path of inquiry - one that examines the effect of wall structure on floor motion. Figure 5, which shows daily variations for each HLS sensor before and after SPEAR was painted white, also shows spatial discontinuities in diurnal floor motion that cannot be explained by temperature fluctuations. A specific example involves the HLS sensors upstream and downstream of BL 7, which recorded far greater daily variation than the sensors on BL10 and BL9. BL10 and 9 are immediately adjacent to BL7 and therefore too close to have a significant ambient temperature difference. BL7 also responded to the white paint more than the surrounding sensors - the two BL7 sensors' variations fell by 35 percent while those at BL10 and BL9 actually increased by two percent. The only known difference between BL7 and the other sensors is that the inner ring wall at BL7 is composed of cast concrete that extends a few feet below ground, whereas the other sections of the surrounding arc are composed of concrete blocks that rest on the asphalt that covers the middle of the ring (See Figures 2 and 6). 


\section{Conclusion and Future Work}

Although each of the three attempts described above have provided insight into the forces and factors that affect ring instability, none have conclusively demonstrated the exact cause and effect relationship between temperature variation and HLS motion. However, the results of the different experiments have eliminated some hypotheses, such as the link between horizontal asphalt expansion and vertical ring motion, and reinforced others, such as the relationship between internal ring temperature and HLS variation. Future work toward improving stability in the ring will focus on quantitatively identifying this relationship between internal temperature and floor motion and designing experiments to minimize both.

The method used to study data for this effort will also have to be re-evaluated, as the analysis expands to include not just the main storage ring, but also the beamlines and experimental floor. A more precise tidal calculator must be used to analyze and extract planar motion from the HLS data. Additional factors, such as ground water levels, humidity and fault line activity must be studied to determine if they also cause uniform fluctuations in HLS data.

In addition, the investigation into the correlation between wall structure and floor motion must expand to include the rest of the ring beyond BL7. It has already been noted that other sections of the ring with discontinuities similar to BL7 do not conform exactly to this wall-type hypothesis, but future work in this area may account for such discrepancies. Since BL7 responded so well to the white paint, areas with similar wall structure could be insulated selectively to test their response in comparison to dissimilar sections of the inner wall. Part of that effort may include the installation of more sensors across definitive features of ring structure, and the extension of the HLS system onto photon beamlines and perhaps even the experimental hall. 


\section{Acknowledgements}

This effort would not have been possible without funding from the Department of Energy Office of Science and the SULI program at SLAC National Laboratory. Special thanks to my mentor, James Safranek, for his unfailing guidance and support. Thanks also to Steve Gierman, Ben Scott, Ann Trautwein, Georg Gassner, Tom Rabedeau, Ray Ortiz, Harvey Rarback, Jim Sebek and the rest of the SSRL Accelerator Systems Division for their very helpful contributions, and to Greg Portman for his PSD code.

\section{References}

[1] Gassner, Georg. "Startup of the HLS in SPEAR3.” <http://www-group.slac.stanford.edu/met/Align/Spear3/... SPEAR_WWW/HLS\%20Startup\%202007.pdf>

[2] T. Katsura, Y. Kamiyat, A. Araki, H. Nakamura and Y. Fujita. "Variation of the Circumference in a Storage Ring and Expansion of the Building.” EPAC92. Third European Particle Accelerator Conference, page 1631, 1992.

[3] Milbert, Dennis. “solid.m” < http://home.comcast.net/ dmilbert/softs/solid.htm> 


\section{Figures and Tables}

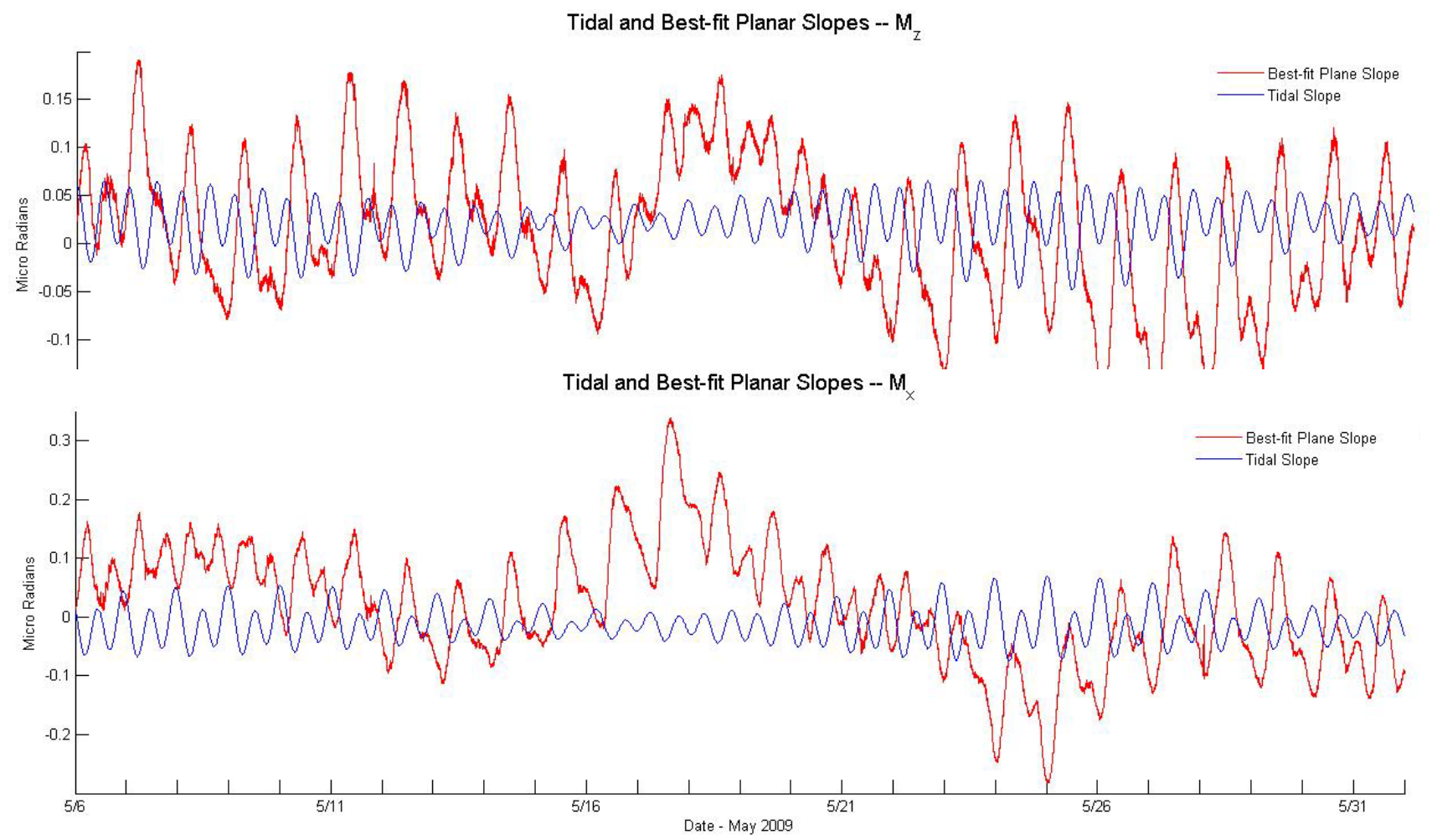

Figure 2: The two plots show the slopes of the major and minor axes of SPEAR versus time, as calculated by the best-fit planar extraction program and a tidal motion calculator. The slopes of the best fit plane across all HLS sensors are on the same order of magnitude as the theoretical slopes that would be caused by tidal motion.

\begin{tabular}{|c|c|c|}
\hline \multirow[t]{2}{*}{ Ratio } & \multicolumn{2}{|c|}{ Amplitude } \\
\hline & 12-hr & 24-hr \\
\hline $\begin{array}{l}\frac{\text { Tidal Slope X }}{\text { Best-fit Slope X }} \\
\text { (Phase Difference) }\end{array}$ & $\begin{array}{l}0.8623 \\
(4.326 \mathrm{hrs})\end{array}$ & $\begin{array}{l}0.3194 \\
(10.055 \mathrm{hrs})\end{array}$ \\
\hline $\begin{array}{l}\frac{\text { Tidal Slope Z }}{\text { Best-fit Slope Z }} \\
\text { (Phase Difference) }\end{array}$ & $\begin{array}{l}0.6806 \\
(3.163 \mathrm{hrs})\end{array}$ & $\begin{array}{l}0.2283 \\
(12.285 \mathrm{hrs})\end{array}$ \\
\hline
\end{tabular}

Table 1: A comparison of the best-fit slopes and theoretical tidal slopes using spectral analysis. The best fit planes were able to approximate 12-hour tidal motion more accurately than the 24 hour content. 


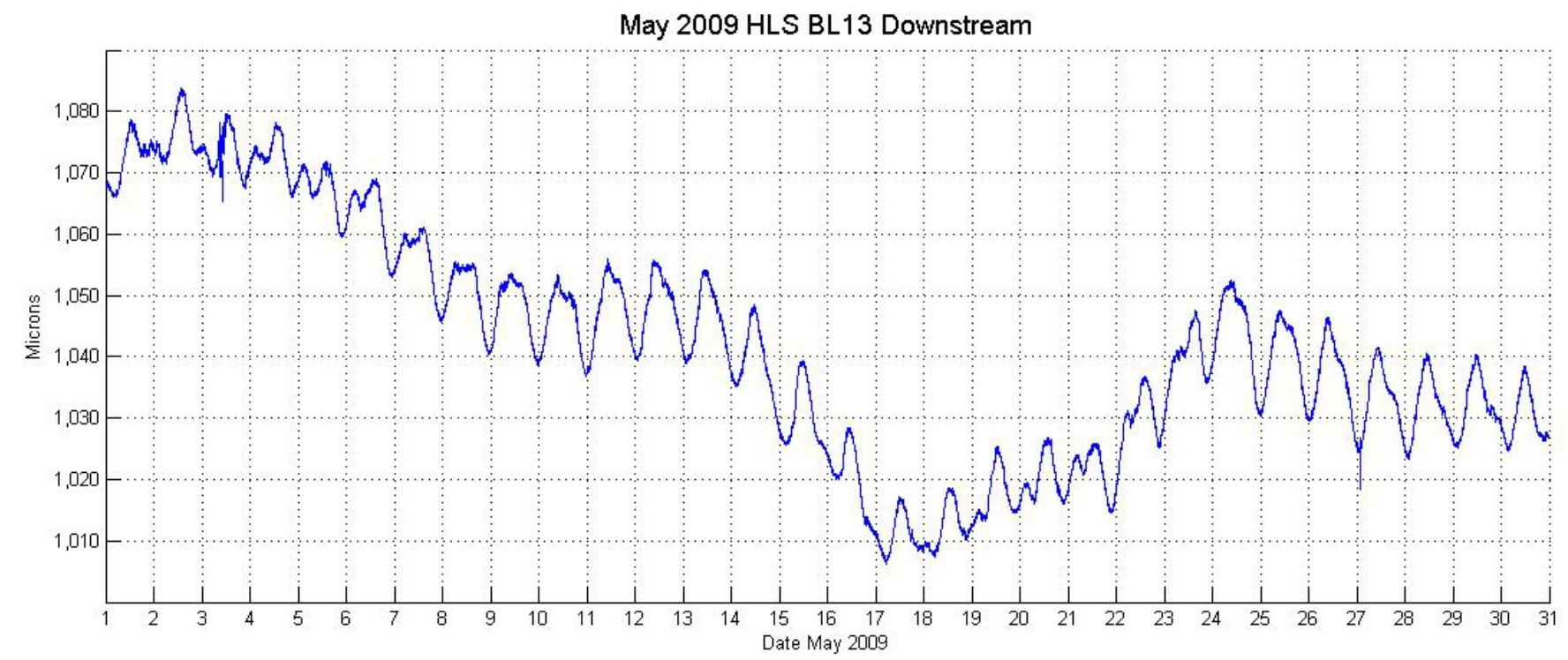

Figure 3: A typical HLS data vector. Each measurement corresponds to the vertical displacement of the ground at one location (in this case, downstream of BL13) in reference to a sensor placed upstream of BL12. 

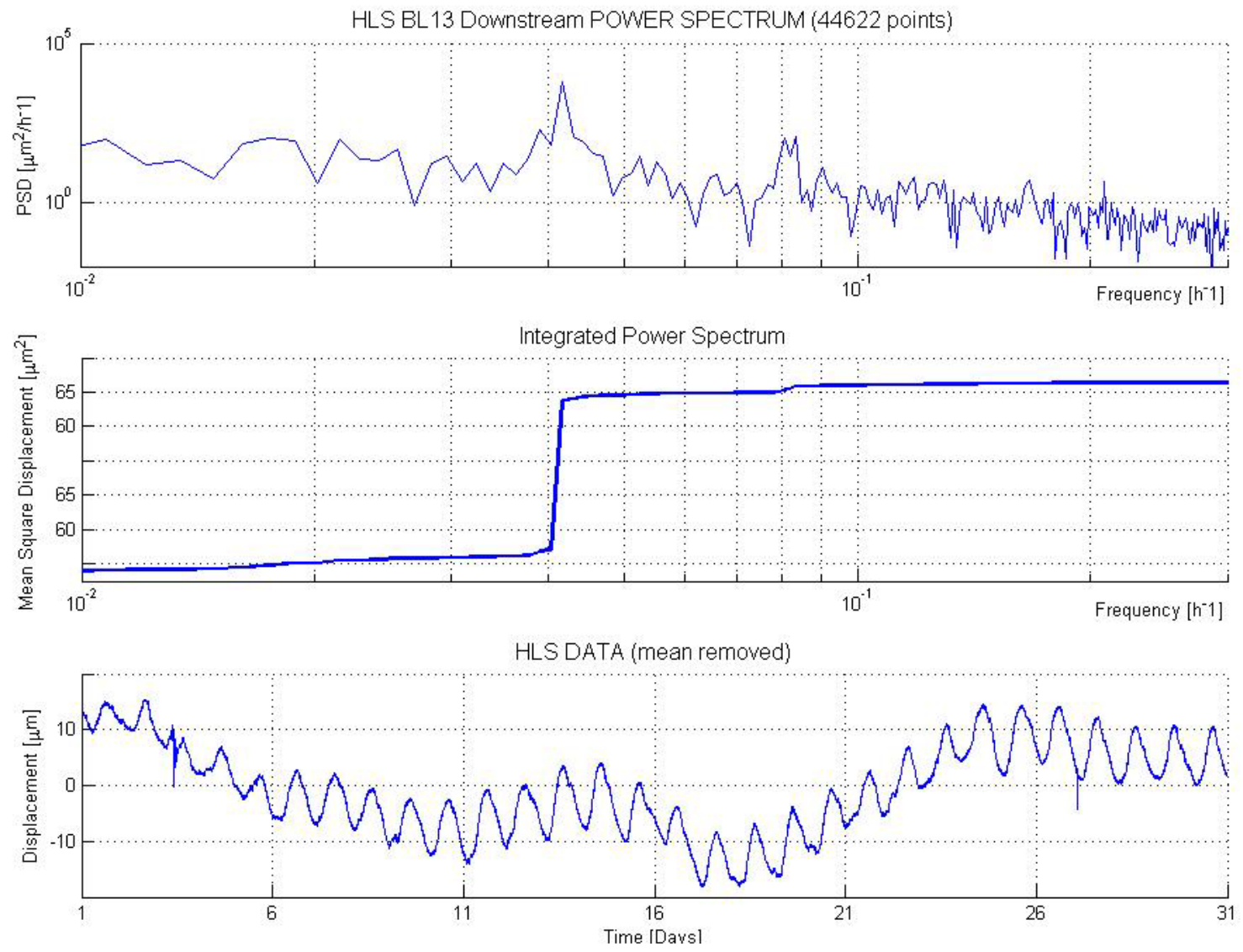

Figure 4: A typical HLS PSD plot. The mean daily variation for this sensor can be determined by calculating the change in the Integrated Power Spectrum across the 24-hour frequency.

\begin{tabular}{|c|c|c|c|c|}
\hline Project & HLS Variation & Outdoor B116 & Roof G13R1C2 & Ring Ambient \\
\hline $\begin{array}{l}\text { WHITEWASH } \\
\text { (May 2009/ } \\
\text { May 2008) }\end{array}$ & 0.846 & 0.999 & 0.5308 & 0.8494 \\
\hline $\begin{array}{l}\text { MYLAR on } \\
\text { ASPHALT } \\
(6 / 13-6 / 30 / 09 / \\
6 / 30-7 / 16 / 09)\end{array}$ & $\begin{array}{l}1.077 \\
\text { RF: } 1.67\end{array}$ & 1.087 & 1.087 & 0.8185 \\
\hline $\begin{array}{l}\text { MYLAR - } \\
\text { Roof/Walls } \\
(6 / 30-7 / 05 / 09 / \\
7 / 29-8 / 03 / 09)\end{array}$ & $\begin{array}{l}\text { (BL } 11 \text { Only } \\
\text { - No plane fit) } \\
\text { Upstream: } 1.314 \\
\text { Downstream: } \\
0.9182\end{array}$ & 0.9473 & 0.8054 & $\begin{array}{l}\text { (Interior Wall TC } \\
\text { Girder 13) } \\
0.4338\end{array}$ \\
\hline
\end{tabular}

Table 2: The results of the three experiments designed to improve SPEAR stability. Each value corresponds to the ratio of the mean daily variation of the signal after an experiment over the variation before an experiment. 


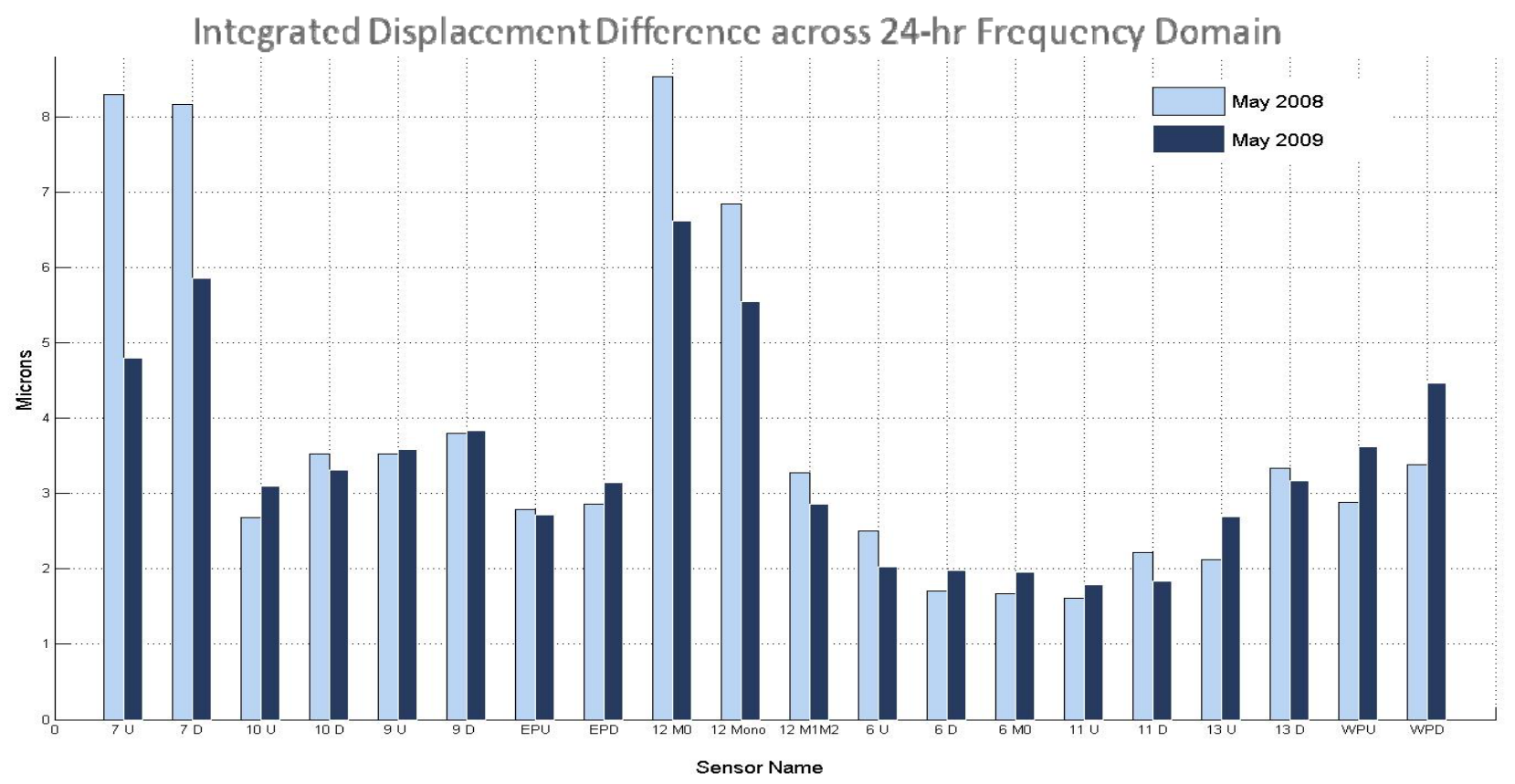

Figure 5: The mean daily variation in each HLS sensor before and after the roof and walls of SPEAR were painted white. Note the lack of a uniform change across all sensors; most of the reduction can be attributed to BL7 and the sensors on the BL12 photon beam floor.

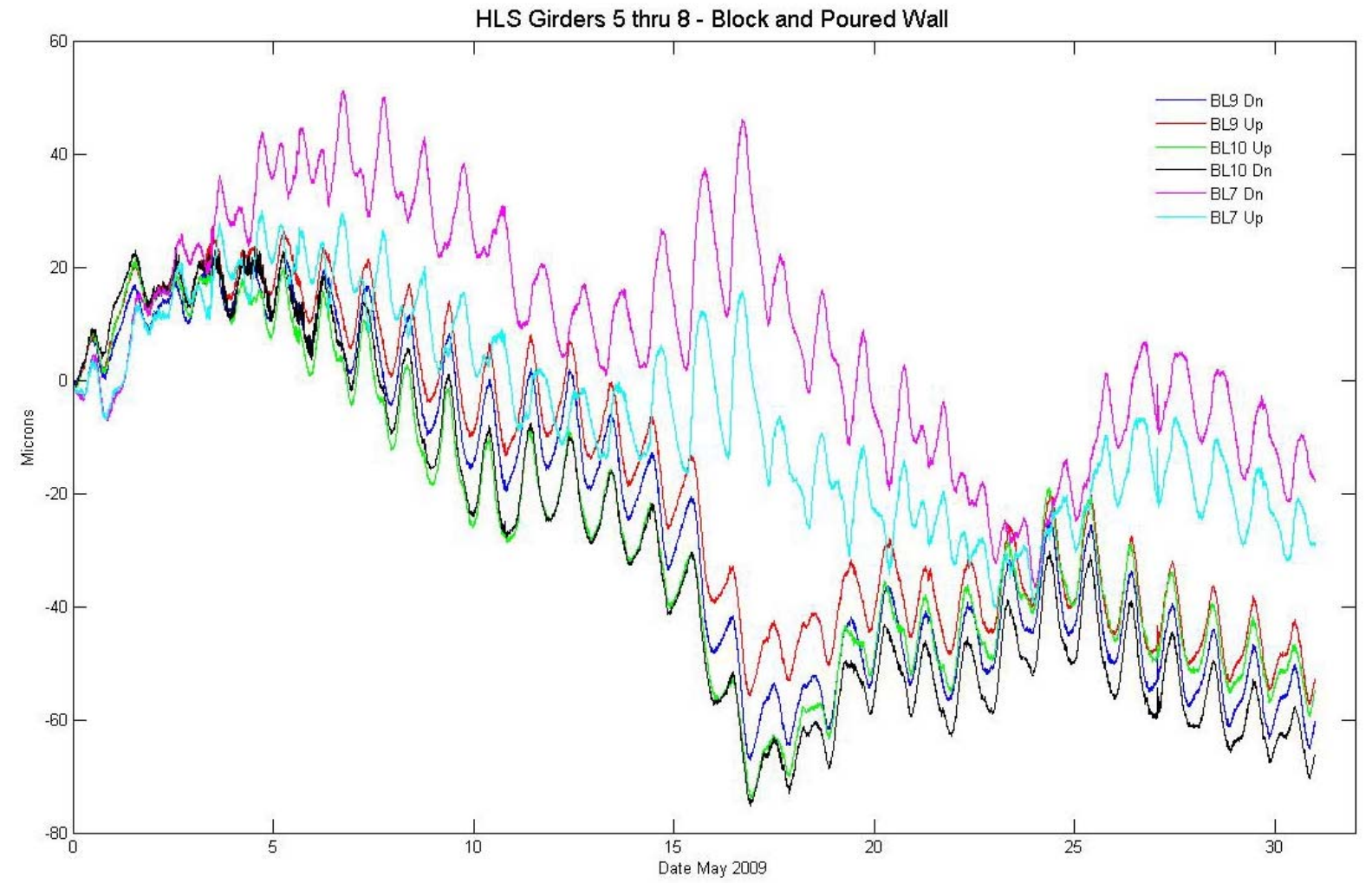

Figure 6: HLS data from sensors up and downstream of BL7, 10 and 9. Although the sensors are adjacent within SPEAR and on a continuous arc of the ring floor, the figure above shows a discontinuity between BL7 and the other sensors. A possible cause for the discontinuity is the non-uniformity of the inner wall structure of SPEAR. 


\title{
Probing the Geometry and Physics of the Emission Region of Active Galactic Nuclei Using Hard X-ray Observations
}

\author{
Natasha Woods \\ Office of Science, Science Undergraduate Laboratory \\ Internship (SULI) \\ The University of Texas at Dallas \\ Stanford Linear Accelerator Center \\ Menlo Park, CA \\ August 13, 2009 \\ Prepared in partial fulfillment of the requirements of the Office \\ of Science, Department of Energy's Science Undergraduate \\ Laboratory Internship under the direction of Marco Ajello and \\ Masaaki Hayashida at the Kavli Institute for Particle \\ Astrophysics and Cosmology, Stanford Linear Accelerator \\ Center.
}

Participant:

Signature

Research Advisors:

Signature

Signature 


\section{Contents}

1 Introduction $\quad 4$

1.1 AGN Classification . . . . . . . . . . . . . . . . 4

1.2 Unified Model for AGN . . . . . . . . . . . . . . . . . 4

1.3 Investigating the Unified Model of AGN using X-rays . . . . . 5

1.4 Relevance ................... . . . 6

$\begin{array}{llr}2 & \text { Methods } & 7\end{array}$

2.1 Swift-BAT Mission . . . . . . . . . . . . . . . . 7

2.2 Sample Population . . . . . . . . . . . . . . . . 7

2.3 Data Analysis . . . . . . . . . . . . . . . . . . 7

2.3.1 Photon Indices \& Simple Power Law Model . . . . . . 7

2.3.2 Stacked Spectra . . . . . . . . . . . . 8

$\begin{array}{llr}3 & \text { Results } & 9\end{array}$

4 Discussion \& Conclusion $\quad 10$

5 Acknowledgments $\quad 11$

$\begin{array}{llr}6 & \text { Figures } & 14\end{array}$ 


\begin{abstract}
Probing the Geometry and Physics of the Emission Region of Active Galactic Nuclei Using Hard X-ray Observations. NATASHA L. WOODS (University of Texas at Dallas, Richardson, TX 75080) MARCO AJELLO \& MASAAKI HAYASHIDA (Kavli Institute for Particle Astrophysics and Cosmology, Stanford Linear Accelerator Center, Menlo Park, CA 94204)
\end{abstract}

The X-ray spectra of $\sim 200$ AGN collected from Swift-BAT were analyzed to test the Unified Model for AGN. Specifically, the photon indices, high energy cutoffs, and reflection components of Sy1 and Sy2 were compared. Under the Unified Model, the photon indices and reflection components for Sy1 should be larger than Sy2 and the high energy cutoffs should be the same. Fitting a simple power law model to the sample spectra proved to be insufficient. The PEXRAV model fit the spectra of the Sy1 and Sy2 significantly better, indicating that a reflection component and/or high energy cutoff exists as the Unified Model expects. Using both the simple power law and PEXRAV models it was concluded that in the population studied, Sy1 had a larger photon index than Sy2, as expected by the Unified Model. For Sy1 and Sy2, the reflection components were found to be compatible, but given the large errors, this finding cannot be said to be evidence against the Unified Model. However, it was concluded that Sy1 and Sy2 have different high energy cutoffs, which is unexpected under the Unified Model. 


\section{Introduction}

The study of Active Galactic Nuclei (AGN) began in 1908 when Arthur Fath noticed six emission lines in the spectra of NGC 1068 that could not be attributed to stellar processes. Less than two decades thereafter, Carl Seyfert recognized similar emission lines in the spectra of spiral galaxies (Fig.1). Over time, galaxies with these emission lines were named AGN. These objects are characterized by the fact that the majority of their energy output is unrelated to stellar processes. Formally, AGN are compact regions of space at the center of galaxies that are exceedingly luminous over all or part of the electromagnetic spectrum [1].

\subsection{AGN Classification}

AGN are classified by their radio luminosity and spectral properties. Radioloud AGN emit radio waves, radio-quiet AGN have minimal or no radio flux. Additionally, if the source has a bright optical continuum with broad emission lines, it is classified as Type 1. Type 2 AGN have a weak optical continuum with narrow emission lines [2].

\subsection{Unified Model for AGN}

The Unified Model for AGN posits that all AGN are intrinsically the same and their perceived differences can be attributed to their viewing orientations. In this model, AGN are axis symmetric and host $10^{6-10} M_{\text {sun }}$ super-massive black holes (SMBH) that actively accrete matter from surrounding accretion disks. Outside this disk is a molecular torus composed of gas and dust (Fig.2). The viewing orientation of the torus is believed to obscure or reveal the luminous continuum emission from the SMBH and its accretion disk.

AGN have a broad line region (BLR) composed of 3,000-30,000K gas clouds orbiting the SMBH at 3,000-10,000 km/s, 0.05pc from the SMBH. Doppler shifting of the photons emitted from the BLR broadens emission lines from this region. The narrow line region (NLR) consists of gas clouds also orbiting the SMBH, but 100-300pc from the black hole at 100-300 km/s, producing much narrower emission lines (Fig. 2) [1]. In this work, Seyfert galaxies, a class of radio-quiet AGN were studied. In Seyfert 1 galaxies (Sy1) the torus is viewed face-on making both the NLR and BLR visible. Conversely, in Seyfert 2 galaxies (Sy2) the torus is viewed edge-on and only 
the NLR is usually observed (Fig.3) [1]. Much evidence has been found to support this model. Antonucci and Miller found polarized broad emission lines in Sy2 spectra in 1985, evidence that Sy2 had a BLR hidden by the torus [13]. Additionally, the luminosity, rapid energy variability, and velocity dispersion of stars near SMBHs best match the observations of the central engine of AGN [1].

However, there are observations which are inconsistent or unexplained in this model. For instance, AGN have been observed to change from Sy1 to Sy2 and vice versa [3]. According to the Unified Model, the classification of AGN depends only on orientation, which is constant. Therefore, AGN cannot change classes under this model. This suggests that other factors also determine AGN classification, such as the density and motion of material in the torus.

Another piece of contradictory evidence is the possible difference in electron temperatures in the coronas of Sy1 and Sy2 [1]. If the electron temperatures are truly different, the magnetic fields of Sy1 and Sy2 might be different, which the Unified Model does not mention. Essentially, this would suggest that these objects are not the same, namely their differences could not be attributed to their orientation alone, as the Unified Model states.

Additionally, the fraction of obscured sources might depend on luminosity as well as orientation [3]. If this is true, more luminous objects would tend to be unobscured due to their increased luminosity, not their orientation alone.

\subsection{Investigating the Unified Model of AGN using X- rays}

To further investigate this model, the X-ray spectra of the sample population, which probes the innermost regions of AGN as the torus is transparent to XRays $^{1}$, was examined. X-ray emission from AGN is believed to be a product of inverse Compton radiation (IC). In this process, photons from the accretion disk are up-scattered by hot (possibly relativistic) electrons in a corona above the disk in the X-ray energy band [1]. The origin and exact geometry of the hot corona remains highly debatable and was further examined.

The IC light observed from AGN falls into three categories: direct, trans-

\footnotetext{
${ }^{1}$ The torus of a Compton-thick source, a source where $\mathrm{N}_{H} \sim 1.5 \times 10^{24} \mathrm{~cm}^{-2}\left(\mathrm{~N}_{H}\right.$ is the density of neutral Hydrogen atoms), is not transparent to X-Rays. However, only $5 \%$ of the sample population was Compton-thick.
} 
mitted, and reflected. Direct light comes from photons scattered off the corona. Transmitted light originates from photons scattered off the corona that travel through the torus. Reflected light is scattered off the corona and reflected off the accretion disk or torus via inverse Compton reflection. This type of reflection peaks at $30 \mathrm{keV}$ and then sharply declines. Consequently, the larger the reflection component the softer the spectrum in the 15-200 $\mathrm{keV}$ band. Due to the mere geometry of the model, Sy1 should have a larger reflection component than Sy2 in the Unified Model [7].

The reflection component of the total light emitted by AGN and the photon index (see Methods) are directly related. By examining the distribution of the photon indices, the Unified model was tested. Under the Unified Model the photon index of Sy1 should be larger than that of Sy2 as Sy1 galaxies have a larger reflection component.

A high energy cutoff is expected in both Sy1 and Sy2 spectra as the corona's electrons, which IC scatter the photons, are expected to be thermal with their own high energy cutoff, due to observations such as Fe emission lines. Moreover, the cutoff for Sy1 and Sy2 should be compatible since the properties of the corona should be independent of orientation.

\subsection{Relevance}

Ultimately, the Unified Model for AGN was tested. The photon indices, high energy cutoffs, and reflection components of Sy1 and Sy2 galaxies were compared to determine if Sy1 and Sy2 are intrinsically different. This research matters as AGN are believed to account for $100 \%$ of the Cosmic X-ray Background (CXB) (Fig.4). However, for this to be true, it is believed that a third of the AGN population should be Compton-thick, and very few of these objects have been detected thus far. Another explanation of the CXB posits that AGN have a larger reflection component than previously estimated, and the sum of all AGN reflection peaks at $\sim 30 \mathrm{keV}$ will reproduce the CXB without the need of more Compton-thick sources. Additionally, better understanding AGN and their relationship to the CXB will help place constraints on cosmological models and black hole physics. 


\section{Methods}

\subsection{Swift-BAT Mission}

Swift is a revolutionary multi-wavelength observatory focused on the GammaRay Burst science (GRB). Launched in 2004, Swift uses three instruments: the Burst Alert Telescope (BAT), the X-ray Telescope (XRT), and the Ultraviolet/Optical Telescope (UVOT), to monitor GRBs and afterglows in gamma-ray, X-ray, optical, and ultraviolet wavebands. BAT, the largest instrument on board, detects $\sim 100$ GRBs per year. Once a GRB is detected, XRT and UVOT are aimed at the burst to help determine the location and spectrum of the GRB. The data collected is circulated publicly worldwide for follow-up observations and studies. BAT is a coded mask telescope that utilizes a photon-counting CdZnTe detector with a $5200 \mathrm{~cm}^{2}$ detecting area in the $15-150 \mathrm{keV}$ range [14]. BAT surveys the X-ray sky with unprecedented sensitivity due to its wide field of view and pointing strategy enabling it to continuously monitor $\sim 80 \%$ of the sky daily. It has reached a sensitivity of $\sim 1 \mathrm{mCrab}$ in $1 \mathrm{Ms}$ of exposure, making it a perfect instrument for studying objects whose emission is faint in hard X-rays such as AGN [13]. The raw data from the telescope is calibrated using various calibration files such as the two seen in Fig. $5 \& 7$.

\subsection{Sample Population}

All the sources in this sample were detected above a $5 \sigma$ significance level from a three year exposure from the Swift-BAT hard X-ray survey (see [13] for more information). As mentioned before, the sources were studied in the 15-200 keV range, in which photons are transparent to the torus, further strengthening the integrity of this sample. This study was different from previous work in that an unbiased sample (blind search) of $\sim 200$ sources from [13] was used (See Fig.6\&8 and Table 6).

\subsection{Data Analysis}

\subsubsection{Photon Indices \& Simple Power Law Model}

Once the sources were chosen, spectra were generated by the methods specified in [13]. Using these spectra, photon indices for each source were determined by fitting the simple power law model to the sample spectra seen in 
Equation (1) using $\mathrm{X}_{\mathrm{spec}}$ 11.3.2. This program fits the data to Equation (1) using the $\chi^{2}$ statistic for the data by varying model parameters (see Fig.12).

$$
\frac{d N}{d E}=A E^{-\Gamma}
$$

In Equation (1) $\Gamma$ is the photon index, $\mathrm{A}$ is the normalization, $\mathrm{N}$ is the number of photons, and $\mathrm{E}$ is energy.

A python script was then written that used Root 5.18 to generate a photon index vs. redshift plot and a photon index histogram for the sample population (see Fig.9\&11). The Gaussian distribution was then fit to the generated histograms using the Binned Likelihood and $\chi^{2}$ methods in Root (see Table 3). Using the fitted histogram, the mean photon index, its error and sigma for Sy1, 1.5, 2 and other sources were determined.

Additionally, weighted averages and their errors were computed for Sy1 and Sy2. This method is particularly applicable in this case as all the individual errors are accounted for and this average does not involve fitting a model to the data.

To decide if the mean photon indices of Sy1 and Sy2 were statistically different, the Kolmogorov-Smirnov test, a non-parametric test, meaning that no assumptions are made about the data's probability distributions, was used. This test determines if two samples come from the same parent population by determining the maximum distance between the two samples' cumulative distribution functions. The p-value of this test was determined by using the same python script mentioned above.

To further ensure the validity of these results, the sample population was then divided into two groups around the mean flux, $2.89 \times 10^{-11} \mathrm{ergs} / \mathrm{cm}^{2} / \mathrm{s}$. The sources that had a flux greater than the mean were classified as bright. The sources with a flux less than or equal to the mean flux were classified as faint. The same analysis listed above for the entire population was then repeated for the faint and bright sources.

\subsubsection{Stacked Spectra}

To further investigate the Unified Model stacked spectra were created and examined. A stacked spectrum is a weighted average of individual spectra based on their errors. By using stacked spectra, the signal to noise ratio $(\mathrm{S} / \mathrm{N})$ increases and the mean properties of populations are more easily studied. Stacked Spectra for Sy1 and 2 were generated. The data for the stacked 
spectra was calculated using another python script that was created.

The simple power law and the PEXRAV models were fit to the Sy1 and Sy2 stacked spectra using Xspec. Utilizing the simple power law model the mean photon index, its error, and sigma were determined. The PEXRAV model, unlike the simple power law model, adds the reflection component and high energy cutoffs to the underlying continuum providing a better fit the majority of the time. First, the stacked spectra were fit ignoring the high energy cutoff, and the photon index and reflection component were determined. The reflection component is the total amount of reflection observed normalized to the directly viewed primary spectrum based on their associated integrated power outputs. When using this model Sy1 and Sy2 were assumed to be at inclination angles ${ }^{2}$ of 30 and 60 degrees, respectively. Then the reflection component was ignored, and the high energy cutoff and photon index were determined. For Sy2 the photon index, reflection component, and high energy cutoff were fit simultaneously as neither of the two previous fits accurately described the data.

\section{Results}

The results from fitting the histograms generated by the simple power law model from all the individual spectra and from the calculated weighted averages and errors are seen in Table 3. The p-values of the Kolmogorov-Smirnov test for Sy1 and Sy2 are listed in Table 2. The weighted averages were more accurate in this instance as significant tails in the histograms caused the Gaussian to not fit the data well (see Fig.10) and the individual errors for all the AGN in the sample are accounted for in this method.

The results of fitting the stacked spectra to the PEXRAV model and the simple power law model are seen in Table 4 . The $\chi^{2}$ values for the PEXRAV model were significantly smaller than those for the simple power law model for the stacked spectra in Table 4 .

The results from both Table $3 \& 4$ showed that the spectra of Sy1 are softer than that of Sy2, meaning the Sy1 have a larger photon index than Sy2. Sy2 seemed to need a high energy cutoff $\sim 360 \mathrm{keV}$, whereas Sy1 had a high energy

\footnotetext{
${ }^{2}$ The inclination angle is the angle between the normal of the accretion disc and the line of sight.
} 
cutoff outside the X-ray energy band. Finally, the reflection components of Sy1 and Sy2 were found to be compatible within their errors.

\section{Discussion \& Conclusion}

From the photon index histograms generated from the individual spectra, it is evident that the mean photon indices of Sy1 and Sy2 are different at a $2 \sigma$ level (Table 2), specifically Sy1 had a larger photon index than Sy2. This trend is in agreement with [3]. Under the Unified Model the mean photon index of Sy1 should be larger than that of Sy2 as the reflection component in Sy1 is larger than that in Sy2, which increases the photon index.

When examining the data from the stacked spectra, it is evident that the PEXRAV model fits the spectra significantly better than the simple power law model based on the lower $\chi^{2}$ values. This indicates that AGN probably do have a high energy cut and/or reflection component, in agreement with the Unified Model. However, accounting for these new parameters did not significantly change the photon index, as the photon index for Sy1 was still larger than that of Sy2 when this model was fit to the stacked spectra.

The reflection component for Sy1 under the Unified Model should be larger than that of Sy2. However, the data in Table 4 indicates that the reflection components for Sy1 and Sy2 are compatible, but given the significant errors, this finding does not disprove the Unified Model. Moreover, Sy2 seemed to have a high energy cutoff at $\sim 360 \mathrm{keV}$, whereas Sy1 had a cutoff

outside the X-ray energy band, suggesting the corona of Sy1 and Sy2 are different, which is not in agreement with the Unified Model.

Overall, in this work the photon index of Sy1 was found to be larger than that of Sy2, as the Unified Model predicts. However, Sy1 and Sy2 had compatible reflection components. Given the significant errors of this finding, this cannot be said to be evidence against the Unified Model. However, it was concluded that Sy1 and Sy2 do have different high energy cutoffs, which is not in agreement with the Unified Model. Given the substantial errors of parts of the data, to make more definite conclusions a larger sample should be used. This is plausible as the data for this study came from Swift-BAT, which is still accumulating data. 


\section{Acknowledgments}

This research, conducted at the Stanford Linear Accelerator Center, could not have been completed without the support, funding, and opportunity provided by the Department of Energy, Office of Science, SLAC, and KIPAC. My mentors, Marco Ajello and Masaaki Hayashida deserve much thanks for their patience, enthusiasm, and expertise. I would also like to thank all the SULI students and staff at SLAC. 


\section{References}

[1] M. Ajello, "Swift/BAT studies of AGN and the Cosmic X-ray Background", Universittsbibliothek der TU Mnchen, 2007.

[2] O. Labiano, "Host galaxies and environments of compact extragalactic radio sources", Kapteyn Astronomical Institute and Space Telescope Science Institute, February 2006.

[3] V. Beckmann, S. Soldi, C. Ricci, J. Alfonso-Garzn, T.J.-L. Courvoisier, A. Domingo, N. Gehrels, P. Lubinsk i, J. M. Mas-Hesse, and A. A. Zdziarski, "The Second INTEGRAL AGN Catalogue", July 2009

[4] S. Kraemer, R. Windhorst, K. Carpenter, M. Crenshaw, M. Elvis,and M. Karovska, "Active Galactic Nuclei and their role in Galaxy Formation and Evolution", February 2009.

[5] P.O. Petrucci, "Models of Comptonization", HAL - CCSD, 2008.

[6] V. Beckmann, S. Soldi, C.R. Shrader, N. Gehrels, and N. Produit, "The Hard X-ray 20-40 keV AGN Luminosity Function”, 2006.

[7] M. Ajello, A. Rau, J. Greiner, G. Kanbach, M. Salvato, A. W. Strong, S. D. Barthelmy, N. Gehrels, C. B. Mar kwardt, J. Tueller, "BAT X-ray Survey - III: X-ray Spectra and Statistical Properties", The Astrophysical Journal, September 2007.

[8] S. Deluit, T. Courvoisier, "The intrinsic emission of Seyfert galaxies observed with BeppoSAX/PDS I. Comparison of the average spectra of the three classes of Seyfert Galaxies", Astron. Astrophys. 399 77, 2003.

[9] P. Ricker and P. Meszaros, "Starburst and Reflection-Dominated AGN Contributions to the Cosmic X-Ray Background", ApJ vol. 418 49, 1993.

[10] C. Caradamone, http://www.astro.yale.edu/ccardamone/index.html

[11] D.E. Gruber, J. Matteson, L. Peterson, and G. Jung, "The Spectrum of Diffuse Cosmic Hard X-Rays Measured with HEAO-1", 1999.

[12] P. Magdziarz, A.A. Zdziarski, "The nature of the X-ray source in NGC 415”, 1995. 
[13] M. Ajello, L. Costamante, R. M. Sambruna, N. Gehrels, J. Chiang, A. Rau, A. Escala, J. Greiner, J. Tueller, J. V. Wall, and R. F. Mushotzky, "The Evolution of Swift/BAT blazars and the o rigin of the MeV background", ApJ, 2009.

[14] BAT Digest, swift.gsfc.nasa.gov, 2005.

[15] Zwillinger D.\& Kokoska S., Standard Probability and Statistics Tables and Formulae. Boca Raton, Florida: CRC Press LLC, -c2000, ISBN 1-58488-059-7. 


\section{$6 \quad$ Figures}

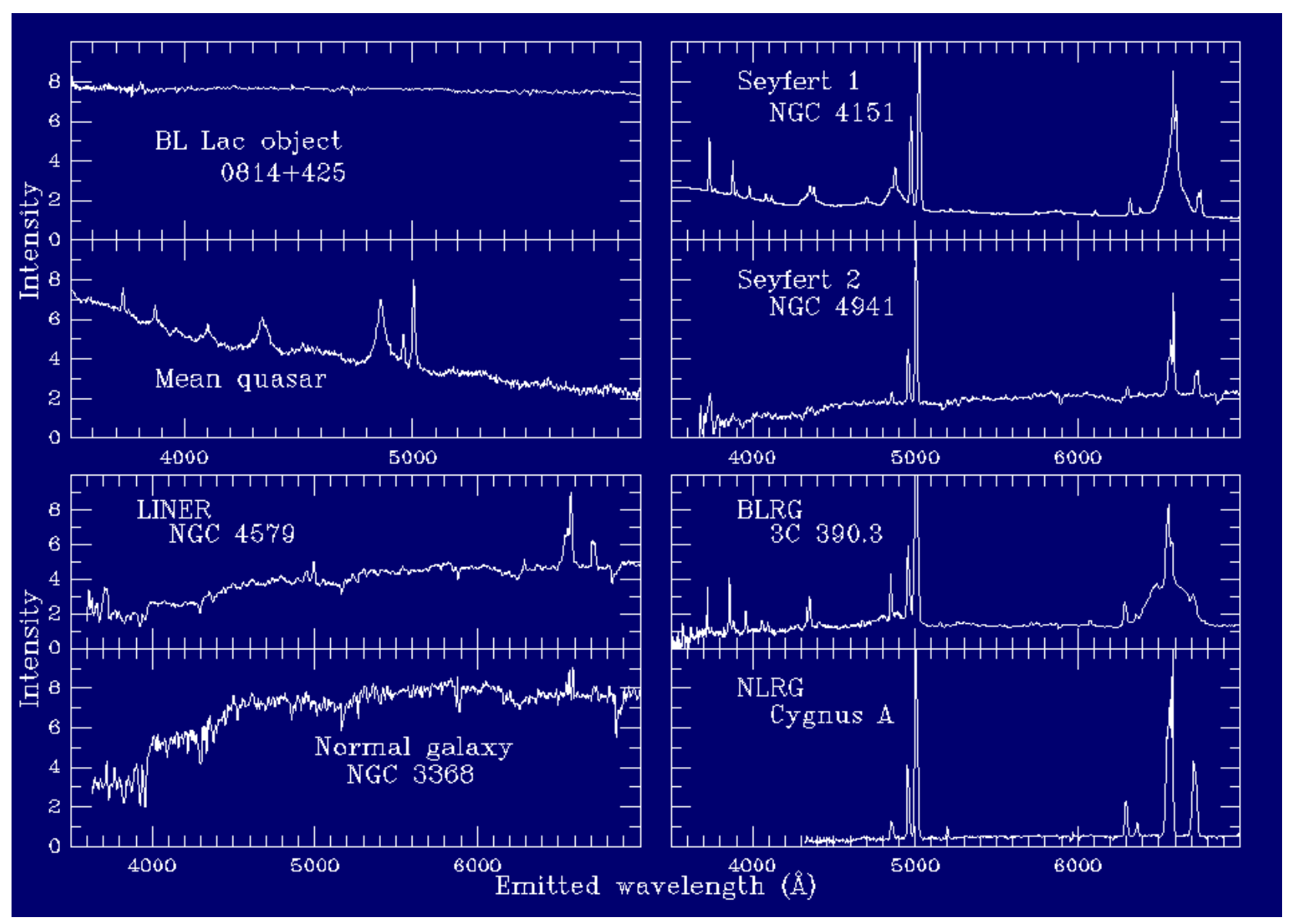

Figure 1: Representative spectra of different types of AGN. The Seyfert 1 spectrum has noticeably broader emission lines than Seyfert 2 spectrum. Image from Ajello [1]. 


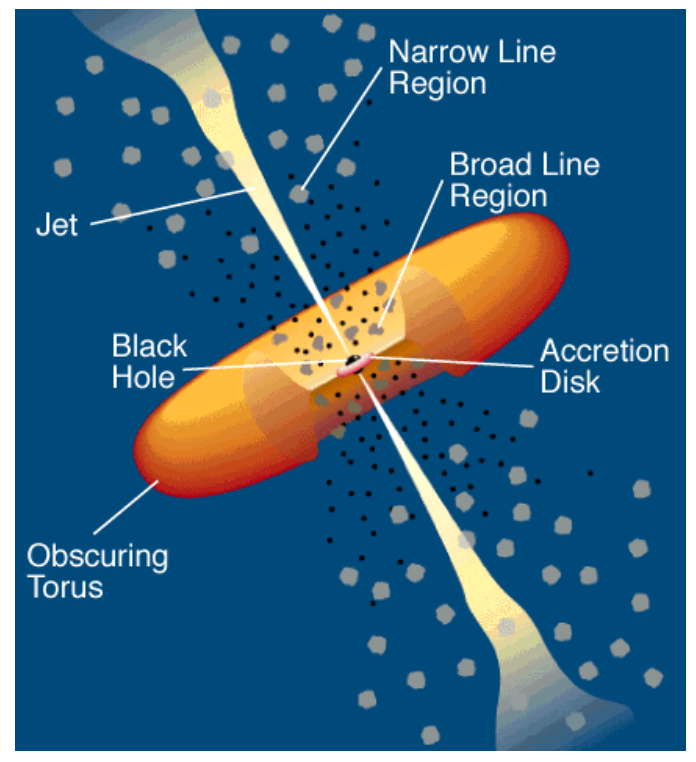

Figure 2: Unified Model. Image from Ajello [1].

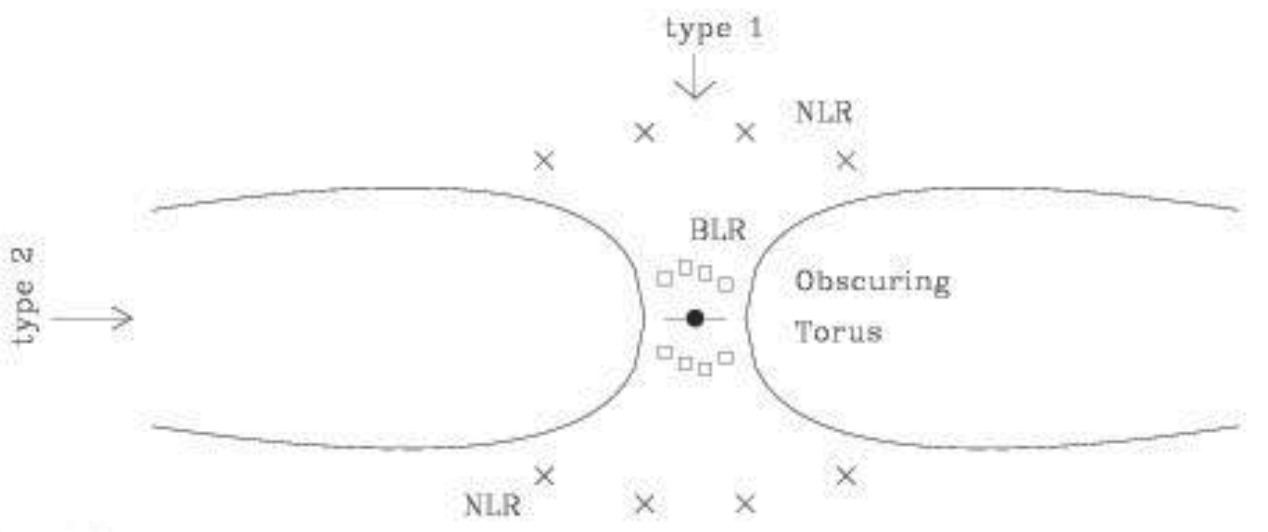

Figure 3: In Sy2 the torus is viewed more edge-on. In Sy1 the torus is viewed more face-on. Image from Cardamone [10]. 


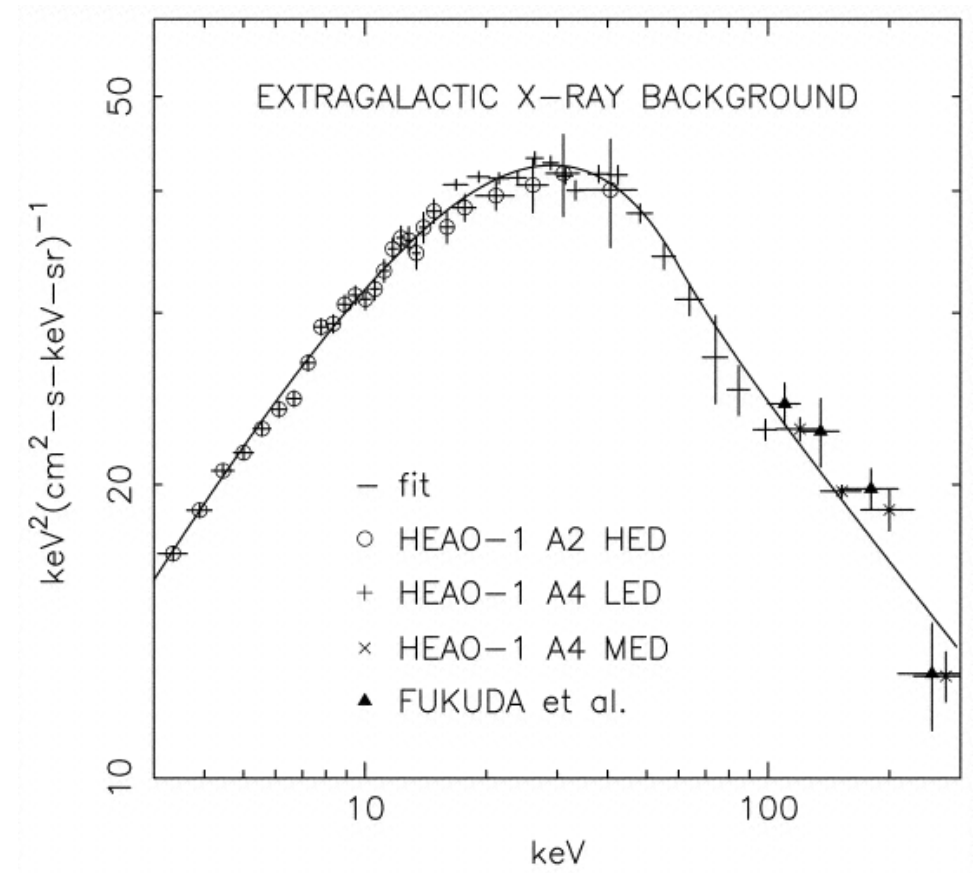

Figure 4: Photon spectrum of CXB compiled from various instruments on HEAO 1. Image from Gruber [11].

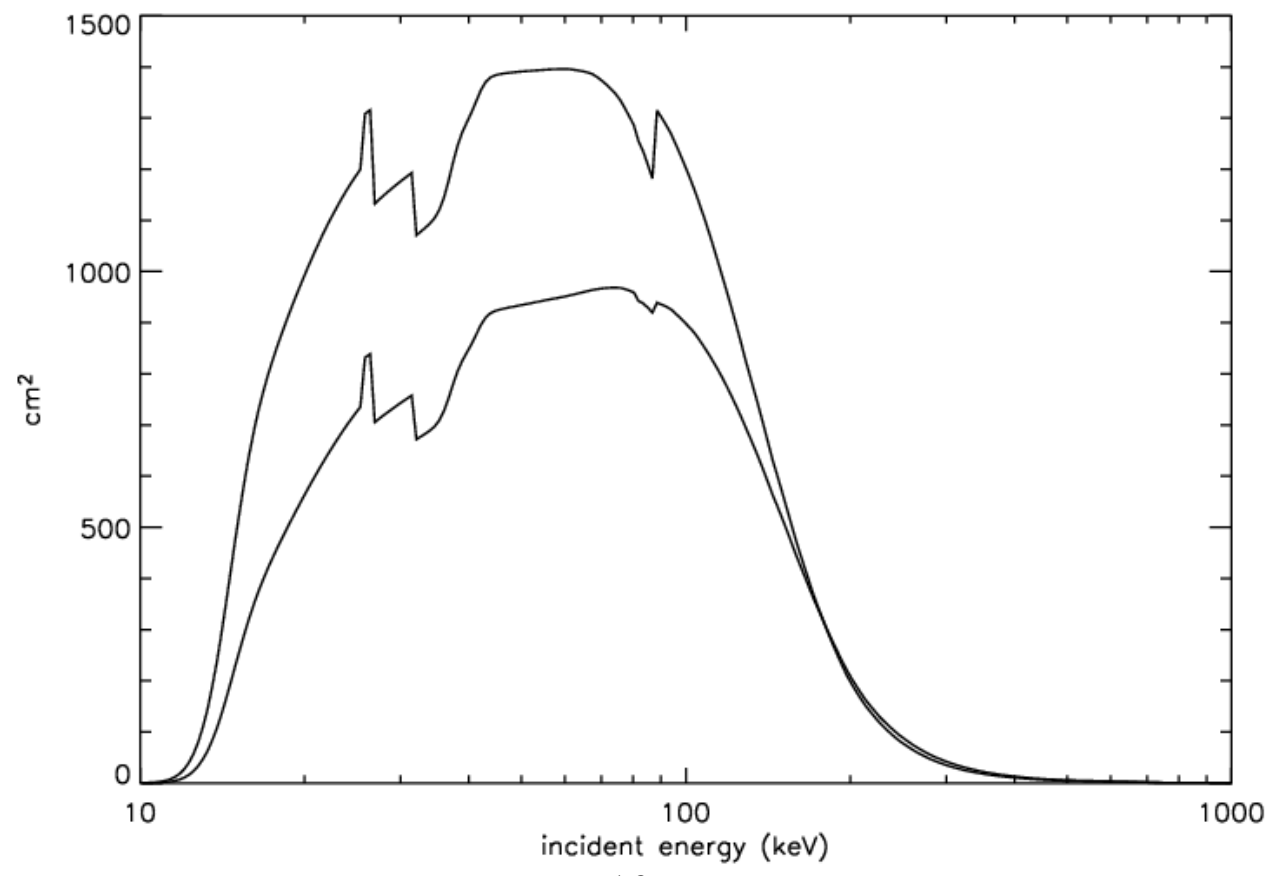

16

Figure 5: Effective area of the BAT for on- and off-axis (45 deg.) sources at different energy levels [14]. 


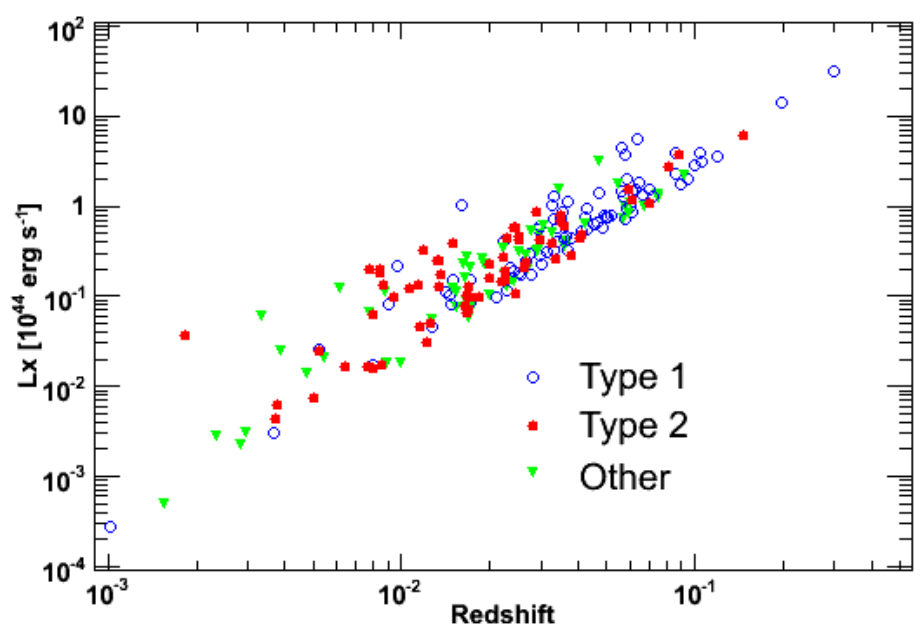

Figure 6: Luminosity vs. Redshift of source population studied.

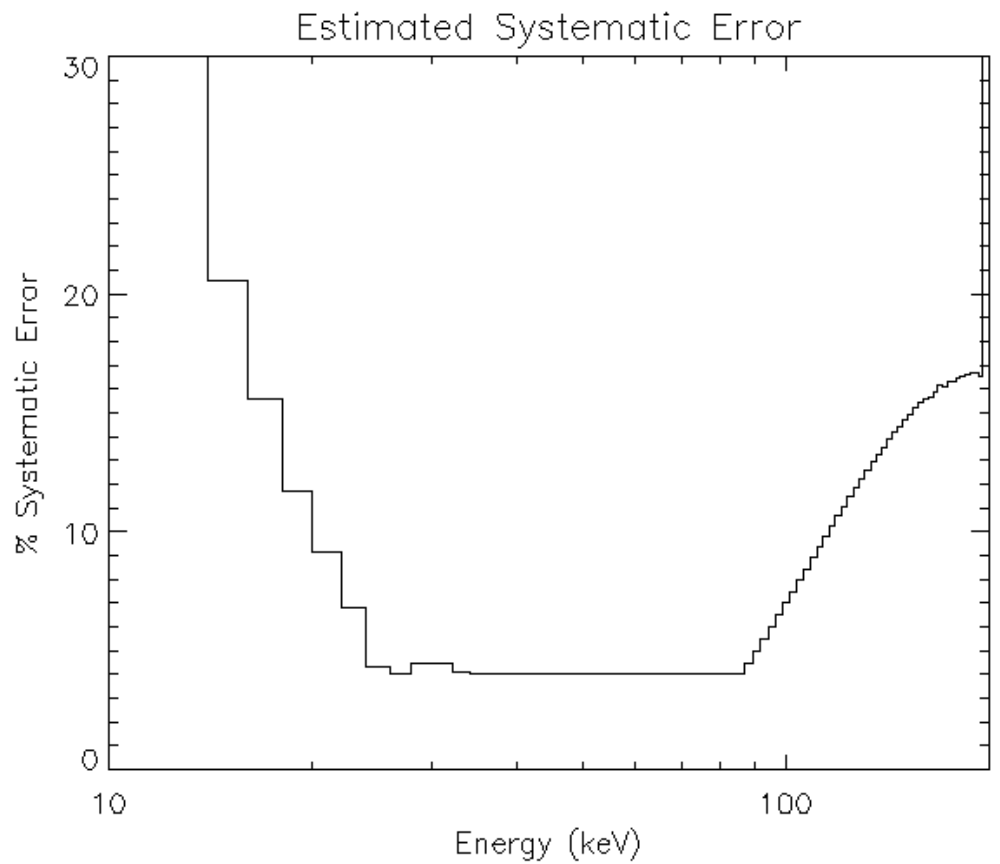

Figure 7: Systematic error of BAT as a function of energy [14]. 


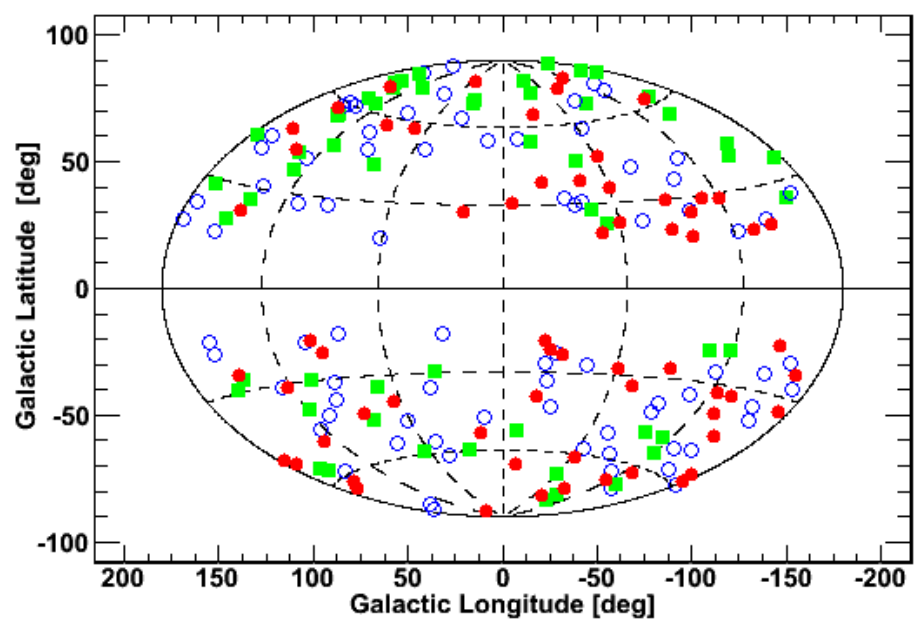

Figure 8: Map of source population.

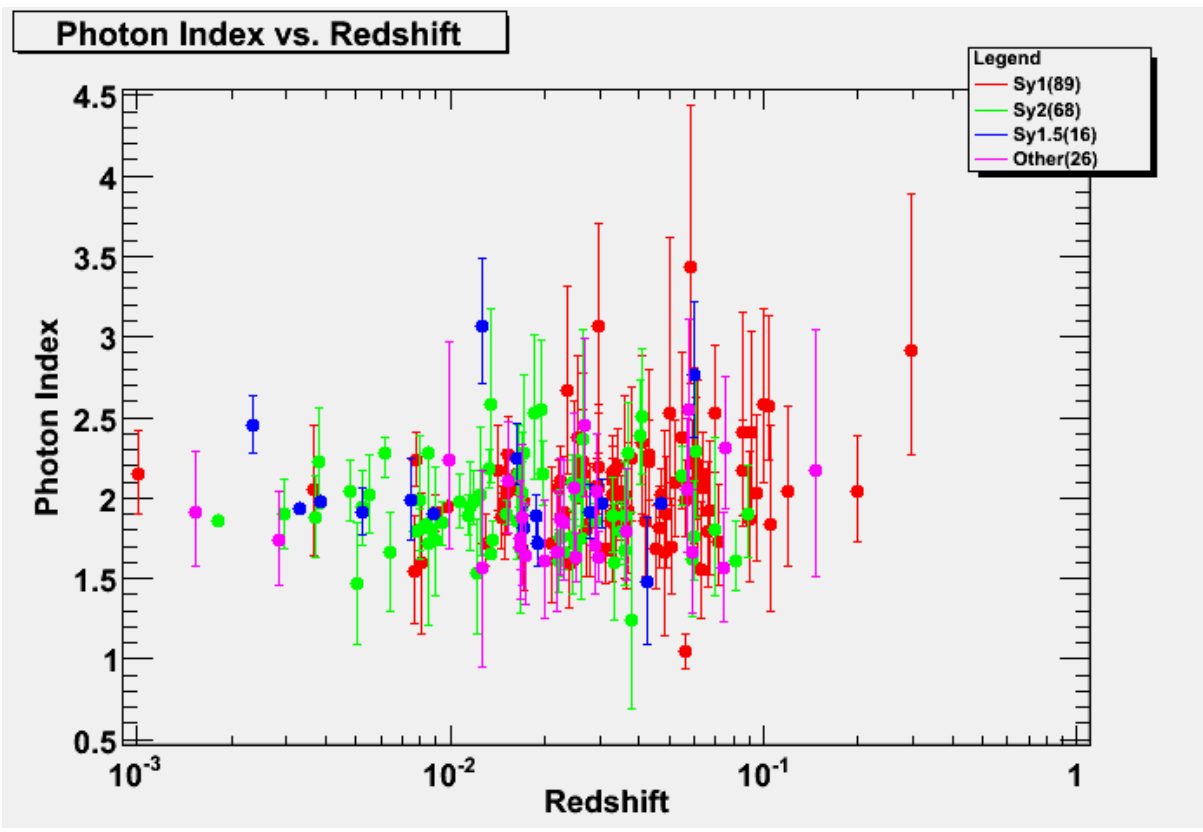

Figure 9: Redshift vs. Photon Index of source population generated using Root. 


\section{Number of Sources vs. Photon Index}

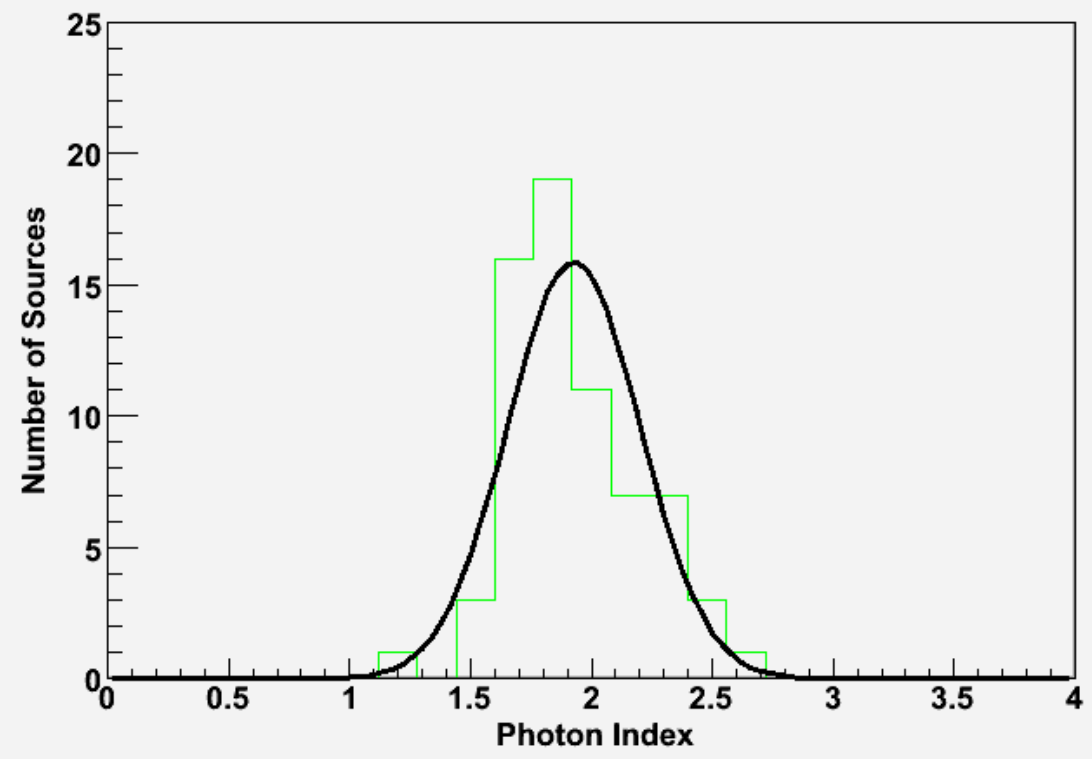

Figure 10: Gaussian Fit of entire Sy2 population using binned likelihood method. The right-hand tail of the data skews the data, causing the Gaussian to not match the data well.

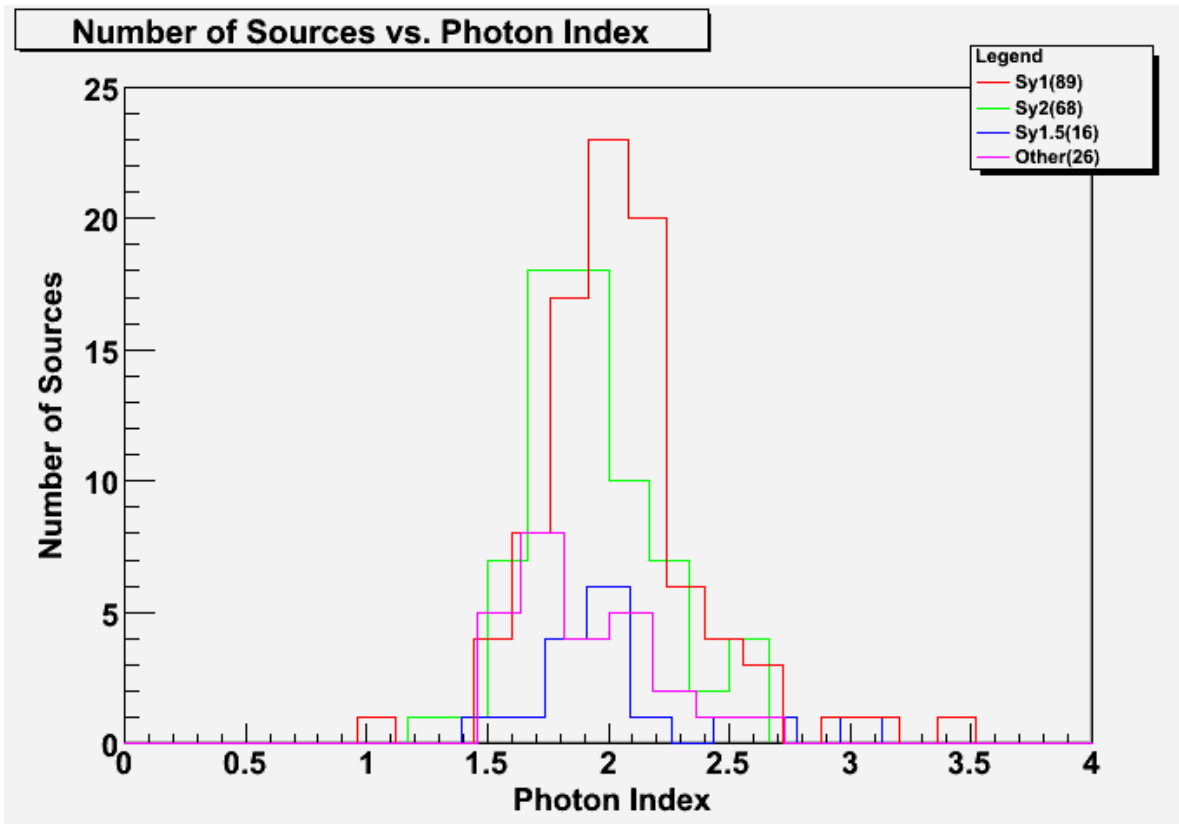

Figure 11: Sample population distribution generated using Root. 


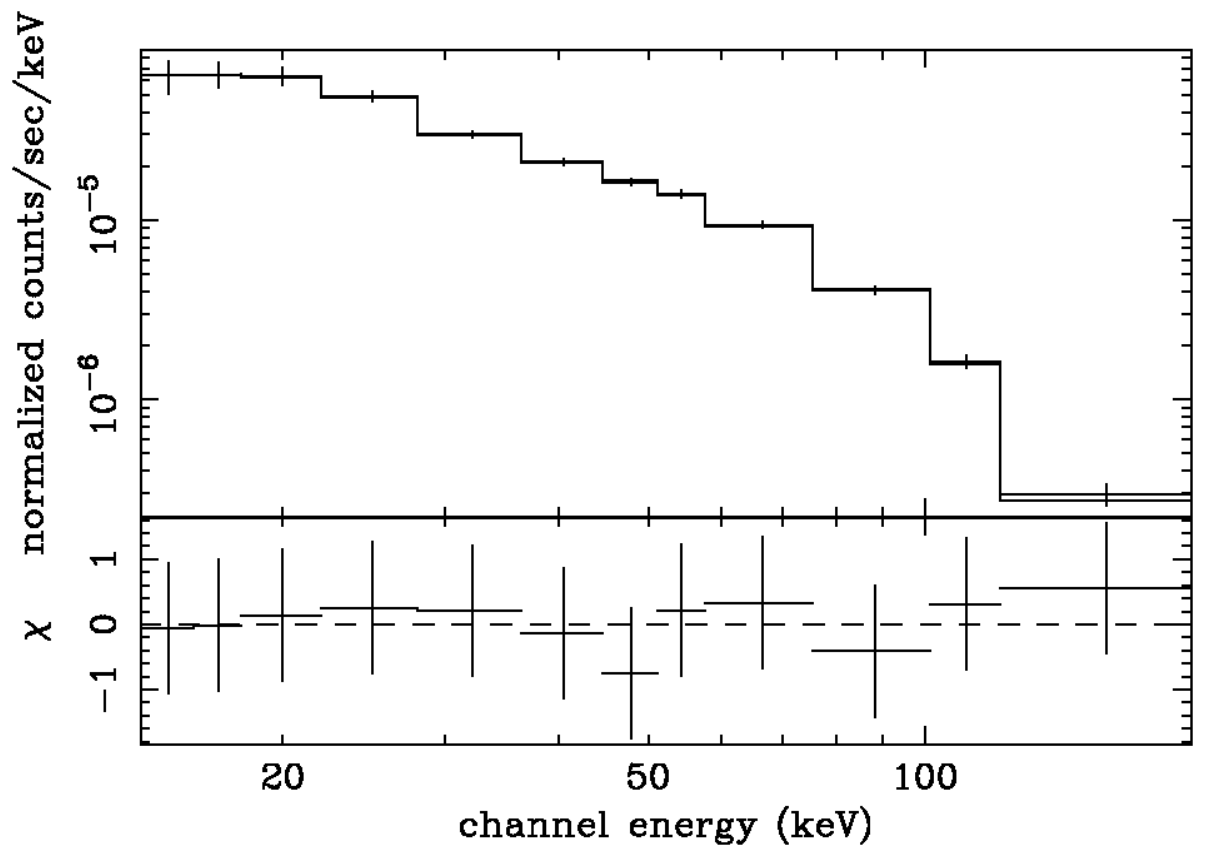

Figure 12: Typical spectra generated by Xspec11.

\begin{tabular}{ccccc}
\hline \hline Type & Sy1 & Sy1.5 & Sy2 & Others \\
\hline Number of Sources & 89 & 16 & 68 & 26 \\
\hline
\end{tabular}

Table 1: Source Population ${ }^{3}$

\footnotetext{
${ }^{3}$ Sy1 class includes Sy1, 1.1, and 1.2. Sy1.5 class includes strictly Sy1.5. Sy2 class includes Sy1.8, 1.9, and 2. All other sources were classified as others.
} 


\begin{tabular}{cc}
\hline \hline Classification & K-S P-Value \\
Sy1 \& Sy2 & 0.027 \\
Bright Sy1 \& Sy2 & 0.074 \\
Faint Sy1 \& Sy2 & 0.260 \\
\hline \hline
\end{tabular}

Table 2: Kolmogorov-Smirnov P-Values

\begin{tabular}{|c|c|c|c|c|c|}
\hline & Type & Source Count & Fit Method & $\begin{array}{l}\Gamma \\
\end{array}$ & $\sigma$ \\
\hline \multirow{6}{*}{ All Sources } & \multirow{2}{*}{ Sy1 } & \multirow{2}{*}{89} & $W A$ & $1.945 \pm 0.263$ & - \\
\hline & & & $\mathrm{BL}$ & $2.049 \pm 0.036$ & $0.342_{-0.033}^{+0.48}$ \\
\hline & \multirow{2}{*}{ Sy1.5 } & \multirow{2}{*}{16} & WA & $1.939 \pm 0.152$ & \\
\hline & & & BL & $2.070 \pm 0.103$ & $0.404_{-0.062}^{+0.084}$ \\
\hline & \multirow{2}{*}{ Sy2 } & \multirow{2}{*}{68} & WA & $1.851 \pm 0.166$ & \\
\hline & & & $\mathrm{BL}$ & $1.927 \pm 0.033$ & $0.274_{0.022}^{+0.025}$ \\
\hline \multirow{6}{*}{ Bright Sources } & \multirow{2}{*}{ Sy1 } & \multirow{2}{*}{17} & WA & $1.943 \pm 0.169$ & \\
\hline & & & $\mathrm{BL}$ & $1.985 \pm 0.060$ & $0.243_{-0.36}^{+0.048}$ \\
\hline & \multirow{2}{*}{ Sy1.5 } & \multirow[b]{2}{*}{9} & WA & $1.938 \pm 0.168$ & \\
\hline & & & BL & $1.935 \pm 0.042$ & $0.123_{-0.024}^{+0.036}$ \\
\hline & \multirow{2}{*}{ Sy2 } & \multirow{2}{*}{24} & WA & $1.851 \pm 0.166$ & \\
\hline & & & BL & $1.940 \pm 0.048$ & $0.235_{-0.030}^{+0.038}$ \\
\hline \multirow{6}{*}{ Faint Sources } & \multirow{2}{*}{ Sy1 } & \multirow{2}{*}{72} & $\overline{\mathrm{WA}}$ & 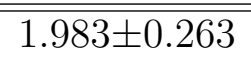 & \\
\hline & & & BL & $2.090 \pm 0.041$ & $0.351_{-0.027}^{+0.031}$ \\
\hline & \multirow{2}{*}{ Sy1.5 } & \multirow{2}{*}{7} & WA & $2.042 \pm 0.152$ & \\
\hline & & & $\mathrm{BL}$ & $2.223_{-0.215}^{+0.219}$ & $0.549_{-0.121}^{+0.201}$ \\
\hline & \multirow{2}{*}{ Sy2 } & \multirow{2}{*}{44} & WA & $1.914 \pm 0.253$ & \\
\hline & & & BL & $1.920 \pm 0.044$ & $0.293_{-0.029}^{+0.034}$ \\
\hline
\end{tabular}

Table 3: Mean Photon Indices of Source Population using Gaussian Fit ${ }^{4}$

\footnotetext{
${ }^{4} \mathrm{BL}$ and WA stand for binned likelihood and weighted average, respectively.
} 


\begin{tabular}{rcccccc}
\hline \hline & & & & & \\
& Type & Source Count & $\Gamma$ & $\mathrm{E}_{C}[\mathrm{keV}]$ & $\mathrm{R}$ & $\chi_{\nu}^{2}$ \\
\hline & & & & & & \\
Sy1 & 89 & $2.143 \pm 0.07$ & - & - & 1.38 \\
Sy 1 & 89 & $2.079 \pm 0.03$ & - & $0.675_{-0.20}^{+0.26}$ & 1.04 \\
& Sy 1 & 89 & $1.522_{-0.07}^{+0.16}$ & $81_{-11}^{+39}$ & - & 1.01 \\
\cline { 2 - 7 } & & & & & & \\
& Sy 2 & 68 & $2.035_{-0.10}^{+0.06}$ & - & - & 1.83 \\
Sy 2 & 68 & $1.956_{-0.04}^{+0.05}$ & - & $1.779_{-1.09}^{+2.07}$ & 0.912 \\
& Sy 2 & 68 & $1.310_{-0.12}^{+0.05}$ & $74_{-12}^{+7}$ & - & 1.27 \\
Sy 2 & 68 & $1.793_{-0.22}^{+0.23}$ & $360_{-46}^{+156}$ & $1.102_{-0.30}^{+0.49}$ & 1.11 \\
\hline
\end{tabular}

Table 4: Fitting Stacked Spectra with PEXRAV Model 
Intelligent Platform Management Interface (IPMI)

Derek S. Wung

Office of Science, Science Undergraduate Laboratory Internship Program

University of California Los Angeles

SLAC National Accelerator Laboratory

Menlo Park, California

August 14, 2009

Prepared in partial fulfillment of the requirement of the Office of Science, Department of Energy's Science Undergraduate Laboratory Internship under the direction of Ronald Johnson in the Controls Department of the SLAC National Accelerator Laboratory.

Participant:

Signature

Research Advisor:

Signature 


\section{Table of Contents}

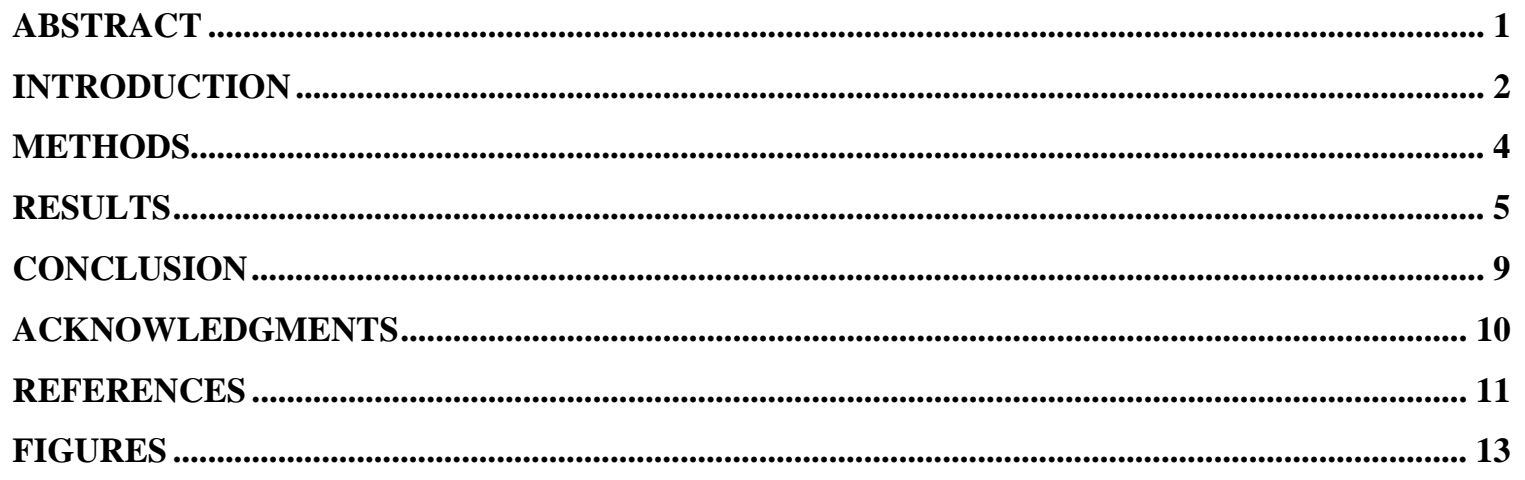




\begin{abstract}
:
Intelligent Platform Management Interface (IPMI). DEREK S. WUNG (University of California Los Angeles, Los Angeles, CA 90024) RON JOHNSON (Controls Department, SLAC National Accelerator Laboratory, Menlo Park, CA 94025).
\end{abstract}

Intelligent Platform Management Interface (IPMI) is a set of specifications that defines interfaces between components of the same system, most notably in servers; these interfaces provide information on the status of environmental variables such as voltage, temperature, and fan status. IPMI, which has grown significantly within the past five years, is generally used in computers to keep the system in check. However, the Controls Department at the SLAC National Accelerator Laboratory wants to find a way to implement IPMI in its standalone chassis. First, it is imperative to study how IPMI is used in a computer before actually going about designing a system that implements IPMI. Next, the proper parts of the system must be determined before research can be conducted on finding these parts. In addition to finding the right chips, figuring out the proper connections between these parts is just as important. While the system wasn't constructed during the course of the project, the general process of designing the system from scratch is outlined. 


\section{Introduction:}

When we buy our first computers, we hope that they don’t break down shortly after we start to use them. Today’s computers, however, can last for several years before malfunctioning. A set of specifications called Intelligent Platform Management Interface (IPMI) plays a major part in maintaining the health of modern-day computers. With the implementation of such an interface, computer owners need not worry much about their hard drives overheating or breaking down since IPMI helps prevent such an occurrence.

Introduced by companies from Dell to Intel, IPMI helps maintain the safety of a computer by defining interfaces to allow different parts of a computer to communicate with one another. Communication is done by way of a protocol called Inter-Integrated Circuit $\left(\mathrm{I}^{2} \mathrm{C}\right)$, which transfers data using two lines: a Serial Clock Line (SCL) and a Serial Data Line (SDA). The SDA physically transmits the data from one location to another while the SCL determines when exactly that data is sent to avoid a massive influx of messages.

Over the past eleven years, the specifications of IPMI have evolved. In 1998, IPMI V 1.0 was introduced. Three years later, IPMI V 1.5 was released. Finally, in 2004, the specifications of the latest version of IPMI (V 2.0) were publicized on Intel's website. Numerous revisions have taken place over the last five years, but the general concept of IPMI has remained the same throughout its history.

With IPMI, one can physically monitor a computer’s temperature, fan status, and power supply voltage; this can be done by utilizing downloadable software programs such as IPMItool, IPMIView, and OpenIPMI. However, not every program can be run on 
every operating system, so it is necessary to check the compatibility of the software with the user's operating system before downloading the program.

One of the advantages of utilizing IPMI is the fact that it operates independently of the main processor as well as the operating system. Therefore, even if the operating system fails or if the processor shuts down, administrators can still use IPMI to diagnose and recover their systems. With IPMI, alert notifications will be sent to administrators before encountering hardware problems [5].

At the heart of the IPMI architecture lies a microcontroller called a Baseboard Management Controller (BMC), which serves as the intelligence of IPMI [8]. The BMC's main job is to help all elements communicate with one another by way of messages to other interfaces; the messages are sent via an Intelligent Platform Management Bus (IPMB), a computer bus that is based on the $\mathrm{I}^{2} \mathrm{C}$ protocol [2]. Embedded on most motherboards, the BMC can interact with every other board component and keep track of logging information in a System Event Log (SEL), which is utilized by an administrator in the event that the main processor fails or the operating system crashes.

One of the advantages of using IPMB is that it can communicate with devices that do not support IPMI [6]. For example, most remote management cards do not typically communicate with other components via $\mathrm{I}^{2} \mathrm{C}$; instead, they are usually connected to an IPMB connector before interacting with the BMC. In addition, IPMB allows platform management to be extended by connecting more management controllers, which are microcontrollers that operate in a similar manner as the BMC; these additional management controllers are typically connected to other boards in the same system and are often called satellite controllers [1]. 
While IPMI is primarily used to monitor the health of computers, it can be used in other systems as well. The main objective was to study IPMI and determine how it could be implemented in standalone electronics chassis.

\section{Methods:}

This research project was conducted in the Controls Department at the SLAC National Accelerator Laboratory. No textbook was consulted during the entire course of the project, but the use of the internet was extensive. Websites that appeared on Google as well as the actual IPMI specifications posted on Intel's website were often referenced. Intel's specifications demonstrated IPMI in more detail than any other site.

After studying the basics of IPMI, a design of such a system was constructed. To plan this system, each individual part was researched; most of these parts were found via www.findchips.com. Information on numerous chips of the same type was gathered and compiled together in the form of datasheets; each chip was then compared and contrasted to determine which chip represented the best choice for its respective part.

At times, certain parts of the system could not be found on www.findchips.com; when that happened, a simple Google search was used. For example, the Find Chips database contained no information about remote management cards, but Google yielded some relatively decent hits about such hardware. Then, after entering the names of the hardware on www.findchips.com, the search would yield a few results.

Upon examination of all necessary chips, some of the datasheets gathered did not contain the pin-out information. Other times, it was discovered that the chips were 
compatible with IPMI V 1.5, but not necessarily with IPMI V 2.0. In such events, different chips were found to make sure they could function properly in the system.

The architecture of a system that utilizes IPMI V 2.0 is shown in Figure 1. The figure not only shows the chips involved in the system, but it also shows how each chip interacts with the BMC. When all the chips, as well as their interfaces with the BMC, were gathered, it was time to create a block diagram. A simple sketch was drawn by hand initially to get a rough idea of what the system would look like in real life. Then, a final block diagram of the necessary hardware was developed.

\section{Results:}

Upon gathering various datasheets of chips from BMCs to numerous sensors, the chips that seemed most promising were chosen. In addition to the BMC, the system design contained sensors that monitor temperature, fan status, and voltage. While the construction of the actual design won't commence until after the project, its foundation was established.

Initially, a PC87431M mini-BMC was found, but because no pinout information was found on its datasheet, it could not be trusted. After additional research, the Renesas H8S/2167 BMC (see Figure 2) was found. This BMC contained 144 pins, of which twelve were used as $\mathrm{I}^{2} \mathrm{C}$ ports. Because an $\mathrm{I}^{2} \mathrm{C}$ port requires two pins for the SCL and SDA lines, up to six components can be connected to the H8S/2167 via $\mathrm{I}^{2} \mathrm{C}$. The H8S/2167 also contains three sets of two pins each that transmit and receive data from other components using different communication protocols. 
A non-volatile storage chip retains data in a system when power is shut off; if manufactured properly, today’s chips can hold data for up to ten years. Data that can be stored in non-volatile storage chips include Sensor Data Records (SDRs), the System Event Log (SEL), and Field Replaceable Unit (FRU) inventories. SDRs typically contain information about the sensors in the system; these records are kept in an SDR repository, which is managed by the BMC [7]. The SEL keeps track of logging information in the event that the main processor crashes or the operating system fails. Field Replaceable Units are interchangeable parts of a system; in other words, an FRU can easily be replaced in case it malfunctions. After researching different chips for NV storage, the PCF8582C-2 (see Figure 3) was found to be optimal in the design mainly because it has a serial input/output $\mathrm{I}^{2} \mathrm{C}$ bus, meaning it has SCL and SDA pins. The PCF8582C-2 can also hold up to 256 x 8 bits of non-volatile storage.

A set of three board temperature sensors from Analog Devices was found: the AD7416, AD7417, and the AD7418. Although each sensor can be connected via $\mathrm{I}^{2} \mathrm{C}$, the AD7416 (see Figure 4) was chosen because of its simplicity and low number of pins. Whereas the AD7416 and AD7418 each have eight pins, the AD7417 has sixteen pins. In addition, the AD7418 had seemingly unnecessary pins for the matter at hand. These sensors can withstand temperatures between $-40^{\circ} \mathrm{C}$ and $125^{\circ} \mathrm{C}$.

The MAX1617A (see Figure 5) is a remote diode temperature sensor that has an SMBus two-wire serial interface; SMBus is a derivation of $\mathrm{I}^{2} \mathrm{C}$, so the sensor also has clock and data pins. In addition to remote temperatures, the MAX1617A can also measure local temperatures and send out alarms in events of abnormally low or high temperatures. 
It was far from easy to search for a suitable voltage sensor, but one chip from Texas Instruments was discovered to sense voltages from 0 to 26 volts. The INA209EVM (see Figure 6) can also monitor current and power in a system; the device can report readings with uncertainties of at most one percent. It also has serial clock and data lines for connections via SMBus.

The ADT7473 fan controller (see Figure 7) can manage up to four fans simultaneously. Like the other sensors aforementioned, this sensor can be connected to the BMC via SMBus. In addition, the ADT7473 has two remote temperature sensors as well as an on-chip temperature sensor that can measure its own internal temperature; the chip can measure temperatures as high as $191^{\circ} \mathrm{C}$.

A remote management card allows administrators to manage their servers from any spot in the world. The MegaRAC G4 (see Figure 8) is one of the few remote management cards that can operate alongside IPMI V 2.0. Unlike most other remote management cards, the G4 can directly connect to the BMC via $\mathrm{I}^{2} \mathrm{C}$, but it does not depend on the BMC to manage system power if it has a special Feature Connector.

Finally, a serial/modem cable as well as a LAN Network controller was needed to serve as interfaces for IPMI messaging, which is how elements of a system communicate with one another. The Microchip ENC28J60 (see Figure 9) is an Ethernet controller that does not communicate via $\mathrm{I}^{2} \mathrm{C}$; instead, it communicates via Serial Peripheral Interface (SPI), a four-wire serial bus. To connect the ENC28J60 to the BMC, an SC18IS602IPW from NXP (see Figure 10) was needed to convert $\mathrm{I}^{2} \mathrm{C}$ to SPI and allow for a connection between the ENC28J60 and the H8S/2167. 
Unlike all of the previous components mentioned, the Serial/Modem cable does not connect via $\mathrm{I}^{2} \mathrm{C}$, SMBus, or SPI. Instead, the cable is connected by a different protocol called RS-232. RS-232 serves as two-line interface, similar to the $\mathrm{I}^{2} \mathrm{C}$ interface. However, the two lines involved in the RS-232 standard are Transmit (Tx) and Receive (Rx). The HN-210 (see Figure 11) is one cable that is actually compatible with this standard.

When everything is put together, there are a total of seven chips that require $\mathrm{I}^{2} \mathrm{C}$ connections to the BMC. Because the Renesas H8S/2167 has only six available $\mathrm{I}^{2} \mathrm{C}$ ports, it is necessary to find an $\mathrm{I}^{2} \mathrm{C}$ hub that allows multiple devices to connect to the same port. The PCA9518 (see Figure 12) is an expandable hub that can take up to five connections via $\mathrm{I}^{2} \mathrm{C}$; it also has two sets of two expansion pins each that allow two devices on the hub to run at the same time. In this design, only one set of expansion pins are needed.

Finally, the design of the system was created using DxDesigner. The design on DxDesigner can be seen in Figure 13; a simplified version of the design can be seen in Figure 14. It is not certain whether or not this specific system design will work. However, any chip that is discovered to be unsuitable for the design can easily be replaced at a relatively low cost. The fact that these parts are interchangeable ensures reliability, availability, and serviceability in today's systems [3].

In addition to microcontrollers, Field-Programmable Gate Arrays (FPGAs) can be incorporated in the system. FPGAs are more flexible than regular microcontrollers; whereas microcontrollers have fixed pin configurations, FPGAs have programmable pins. In other words, FPGAs can be programmed for extended capabilities during the construction of the design [9]. 
There exist software programs that let users keep an eye on the environmental variables of their system; such software can be downloaded for free online. IPMIView, for example, is a program written in Java that allows users to communicate with the BMC via Ethernet [4]. A screenshot of IPMIView can be seen in Figure 15. Most of the programs found online, however, aren't compatible with Windows, so it is desirable to use another operating system like Linux to monitor these variables. Figure 16 displays a screenshot of OpenIPMI while Figure 17 displays five different screenshots of IPMItool.

\section{Conclusion:}

This project not only accomplished the objective of designing such a system, but it also portrayed the general process of accomplishing such a task from scratch.

Upon designing a system, it was not only important to find the right chips, but it was also important to determine how each device was connected to one another since IPMI is a standard. By researching that necessary information, it gave me an idea of what chips could be used.

This project also allowed for open collaboration among colleagues as it posed numerous problems during the research and design processes. Several aspects of the project were difficult to solve at first, but the help of fellow workers made the problem much easier to solve. 


\section{Acknowledgments:}

First and foremost, I would like to thank the U.S. Department of Energy, Office of Science, and the Science Undergraduate Laboratory Internship (SULI) program for funding this wonderful research experience at the Stanford Linear Accelerator Center (SLAC). A special thanks goes to Susie Zheng for allowing me to use her office space to conduct my research as well as guiding me during the entire course of the project.

Additional thanks goes to my mentor Ronald Johnson for his time and effort into helping me with the study of IPMI. I would also like to thank my fellow workers for creating a friendly working atmosphere around my office space: Jeff Olsen, Andrew Young, Dave Anderson, Dave Brown, John Dusatko, Kazuko Onaga, Evgeny Medvedko, Chuck Yee, Vernon Smith, Deborah Lilly, and Kenneth Leung. I would finally like to thank the following for running the SULI program: Program Director Stephen Rock, Residence Assistant Howard Young, Program Manager SueVon Gee, and Program Administrators Vivian Lee and Elizabeth Smith. 


\section{References:}

[1]: Intel, Hewlett-Packard, NEC, and Dell Computer Co. Intelligent Platform Management Interface Specification Second Generation, V 2.0, Feb. 2004.

[2]: “Intelligent Platform Management Interface,” [Online Document], [2009 Jul 7], Available at HTTP: http://en.wikipedia.org/wiki/IPMI

[3]: K. Dua, “Intelligent Platform Management,” [Online Document] (Oct. 2005), [2009 Jul 6], Available at HTTP: http://pcquest.ciol.com/content/search/showarticle.asp?artid=75814

[4]: A. Singh, D. Holmgren, R. Rechenmacher, and S. Epsteyn, “Tools and Techniques for Managing Clusters for SciDAC Lattice QCD at Fermilab,” Computing in High Energy and Nuclear Physics, Mar., pp. 24-28, 2003.

[5]: “IPMI Management,” [Online Document] (2009), [2009 Jul 7], Available at HTTP: http://www.opengear.com/SP-IPMI.html

[6]: B. Suppanz, “Intelligent Platform Starts with Intelligent Power,” [Online Document] (Jul. 2006), Available at HTTP: http://www.powerone.com/technical/articles/IPMI.pdf

[7]: Y. Fang, G. Kochhar, and R. DeRoeck, “High-Performance Computing Clusters with IPMI,” Dell Power Solutions, Oct., pp. 58-63, 2004.

[8]: H. Zhou, J. Yin, and A. Rao, “Remote Management with the Baseboard Management Controller in Eighth-Generation Dell PowerEdge Servers,” Dell Power Solutions, Oct., pp. 26-29, 2004. 
[9]: “Actel Fusion FPGAs Supporting Intelligent Peripheral Management Interface (IPMI) Applications,” [Online Document] (Oct. 2006), Available at HTTP: http://www.actel.com/documents/Fusion_IPMI_AN.pdf 


\section{Figures:}

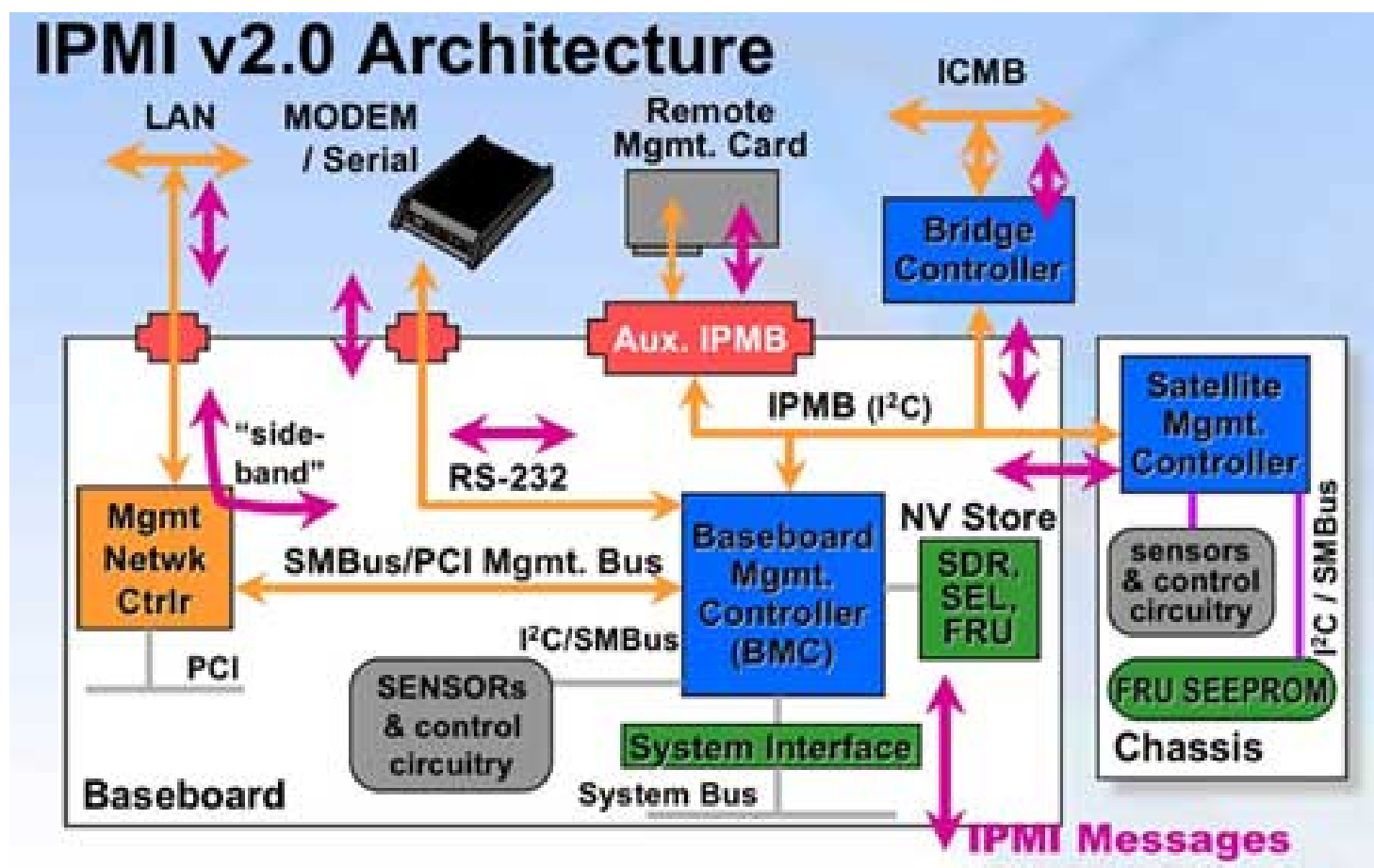

Figure 1: A block diagram of a system that utilizes IPMI. The Baseboard Management Controller (BMC) acts as the heart of this system because it interacts with every other device. The bridge controller was not included in the design because the objective is to work with just one chassis. This design is typically used on computer motherboards, so the actual design of the system in question may vary. 


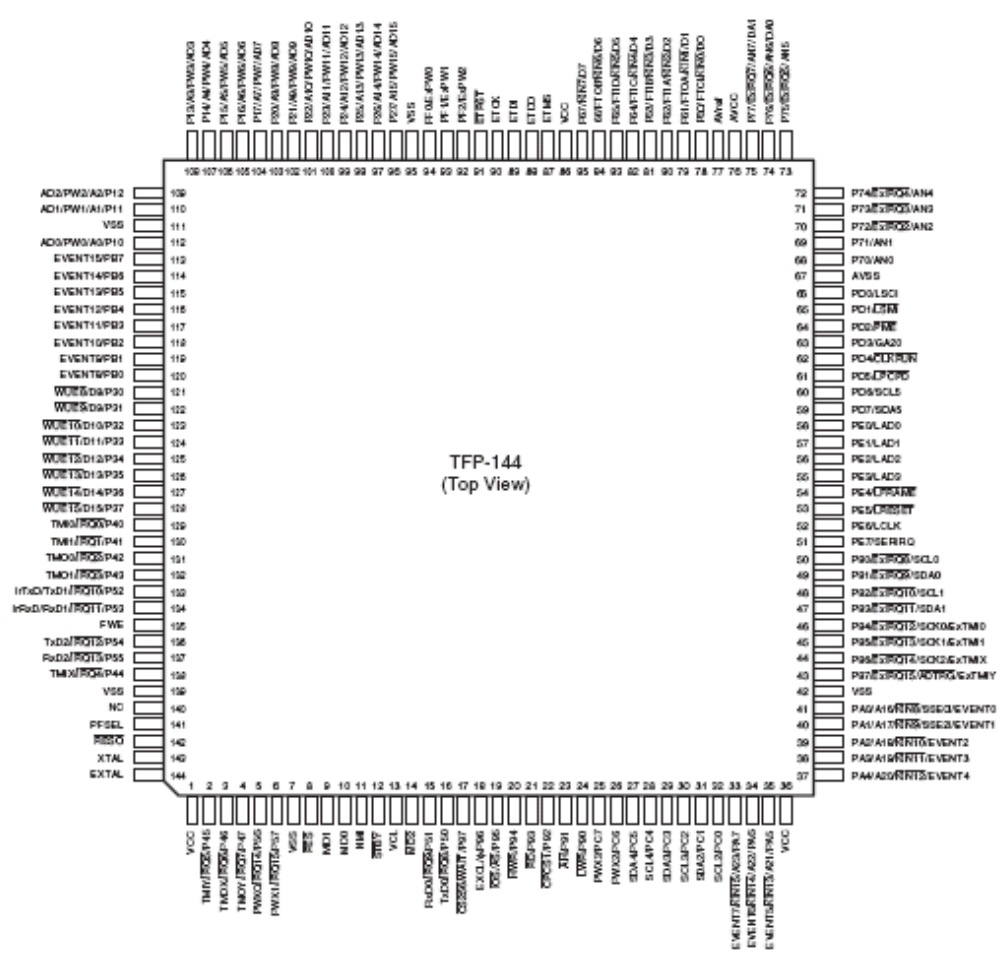

Figure 2: Pinout information of the Renesas H8S/2167 BMC. The microcontroller has a total of six $\mathrm{I}^{2} \mathrm{C}$ ports and three ports for receiving and transmitting data.

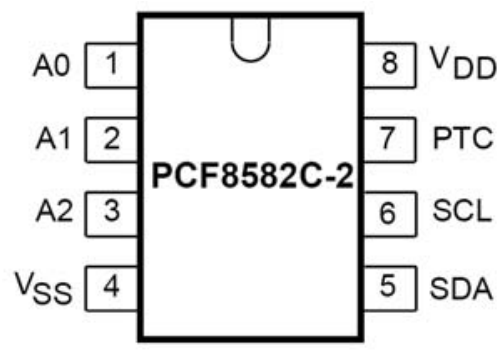

Figure 3: Phillips PCF8582C-2 Non-Volatile Storage

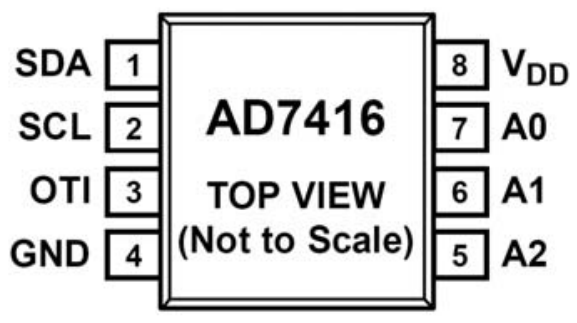

Figure 4: AD7416 Board Temperature Sensor 


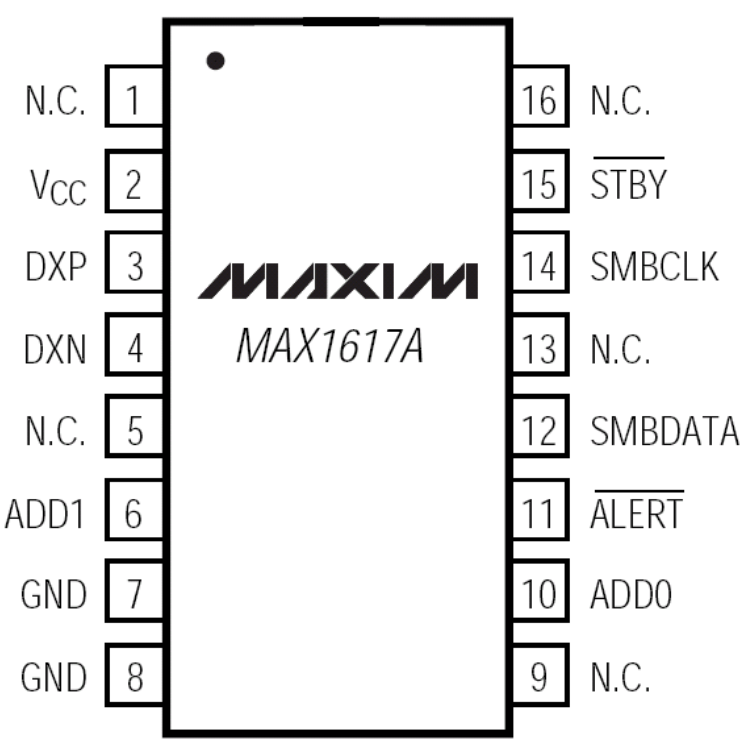

Figure 5: MAX1617A Remote Diode Temperature Sensor

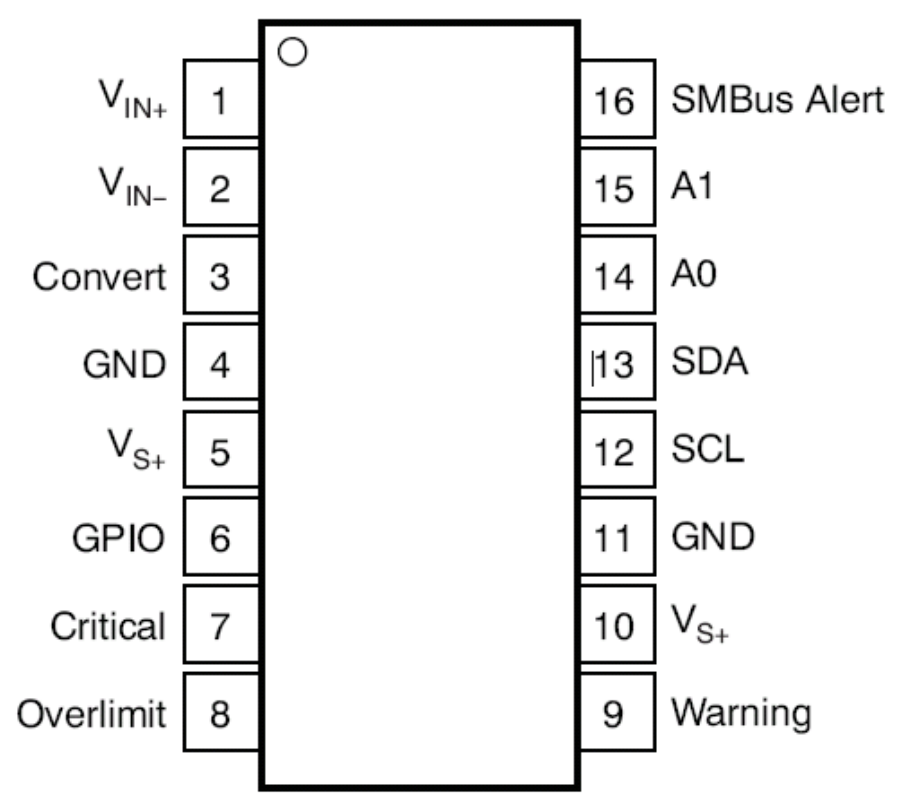

Figure 6: Texas Instruments INA209EVM Voltage Sensor 


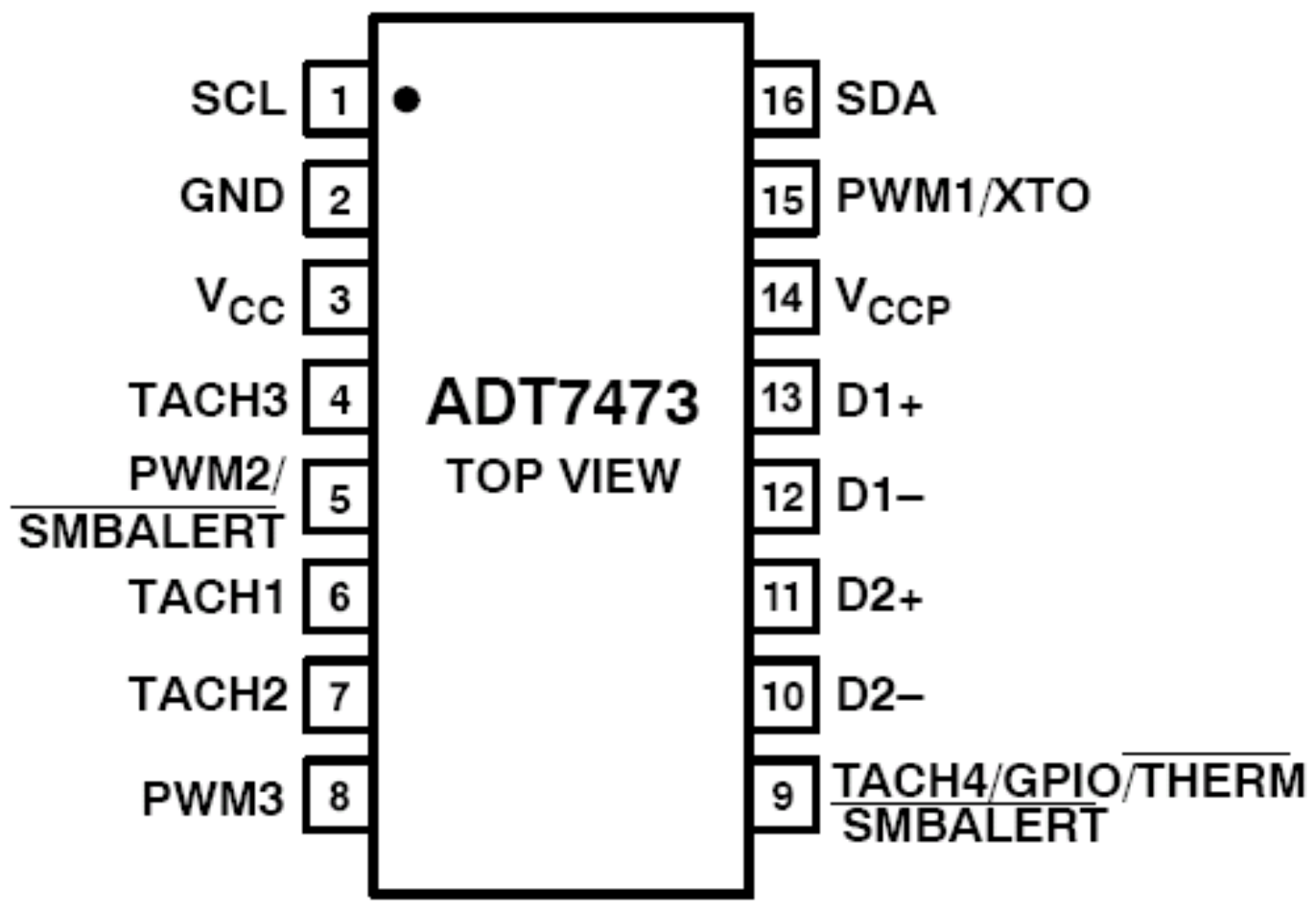

Figure 7: ADT7473 Fan Sensor

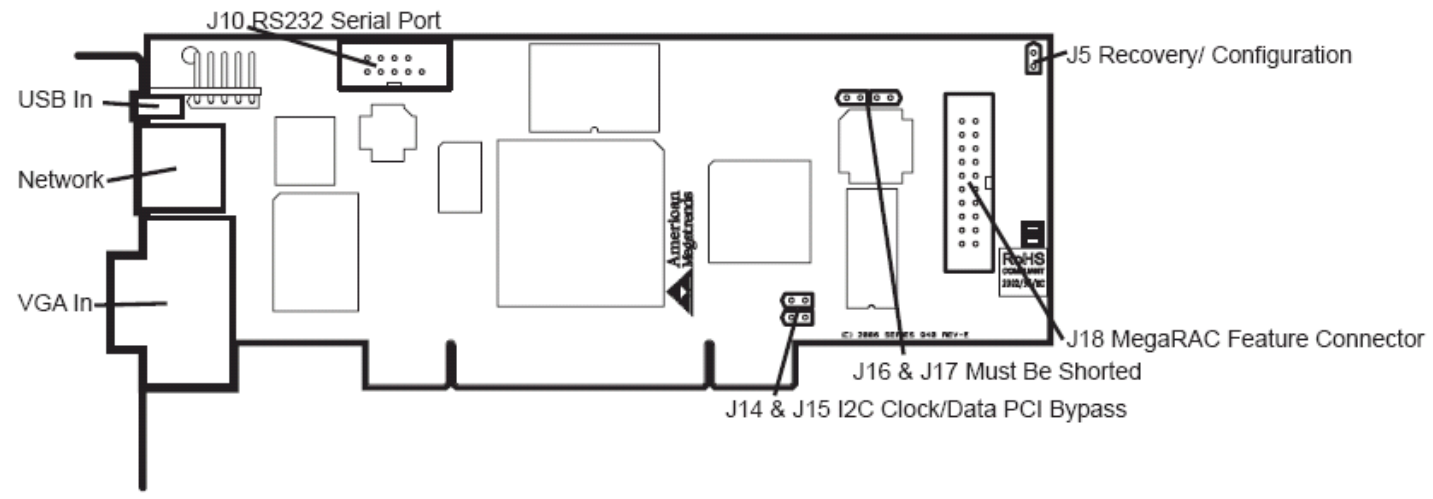

Figure 8: MegaRAC G4 Remote Management Card 


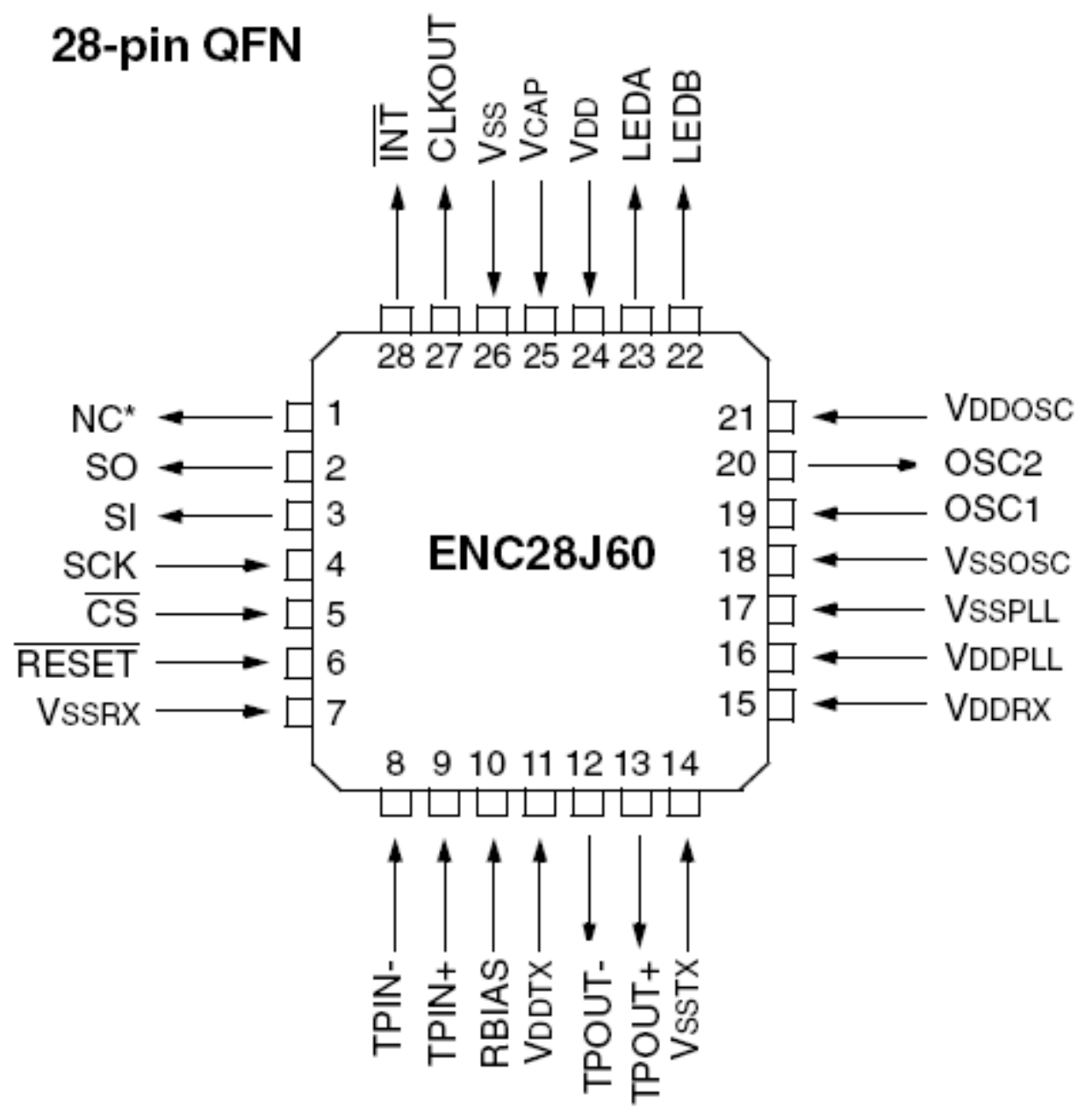

Figure 9: Microchip ENC28J60 Ethernet Controller 


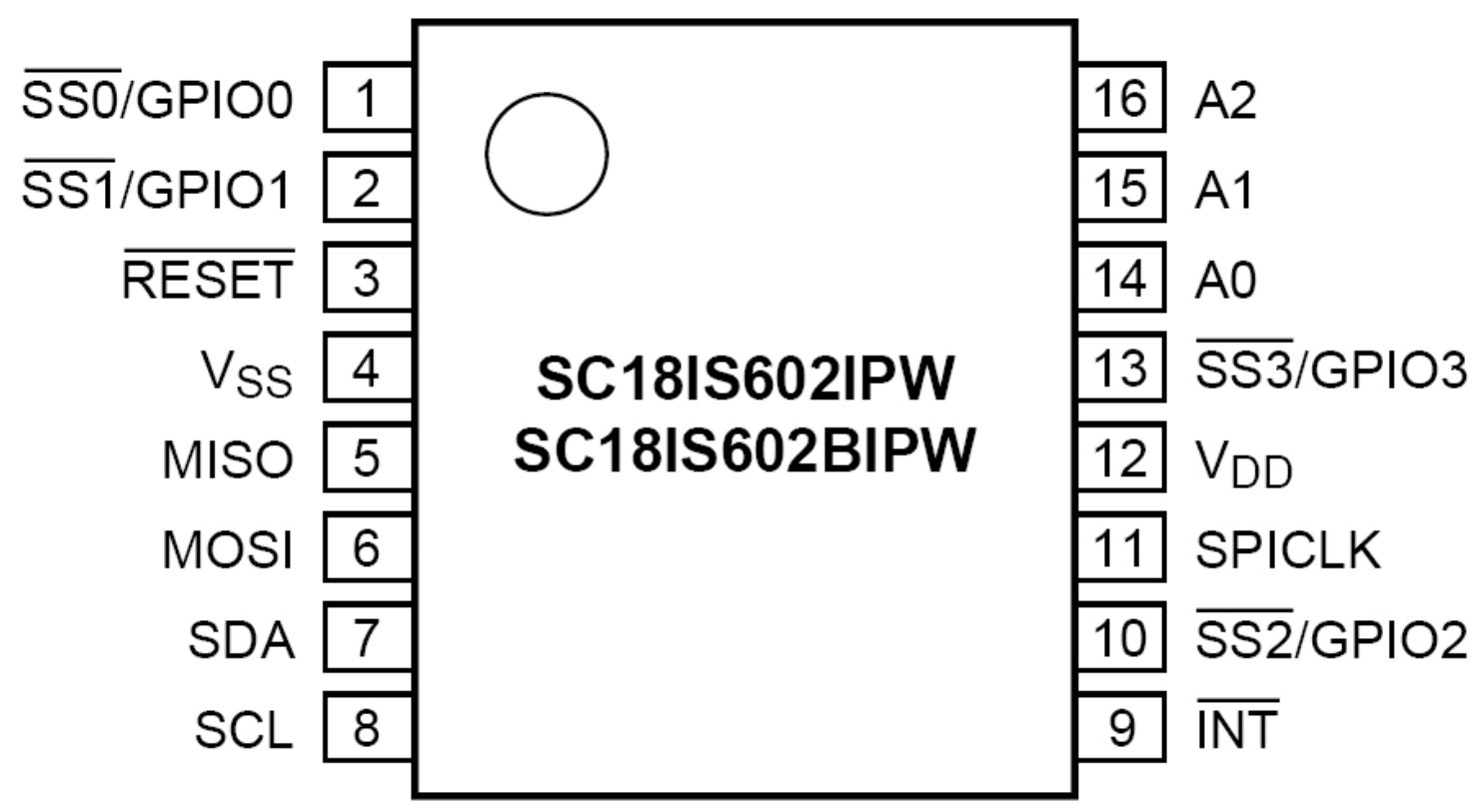

Figure 10: NXP SC18IS602IPW I²C-bus to SPI Bridge

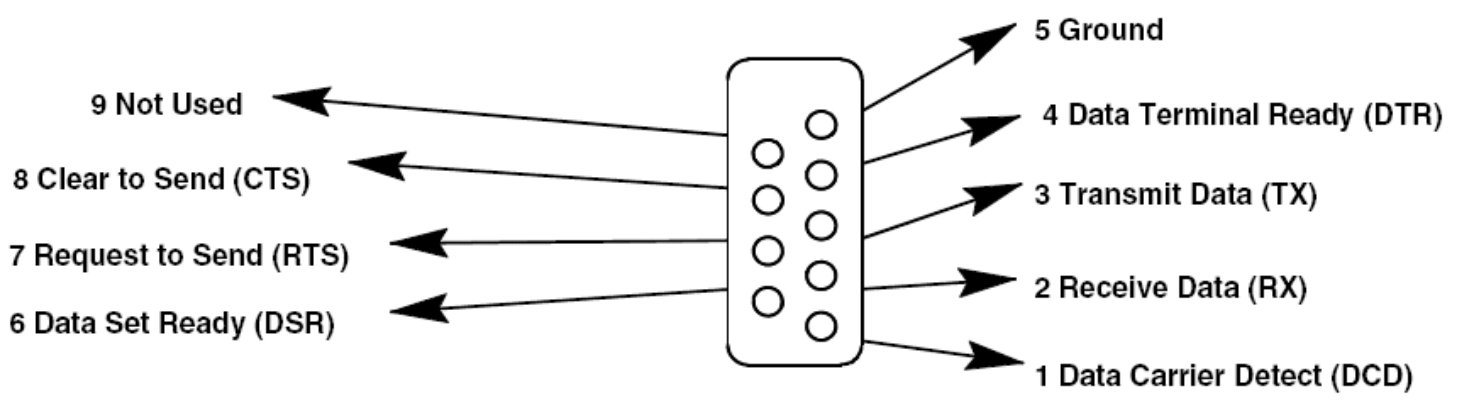

Figure 11: HN-210 Serial/Modem Cable 


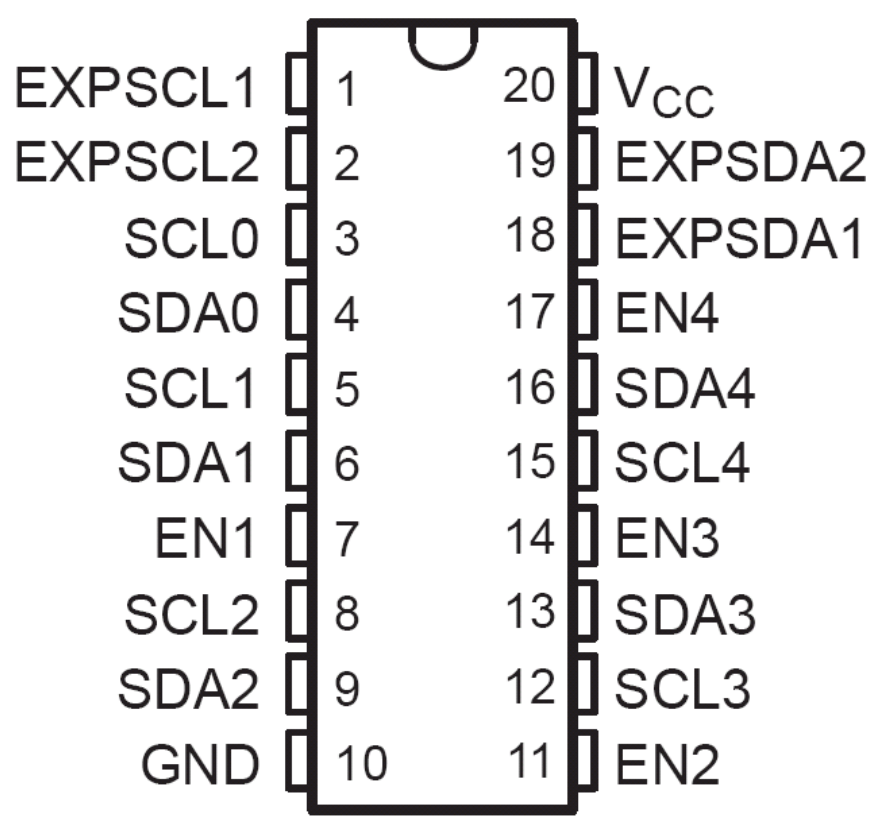

Figure 12: PCA9518 I² C Hub

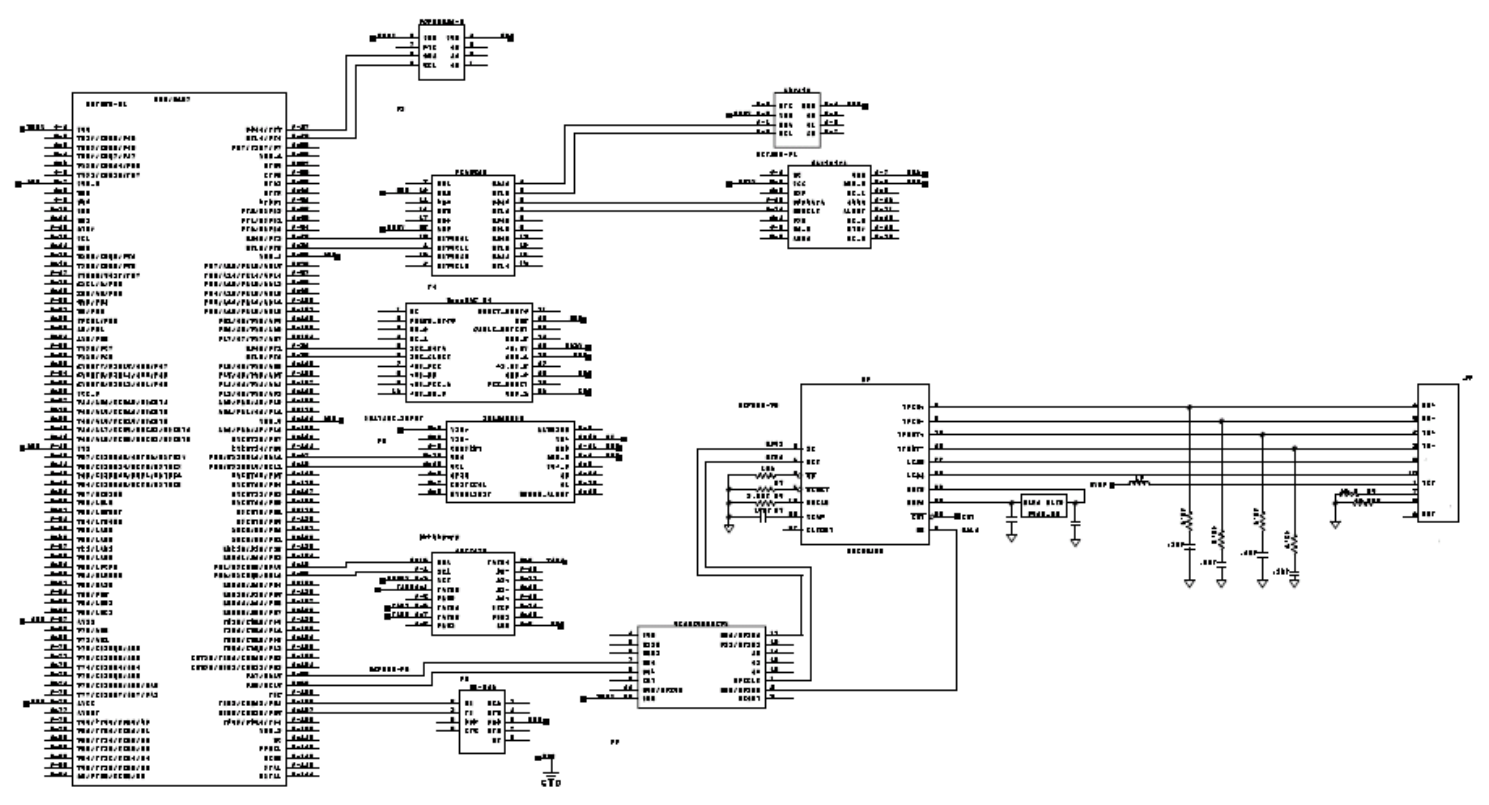

Figure 13: Block Diagram of System Design. Created using DxDesigner 


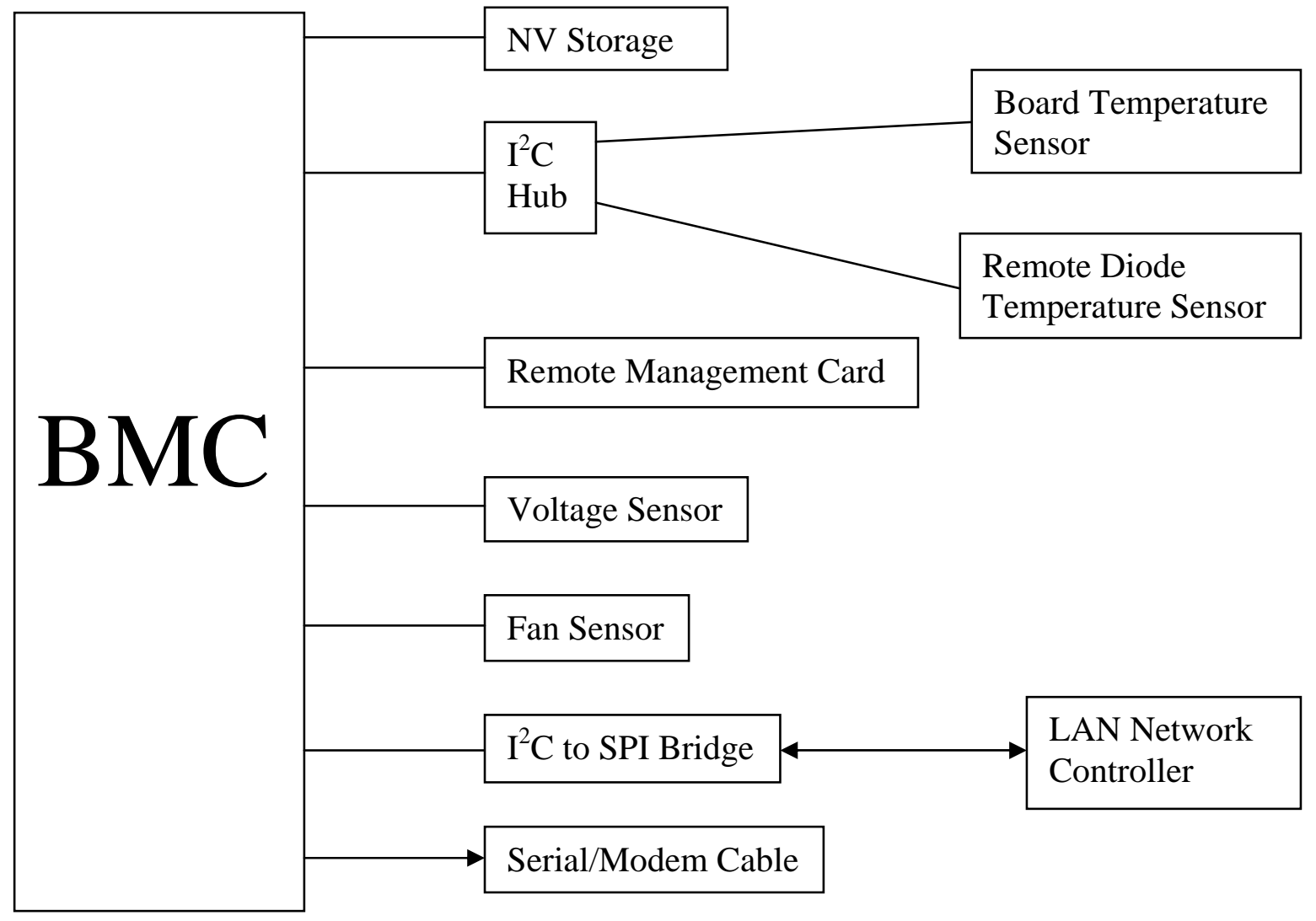

Figure 14: Simplified block diagram of Figure 13. In this diagram, segments depict $\mathrm{I}^{2} \mathrm{C}$ connections, rays depict RS-232 connections, and lines represent SPI connections. 


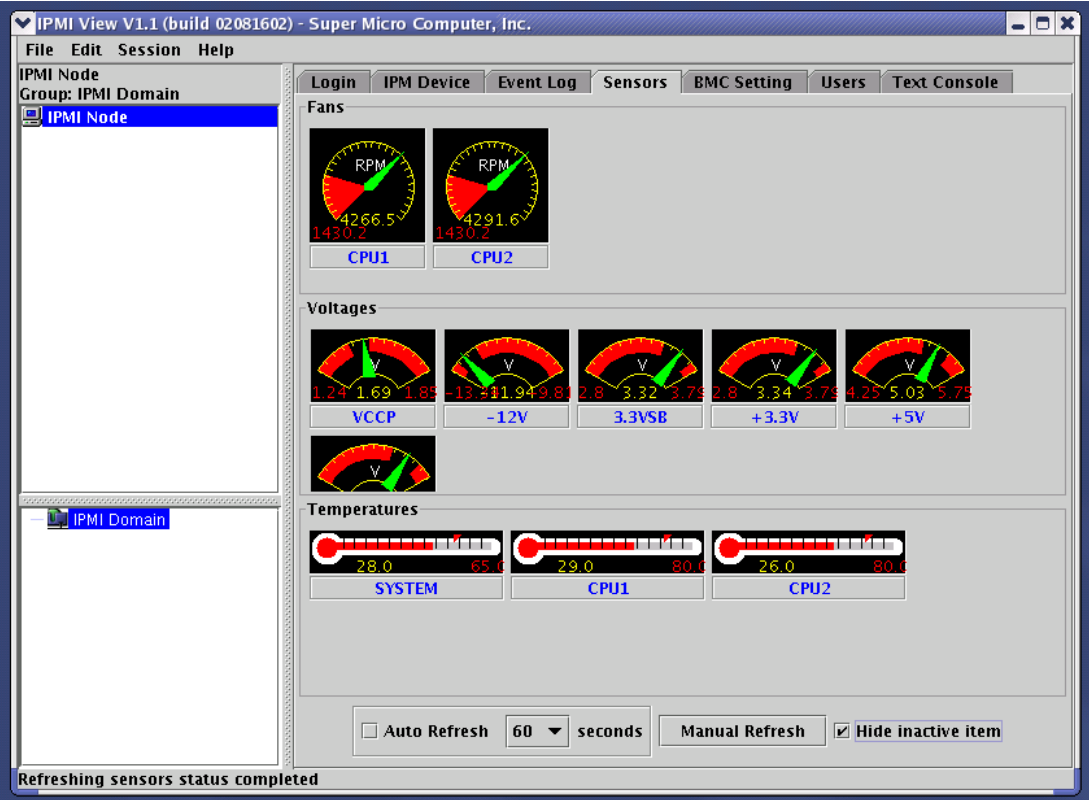

Figure 15: Screenshot of IPMIView

\begin{tabular}{|c|c|c|c|c|}
\hline \multicolumn{2}{|l|}{ Sensor } & \multicolumn{2}{|l|}{ Status } & \multirow[t]{2}{*}{ Reading } \\
\hline \multirow{11}{*}{$\begin{array}{l}\oplus \underset{G}{G} \\
\square\end{array}$} & \multirow{2}{*}{$\begin{array}{l}\text { IBM BladeCenter LS21-[7971AC1] - } \\
\text { Processors }\end{array}$} & \multirow{2}{*}{$\begin{array}{l}000 \\
000\end{array}$} & Normal & \\
\hline & & & \multicolumn{2}{|l|}{ Normal } \\
\hline & Board 1: Package 1 & 000 & \multicolumn{2}{|l|}{ Normal } \\
\hline & Board 1: Package 2 & 000 & \multicolumn{2}{|l|}{ Normal } \\
\hline & Board 1: Package 1 Level-1 C... & 000 & \multicolumn{2}{|l|}{ Normal } \\
\hline & Board 1: Package 1 Level-2 C... & 000 & \multicolumn{2}{|l|}{ Normal } \\
\hline & Board 1: Package 1 Level-3 C... & 000 & \multicolumn{2}{|l|}{ Normal } \\
\hline & Board 1: Package 2 Level-1 C... & 000 & \multicolumn{2}{|l|}{ Normal } \\
\hline & Board 1: Package 2 Level-2 C... & 000 & \multicolumn{2}{|l|}{ Normal } \\
\hline & Board 1: Package 2 Level-3 C... & 000 & \multicolumn{2}{|l|}{ Normal } \\
\hline & Memory & 000 & \multicolumn{2}{|l|}{ Normal } \\
\hline & (1) Temperature & 000 & \multicolumn{2}{|l|}{ Normal } \\
\hline & (1) AvgPwrIns 2 for Power Distribut... & 000 & Normal & OWatts \\
\hline & AvgPwrIns 1 for Power Distribut... & 000 & Normal & 1280 Watts \\
\hline & CPU2 TEMP for Processor 2 & 000 & Normal & 26 Degrees C \\
\hline & BANK2 TEMP for Memory Mod... & 000 & Normal & 24 Degrees C \\
\hline & BANK1 TEMP for Memory Mod... & 000 & Normal & 34 Degrees C \\
\hline & 1] CPU1 TEMP for Processor 1 & 000 & Normal & $O$ Degrees $\mathrm{C}$ \\
\hline \multicolumn{2}{|r|}{$\square>$ Other } & 000 & \multicolumn{2}{|l|}{ Normal } \\
\hline & 1 SEL fullness for System Manag... & 000 & Normal & 1 Percentage \\
\hline \multicolumn{2}{|r|}{$\square$ \& Voltage } & 000 & \multicolumn{2}{|l|}{ Normal } \\
\hline & \& Planar VBAT for Battery 1 & 000 & Normal & $3.05 \mathrm{Volts}$ \\
\hline & IV $12 \mathrm{~V}$ Sense for System Board 3 & 000 & Normal & 12.12 Volts \\
\hline & 35 V Sense for System Board 2 & 000 & Normal & 5.04 Volts \\
\hline & $33.3 v$ Sense for System Board 1 & 000 & Normal & 3.31 Volts \\
\hline \multirow[t]{4}{*}{$\boxminus$} & Software Components & 000 & \multicolumn{2}{|l|}{ Normal } \\
\hline & D VMware Inc. Hypervisor VMwa... & 000 & Normal & \\
\hline & IBM System BIOS-[BAE149AU... & 000 & Normal & \\
\hline & 1 System Management Software... & 000 & Normal & \\
\hline & 岁 Power & 000 & Unknown & \\
\hline & 岁 Power Distribution 1: Not Insta... & 000 & Normal & o Watts \\
\hline & Power Distribution 2: Not Insta... & 000 & Unknown & OWatts \\
\hline
\end{tabular}

Figure 16: Screenshot of OpenIPMI 


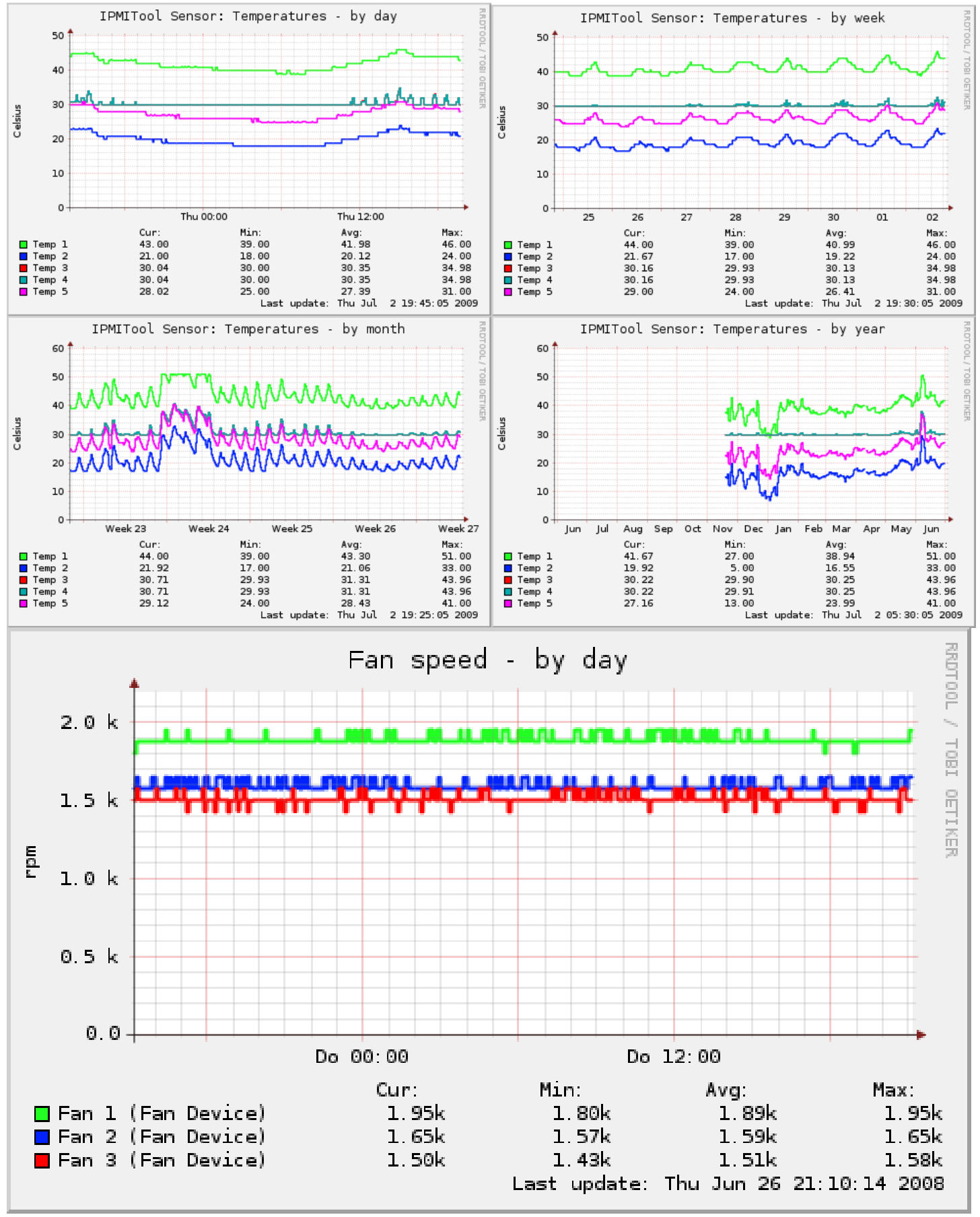

Figure 17: Five different screenshots of IPMItool 